\title{
SEARCH FOR THE STANDARD MODEL HIGGS BOSON IN ASSOCIATION WITH A W BOSON AT D0 \\ By
}

Savanna Marie Shaw

\section{A DISSERTATION}

Submitted to

Michigan State University

in partial fulfillment of the requirements

for the degree of

Physics - Doctor of Philosophy

2013 


\section{ABSTRACT \\ SEARCH FOR THE STANDARD MODEL HIGGS BOSON IN ASSOCIATION WITH A W BOSON AT D0}

By

\section{Savanna Marie Shaw}

I present a search for the standard model Higgs boson, $H$, produced in association with a $W$ boson in data events containing a charged lepton (electron or muon), missing energy, and two or three jets. The data analysed correspond to $9.7 \mathrm{fb}^{-1}$ of integrated luminosity collected at a center-of-momentum energy of $\sqrt{s}=1.96 \mathrm{TeV}$ with the D0 detector at the Fermilab Tevatron $p \bar{p}$ collider. This search uses algorithms to identify the signature of bottom quark production and multivariate techniques to improve the purity of $H \rightarrow b \bar{b}$ production. We validate our methodology by measuring $W Z$ and $Z Z$ production with $Z \rightarrow b \bar{b}$ and find production rates consistent with the standard model prediction. For a Higgs boson mass of $125 \mathrm{GeV}$, we determine a 95\% C.L. upper limit on the production of a standard model Higgs boson of 4.8 times the standard model Higgs boson production cross section, while the expected limit is 4.7 times the standard model production cross section. I also present a novel method for improving the energy resolution for charged particles within hadronic signatures. This is achieved by replacing the calorimeter energy measurement for charged particles within a hadronic signature with the tracking momentum measurement. This technique leads to a $\sim 20 \%$ improvement in the jet energy resolution, which yields a $\sim 7 \%$ improvement in the reconstructed dijet mass width for $H \rightarrow b \bar{b}$ events. The improved energy calculation leads to a $\sim 5 \%$ improvement in our expected 95\% C.L. upper limit on the Higgs boson production cross section. 


\section{ACKNOWLEDGMENTS}

There are many people to thank for their help throughout my education in physics. First and foremost I would like to thank my thesis advisor, Professor Wade Fisher. Wade's intelligence, patience, and good humour made for a very enjoyable career as a graduate student. I will be forever grateful for all his help and support over the years in everything from answering physics questions, to helping debug code, to editing my thesis, to being the only American I know who can actually make decent jokes about Canada.

An analysis as complex as the $\mathrm{WH}$ analysis presented in this thesis requires the hard work of a team of people so I would also like to thank the members of the D0 WH analysis group with whom I had the pleasure of working alongside for the past few years: Gregorio Bernardi, Mike Cooke, Yuji Enari, Sebastien Greder, Ken Herner, Bob Hirosky, Emily Johnson, Dikai Li, Huong Nguyen, and Lidija Živković.

I would also like to thank Aurelio Juste for his supervision and collaboration of the jet energy resolution studies presented in this thesis. This was a technically challenging project that was never guaranteed to result in any sort of improvement, but Aurelio's seemingly never-ending supply of ideas and enthusiasm made it a very interesting and rewarding project to work on.

Many thanks to the other graduate students and post-docs at D0 and at MSU for their help both with questions relating to physics and questions more related to the peculiarities of American culture.

Last, but certainly not least, I would like to thank my family. Without my parents, I certainly would not be here (literally, and figuratively). I can not thank them enough for their support and encouragement over the years. They really did believe that I could do 
anything I wanted, and as a result, I believed that too. I would also like to thank my little sister for putting up with me for the entirety of her life, and never rubbing it in that she is the far cooler one.

It's impossible to to thank everyone who deserves my gratitude in such a small space, and I have left numerous people out. To all of those who have helped me along the way who aren't listed, please accept my sincere gratitude for your help. 


\section{TABLE OF CONTENTS}

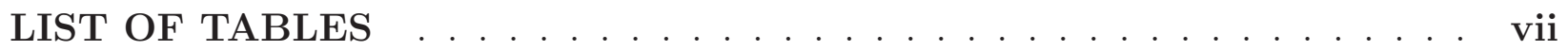

LIST OF FIGURES ...................... . . ix

KEY TO SYMBOLS AND ABBREVIATIONS . . . . . . . . . . . . . xxxii

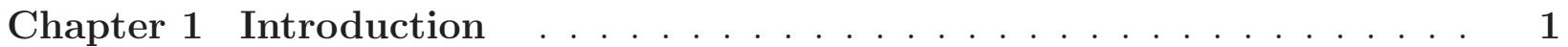

1.1 Standard Model Particles . . . . . . . . . . . . . . . . . . . . . . . . . . . . . . .

1.2 The Standard Model . . . . . . . . . . . . . . . . . . . . . . . . . . . . . . . . . . .

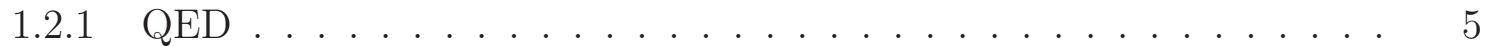

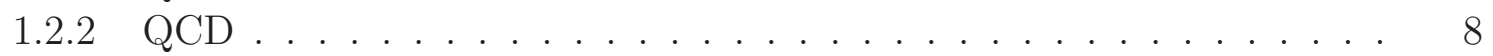

1.2.3 Electroweak Unification and the Higgs Mechanism . . . . . . . . . . . 9

1.2.4 Properties of the Higgs Boson . . . . . . . . . . . . . . . . . . . 17

1.3 Beyond the Standard Model . . . . . . . . . . . . . . . . . . . 20

Chapter 2 Experimental Apparatus . . . . . . . . . . . . . . . 24

2.1 The Tevatron . . . . . . . . . . . . . . . . . . . . . . 24

2.2 The Do Detector . . . . . . . . . . . . . . . . . . . . . . . . . . . . . . . . . . . . .

2.3 Data Acquisition . . . . . . . . . . . . . . . . . . . . . . . . . . . . . . . . . . .

2.4 Data Collection . . . . . . . . . . . . . . . . . 45

Chapter 3 Particle Identification and Reconstruction Algorithms . . . . 46

3.1 Charged Particle Tracks . . . . . . . . . . . . . . . . . . . 46

3.2 Primary Vertices . . . . . . . . . . . . . . . . . . . . . . . . . . . . . . . . . . . . . . . . . .

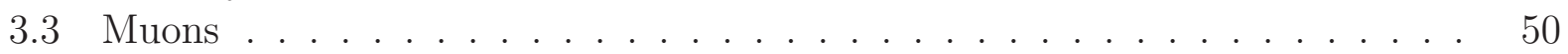

3.4 Electrons and Photons . . . . . . . . . . . . . . . . . . 50

3.5 Jets . . . . . . . . . . . . . . . . . . . . . . 51

3.6 Missing Transverse Energy . . . . . . . . . . . . . . . . . . . . 54

3.7 Bottom Quark Identification . . . . . . . . . . . . . . . . . 55

Chapter 4 Event Simulation _...................... 58

Chapter 5 Statistical Analysis . . . . . . . . . . . . . . . 62

Chapter 6 Data Analysis . . . . . . . . . . . . . . . . 66

6.1 Analysis Strategy . . . . . . . . . . . . . . . . . . 67

6.2 Monte Carlo Simulation . . . . . . . . . . . . . . . . 67 
6.3 Multijet Background Estimation . . . . . . . . . . . . . . . . 72

6.4 Selection . . . . . . . . . . . . . . . . . . 76

6.5 b Tagging . . . . . . . . . . . . . . . . . . . . . . . 81

6.6 Multivariate Classification . . . . . . . . . . . . . . . . . . . 92

6.7 Systematic Uncertainties . . . . . . . . . . . . . . . . . . . . . . . 95

6.8 Diboson $(V Z)$ Production With $Z \rightarrow b \bar{b} \ldots \ldots \ldots \ldots$

6.9 Upper Limits on Higgs Boson Production . . . . . . . . . . . . . . . . . . . 108

6.10 D0 and Tevatron Higgs Boson Searches . . . . . . . . . . . . . . . . 113

6.11 Future Studies of the Higgs Boson . . . . . . . . . . . . . . . . . 117

Chapter 7 Improvements to Jet Energy Resolution . . . . . . . . . . . . . . 118

7.1 Track-Cal Jet Algorithm . . . . . . . . . . . . . . . . . . . . 119

7.2 Energy Resolution Improvement . . . . . . . . . . . . . . . . . . . . . . . . . . . . . . . . . . . . . . . 132

7.3 Improvement in Higgs Boson Analysis . . . . . . . . . . . . . . . . . . . . . . 132

Chapter 8 Conclusion . . . . . . . . . . . . . . . . . 143

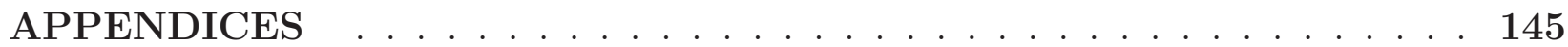

Appendix A Multijet MVA Input Distributions . . . . . . . . . . . . . . . 146

Appendix B Final BDT Input and Output Distributions . . . . . . . . . . . . . . 153

BIBLIOGRAPHY . . . . . . . . . . . . . . . . . . . . 238 


\section{LIST OF TABLES}

Table 1.1 The three generations of Fermions [1] . . . . . . . . . . . . 4

Table 1.2 The force carrying bosons $[1] \ldots \ldots \ldots$. . . . . . . . . . 4

Table 6.1 The cross section times branching fraction for diboson and top-quark simulated processes. . . . . . . . . . . . . . . .

Table 6.2 The cross section times branching fraction for $W+$ jets simulated processes. . . . . . . . . . . . . . . . .

Table 6.3 The cross section times branching fraction for $Z+$ jets simulated processes. . . . . . . . . . . . . . . . . . 70

Table 6.4 Observed number of events in data and expected number of events from each signal and background source (where $V=W, Z$ ) for events with a muon and exactly two jets. The expected signal is quoted at $M_{H}=125 \mathrm{GeV}$. The total background uncertainty includes all sources of systematic uncertainty added in quadrature. . . . . . . .

Table 6.5 Observed number of events in data and expected number of events from each signal and background source (where $V=W, Z$ ) for events with an electron and exactly two jets. The expected signal is quoted at $M_{H}=125 \mathrm{GeV}$. The total background uncertainty includes all sources of systematic uncertainty added in quadrature. . . . . . . .

Table 6.6 Observed number of events in data and expected number of events from each signal and background source (where $V=W, Z$ ) for events with a muon and exactly three jets. The expected signal is quoted at $M_{H}=125 \mathrm{GeV}$. The total background uncertainty includes all sources of systematic uncertainty added in quadrature. . . . . . .

Table 6.7 Observed number of events in data and expected number of events from each signal and background source (where $V=W, Z$ ) for events with an electron and exactly three jets. The expected signal is quoted at $M_{H}=125 \mathrm{GeV}$. The total background uncertainty includes all sources of systematic uncertainty added in quadrature. . . . . . . . 86 
Table 6.8 Input variables for the $\operatorname{MVA}_{\mathrm{MJ}}(V H)$ discriminant, which was trained using $V H \rightarrow \ell \nu b \bar{b}$ events as a signal. Variables are ranked by their importance in the BDT (which is based on how often they are used in the training $[2] \ldots \ldots \ldots \ldots \ldots \ldots$

Table 6.9 Table of input variables for the final signal discriminant. Variables are ranked by their importance in the BDT (which is based on how often they are used in the training) in the two tight $b$-tagged (2T), two medium $b$-tagged $(2 \mathrm{M})$, two loose $b$-tagged $(2 \mathrm{~L})$, and one tight $b$-tagged $(1 \mathrm{~T})$ categories $[2] . \ldots \ldots \ldots \ldots$

Table 6.10 The expected and observed 95\% C.L. limits, as a function of the Higgs boson mass $M_{H}$, presented as ratios of production cross section times branching fraction to the SM prediction $[2] \ldots \ldots \ldots \ldots$. . . . . 113 


\section{LIST OF FIGURES}

Figure 1.1 For the interpretation of the references to colour in this and all other figures, the reader is referred to the electronic version of this dissertation. A global fit to data from precision electroweak experiments can place constraints on the $W$ boson and top quark masses. Shown here are the $68 \%$ and $95 \%$ contours from this fit including the Higgs boson mass measurement in blue, and not including the Higgs boson mass measurement in gray. The horizontal and vertical green bands correspond to 1 standard deviation on the $W$ boson and top quark masses respectively. The diagonal lines show where different values for the Higgs mass would appear in the $W$-top mass plane [3]. . . .

Figure 1.2 The masses of the top quark, $W$ boson, and Higgs boson are related through loop diagrams. . . . . . . . . . . . . . . . .

Figure $1.395 \%$ confidence level upper limit on Higgs boson production cross section as a ratio to the Standard Model cross section from the ATLAS experiment (top) [4] and the CMS experiment (bottom) [5]. Masses up to $500 \mathrm{GeV}$ are excluded, with the exception of a small window

of masses centred around $125 \mathrm{GeV}$. . . . . . . . . . . . . . .

Figure 1.4 The production cross section for the Higgs boson as a function of Higgs boson mass at the Tevatron (where the center-of-momentum energy, $\sqrt{s}$, is $1.96 \mathrm{TeV}) . \ldots \ldots \ldots$

Figure 1.5 The decay rates for the Higgs boson as a function of Higgs boson mass. 22

Figure 1.6 The Feynman diagrams for the most important Higgs boson production modes at the Tevatron: (a) gluon-gluon fusion, (b) associated production of a Higgs boson with a pair of top quarks, and (c) vector boson fusion. . . . . . . . . . . . . . . . . . 22

Figure 2.1 A schematic view of the accelerator chain at Fermilab. . . . . . . . 25

Figure 2.2 A cross sectional view of the D0 detector, showing the tracking system surrounded by the calorimeter and the muon detection system [6]. . 
Figure 2.3 A cross sectional view of the D0 tracking system. Closest to the beampipe is the silicon microstrip tracker, surrounded by the central fibre tracker. The tracking system is contained within a superconducting solenoid $[6] \ldots \ldots \ldots \ldots \ldots \ldots$

Figure 2.4 A view of the SMT . . . . . . . . . . . .

Figure 2.5 The layout of the CFT, showing the fibre layers. . . . . . . . . . 32

Figure 2.6 A diagram showing a cut away view of the D0 calorimeter. The calorimeter is comprised of three pieces: a central calorimeter and two end calorimeters. Each piece is made up of several layers of absorber plates (made of Uranium or copper or steel) inserted in liquid argon. These layers form the electromagnetic, the fine hadronic, and the coarse hadronic calorimeters $[6] . \ldots \ldots \ldots \ldots$

Figure 2.7 A diagram of a calorimeter cell. Each cell consists of an absorber plate, the liquid argon, and a plate to read out the charge. . . . . .

Figure 2.8 The calorimeter cells are arranged in towers along lines of constant $\eta$, illustrated by the alternating shaded areas. . . . . . . . . . . .

Figure 2.9 A cross sectional view of the D0 preshower detectors located between the solenoid and the calorimeters. The preshower detectors are comprised of interleaved scintillator strips with a wavelength shifting fibre at the center $[6] \ldots \ldots \ldots \ldots \ldots \ldots \ldots$

Figure 2.10 An exploded view of the arrangement of the drift tubes in the muon system. . . . . . . . . . . . . . . . . .

Figure 2.11 A schematic view of the flow of data through the D0 trigger system [6]. 41

Figure 2.12 A schematic view of the relationship between the different trigger components and the different detector subsystems $[6] . \ldots . .$.

Figure 3.1 The HTF method for a single $1.5 \mathrm{GeV}$ track with 5 hits. (a) Some of the possible trajectories for a single hit in the x-y plane, (b) translation of a single hit into $\rho-\phi$ space, (c) multiple hits from the same track form lines that intersect at a single point, and (d) the intersection point as a peak in the $\rho-\phi$ histogram. . . . . . . 
Figure 3.2 The b-tagging efficiency (in black) and rate for light jets to be mistagged (in red) as a function of the neural network output for jets with $p_{T}>30 \mathrm{GeV}$. The vertical blue lines indicate points on the neural network output where the efficiencies in simulation have been corrected to match the efficiencies in data. . . . . . . . . . . . . . . .

Figure 6.1 Example Feynman diagrams for processes we consider as backgrounds: $W+$ jets (top left), top quark pair production (top right), $W Z$ production (bottom left), and multijet production (bottom right). . . . 68

Figure 6.2 Feynman diagrams for processes contributing to our Higgs boson signal: associated production of a Higgs with a $\mathrm{W}$ boson, with the Higgs boson decaying to a pair of $b$-quarks (left); $g g \rightarrow H \rightarrow W W$ (middle), and associated production of a Higgs boson with a $W$ boson, with the Higgs boson decaying to a pair of $W$ bosons (right). . . . . . .

Figure 6.3 Reweighting functions to correct for mismodelling in the ALPGEN $\mathrm{V}+$ jets $\mathrm{MC}$ for the second leading jet $\eta$ (top left), leading jet $\eta$ (top right), $W p_{T}$ (middle left), $\Delta R\left(j_{1}, j_{2}\right)$ (middle right), and lepton $\eta$ (bottom). The black points are the data with all non- $\mathrm{V}+$ jets backgrounds subtracted off. The black curves are the reweighting functions fit to the points, and the blue and red are the \pm 1 standard deviations on the black curve. . . . . . . . . . . . . . . . . .

Figure 6.4 Example of the probability for a jet that passes the loose lepton identification requirements to pass the tight lepton isolation requirements for the $15<p_{T}<17 \mathrm{GeV}$ range with $0.4<\min \left[\Delta \phi\left(E_{T}\right.\right.$, jet $\left.)\right]<\pi$ as a function of the electron $\eta$ in the (a) CC and (b) EC. The solid line is a fit to the data, and the dashed lines are the functions with the parameters shifted up and down by their uncertainties.

Figure 6.5 Example of the probability for a jet that passes the loose lepton identification requirements to pass the tight lepton isolation requirements as a function of muon $p_{T}$ for (a) $|\eta|<1.0$ and (b) $1.0<|\eta|<1.6$, with $0<\Delta \phi\left(E_{T}, \mu\right)<1$. The solid line is a fit to the data, and the dashed lines are the functions with the parameters shifted up and down by their uncertainties. 
Figure 6.6 Muon trigger correction, derived from data to account for the gain in efficiency when moving from single muon and muon+jets triggers to inclusive triggers. The corrections are parametrized as a function $H_{T}$, shown for events with muon $|\eta|<1.0$ with a) $E_{T}<50 \mathrm{GeV}$ and b) $E_{T} \geq 50 \mathrm{GeV}$. The black circles represent the correction when the muon passes through the detector support region $(-2<\phi<-1.2)$, and the red triangles represent the correction in all other regions of $\phi[2] \ldots \ldots \ldots \ldots \ldots \ldots \ldots$

Figure 6.7 The average b-jet identification output for events with two jets. We define three categories containing two b-tagged jets based on this average output. The signal is shown for a Higgs boson mass of $125 \mathrm{GeV}$, scaled by a factor of $50[2] \ldots \ldots \ldots \ldots$

Figure 6.8 Distributions for all selected events with two jets of (a) transverse mass of the lepton- $\mathbb{E}_{T}$ system, and (b) charged lepton $p_{T}$. The signal is multiplied by 1000. Overflow events are added to the last bin [2].

Figure 6.9 Distributions for all selected events with two jets of (a) leading jet $p_{T}$, and (b) second-leading jet $p_{T}$. The signal is multiplied by 1000 .

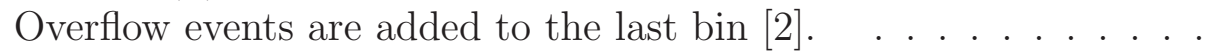

Figure 6.10 Distributions for all selected events with two jets of (a) $E_{T}$, and (b) $\Delta R$ between the leading and second-leading jets. The signal is multiplied by 1000. Overflow events are added to the last bin [2].

Figure 6.11 Invariant mass of the leading and second-leading jets in events with two jets and (a) one tight $b$-tag, and (b) two loose $b$-tags. The signal is multiplied by 200. Overflow events are added to the last bin [2]. .

Figure 6.12 Invariant mass of the leading and second-leading jets in events with two jets and (a) two medium $b$-tags, and (b) two tight $b$-tags. The signal is multiplied by 50. Overflow events are added to the last bin $[2] \ldots \ldots \ldots \ldots \ldots \ldots \ldots$

Figure 6.13 The output distribution for the Higgs boson signal vs multijet discriminant shown for all events containing exactly two jets. The signal for $M_{H}=125 \mathrm{GeV}$ is scaled by a factor of $1000[2]$. . . . . . . . .

Figure 6.14 Distributions of some of the most significant inputs to the final discriminant in events with exactly two jets: (a) $p_{T}^{W} /\left(p_{T}^{\ell}+E_{T}\right)$, shown for events with one tight $b$-tag; (b) $\max \mid \Delta \eta\left(\ell,\left\{j_{1}\right.\right.$ or $\left.\left.j_{2}\right\}\right) \mid$, shown for events with two loose $b$-tags. The $M_{H}=125 \mathrm{GeV}$ signal is multiplied by 200. Overflow events are added to the last bin [2]. . . . . . . . . 97 
Figure 6.15 Distributions of some of the most significant inputs to the final discriminant in events with exactly two jets: (a) $q^{\ell} \times \eta^{\ell}$, shown for events with two medium $b$-tags; (b) $\sum\left(p_{T}\right)^{V I S}$, shown for events with two tight $b$-tags. The $M_{H}=125 \mathrm{GeV}$ signal is multiplied by 50 . Overflow

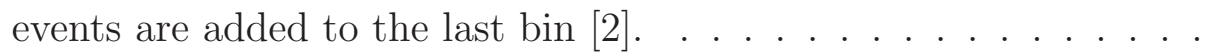

Figure 6.16 Distributions of some of the most significant inputs to the final discriminant in events with exactly three jets: (a) $\max \mid \Delta \eta\left(\ell,\left\{j_{1}\right.\right.$ or $\left.\left.j_{2}\right\}\right) \mid$, shown for events with one tight $b$-tag; (b) $q^{\ell} \times \eta^{\ell}$, shown for events with two loose $b$-tags. The $M_{H}=125 \mathrm{GeV}$ signal is multiplied by 200. Overflow events are added to the last bin $[2]$. . . . . . . . . .

Figure 6.17 Distributions of some of the most significant inputs to the final discriminant in events with exactly three jets: (a) aplanarity, shown for events with two medium $b$-tags; (b) $m_{\ell \nu j_{2}}$, shown for events with two tight $b$-tags. The $M_{H}=125 \mathrm{GeV}$ signal is multiplied by 50 . Overflow

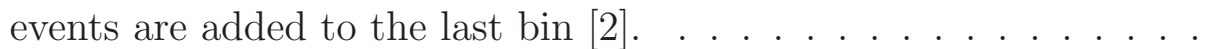

Figure 6.18 Distributions of the final discriminant output, after the maximum likelihood fit (described in Ch. ), in events with exactly two jets and: (a) one tight $b$-tag, and (b) two loose $b$-tags. The $M_{H}=125 \mathrm{GeV}$

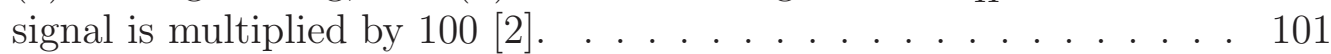

Figure 6.19 Distributions of the final discriminant output, after the maximum likelihood fit (described in Ch. ), in events with exactly two jets and: (a) two medium $b$-tags, and (b) two tight $b$-tags. The $M_{H}=125 \mathrm{GeV}$ signal is multiplied by $20[2]$.

Figure 6.20 Distributions of the final discriminant output, after the maximum likelihood fit (described in Ch. ), in events with exactly three jets and: (a) one tight $b$-tag, and (b) two loose $b$-tags. The $M_{H}=125 \mathrm{GeV}$

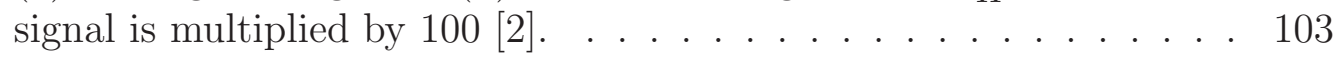

Figure 6.21 Distributions of the final discriminant output, after the maximum likelihood fit (described in Ch. ), in events with exactly three jets and: (a) two medium $b$-tags, and (b) two tight $b$-tags. The $M_{H}=125 \mathrm{GeV}$ signal is multiplied by 20 [2].

Figure 6.22 The dijet mass shown for the expected diboson signal and backgroundsubtracted data after the maximum likelihood fit, summed over $b$-tag channels. The error bars on data points represent the statistical uncertainty only. The post-fit systematic uncertainties are represented by the solid lines. The signal expectation is shown scaled to the best

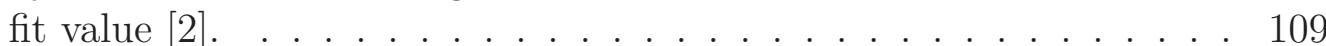


Figure 6.23 The final MVA discriminant output shown for the expected diboson signal and background-subtracted data rebinned as a function of $\log (S / B)$, after the maximum likelihood fit, summed over $b$-tag channels. The error bars on data points represent the statistical uncertainty only. The post-fit systematic uncertainties are represented by the solid lines. The signal expectation is shown scaled to the best fit value. The inset gives an expanded view of the high $\log (S / B)$

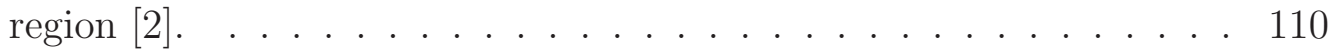

Figure 6.24 The log likelihood ratio as a function of Higgs boson mass for the expected background only hypothesis (dashed black line), expected signal+background hypothesis (red dashed line), and data (solid black line). The green and yellow bands represent, respectively, \pm one and two standard deviations on the background only hypothesis [2]. . . .

Figure 6.25 The upper 95\% confidence level limit on the Higgs boson production cross section times branching ratio as a ratio to the SM cross section times branching ratio. The dashed line shows the expected limit, the solid line shows the limit observed in data, and the green and yellow bands show the \pm 1 and 2 standard deviations on the expected limit [2].112

Figure 6.26 The LLR for the combined D0 Higgs boson searches (top) and the combined Tevatron Higgs searches (bottom), showing the background only expectation (the black dashed line), the signal+background expectation (red dashed line), the observed data (black solid line), and the background plus a $125 \mathrm{GeV}$ Higgs boson signal (blue dashed line). The green and yellow bands are the \pm 1 and 2 standard deviation on the background only expectation $[7,8] \ldots \ldots$. . . . . . . .

Figure 6.27 The 95\% C.L. limit as a function of Higgs boson mass for the combined D0 Higgs boson searches (top) and the combined Tevatron Higgs boson searches (bottom), shown as a ratio the the SM cross section. The black dashed line shows the expected limit, the solid black line shows the observed limit, the blue dashed line shows the limit expected if a Higgs boson with a mass of $125 \mathrm{GeV}$ were present. The green and yellow bands are the \pm 1 and 2 standard deviation on the expectation. Regions where the observed limit is below 1 $\left(90<M_{H}<101,157<M_{H}<178 \mathrm{GeV}\right.$ (D0), and $90<M_{H}<108$, $150<M_{H}<182 \mathrm{GeV}$ (Tevatron)), are regions of $M_{H}$ space that are excluded at the $95 \%$ confidence level $[7,8]$. . . . . . . . . . . . 115 
Figure 6.28 The probability for the background to fluctuate to the observed rate in data as a function of Higgs boson mass for the D0 combination (left) and the Tevatron combination (right). The black dashed line shows the expectation, the solid black line shows the observed data, the blue dashed line shows the expectation for a Higgs boson with $M_{H}=125 \mathrm{GeV}$. The green and yellow bands enclose the \pm 1 and 2 s.d. fluctuations of the background $[7,8] \ldots \ldots \ldots \ldots$

Figure 7.1 The fraction of energy deposited in various cones around a single pion track for a pion energy of $10 \mathrm{GeV} .90 \%$ of the energy is contained within a cone of $\Delta R=0.15 \ldots \ldots \ldots \ldots \ldots$

Figure $7.2 \quad\left[E_{\text {track }}^{\Delta R=0.15}-P_{\text {track }} * R\left(P_{\text {track }}\right)\right] / P_{\text {track }}$ for (a) $5 \mathrm{GeV}$ simulated single charged pion tracks, and (b) tracks within a jet with $4.5<P_{\text {track }}<$ $5 \mathrm{GeV}$ using simulated $\gamma+$ jet events. The vertical lines indicate twice the width of the charged pion distribution $\left(\sigma_{F}\right) \ldots \ldots \ldots$

Figure 7.3 The resolution for the track-cal jet energy $+k_{\mathrm{CPS}}$ in the blue circles and calorimeter jet energy $+k_{\mathrm{CPS}}$ in the red crosses as a function of $k_{\mathrm{CPS}}$, for a) $\left|\eta_{\text {jet }}\right|<0.4$, and b) $0.4<\left|\eta_{\text {jet }}\right|<0.8$. The pink solid and green dashed lines show the track-cal jet energy and calorimeter jet energy resolutions respectively for $k_{\mathrm{cps}}=0 \ldots \ldots \ldots$. . .

Figure 7.4 The resolution for the track-cal jet energy $+k_{\mathrm{CPS}}$ in the blue circles and calorimeter jet energy $+k_{\mathrm{CPS}}$ in the red crosses as a function of $k_{\mathrm{CPS}}$, for a) $0.8<\left|\eta_{\text {jet }}\right|<1.2$, and b) $1.2<\left|\eta_{\text {jet }}\right|<1.6$. The pink solid and green dashed lines show the track-cal jet energy and calorimeter jet energy resolutions respectively for $k_{\mathrm{cps}}=0 . \ldots 126$

Figure 7.5 The ratio of the reconstructed jet $p_{T}$ to the true $\mathrm{MC}$ jet $p_{T}$ for jets with $30<p_{T}<40 \mathrm{GeV}$, and $|\eta|<0.4$, for track-cal jets (in red) and calorimeter jets (in black). The mean of this distribution is taken as the calorimeter response. . . . . . . . . . . . . . . . . . 127

Figure 7.6 The energy response for track-cal jets as a function of true $\mathrm{MC} p_{T}$ for various track multiplicities for a) $\left|\eta_{\text {jet }}\right|<0.4$, and b) $0.4<\left|\eta_{\text {jet }}\right|<$ 0.8. The response for calorimeter jets is also shown for comparison. .

Figure 7.7 The energy response for track-cal jets as a function of true MC $p_{T}$ for various track multiplicities for a) $0.8<\left|\eta_{\text {jet }}\right|<1.2$, and b) $1.2<\left|\eta_{\text {jet }}\right|<1.6$. The response for calorimeter jets is also shown for comparison. . . . . . . . . . . . . . 
Figure 7.8 The energy resolution for track-cal jets as a function of true $\mathrm{MC} p_{T}$ compared to the resolution for calorimeter jets for a) $\left|\eta_{\text {jet }}\right|<0.4$, and b) $0.4<\left|\eta_{\text {jet }}\right|<0.8$. The bottom panels show the ratio of the track-cal jet resolution to the calorimeter jet resolution. . . . . . .

Figure 7.9 The energy resolution for track-cal jets as a function of true $\mathrm{MC} p_{T}$ compared to the resolution for calorimeter jets for a) $0.8<\left|\eta_{\text {jet }}\right|<$ 1.2 , and b) $1.2<\left|\eta_{\text {jet }}\right|<1.6$. The bottom panels show the ratio of the track-cal jet resolution to the calorimeter jet resolution. . . . .

Figure 7.10 Distributions for all selected events with two jets of (a) transverse mass of the lepton- $\mathbb{E}_{T}$ system, and (b) charged lepton $p_{T}$. The $M_{H}=$ $125 \mathrm{GeV}$ signal is multiplied by 1000 . Overflow events are added to the last bin. . . . . . . . . . . . . . . . . . . . . .

Figure 7.11 Distributions for all selected events with two jets of (a) leading jet $p_{T}$, and (b) second-leading jet $p_{T}$. The $M_{H}=125 \mathrm{GeV}$ signal is multiplied by 1000. Overflow events are added to the last bin. . .

Figure 7.12 Distributions for all selected events with two jets of (a) $E_{T}$, and (b) $\Delta R$ between the leading and second-leading jets. The $M_{H}=125 \mathrm{GeV}$ signal is multiplied by 1000. Overflow events are added to the last bin. 135

Figure 7.13 Invariant mass of the leading and second-leading jets in events with two jets and (a) one tight $b$-tag, and (b) two loose $b$-tags. The $M_{H}=$ $125 \mathrm{GeV}$ signal is multiplied by 200 . Overflow events are added to the last bin. . . . . . . . . . . . . . . . . . 136

Figure 7.14 Invariant mass of the leading and second-leading jets in events with two jets and (a) two medium $b$-tags, and (b) two tight $b$-tags. The $M_{H}=125 \mathrm{GeV}$ signal is multiplied by 50 . Overflow events are added to the last bin.

Figure 7.15 The dijet mass for track-cal jets (red) and calorimeter jets (black), for the $W H M_{H}=125 \mathrm{GeV}$ signal (solid lines) and all backgrounds (dashed lines) for events with exactly two jets and two tight b-tags. All curves are normalized to have unit area. The mean and width values quoted are determined by fitting the signal dijet mass peak with a Gaussian distribution. The width of the signal peak is $\sim 7 \%$ narrower for track-cal jets than for calorimeter jets. . . . . . . . . . 138 
Figure 7.16 The dijet mass for track-cal jets (red) and calorimeter jets (black), for $W W$ events with exactly two jets and zero b-tags. The mean and width values quoted are determined by fitting the signal dijet mass peak with a Gaussian distribution. The width of the dijet mass peak is $\sim 7 \%$ narrower for track-cal jets than for calorimeter jets. . . .

Figure 7.17 The dijet mass shown for the expected diboson signal and backgroundsubtracted data rebinned, after the maximum likelihood fit, for events with zero $b$-tagged jets (a) using calorimeter jets and (b) using trackcal jets. The error bars on data points represent the statistical uncertainty only. The post-fit systematic uncertainties are represented by the solid lines. The diboson signal expectation is shown scaled to the best fit value. . . . . . . . . . . . . . . . . . . . . . . . . 140

Figure 7.18 The $95 \%$ C.L. expected Higgs boson production cross section limit (top), presented as a ratio to the SM cross section, for track-cal jets (black dashed line), and calorimeter jets (black solid line). The green and grey shaded areas represent the \pm 1 and 2 standard deviations on the calorimeter jet expectation. The ratio of the expected 95\% C.L. limit for track-cal jets to calorimeter jets (bottom). . . . . . . . . . 142

Figure A.1 Input variables to the multijet BDT shown for all events containing two jets: (top left) $\eta$ of the $E_{T}$, (top right) the $E_{T}$ significance, (bottom left) $\Delta \eta(\ell, \nu)$, and (bottom right) the twist of the $\ell-\nu$ system. The signal is shown for $M_{H}=125 \mathrm{GeV}$ multiplied by a factor of 1000. Overflow events are added to the last bin. . . . . . . 147

Figure A.2 Input variables to the multijet BDT shown for all events containing two jets: (top left) $\cos (\theta)$ in the $\ell \nu$ center-of-momentum frame, (top right) the velocity of the dijet system, (bottom left) the mass asymmetry between the dijet and the $\ell \nu$ system, and (bottom right) the centrality of the $\ell \nu j j$ system. The signal is shown for $M_{H}=125 \mathrm{GeV}$ multiplied by a factor of 1000 . Overflow events are added to the last bin. . . . . . . . . . . . . . . . . . . 148

Figure A.3 Input variables to the multijet BDT shown for all events containing two jets: (left) the magnitude of the vector sum of $p_{T}$ for all visible particles, and (right) the maximum $\Delta \eta(\ell, j)$. The signal is shown for $M_{H}=125 \mathrm{GeV}$ multiplied by a factor of 1000 . Overflow events are added to the last bin. . . . . . . . . . . . . . . . . . . 149 
Figure A.4 Input variables to the multijet BDT shown for all events containing two jets: (top left) $\eta$ of the $\mathbb{E}_{T}$, (top right) the $\mathbb{E}_{T}$ significance, (bottom left) $\Delta \eta(\ell, \nu)$, and (bottom right) the twist of the $\ell-\nu$ system. The signal is shown for $M_{H}=125 \mathrm{GeV}$. The signal is normalized to the sum of the backgrounds. Overflow events are added to the last bin.150

Figure A.5 Input variables to the multijet BDT shown for all events containing two jets: (top left) $\cos (\theta)$ in the $\ell \nu$ center-of-momentum frame, (top right) the velocity of the dijet system, (bottom left) the mass asymmetry between the dijet and the $\ell \nu$ system, and (bottom right) the centrality of the $\ell \nu j j$ system. The signal is shown for $M_{H}=125 \mathrm{GeV}$. The signal is normalized to the sum of the backgrounds. Overflow events are added to the last bin. . . . . . . . . . . . . . . 151

Figure A.6 Input variables to the multijet BDT shown for all events containing two jets: (left) the magnitude of the vector sum of $p_{T}$ for all visible particles, and (right) the maximum $\Delta \eta(\ell, j)$. The signal is shown for $M_{H}=125 \mathrm{GeV}$. The signal is normalized to the sum of the backgrounds. Overflow events are added to the last bin. . . . . . . . 152

Figure B.1 Input variables to the BDT trained to distinguish signal from all backgrounds in events with two jets and one tight b-tag: (top left) the bid MVA output, (top right) the maximum $\Delta \eta(\ell, j)$, (bottom left) $q^{\ell} \times \eta^{\ell}$, and (bottom right) the minimum significance of the dijet system. The signal is shown for $M_{H}=125 \mathrm{GeV}$, and is multiplied by a factor of 200 . Overflow events are added to the last bin. . . .

Figure B.2 Input variables to the BDT trained to distinguish signal from all backgrounds in events with two jets and one tight b-tag. The signal is shown for $M_{H}=125 \mathrm{GeV}$, and is multiplied by a factor of 200 . Overflow events are added to the last bin. . . . . . . . . . . . .

Figure B.3 Input variables to the BDT trained to distinguish signal from all backgrounds in events with two jets and one tight b-tag: (top left) the mass asymmetry between the $\ell \nu$ and dijet systems, (top right) aplanarity, and (bottom) the second leading jet $p_{T}$. The signal is shown for $M_{H}=125 \mathrm{GeV}$, and is multiplied by a factor of 200 . Overflow events are added to the last bin. . . . . . . . . . . 156

Figure B.4 Input variables to the BDT trained to distinguish signal from all backgrounds in events with two jets and two loose b-tag: (top left) the bid MVA output, (top right) the maximum $\Delta \eta(\ell, j$ ), (bottom left) $q^{\ell} \times \eta^{\ell}$, and (bottom right) the minimum significance of the dijet system. The signal is shown for $M_{H}=125 \mathrm{GeV}$, and is multiplied by a factor of 200. Overflow events are added to the last bin. . . . . 157 
Figure B.5 Input variables to the BDT trained to distinguish signal from all backgrounds in events with two jets and two loose b-tag. The signal is shown for $M_{H}=125 \mathrm{GeV}$, and is multiplied by a factor of 200 . Overflow events are added to the last bin. . . . . . . . . . . . 158

Figure B.6 Input variables to the BDT trained to distinguish signal from all backgrounds in events with two jets and two loose b-tag: (top left) the mass asymmetry between the $\ell \nu$ and dijet systems, (top right) aplanarity, and (bottom) the second leading jet $p_{T}$. The signal is shown for $M_{H}=125 \mathrm{GeV}$, and is multiplied by a factor of 200 . Overflow events are added to the last bin. . . . . . . . . . . . . . .

Figure B.7 Input variables to the BDT trained to distinguish signal from all backgrounds in events with two jets and two medium b-tag: (top left) the bid MVA output, (top right) the maximum $\Delta \eta(\ell, j)$, (bottom left) $q^{\ell} \times \eta^{\ell}$, and (bottom right) the minimum significance of the dijet system. The signal is shown for $M_{H}=125 \mathrm{GeV}$, and is multiplied by a factor of 50 . Overflow events are added to the last bin. . . . . .

Figure B.8 Input variables to the BDT trained to distinguish signal from all backgrounds in events with two jets and two medium b-tag. The signal is shown for $M_{H}=125 \mathrm{GeV}$, and is multiplied by a factor of 50. Overflow events are added to the last bin. . . . . . . . . . . . 161

Figure B.9 Input variables to the BDT trained to distinguish signal from all backgrounds in events with two jets and two medium b-tag: (top left) the multijet MVA output, (top right) $\cos \left(\chi^{*}\right)$, (bottom left) $\cos \left(\theta^{*}\right.$, and (bottom right) the dijet transverse mass. The signal is shown for $M_{H}=125 \mathrm{GeV}$, and is multiplied by a factor of 50 . Overflow events are added to the last bin. . . . . . . . . . . . . . . . . . .

Figure B.10 Input variables to the BDT trained to distinguish signal from all backgrounds in events with two jets and two medium b-tags: the magnitude of the vector $p_{T}$ sum for all visible particles. The signal is shown for $M_{H}=125 \mathrm{GeV}$, and is multiplied by a factor of 50 . Overflow events are added to the last bin. . . . . . . . . . . . .

Figure B.11 Input variables to the BDT trained to distinguish signal from all backgrounds in events with two jets and two tight b-tag: (top left) the bid MVA output, (top right) the maximum $\Delta \eta(\ell, j)$, (bottom left) $q^{\ell} \times \eta^{\ell}$, and (bottom right) the minimum significance of the dijet system. The signal is shown for $M_{H}=125 \mathrm{GeV}$, and is multiplied by a factor of 50. Overflow events are added to the last bin. . . . . . 164 
Figure B.12 Input variables to the BDT trained to distinguish signal from all backgrounds in events with two jets and two tight b-tag. The signal is shown for $M_{H}=125 \mathrm{GeV}$, and is multiplied by a factor of 50 . Overflow events are added to the last bin. . . . . . . . . . . . .

Figure B.13 Input variables to the BDT trained to distinguish signal from all backgrounds in events with two jets and two tight b-tag: (top left) the multijet MVA output, (top right) $\cos \left(\chi^{*}\right)$, (bottom left) $\cos \left(\theta^{*}\right.$, and (bottom right) the dijet transverse mass. The signal is shown for $M_{H}=125 \mathrm{GeV}$, and is multiplied by a factor of 50 . Overflow events are added to the last bin. . . . . . . . . . . . . . . . . .

Figure B.14 Input variables to the BDT trained to distinguish signal from all backgrounds in events with two jets and two tight b-tag: the ratio of the $W p_{T}$ to the sum of the lepton $p_{T}$ and $E_{T}$. The signal is shown for $M_{H}=125 \mathrm{GeV}$, and is multiplied by a factor of 50 . Overflow events are added to the last bin. . . . . . . . . . . . . . . .

Figure B.15 The final MVA output for events with two jets and one tight b-tag for (top left) $M_{H}=90 \mathrm{GeV}$, (top right) $M_{H}=95 \mathrm{GeV}$, (bottom left) $M_{H}=100 \mathrm{GeV}$, and (bottom right) $M_{H}=105 \mathrm{GeV} \ldots . . . .168$

Figure B.16 The final MVA output for events with two jets and one tight b-tag for (top left) $M_{H}=110 \mathrm{GeV}$, (top right) $M_{H}=115 \mathrm{GeV}$, (bottom left) $M_{H}=120 \mathrm{GeV}$, and (bottom right) $M_{H}=130 \mathrm{GeV} \ldots \ldots$. .

Figure B.17 The final MVA output for events with two jets and one tight b-tag for (top left) $M_{H}=135 \mathrm{GeV}$, (top right) $M_{H}=140 \mathrm{GeV}$, (bottom left) $M_{H}=145 \mathrm{GeV}$, and (bottom right) $M_{H}=150 \mathrm{GeV}$. . . . . . 170

Figure B.18 The final MVA output for events with three jets and one tight b-tag for (top left) $M_{H}=90 \mathrm{GeV}$, (top right) $M_{H}=95 \mathrm{GeV}$, (bottom left) $M_{H}=100 \mathrm{GeV}$, and (bottom right) $M_{H}=105 \mathrm{GeV}$. . . . . . 171

Figure B.19 The final MVA output for events with three jets and one tight b-tag for (top left) $M_{H}=110 \mathrm{GeV}$, (top right) $M_{H}=115 \mathrm{GeV}$, (bottom left) $M_{H}=120 \mathrm{GeV}$, and (bottom right) $M_{H}=130 \mathrm{GeV} \ldots .$. .

Figure B.20 The final MVA output for events with three jets and one tight b-tag for (top left) $M_{H}=135 \mathrm{GeV}$, (top right) $M_{H}=140 \mathrm{GeV}$, (bottom left) $M_{H}=145 \mathrm{GeV}$, and (bottom right) $M_{H}=150 \mathrm{GeV} \ldots .$.

Figure B.21 The final MVA output for events with two jets and two loose b-tags for (top left) $M_{H}=90 \mathrm{GeV}$, (top right) $M_{H}=95 \mathrm{GeV}$, (bottom left) $M_{H}=100 \mathrm{GeV}$, and (bottom right) $M_{H}=105 \mathrm{GeV}$. . . . . . 174 
Figure B.22 The final MVA output for events with two jets and two loose b-tags for (top left) $M_{H}=110 \mathrm{GeV}$, (top right) $M_{H}=115 \mathrm{GeV}$, (bottom left) $M_{H}=120 \mathrm{GeV}$, and (bottom right) $M_{H}=130 \mathrm{GeV} \ldots$. . .

Figure B.23 The final MVA output for events with two jets and two loose b-tags for (top left) $M_{H}=135 \mathrm{GeV}$, (top right) $M_{H}=140 \mathrm{GeV}$, (bottom left) $M_{H}=145 \mathrm{GeV}$, and (bottom right) $M_{H}=150 \mathrm{GeV} . . . . .176$

Figure B.24 The final MVA output for events with three jets and two loose b-tags for (top left) $M_{H}=90 \mathrm{GeV}$, (top right) $M_{H}=95 \mathrm{GeV}$, (bottom left) $M_{H}=100 \mathrm{GeV}$, and (bottom right) $M_{H}=105 \mathrm{GeV}$. . . . . . 177

Figure B.25 The final MVA output for events with three jets and two loose b-tags for (top left) $M_{H}=110 \mathrm{GeV}$, (top right) $M_{H}=115 \mathrm{GeV}$, (bottom left) $M_{H}=120 \mathrm{GeV}$, and (bottom right) $M_{H}=130 \mathrm{GeV} \ldots$. . . . . 178

Figure B.26 The final MVA output for events with three jets and two loose b-tags for (top left) $M_{H}=135 \mathrm{GeV}$, (top right) $M_{H}=140 \mathrm{GeV}$, (bottom left) $M_{H}=145 \mathrm{GeV}$, and (bottom right) $M_{H}=150 \mathrm{GeV}$. . . . . . 179

Figure B.27 The final MVA output for events with two jets and two medium btags for (top left) $M_{H}=90 \mathrm{GeV}$, (top right) $M_{H}=95 \mathrm{GeV}$, (bottom left) $M_{H}=100 \mathrm{GeV}$, and (bottom right) $M_{H}=105 \mathrm{GeV}$. . . . . . 180

Figure B.28 The final MVA output for events with two jets and two medium b-tags for (top left) $M_{H}=110 \mathrm{GeV}$, (top right) $M_{H}=115 \mathrm{GeV}$, (bottom left) $M_{H}=120 \mathrm{GeV}$, and (bottom right) $M_{H}=130 \mathrm{GeV}$. . . . . . 181

Figure B.29 The final MVA output for events with two jets and two medium b-tags for (top left) $M_{H}=135 \mathrm{GeV}$, (top right) $M_{H}=140 \mathrm{GeV}$, (bottom left) $M_{H}=145 \mathrm{GeV}$, and (bottom right) $M_{H}=150 \mathrm{GeV} \ldots \ldots$

Figure B.30 The final MVA output for events with three jets and two medium btags for (top left) $M_{H}=90 \mathrm{GeV}$, (top right) $M_{H}=95 \mathrm{GeV}$, (bottom left) $M_{H}=100 \mathrm{GeV}$, and (bottom right) $M_{H}=105 \mathrm{GeV} \ldots$. . . . 183

Figure B.31 The final MVA output for events with three jets and two medium b-tags for (top left) $M_{H}=110 \mathrm{GeV}$, (top right) $M_{H}=115 \mathrm{GeV}$, (bottom left) $M_{H}=120 \mathrm{GeV}$, and (bottom right) $M_{H}=130 \mathrm{GeV}$.

Figure B.32 The final MVA output for events with three jets and two medium b-tags for (top left) $M_{H}=135 \mathrm{GeV}$, (top right) $M_{H}=140 \mathrm{GeV}$, (bottom left) $M_{H}=145 \mathrm{GeV}$, and (bottom right) $M_{H}=150 \mathrm{GeV} . \quad 185$ 
Figure B.33 The final MVA output for events with two jets and two tight b-tags for (top left) $M_{H}=90 \mathrm{GeV}$, (top right) $M_{H}=95 \mathrm{GeV}$, (bottom left) $M_{H}=100 \mathrm{GeV}$, and (bottom right) $M_{H}=105 \mathrm{GeV} \ldots \ldots .186$

Figure B.34 The final MVA output for events with two jets and two tight b-tags for (top left) $M_{H}=110 \mathrm{GeV}$, (top right) $M_{H}=115 \mathrm{GeV}$, (bottom left) $M_{H}=120 \mathrm{GeV}$, and (bottom right) $M_{H}=130 \mathrm{GeV} \ldots \ldots 187$

Figure B.35 The final MVA output for events with two jets and two tight b-tags for (top left) $M_{H}=135 \mathrm{GeV}$, (top right) $M_{H}=140 \mathrm{GeV}$, (bottom left) $M_{H}=145 \mathrm{GeV}$, and (bottom right) $M_{H}=150 \mathrm{GeV} \ldots \ldots .188$

Figure B.36 The final MVA output for events with three jets and two tight b-tags for (top left) $M_{H}=90 \mathrm{GeV}$, (top right) $M_{H}=95 \mathrm{GeV}$, (bottom left) $M_{H}=100 \mathrm{GeV}$, and (bottom right) $M_{H}=105 \mathrm{GeV} \ldots \ldots$.

Figure B.37 The final MVA output for events with three jets and two tight b-tags for (top left) $M_{H}=110 \mathrm{GeV}$, (top right) $M_{H}=115 \mathrm{GeV}$, (bottom left) $M_{H}=120 \mathrm{GeV}$, and (bottom right) $M_{H}=130 \mathrm{GeV} \ldots \ldots$.

Figure B.38 The final MVA output for events with three jets and two tight b-tags for (top left) $M_{H}=135 \mathrm{GeV}$, (top right) $M_{H}=140 \mathrm{GeV}$, (bottom left) $M_{H}=145 \mathrm{GeV}$, and (bottom right) $M_{H}=150 \mathrm{GeV} \ldots$. . .

Figure B.39 Input variables to the BDT trained to distinguish signal from all backgrounds in events with two jets and one tight b-tag: (top left) the bid MVA output, (top right) the maximum $\Delta \eta(\ell, j)$, (bottom left) $q^{\ell} \times \eta^{\ell}$, and (bottom right) the minimum significance of the dijet system. The signal is shown for $M_{H}=125 \mathrm{GeV}$, and is normalized to the sum of the backgrounds. Overflow events are added to the last bin. . . . . . . . . . . . . . . . . . . .

Figure B.40 Input variables to the BDT trained to distinguish signal from all backgrounds in events with two jets and one tight b-tag. The signal is shown for $M_{H}=125 \mathrm{GeV}$, and is normalized to the sum of the backgrounds. Overflow events are added to the last bin. . . . . . . .

Figure B.41 Input variables to the BDT trained to distinguish signal from all backgrounds in events with two jets and one tight b-tag: (top left) the mass asymmetry between the $\ell \nu$ and dijet systems, (top right) aplanarity, and (bottom) the second leading jet $p_{T}$. The signal is shown for $M_{H}=125 \mathrm{GeV}$, and is normalized to the sum of the backgrounds. Overflow events are added to the last bin. . . . . . . . 
Figure B.42 Input variables to the BDT trained to distinguish signal from all backgrounds in events with two jets and two loose b-tag: (top left) the bid MVA output, (top right) the maximum $\Delta \eta(\ell, j$ ), (bottom left) $q^{\ell} \times \eta^{\ell}$, and (bottom right) the minimum significance of the dijet system. The signal is shown for $M_{H}=125 \mathrm{GeV}$, and is normalized to the sum of the backgrounds. Overflow events are added to the last bin. . . . . . . . . . . . . . .

Figure B.43 Input variables to the BDT trained to distinguish signal from all backgrounds in events with two jets and two loose b-tag. The signal is shown for $M_{H}=125 \mathrm{GeV}$, and is normalized to the sum of the backgrounds. Overflow events are added to the last bin. . . . . . . .

Figure B.44 Input variables to the BDT trained to distinguish signal from all backgrounds in events with two jets and two loose b-tag: (top left) the mass asymmetry between the $\ell \nu$ and dijet systems, (top right) aplanarity, and (bottom) the second leading jet $p_{T}$. The signal is shown for $M_{H}=125 \mathrm{GeV}$, and is normalized to the sum of the backgrounds. Overflow events are added to the last bin. . . . . . . . 197

Figure B.45 Input variables to the BDT trained to distinguish signal from all backgrounds in events with two jets and two medium b-tag: (top left) the bid MVA output, (top right) the maximum $\Delta \eta(\ell, j)$, (bottom left) $q^{\ell} \times \eta^{\ell}$, and (bottom right) the minimum significance of the dijet system. The signal is shown for $M_{H}=125 \mathrm{GeV}$, and is normalized to the sum of the backgrounds. Overflow events are added to the last bin. . . . . . . . . . . . . . . . . . . .

Figure B.46 Input variables to the BDT trained to distinguish signal from all backgrounds in events with two jets and two medium b-tag. The signal is shown for $M_{H}=125 \mathrm{GeV}$, and is normalized to the sum of the backgrounds. Overflow events are added to the last bin. . . . . .

Figure B.47 Input variables to the BDT trained to distinguish signal from all backgrounds in events with two jets and two medium b-tag: (top left) the multijet MVA output, (top right) $\cos \left(\chi^{*}\right)$, (bottom left) $\cos \left(\theta^{*}\right.$, and (bottom right) the dijet transverse mass. The signal is shown for $M_{H}=125 \mathrm{GeV}$, and is normalized to the sum of the backgrounds. Overflow events are added to the last bin. . . . . . . . . . . . 200

Figure B.48 Input variables to the BDT trained to distinguish signal from all backgrounds in events with two jets and two medium b-tags: the magnitude of the vector $p_{T}$ sum for all visible particles. The signal is shown for $M_{H}=125 \mathrm{GeV}$, and is normalized to the sum of the backgrounds. Overflow events are added to the last bin. . . . . . . . 201 
Figure B.49 Input variables to the BDT trained to distinguish signal from all backgrounds in events with two jets and two tight b-tag: (top left) the bid MVA output, (top right) the maximum $\Delta \eta(\ell, j$ ), (bottom left) $q^{\ell} \times \eta^{\ell}$, and (bottom right) the minimum significance of the dijet system. The signal is shown for $M_{H}=125 \mathrm{GeV}$, and is normalized to the sum of the backgrounds. Overflow events are added to the last bin. . . . . . . . . . . . . . . . 202

Figure B.50 Input variables to the BDT trained to distinguish signal from all backgrounds in events with two jets and two tight b-tag. The signal is shown for $M_{H}=125 \mathrm{GeV}$, and is normalized to the sum of the backgrounds. Overflow events are added to the last bin. . . . . . . . 203

Figure B.51 Input variables to the BDT trained to distinguish signal from all backgrounds in events with two jets and two tight b-tag: (top left) the multijet MVA output, (top right) $\cos \left(\chi^{*}\right)$, (bottom left) $\cos \left(\theta^{*}\right.$, and (bottom right) the dijet transverse mass. The signal is shown for $M_{H}=125 \mathrm{GeV}$, and is normalized to the sum of the backgrounds. Overflow events are added to the last bin. . . . . . . . . . . . . . 204

Figure B.52 Input variables to the BDT trained to distinguish signal from all backgrounds in events with two jets and two tight b-tag: the ratio of the $W p_{T}$ to the sum of the lepton $p_{T}$ and $E_{T}$. The signal is shown for $M_{H}=125 \mathrm{GeV}$, and is normalized to the sum of the backgrounds. Overflow events are added to the last bin. . . . . . . . . . . . . 205

Figure B.53 The final MVA output for events with two jets and one tight b-tag for (top left) $M_{H}=90 \mathrm{GeV}$, (top right) $M_{H}=95 \mathrm{GeV}$, (bottom left) $M_{H}=100 \mathrm{GeV}$, and (bottom right) $M_{H}=105 \mathrm{GeV}$. The signal is normalized to the sum of the backgrounds. . . . . . . . . . . .

Figure B.54 The final MVA output for events with two jets and one tight b-tag for (top left) $M_{H}=110 \mathrm{GeV}$, (top right) $M_{H}=115 \mathrm{GeV}$, (bottom left) $M_{H}=120 \mathrm{GeV}$, and (bottom right) $M_{H}=125 \mathrm{GeV}$. The signal is normalized to the sum of the backgrounds. . . . . . . . . . 207

Figure B.55 The final MVA output for events with two jets and one tight b-tag for (top left) $M_{H}=130 \mathrm{GeV}$, (top right) $M_{H}=135 \mathrm{GeV}$, (bottom left) $M_{H}=140 \mathrm{GeV}$, and (bottom right) $M_{H}=145 \mathrm{GeV}$. The signal is normalized to the sum of the backgrounds. . . . . . . . . . . 208

Figure B.56 The final MVA output for events with two jets and one tight b-tag for $M_{H}=150 \mathrm{GeV}$. The signal is normalized to the sum of the backgrounds. . . . . . . . . . . . . . . . . . 209 
Figure B.57 The final MVA output for events with three jets and one tight b-tag for (top left) $M_{H}=90 \mathrm{GeV}$, (top right) $M_{H}=95 \mathrm{GeV}$, (bottom left) $M_{H}=100 \mathrm{GeV}$, and (bottom right) $M_{H}=105 \mathrm{GeV}$. The signal is normalized to the sum of the backgrounds. . . . . . . . . . . 210

Figure B.58 The final MVA output for events with three jets and one tight b-tag for (top left) $M_{H}=110 \mathrm{GeV}$, (top right) $M_{H}=115 \mathrm{GeV}$, (bottom left) $M_{H}=120 \mathrm{GeV}$, and (bottom right) $M_{H}=125 \mathrm{GeV}$. The signal is normalized to the sum of the backgrounds. ..............

Figure B.59 The final MVA output for events with three jets and one tight b-tag for (top left) $M_{H}=130 \mathrm{GeV}$, (top right) $M_{H}=135 \mathrm{GeV}$, (bottom left) $M_{H}=140 \mathrm{GeV}$, and (bottom right) $M_{H}=145 \mathrm{GeV}$. The signal is normalized to the sum of the backgrounds. . . . . . . . . . . . .

Figure B.60 The final MVA output for events with three jets and one tight btag for $M_{H}=150 \mathrm{GeV}$. The signal is normalized to the sum of the backgrounds. . . . . . . . . . . . . . . . . . .

Figure B.61 The final MVA output for events with two jets and two loose b-tags for (top left) $M_{H}=90 \mathrm{GeV}$, (top right) $M_{H}=95 \mathrm{GeV}$, (bottom left) $M_{H}=100 \mathrm{GeV}$, and (bottom right) $M_{H}=105 \mathrm{GeV}$. The signal is normalized to the sum of the backgrounds. . . . . . . . . . . . . 214

Figure B.62 The final MVA output for events with two jets and two loose b-tags for (top left) $M_{H}=110 \mathrm{GeV}$, (top right) $M_{H}=115 \mathrm{GeV}$, (bottom left) $M_{H}=120 \mathrm{GeV}$, and (bottom right) $M_{H}=125 \mathrm{GeV}$. The signal is normalized to the sum of the backgrounds. ...................

Figure B.63 The final MVA output for events with two jets and two loose b-tags for (top left) $M_{H}=130 \mathrm{GeV}$, (top right) $M_{H}=135 \mathrm{GeV}$, (bottom left) $M_{H}=140 \mathrm{GeV}$, and (bottom right) $M_{H}=145 \mathrm{GeV}$. The signal is normalized to the sum of the backgrounds. . . . . . . . . . . 216

Figure B.64 The final MVA output for events with two jets and two loose b-tags for $M_{H}=150 \mathrm{GeV}$. The signal is normalized to the sum of the backgrounds. . . . . . . . . . . . . . . . . . . . 217

Figure B.65 The final MVA output for events with three jets and two loose b-tags for (top left) $M_{H}=90 \mathrm{GeV}$, (top right) $M_{H}=95 \mathrm{GeV}$, (bottom left) $M_{H}=100 \mathrm{GeV}$, and (bottom right) $M_{H}=105 \mathrm{GeV}$. The signal is normalized to the sum of the backgrounds. . . . . . . . . 218 
Figure B.66 The final MVA output for events with three jets and two loose b-tags for (top left) $M_{H}=110 \mathrm{GeV}$, (top right) $M_{H}=115 \mathrm{GeV}$, (bottom left) $M_{H}=120 \mathrm{GeV}$, and (bottom right) $M_{H}=125 \mathrm{GeV}$. The signal is normalized to the sum of the backgrounds. . . . . . . . . . .

Figure B.67 The final MVA output for events with three jets and two loose b-tags for (top left) $M_{H}=130 \mathrm{GeV}$, (top right) $M_{H}=135 \mathrm{GeV}$, (bottom left) $M_{H}=140 \mathrm{GeV}$, and (bottom right) $M_{H}=145 \mathrm{GeV}$. The signal is normalized to the sum of the backgrounds. .............

Figure B.68 The final MVA output for events with three jets and two loose btags for $M_{H}=150 \mathrm{GeV}$. The signal is normalized to the sum of the backgrounds. . . . . . . . . . . . . . . . . 221

Figure B.69 The final MVA output for events with two jets and two medium btags for (top left) $M_{H}=90 \mathrm{GeV}$, (top right) $M_{H}=95 \mathrm{GeV}$, (bottom left) $M_{H}=100 \mathrm{GeV}$, and (bottom right) $M_{H}=105 \mathrm{GeV}$. The signal is normalized to the sum of the backgrounds. . . . . . . . . . . . .

Figure B.70 The final MVA output for events with two jets and two medium b-tags for (top left) $M_{H}=110 \mathrm{GeV}$, (top right) $M_{H}=115 \mathrm{GeV}$, (bottom left) $M_{H}=120 \mathrm{GeV}$, and (bottom right) $M_{H}=125 \mathrm{GeV}$. The signal is normalized to the sum of the backgrounds. . . . . . . . . . . .

Figure B.71 The final MVA output for events with two jets and two medium b-tags for (top left) $M_{H}=130 \mathrm{GeV}$, (top right) $M_{H}=135 \mathrm{GeV}$, (bottom left) $M_{H}=140 \mathrm{GeV}$, and (bottom right) $M_{H}=145 \mathrm{GeV}$. The signal is normalized to the sum of the backgrounds. . . . . . . . . . . .

Figure B.72 The final MVA output for events with two jets and two medium btags for $M_{H}=150 \mathrm{GeV}$. The signal is normalized to the sum of the backgrounds. . . . . . . . . . . . . . . 225

Figure B.73 The final MVA output for events with three jets and two medium btags for (top left) $M_{H}=90 \mathrm{GeV}$, (top right) $M_{H}=95 \mathrm{GeV}$, (bottom left) $M_{H}=100 \mathrm{GeV}$, and (bottom right) $M_{H}=105 \mathrm{GeV}$. The signal is normalized to the sum of the backgrounds. . . . . . . . . . . . 226

Figure B.74 The final MVA output for events with three jets and two medium b-tags for (top left) $M_{H}=110 \mathrm{GeV}$, (top right) $M_{H}=115 \mathrm{GeV}$, (bottom left) $M_{H}=120 \mathrm{GeV}$, and (bottom right) $M_{H}=125 \mathrm{GeV}$. The signal is normalized to the sum of the backgrounds. . . . . . . . 227 
Figure B.75 The final MVA output for events with three jets and two medium b-tags for (top left) $M_{H}=130 \mathrm{GeV}$, (top right) $M_{H}=135 \mathrm{GeV}$, (bottom left) $M_{H}=140 \mathrm{GeV}$, and (bottom right) $M_{H}=145 \mathrm{GeV}$. The signal is normalized to the sum of the backgrounds. . . . . . . . 228

Figure B.76 The final MVA output for events with three jets and two medium b-tags for $M_{H}=150 \mathrm{GeV}$. The signal is normalized to the sum of the backgrounds. . . . . . . . . . . . . . . . . .

Figure B.77 The final MVA output for events with two jets and two tight b-tags for (top left) $M_{H}=90 \mathrm{GeV}$, (top right) $M_{H}=95 \mathrm{GeV}$, (bottom left) $M_{H}=100 \mathrm{GeV}$, and (bottom right) $M_{H}=105 \mathrm{GeV}$. The signal is normalized to the sum of the backgrounds. . . . . . . . . . . . . 230

Figure B.78 The final MVA output for events with two jets and two tight b-tags for (top left) $M_{H}=110 \mathrm{GeV}$, (top right) $M_{H}=115 \mathrm{GeV}$, (bottom left) $M_{H}=120 \mathrm{GeV}$, and (bottom right) $M_{H}=125 \mathrm{GeV}$. The signal is normalized to the sum of the backgrounds. . . . . . . . . . . .

Figure B.79 The final MVA output for events with two jets and two tight b-tags for (top left) $M_{H}=130 \mathrm{GeV}$, (top right) $M_{H}=135 \mathrm{GeV}$, (bottom left) $M_{H}=140 \mathrm{GeV}$, and (bottom right) $M_{H}=145 \mathrm{GeV}$. The signal is normalized to the sum of the backgrounds. . . . . . . . . . . . 232

Figure B.80 The final MVA output for events with two jets and two tight b-tags for $M_{H}=150 \mathrm{GeV}$. The signal is normalized to the sum of the backgrounds. . . . . . . . . . . . . . . . .

Figure B.81 The final MVA output for events with three jets and two tight b-tags for (top left) $M_{H}=90 \mathrm{GeV}$, (top right) $M_{H}=95 \mathrm{GeV}$, (bottom left) $M_{H}=100 \mathrm{GeV}$, and (bottom right) $M_{H}=105 \mathrm{GeV}$. The signal is normalized to the sum of the backgrounds. . . . . . . . . . . . . . 234

Figure B.82 The final MVA output for events with three jets and two tight b-tags for (top left) $M_{H}=110 \mathrm{GeV}$, (top right) $M_{H}=115 \mathrm{GeV}$, (bottom left) $M_{H}=120 \mathrm{GeV}$, and (bottom right) $M_{H}=125 \mathrm{GeV}$. The signal is normalized to the sum of the backgrounds. . . . . . . . . . . .

Figure B.83 The final MVA output for events with three jets and two tight b-tags for (top left) $M_{H}=130 \mathrm{GeV}$, (top right) $M_{H}=135 \mathrm{GeV}$, (bottom left) $M_{H}=140 \mathrm{GeV}$, and (bottom right) $M_{H}=145 \mathrm{GeV}$. The signal is normalized to the sum of the backgrounds. . . . . . . . . . . . 236 
Figure B.84 The final MVA output for events with three jets and two tight btags for $M_{H}=150 \mathrm{GeV}$. The signal is normalized to the sum of the backgrounds. . . . . . . . . . . . . . . . . 237 


\section{KEY TO SYMBOLS AND ABBREVIATIONS}

$\Delta R$ The distance in $\eta-\phi$ space, $\Delta R=\sqrt{\eta^{2}+\phi^{2}}$

$\ell \quad$ Lepton, $\ell=e, \mu$

$\eta \quad$ Pseudorapidity, $\eta=-\ln \left(\tan \frac{\theta}{2}\right)$

$\gamma \quad$ Photon

$E_{T} \quad$ Missing energy in the plane transverse to the beam line.

$\mu \quad$ Muon

$\nu \quad$ Neutrino

$\phi \quad$ The detector azimuthal angle.

$\tau \quad$ Tau

$\theta \quad$ The detector polar angle measured from the beam axis.

$b \quad$ Bottom quark

c Charm quark

$C L_{S}$ The ratio of the signal+background confidence level to the background-only confidence level, $C L_{S}=\frac{C L_{S}}{C L_{B}}$

d Down quark

e Electron

g Gluon

$H_{0} \quad$ The null (or background-only) hypothesis

$H_{1} \quad$ The null (or signal+background) hypothesis

$H_{T} \quad$ The sum over jet $p_{T}$ 
ID Identification

$j_{1} \quad$ Jet with the highest $p_{T}$

$j_{2} \quad$ Jet with the second highest $p_{T}$

$M_{H}$ The mass of the Higgs boson

$M_{T}^{W}$ Transverse mass of the reconstructed $W$ boson

$p_{T} \quad$ Momentum in the plane transverse to the beam line.

$s \quad$ Strange quark

$t \quad$ Top quark

$T_{\mu O R}$ Logical OR of single-muon and muon+jets triggers

$u \quad$ Up quark

$V \quad$ Vector boson, $V=W, Z$

AA Alternative Algorithm

BDT Boosted decision Tree

bid Identification of mesons containing a b-quark

CC Central Calorimeter

CFT Central Fibre Tracker

CL Confidence Level

CPS Central Preshower Detector

EC End Calorimeter

EM Electromagnetic

FNAL Fermi National Accelerator Laboratory

FPS Forward PreShower Detector

hf Heavy Flavour: jets originating from heavy partons: $b, c$.

HTF Histogramming Track Finder

ICD Inter-Cryostat Detector

ICR Inter-Cryostat Region

JES Jet Energy Scale 
JESMU Jet Energy Scale for jets containing a muon from the decay of a B meson

JSSR Jet Smearing Shifting and Removal

L1 Trigger Level 1

L1Cal Level 1 Calorimeter Trigger

L1CalTrack Level 1 Calorimeter Track Trigger

L1CTT Level 1 Central Track Trigger

L1Mu Level 1 Muon Trigger

L1PS Level 1 Preshower Detector

L2 Trigger Level 2

L2Cal Level 2 Calorimeter Trigger

L2CTT Level 2 Central Track Trigger

L2Mu Level 2 Muon Trigger

L2PS Level 2 Preshower Trigger

L3 Trigger Level 3

Level 2 Silicon Track Trigger ,

If Light Flavour: jets originating from light partons: $u, d, s, g$.

LLR Log Likelihood Ratio

LO Leading Order

MC Monte-Carlo

MJ Multijet

MVA Multivariate Analysis

NLO Next to Leading Order

PDF Parton Distribution Function

PDT Proportional Drift Tubes

PV Primary Vertex

QCD Quantum Chromodynamics

QED Quantum Electrodynamics 
s. d. Standard deviation

SM Standard Model

SMT Silicon Microstrip Tracker 


\section{Chapter 1}

\section{Introduction}

Particle physics is the study of the most fundamental pieces of the universe. These fundamental pieces, or particles, and the interactions between them, in principle should be able to describe all matter in the universe. It is impractical from a mathematical standpoint to describe macroscopic systems of particles by their constituent single particles, so we limit the discussion to systems of relatively few particles that interact over distances smaller than that of an atomic nucleus. These particles, and their interactions are described in Chapter 1.1.

The standard model (SM) of particle physics is the quantum field theory that governs the dynamics of the fundamental particles. The theoretical framework of the standard model is described in Chapter 1.2.1-1.2.3. The standard model has been incredibly successful, with many predictions experimentally verified. The discovery of the $Z$ boson and the top quark, and many other experimental results have agreed with the SM to high precision. As successful as the SM has been, it is not complete. It does not include any explanation for gravitational interactions, or the asymmetry between the amount of matter and anti-matter in the universe, and also explains only the visible matter in the universe (which accounts for only $\sim 5 \%$ of the total matter density of the universe). The relatively recent observation of neutrino flavour oscillations is also at odds with the SM, as neutrinos in the SM are massless and can therefore not oscillate.

The recent observation of the Higgs boson, which is responsible for generating the masses 
of the gauge bosons as well as the fermions, is the last remaining prediction of the SM to be experimentally verified, and is the main topic of this thesis. The current status of the Higgs boson measurements discussed in Chapter 1.2.4.

In order to study the Higgs boson experimentally, we first need to be able to produce it. This is done by colliding particles with high energies using sophisticated particle accelerators. Observing the results of these high energy collisions with high precision requires intricate detectors. The work described in this thesis was carried out at the Tevatron collider at Fermilab, which collided protons and anti-protons at a center-of-momentum energy of 1.96 TeV. The D0 detector was used to collect the data analysed in this work. The Tevatron and the D0 detector are described in Chapter 2.1 and 2.2 respectively. The particle identification and reconstruction algorithms are described in Chapter. Following this, is a discussion of event simulation in Chapter .

These different components are brought together in Chapter, where I describe how they are used in the search for a Higgs boson produced in association with a $W$ boson. This search uses sophisticated multivariate techniques to maximize our sensitivity to the Higgs boson signal while accepting as many potential signal events as possible. This search is mildly sensitive to the Higgs boson signal, but greater sensitivity can be achieved by combining this result with the other Higgs boson searches at the Tevatron in a combined search for the Higgs boson. I discuss how this analysis fits into the more global D0 and Tevatron Higgs boson picture in Chapter 6.10. Finally I discuss a method to improve the sensitivity for this Higgs boson search by improving the jet energy resolution in Chapter . 


\subsection{Standard Model Particles}

The standard model of particle physics combines special relativity and quantum mechanics into a theory framework that is used to describe the known fundamental particles and the interactions between them (with the exception of gravity). The list of SM particles is comprised of matter particles; leptons, quarks, and neutrinos; gauge bosons, which mediate the interactions between particles; and the Higgs boson, which is a result of electroweak symmetry breaking and is responsible for generating the masses of the massive gauge bosons and matter particles. These particles are said to be fundamental as they do not contain any known substructure.

Leptons and quarks are fermions. That is to say, they have an intrinsic angular momentum, known as spin, that is a half integer multiple of the reduced Plank constant, $\hbar$. These fermions can be arranged into three generations containing one lepton, one neutrino, and two quarks as shown in Table 1.1. Across generations, particles have the same quantum numbers. For example, the leptons: electron $(e)$, muon $(\mu)$, tau $(\tau)$ all have the same charge and spin, but the $\tau$ is more massive than the $\mu$, which is more massive than the $e$.

The matter particles interact with each other through the exchange of force-carrying particles corresponding to the electromagnetic, weak, and strong forces. These particles are bosons, that is they are particles which have an intrinsic spin equal to integer multiples of $\hbar$, and are summarized in Table 1.2 .

All particles with electric charge interact electromagnetically through the exchange of a photon, which is a massless particle with a spin of 1 . Because the photon is massless, the electromagnetic force has an infinite range. All fermions also interact via the weak force. The weak force is mediated by the massive $W^{ \pm}$and $Z$ bosons. The masses of the weak 
Table 1.1: The three generations of Fermions [1].

\begin{tabular}{|c|c|c|c|}
\hline Fermion & Generation 1 & Generation 2 & Generation 3 \\
\hline Lepton & Electron $(\mathrm{e})$ & Muon $(\mu)$ & Tau $(\tau)$ \\
Electric Charge & -1 & -1 & -1 \\
Mass (MeV) & $0.510998910 \pm 0.000000013$ & $105.658367 \pm 0.000004$ & $1176.86 \pm 0.16$ \\
\hline Neutrino & Electron $\left(\nu_{e}\right)$ & Muon $\left(\nu_{\mu}\right)$ & Tau $\left(\nu_{\tau}\right)$ \\
Electric Charge & 0 & 0 & 0 \\
Mass (MeV) & $<2 \times 10^{-6}$ & $<0.19$ & $<18.2$ \\
\hline Quark & up $(u)$ & charm $(c)$ & top $(t)$ \\
Electric Charge & $2 / 3$ & $2 / 3$ & $2 / 3$ \\
Mass (MeV) & $2.3_{-0.5}^{+0.7}$ & $1.275 \pm 0.025 \times 10^{3}$ & $173.07 \pm 0.89 \times 10^{3}$ \\
\hline Quark & down $(d)$ & strange $(s)$ & bottom $(b)$ \\
Electric Charge & $-1 / 3$ & $-1 / 3$ & $-1 / 3$ \\
Mass (MeV) & $4.8_{-0.3}^{+0.5}$ & $95 \pm 5$ & $4.18 \pm 0.03 \times 10^{3}$ \\
\hline
\end{tabular}

Table 1.2: The force carrying bosons [1].

\begin{tabular}{|c|c|c|}
\hline Particle & Force & Mass $(\mathrm{GeV})$ \\
\hline & & \\
photon $(\gamma)$ & Electromagnetic & $<10^{-27}$ \\
$Z^{0}$ & Weak & $91.1876 \pm 0.0021$ \\
$W^{ \pm}$ & Weak & $80.399 \pm 0.023$ \\
gluon $(g)$ & Strong & $<O\left(10^{-3}\right)$ \\
\hline
\end{tabular}

bosons lead to a short interaction range $\left(<10^{-16} \mathrm{~m}\right)$. The strong force is mediated by the massless gluon. Gluons are electrically neutral but carry colour charge and interact with quarks (which also carry a colour charge). Because gluons themselves have a colour charge, they can interact with themselves.

While the electromagnetic and weak interactions look quite different, they are actually two aspects of the same interaction. The "weakness" of the weak interactions is due to the limited interaction range rather than the inherent interaction strength. The masses of the 
$W$ and $Z$ bosons, and thus the differences between electromagnetic and weak interaction strengths, require an explanation. In the standard model, this is achieved through spontaneous electroweak symmetry breaking and the introduction of another boson called the Higgs boson.

\subsection{The Standard Model}

The standard model combines quantum mechanics and special relativity into a theory framework to describe the interactions amongst the fundamental particles. In this framework particles are interpreted as excitations of relativistic quantum fields. The behaviour of these fields is described by the SM Lagrangian. This Lagrangian is a function of the fields, and is described below $[9,10,11]$.

\subsubsection{QED}

To describe the theory that governs the electromagnetic interactions, we will start with the simple theory of a free electron. The Lagrangian for such a theory is:

$$
L=\bar{\psi}\left(i \gamma^{\mu} \partial_{\mu}-m\right) \psi
$$

where $\psi$ is a spinor field for the spin $1 / 2$ electron of mass $m$, and $\gamma^{\mu}$ are the Dirac matrices ${ }^{1}$. This Lagrangian is invariant under a global gauge transformation. That is, if we transform

${ }^{1}$ The Dirac matrices are $4 \times 4$ matrices defined as: $\gamma^{0}=\left(\begin{array}{ll}1 & 0 \\ 0 & 1\end{array}\right), \gamma^{i=1,2,3}=\left(\begin{array}{cc}0 & \sigma_{i} \\ -\sigma_{i} & 0\end{array}\right)$, where $\sigma_{i}$ are the Pauli matrices 
the phase of $\psi$ globally:

$$
\psi(x) \rightarrow \psi(x)^{\prime}=e^{-i \alpha} \psi(x)
$$

and

$$
\bar{\psi}(x) \rightarrow \bar{\psi}(x)^{\prime}=e^{i \alpha} \bar{\psi}(x)
$$

the Lagrangian remains unchanged. Now consider the Lagrangian under a local gauge transformation:

$$
\psi(x) \rightarrow \psi(x)^{\prime}=e^{-i \alpha(x)} \psi(x)
$$

and

$$
\bar{\psi}(x) \rightarrow \bar{\psi}(x)^{\prime}=e^{i \alpha(x)} \bar{\psi}(x)
$$

The $\bar{\psi} m \psi$ term in Eq. 1.1 is clearly invariant under a local gauge transformation, but the $\bar{\psi} \partial_{\mu} \psi$ term is not. Thus, if we want our Lagrangian to be invariant under a local gauge transformation, we require a gauge covariant derivative, $D_{\mu}$ to replace $\partial_{\mu}$ such that:

$$
\bar{\psi}(x) D_{\mu} \psi(x) \rightarrow \bar{\psi}^{\prime}(x) D_{\mu} \psi^{\prime}(x)=\bar{\psi}(x) D_{\mu} \psi(x)
$$

We can accomplish this by introducing a vector field $A_{\mu}(x)$ with coupling strength $e$ and write the covariant derivative as:

$$
D_{\mu}=\partial_{\mu}+i e A_{\mu}
$$

with the requirement that $A_{\mu}$ transforms as: 


$$
A_{\mu} \rightarrow A_{\mu}^{\prime}=A_{\mu}+\frac{1}{e} \partial_{\mu} \alpha(x)
$$

If we also introduce the field tensor to include the dynamics of the gauge field:

$$
F_{\mu \nu}=\partial_{\mu} A_{\nu}-\partial_{\nu} A_{\mu}
$$

we can then write our gauge invariant Lagrangian as:

$$
L=\bar{\psi}\left(i \gamma^{\mu} D_{\mu}-m\right) \psi-\frac{1}{4} F_{\mu \nu} F^{\mu \nu}
$$

Alternatively, if we expand out the covariant derivative:

$$
L=\bar{\psi}\left(i \gamma^{\mu} \partial_{\mu}-m\right) \psi-e \bar{\psi} \gamma^{\mu} \psi A_{\mu}-\frac{1}{4} F_{\mu \nu} F^{\mu \nu}
$$

From this we can clearly see three different pieces. The first piece, $\bar{\psi}\left(i \gamma^{\mu} \partial_{\mu}-m\right) \psi$, is the free electron Lagrangian we started with. The third part, $\frac{1}{4} F_{\mu \nu} F^{\mu \nu}$ describes the dynamics of the gauge field, $A_{\mu}$. The second part, $e \bar{\psi} \gamma^{\mu} \psi A_{\mu}$, describes the interaction between the spinor field $\psi$ and the gauge field $A_{\mu}$. We can identify $A_{\mu}$ as the field for the photon, that interacts with our spinor field with strength $e$, which we can identify as the electric charge. Note that there are no terms of the form $A^{\mu} A_{\mu}$. These terms would be photon mass terms, but can not be included in our Lagrangian as they are not invariant under local gauge transformations. 


\subsubsection{QCD}

Quantum Chromodynamics (QCD) describes strong force interactions between quarks and gluons. The QCD Lagrangian can be constructed in an analogous way to the QED Lagrangian by requiring invariance under $\mathrm{SU}(3)$ local gauge transformations of the form:

$$
\psi \rightarrow U \psi
$$

where $U$ is a unitary $3 \times 3$ matrix. The QCD Lagrangian is given by:

$$
L=\sum_{j} \bar{\psi}\left(i \gamma^{\mu} D_{\mu}-m_{j}\right) \psi-\frac{1}{4} G_{\mu \nu}^{a} G_{a}^{\mu \nu}
$$

where $j$ corresponds to one of the six quark flavours, and the index $a$ is summed over the eight colour degrees of freedom. $G_{\mu \nu}^{a}$ is the gluon field tensor, equivalent to $F_{\mu \nu}$ in QED, and is given by:

$$
G_{\mu \nu}^{a}=\partial_{\mu} C_{\nu}^{a}-\partial_{\nu} C_{\mu}^{a}+g f^{a b c} C_{\mu}^{b} C_{\nu}^{c}
$$

and the covariant derivative is:

$$
D_{\mu}=\partial_{\mu}+i g_{s} C_{\mu}^{a} t^{a}
$$

Here $C_{\mu}$ is the vector field of the gluons, and $t^{a}$ are the generators of the $\mathrm{SU}(3)$ gauge group. The full QCD Lagrangian can be written in three pieces: a kinetic piece, $L_{k i n e t i c}$; a quark-gluon interaction piece, $L_{\text {quark-gluon }}$; and a gluon self-interaction piece $L_{\text {gluon-gluon }}$.

$$
L_{\text {kinetic }}=-\frac{1}{4}\left(\partial_{\mu} C_{\nu}^{a}-\partial_{\nu} C_{\mu}^{a}\right)+\sum_{j}^{\text {flavours }} \bar{\psi}_{j}\left(i \gamma^{\mu} \partial_{\mu}-m_{j}\right) \psi_{j}
$$




$$
\begin{aligned}
L_{\text {quark-gluon }} & =-g_{s} \sum_{j}^{\text {flavours }} C_{\mu}^{a} \bar{\psi}_{j} \gamma^{\mu} C_{\mu}^{a} t^{a} \psi_{j} \\
L_{\text {gluon-gluon }} & =\frac{g_{s}}{2} f^{a b c}\left(\partial_{\mu} C_{\nu}^{a}-\partial_{\nu} C_{\mu}^{a}\right)\left(C_{b}^{\mu} C_{c}^{\nu}\right)-\frac{g_{s}}{2} f^{a b c} f_{a d e} C_{b}^{\mu} C_{c}^{\nu} C_{\mu}^{d} C_{\nu}^{e}
\end{aligned}
$$

The gluon self interaction term comes from the gluons themselves carrying colour charge, and does not have an equivalent in QED. There are a couple of properties of QCD that are not obvious from the Lagrangian. The first is that quarks are confined to live within colour neutral hadrons (either as a colour-anticolour pair, or as a triplet of the three different colours). From an experimental perspective, this has the implication that we don't measure individual quarks within our detector, but rather composite particles called hadrons. The second property is known as asymptotic freedom. At high energies, QCD becomes a perturbative theory, and quarks and gluons behave as though they are free particles. The implication of this is that we can treat the interaction of protons and anti-protons at high energy as an interaction between a single quark or gluon from each proton and anti-proton.

\subsubsection{Electroweak Unification and the Higgs Mechanism}

The electromagnetic and weak forces are actually two facets of the same force. The unification of the two forces is completed within the $S U(2)_{L} \times U(1)_{Y}$ gauge group, with the $S U(2)_{L}$ group representing weak isospin space and $U(1)_{Y}$ representing hypercharge space. This representation is consistent with only left handed fermions being able to undergo flavour changing weak interactions. The three gauge fields of $S U(2)_{L}$ are $W_{\mu}^{i=1,2,3}$ with coupling $g$,

and the gauge field of $U(1)_{Y}$ is $B_{\mu \nu}$ with coupling $g^{\prime}$. The kinetic term of the Lagrangian is given by:

$$
L^{\text {kinetic }}=-\frac{1}{4} W_{a}^{\mu \nu} W_{\mu \nu}^{a}-\frac{1}{4} B^{\mu \nu} B_{\mu \nu}
$$


where

$$
\begin{gathered}
W_{\mu \nu}^{i}=\partial_{\mu} W_{\nu}^{i}-\partial_{\nu} W_{\mu}^{i}+g \epsilon^{i j k} A_{\mu}^{j} A_{\nu}^{k}, \\
B_{\mu \nu}=\partial_{\mu} B_{\nu}-\partial_{\nu} B_{\mu} .
\end{gathered}
$$

Since leptons have both right and left handed components but only left handed neutrinos exist, it is natural to write the interactions with the gauge fields in two pieces. The first is a purely right handed interaction with a singlet of a lepton field, $R$, that interacts only with the $B_{\mu}$ field:

$$
L^{\text {singlet }}=i \bar{R} \gamma^{\mu}\left(\partial_{\mu}+i g^{\prime} \frac{Y}{2} B_{\mu}\right) R
$$

The left handed lepton doublet of the lepton and neutrino fields:

$$
L=\left(\begin{array}{c}
\nu \\
\ell^{-}
\end{array}\right)
$$

interacts with fields from both groups:

$$
L^{\text {doublet }}=i \bar{L} \gamma^{\mu}\left(\partial_{\mu}+i g^{\prime} \frac{Y}{2} B_{\mu}+i g \frac{\sigma_{a}}{2} W_{\mu}^{a}\right) L
$$

The physical fields that we observe for the photon, $W^{ \pm}$, and $Z$ can be written in terms of these gauge fields and their couplings:

$$
\begin{array}{r}
W_{\mu}^{ \pm}=\frac{1}{\sqrt{2}}\left(W_{\mu}^{1} \pm W_{\mu}^{2}\right) \\
Z_{\mu}=-\sin \left(\theta_{W}\right) B_{\mu}+\cos \left(\theta_{W}\right) W_{\mu}^{3} \\
A_{\mu}=\cos \left(\theta_{W}\right) B_{\mu}+\sin \left(\theta_{W}\right) W_{\mu}^{3}
\end{array}
$$


where $\theta_{W}$ is the weak mixing angle (also called the Weinberg angle) which mixes the $B_{\mu}$ and $W_{\mu}^{3}$ fields is defined as:

$$
\cos \left(\theta_{W}\right)=\frac{g}{\sqrt{g^{2}+g^{\prime 2}}}
$$

One important thing to notice is that, so far, we haven't included any mass terms for the gauge bosons. However, we know from experimental observations that the $W$ and $Z$ bosons do indeed have mass. Explicitly adding such terms to the Lagrangian would not preserve local gauge invariance, so we require some mechanism to give the weak bosons mass. One such method is to spontaneously break a symmetry. Electroweak spontaneous symmetry breaking was proposed as a mechanism to include massive gauge bosons within the standard model in 1964 by Higgs, Englert, Brout, Guralnik, Hagen, and Kibble [12, 13, 14], for which Higgs and Englert were recently awarded the Nobel prize in physics [15].

To illustrate this phenomenon, consider the Lagrangian for a real scalar field, $\phi$, in a potential $V(\phi)=\frac{1}{2} \mu^{2} \phi^{2}+\frac{1}{4} \lambda \phi^{4}$ :

$$
L=\frac{1}{2}\left(\partial_{\mu} \phi\right)^{2}-\left(\frac{1}{2} \mu^{2} \phi^{2}+\frac{1}{4} \lambda \phi^{4}\right)
$$

If $\mu^{2}>0$, then the particle has a mass of $\mu$ and the fourth order self-interaction strength of the field, $\phi$, is $\lambda$. If we minimize the potential $V(\phi)$, we find that the ground state, or vacuum, corresponds to $\phi=0$. We can also consider the case where $\mu^{2}<0$. In this case we find that the ground state of the potential corresponds to $\phi= \pm v$, with $v=\sqrt{\mu^{2} / \lambda}$. The two ground states are completely equivalent, and when we choose one or the other as a point to perturbatively expand about, we spontaneously break the symmetry. We will choose to 
perturbatively expand our Lagrangian around the $+v$ ground state, and will write the field as $\phi(x)=v+\eta(x)$. Our Lagrangian can then be written in terms of $v, \eta$ and $\lambda$ :

$$
L^{\prime}=\frac{1}{2}\left(\partial_{\mu} \eta\right)^{2}-\lambda v^{2} \eta^{2}-\lambda v \eta^{3}-\frac{1}{4} \lambda \eta^{4}+\frac{1}{4} \lambda v^{4} .
$$

We can now see that we have a field $\eta$ with a mass of $\sqrt{2 \lambda} v$. Ultimately, we would like a massive gauge field, $a^{\mu}$, with charge, $q$. Luckily, our example can be easily taken a step further by considering a locally gauge invariant complex scalar field, $\phi=\frac{1}{2}\left(\phi_{1}+i \phi_{2}\right)$ :

$$
L=D^{\mu} \phi^{*} D_{\mu} \phi-\frac{1}{4} F^{\mu \nu} F_{\mu \nu}-\mu^{2} \phi^{*} \phi-\lambda\left(\phi^{*} \phi\right)^{2},
$$

where:

$$
\begin{gathered}
D_{\mu}=\partial_{\mu}+i q a_{\mu}, \\
F_{\mu \nu}=\partial_{\mu} a_{\nu}-\partial_{\nu} a_{\mu} .
\end{gathered}
$$

If we again consider the case where $\mu^{2}<0$, we see that the minimum is a circle in $\phi_{1}-\phi_{2}$ space:

$$
\phi_{1}^{2}+\phi_{2}^{2}=v^{2}=\sqrt{\frac{-\mu^{2}}{\lambda}}
$$

We can spontaneously break the symmetry and choose $\phi_{1}=v$ and $\phi_{2}=0$, and expand around this minimum:

$$
\phi(x)=\frac{1}{\sqrt{2}}(v+\eta(x)+i \epsilon(x)),
$$


and

$$
L^{\prime}=\frac{1}{2}\left[\left(\partial_{\mu} \eta\right)^{2}+\left(\partial_{\mu} \epsilon\right)^{2}\right]-\frac{1}{4} F^{\mu \nu} F_{\mu \nu}-v^{2} \lambda \eta^{2}+\frac{1}{2} q^{2} v^{2} a_{\mu} a^{\mu}-q v a_{\mu} \partial^{\mu} \epsilon .
$$

Similar to the previous example, we can see that we have a scalar field $\eta$ with mass $\sqrt{2 \lambda} v$. We also see that we have a massless scalar field, $\epsilon$. Additionally, our gauge field, $a_{\mu}$, now has a mass of $q v$. The masslessness of the $\epsilon$ field comes from the process of breaking the symmetry, and is somewhat intuitive. The potential in the tangential $(\epsilon)$ direction is flat, which means there is no resistance to oscillations in the $\epsilon$ direction, which leads to a massless field. Whenever we break a continuous symmetry spontaneously, we will end up with a massless scalar field. We don't observe such extra massless fields in nature, so how should we interpret them? We can note that in the lowest order of $\epsilon$, our complex scalar field can be written as:

$$
\begin{aligned}
\phi & =\sqrt{\frac{1}{2}}(v+\eta+i \epsilon) \\
& \simeq \sqrt{\frac{1}{2}}(v+\eta) e^{i \epsilon / v} .
\end{aligned}
$$

We can consider rewriting our Lagrangian, choosing a specific gauge, by expressing everything in terms of real fields $h, \theta$, and $a_{\mu}$ with:

$$
\begin{array}{r}
\phi \quad \rightarrow \sqrt{\frac{1}{2}}(v+h) e^{i \theta / v}, \\
a_{\mu} \quad \rightarrow a_{\mu}+\frac{1}{q v} \partial_{\mu} \theta .
\end{array}
$$

Here, $\theta$ is chosen in such a way that $h$ is real. This choice of gauge renders our Lagrangian 
independent of $\theta$, so that the non-physical massless field that appeared from the spontaneous symmetry breaking is now nowhere to be seen:

$L^{\prime \prime}=\frac{1}{2}\left(\partial_{\mu} h^{2}\right)-\frac{1}{4} F^{\mu \nu} F_{\mu \nu}-v^{2} \lambda h^{2}+\frac{1}{2} q^{2} v^{2} a_{\mu} a^{\mu}-v \lambda h^{3}-\frac{1}{4} \lambda h^{4}+\frac{1}{2} q^{2} a_{\mu} a^{\mu} h^{2}+v q^{2} a_{\mu} a^{\mu}$.

This is because the apparent extra degree of freedom of the massless field was actually just the freedom to make a gauge choice. We are now left with a Lagrangian for two massive fields: a massive gauge field $a_{\mu}$, and a massive scalar field $h$. This process is known as the Higgs mechanism, and the field $h$ is called the Higgs field.

We can now go even further, and apply this same procedure to a Lagrangian invariant under $S U(2)_{L} \times U(1)_{Y}$ transformations. In this case, we will need to couple to $\mathrm{SU}(2)$ doublets with four degrees of freedom, so our field, $\Phi$ is now a doublet of complex scalar fields:

$$
\Phi=\left(\begin{array}{c}
\phi^{+} \\
\phi^{0}
\end{array}\right)
$$

with

$$
\begin{aligned}
\phi^{+} & =\sqrt{\frac{1}{2}}\left(\phi_{1}+i \phi_{2}\right), \\
\phi^{0} & =\sqrt{\frac{1}{2}}\left(\phi_{3}+i \phi_{4}\right) .
\end{aligned}
$$

We again consider the $\mu^{2}<0$ case, and find the minimum of our potential. We choose 
the minimum point to do our perturbative expansion around to be:

$$
\Phi=\sqrt{\frac{1}{2}}\left(\begin{array}{l}
0 \\
v
\end{array}\right)
$$

The choice of only allowing the neutral component to be non-zero is to conserve electric charge. If we expand our Lagrangian about this minimum, we find that we get a massless field, $A_{\mu}$, corresponding to the photon, and two massive gauge bosons. In the end, we find:

$$
\begin{array}{cc}
M_{W} & =\frac{1}{2} v g \\
M_{A} & =0 \\
M_{Z} & =\frac{1}{2} v \sqrt{g^{2}+g^{\prime 2}} \\
\frac{M_{W}}{M_{Z}} & =\cos \theta_{W}
\end{array}
$$

We now have our weak bosons with non-zero masses, a massless photon, plus an additional massive scalar boson, the Higgs boson. It is worth noting that the masses of the $W$ and $Z$ bosons are predicted theoretically, however the Higgs boson mass is not. Also, our fermions are still massless, as we could not simply add in an explicit mass term to our electroweak Lagrangian and still preserve gauge invariance. The Higgs mechanism comes to the rescue again. Let's consider including the following term in our electroweak Lagrangian:

$$
L_{\ell}=-g_{e}\left[\left(\overline{\nu_{\ell}}, \bar{\ell}\right)_{L}\left(\begin{array}{c}
\phi^{+} \\
\phi^{0}
\end{array}\right) \ell_{R}+\bar{\ell}_{R}\left(\phi^{-}, \phi^{0}\right)\left(\begin{array}{c}
\nu_{\ell} \\
\ell
\end{array}\right)_{L}\right] .
$$


We, as before, break the symmetry spontaneously with:

$$
\phi=\sqrt{\frac{1}{2}}\left(\begin{array}{c}
0 \\
v+h
\end{array}\right)
$$

so that out Lagrangian becomes:

$$
L_{\ell}=-m_{\ell} \overline{\ell \ell}-\frac{m_{\ell}}{v} \bar{\ell} \ell h
$$

where we have defined the lepton mass:

$$
m_{\ell}=\frac{g_{\ell} v}{\sqrt{2}}
$$

Notice that the lepton masses are not predicted, as $g_{\ell}$ is not given.

Quark masses arise similarly from starting with the Lagrangian:

$$
L_{q}=-g_{d}^{i j}\left(\bar{u}_{i}, \bar{d}_{i^{\prime}}\right)_{L}\left(\begin{array}{c}
\phi^{+} \\
\phi^{0}
\end{array}\right) d_{j R}-g_{u}^{i j}\left(\bar{u}_{i}, \bar{d}_{i^{\prime}}\right)_{L}\left(\begin{array}{c}
-\bar{\phi}^{0} \\
\phi^{-}
\end{array}\right) u_{j R}+\text { hermetian conjugate }
$$

where $u$ and $d$ correspond to up-type and down-type quarks respectively. After spontaneous symmetry breaking this becomes:

$$
L_{q}=-m_{d}^{i} \bar{d}_{i} d_{i}\left(1+\frac{h}{v}\right)-m_{u}^{i} \bar{u}_{i} u_{i}\left(1+\frac{h}{v}\right) .
$$




\subsubsection{Properties of the Higgs Boson}

While the Higgs boson was first predicted to exist about 50 years ago, we are only now reaching the point where we can experimentally probe it. The standard model does not predict the mass of this Higgs boson, so typically experiments look over a large range of masses.

This mass range can be constrained experimentally by precision electroweak measurements and by direct searches for the Higgs boson. Indirect constraints on the Higgs boson mass come from measuring precisely the mass of the $W$ boson and top quark as shown in Fig. 1.1 [3]. Diagrams such as those in Fig. 1.2 show how the $W$ boson mass is related to the top quark mass and the Higgs boson mass. Direct constraints have also been placed on the Higgs boson mass. Searches for the Higgs boson at LEP constrain the Higgs boson mass to be greater than $114.4 \mathrm{GeV}$ [16]. The Tevatron additionally excludes masses $150<M_{H}<180 \mathrm{GeV}[8]$

In July 2012, the ATLAS and CMS experiments excluded masses up to about $500 \mathrm{GeV}$ with the exception of a narrow region centred on about $125 \mathrm{GeV}[4,5]$ as shown in Fig. 1.3. In this narrow region, both experiments reported discovery ${ }^{2}$ of a new particle with a significance of 5 standard deviations above the background-only expectation. At the same time the Tevatron experiments reported evidence of a particle decaying to b-quarks with a significance of three standard deviations above the background only expectation in the range of 115$140 \mathrm{GeV}[17]$.

For a given mass of the Higgs boson, the SM does predict the production cross section,

\footnotetext{
2In particle physics, the words "evidence" and "discovery" have specific statistical meanings. If the data are 3 standard deviations above the background-only expectation, that corresponds to "evidence" of something new. If the data are 5 standard deviations above the background-only expectation, that is referred to as a "discovery".
} 


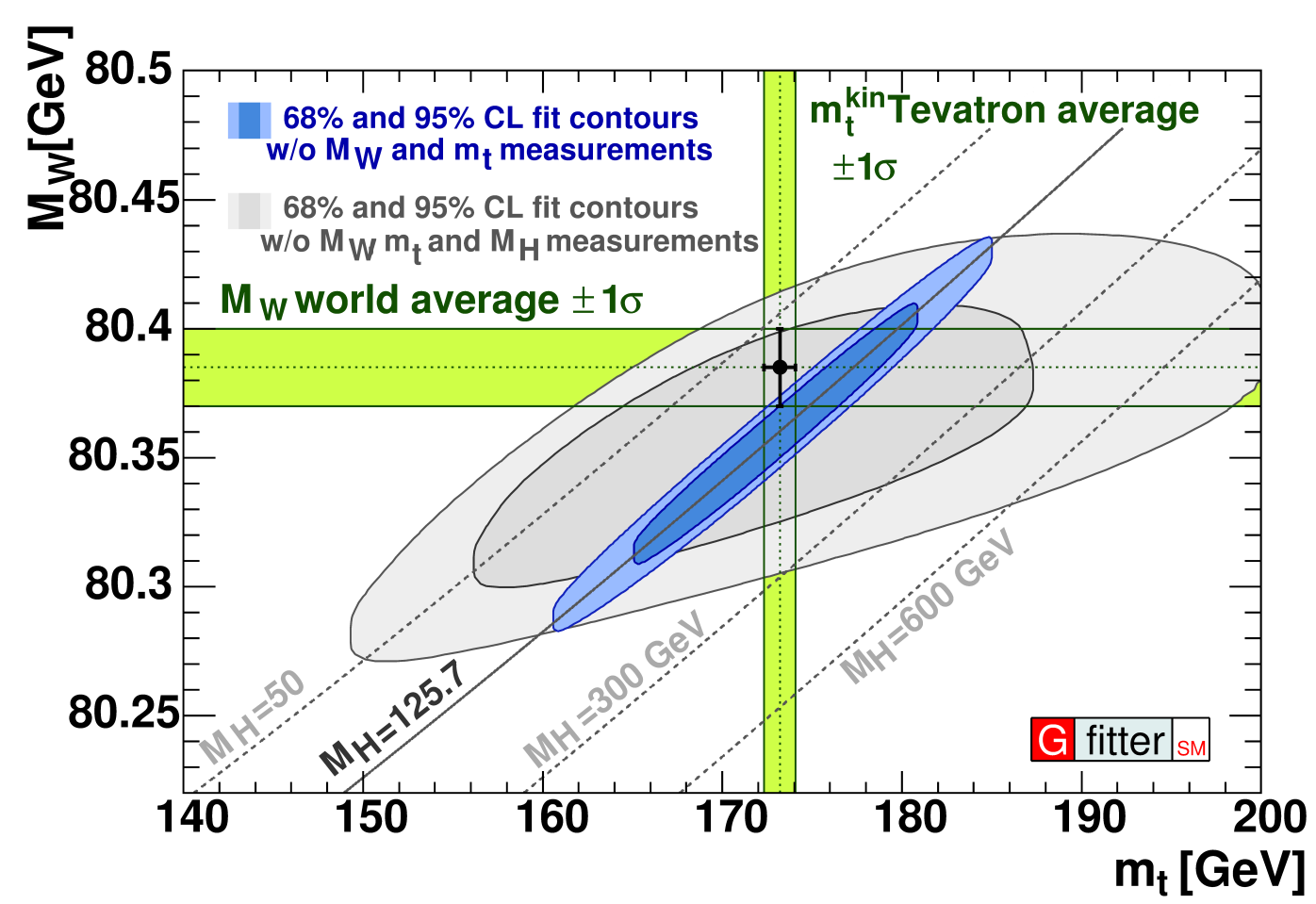

Figure 1.1: For the interpretation of the references to colour in this and all other figures, the reader is referred to the electronic version of this dissertation. A global fit to data from precision electroweak experiments can place constraints on the $W$ boson and top quark masses. Shown here are the $68 \%$ and $95 \%$ contours from this fit including the Higgs boson mass measurement in blue, and not including the Higgs boson mass measurement in gray. The horizontal and vertical green bands correspond to 1 standard deviation on the $W$ boson and top quark masses respectively. The diagonal lines show where different values for the Higgs mass would appear in the $W$-top mass plane [3].
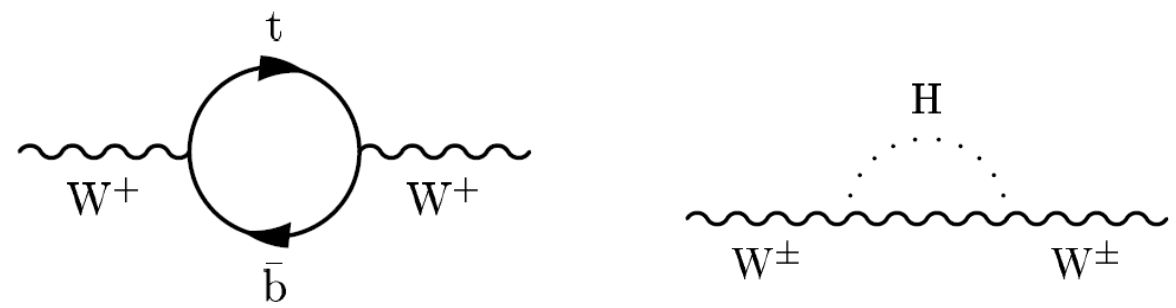

Figure 1.2: The masses of the top quark, $W$ boson, and Higgs boson are related through loop diagrams. 

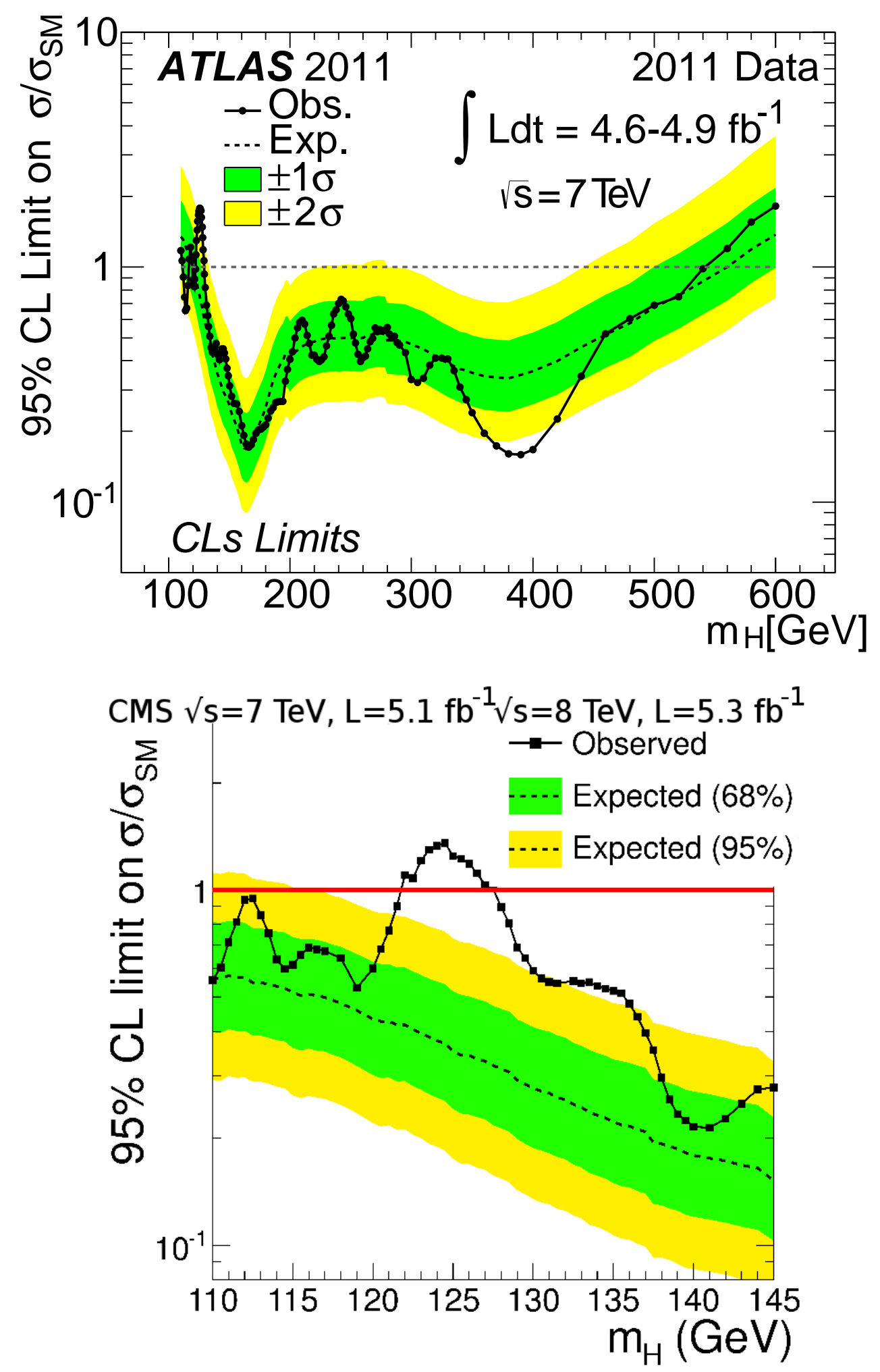

Figure 1.3: 95\% confidence level upper limit on Higgs boson production cross section as a ratio to the Standard Model cross section from the ATLAS experiment (top) [4] and the CMS experiment (bottom) [5]. Masses up to $500 \mathrm{GeV}$ are excluded, with the exception of a small window of masses centred around $125 \mathrm{GeV}$. 
and the decay rate for the Higgs boson into all allowed decay particles as is shown in Figs. 1.4 and 1.5 respectively. The dominant production mode for a Higgs boson at the Tevatron with mass of $M_{H}=125 \mathrm{GeV}$ is through gluon-gluon fusion, followed by associated production of the Higgs boson with a vector boson $V=W, Z$, fusion of two vector bosons, and production of a Higgs boson produced in association with a pair of top quarks (see Fig. 1.6). The dominant decay mode is into a pair of $b$ quarks, followed by decay into $W$ bosons. Searches for $g g \rightarrow H \rightarrow b \bar{b}$ tend to be overwhelmed by jet production in hadron colliders. For this

reason, when searching for $H \rightarrow b \bar{b}$, it is common to look for the Higgs boson produced in association with a $W$ or $Z$ boson, where the $W$ or $Z$ decays to leptons (which are produced less frequently in a hadron collider, and are thus a unique signature relative to the background of multijet production). This thesis focusses on the search for a Higgs boson produced in association with a $W$ boson, where the Higgs boson decays to b quarks, and the $W$ boson decays to a lepton, $\ell=\mu, e$ and a neutrino.

\subsection{Beyond the Standard Model}

While the SM has been incredibly successful, it does have some shortcomings. It does not explain dark matter or dark energy, or the asymmetry in the amount of matter over antimatter in the universe. It also does not include a description of gravity, or neutrino masses. Additionally, there are two energy scales within the SM that are very different. The first is the scale of electroweak symmetry breaking at $\sim 100 \mathrm{GeV}$. The second is the scale at which the electroweak and strong forces would be unified, $\sim 10^{16} \mathrm{GeV}$. Typically, the unification of electroweak and strong forces would mean that the Higgs boson mass would be on the order of the second scale. To get the mass back down to the first energy scale, one needs 


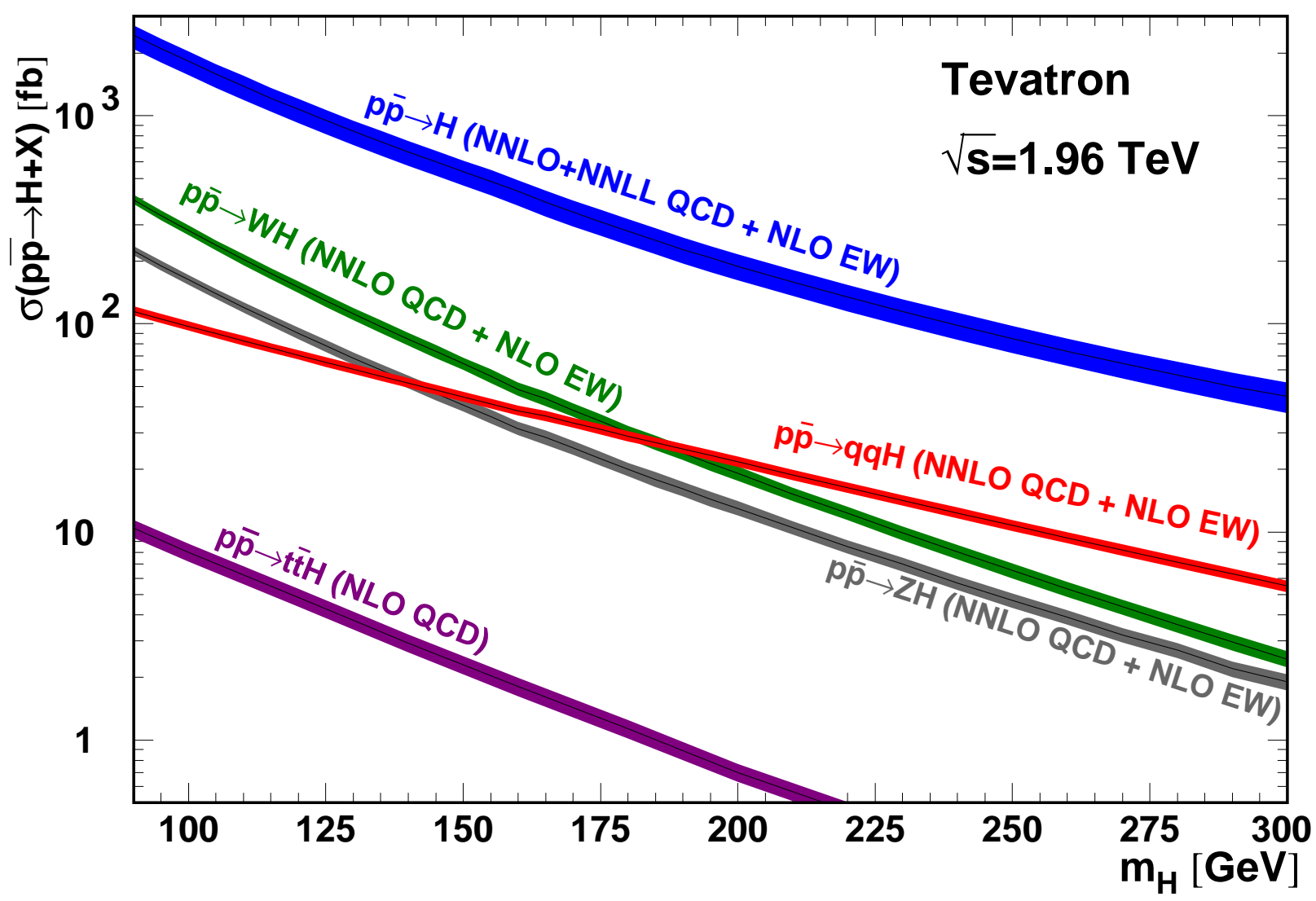

Figure 1.4: The production cross section for the Higgs boson as a function of Higgs boson mass at the Tevatron (where the center-of-momentum energy, $\sqrt{s}$, is $1.96 \mathrm{TeV}$ ). 


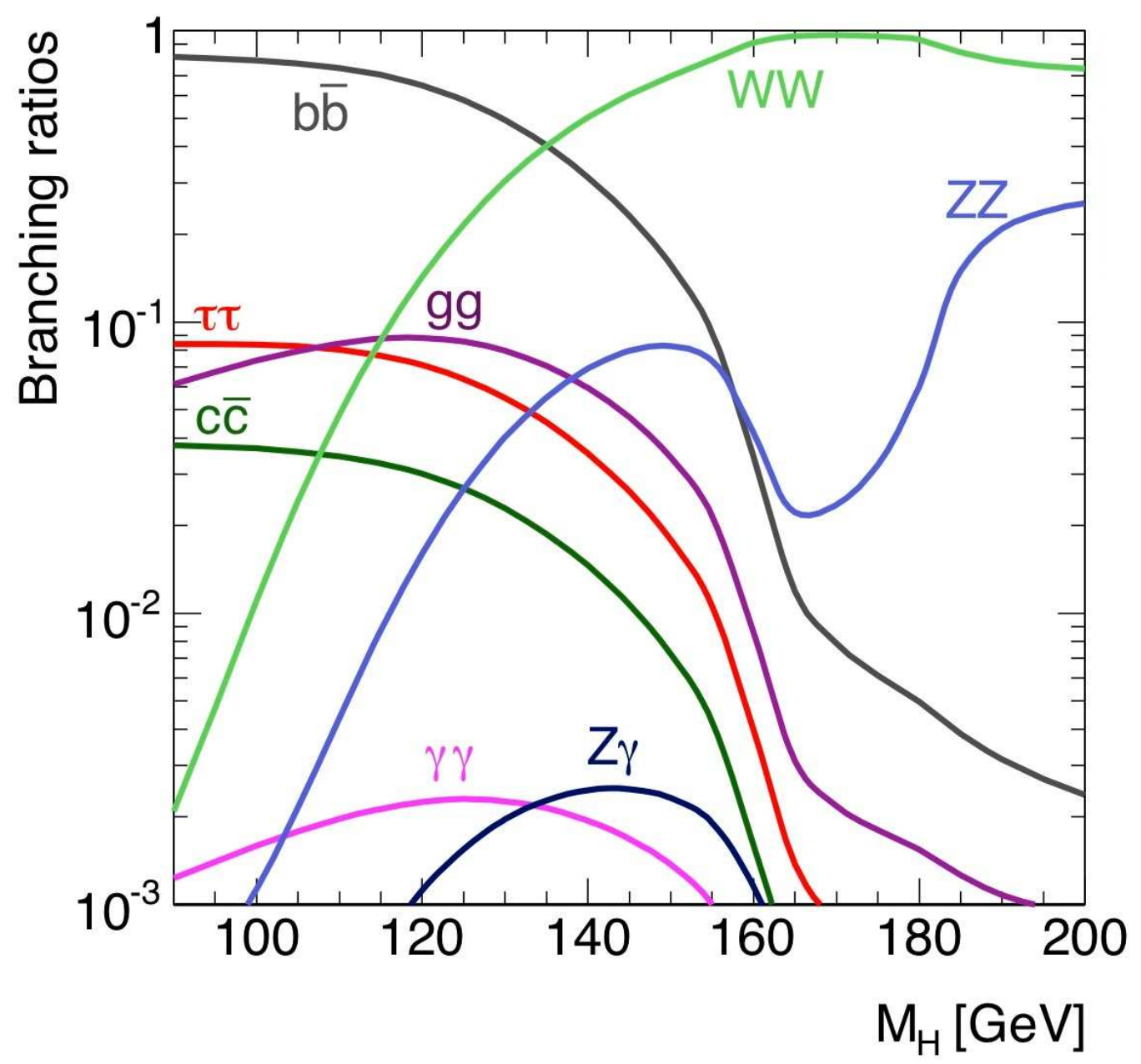

Figure 1.5: The decay rates for the Higgs boson as a function of Higgs boson mass.

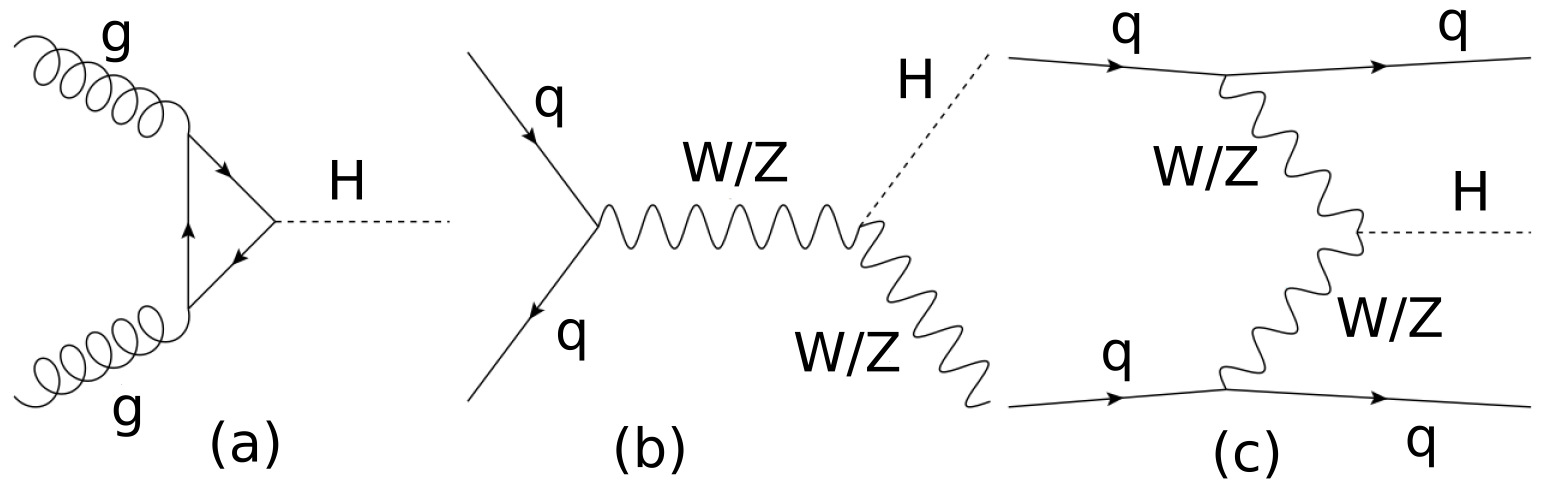

Figure 1.6: The Feynman diagrams for the most important Higgs boson production modes at the Tevatron: (a) gluon-gluon fusion, (b) associated production of a Higgs boson with a pair of top quarks, and (c) vector boson fusion. 
very finely tuned cancellations. Because the SM is not complete, there is motivation to look for something beyond the SM. There are many theories positing mechanisms and particles to address some of the SM's shortcomings.

Supersymmetry theories propose that all SM particles have supersymmetric partner particles, where fermionic particles have a bosonic partner particle, and bosonic SM particles have fermionic particles. These theories tend to contain multiple Higgs bosons, with one of the Higgs bosons strongly resembling the SM Higgs boson. There are also theories that predict a Higgs boson that couples only to some particles and not others (a common example is a Higgs boson that couples only to bosons with stronger bosonic couplings than are predicted in the SM). Some theories do not include a Higgs boson at all. For example, technicolour theories break electroweak symmetry and introduce the $W$ and $Z$ boson masses through new gauge interactions. Since we have now observed the Higgs boson, these theories now contradict the observed data. Based on the current experimental results, the favoured theory for mass generation is the Higgs mechanism, so I won't discuss these additional theories further. 


\section{Chapter 2}

\section{Experimental Apparatus}

In order to study the Higgs boson experimentally, one first needs to be able to produce and detect its decay products. To do this we need a carefully controlled high energy environment and the ability to measure such an environment. The Tevatron collider provided such an environment by colliding protons with anti-protons at high energies. The proton and antiproton collisions occurred at two points along the ring, and a detector was built around each of these collision points to detect the particles resulting from the collisions. Below, I will describe the accelerator and the D0 detector, which was used to record the data used in this thesis.

\section{$2.1 \quad$ The Tevatron}

The Tevatron [18] is the largest in a series of accelerators [19] located at the Fermi National Accelerator Laboratory (FNAL) complex in Batavia, Illinois, shown in Fig. 2.1. The Tevatron is a circular synchrotron that collided protons and anti-protons with a centerof-momentum energy of $1.96 \mathrm{TeV}$ at two locations around a ring with a radius of $1 \mathrm{~km}$. Multi-purpose detectors around each of these collision points measure properties of the particles resulting from the proton and anti-proton collisions. The running time of the Tevatron can be split into different data collection epochs. Run I took place from 1992-1996, and Run II from 2001-2011. Between Run I and Run II, upgrades to the accelerator made it possible 
to run at higher center-of-momentum energies and at higher instantaneous luminosities. The luminosity also increased over the course of Run II, and so Run II can also be divided into different data taking epochs: Run IIa, Run IIb1, Run IIb2, Run IIb3, and Run IIb4.

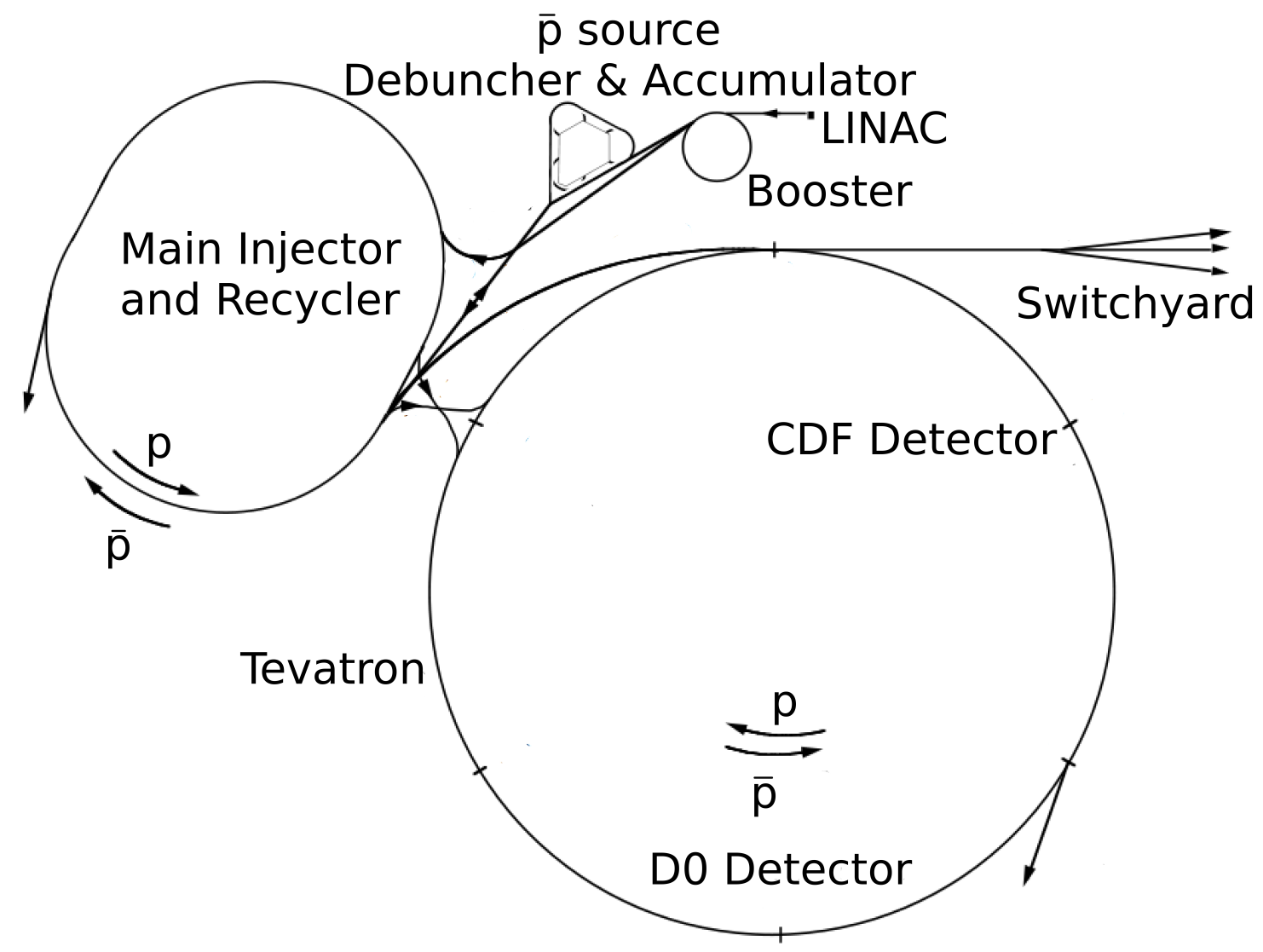

Figure 2.1: A schematic view of the accelerator chain at Fermilab.

The colliding protons start as hydrogen gas. The gas enters a magnetron ion source where the hydrogen is negatively ionized by a pulsed electric field. The $\mathrm{H}^{-}$ions then move to the first stage of acceleration. A Cockroft-Walton electrostatic accelerator accomplishes this initial acceleration, giving the $H^{-}$ions $750 \mathrm{keV}$ of kinetic energy. From the CockroftWalton accelerator, the ions go through a linear accelerator that accelerates the ions to 400 $\mathrm{MeV}$. When the ions leave the linear accelerator, they pass through a carbon foil which strips 
the electrons from the hydrogen ions leaving a beam of protons $\left(H^{+}\right)$. The protons then enter a circular synchrotron with a radius of $75 \mathrm{~m}$, called the booster, which accelerates the protons to $8 \mathrm{GeV}$ and groups the protons into bunches.

The protons then travel to the main injector from the booster. The main injector is another synchrotron that can accept both protons and anti-protons, accelerate them, change the beam structure, and then send the beam towards multiple destinations. Protons can either be injected into the Tevatron (after being accelerated to $150 \mathrm{GeV}$ ), or sent to the anti-proton source (after accelerating the protons to $120 \mathrm{GeV}$ ). To create anti-protons, the $120 \mathrm{GeV}$ proton beam from the main injector is collided with a nickel-iron-chromium alloy. This results in a spray of particles; about one in one million of which are anti-protons with an energy of about $8 \mathrm{GeV}$. The anti-protons are focussed into a beam and sent to the debuncher. The debuncher reduces the momentum spread of the beam, before the anti-protons head to the accumulator. The accumulator is a synchrotron that is used to store anti-protons until the desired beam intensity is reached. Once the desired intensity of anti-protons is reached, the anti-protons can be extracted into the main injector where they are accelerated to be sent to the Tevatron.

Once in the Tevatron, the protons and anti-protons are each accelerated to $980 \mathrm{GeV}$, and focused to collide at two points along the ring.

\subsection{The D0 Detector}

The D0 detector first started recording $p \bar{p}$ collisions during RunI of the Tevatron (19921996). Between 1996 and 2001, the accelerators went through several upgrades. The main injector and booster were built, the maximum center-of-momentum energy was increased 
from 1.8 to $1.96 \mathrm{TeV}$, and the number of colliding bunches was increased from 6 to 36 . The D0 detector also went through significant upgrades to handle this new collision environment. RunII of the Tevatron commenced in 2001 and continued through September 2011. The data presented in this thesis are from RunII, so the RunII detector is described below.

The D0 detector is one of two general purpose detectors located at one of the two collision points of the Tevatron [6], where protons and anti-protons collide once every 396 ns. The detector has a cylindrical design with concentric sub-detectors centred on the collision point, as shown in Fig. 2.2. Closest to the collision point is the tracking system which is enclosed in a superconducting solenoid magnet which creates a $1.92 \mathrm{~T}$ magnetic field. Surrounding this is a calorimeter, then the muon detection system, which includes a toroidal magnetic field.

The coordinate system of the detector is defined as having positive $z$ along the direction of travel of the proton beam, and positive $y$ pointing away from the center of the earth. The distance perpendicular to the $z$-axis is labelled by $r$, and the polar and azimuthal angles are labelled by $\theta$ and $\phi$ respectively. An useful quantity to consider is rapidity, $\mathcal{Y}$, which is invariant under Lorentz boosts along the z direction:

$$
\mathcal{Y}=\tanh ^{-1}\left(\frac{v}{c}\right),
$$

where $v$ is the speed of the particle, and $c$ is the speed of light. The pseudo-rapidity, $\eta$, is given by:

$$
\eta=-\ln \left(\tan \frac{\theta}{2}\right)=\frac{1}{2} \ln \left(\frac{|\vec{p}|+p_{z}}{|\vec{p}|-p_{z}}\right) .
$$

In the relativistic limit $\left(E>>m c^{2}\right)$, the pseudorapidity is approximately equal to the rapidity. Values of $\eta$ that are near 0 correspond to the central part of the D0 detector, and 


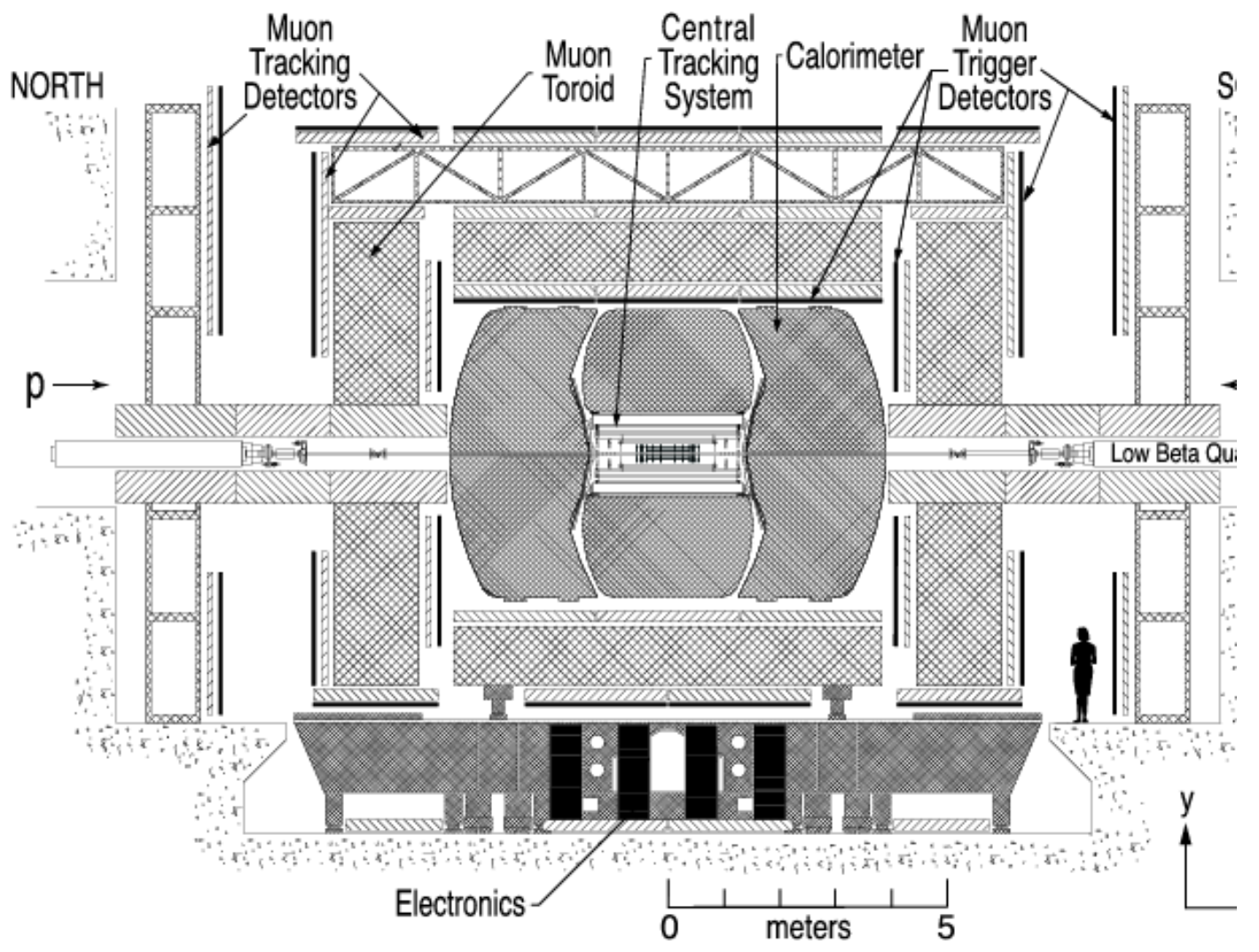

Figure 2.2: A cross sectional view of the D0 detector, showing the tracking system surrounded by the calorimeter and the muon detection system [6]. 
larger values of $|\eta|$ correspond to the ends (or forward regions) of the detector. Distances between objects are calculated in $\eta-\phi$ space using a distance:

$$
\Delta R=\sqrt{\Delta \eta^{2}+\Delta \phi^{2}} .
$$

The trajectories of charged particles are measured using the Silicon Microstrip Tracker (SMT) and the Central Fibre Tracker (CFT). The SMT is located directly outside of the beam pipe, and has an inner radius of $2.7 \mathrm{~cm}$ and an outer radius of $10.5 \mathrm{~cm}$. The $\mathrm{CFT}$ is outside located of the SMT, and has an inner radius of $20 \mathrm{~cm}$ and an outer radius of $52 \mathrm{~cm}$. Surrounding both detectors is a $1.92 \mathrm{~T}$ superconducting solenoid magnet that is $2.7 \mathrm{~m}$ thick, and has an inner (outer) radius of $1.1(1.4) \mathrm{m}$. The tracking system is shown in Fig. 2.3. The momentum of charged particles can be determined by measuring how much a particle curves as it moves through the detectors within the magnetic field. The tracking system is also used to determine the location of the primary interaction vertex (PV) with a resolution of $35 \mu \mathrm{m}$, as well as positions of secondary decay vertices from particles that decay within the tracking system.

The SMT is constructed from barrel and disk modules such that particle trajectories tend to be perpendicular to the detector surface [20]. The modules are made up of a series of silicon-doped wafers. A charged particle passing through the positive-negative (p-n) junction creates electron-hole pairs. A voltage difference across the wafers causes the electrons to drift to one side, where the charge is collected, digitized, and read out. The barrels measure 12 $\mathrm{cm}$ in length and are comprised of five layers of rectangular silicon wafers. Some of the wafers are single sided, while some are double sided to determine the particle position in the direction parallel to the strips. The double sided wafers have either a $2^{\circ}$ or $90^{\circ}$ angle 


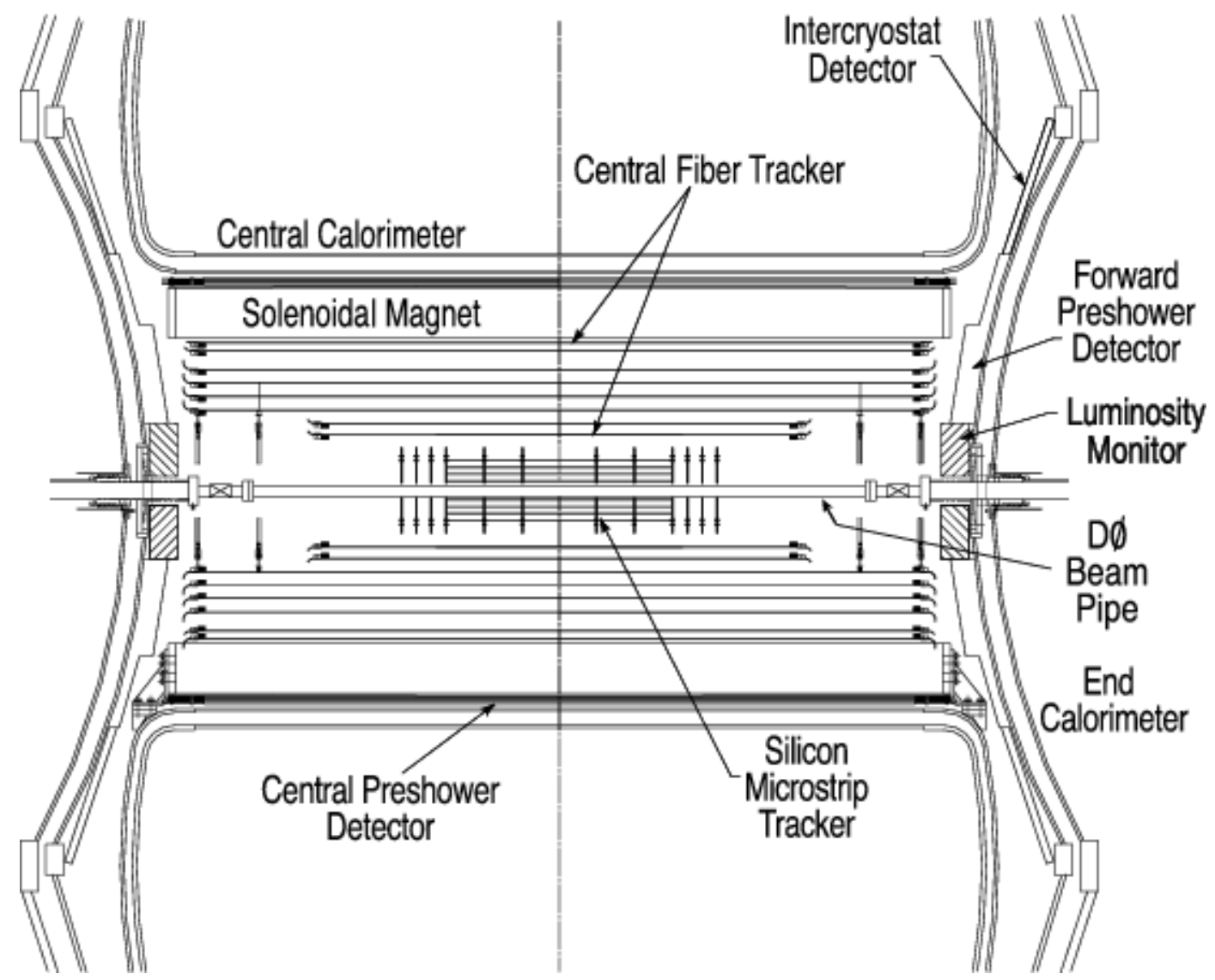

Figure 2.3: A cross sectional view of the D0 tracking system. Closest to the beampipe is the silicon microstrip tracker, surrounded by the central fibre tracker. The tracking system is contained within a superconducting solenoid [6]. 


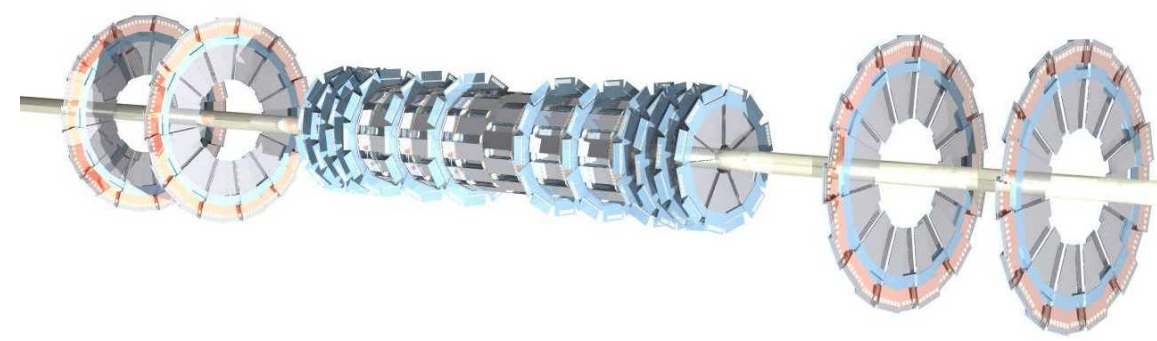

Figure 2.4: A view of the SMT.

between the axial (parallel to the $\mathrm{z}$ axis), and the stereo sides. The single sided wafers are all arranged axially. This allows positions to be measured in both the $r-\phi$ plane and the $r-z$ plane.

In addition to the barrels, there are also 12 disks, or F-disks to make measurements in the $r-z$ plane. The disks are comprised of wedge shaped double sided silicon modules. Each barrel is capped by a disk, with additional disks farther out in z, to detect forward tracks. The SMT system is shown in Fig. 2.4.

The CFT is made up of eight concentric layers of scintillating fibre. Each layer arranges four fibres into an axial and stereo layer doublet, with the axial fibres oriented parallel to the $\mathrm{z}$ axis, and the stereo fibres oriented at a $3^{\circ}$ angle. Charged particles travelling through the fibres excite the fibre core and photons are emitted with a wavelength of $340 \mathrm{~nm}$. The photons propagate down the internally reflective fibres, where they are read out by photon counters which can detect a single photon. The layout of the CFT is illustrated in Fig. 2.5.

The calorimeter is used to measure the energy of photons, electrons, and hadrons, as well as any imbalance in the transverse energy from which we infer the existence of neutrinos. The calorimeter consists of absorber plates of either Uranium, copper, or steel inserted in liquid argon, and is split into three pieces: the central calorimeter $(\mathrm{CC})$, which covers $|\eta|<1$; and two end cap calorimeters (EC), which extend coverage to $|\eta| \sim 4$, and is shown in Fig. 2.6. 


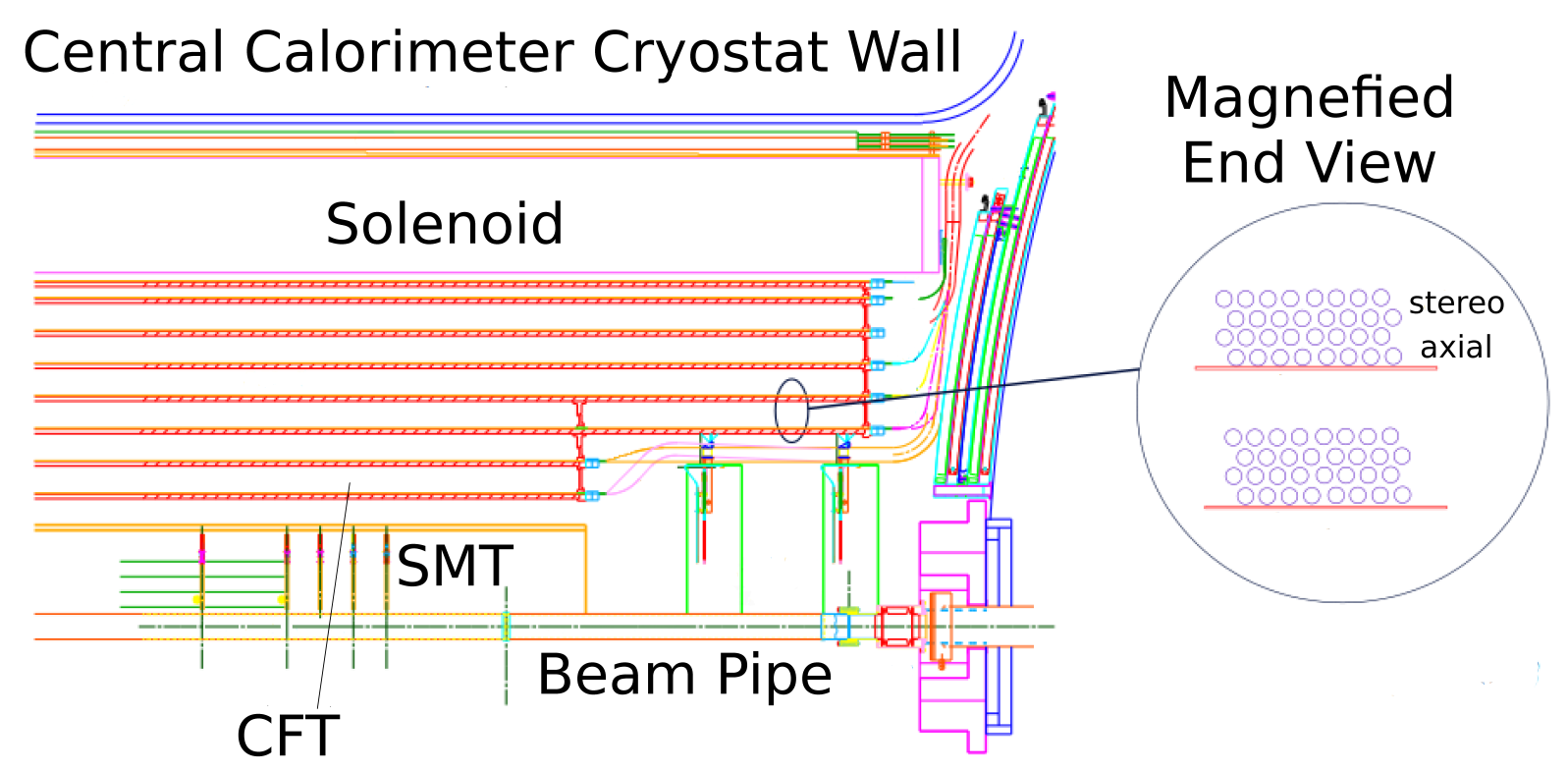

Figure 2.5: The layout of the CFT, showing the fibre layers.

Each of these pieces is contained within a cryostat to maintain a temperature of $90 \mathrm{~K}$.

A particle travelling through the calorimeter will interact either through the electromagnetic or nuclear forces with the absorber plates which will produce a spray of additional particles. These particles then ionize the liquid argon. The resulting electrons are collected and the magnitude of the charge is read out. The absorber plates, liquid argon, and the charge read out constitute a "cell", shown in Fig. 2.7. These cells are arranged such that their boundaries are centred along lines of constant pseudorapidity. "Towers" of cells are defined along such lines of pseudorapidity as illustrated in Fig. 2.8 with a size of $\eta \times \phi=0.1 \times 0.1$.

The innermost, or electromagnetic (EM), layers of the calorimeter are where most of the electromagnetically interacting particles (such as electrons and photons) deposit most of their energy through electromagnetic interactions. Particles are produced either through pair production $\left(\gamma \rightarrow e^{+} e^{-}\right)$or bremsstrahlung $\left(e^{ \pm} \rightarrow e^{ \pm} \gamma\right)$. The length of interest when describing electromagnetic cascades is the radiation length, $X_{0}$. The radiation length is the length over which the electron's energy is reduced to $1 / e$ of it's original energy through 


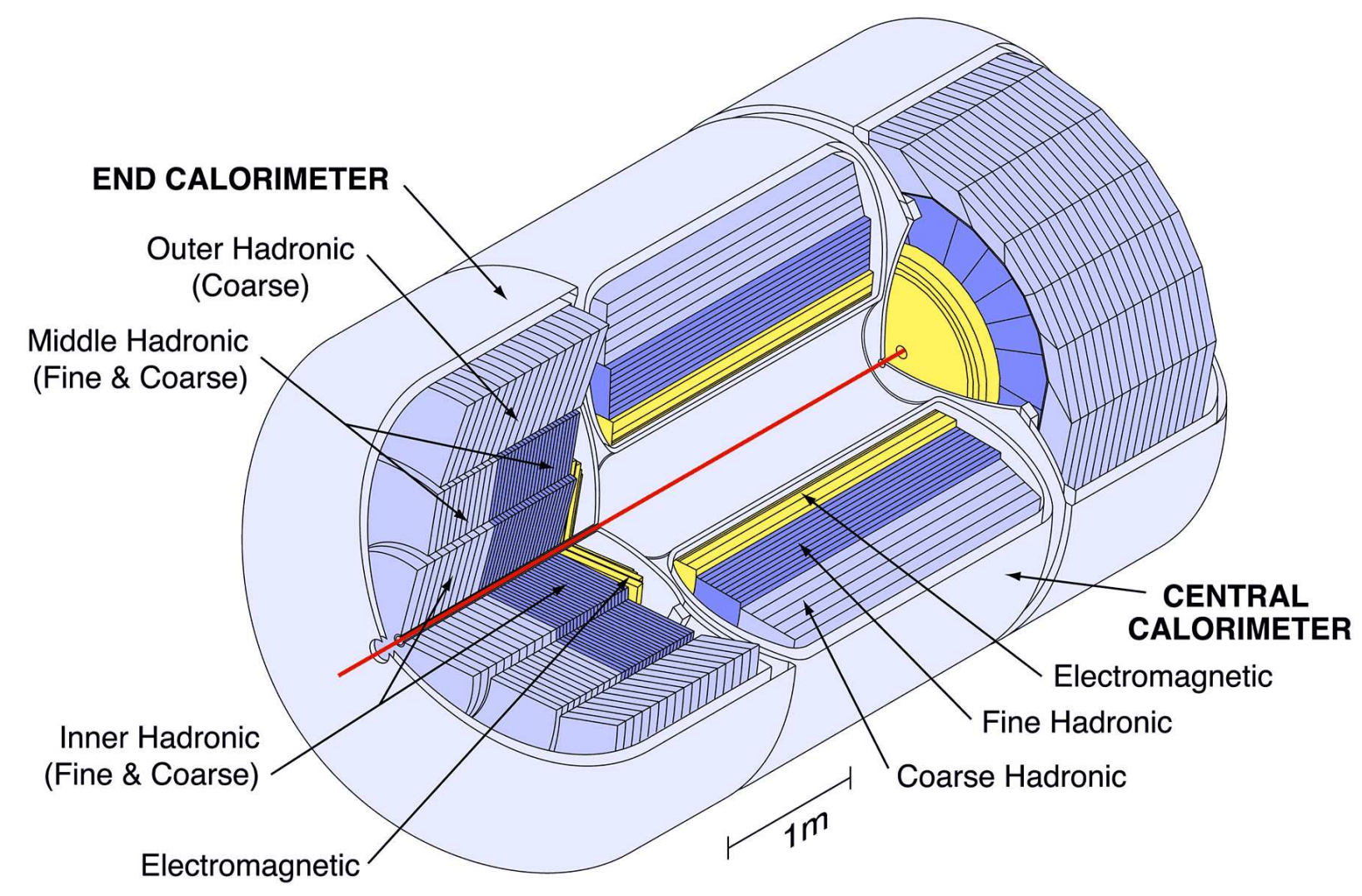

Figure 2.6: A diagram showing a cut away view of the D0 calorimeter. The calorimeter is comprised of three pieces: a central calorimeter and two end calorimeters. Each piece is made up of several layers of absorber plates (made of Uranium or copper or steel) inserted in liquid argon. These layers form the electromagnetic, the fine hadronic, and the coarse hadronic calorimeters [6]. 


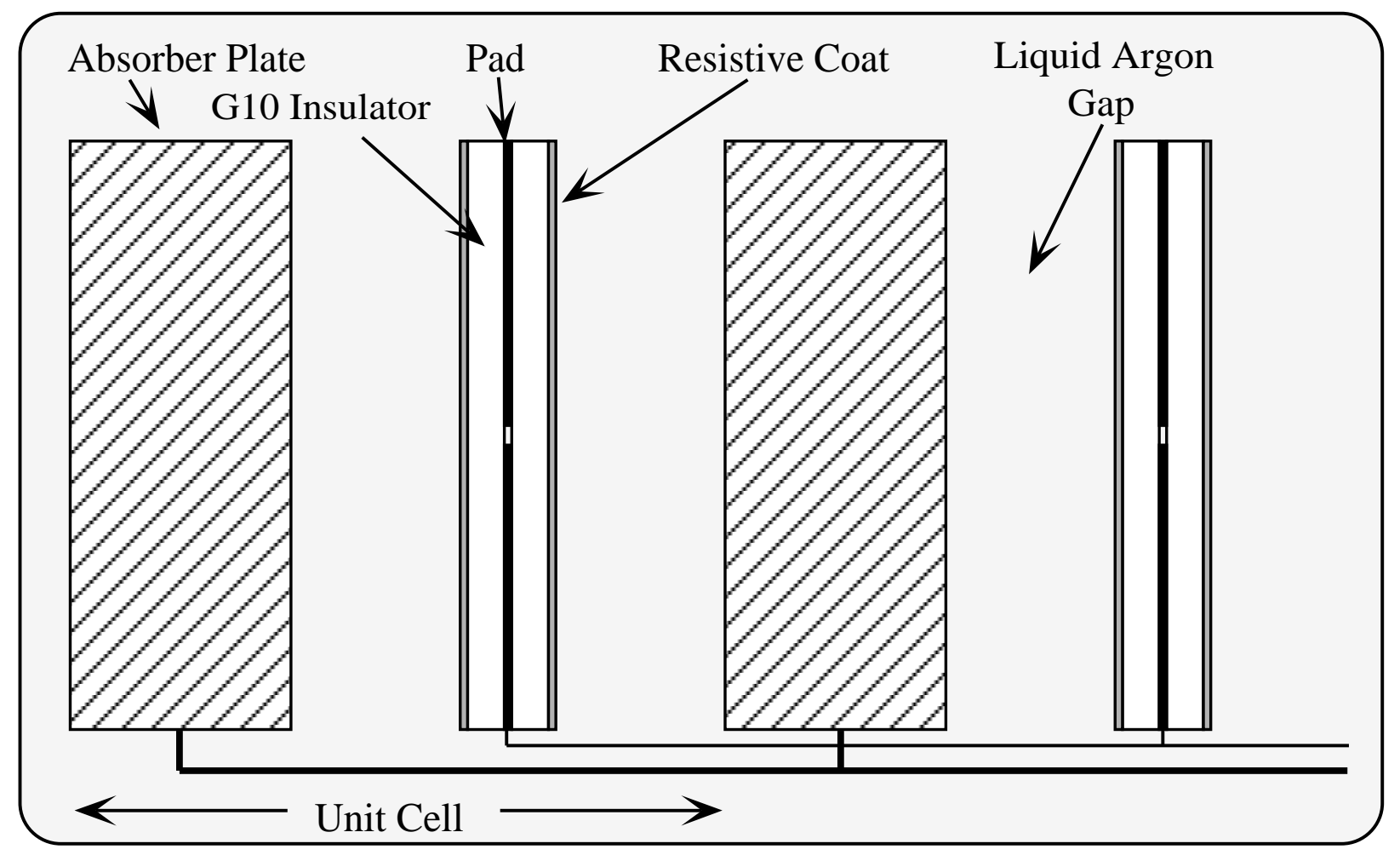

Figure 2.7: A diagram of a calorimeter cell. Each cell consists of an absorber plate, the liquid argon, and a plate to read out the charge.

bremsstrahlung, or $7 / 9$ of the mean free path for a photon to produce an electron-positron pair. The EM layers of the calorimeter have a total depth of about $20 X_{0}$.

Hadronic particles tend to deposit their energy deeper in the calorimeter, as they loose energy through inelastic interactions with a nucleus in the absorber plates. The length scale of interest here is the absorption length $\lambda$, which is the mean free path of a particle before it inelastically interacts with a nucleus. The absorption length is generally larger than the radiation length, so hadronic particles tend to deposit energy deeper in the calorimeter than electromagnetic particles do (in uranium, the radiation length is $0.32 \mathrm{~cm}$, while the absorption length is $11.03 \mathrm{~cm}$ [1]). The EM layers of the calorimeter have an interaction length of about $0.57 \lambda$, and the hadronic layers of the calorimeter have an interaction length of about $6 \lambda$ in the CC and up to about $10 \lambda$ in the EC. The D0 calorimeter is known as 


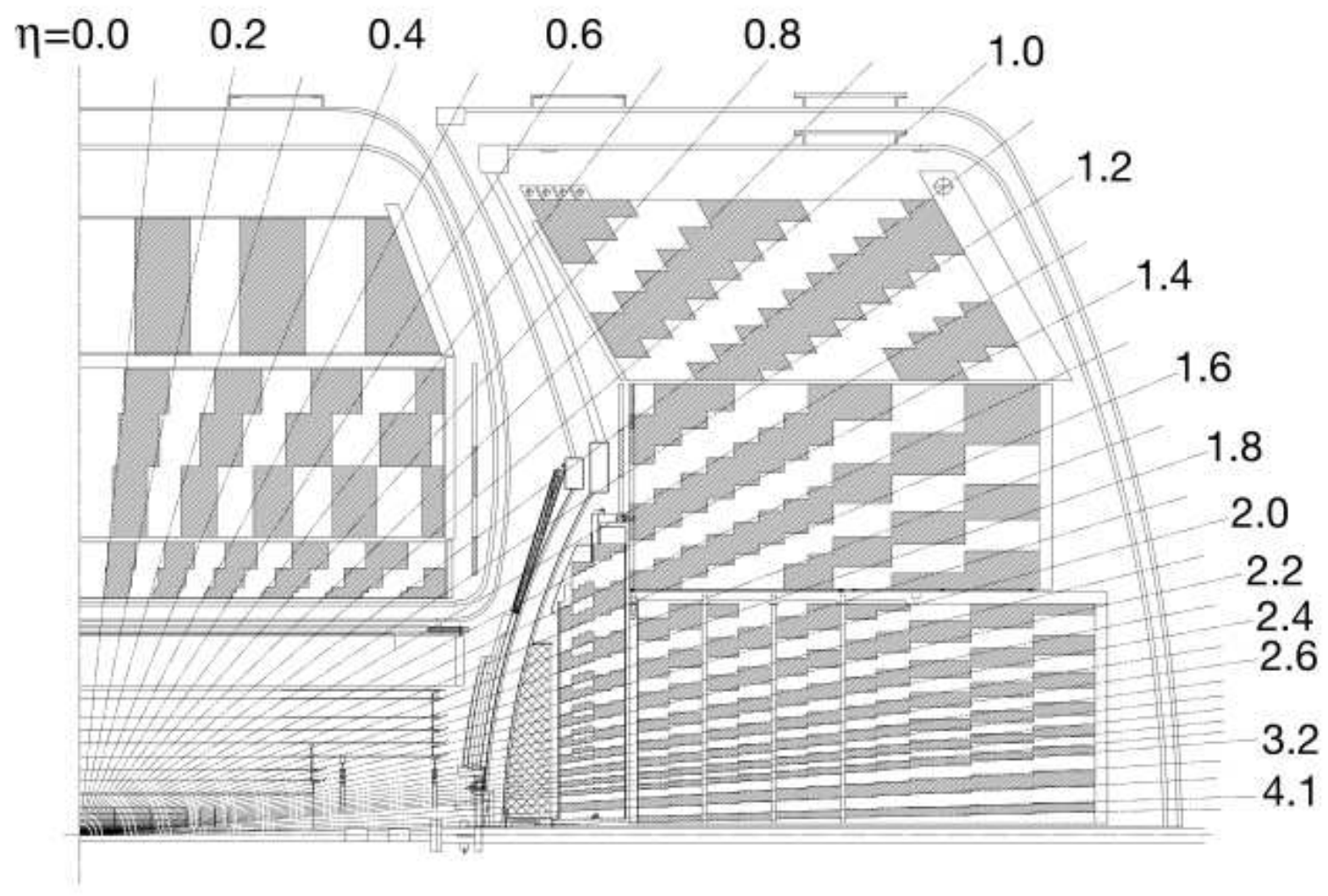

Figure 2.8: The calorimeter cells are arranged in towers along lines of constant $\eta$, illustrated by the alternating shaded areas. 
a compensating calorimeter, which means that the ratio of the electromagnetic to hadronic responses is approximately one. While this is true for the D0 detector, it depends on the material used as an absorber, and is thus not true for a general calorimeter.

The cryostat walls between the central and end calorimeters lead to poor coverage by the calorimeter for $0.8<|\eta|<1.4$. To improve coverage in this inter-cryostat region (ICR), an inter-cryostat detector (ICD) was added. The ICD is made up of two rings of scintillating tiles mounted on the inner faces of the EC cryostats. The tiles increase coverage in the range $1.1<|\eta|<1.4$

Between the solenoid and the central calorimeter are the preshower detectors, shown in Fig. 2.9. The central preshower detector (CPS) covers $|\eta|<1.3$, while the forward preshower detector (FPS) covers $1.5<|\eta|<2.5$. The preshower detectors are designed to act as both a tracking system and as calorimeters, and can be used to distinguish between photons and pions. The detectors are made up of triangular scintillator strips interleaved together. At the center of each strip is a wavelength shifting fibre that collects and transports the collected light for readout. The CPS consists of a lead radiator that is approximately one radiation length thick, and three triangular scintillator strips. The scintillator strips are arranged such that there is one axial layer aligned with the $\mathrm{z}$ axis, and two stereo layers aligned at $\pm 24^{\circ}$. Each of the FPS detectors consist of two layers of scintillator strips with a lead-stainless-steel absorber, that is two radiation lengths thick, in between. 


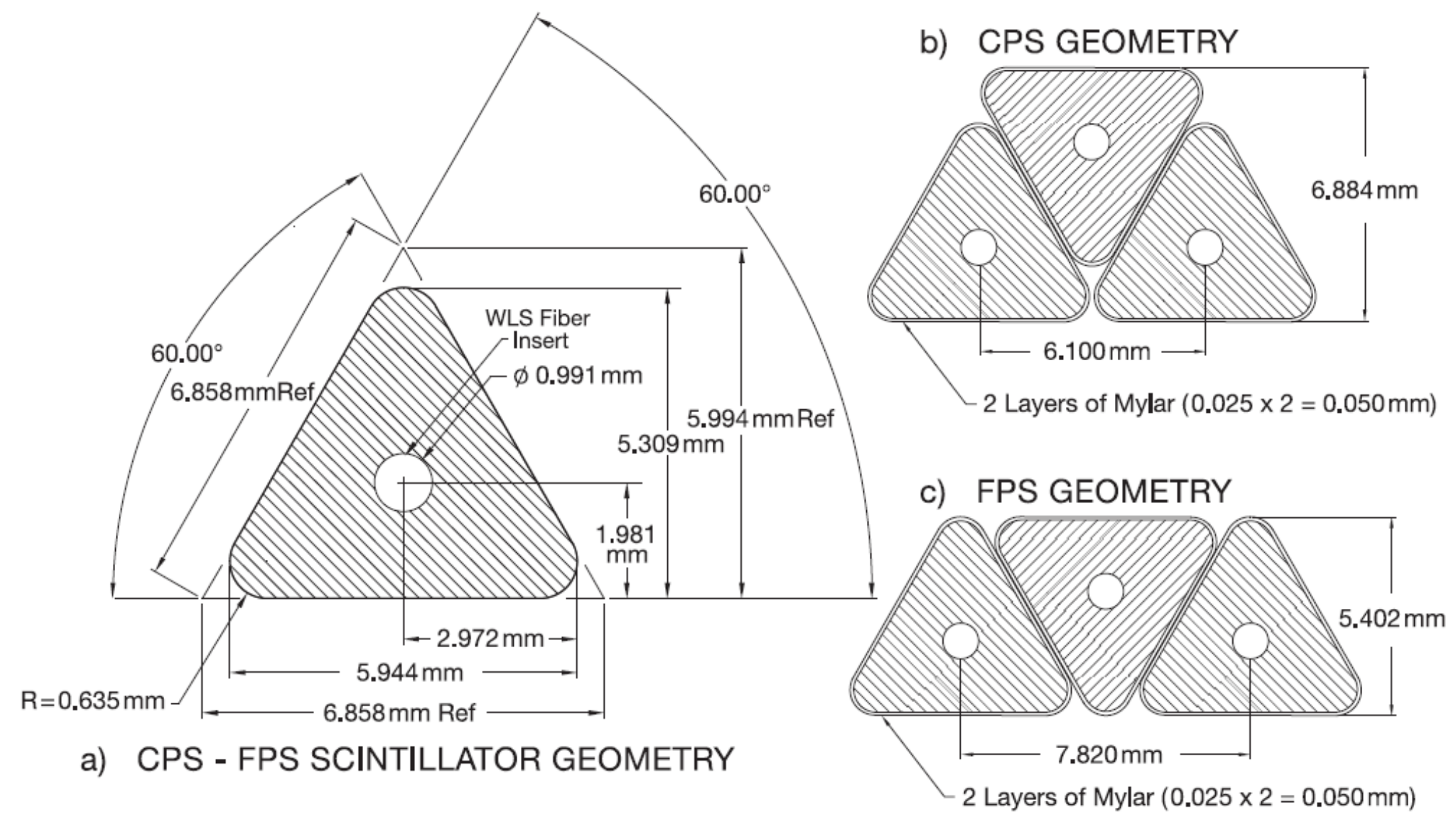

Figure 2.9: A cross sectional view of the D0 preshower detectors located between the solenoid and the calorimeters. The preshower detectors are comprised of interleaved scintillator strips with a wavelength shifting fibre at the center [6]. 
While the calorimeter absorbs the energy from most particles that travel through it, there are some particles that pass through completely without depositing very much of their energy. Neutrinos interact only through the weak force, and so the detector is mostly transparent to neutrinos. High energy muons will also tend to pass through the detector. The muon's large mass relative to the electron and the fact that power radiated through bremsstrahlung is inversely proportional to the mass of the particle to fourth power, means bremsstrahlung tends to be suppressed. Since muons do not have colour charge, strong interactions with the nuclei in the calorimeter do not happen. However, muons do deposit some energy in the calorimeter through ionization. Typically the amount of energy a muon loses to ionization is about $3 \mathrm{GeV}$, spread uniformly along its trajectory. Since muons are charged, they will produce a track in the tracking system. While the track plus the small, collimated energy deposit in the calorimeter is a unique signature, muon identification, and suppression of pions mimicking a muon signature can be improved by the additional muon detectors [21].

The muon tracking system includes a $1.9 \mathrm{~T}$ toroidal magnetic field which points approximately along the $\phi$ direction. This causes the muon's trajectory to curve to higher or lower $\eta$, depending on the muon charge. To measure the deflection of the muon's path in this magnetic field, we look at track segments in layers both before and after the toroid. There are four tracking layers before the toroid, which together are called layer A. After the toroid are layer B and layer $\mathrm{C}$, which have three tracking layers each. The tracking layers are comprised of proportional drift tubes (PDTs) to measure the muon's trajectory through the trackers. A PDT consists of a thin wire in the center of a metal tube filled with an ionizing gas, with the wire held at a large electric potential relative to the tube. When the muon moves through the PDT, it ionizes the gas in the tube. The free electrons from the ionization drift to the wire and produce an electrical pulse. The shape of the electrical pulse 
can be used to determine the distance of closest approach to the axis of the tube, and the time difference between when opposite ends of the tube receive the pulse can be used to determine the longitudinal placement.

In the central region of the detector $(|\eta|<1.0)$, the PDTs have a cross section of $10 \times 5.5 \mathrm{~cm}^{2}$, and the gas composition is $84 \%$ argon, $8 \%$ methane, and $8 \% \mathrm{CF}_{4}$. The maximum electron drift time in the PDTs is $500 \mathrm{~ns}$. In the forward regions (up to $|\eta| \approx 2$ ), where the particle density tends to be high, the drift tubes are rather smaller, with a cross section of $9.4 \times 9.4 \mathrm{~mm}^{2}$. These so-called mini drift tubes have a drift time of about $60 \mathrm{~ns}$, and the ionizing gas is composed of $90 \% \mathrm{CF}_{4}$ and $10 \%$ methane. The drift tubes in all regions are aligned to be roughly parallel with the toroidal field. The arrangement of the muon drift tubes is shown in Fig. 2.10

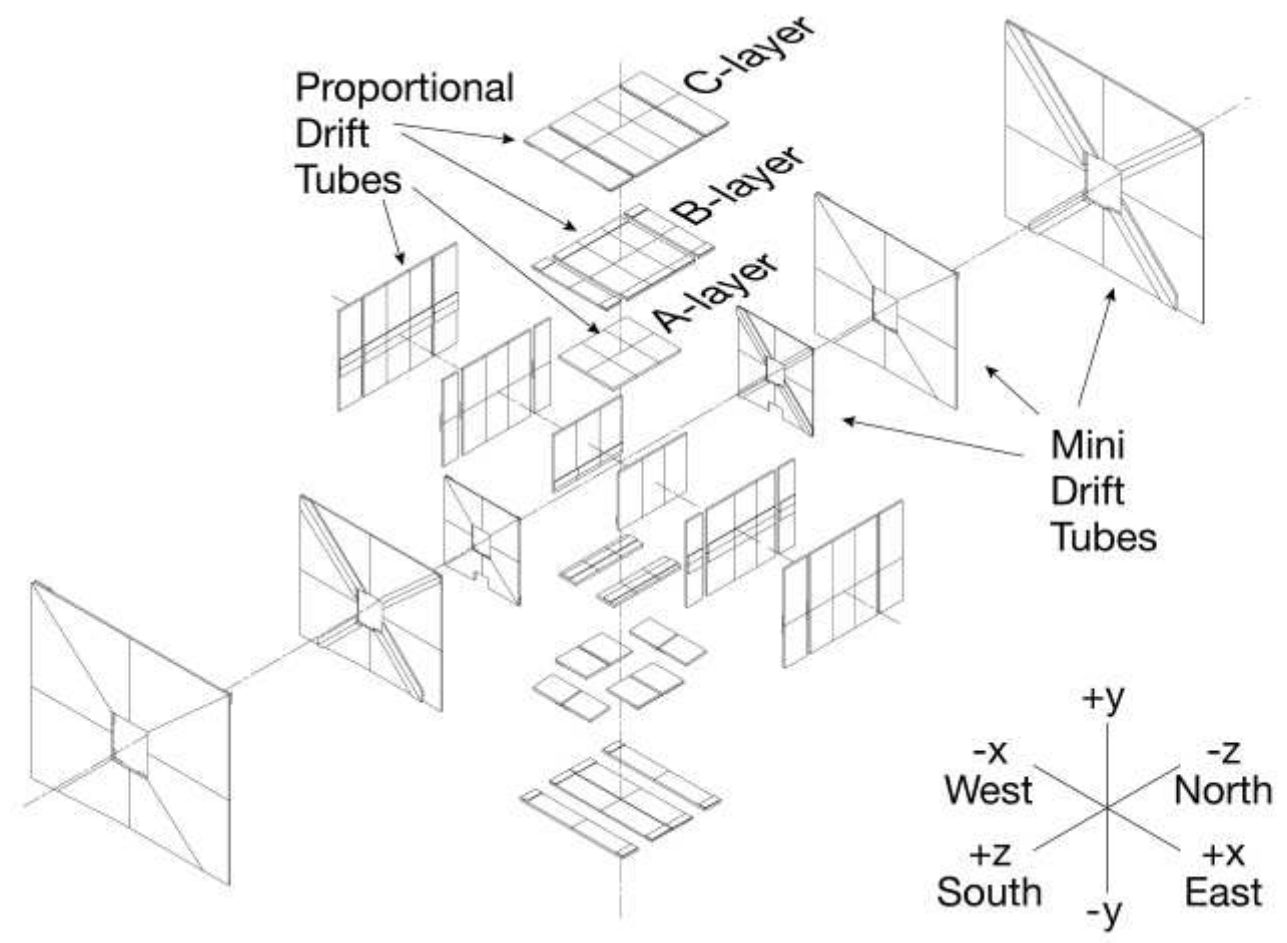

Figure 2.10: An exploded view of the arrangement of the drift tubes in the muon system.

Additionally, scintillator tiles are placed in the muon trackers to gain timing information. 
Hits in the scintillator can be spatially matched to the PDTs to associate the muon track with the appropriate collision. Without this timing information, there is ambiguity in the bunch crossing to which the muon track corresponds, as the $500 \mathrm{~ns}$ drift time is larger than the $396 \mathrm{~ns}$ bunch crossing time. The scintillator timing information can also be used to reject non-collision muons occurring out-of-time with respect to the beam, such as those coming from cosmic rays.

\subsection{Data Acquisition}

On average, 1.7 million beam bunches collide at the D0 detector every second. We can't feasibly record every bunch crossing. Even if we could record them all, the vast majority of the collisions involve the partons within the protons and anti-protons scattering elastically. These elastic collisions aren't that interesting for analyses to consider. Instead we try to record collisions containing an inelastic interaction. To select collisions of interest, we employ a series of filters, or triggers [22]. D0 has three levels of triggers that, when combined, pass

about 100 events per second to be considered for analyses. Schematic views of the triggers can be seen in Fig. 2.11 and 2.12. 


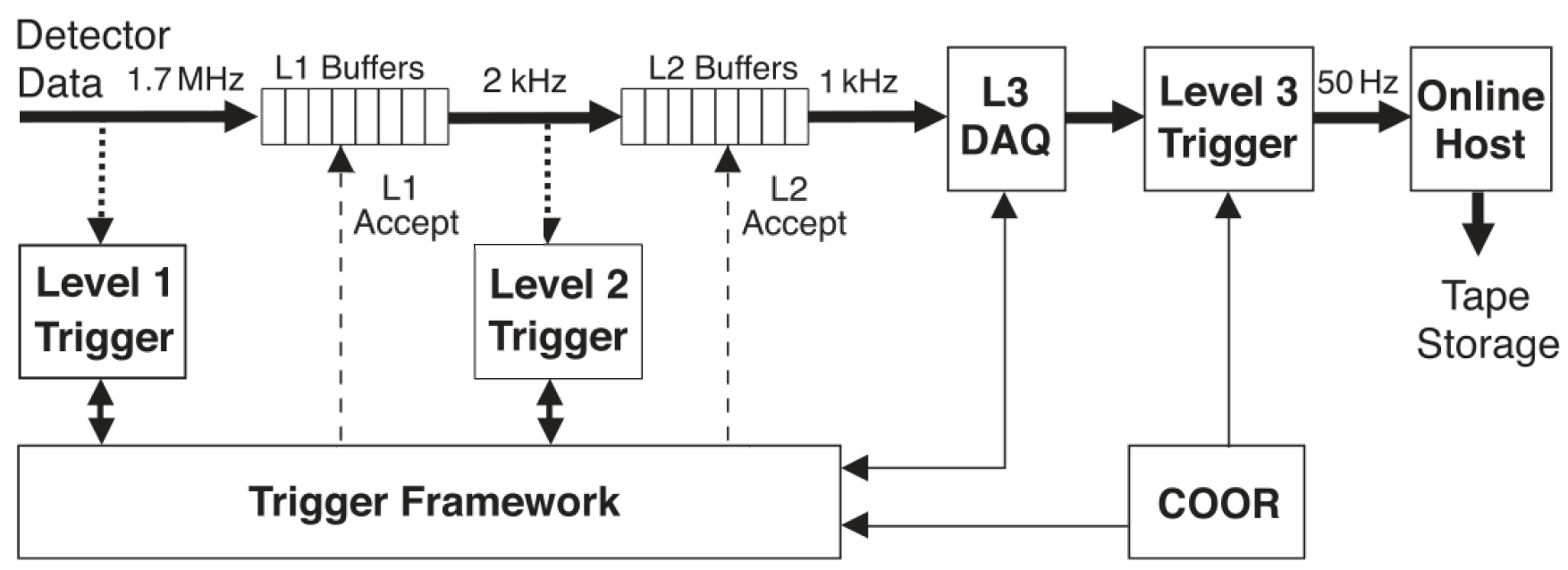

Figure 2.11: A schematic view of the flow of data through the D0 trigger system [6]. 
The first trigger level, called Level 1 (L1), uses coarse information from the muon system, the calorimeter, the preshower detectors, and the CFT. Approximately 2000 events per second pass this first level. The incoming data are held in a buffer for $3.7 \mu \mathrm{s}$, so that the subdetectors can provide the information needed for a decision on whether to have the data move to the next trigger level. The Level 1 trigger is made up of several components: the L1 central track trigger (L1CTT), the L1 preshower trigger (L1PS), the L1 calorimeter trigger (L1Cal), the L1 calorimeter track trigger (L1CalTrack), and the L1 muon trigger (L1Mu). The L1CTT uses the axial layers of the CFT and CPS detectors to help reject fake tracks. Hits from tracks in the axial layers of the CFT are matched to clusters in the axial layers of the CPS, and this information is sent to the L1Mu and L1CalTrack systems. Thresholds can be applied to clusters of energy found in the FPS detectors in the L1PS trigger. The L1Cal triggers on estimates of the EM and hadronic energy in $\Delta \eta \times \Delta \phi=0.2 \times 0.2$ towers. These towers can be used to reconstruct quantities such as the total transverse energy or the number of towers above an energy threshold. The L1CalTrak trigger matches the positions of tracks from the L1CTT trigger with the objects formed in the L1Cal trigger. Similarly, the L1Mu trigger considers hits in the muon wire chambers and scintillation counters, and integrates this with the information from the L1CTT triggers to find patterns consistent with the passage of a muon.

The second level of triggering has an increased decision time of $100 \mu \mathrm{s}$. This allows for more detailed information and more sophisticated algorithms to be used in the triggers. The Level 2 (L2) trigger uses information correlated across different detector subsystems. In addition to the CFT and preshower detectors used in L1, L2 also uses information from the SMT. This allows the track list from L1 to be refined. The output event rate at L2 is about half that of L1, 1000 events per second. The L2 trigger is comprised of the L2 silicon track 


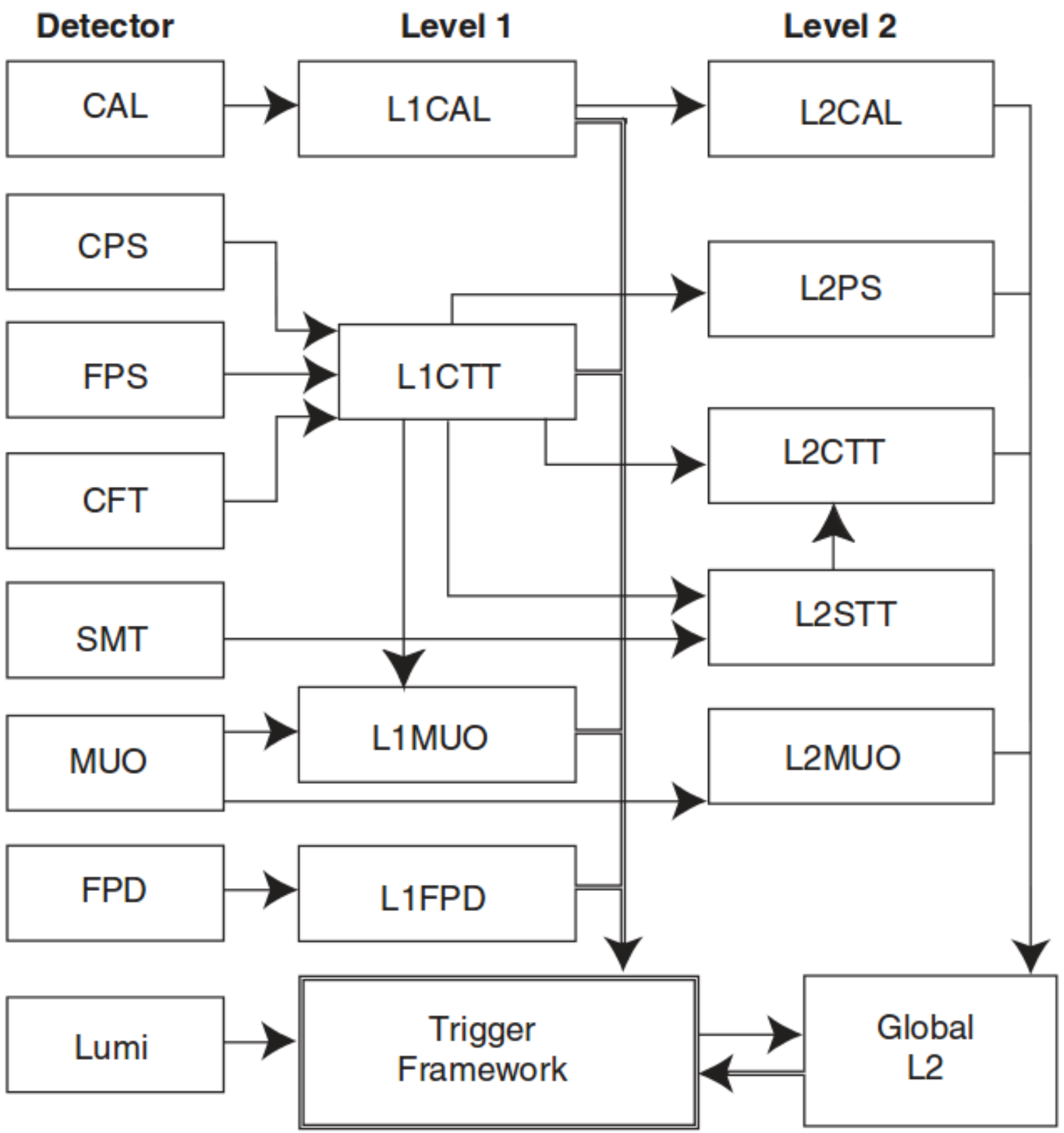

Figure 2.12: A schematic view of the relationship between the different trigger components and the different detector subsystems [6]. 
trigger (L2STT) the L2 central track trigger (L2CTT), the L2 preshower trigger (L2PS), the L2 calorimeter trigger (L2Cal), and the L2 muon trigger (L2Mu). The L2STT system uses the SMT, which has a finer spatial resolution than the CFT, and refines the track list found by the L1CTT trigger. This results in higher resolution track information, which in turn results in higher resolution impact parameter measurements which allows us to identify long lived particles. The L2CTT recalculates the tracks from the L1CTT triggers using additional hit information. This information and the information from L2STT allow for track isolation information to be calculated. The L2PS system identifies preshowers for electrons and photons by looking for clusters with and without a L1CTT track match to clusters in the preshower detectors respectively. The L2Cal system uses the towers constructed in L1Cal and forms jets from groups of $5 \times 5$ towers. EM objects can also be calculated based on towers in the EM layers of the calorimeter. An estimate of the $E_{T}$ can also be determined from the vectorial sum of the calorimeter towers. The L2Mu trigger adds in more precise timing and calibration information to the L1Mu information.

The third, and final, level of triggering is level 3 (L3). The L3 decision time is about one second. The decisions are made based on algorithms that reconstruct physics objects with almost as much sophistication as is done in analyses. Tracks are used to locate the primary vertex, which also improves the calorimeter-based $\#_{T}$ measurements. Secondary vertices can also be identified, which means b-jet identification can be included in L3 triggers. Decisions can be made on single objects, or on variables that combine several objects. Events pass the L3 triggers at a rate of 100 events per second. The events that do make it past this final trigger level are recorded for analysis. 


\subsection{Data Collection}

Over approximately ten years of running, the Tevatron delivered a total of about $12 \mathrm{fb}^{-1}$ of luminosity to the D0 detector. The D0 detector collected $10.7 \mathrm{fb}^{-1}$ of this (the detector is not actively recording due to electronics latency or malfunctions for $100 \%$ of the collisions). At the data analysis level, quality requirements are applied. For example, sometimes certain components of the detector cease functioning temporarily. Events are not considered if any part of the detector that is used in calculating particle identification was malfunctioning or not operating. After data quality requirements, the analysis discussed in this thesis uses 9.7 $f b^{-1}$ of data. 


\section{Chapter 3}

\section{Particle Identification and}

\section{Reconstruction Algorithms}

Particles that travel through and interact with the detector leave behind energy deposits. These different signatures are reconstructed by algorithms designed to identify elementary particles.

\subsection{Charged Particle Tracks}

There are two algorithms used to reconstruct tracks for charged particles in the tracking system: the histogrammming track finder (HTF), and the alternative algorithm (AA). The HTF algorithm is designed to be more efficient at reconstructing tracks with high transverse momentum $\left(p_{T}\right)$, in the forward $\eta$ regions, and at high luminosities, whereas the AA algorithm is designed to be more efficient at low $p_{T}$.

The HTF method operates based on pattern recognition. A particle with charge, $q$, and transverse momentum, $p_{T}$, travelling through a magnetic field, $B$, will have a trajectory curvature of:

$$
\rho=\frac{q B}{p_{T}} .
$$


The trajectory of the particle through the detector is determined by the curvature, the distance of closest approach, $d_{0}$, and the direction, $\phi$. We are interested in tracks coming from the interaction vertex, so we assume $d_{0} \approx 0$. A series of paths can then be drawn from the origin to a given hit with position $\left(x_{i}, y_{i}\right)$, which is transformed into $(\rho, \phi)$ space. Two tracking hits correspond to a single point in $(\rho, \phi)$ space, but a single tracking hit corresponds to a line as illustrated in Fig. 3.1. Different hits from the same track will produce multiple lines (with different slopes) that intersect at a single point. Similarly, the $(r, z)$ coordinate is translated to the coordinate space of $(z, d z / d r)$. From this a list of template hits is produced separately for the SMT and CFT. The hits are passed through a Kalman filter. The Kalman filter (or Kalman fit) is an iterative algorithm that is used to estimate unknown variables using measurements of known variables over time. Each iteration of the Kalman fit contains two steps. The first step estimates the unknown parameters (for example in the case of tracks, these would be the curvature and direction) and their uncertainties based on the current available information. Once the next measurement is taken, the estimates are updated with the new information using a weighted average (with larger weights for estimates with smaller uncertainties). The Kalman filter builds up a list of tracks based on hits in the SMT, and the $\chi^{2}$ of the fit to track hits. These tracks are then extrapolated to the CFT to build a complete track. Alternatively, the track can be started in the CFT, then propagated to the SMT. The final list of tracks is the combination of the two approaches with duplicate tracks merged.

The AA approach does not make the assumption that $d_{0}$ is approximately zero, which makes it the better choice for particles that have a large impact parameter, such as particles that travel some distance through the tracker before decaying. The algorithm starts with a seed track comprised of the three innermost hits in the SMT. The track is extrapolated 

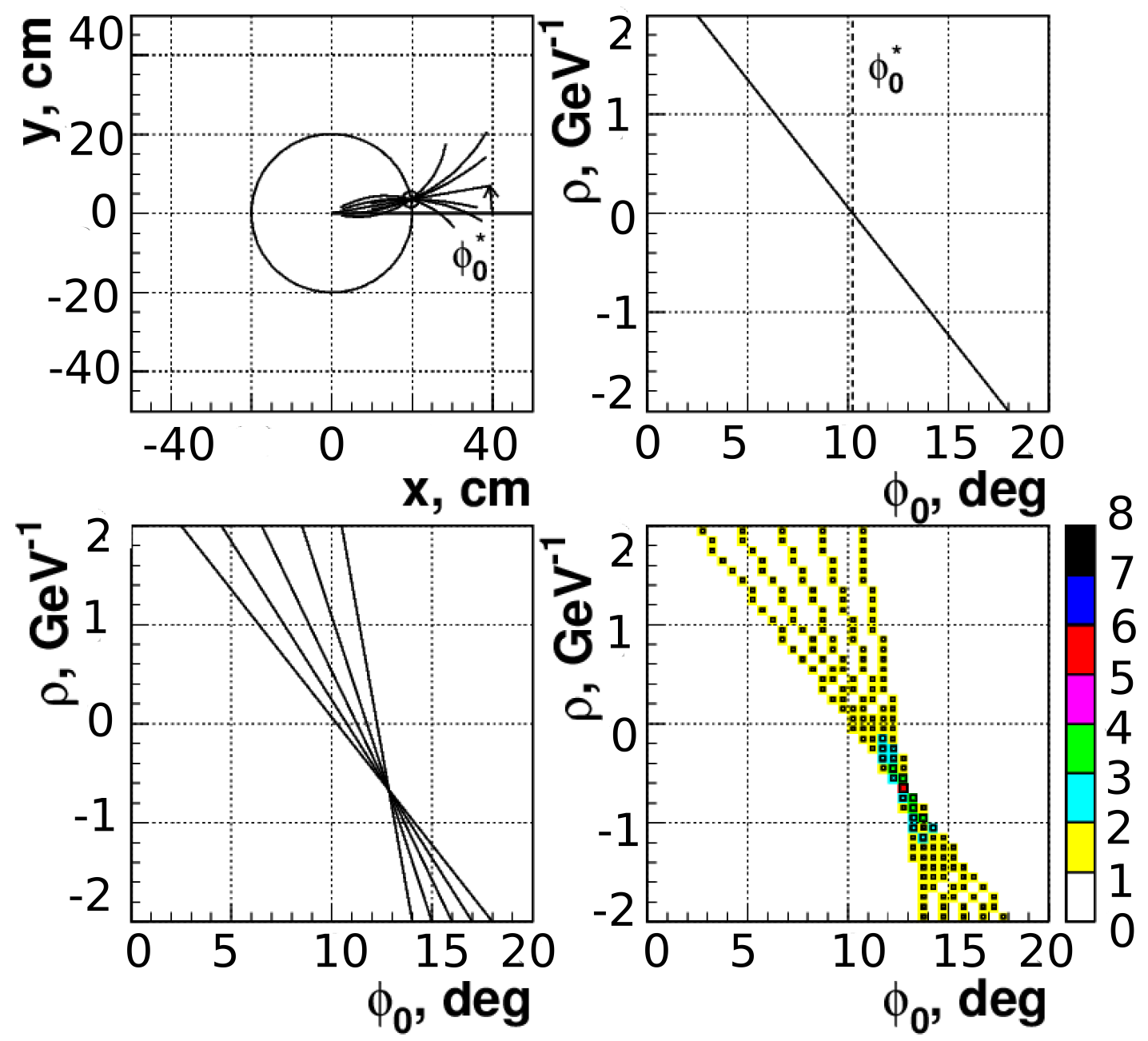

Figure 3.1: The HTF method for a single $1.5 \mathrm{GeV}$ track with 5 hits. (a) Some of the possible trajectories for a single hit in the $\mathrm{x}-\mathrm{y}$ plane, (b) translation of a single hit into $\rho-\phi$ space, (c) multiple hits from the same track form lines that intersect at a single point, and (d) the intersection point as a peak in the $\rho-\phi$ histogram.

outwards, and additional hits are looked for in a narrow window around the extrapolation. If no hit is found the layer is counted as a miss. If a is hit found, it is added to the track if the fit $\chi^{2}$ increases by less than 16. If multiple hits are found, multiple track candidates arise. The AA proceeds to move through all layers until it reaches the last layer, or until three missed layers are encountered. The AA is also started with three hits in the CFT, with the PV assumed as a fourth hit. The algorithm then proceeds iteratively inwards through the detector. The final list is a combination of the SMT and CFT seeded tracks, with duplicate 
tracks merged.

Neither the HTF nor the AA algorithm account for tracks losing energy to ionization within the tracking system, or for the possibility of multiple scattering to change the track direction. To deal with these effects, the tracks are run through a Kalman filter that fits a more sophisticated track model to the hits for each track candidate. Placing an upper limit on the $\chi^{2}$ from this fit helps cut down on the number of mis-reconstructed tracks.

\subsection{Primary Vertices}

At each beam crossing, it is possible to have multiple $p \bar{p}$ interactions. Usually only one interaction results in an inelastic collision, in which we are interested. The interactions that produce only elastic collisions are referred to as minimum bias interactions. The first step to determining the primary vertex is to determine all vertices from a $p \bar{p}$ interaction. To do this, tracks with $p_{T}>0.5 \mathrm{GeV}$ that have at least 2 hits in the SMT are clustered together such that the distance in the $\mathrm{z}$ direction between a track and the cluster is less than $2 \mathrm{~cm}$.

Kalman fits are performed to find the vertex for each cluster. If the fit $\chi^{2}$ per degree of freedom is greater than 10, the track that contributes the most to the $\chi^{2}$ is removed iteratively until $\chi^{2} / n_{\text {dof }}<10$. These clusters then become a list of possible PV. Since we generally expect tracks from a minimum bias interaction to have low $p_{T}$, the probability for a track to be from a minimum bias vertex can be constructed. The probabilities for tracks to be from a minimum bias interaction can be combined to get a probability for each vertex to be from a minimum bias interaction. The vertex that has the lowest probability of being from a minimum bias interaction is selected as the PV. 


\subsection{Muons}

Muons are identified using the tracking system, the calorimeter (through ionization), and the outer muon system [23]. Tracks in the central tracking system are extrapolated outward to the track segments in the muon system. A muon track will bend between layer A and layer $\mathrm{B}$ of the muon system, due to the toroidal magnetic field. However tracks in layer B and layer $\mathrm{C}$ will be collinear, so for muon identification layers B and $\mathrm{C}$ are considered as merged. Muons that originate from semi-leptonic decays of b-quarks are also identified, through the muon's isolation. The isolation depends on the tracks near the muon, and the amount of energy in the calorimeter near the muon, and can also be used to reject pions that mimic a muon signature. Cosmic rays can be rejected by looking for scintillator hits that correspond to the bunch crossing time plus the time of flight of the muon.

\subsection{Electrons and Photons}

The first step to identify electrons and photons is to form clusters of isolated energy deposits in the calorimeter. Cells within $\Delta R=0.2$ of a seed cell make up these clusters. Next, we try to identify a track matched to the cluster. If such a track is found, the cluster is assumed to be an electron, otherwise it is assumed to be a photon. To differentiate between electrons and hadronic particles, a Boosted Decision Tree (described in Ch. 6.6) is used to classify distinct signatures of electrons and hadronic particles. 


\subsection{Jets}

The quarks we see in our detector are coming from the decay of a particle (for example, a higgs boson), or from a gluon splitting into a quark-antiquark pair. When moving through the detector, the quarks start to move apart from each other. Because the colour potential in the strong interaction grows as the quarks move away from each other, at some point the potential energy will be larger than the energy required to produce a quark-antiquark pair out of the vacuum. This process produces colour-neutral mesons and baryons. It is this highly-collimated collection of particles, which are called jets, that is actually seen by the detector. We associate this signature to the original quark.

Jet identification starts by building a tower out of calorimeter cells with energies above a certain threshold. The towers are then formed into preclusters based on a simple cone algorithm. The towers with $p_{T}>0.5 \mathrm{GeV}$ are ordered in a list from highest $p_{T}$ towers to lowest. The first item of the list is considered as a precluster seed, and removed from the list. The remaining objects in the list are processed and added to the precluster seed if they are within $\Delta R=0.3$ of the seed. This continues until all objects in the list are part of a precluster. From here, preclusters with $p_{T}>1 \mathrm{GeV}$ are used to seed protojets. The protojets are constructed with a cone size of $\Delta R=0.5$ seeded by the preclusters separated from the nearest protojet by at least $\Delta R=0.25$. The preclusters are iteratively added to the protojets until the centroid of the prototjet changes by less than 0.001 when a precluster is added to it, or until 50 iterations have occurred. Protojets with $p_{T}>4 \mathrm{GeV}$ are kept, and to avoid double counting energy, protojets with greater than $50 \%$ of their preclusters shared are merged, otherwise shared preclusters go to the closest protojet.

The jet energy that we measure with our detector is corrected to more accurately reflect 
the total energy of the particles in the jet. This correction is called the jet energy scale correction (JES), and it is comprised of several pieces to account for various inaccuracies in the jet energy measurement [24]. The JES corrects both data and MC events according to:

$$
E_{\text {jet }}=\frac{E_{\text {meas }}-E_{0}}{R_{\text {jet }} S_{\text {jet }}},
$$

where $E_{\text {meas }}$ is the raw jet energy measured with the detector. $E_{0}$ is an offset energy that compensates for uranium decay, energy from minimum bias interactions, energy from previous bunch crossings, and noise in the electronics. The parameter $R_{\text {jet }}$ is the calorimeter energy response and is affected by the amount of energy deposited in the layers of the detector before the calorimeter, or areas of the detector where there is no instrumentation, and various non-linearities in the detector. The parameter $S_{\text {jet }}$ corrects for showers that have particles entering or leaving the jet cone.

The offset energy is measured in two parts. First the calorimeter response component is measured by collecting data events at random and measuring the average energy. Effects from additional interactions and energy from previous bunch crossings are measured by taking a random sample of data events with an inelastic collision. The response $R_{\text {jet }}$ and $S_{\text {jet }}$ are functions of where in the detector the jet is and the energy of the jet. The response is measured using events where a photon and a jet are produced back to back. Because we can use $Z \rightarrow e e$ events to obtain a precise calibration for the calorimeter response to electromagnetic particles, the photon energy can be measured to high precision. Then the fact that there should be no imbalance in transverse energy means that the jet energy correction can be derived. The showering correction comes from MC studies which compare the energy inside and outside cones of various radii. This is used to fit shower templates 
to photon plus jet data to determine the ratio of energy inside the jet cone to the true energy within the cone. Muons that are identified within the jet cone typically come from semi-leptonic decays of B mesons where a neutrino carries off some of the energy. A separate correction (JESMU) has been derived on jets containing muons to account for this.

Jet shifting, smearing, and removal (JSSR) allows jets in simulation to be recalibrated, have their resolution smeared, and possibly discarded, in a consistent way to match behaviour observed in data. This process has been calibrated using $\gamma+$ jet and $Z+$ jet events. First the $p_{T}$ imbalance between the $Z / \gamma$ and the jet is calculated in bins of $p_{T}^{Z / \gamma}$ :

$$
\Delta S=\frac{p_{T}^{\mathrm{jet}}-p_{T}^{Z / \gamma}}{p_{T}^{Z / \gamma}}
$$

To extract the shifting and smearing parameters of interest, a three step fitting procedure is performed. The $\Delta S$ distribution is fit in different bins of $p_{T}^{Z / \gamma}$ with the function:

$$
f(\Delta S)=N \times \exp \left(-\frac{(\Delta S-\langle\Delta S\rangle)^{2}}{\sigma^{2}}\right) \times\left(1+\operatorname{erf}\left(\frac{p_{T}^{j e t}-\alpha}{\sqrt{2} \beta}\right)\right)
$$

Here, the second term is a turn on term used to model the jet reconstruction threshold, and is assumed to be independent of $p_{T}^{Z / \gamma}$. This term is fit simultaneously over all jet $p_{T}$ bins, and thus fixes $\alpha$ and $\beta$, leaving the resolution, $\sigma$, and imbalance, $\langle\Delta S\rangle$, as free parameters. Next, the resolutions are fit in each $p_{T}^{Z / \gamma}$ bin, plotted as a function of $p_{T}^{Z}$, and fit according to:

$$
\sigma\left(p_{T}\right)=\sqrt{\frac{a^{2}}{\left(p_{T}^{Z}\right)^{2}}+\frac{b^{2}}{p_{T}^{Z}}+c^{2}}
$$

The $a$ term describes instrumental effect, such as noise and multiple interaction within 
the calorimeter. It is most important for low energy jets. The $b$ term is a stochastic response term. It describes fluctuations inherent in developing showers. The constant term describes calibration errors, dead material in front of the calorimeter, and non-uniformities in the calorimeter.

Finally, with the resolution fixed, the imbalance term is fit according to:

$$
<\Delta S>=A+B \exp \left(-C p_{T}^{Z}\right)+D \exp \left(-E\left(p_{T}^{Z}\right)^{2}\right)
$$

Jets in simulation are smeared such that the resolution agrees with the data on average. The energy level of the jets is then shifted to account for the differences in the mean, $<\Delta S>$.

\subsection{Missing Transverse Energy}

Because we are colliding composite particles (protons and anti-protons), most of the particles inside the protons do not contribute directly to the interaction, and end up travelling down the beam pipe undetected. For this reason, we can not enforce momentum conservation in the beam direction. However, we assume that the beam has zero momentum in the plane transverse to the beam direction ${ }^{1}$. This means that the total transverse energy resulting from a collision should be zero. Neutrinos interact only weakly, and do not deposit energy in the detector. We can infer their existence by looking for an imbalance in the transverse energy. This missing transverse energy, or $E_{T}$, is calculated as the negative of the sum of

\footnotetext{
${ }^{1}$ Actually, not only do the protons and anti-protons in the beam have some momentum in the plane transverse to the beam line, but the quarks and gluons inside the protons and anti-protons also have non-zero transverse momentum. This transverse energy tends to be small (a few $\mathrm{GeV}$ ) compared to the energy of the particles in an event under consideration.
} 
the energy deposited in the cells of the calorimeter:

$$
E_{T} x, y=-E_{T}^{x, y}=-\sum_{i}^{\text {cells }} E_{i}^{x, y}
$$

The sum over calorimeter cells does not include the coarse hadronic calorimeter cells, as these tend to be noisy and reduce the resolution of the measurement. Since the simulated jet energy needs to be corrected to match data, these corrections must also be propagated to the $E_{T}$ measurement. This is done by replacing the energy of the cells associated with a jet by the corrected energy in the sum. Since muons leave very little energy in the calorimeter, they mimic the signature of a neutrino from the point of view of the calorimeter. This means that the muon energy is included in the above definition of $E_{T}$. This is included at the final data analysis level.

\subsection{Bottom Quark Identification}

Jets arising from a hadron containing a b-quark tend to have a relatively long lifetime of $\sim 10^{-12} \mathrm{~s}$. This results in B hadrons travelling a few millimetres before they decay, leading to a decay vertex that is displaced from the primary interaction vertex. This displaced interaction vertex helps us identify, or tag, jets originating from a B hadron. The b-tagging algorithm is based on a multivariate discriminant that uses information about tracks and secondary vertices to distinguish jets containing b-quarks from those originating from c quarks or light quarks (u, d, s) and gluons.

Before considering whether a jet is a b-jet or not, we first require that the jet is "taggable". This is a requirement that the jet must contain at least two tracks, each of which must have 
at least one hit in the SMT. The determination of whether a jet contains a b-quark is done in two steps using multivariate techniques. The first step uses six random forests [25] to separate out jets containing b-quarks from jets not originating from b-quarks. One random forest uses information about the track impact parameters, such as a the number of tracks in a jet that pass various impact parameter thresholds, or the jet lifetime impact parameter, which uses the impact parameters for all tracks in a jet to construct a probability that it is a light jet. The other five random forests use information about the secondary vertex such as the number of tracks associated with the secondary vertex, the distance of closest approach of the secondary vertex to the primary vertex, and the maximum $\Delta R$ between tracks. The outputs of these five random forests are combined using a neural network, which helps exploit non-linear correlations between the random forests.

The b-tagging efficiency, and the rate for a light jet to be mistagged as a b-jet is measured in data using a sample enriched in b-jets, and the MC efficiencies are corrected to match the measured efficiencies. The efficiency and mistag rate depends on the jet $p_{T}$ and $\eta$, as well as the value of the neural network output being considered. Figure 3.2 shows the btagging efficiency and mistag rates as a function of the neural network output for jets with $p_{T}>30 \mathrm{GeV}$. In the $W H \rightarrow \ell \nu b \bar{b}$ analysis, the b-tag efficiency ranges from $\sim 50 \%$ to $\sim 80 \%$, and the mistag rate ranges from $0.15 \%$ to $11 \%$. 


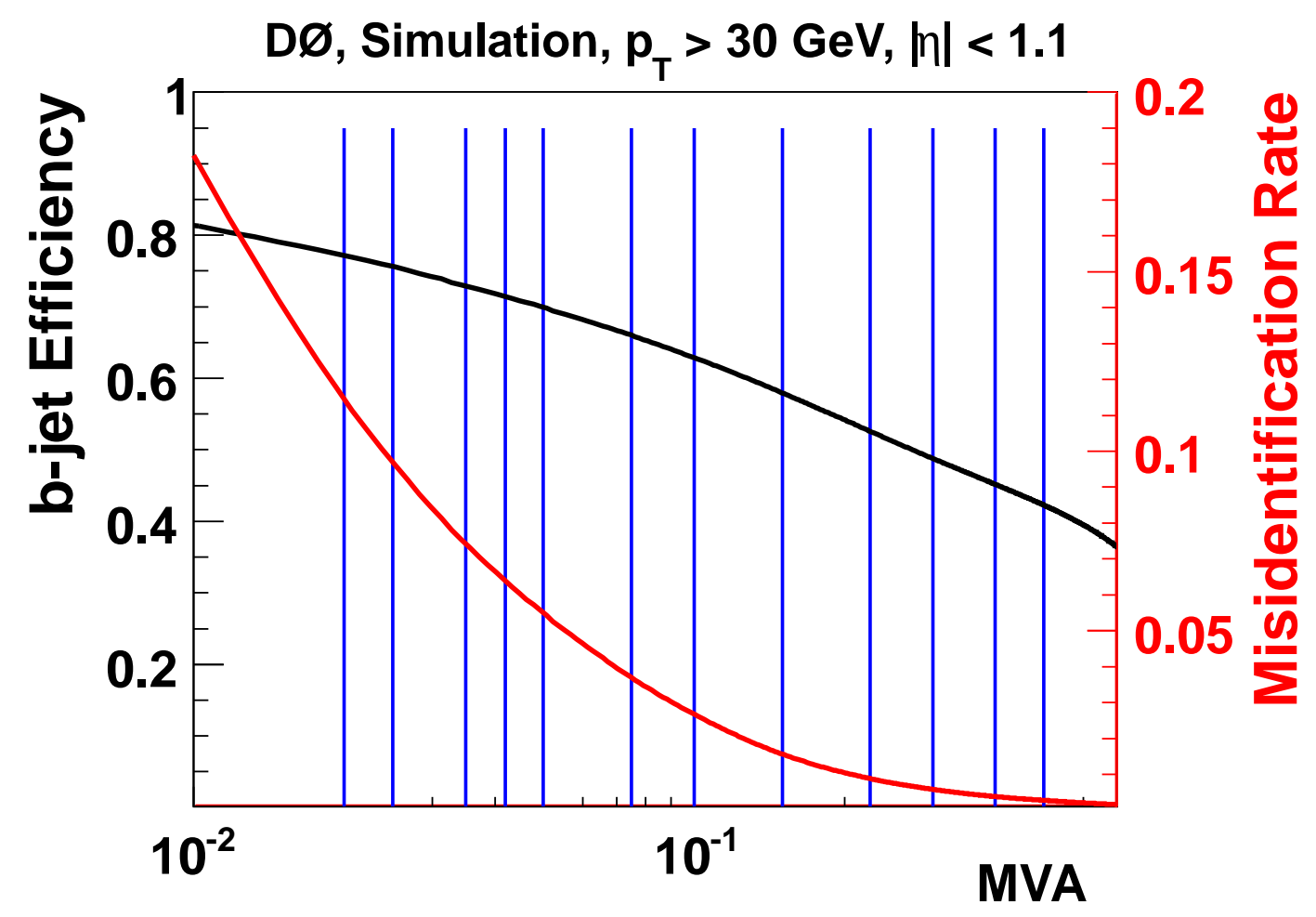

Figure 3.2: The b-tagging efficiency (in black) and rate for light jets to be mistagged (in red) as a function of the neural network output for jets with $p_{T}>30 \mathrm{GeV}$. The vertical blue lines indicate points on the neural network output where the efficiencies in simulation have been corrected to match the efficiencies in data. 


\section{Chapter 4}

\section{Event Simulation}

Since any given $p \bar{p}$ collision can produce a multitude of different particles, and those particles can decay to stable particles, the final topology of an event can match the topology of several processes. Differentiating between processes with the same topology is difficult, and will be discussed in Ch. 6.6. Before we can even try to differentiate between different processes, we must estimate the relative contribution from each process to our data. To do this, we simulate possible outcomes for a $p \bar{p}$ collision and the detector response to the particles produced. This is then compared to the data.

To simulate events, we use a monte-carlo (MC) random generator to expand the quantum field theory equations perturbatively. The expansion, in principle, can be expanded to many orders, but due to numerical or theoretical challenges it is usually cut off at leading order (LO) or next-to-leading order (NLO). At D0, we usually calculate the LO terms to describe an object's kinematics, but use the NLO calculation to scale the cross section of a given process or correct the kinematics.

There are a few effects to account for when performing the simulation. We need to take into account that when the protons and anti-protons collide, it is really the constituent quarks and gluons interacting, and these constituent particles carry some fraction of the proton or anti-proton momentum. Additionally, we need to consider the possibility that the interacting and/or resulting particles can radiate gluons or photons. We also need to 
simulate the process of quarks forming hadrons (known as hadronization). Finally, we need to simulate the passage of particles through our detector.

PYTHIA [26] is a LO generator that is used widely in the field of high energy physics. We use the CTEQ6L1 [27, 28] parton distribution function to model the quarks and gluons contained in the incoming protons and anti-protons. Following the LO event simulation, we use PYTHIA to model the hadronization of quarks, and the radiation of low energy photons or gluons from the incoming and final state particles. While PYTHIA works well for modelling low energy radiation, it does not model very well the radiation of high energy photons or gluons. This higher energy radiation is handled well by a LO matrix element event generator called ALPGEN [29]. ALPGEN calculates the LO term exactly, which gets the high energy radiation correct. However, it doesn't well describe the hadronization of quarks.

We can obtain the best of both worlds by using ALPGEN to provide final state partons, which are then showered with PYTHIA. In doing this, we may generate radiated jets with PYTHIA that are counted in the ALPGEN processing. To avoid double counting these jets, we use the MLM matching scheme [30] to determine if there are jets corresponding to the original final state partons from ALPGEN. When doing the showering with PYTHIA, it is possible to produce heavy-flavour jets, but these are also all ready accounted for by generating specific heavy flavour samples using ALPGEN. To avoid double counting, in samples that should contain only light jets, we veto events that have a $b$ or $c$ quark, and in samples that should have only $c$ quarks we veto events containing $b$ quarks.

We use another LO matrix element event generator called SINGLETOP $[31,32]$ to simulate single top events. It describes NLO distributions reasonably well, and also maintains a good description of the spin correlations between the top quarks and resulting $W$ boson.

When particles travel through the detector, they interact with the material inside of it, 
as well as the magnetic fields from the solenoid and toroid. This needs to be simulated. The simulation of the particles passing through the detector is done using the program GEANT [33], which uses precise information about the geometry and composition of the detector and magnetic fields to evolve the passage of particles through the detector. After this, the electronic read out is also simulated to take into account electronic noise and known inefficiencies. The final result is information in the same format as the data.

This simulation process is not perfect, and thus does not reproduce exactly the data. Some of these differences are well known, and can be corrected for. Our simulation assumes only one proton interacting with one anti-proton in each event. In reality, with higher luminosity events, we can have multiple interactions. To account for this, we overlay the simulation with data events from randomly chosen minimum bias events from different instantaneous luminosities. In the simulation, the primary interaction location along the $\mathrm{z}$ axis is assumed to be a Gaussian distribution. In data, the distribution is not quite Gaussian, so the simulation is reweighted to match the distribution in data.

By looking at $Z / \gamma^{*}$ events that decay to two leptons, the description of the $Z$ boson $p_{T}$ can be tested. It was found that at low $Z$ boson $p T$, the simulation does not describe the data well in either ALPGEN or PYTHIA. A reweighting was derived such that simulated $p_{T}$ spectrum matches that in data [34]. There is no measurement of the $W$ boson $p_{T}$ to derive an equivalent reweighting, so the $W p_{T}$ is corrected using the $Z p_{T}$ reweighting, adjusted by the ratio of the $W$ to $Z$ differential cross sections at next-to-next-to-leading-order.

For each process that we simulate, we generate many thousands of events. We then must normalize each process to the rates present in our data. Classically, if we have particles with a density $\rho$, colliding with a speed $v$, the rate at which we expect a process with a cross 
section $\sigma$ to occur is given by:

$$
R^{\text {classical }}=v \rho \sigma
$$

We can express the analogous rate in quantum mechanics as:

$$
R^{\mathrm{QM}}=\mathscr{L} \sigma \epsilon
$$

where $\mathscr{L}$ is the luminosity of the colliding beams, $\sigma$ is the cross section for the process under consideration, and $\epsilon$ is the detector efficiency. The cross section is calculated from theory, and the luminosity and efficiency are both measured from data, giving us everything we need to normalize our simulated samples. 


\section{Chapter 5}

\section{Statistical Analysis}

To make any statistical statements about our data, we perform a statistical analysis as is outlined below $[35,36,37]$. We are looking for events that have a small probability of occurring (given the small cross section for Higgs boson production), but many chances to occur (given the large number of collisions occurring at D0), so we can describe the probability of observing $d$ events given $p$ predicted events by a Poisson distribution:

$$
P(d, p)=\frac{p^{d}}{d !} e^{-p} .
$$

We have two hypotheses we would like to compare: the null (or background-only) hypothesis $H_{0}$, and the test (or signal+background) hypothesis $H_{1}$. The probabilities for each of these hypotheses are then:

$$
\begin{aligned}
& P\left(d \mid H_{0}\right) \quad=\frac{b^{d}}{d !} e^{-b}, \\
& P\left(d \mid H_{1}\right)=\frac{(s+b)^{d}}{d !} e^{-(s+b),}
\end{aligned}
$$

where $b$ and $s$ correspond to the number of predicted background and signal events respectively. To combine the two hypotheses into one metric to test the difference between the two 
hypotheses, we form the log likelihood ratio:

$$
\begin{gathered}
\text { LLR }=-2 \ln \left(\frac{P\left(d \mid H_{1}\right)}{P\left(d \mid H_{0}\right)}\right), \\
\text { LLR }=2 \sum_{i}^{N_{c}} \sum_{j}^{N_{b}}\left[s_{i j}-d_{i j} \ln \left(1+\frac{s_{i j}}{b_{i j}}\right)\right],
\end{gathered}
$$

where the sum over $i$ is over the different categories (lepton flavour, number of jets, and b-tag categories), and the sum over $j$ is over the bins of the final MVA distribution. The sums come about because the probability for multiple independent bins is the product of the individual bin probabilities.

In reality, the predicted number of events in a given hypothesis is uncertain, and we need to take into account systematic uncertainties on our predictions. This is done by considering the various parameters that affect the predictions of $H_{0}$ and $H_{1}$ as described in Sec. 6.7. Each of these parameters (referred to as nuisance parameters) has a range of possible values around some central value, and are considered as Gaussian distribution with the mean corresponding to the central value, and width corresponding to the uncertainty size. Thus for each hypothesis, we allow the number of predicted events to vary within the systematic uncertainties, and maximize the compatibility with our data. This is equivalent to minimizing a $\chi^{2}$ :

$$
\chi^{2}=-2 \ln P(d \mid H, \theta)=2 \sum_{i}^{N_{c}} \sum_{j}^{N_{b}}\left[p(H)_{i j}^{\prime}-d_{i j}-d_{i j} \ln \left(\frac{p(H)_{i}^{\prime}}{d_{i}}\right)\right]+\sum_{k}^{N_{n}} R(H)_{k}^{2} .
$$

Here, $p(H)_{i}^{\prime}$ is the predicted yields for a set of nuisance parameters, $N_{n}$, and $R(H)_{k}$ is the deviation of the $k^{t h}$ nuisance parameter from the central value in units of the Gaussian 
probability distribution width, $\sigma_{k}$ :

$$
R_{k}=\frac{\theta_{k}-\theta_{k}^{0}}{\sigma_{k}} .
$$

Our log-likelihood ratio is then:

$$
\begin{aligned}
\text { LLR } & =-2 \ln \left(\frac{P\left(d \mid H_{1}, \theta_{1}\right)}{P\left(d \mid H_{0}, \theta_{0}\right)}\right) \\
= & \chi^{2}\left(H_{0}\right)_{\text {min }}-\chi^{2}\left(H_{1}\right)_{\text {min }},
\end{aligned}
$$

where $\theta_{0}$ are the set of nuisance parameters that maximize the likelihood for $H_{0}$, and $\theta_{1}$ are the set of nuisance parameters that maximize the likelihood for $H_{1}$.

From here, we want to be able to quantify any excess that we observe above our background only prediction. We do this by considering confidence levels and p-values. The confidence level is a statement of how often the true value lies within a given interval. So a confidence interval with confidence level of $1-\alpha$ contains all values for which $H_{0}$ is not rejected at a significance level $\alpha$. The p-value is the probability that a hypothesis will fluctuate resulting in the observed data. We can relate the confidence levels and p-values for the two hypotheses by:

$$
\begin{aligned}
C L_{B} & =1-P V_{B}=\int_{x_{0}}^{\infty} H_{S+B}(\mathrm{LLR}) \mathrm{dLLR} \\
C L_{S+B} & =P V_{S+B}=\int_{x_{0}}^{\infty} H_{B}(\mathrm{LLR}) \mathrm{dLLR},
\end{aligned}
$$

where $x_{0}$ is the reference LLR (for example the data LLR). Since the LLR is a function of the signal rate, the confidence level also must be a function of the signal rate. 
In the absence of a significant signal, we want to set an upper limit on the signal cross section. The most straight forward metric to do this would be to simply consider $C L_{S+B}$. However, this does not protect against against making false exclusion statements from background-like fluctuations in our data. To prevent such false exclusions, we instead consider:

$$
C L_{S}=\frac{C L_{S+B}}{C L_{B}}
$$

This allows us to exclude the signal-plus-background hypothesis at a confidence level of 1- $\alpha$ when $C L_{S}<\alpha$. An upper limit on the cross cross section can be set (with a C.L. of $1-\alpha$ ) by determining how much we would need to scale up the signal rate by until $C L_{S}=\alpha$. If the rate does not need to be scaled for $C L_{S}$ to reach $\alpha$, then we can exclude the signal plus background hypothesis at a confidence level of 1- $\alpha$. 


\section{Chapter 6}

\section{Data Analysis}

The goal of this research is to search for the Higgs boson. Since there are multiple production mechanisms and decay modes for the Higgs boson, we first need to pick one production mode and one decay mode to consider for our search. At the Tevatron, the Higgs boson has three dominant production modes (Fig. 1.4): gluon-gluon fusion, and associated production with either a $W$ or a $Z$ boson. The Higgs boson decays into different particles based on its mass (Fig. 1.5). For a Higgs boson with a mass $M_{H}=125 \mathrm{GeV}$, the dominant decay mode is to a pair of bottom quarks, $H \rightarrow b \bar{b}$. While the gluon-gluon fusion production mode is about an order of magnitude higher than associated production with a $W$ or $Z$ boson, the amount of background events from the continuum of QCD $b \bar{b}$ production for $g g \rightarrow H \rightarrow b \bar{b}$ is far

too large compared to the Higgs boson production rate to make a search for $g g \rightarrow H \rightarrow b \bar{b}$ feasible. Instead, we search for a Higgs boson produced in association with a vector boson (either a $W$ or a $Z$ ), where the vector boson decays leptonically. Leptons are produced less frequently than hadronic particles in a hadron collider, so by triggering on a lepton we can greatly reduce the number of background event coming from multijet production. Presented here is the search for a Higgs boson produced in association with a $W$ boson, where the Higgs boson decays to a pair b quarks, and the $W$ decays to a lepton and a neutrino. 


\subsection{Analysis Strategy}

Once we have decided on a final state topology in which to search for the Higgs boson, we need to select events the events in data that match the topology of our expected final state. Then we need to estimate the contribution to our data from various processes that have the same final state topology as the Higgs boson signal. After we have selected the data events for analysis and simulated the contributions from the different processes, we try to distinguish our signal events from our background events. Since we are searching for a Higgs boson that decays to a pair of bottom quarks, we categorize our events based on b-tagging algorithms. We then distinguish signal and background using multivariate techniques. Finally, we perform a statistical analysis to determine whether our data includes the Higgs boson signal. To validate our search methodology, we perform a cross check by measuring the cross section of $V Z \rightarrow \ell \nu b \bar{b}$.

\subsection{Monte Carlo Simulation}

There are multiple processes that will produce the same final state topology as our $W H \rightarrow$ $\ell \nu b b$ signal. These processes include production of a $W$ boson with jets, $W+$ jets; production of a $Z$ boson with jets, $Z+$ jets (where one of the leptons from the $Z$ decay is not identified); single-top quark and top quark pair production; diboson ( $W W, W Z, Z Z)$ production; and multijet production. Example Feynman diagrams for the processes that we consider as background for our Higgs boson search are shown in Fig. 6.1. Additionally, there are other Higgs boson signals that result in a similar final state that are included in our signal sample: $Z H \rightarrow \ell \ell b b$ where one of the leptons is not reconstructed, $g g \rightarrow H \rightarrow W W \rightarrow \ell \nu j j$

$g g \rightarrow H \rightarrow Z Z \rightarrow \ell \ell j j$ (where again one of the leptons is not reconstructed), and $V H \rightarrow$ 
$V W W \rightarrow \ell \nu j j j j$ (where $V=W, Z$ and we don't reconstruct all four jets). Figure 6.2 shows example Feynman diagrams for our signal processes. The multijet events are estimated from data as is described in section 6.3. The other processes are simulated with MC event generators. The signal and diboson samples are generated with PYTHIA. The single top samples are produced with SINGLETOP. The $\mathrm{V}+$ jets and top pair samples are generated with ALPGEN and showered with PYTHIA. The cross sections multiplied by the relevant branching fractions for the simulated processes are given in Tables 6.1 to 6.3 .
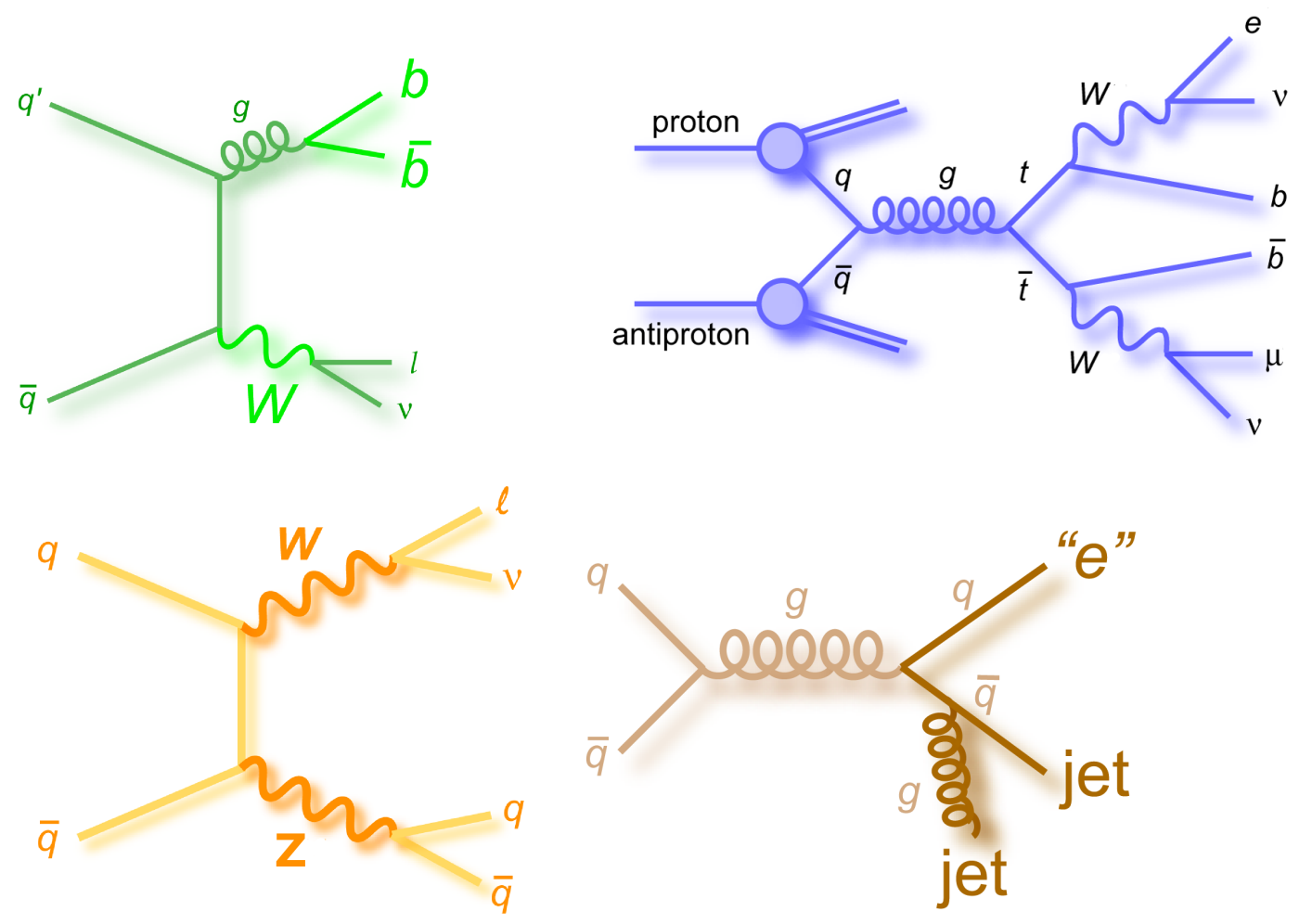

Figure 6.1: Example Feynman diagrams for processes we consider as backgrounds: $W+$ jets (top left), top quark pair production (top right), $W Z$ production (bottom left), and multijet production (bottom right).

In addition to the D0 common corrections described in the previous chapter, we derive and apply some corrections for our simulated samples. Past studies have shown that ALPGEN poorly reproduces certain kinematic distributions. To correct for this deficiency, we derive 
Table 6.1: The cross section times branching fraction for diboson and top-quark simulated processes.

\begin{tabular}{|l|c|}
\hline MC Process & $\sigma \times B[\mathrm{pb}]$ \\
\hline$W W$ & 11.34 \\
$W Z$ & 3.22 \\
$Z Z$ & 1.20 \\
$t \bar{t} \rightarrow b b+\ell^{+} \nu \ell^{\prime-} \bar{\nu}_{\ell^{\prime}}$ & 0.4900 \\
$t \bar{t} \rightarrow b b+\ell^{+} \nu \ell^{\prime-} \bar{\nu}_{\ell^{\prime}+1 \text {-jet }}$ & 0.1980 \\
$t \bar{t} \rightarrow b b+\ell^{+} \nu \ell^{\prime-} \bar{\nu}_{\ell^{\prime}+2 \text {-jets }}$ & 0.0941 \\
$t \bar{t} \rightarrow b b+2 j+\ell \nu$ & 2.0340 \\
$t \bar{t} \rightarrow b b+2 j+\ell \nu+1$-jet & 0.8270 \\
$t \bar{t} \rightarrow b b+2 j+\ell \nu+2$-jets & 0.4050 \\
Single-top $s$-channel $(t b \rightarrow e \nu b b)$ & 0.1050 \\
Single-top $s$-channel $(t b \rightarrow \mu \nu b b)$ & 0.1180 \\
Single-top $s$-channel $(t b \rightarrow \tau \nu b b)$ & 0.1260 \\
Single-top $t$-channel $(t q b \rightarrow e \nu b q b)$ & 0.2520 \\
Single-top $t$-channel $(t q b \rightarrow \mu \nu b q b)$ & 0.2470 \\
Single-top $t$-channel $(t q b \rightarrow \tau \nu b q b)$ & 0.2630 \\
\hline
\end{tabular}

Table 6.2: The cross section times branching fraction for $W+$ jets simulated processes.

\begin{tabular}{|l|c|}
\hline MC Process & $\sigma \times B[\mathrm{pb}]$ \\
\hline$W+1$-jet, $(W \rightarrow \ell \nu)$ & 1656.399 \\
$W+2$-jets & 388.983 \\
$W+3$-jets & 91.519 \\
$W+4$-jets & 20.920 \\
$W+5$-jets & 6.599 \\
$W b b,(W \rightarrow \ell \nu)$ & 17.828 \\
$W b b+1$-jet & 8.127 \\
$W b b+2$-jets & 2.971 \\
$W b b+3$-jets & 1.392 \\
$W c c,(W \rightarrow \ell \nu)$ & 45.684 \\
$W c c+1$-jet & 25.639 \\
$W c c+2$-jets & 10.463 \\
$W c c+3$ jets & 5.08 \\
\hline
\end{tabular}


Table 6.3: The cross section times branching fraction for $Z+$ jets simulated processes.

\begin{tabular}{|l|c|}
\hline$Z+1$-jet, $(Z \rightarrow \ell \ell), 15<M_{Z}<75 \mathrm{GeV}$ & 50.6160 \\
$Z+2$-jets, $15<M_{Z}<75 \mathrm{GeV}$ & 12.3227 \\
$Z+3$-jets, $15<M_{Z}<75 \mathrm{GeV}$ & 3.4067 \\
$Z+1$-jet, $Z \rightarrow \ell \ell), 75<M_{Z}<130 \mathrm{GeV}$ & 51.6103 \\
$Z+2$-jets, $75<M_{Z}<130 \mathrm{GeV}$ & 12.4390 \\
$Z+3$-jets, $75<M_{Z}<130 \mathrm{GeV}$ & 3.9607 \\
$Z+1$-jet, $(Z \rightarrow \ell \ell), 130<M_{Z}<250 \mathrm{GeV}$ & 0.4700 \\
$Z+2$-jets, $130<M_{Z}<250 \mathrm{GeV}$ & 0.1229 \\
$Z+3$-jets, $130<M_{Z}<250 \mathrm{GeV}$ & 0.0418 \\
$Z+1$-jet, $Z \rightarrow \ell \ell), 250<M_{Z}<1950 \mathrm{GeV}$ & 0.04472 \\
$Z+2$-jets, $250<M_{Z}<1950 \mathrm{GeV}$ & 0.0131 \\
$Z+3$-jets, $250<M_{Z}<1950 \mathrm{GeV}$ & 0.0048 \\
$Z b b,(Z \rightarrow \ell \ell), 15<M_{Z}<75 \mathrm{GeV}$ & 0.9853 \\
$Z b b+1$-jet, $15<M_{Z}<75 \mathrm{GeV}$ & 0.3810 \\
$Z b b+2$-jets, $15<M_{Z}<75 \mathrm{GeV}$ & 0.1497 \\
$Z b b,(Z \rightarrow \ell \ell), 75<M_{Z}<130 \mathrm{GeV}$ & 0.8050 \\
$Z b b+1$-jet, $75<M_{Z}<130 \mathrm{GeV}$ & 0.3643 \\
$Z b b+2$-jets, $75<M_{Z}<130 \mathrm{GeV}$ & 0.1969 \\
$Z b b,(Z \rightarrow \ell \ell), 130<M_{Z}<250 \mathrm{GeV}$ & 0.0066 \\
$Z b b+1$-jet, $130<M_{Z}<250 \mathrm{GeV}$ & 0.0034 \\
$Z b b+2$-jets, $130<M_{Z}<250 \mathrm{GeV}$ & 0.0018 \\
$Z b b,(Z \rightarrow \ell \ell), 250<M_{Z}<1960 \mathrm{GeV}$ & 0.0006 \\
$Z b b+1$-jet, $250<M_{Z}<1960 \mathrm{GeV}$ & 0.0003 \\
$Z b b+2$-jets, $250<M_{Z}<1960 \mathrm{GeV}$ & 0.0002 \\
$Z c c,(Z \rightarrow \ell \ell), 15<M_{Z}<75 \mathrm{GeV}$ & 8.6933 \\
$Z c c+1$-jet, $15<M_{Z}<75 \mathrm{GeV}$ & 2.1390 \\
$Z c c+2$-jets, $15<M_{Z}<75 \mathrm{GeV}$ & 0.7797 \\
$Z c c,(Z \rightarrow \ell \ell), 75<M_{Z}<130 \mathrm{GeV}$ & 1.9343 \\
$Z c c+1$-jet, $75<M_{Z}<130 \mathrm{GeV}$ & 1.0920 \\
$Z c c+2$-jets, $75<M_{Z}<130 \mathrm{GeV}$ & 0.6120 \\
$Z c c,(Z \rightarrow \ell \ell), 130<M_{Z}<250 \mathrm{GeV}$ & 0.0159 \\
$Z c c+1$-jet, $130<M_{Z}<250 \mathrm{GeV}$ & 0.0093 \\
$Z c c+2$-jets, $130<M_{Z}<250 \mathrm{GeV}$ & 0.0070 \\
$Z c c,(Z \rightarrow \ell \ell), 250<M_{Z}<1960 \mathrm{GeV}$ & 0.0013 \\
$Z c c+1$-jet, $250<M_{Z}<1960 \mathrm{GeV}$ & 0.0010 \\
$Z c c+2$-jets, $250<M_{Z}<1960 \mathrm{GeV}$ & 0.0005 \\
\hline
\end{tabular}



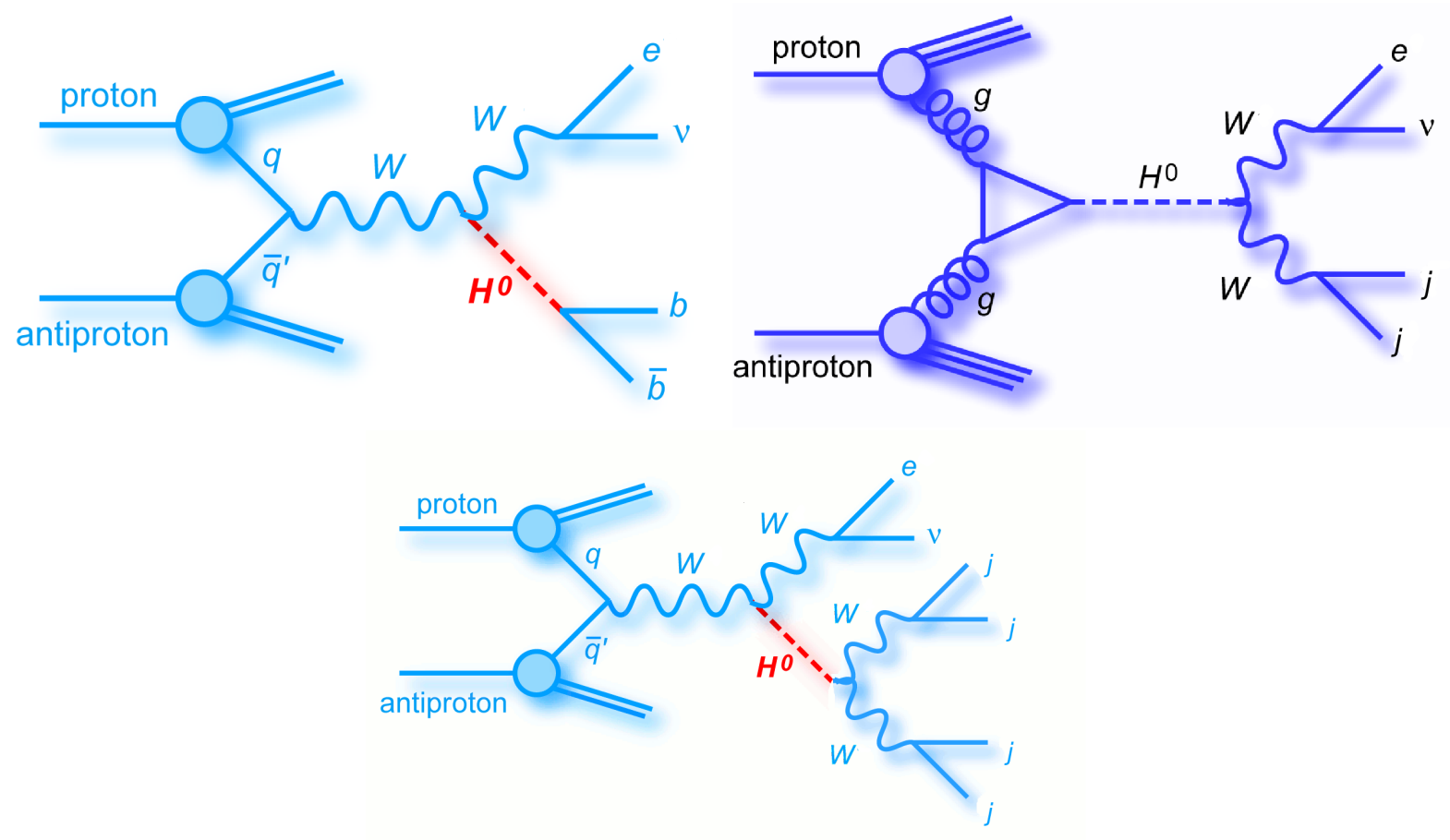

Figure 6.2: Feynman diagrams for processes contributing to our Higgs boson signal: associated production of a Higgs with a $\mathrm{W}$ boson, with the Higgs boson decaying to a pair of $b$-quarks (left); $g g \rightarrow H \rightarrow W W$ (middle), and associated production of a Higgs boson with a $W$ boson, with the Higgs boson decaying to a pair of $W$ bosons (right). 
reweightings for our $W / Z+$ jets samples. The reweightings are derived before the application of b-tagging so that any possible signal contamination is very small. To improve the description of jet angles, we correct the $\eta$ distributions for the two highest $p_{T}$ jets. The correction has the form of a fourth-order polynomial, determined by fitting the ratio of $\mathrm{V}+$ jet events in $\mathrm{MC}$ to data minus the non- $\mathrm{V}+$ jet backgrounds. The lepton $\eta$ distribution is corrected in $W+$ jet events by a second-order polynomial. We also correct for an observed discrepancy between our data and simulation that is correlated between the $W$ boson $p_{T}\left(p_{T}^{W}\right)$ and $\Delta R\left(j_{1}, j_{2}\right)$. This two dimensional function is a product of a third-order polynomial in $\Delta R$, and an error function plus a Gaussian plus a constant in $p_{T}^{W}$. The $p_{T}^{W}$ reweighting is applied to $W+$ jet events, and the $\Delta R$ correction is applied to both $W$ and $Z+$ jet events. Each of these corrections is designed to alter the shape of the distributions, but preserve overall normalizations. These reweighting functions can be seen in Fig. 6.3.

\subsection{Multijet Background Estimation}

When colliding protons and anti-protons, a large amount of the time the result will be production of multiple jets. By considering events triggered by a lepton we can cut down on this background. However it is possible for a jet to mimic a lepton signature in our detector such that a jet is misidentified as a lepton. Mostly this occurs if most of the jet's energy upon hadronization goes into a single pion. The favoured decay mode for a charged pion is $\pi^{+} \rightarrow \mu^{+} \nu_{\mu}$, which will lead to a jet being misidentified as a muon. Neutral pions favour the

decay to a pair of photons, which can lead to a misidentified electron. It is also possible for the jet to simply produce a shower shape similar to that expected for an electron so that we would reconstruct an electron instead of a jet. The estimation of our multijet background 

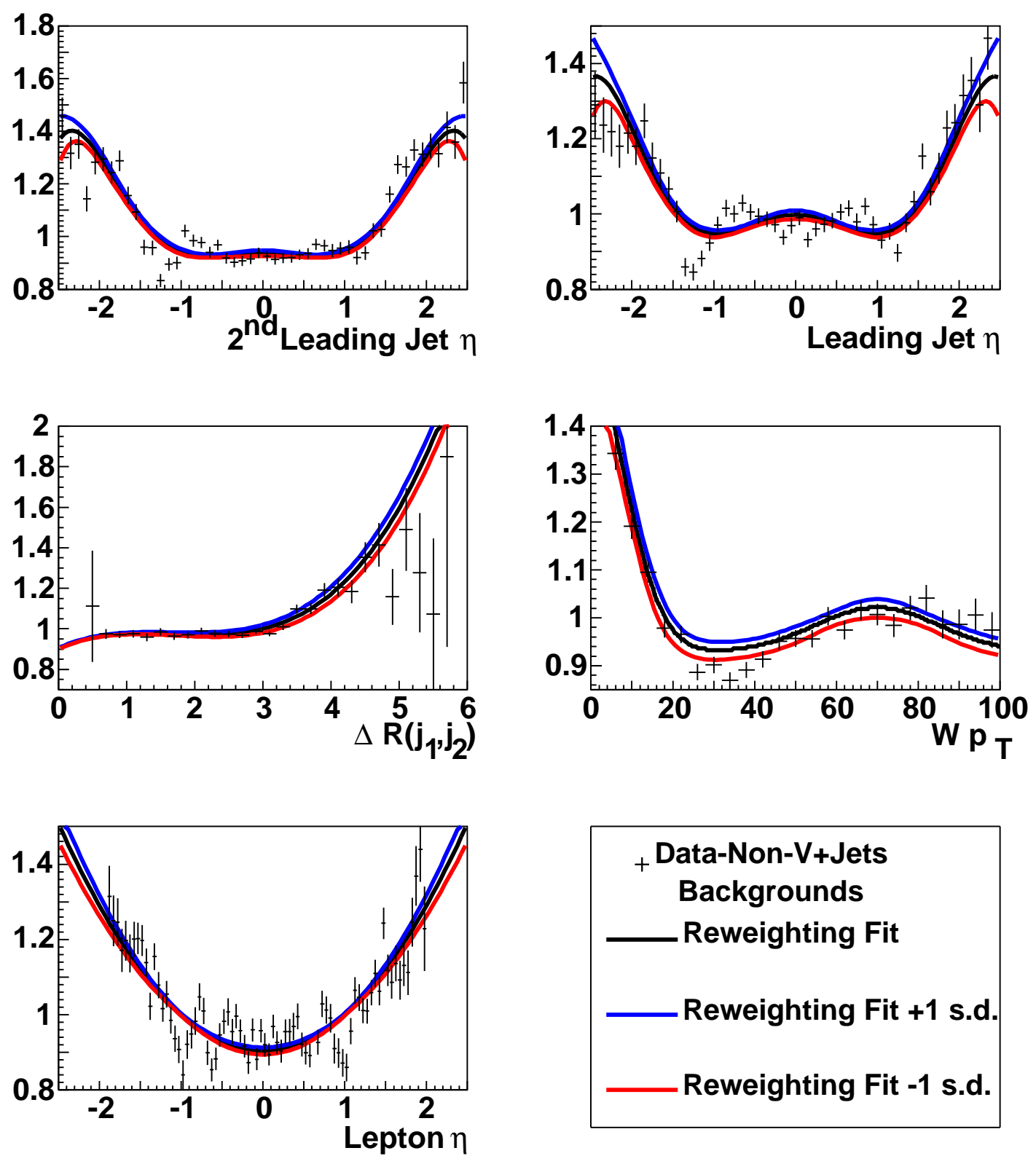

Figure 6.3: Reweighting functions to correct for mismodelling in the ALPGEN V+jets MC for the second leading jet $\eta$ (top left), leading jet $\eta$ (top right), $W p_{T}$ (middle left), $\Delta R\left(j_{1}, j_{2}\right.$ ) (middle right), and lepton $\eta$ (bottom). The black points are the data with all non-V+jets backgrounds subtracted off. The black curves are the reweighting functions fit to the points, and the blue and red are the \pm 1 standard deviations on the black curve. 
comes from data. We use the lepton isolation to define two orthogonal samples with similar kinematic distributions. Events with a lepton that passes "loose" isolation requirements are used to estimate the multijet contribution, and events with a lepton that passes "tight" isolation requirements are used in the analysis sample. In multijet events, we expect that usually the $E_{T}$ we measure to be from a mismeasurement instead of from a neutrino from a $W$ boson decay, so we use events with relatively low $E_{T}, 5<E_{T}<15 \mathrm{GeV}$, to estimate the multijet contribution. Then we weight the events so that we can properly estimate the number of events contributing to our analysis sample. The template is created using a modified version of the Matrix Method to reweight events in data. The matrix method works by solving the following pair of equations for the number of real lepton and multijet events:

$$
\begin{aligned}
& N_{L}=N_{\ell}+N_{M J}, \\
& N_{T}=\epsilon_{\ell} N_{\ell}+f_{j} N_{M J},
\end{aligned}
$$

where $N_{L / T}$ are the number of events that pass loose/tight lepton isolation requirements, $N_{\ell / M J}$ is the number of real lepton/misidentified multijet events in the data sample with the loose isolation requirements, $\epsilon_{\ell}$ is the efficiency for a real lepton that passes the loose isolation requirements to subsequently pass the tight isolation requirements, and $f_{j}$ is the efficiency for a jet that passes the loose isolation requirements to subsequently pass the tight isolation requirements. Solving this system of equations for the number of multijet events in the sample with tight isolation requirements:

$$
N_{M J}^{T}=\frac{f_{j}}{\epsilon_{\ell}-f_{j}}\left(\epsilon_{\ell} N_{L}-N_{T}\right) .
$$


We can then weight each event in our templates by:

$$
w=\frac{f_{j}}{\epsilon_{\ell}-f_{j}}\left(\epsilon_{\ell}-\Theta^{T}\right),
$$

where $\Theta^{T}=1$ if the event satisfies tight isolation requirements, and 0 otherwise. The total number of multijet events in the tight sample is then the sum of weights over all events that pass the loose isolation requirements:

$$
N_{M J}^{T}=\sum_{i=1}^{N^{L}} w_{i}
$$

Since this method uses all events that pass the loose isolation requirements, events that have a lepton that also passes the tight isolation requirements will contribute with a negative weight, and the multijet sample is not statistically independent from the data sample, which could potentially result in correlated fluctuations. To avoid this, we employ a slightly modified version of the matrix method where we consider only the events that pass the loose lepton identification requirements, but fail the tight lepton identification requirements (so-called loose-not-tight events). These events are weighted by:

$$
W_{i}=\frac{f_{j}}{1-f_{j}}
$$

This will result in a multijet prediction of:

$$
N_{M J}^{T}=\sum_{i=1}^{N^{L-n-T}} W_{i}
$$

where, $N^{L-n-T}$ is the number of events in the loose-not-tight sample. 
The efficiencies for real leptons and jets misidentified as leptons depend on the event kinematics. The real lepton efficiencies for electrons, $\epsilon_{e}$, are parametrized as a function of detector $\eta$, electron $p_{T}$, and instantaneous luminosity. For muons, $\epsilon_{\mu}$ is parametrized as a function of muon $p_{T}$. The efficiency rates are determined by studying $Z \rightarrow \ell \ell$ events. The efficiency with which a jet is misidentified as a lepton, $f_{j}$, is determined by studying events with $5<E_{T}<15 \mathrm{GeV}$ by considering the ratio of loose to tight MC-subtracted data events. For electrons, $f_{j}^{e}$ is parametrized as a function of electron $p_{T}$, detector $\eta$, and $\min \Delta \phi\left(E_{T}, j e t\right)$. For muons, $f_{j}^{\mu}$ is parametrized as a function of muon $p_{T}$, detector $\eta$, and $\Delta \phi\left(E_{T}, \mu\right)$. An example $f_{j}^{e}$ is shown in Fig. 6.4, and an example of $f_{j}^{\mu}$ is shown in Fig. 6.5.

\subsection{Selection}

Since we are trying to find a Higgs boson that decays to b-quarks, and a $W$ that decays leptonically, we want to look for exactly one high energy lepton (either an electron or muon), a large amount of $\mathbb{E}_{T}$ from the neutrino in the $W$ decay, and two or three jets. We require the lepton $p_{T}$ to be greater than $15 \mathrm{GeV}$ to cut down on the number of multijet events without cutting out too many signal events from our sample, since the $p_{T}$ of a lepton from a $W$ boson decay tends to be large compared to the misidentified leptons in the multijet sample. The lepton $\eta$ is required to be less than 2 for muons so that the muon passes through the acceptance of the muon system. We require the electron to pass through either the $\mathrm{CC},|\eta|<1.1$, or the EC, $1.5<|\eta|<2.5$. The $E_{T}$ is required to be greater than $15 \mathrm{GeV}$ in electron events to ensure orthogonality with the sample in which we estimate the multijet contribution to our data, and $20 \mathrm{GeV}$ in muon events to further suppress the multijet background. Since jets in our signal are coming from the decay of a Higgs, they 

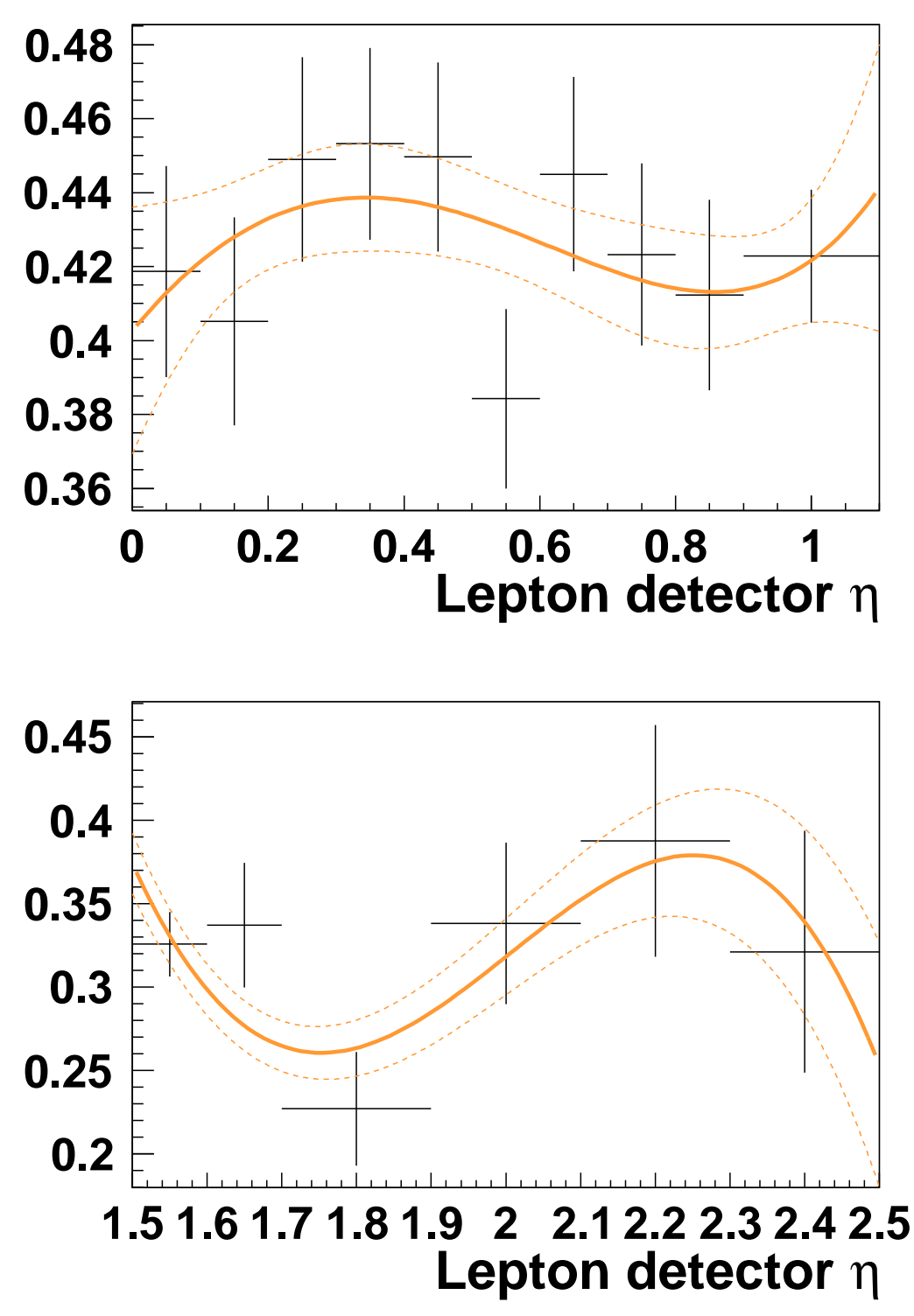

Figure 6.4: Example of the probability for a jet that passes the loose lepton identification requirements to pass the tight lepton isolation requirements for the $15<p_{T}<17 \mathrm{GeV}$ range with $0.4<\min \left[\Delta \phi\left(E_{T}, j e t\right)\right]<\pi$ as a function of the electron $\eta$ in the (a) CC and (b) EC. The solid line is a fit to the data, and the dashed lines are the functions with the parameters shifted up and down by their uncertainties. 

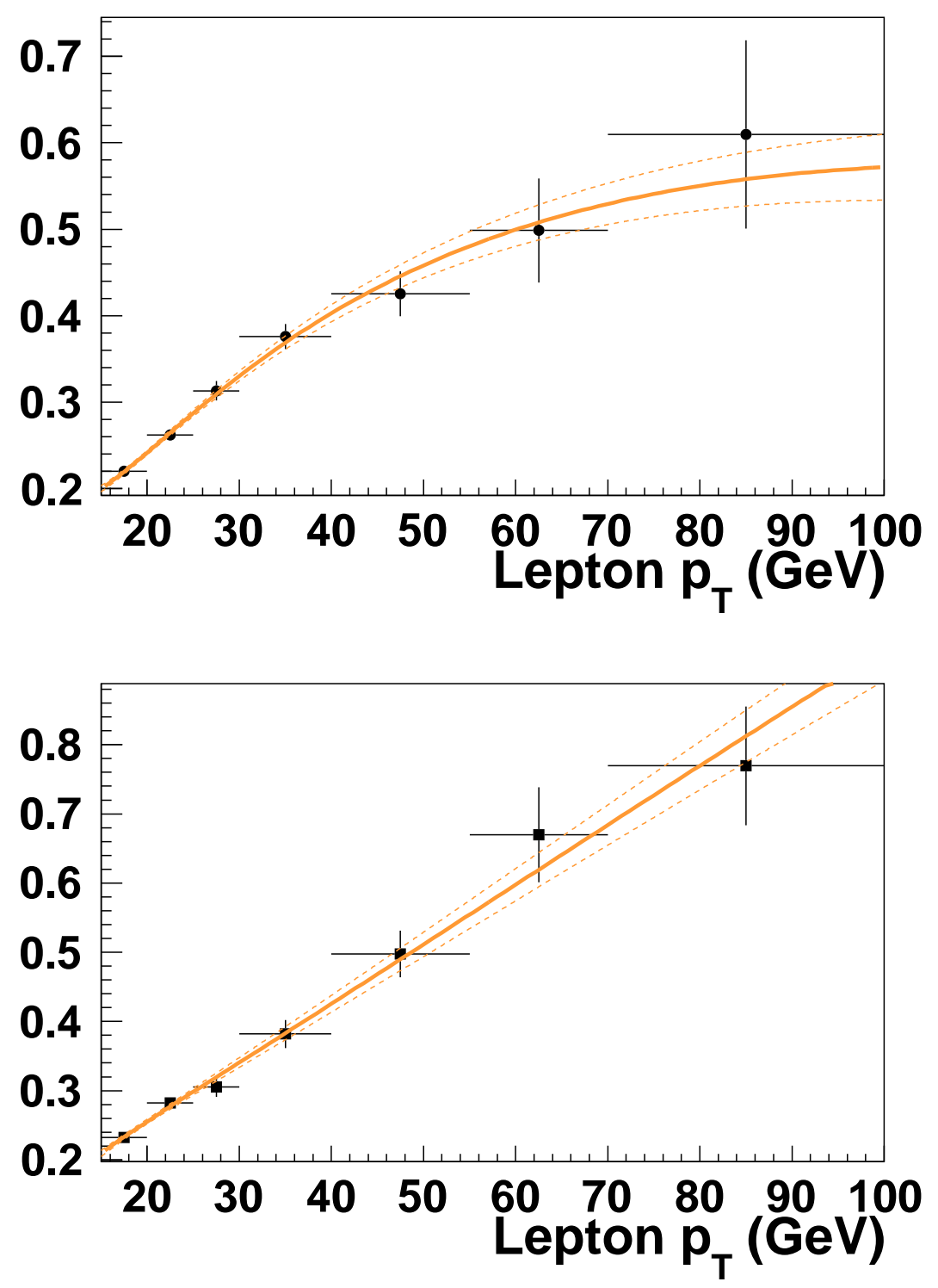

Figure 6.5: Example of the probability for a jet that passes the loose lepton identification requirements to pass the tight lepton isolation requirements as a function of muon $p_{T}$ for (a) $|\eta|<1.0$ and (b) $1.0<|\eta|<1.6$, with $0<\Delta \phi\left(E_{T}, \mu\right)<1$. The solid line is a fit to the data, and the dashed lines are the functions with the parameters shifted up and down by their uncertainties. 
will tend to have higher $p_{T}$ than jets coming from gluon splitting as the jets in our $W b \bar{b}$ background do. We therefore require that the jet $p_{T}$ be larger than $20 \mathrm{GeV}$. Since we are looking for a charged lepton, and two charged jets (from the b-quarks from the Higgs boson decay), we require that the event have a primary vertex with at least three associated tracks, and that the z position be within the coverage of the SMT, $|z|<60 \mathrm{~cm}$. We also require that the primary vertex be matched to the interaction point by requiring that the radial distance between the primary vertex and the interaction point be less than $1 \mathrm{~cm}$. We also impose a two dimensional requirement on the $E_{T}$ and transverse mass of the reconstructed $W$ boson, $M_{T}^{W}$, such that $M_{T}^{W}>40 \mathrm{GeV}-0.5 E_{T}$. This helps to reduce the multijet background, since the lepton and neutrino in a multijet event tend to not come from a real $W$, the transverse mass of the reconstructed $W$ will tend to be low for these events. We have two categories of leptons based on the lepton isolation. The leptons with a "loose" isolation are used to estimate the multijet background as described above, and the leptons with a "tight" isolation are used to perform the analysis. For electrons, the loose and tight isolation is defined by different requirements on the electron ID MVA output. For muons, the loose isolation requires that the lepton be outside of a reconstructed jet, $\Delta R(\mu$, jet $)>0.5$. For an isolated muon, we expect that most of the momentum from charged particles near the muon should come from the muon itself. To account for this, the tight isolation additionally requires that the ratio of the scalar sum of the $p_{T}$ of the tracks within $\Delta R=0.5$ of the muon to the muon $p_{T}$ be less than 0.4 . We also require that the ratio of the transverse energy deposited in a hollow cone of $0.1<\Delta R<0.4$ around the muon to the muon $p_{T}$ must be less than 0.12 . This reduces the probability that a neutral particle has deposited energy in the calorimeter near the muon.

The $W / Z+$ jets cross sections have a large uncertainty, so we apply an additional scaling 
factor to our $W+$ jets and $Z+$ jets samples based on a fit to data. The fit is done before b-tagging by subtracting the non- $\mathrm{V}+$ jets $\mathrm{MC}$ (tt, single top, and diboson) from the data. Then using the transverse mass of the $W$ boson, the $\mathrm{V}+$ jets $\mathrm{MC}$ and multijet contributions are simultaneously fit to the data, and overall scale factors are obtained. These fits are done separately for electron and muon events, and separately for events with two jets and three jets.

Events containing an electron are selected by a logical OR of triggers that require an electromagnetic object. The efficiencies of the triggers measured in data are applied to the MC simulation. These efficiencies are parametrized in electron $\eta, \phi$, and $p_{T}$. Depending on the trigger, and the electron's position within the detector, these efficiencies are $90-100 \%$. Events containing a muon are selected based on the logical OR of all available triggers, except those that contain lifetime based requirements that are used for b-jet identification. To determine the trigger efficiency in this inclusive trigger sample, we consider a subset of triggers which are about $70 \%$ efficient where the simulation models the data well, based on the logical OR of single muon and muon+jets triggers $\left(T_{\mu O R}\right)$. By comparing the data in this subset to that in the inclusive trigger sample, we can determine an inclusive trigger correction for the $\mathrm{MC}$ trigger efficiency, $P_{\text {corr }}$ :

$$
P_{\text {corr }}=\frac{\left(N_{\text {data }}-N_{\mathrm{MJ}}\right)_{\text {incl }}-\left(N_{\text {data }}-N_{\mathrm{MJ}}\right)_{T_{\mu \mathrm{OR}}}}{N_{\mathrm{MC}}},
$$

where the numerator is the difference (after subtracting off the multijet background component) between the number of data events in the inclusive and $T_{\mu \mathrm{OR}}$ trigger samples, and the denominator is the total number of MC events. The total trigger efficiency is estimated as the efficiency in the $T_{\mu \mathrm{OR}}$ sample $+P_{\text {corr }}$, and is limited to be no more than 1 . 
The correction, $P_{\text {corr }}$ is derived as a function of the sum of jet $p_{T}, H_{T}$, and the $E_{T}$, in bins of muon $\eta$. The $\eta$ bins are chosen based on different triggers dominating in different regions of the detector. Single muon triggers dominate for $|\eta|<1.0$, while muon + jets triggers are dominant for $|\eta|>1.6$. The intermediate region, $1.0<|\eta|<1.6$, is a mixture of single muon and muon+jets triggers. Detector supports allow only partial coverage of the muon detector when the muon $|\eta|<1.6$ and $-2<\phi<-1.2$. This support structure impacts the trigger efficiency in these regions, so we derive separate corrections for events that pass through the region where the supports are and events that do not encounter the support region. Examples of these corrections can be seen in Fig. 6.6. This strategy results in a trigger efficiency of $\sim 80-100 \%$, depending on the $H_{T}$ and the muon's position in the detector.

\section{5 b Tagging}

Since we are looking for a Higgs boson that decays to b-quarks, we employ b-tagging to categorize our candidate events based on the likelihood that the event contains either one or two b-tagged jets, with one category for events with one b-tagged jet and three categories for events with two b-tagged jets. The categories for events with two b-tagged jets are chosen based on the MVA b-jet identification (bid) output shown in Fig. 6.7. We choose the categories such that they each contain a different relative contribution of backgrounds, and a different ratio of signal to background events. The category with one "tight" b-tagged jet, requires that the bid MVA output is greater than 0.15. In Fig. 6.7, we see that below a bid output of 0.35 , the background is dominated by $V+$ light jet $(V+$ lf $)$ and multijet events with a small contribution from diboson $(V V)$ events, and above 0.55 , the dominant 

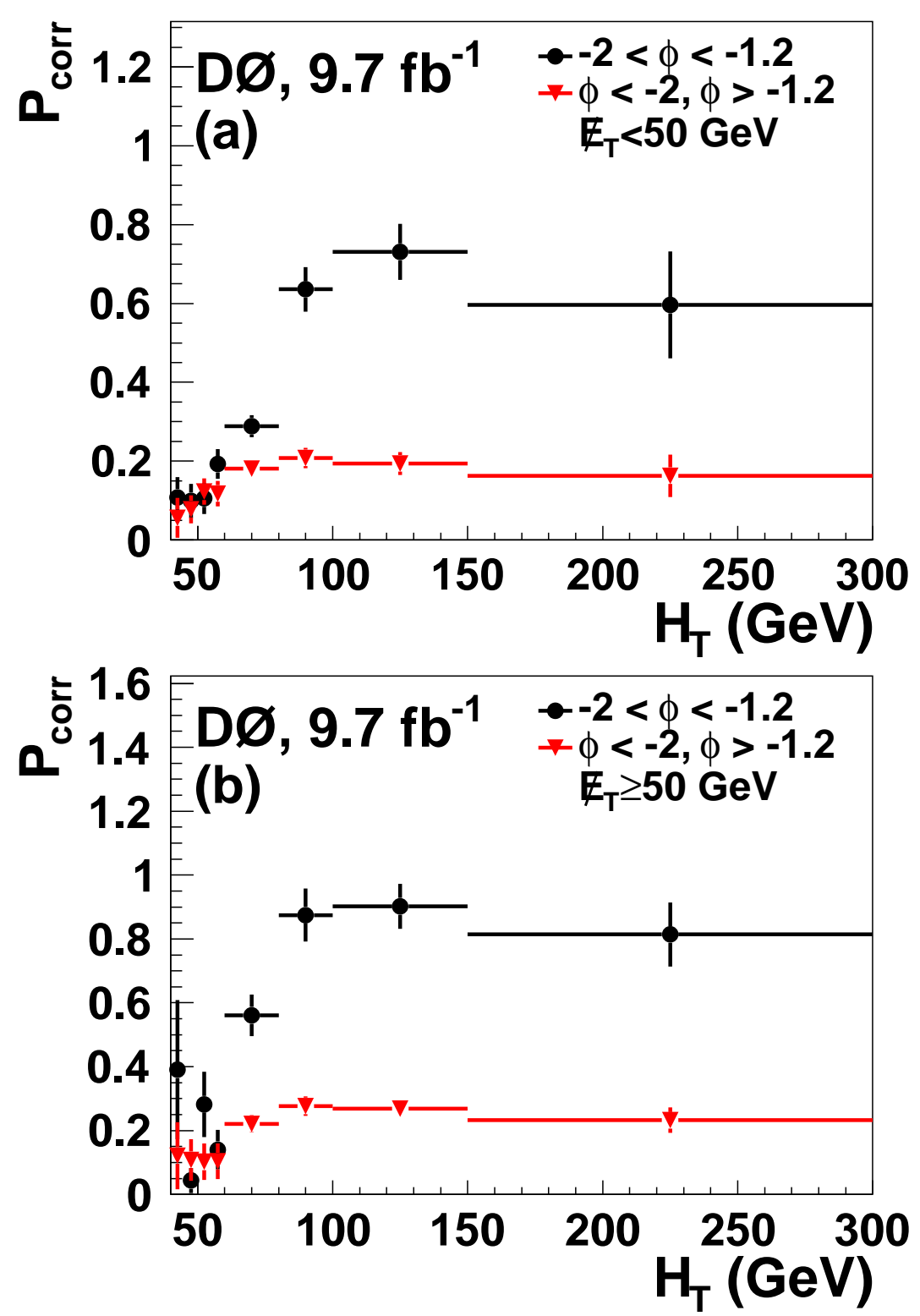

Figure 6.6: Muon trigger correction, derived from data to account for the gain in efficiency when moving from single muon and muon + jets triggers to inclusive triggers. The corrections are parametrized as a function $H_{T}$, shown for events with muon $|\eta|<1.0$ with a) $\mathbb{E}_{T}<$ $50 \mathrm{GeV}$ and b) $E_{T} \geq 50 \mathrm{GeV}$. The black circles represent the correction when the muon passes through the detector support region $(-2<\phi<-1.2)$, and the red triangles represent the correction in all other regions of $\phi[2]$. 
contribution is from $V+$ heavy jet $(V+\mathrm{hf})$ and top events. We also see that the signal is peaking near 1 and also near 0.5. Thus, for events with two b-tagged events we define three categories based on the average bid MVA output for the two jets: two "loose" b-tags $\left(0.02<\left(\operatorname{bid}_{j 1}+\operatorname{bid}_{j 2}\right) / 2<0.35\right)$, two "medium" b-tags $\left(0.35<\left(\operatorname{bid}_{j 1}+\operatorname{bid}_{j 2}\right) / 2<0.55\right)$, and two "tight" b-tags $\left(0.55<\left(\operatorname{bid}_{j 1}+\operatorname{bid}_{j 2}\right) / 2\right)$. Events that fall into more than one b-tag category, are considered in the most stringent tag category. For example, events that pass the two tight b-tag requirements will also pass the two loose b-tag requirements, but are only counted once, in the two tight b-tag category. The requirements for individual jets to pass the loose (medium, tight) threshold, averaged over jet $p_{T}$ and $\eta$, have an identification efficiency of $79 \%(57 \%, 47 \%)$, with a b-tagging misidentification rate of $11 \%(0.6 \%, 0.15 \%)$ for light quark jets.

All events that pass our selection requirements discussed in Sec. 6.4 before being classified into the different b-tagging categories make up our pretag sample. Figures 6.8 to 6.10 show some selected distributions of various kinematic at the pretag level. We see that our expected background events describe the data well. The reconstructed dijet mass is shown in Figs. 6.11 and 6.12 for the four different tag categories. From comparing the dijet mass in the different b-tag categories, we can see that the ratio of the number of signal events to the number of background events gets larger as we require stricter b-tag requirements, and also that the relative background contributions changes as we look at the different b-tag categories. For example the two tight b-tag category is dominated by top and $V$ theavy jet production, whereas the two loose b-tag category has a larger contribution from $V+$ light jet production. The signal, data, and background yields are given in Tables 6.4 to 6.7 for events in the pretag and all four tagging categories. 


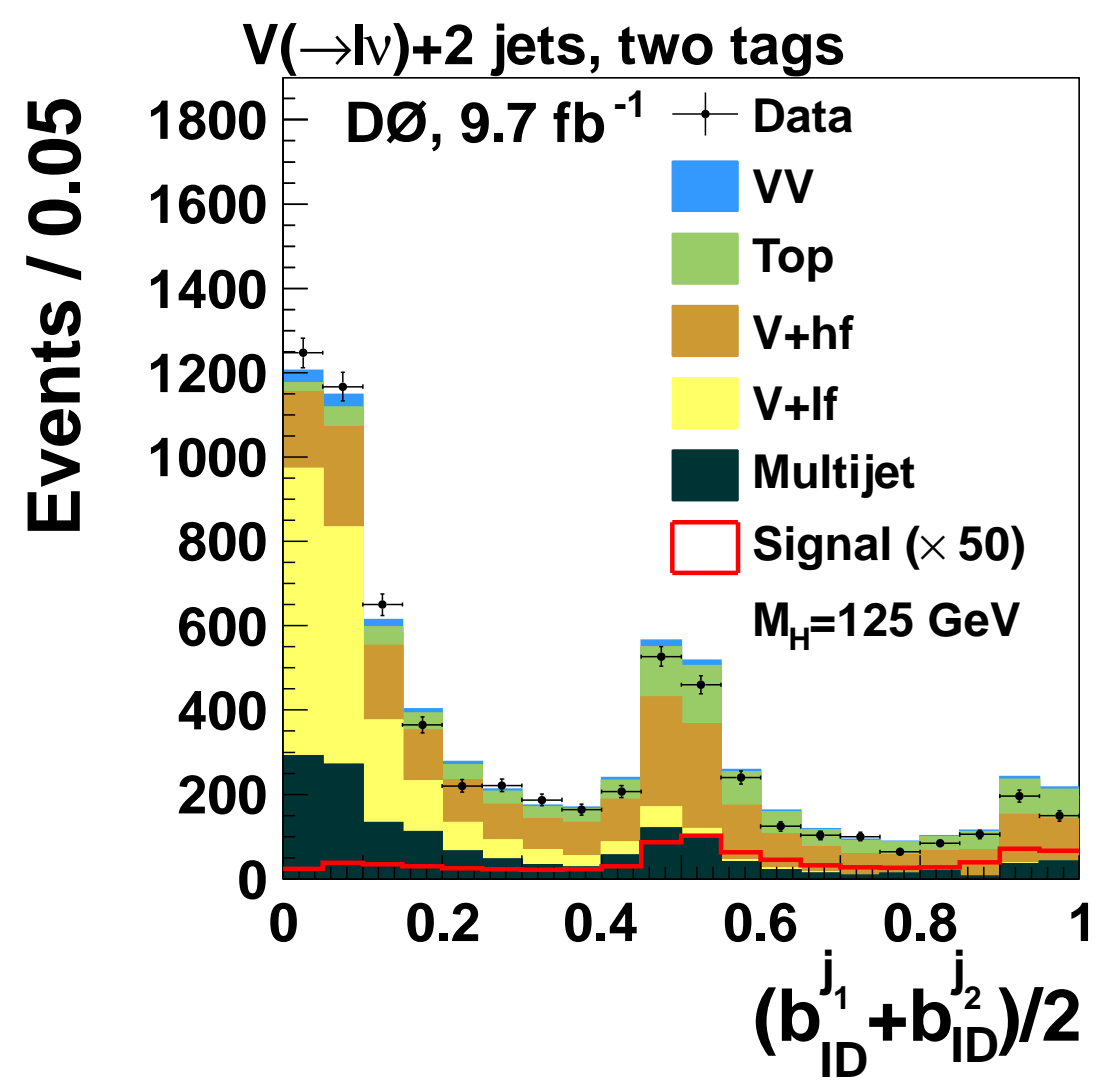

Figure 6.7: The average b-jet identification output for events with two jets. We define three categories containing two b-tagged jets based on this average output. The signal is shown for a Higgs boson mass of $125 \mathrm{GeV}$, scaled by a factor of 50 [2]. 
Table 6.4: Observed number of events in data and expected number of events from each signal and background source (where $V=W, Z$ ) for events with a muon and exactly two jets. The expected signal is quoted at $M_{H}=125 \mathrm{GeV}$. The total background uncertainty includes all sources of systematic uncertainty added in quadrature.

\begin{tabular}{|l|r|r|r|r|r|}
\hline & Pretag & $\begin{array}{r}1 \text { tight } \\
b \text {-tag }\end{array}$ & $\begin{array}{r}2 \text { loose } \\
b \text {-tags }\end{array}$ & $\begin{array}{r}2 \text { med. } \\
b \text {-tags }\end{array}$ & $\begin{array}{r}2 \text { tight } \\
b \text {-tags }\end{array}$ \\
\hline$V H \rightarrow \ell \nu b b$ & 17.4 & 5.0 & 1.4 & 2.1 & 3.5 \\
$H \rightarrow V V \rightarrow \ell \nu j j$ & 10.9 & 0.8 & 0.2 & 0.04 & 0 \\
$V H \rightarrow V V V \rightarrow \ell \nu j j j j$ & 5.1 & 0.4 & 0.1 & 0.02 & 0 \\
\hline Diboson & 2904 & 383 & 68.5 & 39.1 & 44.6 \\
$V+(g, u, d, s)$-jets & 78109 & 2645 & 807 & 60.9 & 5.5 \\
$V+(b \bar{b} / c \bar{c})$ & 13445 & 2531 & 486 & 341 & 335 \\
top $(t \bar{t}+$ single top) & 3448 & 688 & 126 & 156 & 202 \\
Multijet & 28486 & 1560 & 367 & 121 & 87.6 \\
\hline Total background & & & & & \\
expectation & 126425 & 7813 & 1856 & 720 & 678 \\
Total background & & & & & \\
uncertainty & 7576 & 835 & 177 & 87 & 85 \\
Observed events & 126811 & 7460 & 1870 & 656 & 544 \\
\hline
\end{tabular}

Table 6.5: Observed number of events in data and expected number of events from each signal and background source (where $V=W, Z$ ) for events with an electron and exactly two jets. The expected signal is quoted at $M_{H}=125 \mathrm{GeV}$. The total background uncertainty includes all sources of systematic uncertainty added in quadrature.

\begin{tabular}{|l|r|r|r|r|r|}
\hline & Pretag & $\begin{array}{r}1 \text { tight } \\
b \text {-tag }\end{array}$ & $\begin{array}{r}2 \text { loose } \\
b \text {-tags }\end{array}$ & $\begin{array}{r}2 \text { med. } \\
b \text {-tags }\end{array}$ & $\begin{array}{r}2 \text { tight } \\
b \text {-tags }\end{array}$ \\
\hline$V H \rightarrow \ell \nu b \bar{b}$ & 19.2 & 6.1 & 1.6 & 2.3 & 3.9 \\
$H \rightarrow V V \rightarrow \ell \nu j j$ & 13.2 & 1.0 & 0.2 & 0.03 & 0 \\
$V H \rightarrow V V V \rightarrow \ell \nu j j j j$ & 7.1 & 0.7 & 0.1 & 0.02 & 0.01 \\
\hline Diboson & 2877 & 269 & 55.7 & 21.5 & 18.6 \\
$V+(g, u, d, s)$-jets & 89728 & 3121 & 892 & 63.6 & 6.4 \\
$V+(b \bar{b} / c \bar{c})$ & 13596 & 2595 & 480 & 338 & 343 \\
top $(t \bar{t}+$ single top) & 1932 & 703 & 137 & 184 & 252 \\
Multijet & 41986 & 2407 & 619 & 169 & 104 \\
\hline Total background & & & & & \\
expectation & 150158 & 9103 & 2187 & 779 & 727 \\
Total background & & & & & \\
uncertainty & 7423 & 887 & 192 & 92 & 92 \\
Observed events & 150118 & 8946 & 2187 & 702 & 621 \\
\hline
\end{tabular}


Table 6.6: Observed number of events in data and expected number of events from each signal and background source (where $V=W, Z$ ) for events with a muon and exactly three jets. The expected signal is quoted at $M_{H}=125 \mathrm{GeV}$. The total background uncertainty includes all sources of systematic uncertainty added in quadrature.

\begin{tabular}{|l|r|r|r|r|r|}
\hline & Pretag & $\begin{array}{r}1 \text { tight } \\
b \text {-tag }\end{array}$ & $\begin{array}{r}2 \text { loose } \\
b \text {-tags }\end{array}$ & $\begin{array}{r}2 \text { med. } \\
b \text {-tags }\end{array}$ & $\begin{array}{r}2 \text { tight } \\
b \text {-tags }\end{array}$ \\
\hline$V H \rightarrow \ell \nu b b$ & 4.1 & 1.0 & 0.4 & 0.5 & 0.8 \\
$H \rightarrow V V \rightarrow \ell \nu j j$ & 4.4 & 0.4 & 0.1 & 0.03 & 0 \\
$V H \rightarrow V V V \rightarrow \ell \nu j j j j$ & 2.9 & 0.3 & 0.1 & 0.02 & 0 \\
\hline Diboson & 608 & 85.7 & 25.2 & 13.4 & 11.2 \\
$V+(g, u, d, s)$-jets & 10689 & 443 & 285 & 14.9 & 2.3 \\
$V+(b \bar{b} / c \bar{c})$ & 3577 & 682 & 228 & 135 & 109 \\
top $(t \bar{t}+$ single top) & 1845 & 508 & 163 & 201 & 247 \\
Multijet & 4253 & 315 & 127 & 41.4 & 14.6 \\
\hline Total background & & & & & \\
expectation & 20983 & 2034 & 829 & 406 & 385 \\
Total background & & & & & \\
uncertainty & 1360 & 205 & 73 & 41 & 40 \\
Observed events & 21297 & 2027 & 828 & 413 & 401 \\
\hline
\end{tabular}

Table 6.7: Observed number of events in data and expected number of events from each signal and background source (where $V=W, Z$ ) for events with an electron and exactly three jets. The expected signal is quoted at $M_{H}=125 \mathrm{GeV}$. The total background uncertainty includes all sources of systematic uncertainty added in quadrature.

\begin{tabular}{|l|r|r|r|r|r|}
\hline & Pretag & $\begin{array}{r}1 \text { tight } \\
b \text {-tag }\end{array}$ & $\begin{array}{r}2 \text { loose } \\
b \text {-tags }\end{array}$ & $\begin{array}{r}2 \text { med. } \\
b \text {-tags }\end{array}$ & $\begin{array}{r}2 \text { tight } \\
b \text {-tags }\end{array}$ \\
\hline$V H \rightarrow \ell \nu b b$ & 4.4 & 1.1 & 0.5 & 0.5 & 0.8 \\
$H \rightarrow V V \rightarrow \ell \nu j j$ & 4.5 & 0.4 & 0.1 & 0.04 & 0.01 \\
$V H \rightarrow V V V \rightarrow \ell \nu j j j j$ & 3.4 & 0.4 & 0.2 & 0.02 & 0.01 \\
\hline Diboson & 591 & 77.5 & 24.8 & 11.7 & 8.9 \\
$V+(g, u, d, s)$-jets & 9526 & 395 & 251 & 15.0 & 0.4 \\
$V+(b \bar{b} / c \bar{c})$ & 3240 & 639 & 204 & 124 & 104 \\
top $(t \bar{t}+$ single top) & 2979 & 609 & 198 & 235 & 274 \\
Multijet & 8197 & 629 & 259 & 75.6 & 57.2 \\
\hline Total background & & & & & \\
expectation & 24545 & 2351 & 937 & 461 & 445 \\
Total background & & & & & \\
uncertainty & 1225 & 455 & 195 & 115 & 106 \\
Observed events & 24610 & 2251 & 987 & 466 & 396 \\
\hline
\end{tabular}



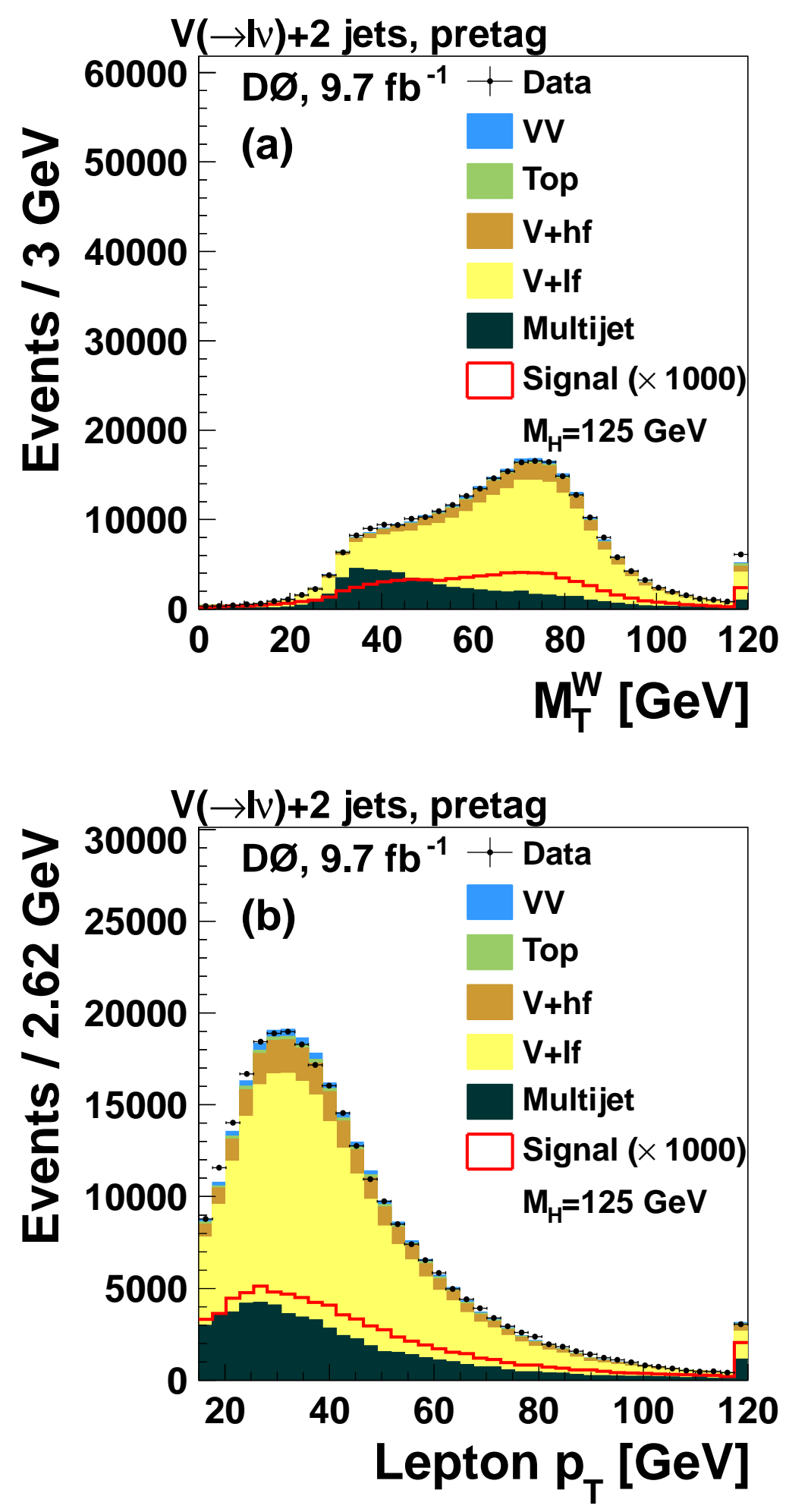

Figure 6.8: Distributions for all selected events with two jets of (a) transverse mass of the lepton- $E_{T}$ system, and (b) charged lepton $p_{T}$. The signal is multiplied by 1000 . Overflow events are added to the last bin [2]. 

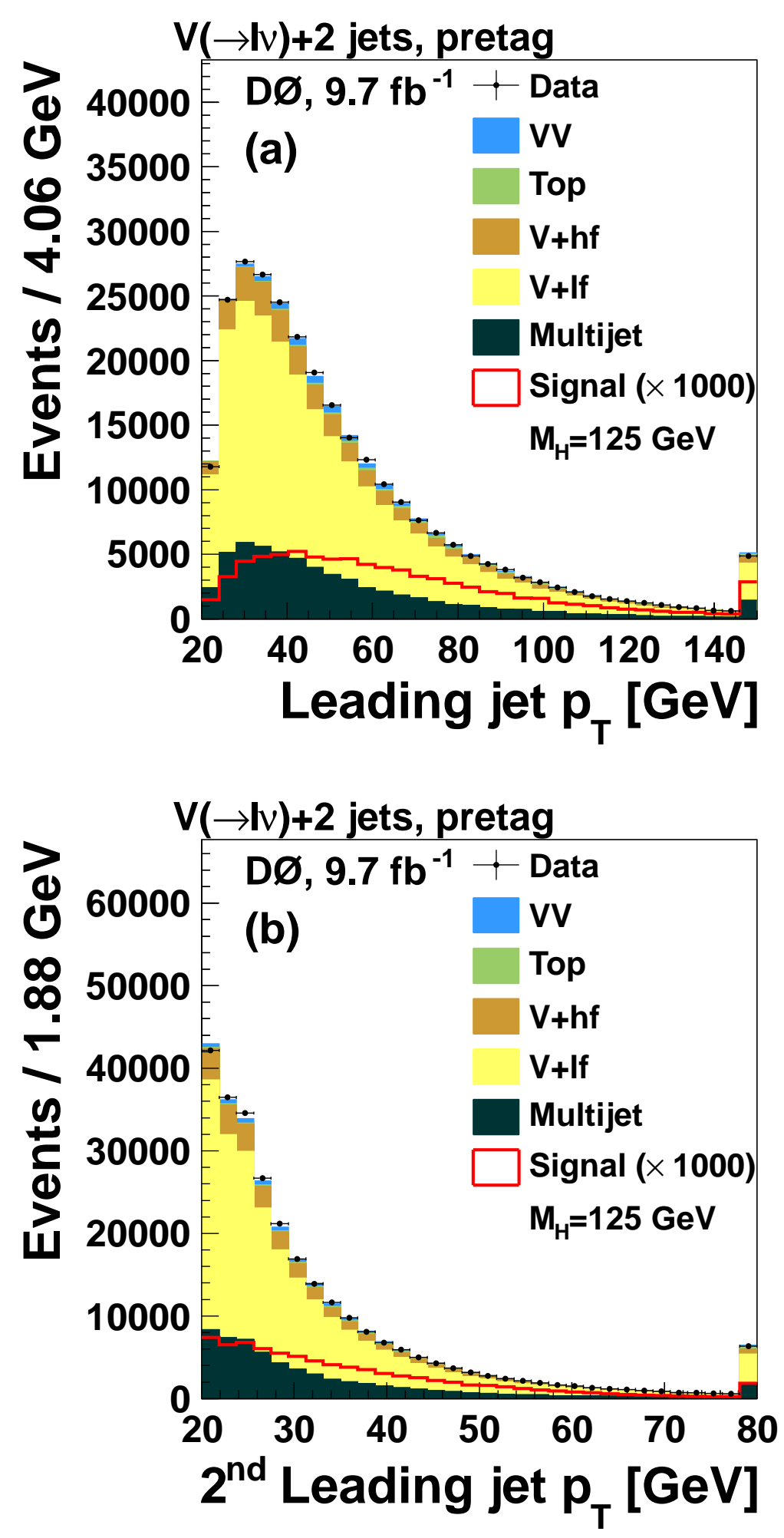

Figure 6.9: Distributions for all selected events with two jets of (a) leading jet $p_{T}$, and (b) second-leading jet $p_{T}$. The signal is multiplied by 1000 . Overflow events are added to the last bin [2]. 

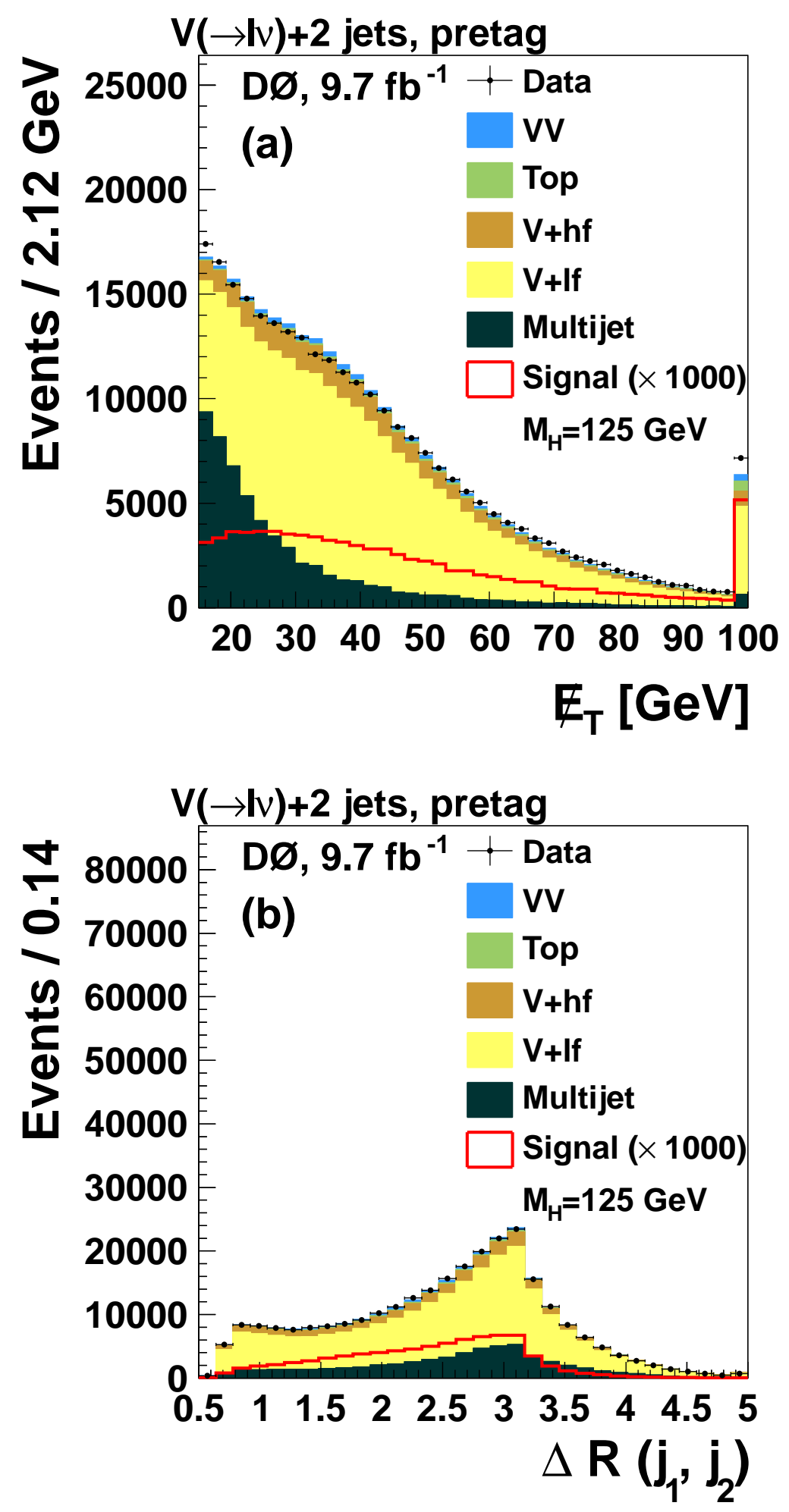

Figure 6.10: Distributions for all selected events with two jets of (a) $E_{T}$, and (b) $\Delta R$ between the leading and second-leading jets. The signal is multiplied by 1000 . Overflow events are added to the last bin [2]. 

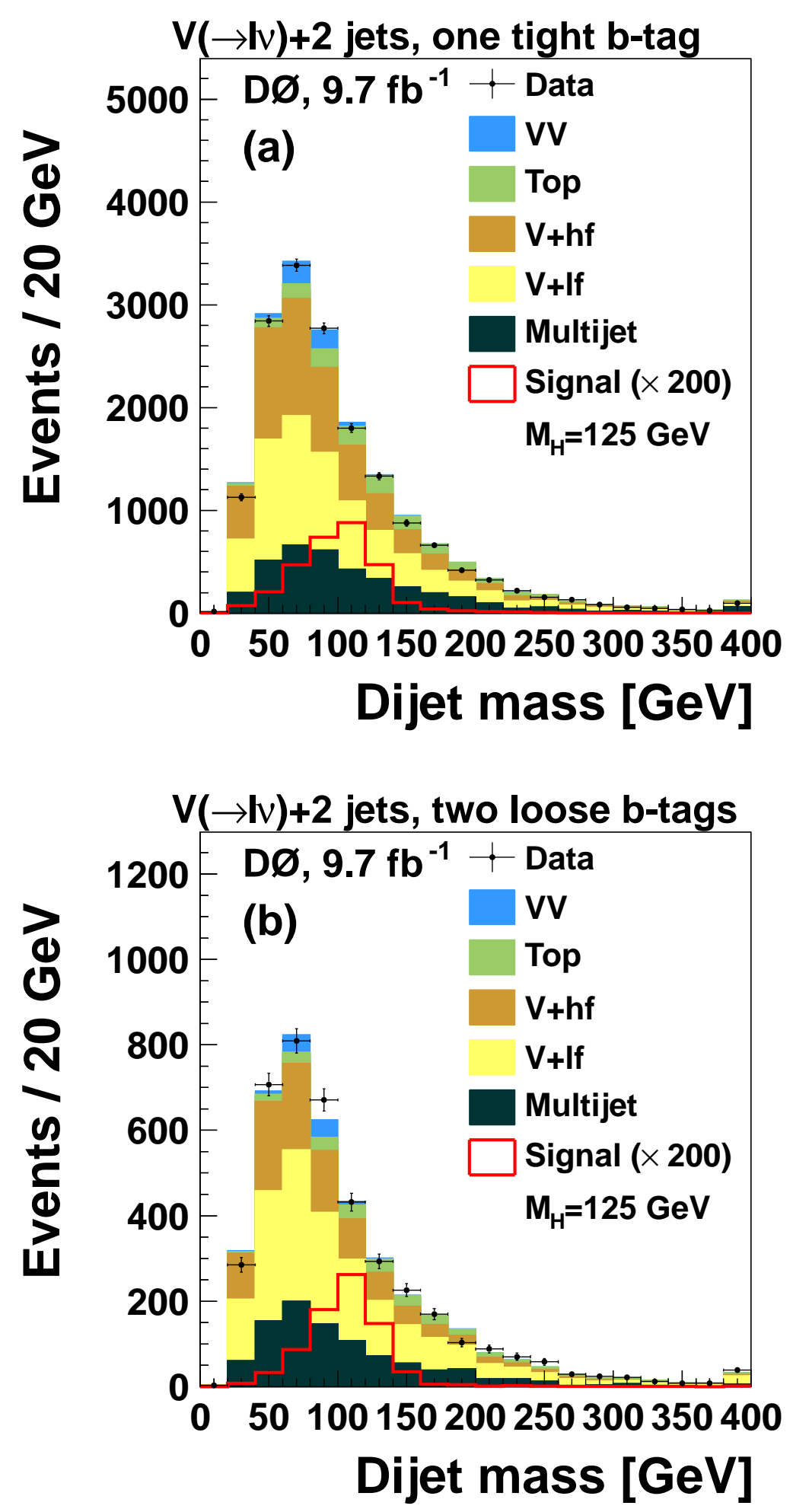

Figure 6.11: Invariant mass of the leading and second-leading jets in events with two jets and (a) one tight $b$-tag, and (b) two loose $b$-tags. The signal is multiplied by 200 . Overflow events are added to the last bin [2]. 

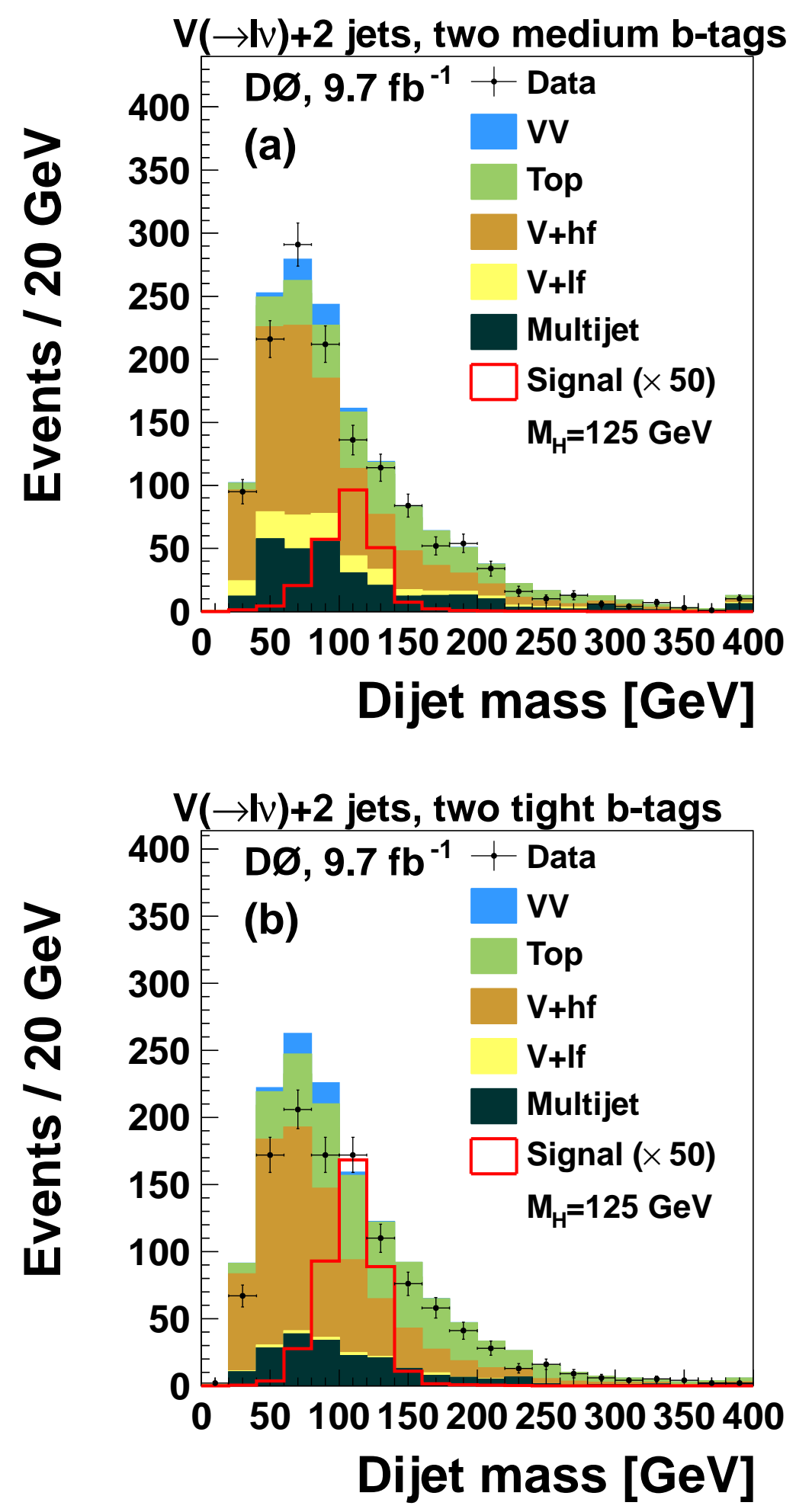

Figure 6.12: Invariant mass of the leading and second-leading jets in events with two jets and (a) two medium $b$-tags, and (b) two tight $b$-tags. The signal is multiplied by 50 . Overflow events are added to the last bin [2]. 


\subsection{Multivariate Classification}

We employ multivariate analysis (MVA) techniques to classify signal and background events.

We use a boosted decision tree $[38,39,40,41,42]$ implemented in the TMVA package [43]

both to distinguish our signal from our multijet background, to distinguish our signal from all backgrounds .

The BDT is a form of a machine learning technique known as a decision tree. We start with a list of variables that show good agreement between the simulation and the data, and have some difference in the distribution between signal and at least one background process. The decision tree uses these variables to create a series of yes/no splits on events that are known to be classified as either signal or background. The splitting is done to maximally separate signal from background, and stops when either a node contains events that are pure signal or background, or when a minimum number of events in a node is reached. Boosting involves building up a series of trees, where each tree is retrained, boosting the weights for events that were misclassified in previous trainings. Our events are split into three statistically independent subsamples for training, testing, and performing our final statistical analysis. After the BDT is trained, it is applied to the testing sample to ensure that our training does not pick up on statistical fluctuations in the training sample (known as overtraining). Having an independent sample to perform our final statistical analysis helps reduce the chance that optimizations performed on our training sample do not bias our final result.

We train a BDT to distinguish our $V H \rightarrow V b b$ signal from our multijet background. The variables used for the training were selected to exploit differences in the kinematics between the multijet and signal events, are listed in Table 6.8, and are shown in Appendix A. This 
BDT is trained using our pretag sample, so we have a large enough sample of multijet events to perform the training. The output from this BDT is shown in Fig. 6.13, and is used as an input to the final Higgs boson MVA which is used to distinguish our signal from all background processes.

Table 6.8: Input variables for the $\mathrm{MVA}_{\mathrm{MJ}}(V H)$ discriminant, which was trained using $V H \rightarrow$ $\ell \nu b \bar{b}$ events as a signal. Variables are ranked by their importance in the BDT (which is based on how often they are used in the training) [2].

\begin{tabular}{|c|c|}
\hline MVA $_{M J}$ Input Variables & Description \\
\hline$\eta^{\nu}$ & Pseudorapidity of the missing $E_{T}$ vector \\
\hline$E_{T}^{S i g}$ & $\begin{array}{l}E_{T} \text { significance, a measure of the consistency of the } \\
\text { observed } \mathbb{E}_{T} \text { with respect to zero } \mathbb{E}_{T} \text {, accounting for } \\
\text { the uncertainty in the calorimeter objects that con- } \\
\text { tribute to } \mathbb{E}_{T}\end{array}$ \\
\hline$\Delta \eta(\ell, \nu)$ & $\begin{array}{l}\text { Separation in } \eta \text { between the lepton and the recon- } \\
\text { structed neutrino, }\left|\eta^{\ell}-\eta^{\nu}\right|\end{array}$ \\
\hline $\mathcal{T}_{W \rightarrow \ell \nu}$ & Twist of the $\ell \nu$ system $=\arctan (\Delta \phi(\ell, \nu) / \Delta \eta(\ell, \nu))$ \\
\hline $\cos \theta(\ell)_{\ell \nu C M}$ & $\begin{array}{l}\text { Cosine of the angle between the charged lepton and } \\
\text { the proton beam axis in the CM of } \ell \nu \text { system }\end{array}$ \\
\hline $\mathcal{V}\left(j_{12}\right)$ & Velocity of the dijet system \\
\hline$m^{\text {Asym }}$ & $\begin{array}{l}\text { Mass asymmetry between } \ell \nu \text { system and the dijet } \\
\text { system: }\left(M_{\ell \nu}-m_{j_{12}}\right) /\left(M_{\ell \nu}+m_{j_{12}}\right)\end{array}$ \\
\hline $\mathcal{C}$ & $\begin{array}{l}\text { Centrality is }\left(\sum_{i} p_{T}^{i}\right) /\left(\sum_{i}\left|\vec{p}_{i}\right|\right) \text {, where } i \text { runs over } \ell \\
\text { and all jets }\end{array}$ \\
\hline$E_{T}$ & Missing transverse energy \\
\hline$p_{T}^{V I S}$ & $\begin{array}{l}\text { Magnitude of the vector sum of the } \vec{p}_{T} \text { of the visible } \\
\text { particles }\end{array}$ \\
\hline $\max \mid \Delta \eta\left(\ell,\left\{j_{1}\right.\right.$ or $\left.\left.j_{2}\right\}\right) \mid$ & $\begin{array}{l}\text { Maximum } \Delta \eta \text { between the charged lepton and the } \\
\text { leading or second leading jet }\end{array}$ \\
\hline
\end{tabular}

A final BDT is trained to distinguish signal from all backgrounds. This is done separately in events with 2 jets, and events with three jets; each b-tagging category; and for muons and electrons. Since the event kinematics for our signal depend on the mass of the Higss we are considering, a separate BDT is trained for each possible Higgs boson mass between 90 and $150 \mathrm{GeV}$ in steps of $5 \mathrm{GeV}$. The variables used to train these final BDTs are given in Table 6.9 (ranked by how often a given variable is used in the MVA), and examples of variables used 


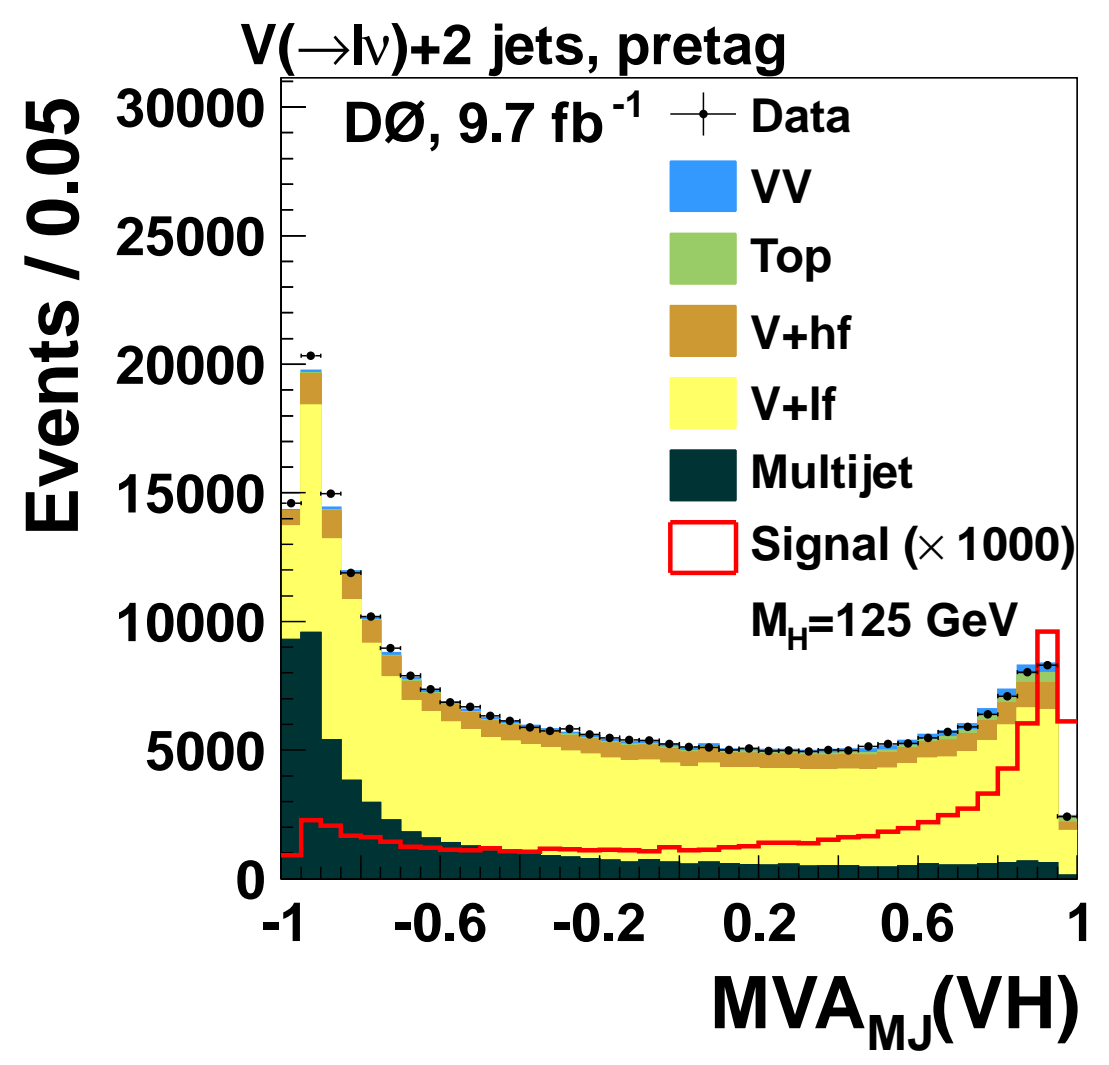

Figure 6.13: The output distribution for the Higgs boson signal vs multijet discriminant shown for all events containing exactly two jets. The signal for $M_{H}=125 \mathrm{GeV}$ is scaled by a factor of 1000 [2]. 
in the training are shown for each jet and b-tag category in Fig. 6.14 to 6.17. One of the most important variables used in the BDT is the dijet mass, as the signal is expected to peak at the mass of the Higgs boson, and the backgrounds tend to peak at lower dijet mass values. In the two tight and two medium b-tag categories, the output from the multijet discriminant is also important in the BDT. While there are very few multijet events in these b-tag categories, the other background processes tend to peak near zero in the multijet BDT

output. In the two loose and 1 b-tag categories, the bid MVA output distribution plays a strong role. This is because in these b-tag categories, the dominant background is from $V+$ light jet production which tends to have lower bid output values than the $H \rightarrow b \bar{b}$ signal does. Figures 6.18 to 6.21 show the final MVA output for $M_{H}=125 \mathrm{GeV}$. The additional input variables and the BDT outputs for the other Higgs boson mass values are shown in Appendix B. In all categories, we see the signal peaking near one, while the background peaks near -1 . We also observe that our data agrees well with our background prediction. These distributions are used to perform our final statistical analysis as discussed in Ch. .

\subsection{Systematic Uncertainties}

We must account for sources of uncertainty in our analysis. This includes both statistical and systematic uncertainties. The statistical uncertainties come from the number of data and MC events we have available to describe kinematic distributions. The systematic uncertainties are uncertainties resulting from object reconstruction and calibration and $\mathrm{MC}$ generation or theory. To determine the systematic uncertainties on signals and backgrounds, we repeat the full analysis with each source of uncertainty varied by \pm 1 standard deviation for the parameter on which we are uncertain. We can divide the uncertainties into two categories: 
Table 6.9: Table of input variables for the final signal discriminant. Variables are ranked by their importance in the BDT (which is based on how often they are used in the training) in the two tight $b$-tagged (2T), two medium $b$-tagged (2M), two loose $b$-tagged (2L), and one tight $b$-tagged (1T) categories [2].

\begin{tabular}{|c|c|c|c|c|c|}
\hline Variable & Description & $2 \mathrm{~T}$ & $2 \mathrm{M}$ & $2 \mathrm{~L}$ & $1 \mathrm{~T}$ \\
\hline $\mathrm{MVA}_{\mathrm{MJ}}(V H)$ & $\begin{array}{l}\text { Output of the MVA trained to distinguish } \\
W H \rightarrow \ell \nu b \bar{b} \text { from the MJ background }\end{array}$ & 1 & 1 & & \\
\hline$m_{b 1 b 2}$ & The dijet invariant mass & 2 & 4 & 3 & 1 \\
\hline$p_{T}^{W} /\left(p_{T}^{\ell}+E_{T}\right)$ & $\begin{array}{l}\text { The transverse momentum of the recon- } \\
\text { structed } W \text { divided by the sum of the trans- } \\
\text { verse momentum of the lepton and the } \mathbb{E}_{T}\end{array}$ & 3 & 6 & 4 & 2 \\
\hline$b_{I D}^{j}$ & $\begin{array}{l}\text { Averaged } b \text {-jet identification output for the } \\
\text { highest energy } b \text {-tagged jets }\end{array}$ & 4 & 13 & 1 & 4 \\
\hline $\cos \left(\chi^{*}\right)$ & $\begin{array}{l}\chi^{*}=\angle(\ell, \text { the direction of the } W \text { boson spin }) \\
\text { in } \ell \nu \text { system CM frame }[44]\end{array}$ & 5 & 3 & & \\
\hline $\max \left|\Delta \eta\left(\ell, j_{1}, j_{2}\right)\right|$ & $\begin{array}{l}\text { Maximum } \Delta \eta \text { between the charged lepton } \\
\text { and the leading or second leading jet }\end{array}$ & 6 & 11 & 2 & 3 \\
\hline$q^{\ell} \times \eta^{\ell}$ & $\begin{array}{l}\text { Product of the lepton charge and its pseudo- } \\
\text { rapidity }\end{array}$ & 7 & 2 & 6 & 6 \\
\hline$\Delta \mathcal{R}\left(\ell, j_{1}\right)$ & $\begin{array}{l}\Delta \mathcal{R} \text { between the charged lepton and the lead- } \\
\text { ing jet }\end{array}$ & 8 & 5 & & \\
\hline $\min \left[\mathcal{S I G}\left(j_{12},\left\{j_{1}, j_{2}\right\}\right)\right]$ & $\begin{array}{l}\text { Minimum } \mathcal{S I G} \text { of the two leading jets de- } \\
\text { fined as } p_{T}^{\min }\left(j_{1}, j_{2}\right) \Delta \mathcal{R}\left(j_{1}, j_{2}\right) / \sum_{i=1}^{2} p_{T}^{j_{i}} \text { with } \\
\text { respect to the dijet system. Based on the } \\
\text { pull variables described in [45] }\end{array}$ & 9 & 15 & 9 & 5 \\
\hline$q^{\ell} \times \eta^{j_{1}}$ & $\begin{array}{l}\text { Product of the the lepton charge and } \eta \text { of the } \\
\text { leading jet }\end{array}$ & 10 & 7 & 11 & 9 \\
\hline $\mathcal{V}\left(j_{12}\right)$ & Velocity of the dijet system & 11 & 12 & 7 & 11 \\
\hline $\cos \left(\theta^{*}\right)$ & $\begin{array}{l}\theta^{*}=\angle(W, u-\text { type quark }) \text { in the Higgs CM } \\
\text { frame }[44]\end{array}$ & 12 & 10 & & \\
\hline$m_{\ell \nu j_{2}}$ & $\begin{array}{l}\text { Invariant mass of the charged lepton, } E_{T} \text {, } \\
\text { and second leading jet system }\end{array}$ & 13 & 16 & 12 & 13 \\
\hline$m_{T}^{j_{12}}$ & Transverse mass of the two leading jets & 14 & 14 & & \\
\hline $\mathcal{C}$ & $\begin{array}{l}\text { Centrality is }\left(\sum_{i} p_{T}^{i}\right) /\left(\sum_{i}\left|\vec{p}_{i}\right|\right) \text {, where } i \text { runs } \\
\text { over } \ell \text { and all jets }\end{array}$ & 15 & 8 & 8 & 10 \\
\hline$\sum\left(p_{T}\right)^{V I S}$ & Scalar sum of the $p_{T}$ of the visible particle & 16 & 9 & & \\
\hline$m^{\text {Asym }}$ & $\begin{array}{l}\text { Mass asymmetry between } \ell \nu \text { system and the } \\
\text { dijet system: }\left(M_{\ell \nu}-m_{j_{12}}\right) /\left(M_{\ell \nu}+m_{j_{12}}\right)\end{array}$ & & & 5 & 8 \\
\hline $\mathcal{A}$ & $\begin{array}{l}3 \lambda_{3} / 2 \text { where } \lambda_{3} \text { is the smallest eigenvalue } \\
\text { of the normalized momentum tensor } S^{\alpha \beta}= \\
\left(\sum_{i} p_{i}^{\alpha} p_{i}^{\beta}\right) /\left(\sum_{i}\left|\overrightarrow{p_{i}}\right|^{2}\right), \text { where } \alpha, \beta=1,2,3 \\
\text { correspond to the } x, y, z \text { momentum compo- } \\
\text { nents, and } i \text { runs over selected objects. }\end{array}$ & & & 10 & 12 \\
\hline$p_{T}^{j 2}$ & $p_{T}$ of the second leading jet & & & 13 & 7 \\
\hline
\end{tabular}



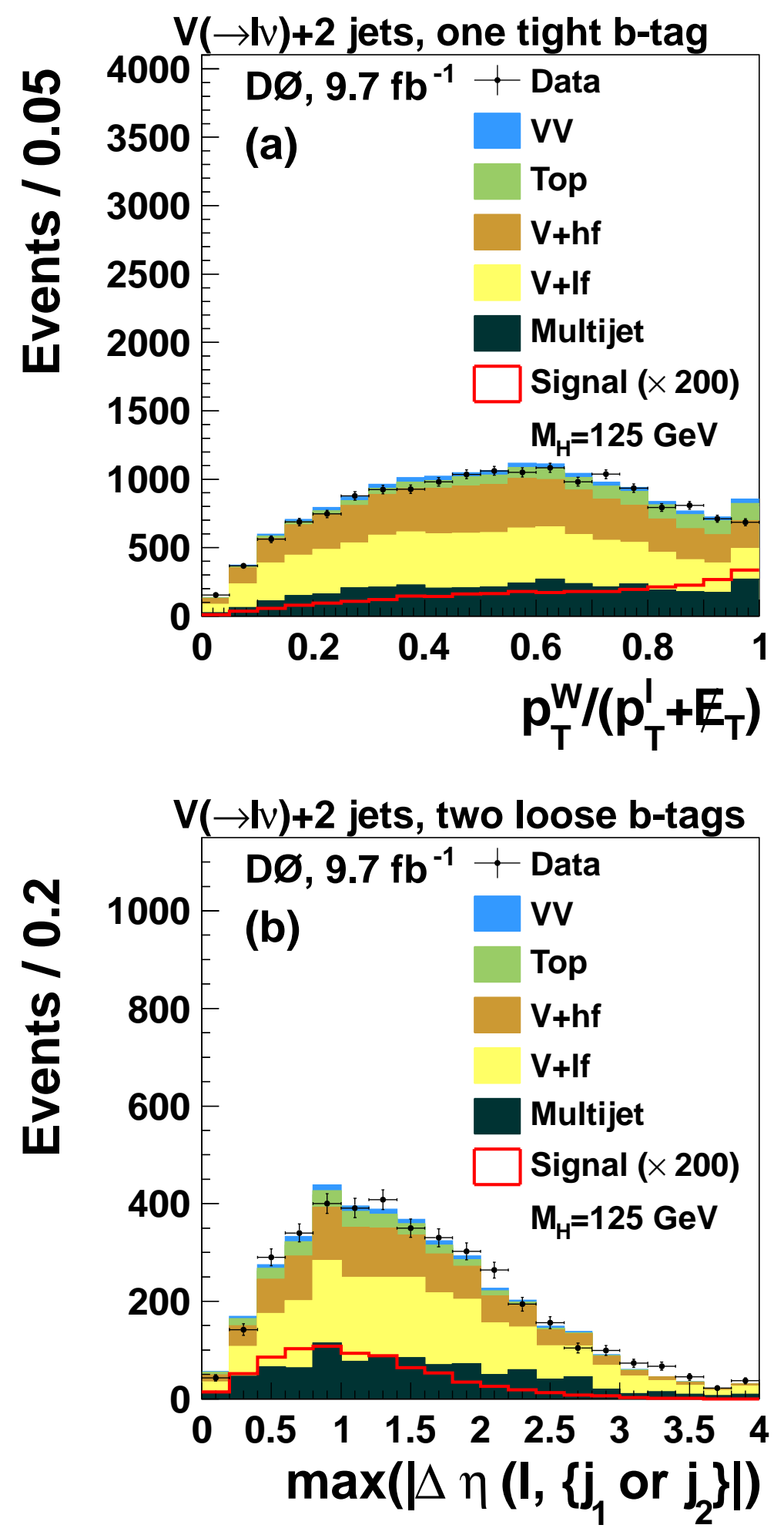

Figure 6.14: Distributions of some of the most significant inputs to the final discriminant in events with exactly two jets: (a) $p_{T}^{W} /\left(p_{T}^{\ell}+E_{T}\right)$, shown for events with one tight $b$-tag; (b) $\max \mid \Delta \eta\left(\ell,\left\{j_{1}\right.\right.$ or $\left.\left.j_{2}\right\}\right) \mid$, shown for events with two loose $b$-tags. The $M_{H}=125 \mathrm{GeV}$ signal is multiplied by 200. Overflow events are added to the last bin [2]. 

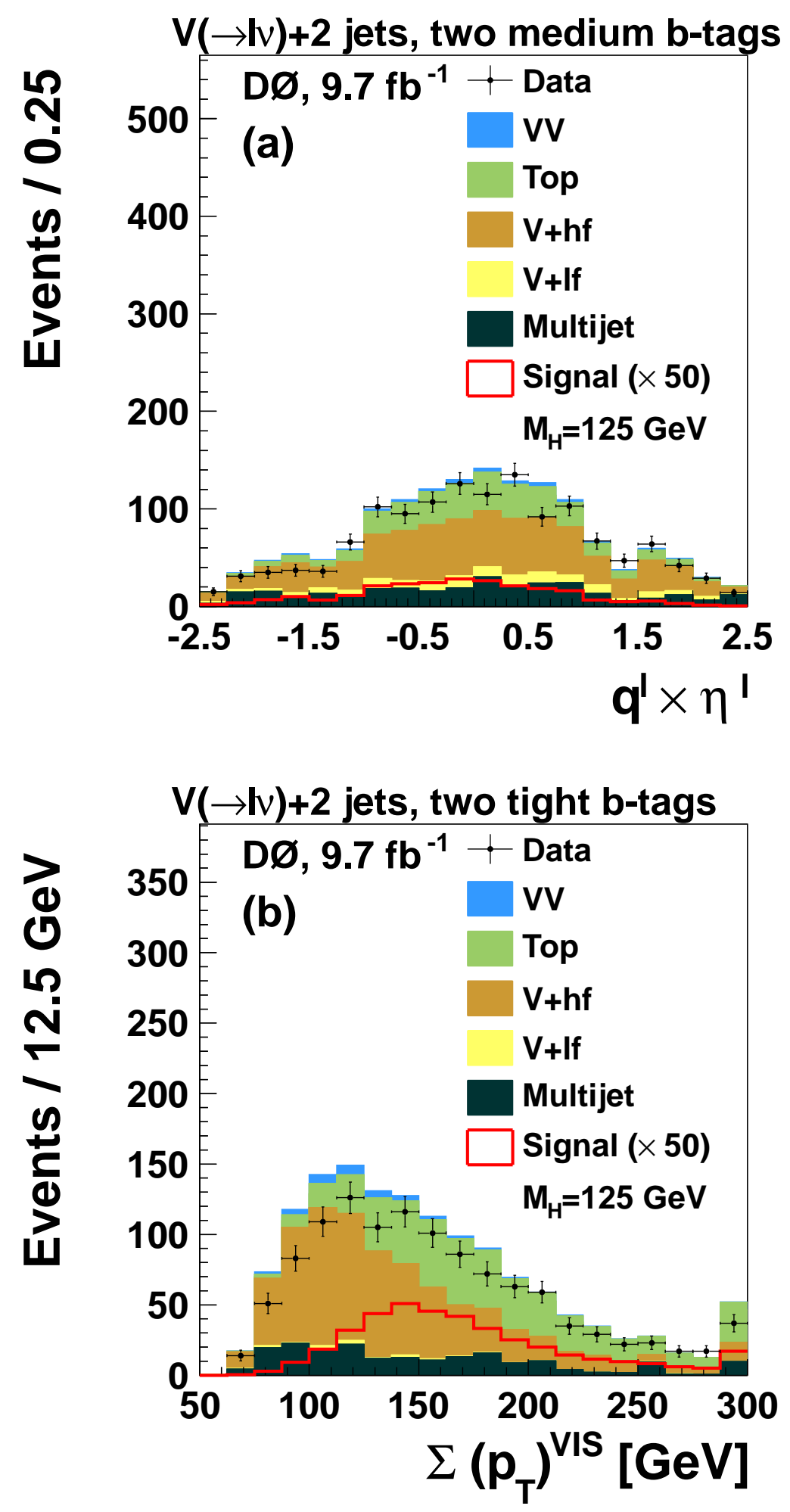

Figure 6.15: Distributions of some of the most significant inputs to the final discriminant in events with exactly two jets: (a) $q^{\ell} \times \eta^{\ell}$, shown for events with two medium $b$-tags; (b) $\sum\left(p_{T}\right)^{V I S}$, shown for events with two tight $b$-tags. The $M_{H}=125 \mathrm{GeV}$ signal is multiplied by 50 . Overflow events are added to the last bin [2]. 

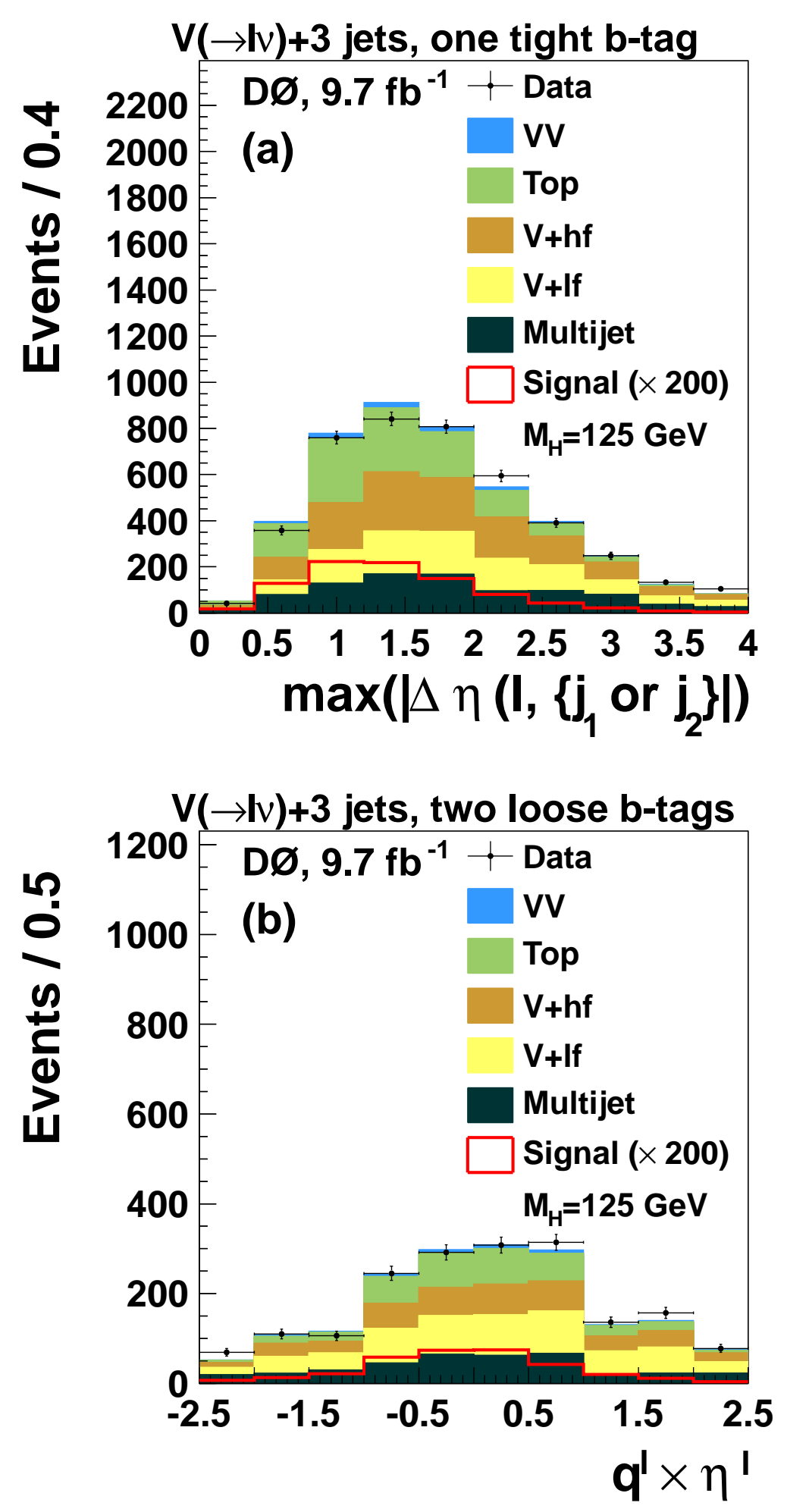

Figure 6.16: Distributions of some of the most significant inputs to the final discriminant in events with exactly three jets: (a) $\max \mid \Delta \eta\left(\ell,\left\{j_{1}\right.\right.$ or $\left.\left.j_{2}\right\}\right) \mid$, shown for events with one tight $b$-tag; (b) $q^{\ell} \times \eta^{\ell}$, shown for events with two loose $b$-tags. The $M_{H}=125 \mathrm{GeV}$ signal is multiplied by 200. Overflow events are added to the last bin [2]. 

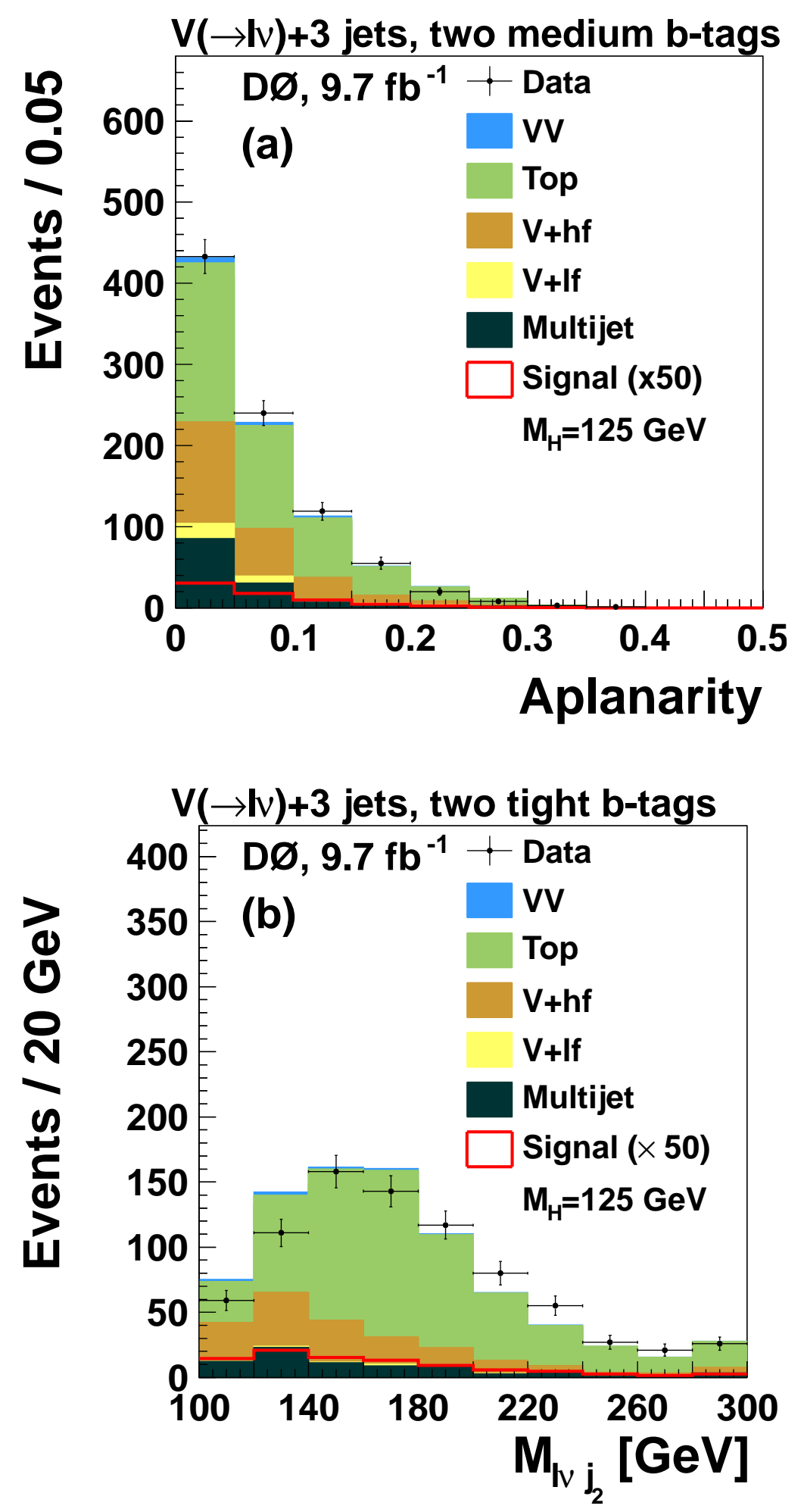

Figure 6.17: Distributions of some of the most significant inputs to the final discriminant in events with exactly three jets: (a) aplanarity, shown for events with two medium $b$-tags; (b) $m_{\ell \nu j_{2}}$, shown for events with two tight $b$-tags. The $M_{H}=125 \mathrm{GeV}$ signal is multiplied by 50. Overflow events are added to the last bin [2]. 

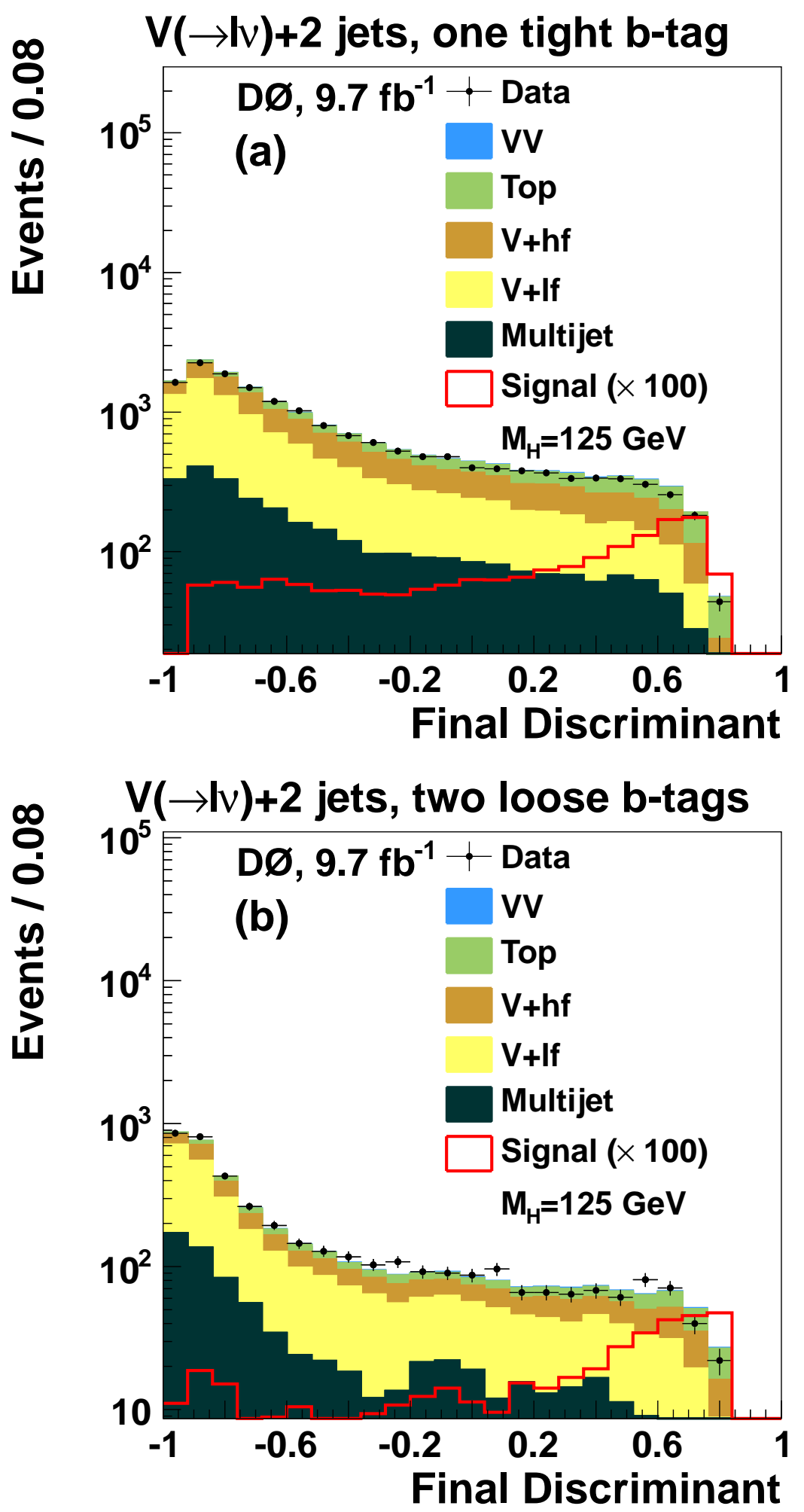

Figure 6.18: Distributions of the final discriminant output, after the maximum likelihood fit (described in Ch. ), in events with exactly two jets and: (a) one tight $b$-tag, and (b) two loose $b$-tags. The $M_{H}=125 \mathrm{GeV}$ signal is multiplied by 100 [2]. 

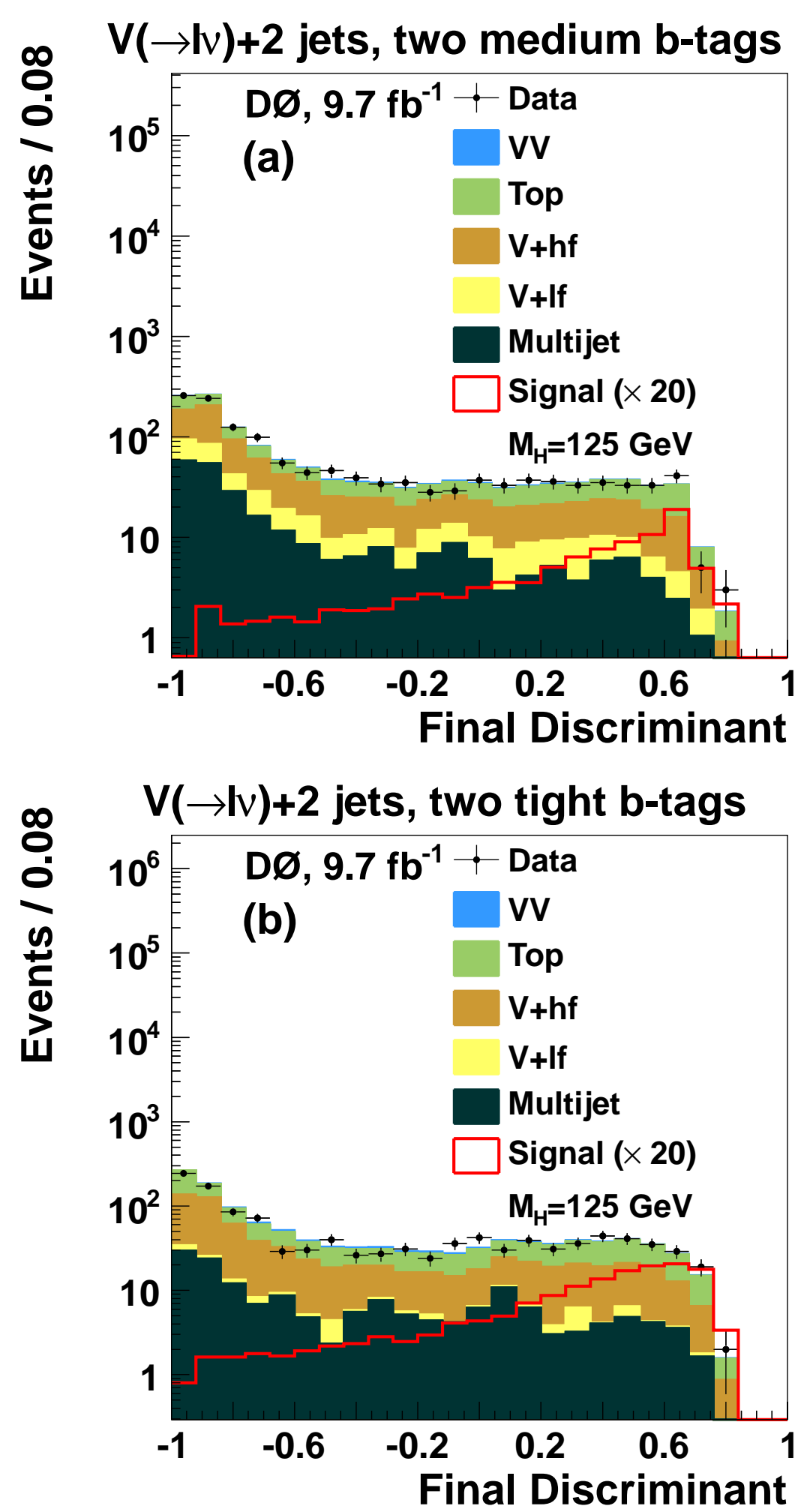

Figure 6.19: Distributions of the final discriminant output, after the maximum likelihood fit (described in Ch. ), in events with exactly two jets and: (a) two medium $b$-tags, and (b) two tight $b$-tags. The $M_{H}=125 \mathrm{GeV}$ signal is multiplied by 20 [2]. 

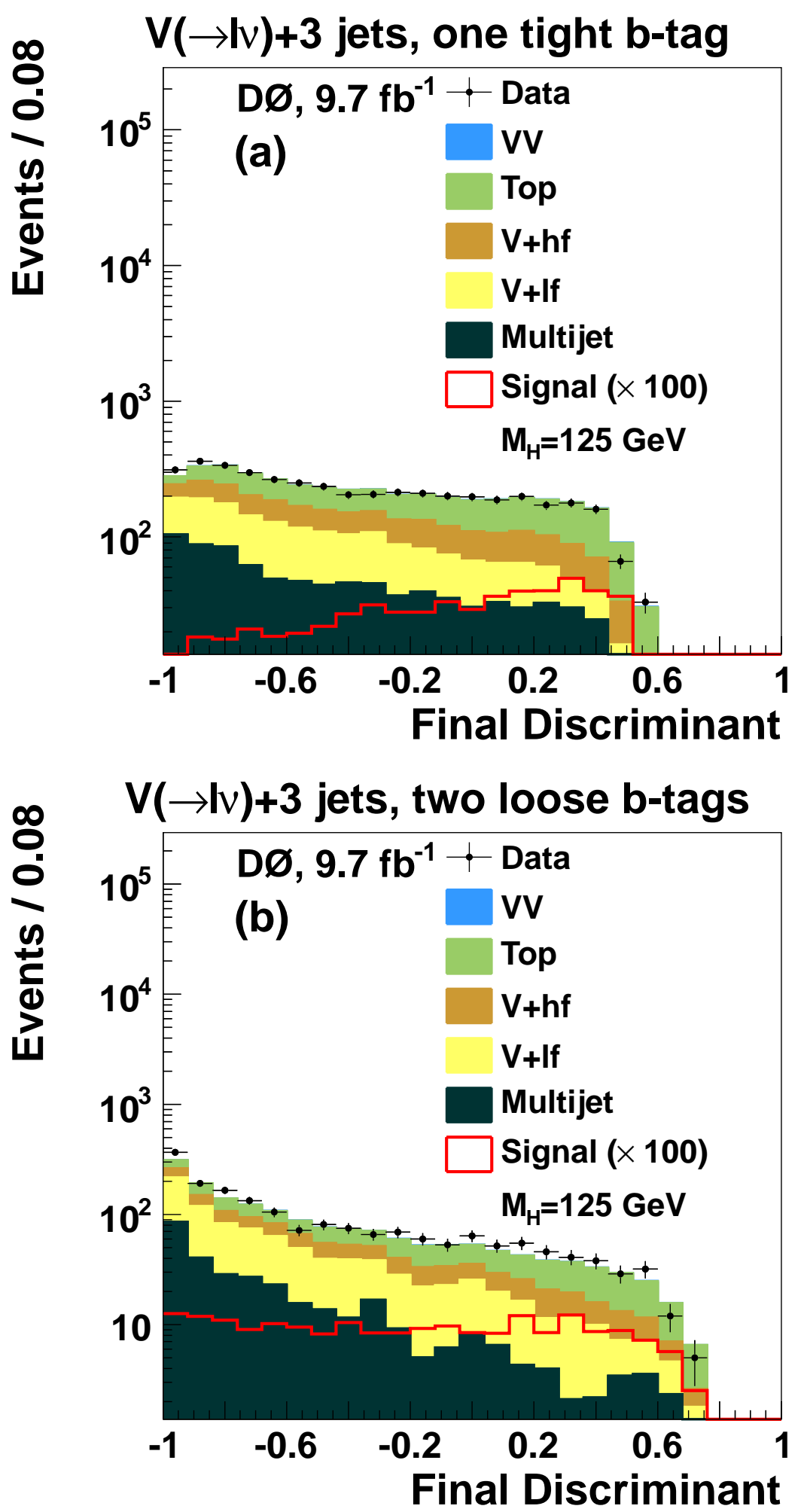

Figure 6.20: Distributions of the final discriminant output, after the maximum likelihood fit (described in Ch. ), in events with exactly three jets and: (a) one tight $b$-tag, and (b) two loose $b$-tags. The $M_{H}=125 \mathrm{GeV}$ signal is multiplied by 100 [2]. 

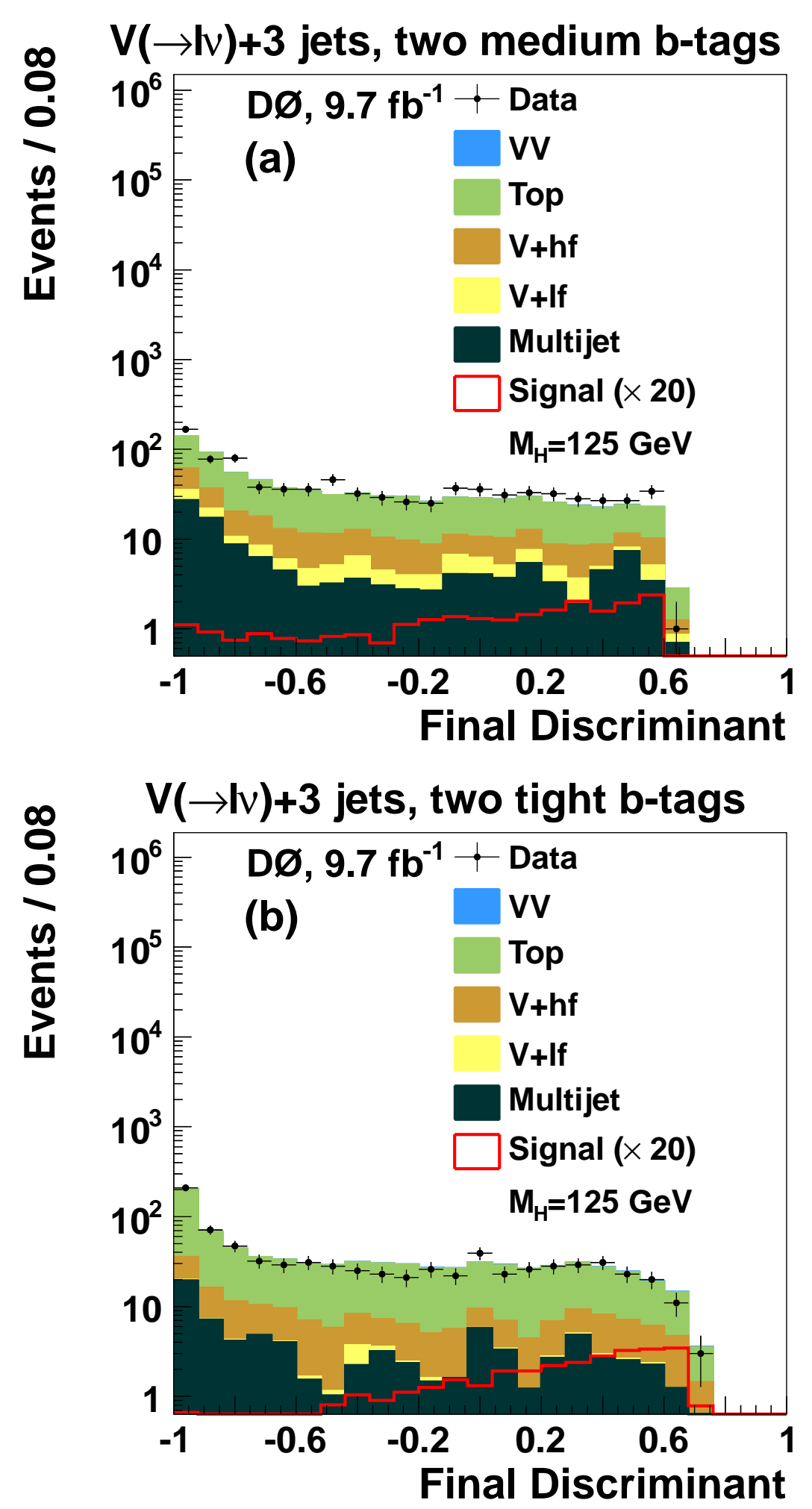

Figure 6.21: Distributions of the final discriminant output, after the maximum likelihood fit (described in Ch. ), in events with exactly three jets and: (a) two medium b-tags, and (b) two tight $b$-tags. The $M_{H}=125 \mathrm{GeV}$ signal is multiplied by 20 [2]. 
uncertainties that affect only the rate of a signal or background process, and/or uncertainties that affect the shape of our MVA output distribution.

Uncertainties that affect only the rates of given processes:

- Theoretical uncertainties on the production cross sections times branching ratios of background processes: $t \bar{t}$ and single top quark production $(7 \%[46,47])$, diboson production (6\% [48]), $V+l f$ production (6\%), and $V+h f$ production (20\%, estimated from MCFM $[49,50])$.

- The parton distribution functions (PDF) used in MC generation affects signal and background acceptances, so we include a $2 \%$ uncertainty to account for this.

- Since the $V+$ jets experimental scaling factor for the three-jet channel is different from unity, we apply an additional systematic uncertainty on the $V+$ jets samples that is uncorrelated across jet multiplicity and lepton flavour bins.

- Since there is some uncertainty in the measurement of the luminosity, we include an uncertainty on the total integrated luminosity of $6.1 \%$ [51], which affects the expected rates of signal and background processes.

- We include a $3 \%$ uncertainty on the efficiency of muon and electron identification, and a $2 \%$ uncertainty on the efficiency of jet identification.

- We apply an uncertainty on our estimate of the multijet rate of $15 \%$ for electrons and $20 \%$ for muons. Since the multijet sample is statistically limited, the uncertainty on the rate is determined by the number of events that end up in our final MVA in each jet multiplicity and b-tag category. This is not correlated across b-tag categories, and thus this uncertainty is uncorrelated across lepton, jet multiplicity, and b-tag categories. 
Since we fit the multijet and V+jet background rates to data simultaneously, we include an uncertainty on the $\mathrm{V}+$ jets rate that is anti-correlated with the multijet rate.

Uncertainties that affect the shape of our final MVA output include:

- The uncertainty on the jet taggability ( $\sim 3 \%$ per jet) comes from the difference between the taggability scale factors being derived with one jet and being derived with two jets. These scale factors should not depend on jet multiplicity, so the uncertainty is taken to be large enough to cover this difference.

- The uncertainty on the b-tag efficiency is correlated between b- and c-jets, and not correlated between light and heavy jets, so we include uncertainties on the b-tagging efficiency by varying the heavy flavour tag rate functions up and down by one standard deviation in samples containing heavy quark jets, and we vary the light quark tag rate functions up and down by one standard deviation in samples devoid of heavy quark jets. The size of these uncertainties is $\sim 2-3 \%$ per heavy quark jet, and $\sim 10 \%$ for light quark jets.

- The trigger uncertainty in the muon channel is calculated as the difference between applying a trigger correction calculated using the ALPGEN reweightings derived on the $T_{\mu O R}$ trigger sample and applying the nominal trigger correction.

- The uncertainty on the jet energy scale is taken by shifting the JES parameters up and down by one standard deviation.

- Similarly, the uncertainty on the jet energy resolution is taken by shifting the JSSR parameters up and down by one standard deviation. 
- We also include uncertainties on the ALPGEN MC generation from the MLM matching [29] applied to $V+$ light-flavour events $(\approx 0.5 \%)$, the ALPGEN renormalization and factorization scales.

- Since we reweight our ALPGEN samples, we include separate uncertainties on each of the five functions used to apply the reweighting. The adjusted functions are calculated by shifting the parameter responsible for the largest shape variation of the fit by \pm 1 s.d. then calculating the remaining parameters for the function using the covariance matrix obtained from the functional fit.

- The uncertainty on the shape of our multijet estimate is determined by relaxing the requirement from Sec. 6.4 on $M_{T}^{\ell \nu}$ to $M_{T}^{\ell \nu}>30 \mathrm{GeV}-0.5 \times \mathbb{E}_{T}$ and repeating the analysis with this selection in place. The positive and negative variations are taken to be symmetric. As with the uncertainty on the multijet rate, we do not correlate the multijet shape uncertainty across lepton, jet multiplicity, and b-tag categories.

\subsection{Diboson $(V Z)$ Production With $Z \rightarrow b \bar{b}$}

Since the SM processes $V Z \rightarrow V b b$ have the same final state as the Higgs boson signature in this search, and also have a cross section small compared to the other background processes (although still approximately 20 times larger than the $W H$ production cross section), it is a good candidate to use as a validation of our search methodology. The only change to the analysis when performing this validation is to train the final MVA discriminant using the $W Z$ and $Z Z$ processes in place of the Higgs boson as signal. Using the output of this discriminant, we measure the the combined $W Z$ and $Z Z$ cross section by performing a maximum likelihood fit to data as described in Ch. . We also perform the cross section 
measurement using the dijet mass distribution, to verify the MVA procedure. The expected significance of the measurement using the dijet mass distribution is 1.4 s.d. We measure a cross section of $1.04 \pm 0.39$ (stat.) \pm 0.28 (syst.) times the SM cross section of $4.4 \pm 0.3 \mathrm{pb}$. The expected significance of the measurement using the MVA output is 1.8 s.d., and we measure a cross section of $0.50 \pm 0.34$ (stat.) \pm 0.36 (syst.) times the expected SM cross section. The dijet mass and MVA measurements are both consistent with each other, which suggests that the MVA methodology is sound. Both values are also consistent with the SM value, thus we have additional trust in our full analysis strategy when it is extended to the Higgs boson search. Figures 6.22 and 6.23 shows the dijet mass and MVA discriminant output for diboson events $(W Z+Z Z)$ after subtracting the background from the data with the $V Z$ signal scaled to the best fit value. The MVA output distribution has been rebinned as a function of $\log (S / B)$. We see that the background-subtracted-data agrees well with our $V Z$ prediction in both the MVA and dijet mass distributions.

\subsection{Upper Limits on Higgs Boson Production}

Once we have some distribution that distinguishes the signal from our expected background, as we do for the MVAs described earlier, we want to be able to make some statistical statement about our data (ie, we want to know how much our data resembles a model that contains our signal). Since we do not see a significant excess in our data over our background expectation, we set an upper limit on the $W H$ production cross section at a confidence level of $95 \%$ using the LLR as a test statistic as described in Ch. .

Typically in Higgs boson searches, the LLR is presented by displaying the LLR for each of the two hypotheses and the data LLR value as a function of Higgs boson mass. The LLR 


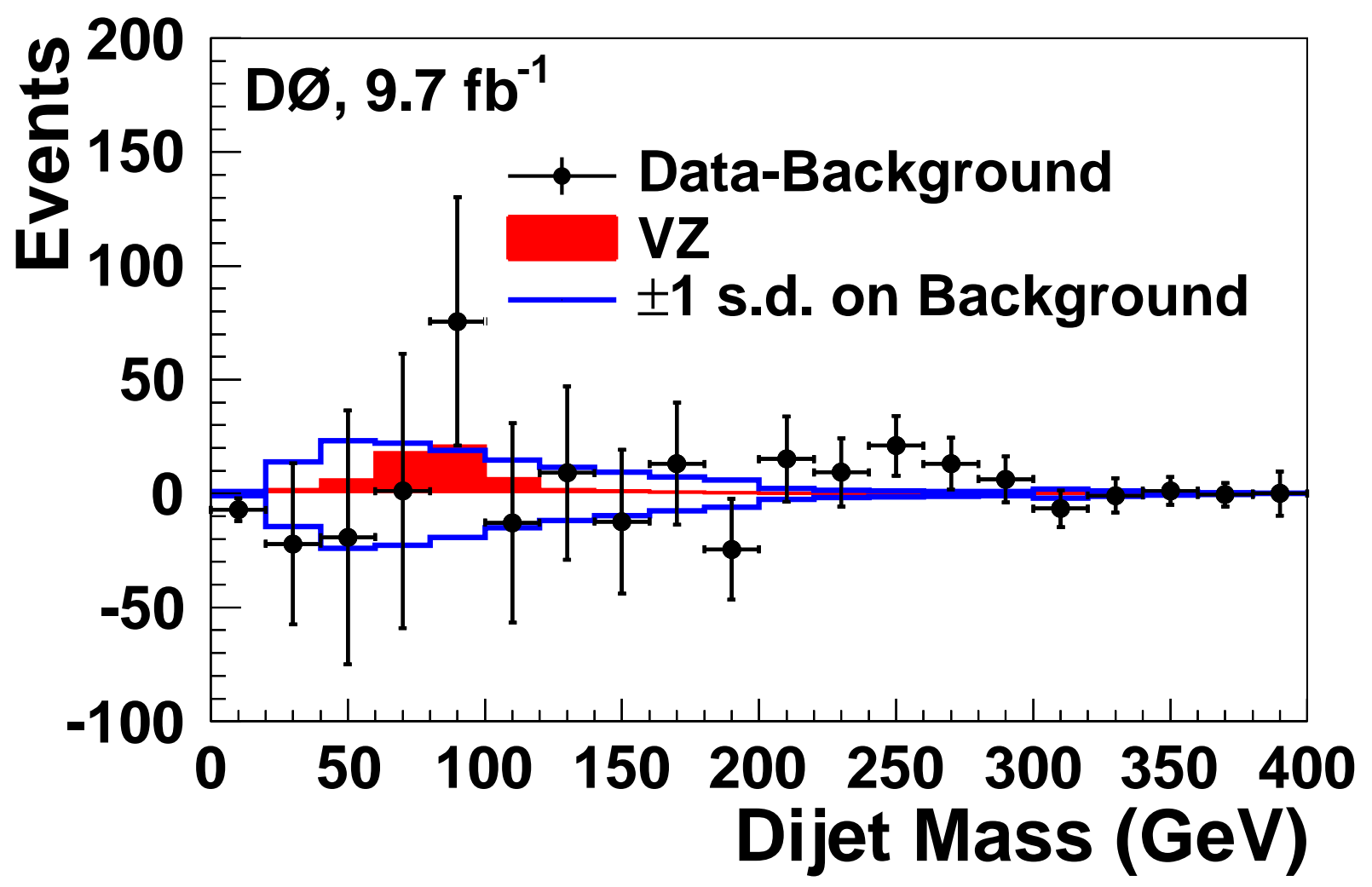

Figure 6.22: The dijet mass shown for the expected diboson signal and backgroundsubtracted data after the maximum likelihood fit, summed over $b$-tag channels. The error bars on data points represent the statistical uncertainty only. The post-fit systematic uncertainties are represented by the solid lines. The signal expectation is shown scaled to the best fit value [2]. 


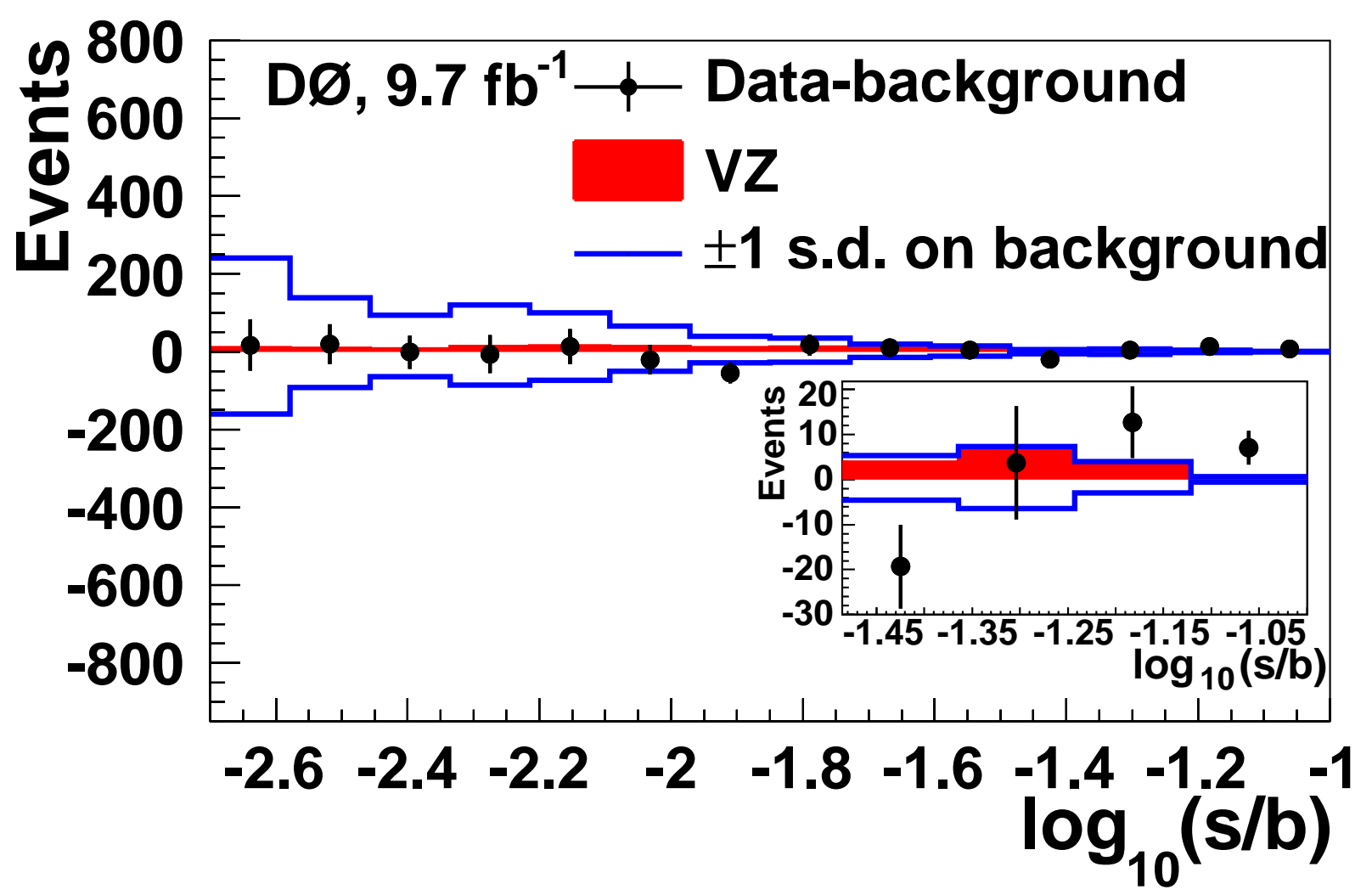

Figure 6.23: The final MVA discriminant output shown for the expected diboson signal and background-subtracted data rebinned as a function of $\log (S / B)$, after the maximum likelihood fit, summed over $b$-tag channels. The error bars on data points represent the statistical uncertainty only. The post-fit systematic uncertainties are represented by the solid lines. The signal expectation is shown scaled to the best fit value. The inset gives an expanded view of the high $\log (S / B)$ region [2]. 
for the $W H \rightarrow \ell \nu b \bar{b}$ search is shown in Fig. 6.24. We see that for a Higgs boson with a mass less than $115 \mathrm{GeV}$, our observed LLR agrees more with the background-only prediction, while for $M_{H}>115 \mathrm{GeV}$, the LLR agrees with the signal+background prediction.

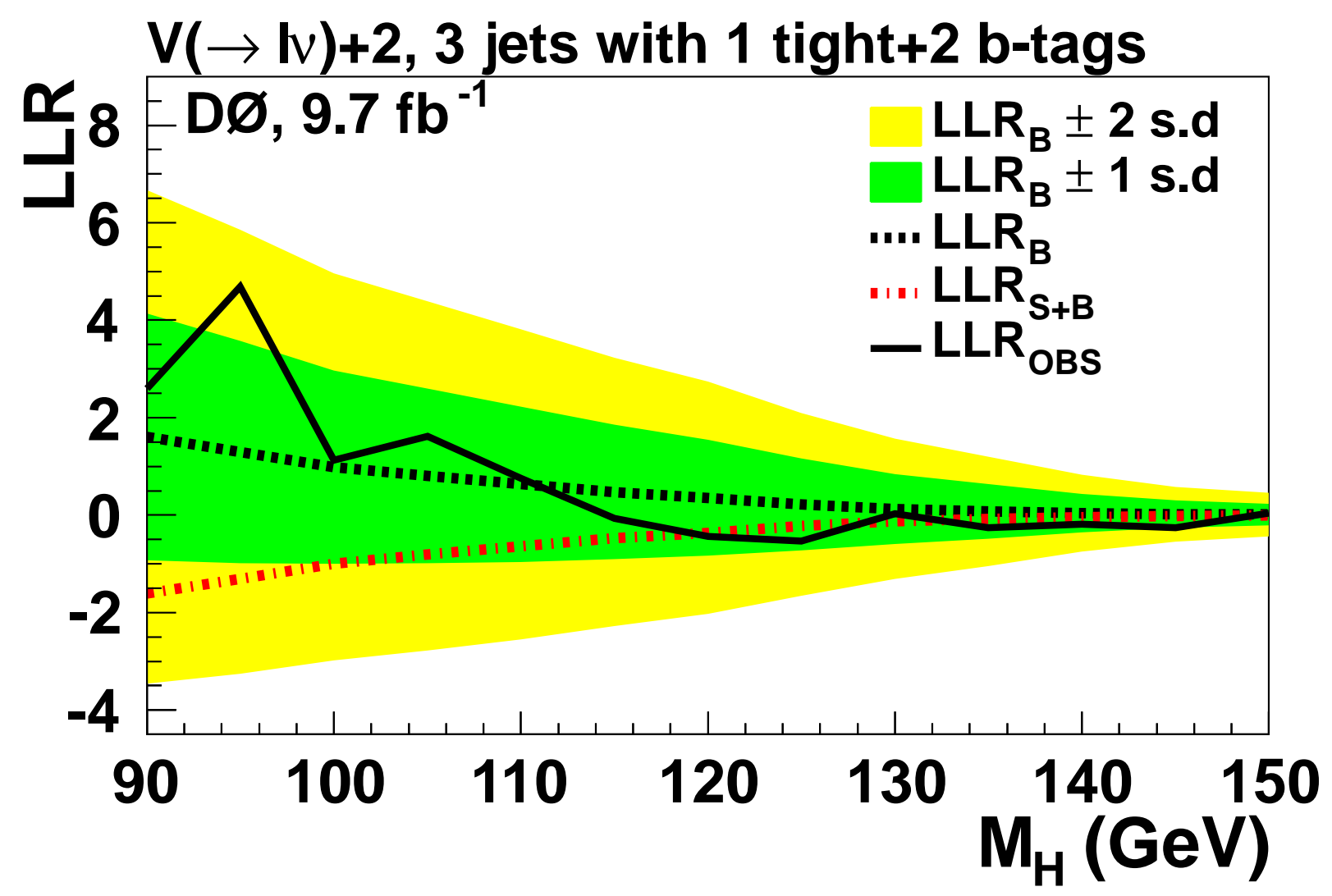

Figure 6.24: The log likelihood ratio as a function of Higgs boson mass for the expected background only hypothesis (dashed black line), expected signal+background hypothesis (red dashed line), and data (solid black line). The green and yellow bands represent, respectively, \pm one and two standard deviations on the background only hypothesis [2].

We calculate the $95 \%$ confidence level $(\alpha=0.05)$, and present the upper limit on Higgs boson production cross section times branching ratio for each Higgs boson mass point considered as a ratio to the SM Higgs boson production cross section times branching ratio for the $W H \rightarrow \ell \nu b \bar{b}$ search in Fig. 6.25 and Table 6.10. For a Higgs with $M_{H}=125 \mathrm{GeV}$, we set an observed (expected) limit of 4.8 (4.7) times the standard model cross section. 


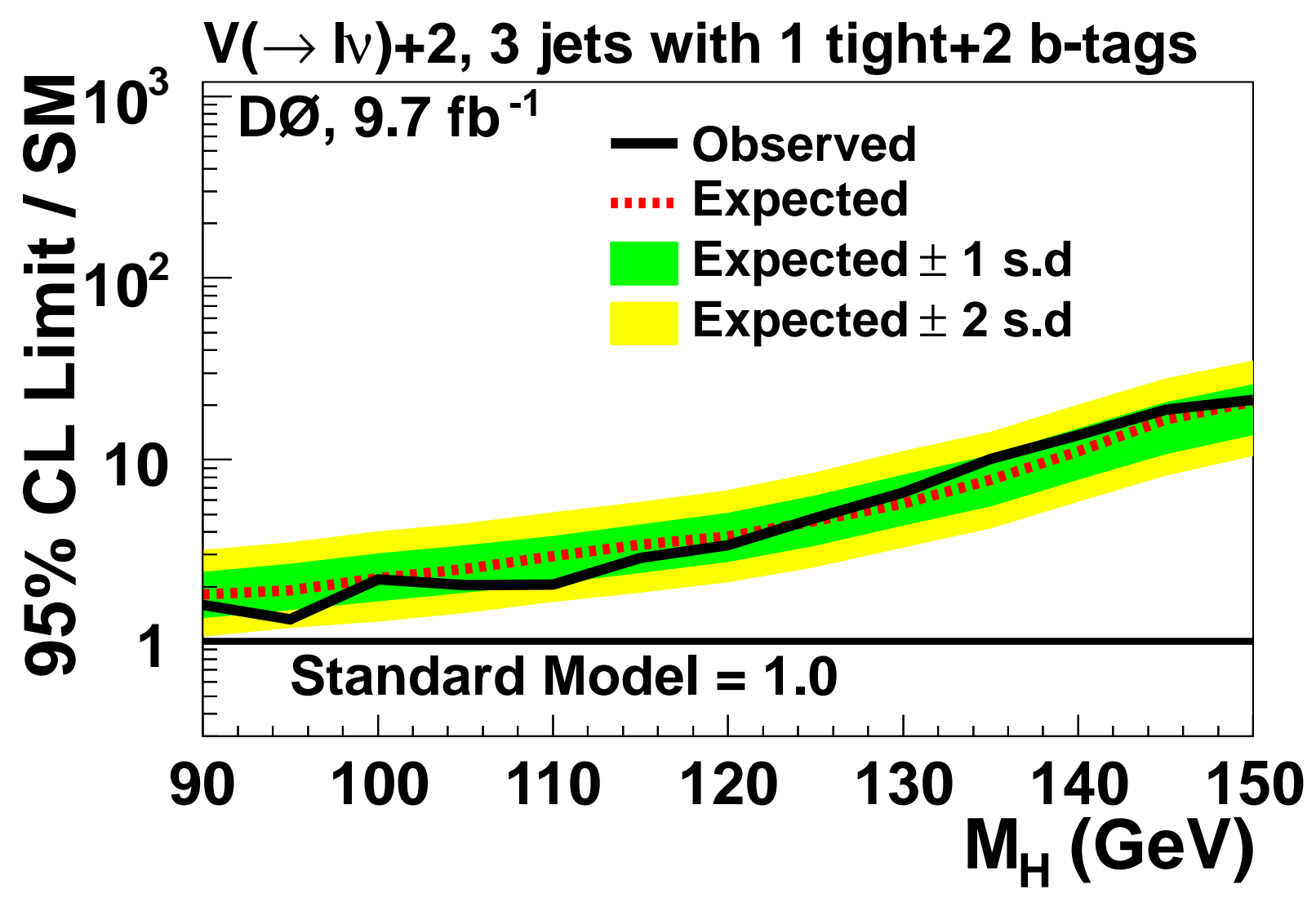

Figure 6.25: The upper 95\% confidence level limit on the Higgs boson production cross section times branching ratio as a ratio to the SM cross section times branching ratio. The dashed line shows the expected limit, the solid line shows the limit observed in data, and the green and yellow bands show the \pm 1 and 2 standard deviations on the expected limit [2]. 
Table 6.10: The expected and observed 95\% C.L. limits, as a function of the Higgs boson mass $M_{H}$, presented as ratios of production cross section times branching fraction to the SM prediction [2].

\begin{tabular}{cccccccccccccc}
\hline$M_{H}(\mathrm{GeV})$ & 90 & 95 & 100 & 105 & 110 & 115 & 120 & 125 & 130 & 135 & 140 & 145 & 150 \\
\hline Expected & 1.8 & 1.9 & 2.2 & 2.5 & 2.9 & 3.4 & 3.8 & 4.7 & 5.8 & 7.9 & 11.1 & 16.7 & 20.8 \\
Observed & 1.6 & 1.3 & 2.2 & 2.0 & 2.1 & 2.9 & 3.4 & 4.8 & 6.6 & 10.1 & 13.6 & 18.8 & 18.5 \\
\hline
\end{tabular}

\subsection{D0 and Tevatron Higgs Boson Searches}

While do not see a significant excess in data over the background only prediction in the $W H \rightarrow \ell \nu b \bar{b}$ search on its own, we combine all Higgs boson searches at D0, and also combine the D0 results with those from CDF, to improve our statistical power. We combine all searches for Higgs boson decaying to $b \bar{b}$ (which includes the search described in detail in this thesis), $W^{+} W^{-}, \tau^{+} \tau^{-}$, and $\gamma \gamma$. The LLR and cross section limits can be seen for the D0 Higgs boson combination, and for the full Tevatron (D0+CDF) Higgs boson combination in Fig. 6.27. When we add together multiple channels which each see a small excess in the data above the background prediction, we see a larger excess from $M_{H} \sim 115 \mathrm{GeV}$ to $M_{H} \sim 145 \mathrm{GeV}$.

We can quantify the excess we see in our data by asking what the probability is that our background would fluctuate up to result in the data we observe. That is we can calculate the background p-value. Figure 6.28 shows the p-values for the D0 and Tevatron Higgs boson combinations as a function of Higgs boson mass. The p-value for the full Tevatron combination for $M_{H}=125 \mathrm{GeV}$ corresponds to a 3 standard deviation significance. Additionally, we can measure the cross section time branching ratio for $H \rightarrow b \bar{b}$. The full Tevatron $H \rightarrow b \bar{b}$ combination results in a cross section of $1.59_{-0.72}^{+0.69}$ times the SM cross section. 

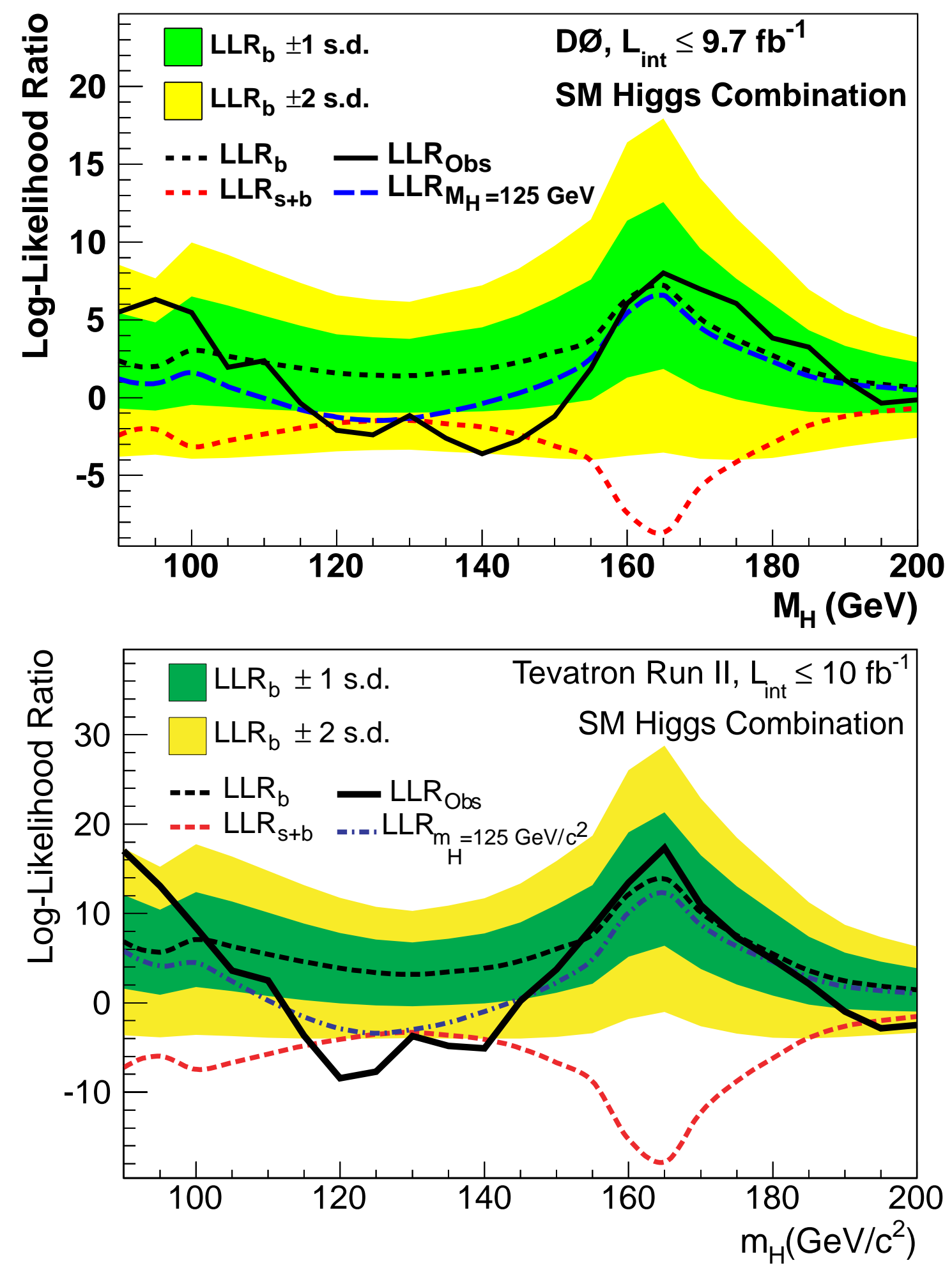

Figure 6.26: The LLR for the combined D0 Higgs boson searches (top) and the combined Tevatron Higgs searches (bottom), showing the background only expectation (the black dashed line), the signal+background expectation (red dashed line), the observed data (black solid line), and the background plus a $125 \mathrm{GeV}$ Higgs boson signal (blue dashed line). The green and yellow bands are the \pm 1 and 2 standard deviation on the background only expectation $[7,8]$. 

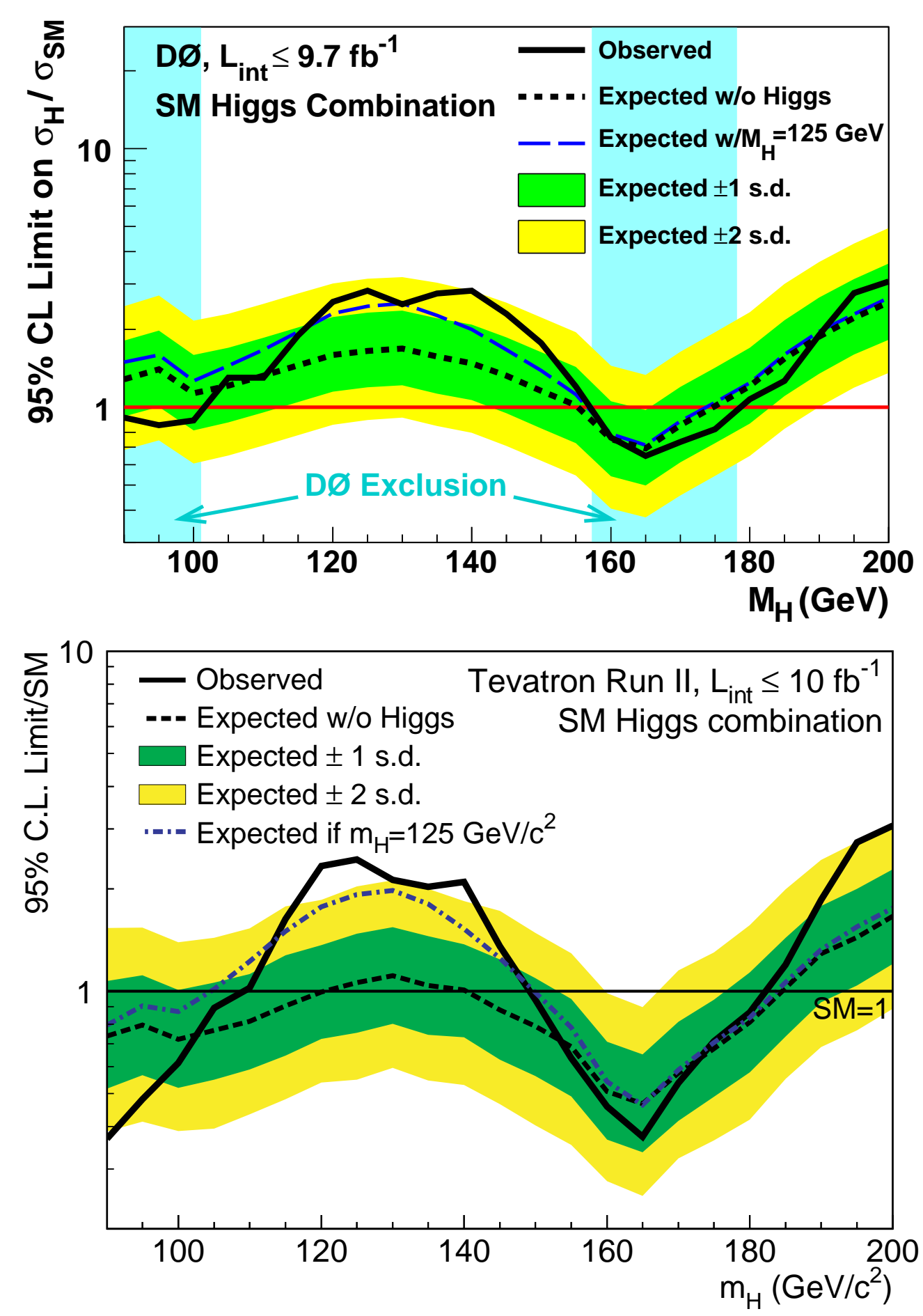

Figure 6.27: The 95\% C.L. limit as a function of Higgs boson mass for the combined D0 Higgs boson searches (top) and the combined Tevatron Higgs boson searches (bottom), shown as a ratio the the SM cross section. The black dashed line shows the expected limit, the solid black line shows the observed limit, the blue dashed line shows the limit expected if a Higgs boson with a mass of $125 \mathrm{GeV}$ were present. The green and yellow bands are the \pm 1 and 2 standard deviation on the expectation. Regions where the observed limit is below 1 $\left(90<M_{H}<101,157<M_{H}<178 \mathrm{GeV}(\mathrm{D} 0)\right.$, and $90<M_{H}<108,150<M_{H}<182 \mathrm{GeV}$ (Tevatron)), are regions of $M_{H}$ space that are excluded at the $95 \%$ confidence level $[7,8]$. 

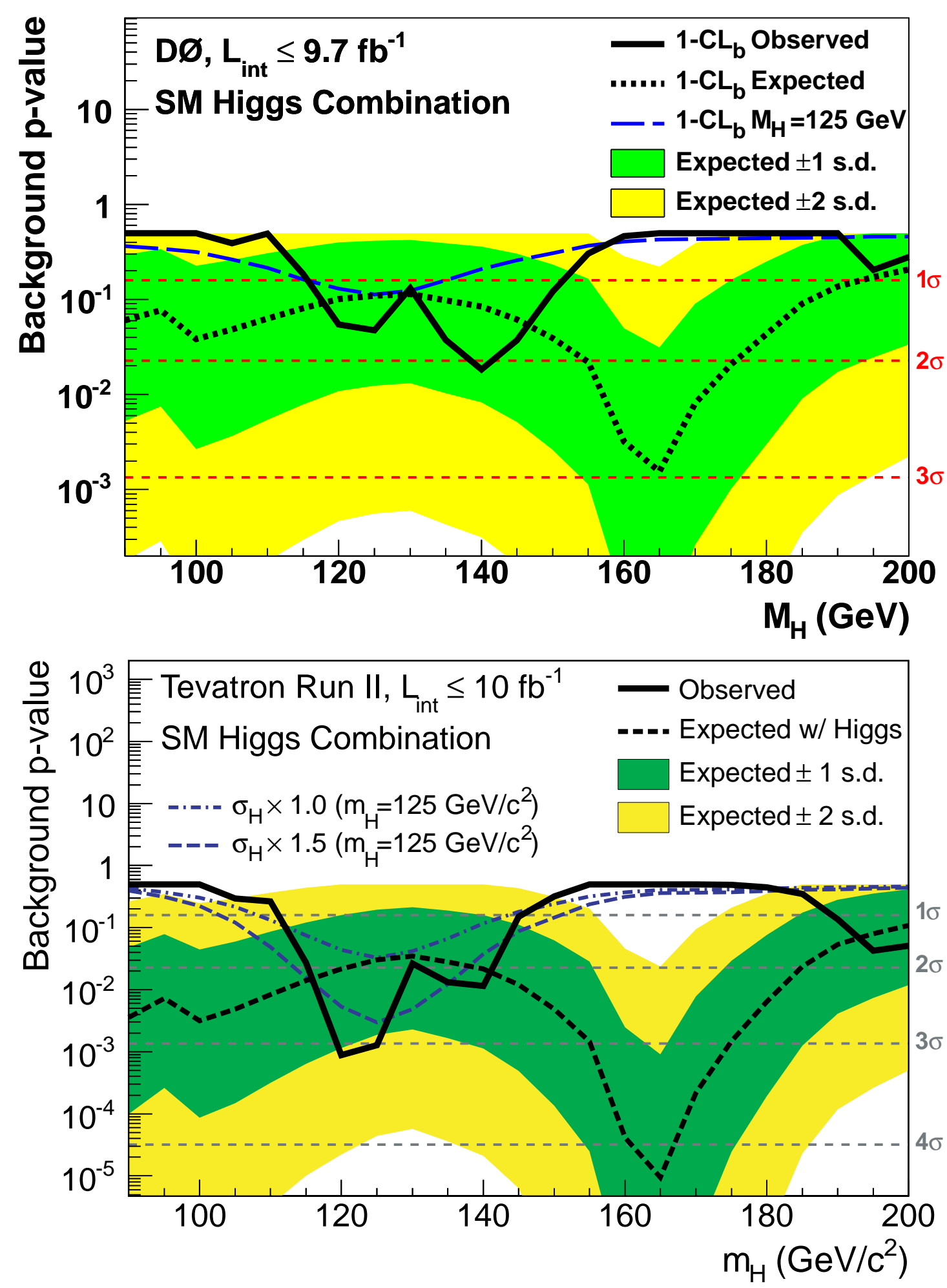

Figure 6.28: The probability for the background to fluctuate to the observed rate in data as a function of Higgs boson mass for the D0 combination (left) and the Tevatron combination (right). The black dashed line shows the expectation, the solid black line shows the observed data, the blue dashed line shows the expectation for a Higgs boson with $M_{H}=125 \mathrm{GeV}$. The green and yellow bands enclose the \pm 1 and 2 s.d. fluctuations of the background $[7,8]$. 


\subsection{Future Studies of the Higgs Boson}

Now that the Higgs boson has been discovered, the next steps are to measure its properties with high precision to determine if it really is the Higgs boson described in the standard model. In addition to measuring the mass of the Higgs boson, it is important to measure the strength with which the Higgs boson couples to all the different particles. Both the LHC experiments and the Tevatron experiments have measured the coupling strength of the Higgs to bosons and fermions $[8,52,53]$, however, the precision of these measurements still allows for many non-SM scenarios. Additionally, the Higgs boson in the standard model is expected to have a spin of 0 and positive parity. So far results from ATLAS, CMS, and D0 suggest that this is true, when comparing the standard model Higgs bosons to specific alternative models [54, 55, 56, 57]. Future measurements of the Higgs boson properties will improve our understanding of whether we are looking at the Higgs boson predicted by the standard model, or if we are looking at a Higgs boson that is merely similar to the standard model Higgs boson. 


\section{Chapter 7}

\section{Improvements to Jet Energy}

\section{Resolution}

In searches for $H \rightarrow b \bar{b}$, the variable that best discriminates between signal and background is the dijet invariant mass. The resolution of the dijet mass depends on the resolution with which we can measure the energy of jets ${ }^{1}$, and therefore an improvement to the jet energy resolution would improve the dijet mass resolution. This improvement would increase the sensitivity to the Higgs boson search discussed in this thesis. The resolution for the jet energy, $\sigma(E)$, as measured by the calorimeter is:

$$
\frac{\sigma(E)}{E}=\frac{a}{\sqrt{E}} \oplus \frac{b}{E} \oplus c .
$$

The $a / \sqrt{E}$ term is the stochastic response. It comes from fluctuations in shower development within the calorimeter, which are due to individual jet fragmentation and fluctuations of individual particles inside the jet shower. This is the dominant term for the jet energy resolution over most of the range of jet energies used in physics analyses. The $b / E$ term describes the noise of the calorimeter measurement and is due to instrumental effects such as electronic noise and the effects of multiple interactions. For low energy jets, this becomes

\footnotetext{
${ }^{1}$ The dijet mass resolution is defined as the width of the resonant peak of the dijet mass distribution. The energy resolution for individual jets is defined in Eq. 7.1.
} 
the limiting factor in the resolution of the calorimeter. The constant term, $c$, describes errors in calibration, non-uniformities in the calorimeter response, dead material, etc. This is the limiting factors for high jet energies.

The tracking system can measure the momenta of individual charged particles that are within a jet. At low energies, the tracking system provides a more precise momentum measurement than the energy measurement of the calorimeter. If we use the track momentum measurement instead of the calorimeter energy measurement for charged particles within a jet, we can improve the jet energy measurement. From here forward in this text, jets that use both the tracking system and the calorimeter to measure the jet energy will be called "track-cal jets", and jets that rely only on the calorimeter for the energy measurement will be called "calorimeter jets".

\subsection{Track-Cal Jet Algorithm}

The general idea behind track-cal jets is to replace the energy measurement from the calorimeter for a charged particle with the momentum measurement from the tracking system, which has a better resolution. This is done by starting with the nominal calorimeter jet energy measurement, then for each charged track in the jet we add in the momentum from the track, and (to avoid double counting energy) subtract out the energy in the calorimeter that is associated with the particle that made the track. We define the track-cal jet energy as:

$$
E_{\text {track-cal }}=E_{\text {raw }}^{\text {calo }}+\sum_{\text {tracks }}\left(P_{\text {track }}-E_{\text {track }}^{\Delta R=0.15}\right)
$$

where $E_{\text {raw }}^{\text {calo }}$ is the nominal calorimeter jet energy, $P_{\text {track }}$ is the track momentum, and $E_{\text {track }}^{\Delta R=0.15}$ is the energy deposited in a cone of $\Delta R=0.15$ (where $\Delta R=\sqrt{\Delta \eta^{2}+\Delta \phi^{2}}$ ) 
around the charged track ${ }^{2}$. The cone size used when subtracting the calorimeter energy near the track was chosen based on studying single pion MC events. The cone size was chosen to be large enough so that most of the energy from the track would be included, but small enough to keep energy not coming from the track minimal. We found that a cone size of $\Delta R=0.15$ contained $90 \%$ of the track energy for single pion events on average, as can be seen in Fig. 7.1.

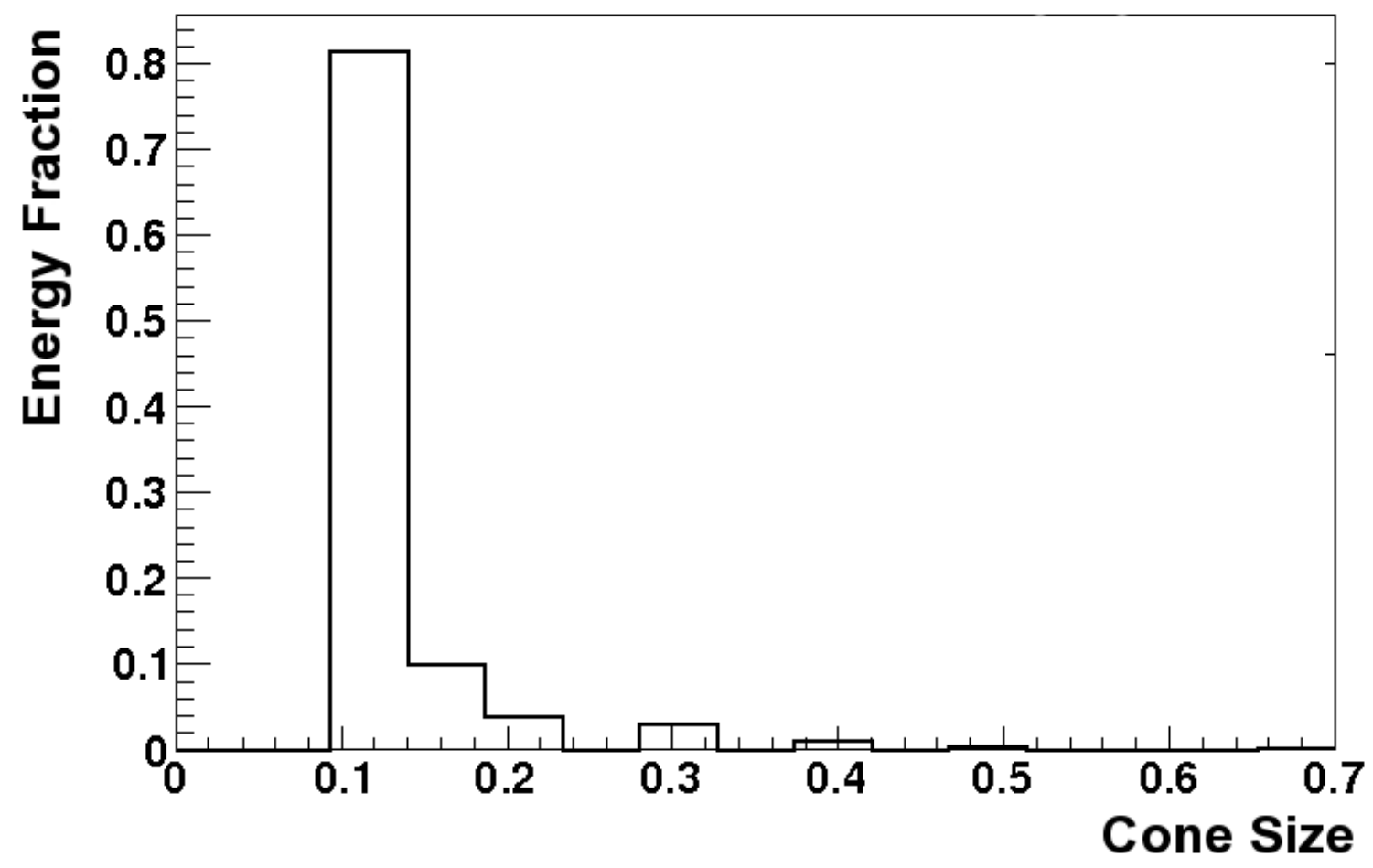

Figure 7.1: The fraction of energy deposited in various cones around a single pion track for a pion energy of $10 \mathrm{GeV} .90 \%$ of the energy is contained within a cone of $\Delta R=0.15$.

We want to make sure that the tracks we are considering for this calculation are coming from real tracks (and not misreconstructed tracks) with a high resolution. To suppress tracks arising from spurious signatures, we require that the the track mis-identification likelihood

${ }^{2}$ Note that because the energy of the particles is much larger than the mass of the particles, we can use energy and momentum interchangeably. 
be larger than zero. To ensure that we select high resolution tracks, we require that the track's z position is within $0.5 \mathrm{~cm}$ of the PV, that the distance of closest approach of the track in the radial direction is less than $0.05 \mathrm{~cm}$, and that the significance of curvature of the track (the ratio of the track $p_{T}$ to the resolution of the track $p_{T}$ ) is greater than 5 .

In addition to selecting well measured tracks, we also need to carefully consider the calorimeter energy we subtract from around the track. Of particular concern is the possibility of having neutral particles overlapping with the track. If this happens, simply subtracting all energy within the $\Delta R=0.15$ cone around the track is not correct, as we would be subtracting energy that isn't associated with the charged particle track. This is the limiting factor of the track-cal jet correction, as it is impossible to tell how much of the energy deposited in the calorimeter is coming from a charged particle vs a neutral one. The most practical solution is to only modify the energy with the track momentum if we don't think there is significant energy from neutral particles deposited within the $\Delta R=0.15$ cone around the track. To determine if there is significant energy coming from neutral particles, we first study single pion events, where we have a jet containing a single charged track. Using these events, we consider:

$$
F_{\mathrm{SP}}=\frac{E_{\text {track }}^{\Delta R=0.15}-P_{\text {track }} * R\left(P_{\text {track }}\right)}{P_{\text {track }}},
$$

where $R\left(P_{\text {track }}\right)$ is the average calorimeter response for a single pion, which depends on the track momentum, and location of the pion in the detector. $F_{\mathrm{SP}}$ should be zero if we are replacing the calorimeter energy by the track momentum correctly on average. Therefore, for a single charged track, we expect $F_{\mathrm{SP}}$ to peak at zero, with a non-zero width due to fluctuations in the jet formation and calorimeter energy measurement. If $F_{\mathrm{SP}}$ is large and negative, we consider that a sign that we misidentified a charged track. In this case we do not 
want to apply the track-cal jet correction, as we would be overestimating the charged track momentum. If $F_{S P}$ is large and positive, that is an indication that we are overestimating the calorimeter energy associated with a charged track. We consider this a sign that there are neutral particles depositing energy in the calorimeter near the charged particle track. We calculate $F_{S P}$ in bins of $P_{\text {track }}$ and jet $\eta$, and calculate the width of $F_{\mathrm{SP}}, \sigma_{F}\left(P_{\text {track }}, \eta_{\text {rmjet }}\right)$, to use as a metric to determine if there is significant energy from neutral particles deposited near the charged track. Then for each track within a jet we calculate:

$$
\left|f_{\text {track }}\right|=\left|\frac{\left[E_{\text {track }}^{\Delta R=0.15}-P_{\text {track }} * R\left(P_{\text {track }}\right)\right]}{P_{\text {track }}}\right|<2 \sigma_{F} .
$$

If $\left|f_{\text {track }}\right|<2 \sigma_{F}$, the energy replacement is performed. Otherwise the energy replacement is not performed (ie, the calorimeter energy measurement is used instead of the tracking momentum measurement). Examples of $F_{\mathrm{SP}}$ and $f_{\text {track }}$ are shown in Fig. 7.2 with vertical lines indicating $2 \sigma_{F}$. This track-cal jet algorithm doesn't perform well for jets with large $|\eta|$, as the tracking resolution gets worse in this region, so we only apply this algorithm to jets with $|\eta|<1.6$.

We can also gain in the energy resolution by adding in the energy from the CPS detector, multiplied by a calibration factor, $k_{\mathrm{CPS}}$, where $k_{\mathrm{CPS}}$ was chosen to minimize the energy resolution. This corrects for energy lost in dead material before the calorimeter (the solenoid, cables, etc.). We found that adding $k_{\mathrm{CPS}} \times E_{\mathrm{CPS}}$ to the track-cal jet energy improved the energy resolution when the jet $|\eta|<1.2$, however, for $1.2<|\eta|<1.6$, we found that correcting the calorimeter energy by adding $k_{\mathrm{CPS}} \times E_{\mathrm{CPS}}$ to the calorimeter energy instead of the track-cal energy resulted in a better jet energy resolution. Figures 7.3 and 7.4 show the resolution as a function of $k_{\mathrm{CPS}}$ for the different jet $\eta$ regions. The full energy correction, 

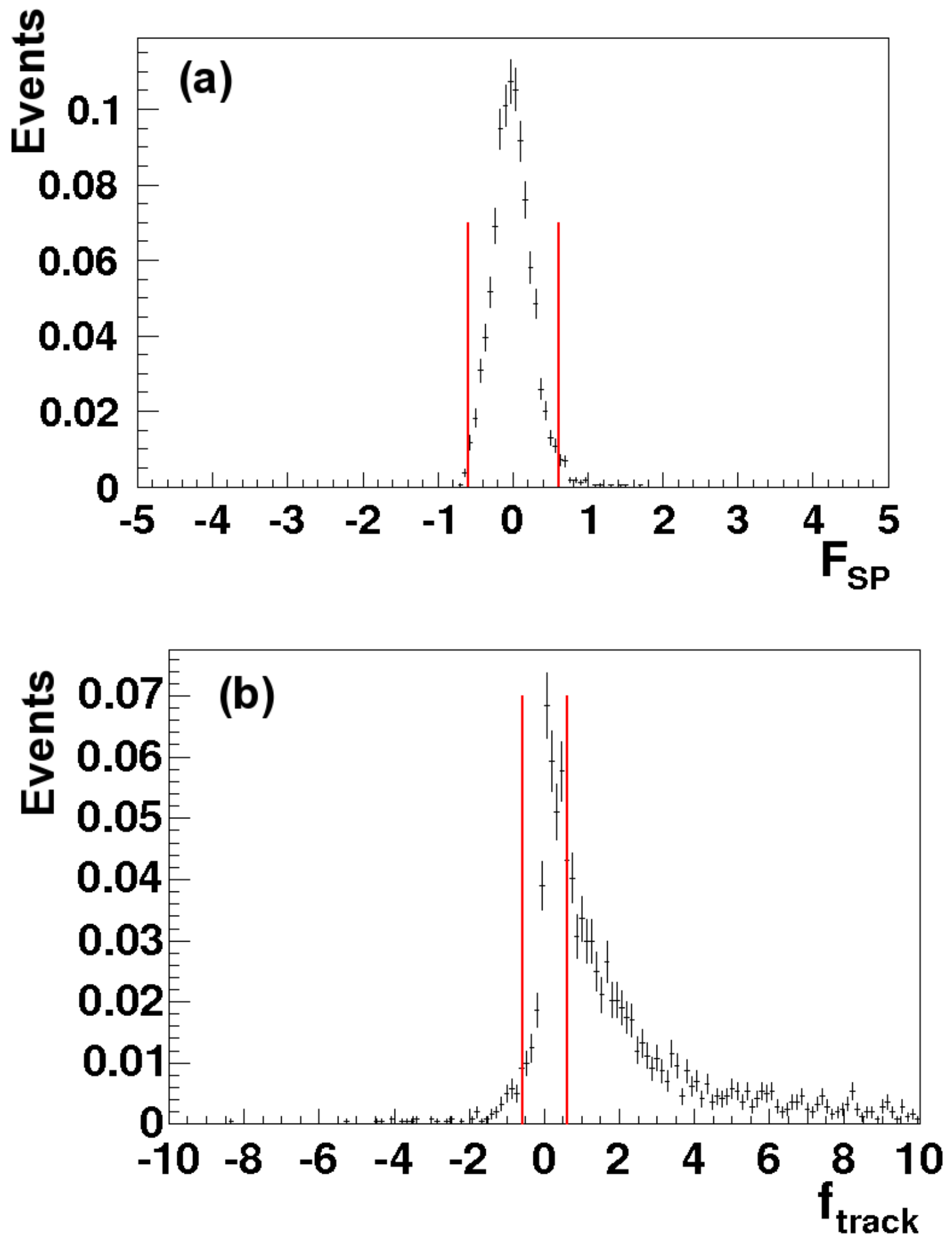

Figure 7.2: $\left[E_{\text {track }}^{\Delta R=0.15}-P_{\text {track }} * R\left(P_{\text {track }}\right)\right] / P_{\text {track }}$ for (a) $5 \mathrm{GeV}$ simulated single charged pion tracks, and (b) tracks within a jet with $4.5<P_{\text {track }}<5 \mathrm{GeV}$ using simulated $\gamma+$ jet events. The vertical lines indicate twice the width of the charged pion distribution $\left(\sigma_{F}\right)$. 
with optimal values of $k_{\mathrm{CPS}}$, is given by:

$$
\begin{gathered}
E_{\text {track-cal }}=E_{\text {raw }}^{\text {calo }}+\sum_{\text {tracks }}\left(P_{\text {track }}-E_{\text {track }}^{\Delta R=0.15}\right)+18 * E_{\mathrm{CPS}}, \text { if }\left|\eta_{\text {jet }}\right|<1.2, \\
E_{\text {track-cal }}=E_{\text {raw }}^{\text {calo }}+13 * E_{\text {CPS }}, \text { if } 1.2<\left|\eta_{\text {jet }}\right|<1.6, \\
E_{\text {track-cal }} \\
=E_{\text {raw }}^{\text {calo }} \text { if }\left|\eta_{\text {jet }}\right|>1.6 .
\end{gathered}
$$

\subsection{Energy Resolution Improvement}

Because the track-cal jets algorithm acts as a jet energy correction, the calorimeter response used in the JES is not necessarily the same for track-cal jets as it is for calorimeter jets as discussed in Ch. 3.5. Therefore, a response function was derived for track-cal jets using photon+jet MC, by taking the ratio of the track-cal jet $p_{T}$ to the true $\mathrm{MC} p_{T}$ in bins of the true $\mathrm{MC}$ particle $p_{T}$, number of tracks in the jet, and jet $\eta$. An example of this distribution can be seen in Fig. 7.5 for jets with $30<p_{T}<40 \mathrm{GeV}$ and $|\eta|<0.4$. The mean of these distributions is then plotted as a function of $\mathrm{MC}$ particle $p_{T}$ in bins of the number of tracks, and jet $\eta$, and fit with logarithmic functions.

These response functions are applied back to the photon+jet MC, and again the ratio of the track-cal jet $p_{T}$ to the true MC $p_{T}$ is considered, with the RMS of these distributions being the resolution. The response functions are shown in Fig. 7.6 and 7.7, and the resolution is shown in Fig. 7.8 and 7.9. The jet energy resolution with track-cal jets is $20 \%$ better than

for calorimeter jets on average. For a typical jet from $H \rightarrow b \bar{b}$ events with $p_{T}=45 \mathrm{GeV}$ and $|\eta|<1.2$, the improvement in the jet energy resolution is $25 \%$. 

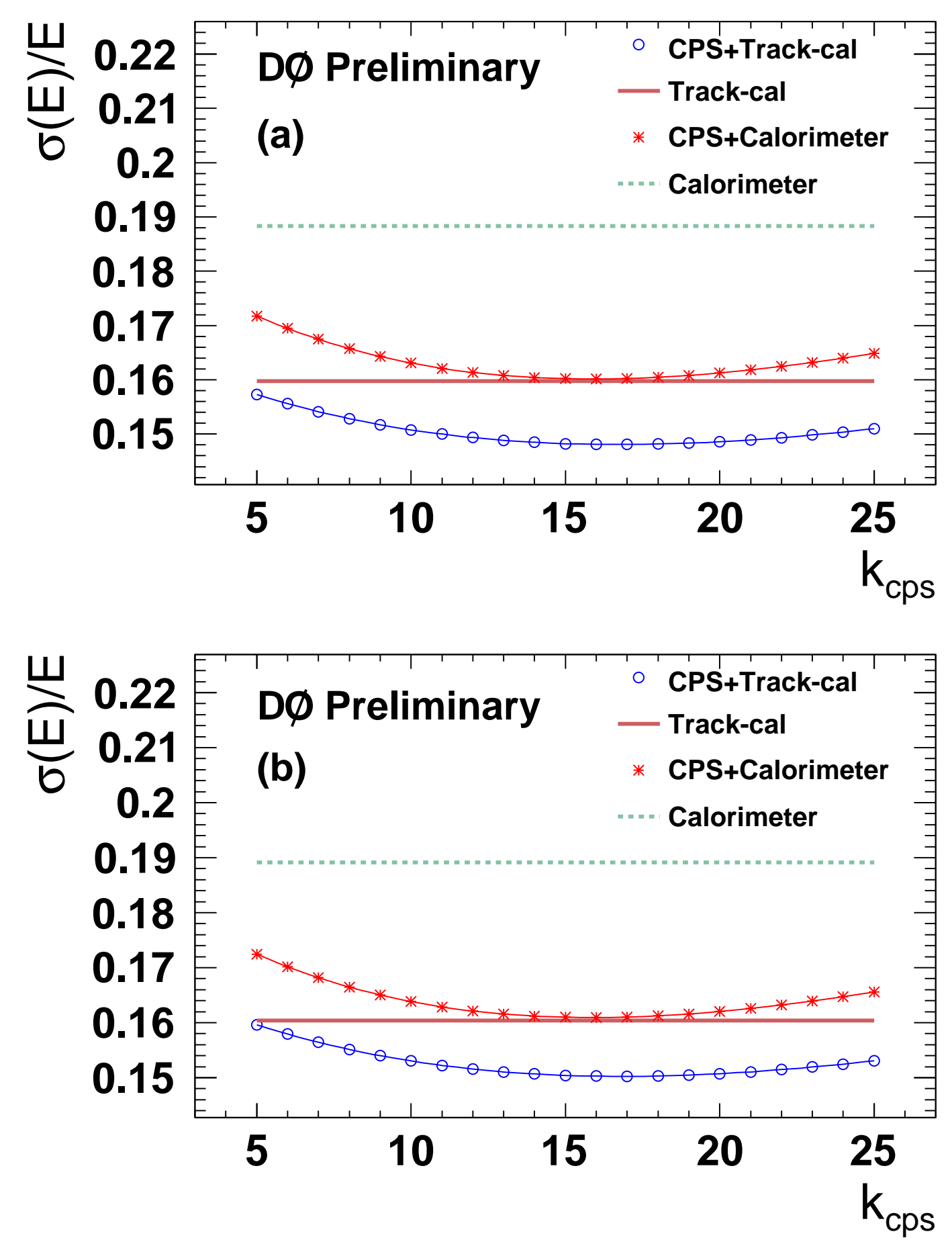

Figure 7.3: The resolution for the track-cal jet energy $+k_{\mathrm{CPS}}$ in the blue circles and calorimeter jet energy $+k_{\mathrm{CPS}}$ in the red crosses as a function of $k_{\mathrm{CPS}}$, for a) $\left|\eta_{\text {jet }}\right|<0.4$, and b) $0.4<\left|\eta_{\text {jet }}\right|<0.8$. The pink solid and green dashed lines show the track-cal jet energy and calorimeter jet energy resolutions respectively for $k_{\mathrm{cps}}=0$. 

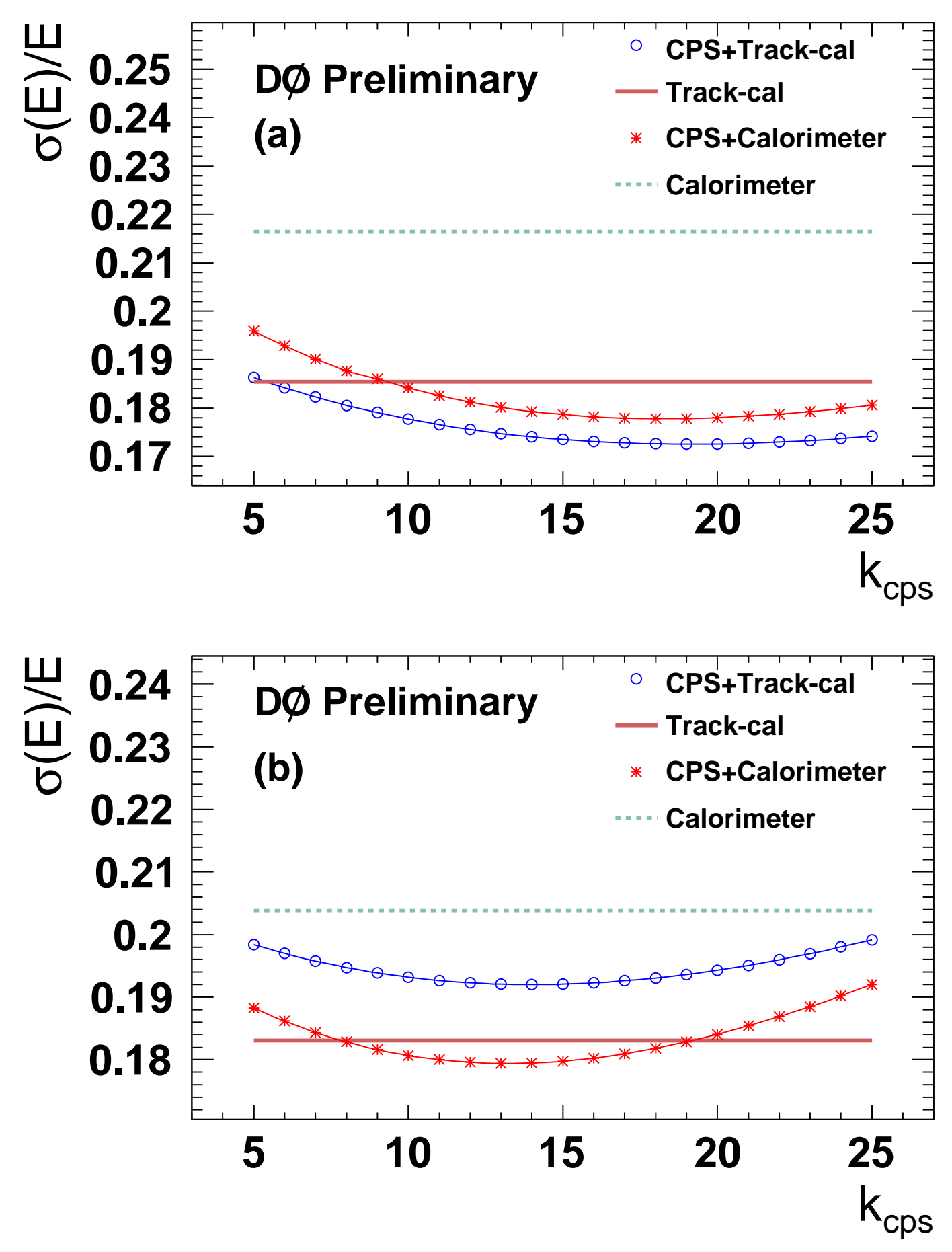

Figure 7.4: The resolution for the track-cal jet energy $+k_{\mathrm{CPS}}$ in the blue circles and calorimeter jet energy $+k_{\text {CPS }}$ in the red crosses as a function of $k_{\mathrm{CPS}}$, for a) $0.8<\left|\eta_{\text {jet }}\right|<1.2$, and b) $1.2<\left|\eta_{\text {jet }}\right|<1.6$. The pink solid and green dashed lines show the track-cal jet energy and calorimeter jet energy resolutions respectively for $k_{\mathrm{cps}}=0$. 


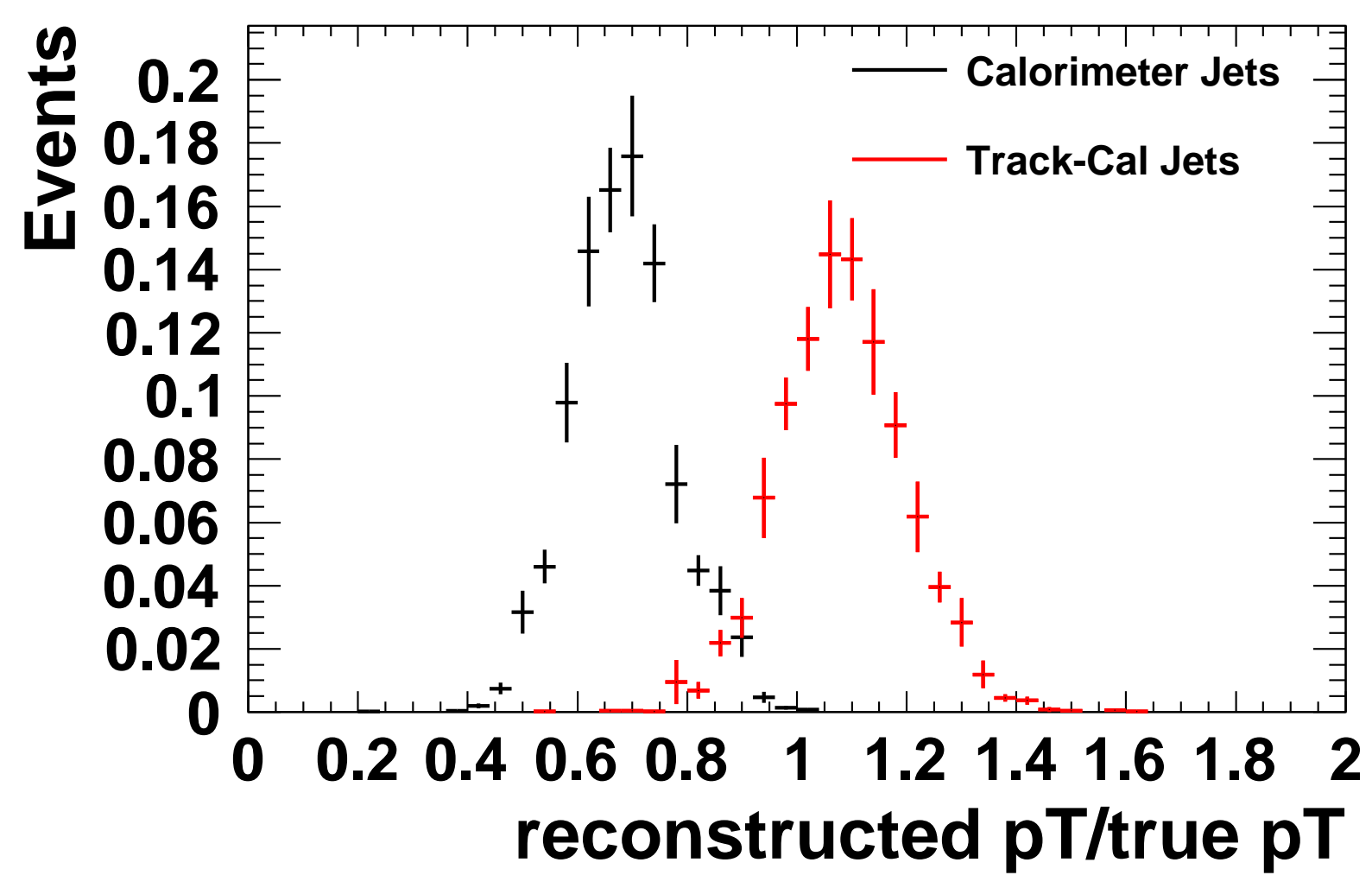

Figure 7.5: The ratio of the reconstructed jet $p_{T}$ to the true MC jet $p_{T}$ for jets with $30<p_{T}<40 \mathrm{GeV}$, and $|\eta|<0.4$, for track-cal jets (in red) and calorimeter jets (in black). The mean of this distribution is taken as the calorimeter response. 

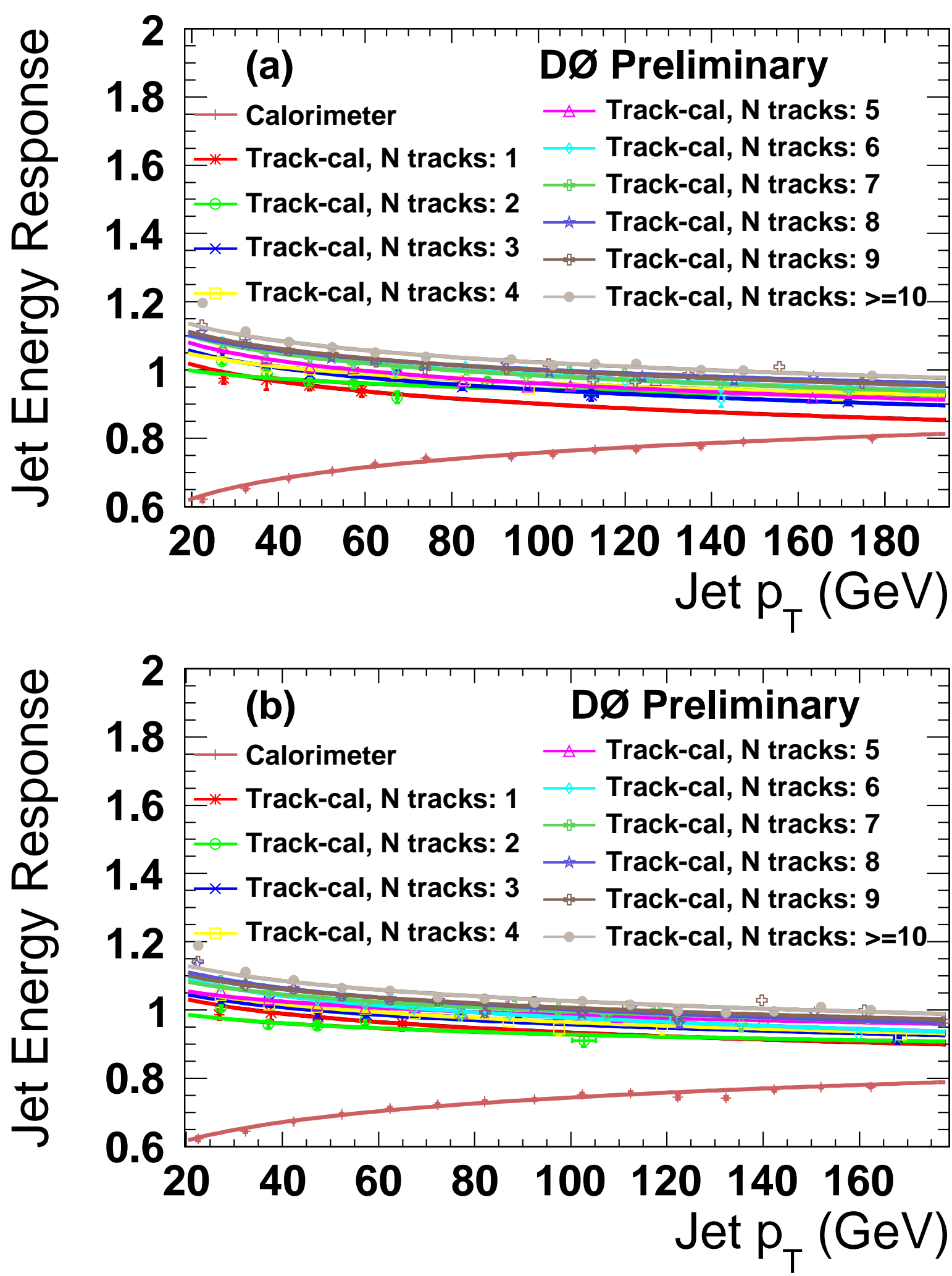

Figure 7.6: The energy response for track-cal jets as a function of true $\mathrm{MC} p_{T}$ for various track multiplicities for a) $\left|\eta_{\text {jet }}\right|<0.4$, and b) $0.4<\left|\eta_{\text {jet }}\right|<0.8$. The response for calorimeter jets is also shown for comparison. 

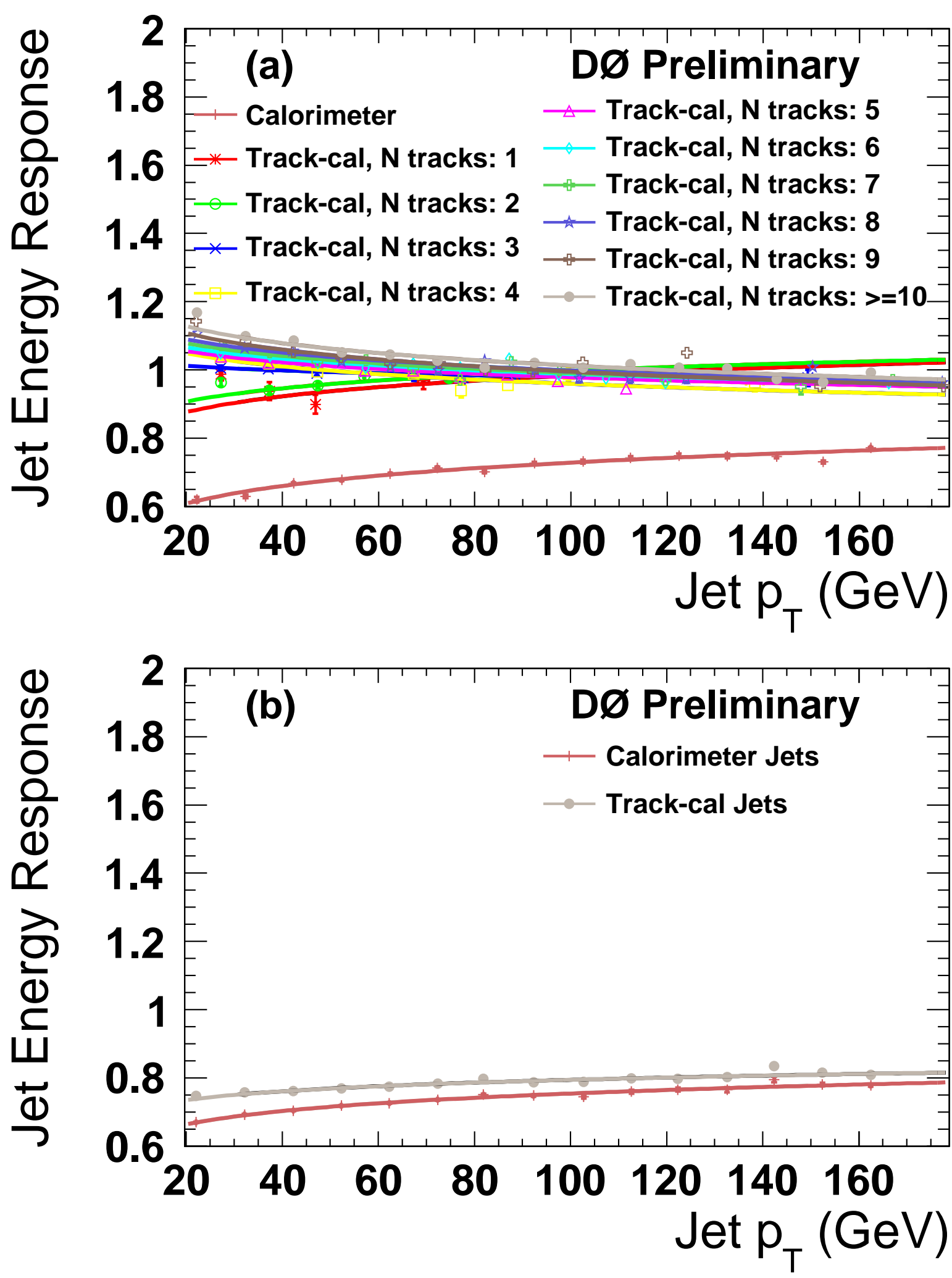

Figure 7.7: The energy response for track-cal jets as a function of true MC $p_{T}$ for various track multiplicities for a) $0.8<\left|\eta_{\text {jet }}\right|<1.2$, and b) $1.2<\left|\eta_{\text {jet }}\right|<1.6$. The response for calorimeter jets is also shown for comparison. 

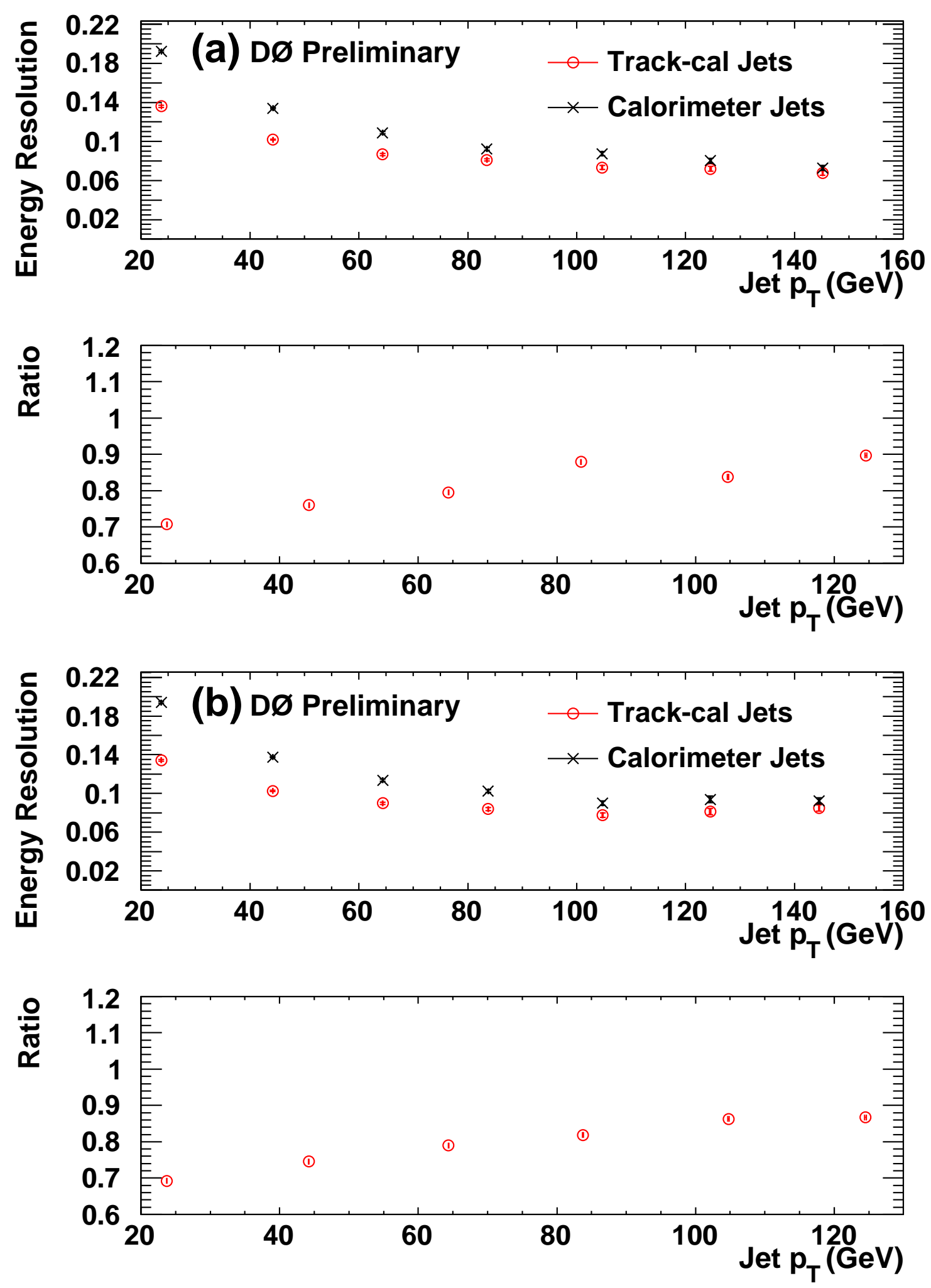

Figure 7.8: The energy resolution for track-cal jets as a function of true $\mathrm{MC} p_{T}$ compared to the resolution for calorimeter jets for a) $\left|\eta_{\text {jet }}\right|<0.4$, and b) $0.4<\left|\eta_{\text {jet }}\right|<0.8$. The bottom panels show the ratio of the track-cal jet resolution to the calorimeter jet resolution. 

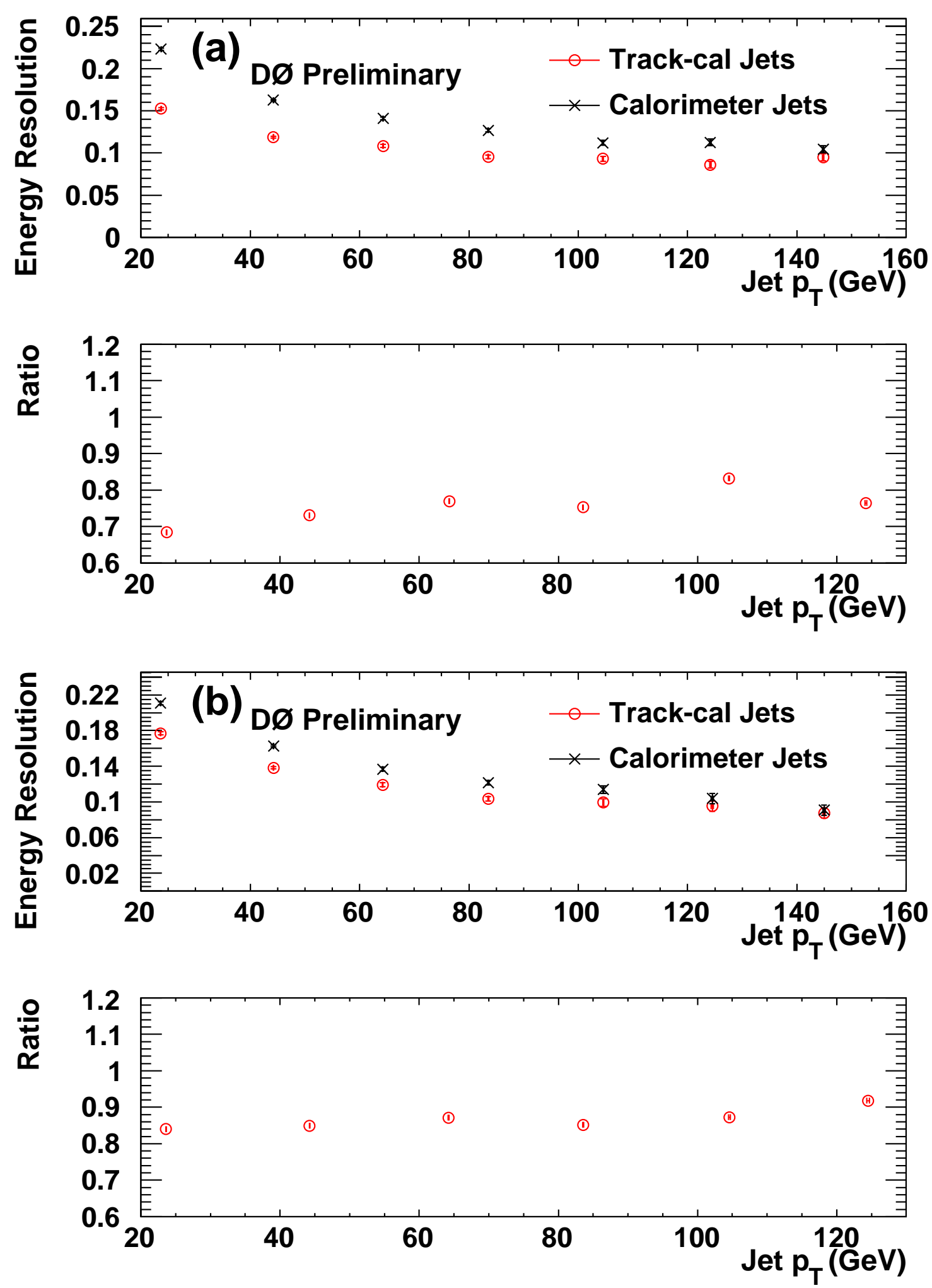

Figure 7.9: The energy resolution for track-cal jets as a function of true $\mathrm{MC} p_{T}$ compared to the resolution for calorimeter jets for a) $0.8<\left|\eta_{\text {jet }}\right|<1.2$, and b) $1.2<\left|\eta_{\text {jet }}\right|<1.6$. The bottom panels show the ratio of the track-cal jet resolution to the calorimeter jet resolution. 


\subsection{Improvement in Higgs Boson Analysis}

The track-cal jets correction is tested with the $W H \rightarrow \ell \nu b \bar{b}$ search, described in the preceding Chapter, to determine the impact on a resonant dijet signal and non resonant background processes. The full analysis is repeated using track-cal jets instead of calorimeter jets. Figures 7.10 to 7.12 show kinematic distributions after our full selection with track-cal jets in place of calorimeter jets. The reconstructed dijet mass is shown in Figs. 7.13 and 7.14 for the four different b-tag categories. From these plots, we can see that our background prediction matches our data well, when using track-cal jets instead of calorimeter jets. Figure 7.16 shows the dijet mass for calorimeter jets and track-cal jets overlayed for the signal and the sum of backgrounds. The signal dijet mass peak for track-cal jets is $\sim 7 \%$ narrower than for calorimeter jets.

We can also look at the improvement in events with jets originating from light quarks, by considering diboson production, in events with zero b-tags. Figure 7.16 shows the dijet mass for $W W \rightarrow \ell \nu q \bar{q}$ events with zero b-tags, and Fig. 7.17 shows the dijet mass for $V Z$ production in events with zero b-tags with the background subtracted from the data. The reconstructed vector boson mass peak is $\sim 7 \%$ narrower when using track-cal jets compared to using calorimeter jets.

To quantify the improvement to the $W H \rightarrow \ell \nu b \bar{b}$ analysis, we look at the expected $95 \%$ C.L. upper limit on the Higgs boson production cross section, with and without the track-cal jet correction. Figure 7.18 shows the expected limit as a ratio to the SM cross section with and without using the track-cal jets as well as the ratio of using the track-cal jets to using the calorimeter jets. We find that the expected limit improves by $\sim 5 \%$ when using track-cal jets for $M_{H}=125 \mathrm{GeV}$. In Fig. 7.15, we can see that there is a larger fraction of background 

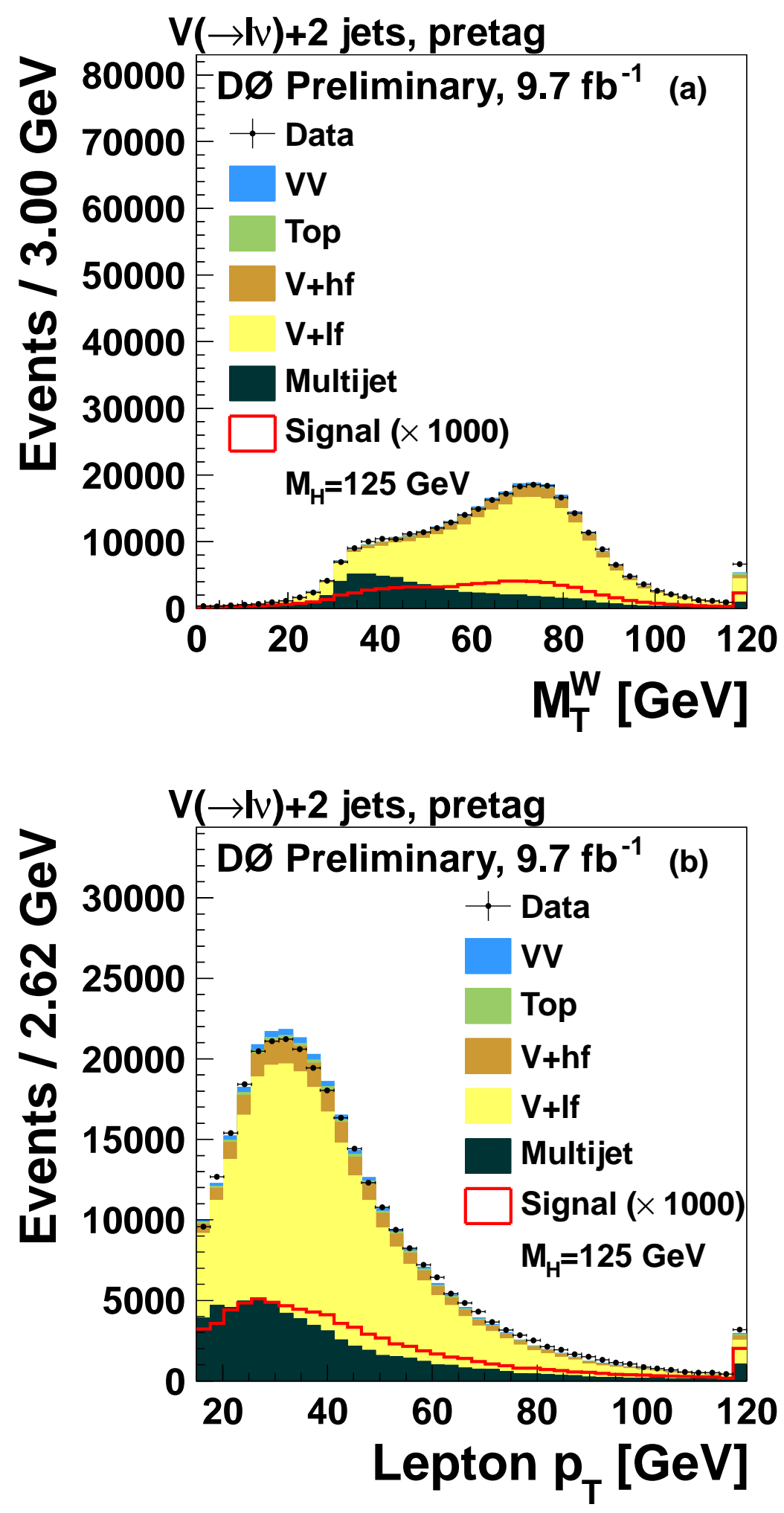

Figure 7.10: Distributions for all selected events with two jets of (a) transverse mass of the lepton- $E_{T}$ system, and (b) charged lepton $p_{T}$. The $M_{H}=125 \mathrm{GeV}$ signal is multiplied by 1000. Overflow events are added to the last bin. 

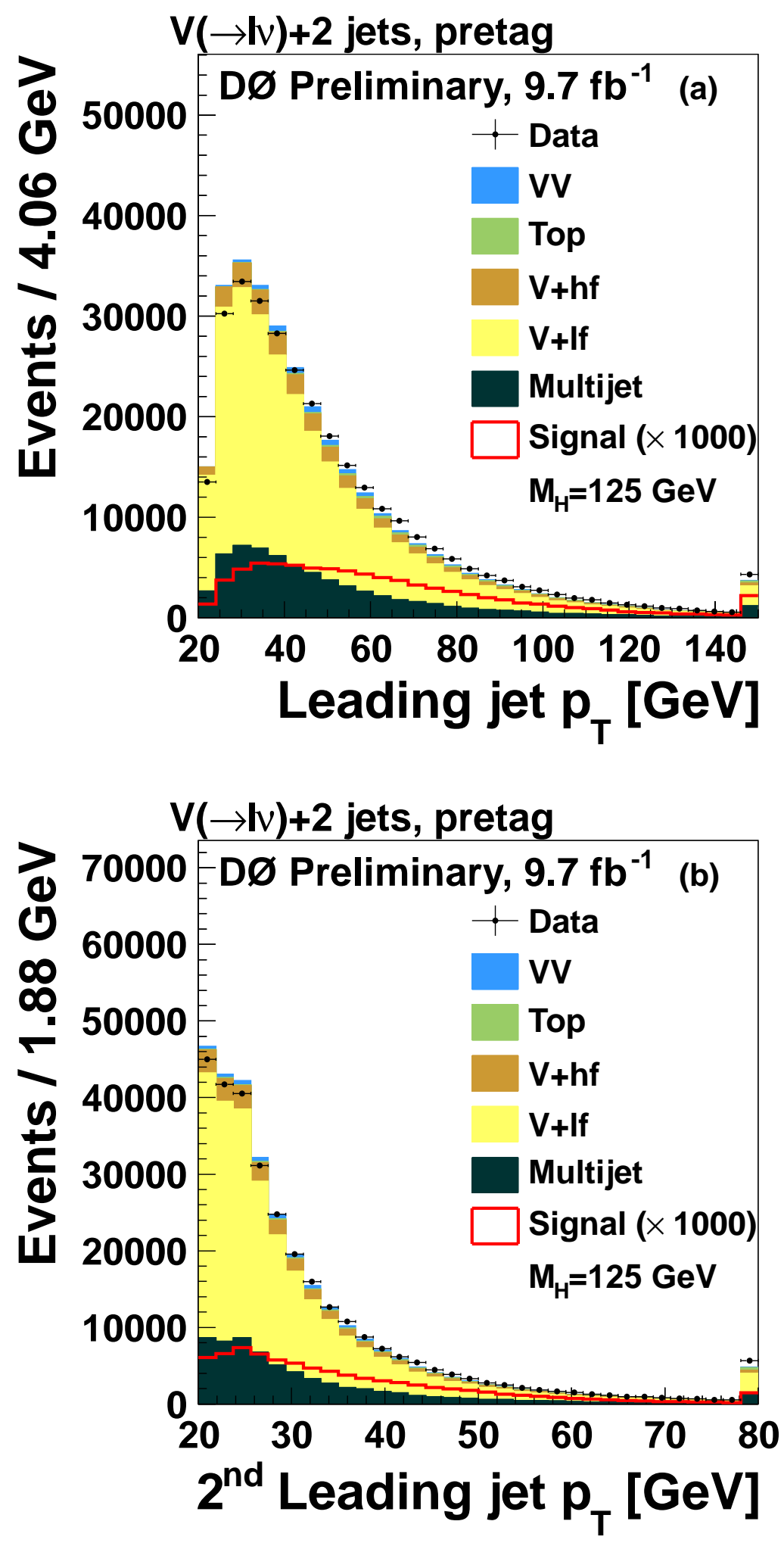

Figure 7.11: Distributions for all selected events with two jets of (a) leading jet $p_{T}$, and (b) second-leading jet $p_{T}$. The $M_{H}=125 \mathrm{GeV}$ signal is multiplied by 1000 . Overflow events are added to the last bin. 

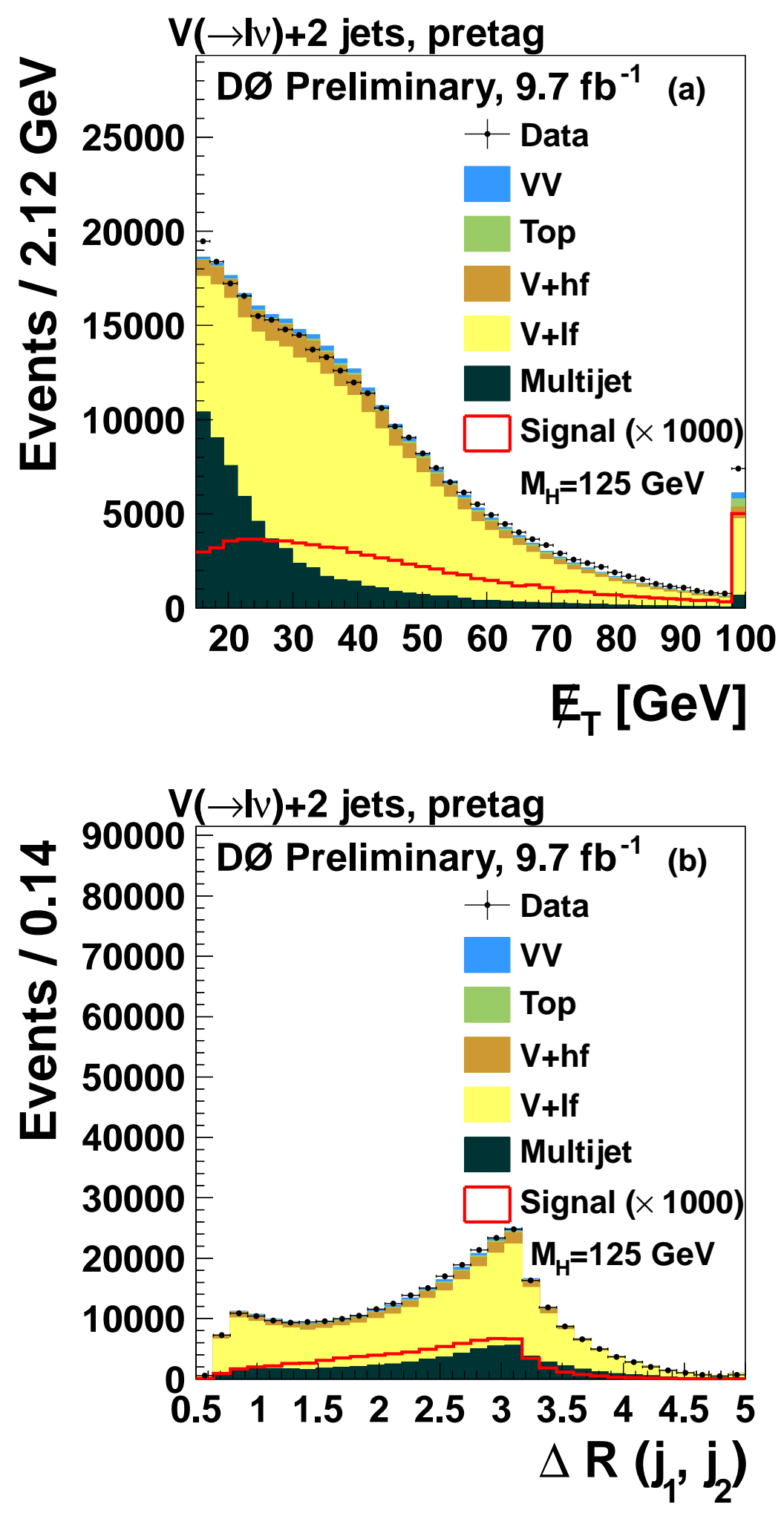

Figure 7.12: Distributions for all selected events with two jets of (a) $E_{T}$, and (b) $\Delta R$ between the leading and second-leading jets. The $M_{H}=125 \mathrm{GeV}$ signal is multiplied by 1000. Overflow events are added to the last bin. 

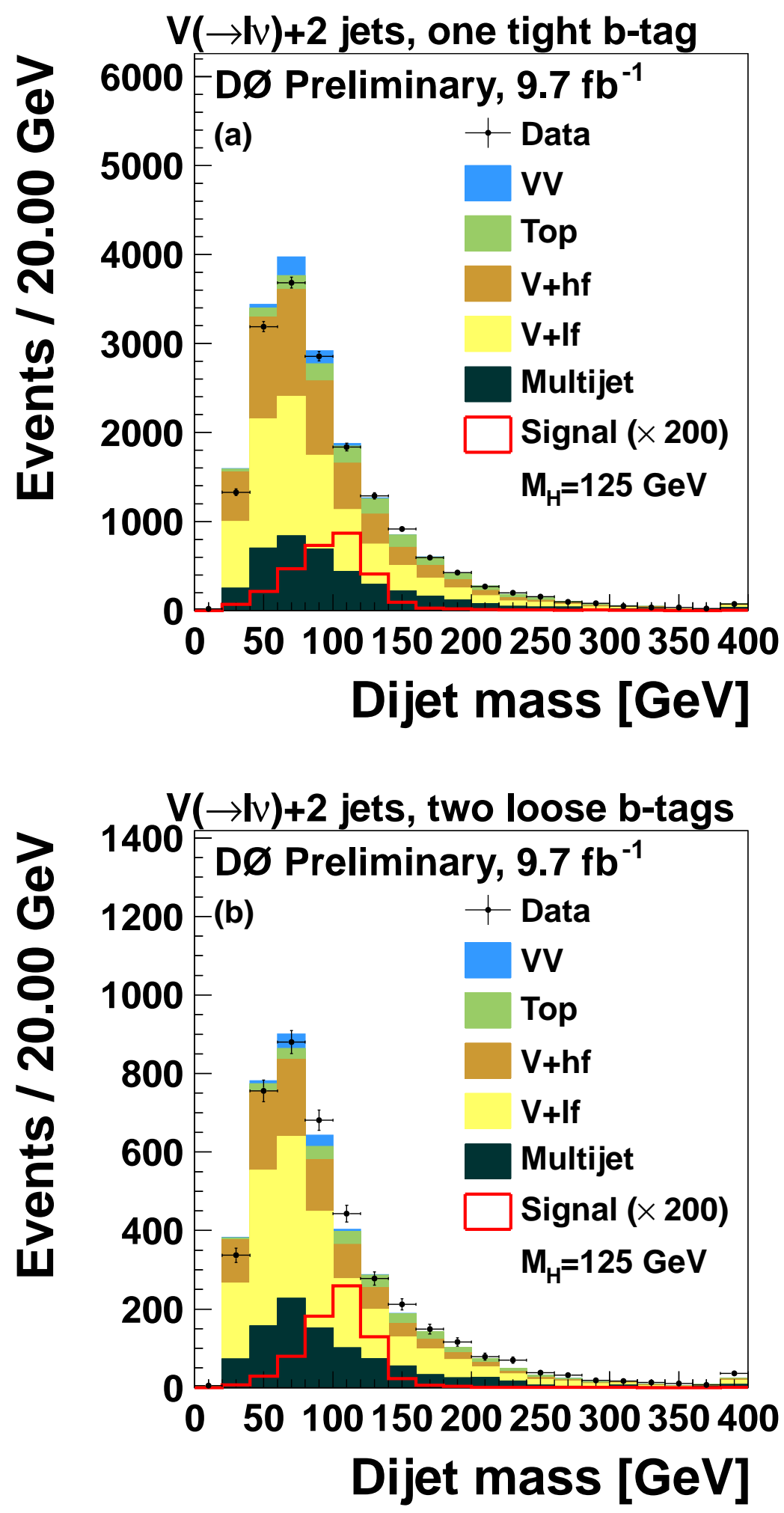

Figure 7.13: Invariant mass of the leading and second-leading jets in events with two jets and (a) one tight $b$-tag, and (b) two loose $b$-tags. The $M_{H}=125 \mathrm{GeV}$ signal is multiplied by 200 . Overflow events are added to the last bin. 

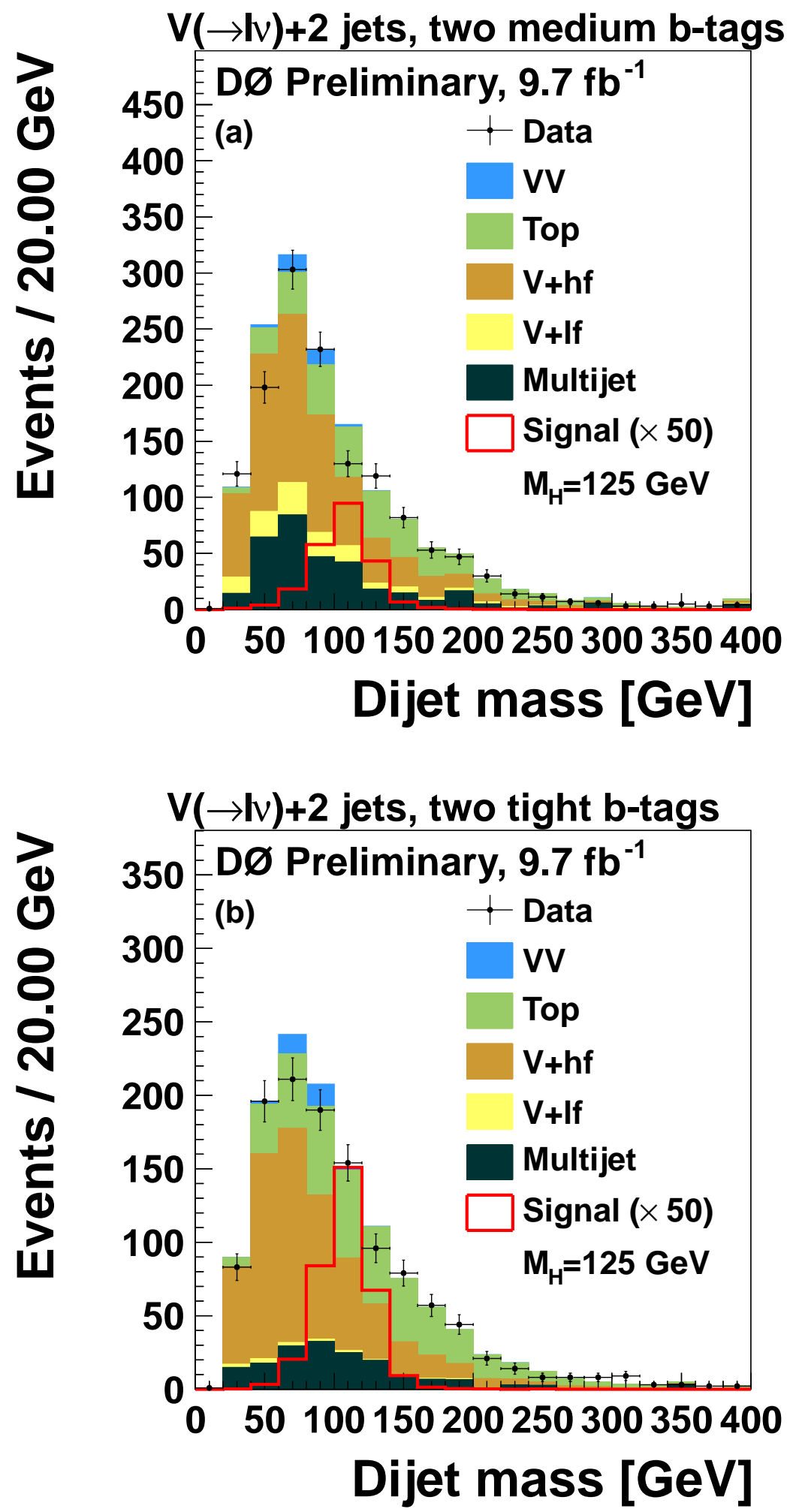

Figure 7.14: Invariant mass of the leading and second-leading jets in events with two jets and (a) two medium $b$-tags, and (b) two tight $b$-tags. The $M_{H}=125 \mathrm{GeV}$ signal is multiplied by 50 . Overflow events are added to the last bin. 


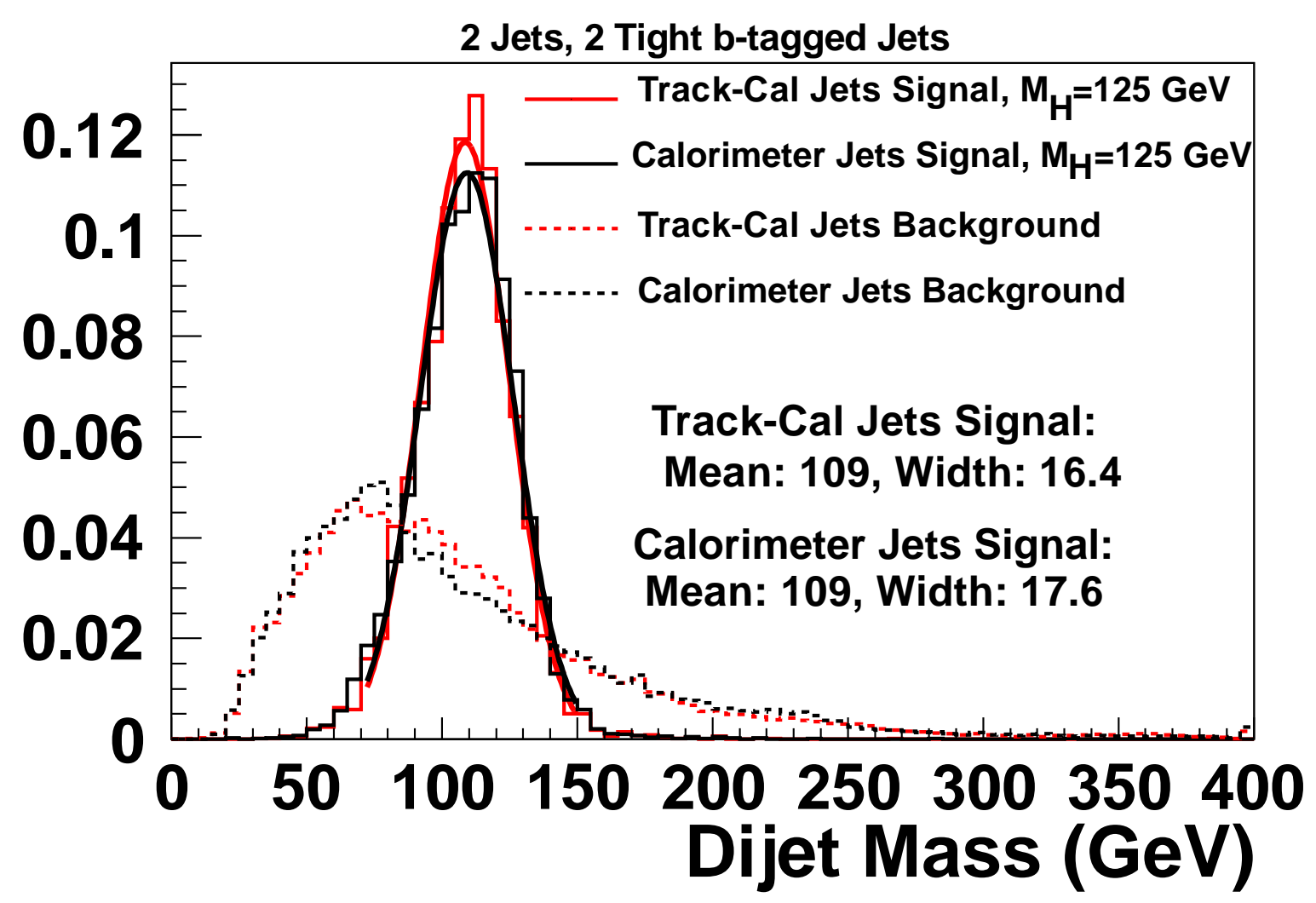

Figure 7.15: The dijet mass for track-cal jets (red) and calorimeter jets (black), for the $W H M_{H}=125 \mathrm{GeV}$ signal (solid lines) and all backgrounds (dashed lines) for events with exactly two jets and two tight b-tags. All curves are normalized to have unit area. The mean and width values quoted are determined by fitting the signal dijet mass peak with a Gaussian distribution. The width of the signal peak is $\sim 7 \%$ narrower for track-cal jets than for calorimeter jets. 


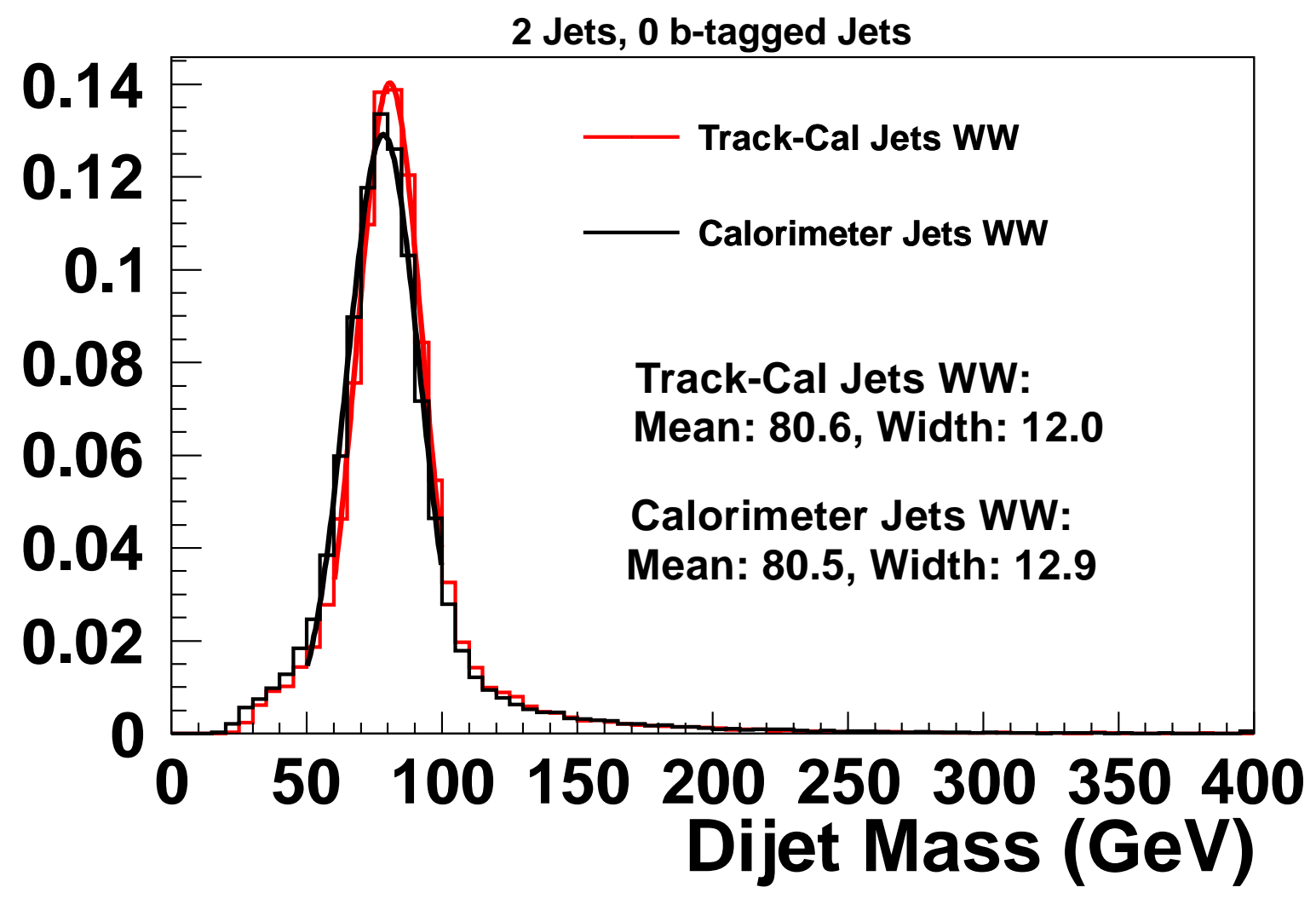

Figure 7.16: The dijet mass for track-cal jets (red) and calorimeter jets (black), for $W W$ events with exactly two jets and zero b-tags. The mean and width values quoted are determined by fitting the signal dijet mass peak with a Gaussian distribution. The width of the dijet mass peak is $\sim 7 \%$ narrower for track-cal jets than for calorimeter jets. 

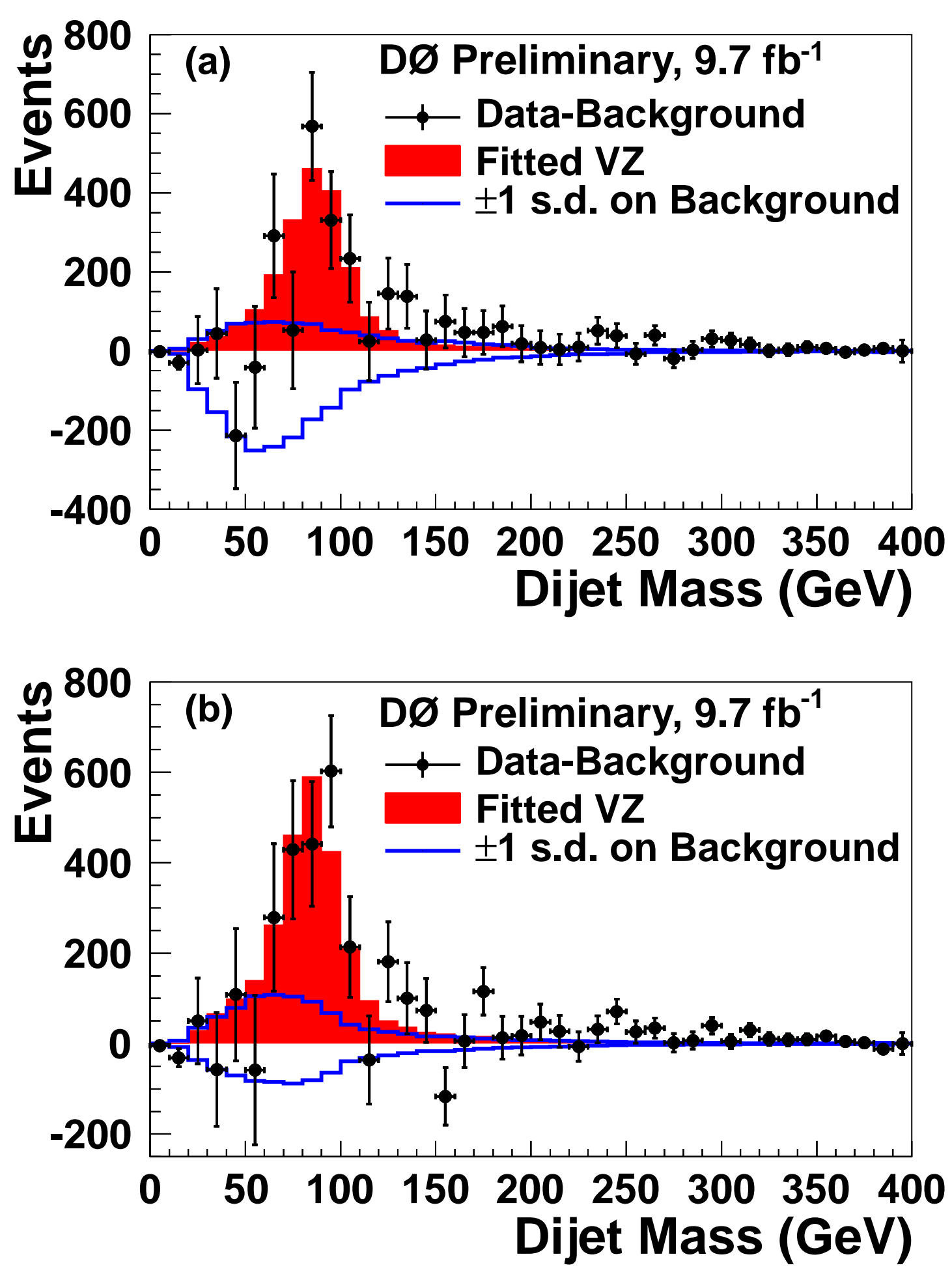

Figure 7.17: The dijet mass shown for the expected diboson signal and backgroundsubtracted data rebinned, after the maximum likelihood fit, for events with zero $b$-tagged jets (a) using calorimeter jets and (b) using track-cal jets. The error bars on data points represent the statistical uncertainty only. The post-fit systematic uncertainties are represented by the solid lines. The diboson signal expectation is shown scaled to the best fit value. 
events under the signal dijet mass peak for track-cal jets than for calorimeter jets. As the Higgs boson mass increases, the signal dijet mass peak will move to larger values, farther away from the background peak, resulting in a larger improvement for in the limit for larger $M_{H}$. 

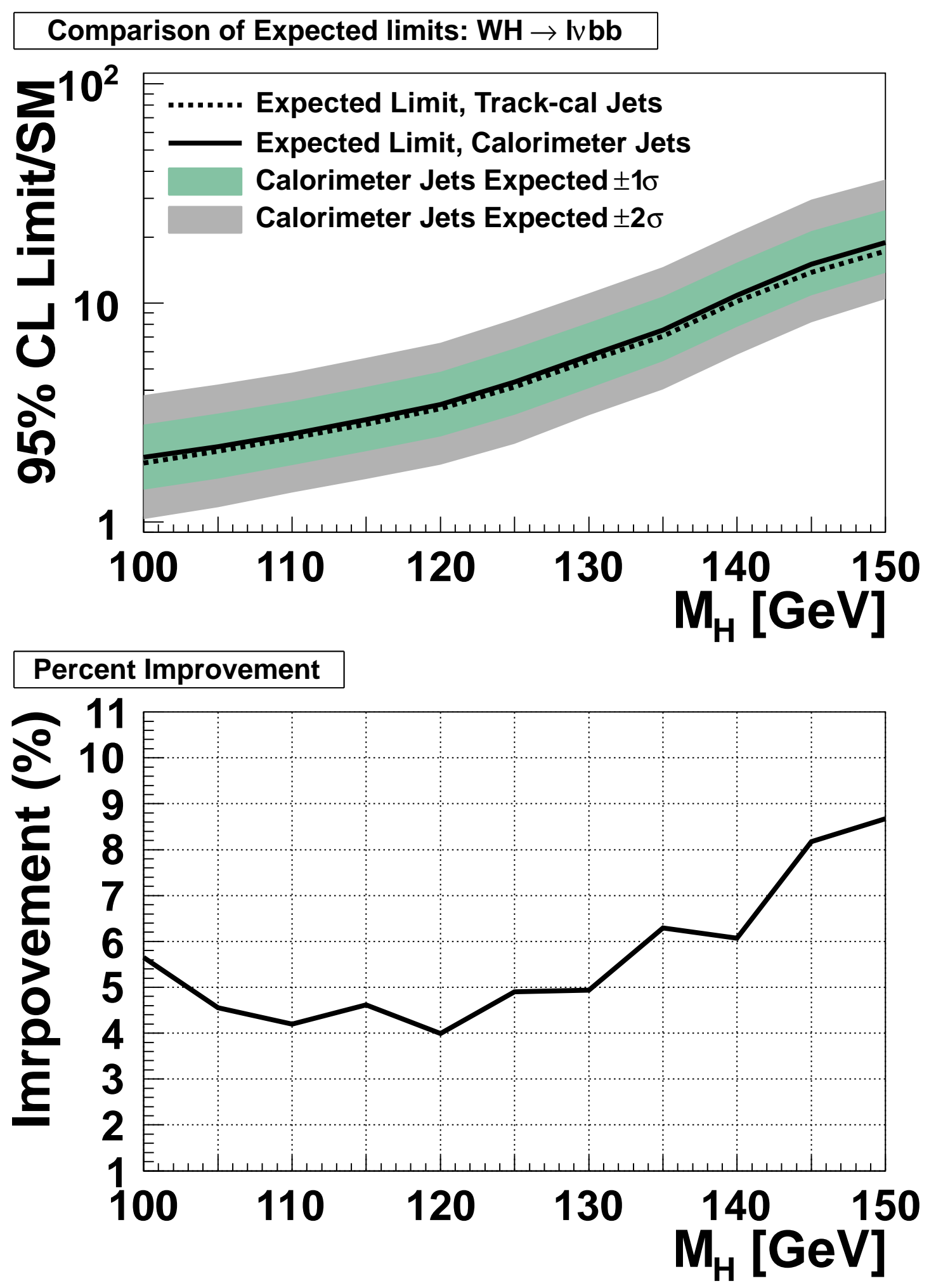

Figure 7.18: The $95 \%$ C.L. expected Higgs boson production cross section limit (top), presented as a ratio to the SM cross section, for track-cal jets (black dashed line), and calorimeter jets (black solid line). The green and grey shaded areas represent the \pm 1 and 2 standard deviations on the calorimeter jet expectation. The ratio of the expected 95\% C.L. limit for track-cal jets to calorimeter jets (bottom). 


\section{Chapter 8}

\section{Conclusion}

I presented in this thesis the search for a Higgs boson produced in association with a $W$ boson where the $W$ boson decays to a lepton and neutrino and the Higgs boson decays to a pair of bottom quarks. Events were selected to match the $W H \rightarrow \ell \nu b \bar{b}$ final state topology, and sorted into categories based on the number and quality of jets coming from b-quarks. Signal events were separated from background events using a Boosted Decision Tree machine learning technique. To test the analysis methodology, we performed a measurement of the $V Z \rightarrow V b \bar{b}$ cross section, which has the same final state as our Higgs boson signal, and a small cross section relative to our other background processes. By using the dijet invariant mass, we measure a cross section of $4.6 \pm 1.5$ (stat.) \pm 1.6 (syst.) pb, and using the MVA output we measure a cross section of $2.2 \pm 1.5$ (stat.) \pm 1.6 (syst.) pb. These values are consistent with each other (within their uncertainties), which supports the validity of our MVA approach. They are also consistent with the SM value of $4.4 \pm 0.3 \mathrm{pb}$, which verifies that, with our analysis strategy, we can measure processes with a small cross section relative to background processes. Since the $W H \rightarrow \ell \nu b \bar{b}$ search on its own does not see a significant excess in data above the background prediction, we set an 95\% C.L. limit on the production cross section for Higgs boson masses between 90 and $150 \mathrm{GeV}$ in steps of $5 \mathrm{GeV}$. For $M_{H}=125 \mathrm{GeV}$ we expect (observe) a limit of 4.7 (4.8) times the SM cross section. This search was included in the combined Tevatron Higgs boson search which measured an excess of data events with a 
significance of 3 s.d. above the background only expectation, and a measured cross section of 1.5 times the SM cross section. The sensitivity to $H \rightarrow b \bar{b}$ strongly depends on the dijet mass, and the resolution with which we can reconstruct the dijet mass. The dijet mass resolution depends on the resolution with which we can determine the energy of jets. We implemented a correction to the jet energy that replaces the calorimeter energy measurement with the higher resolution tracking momentum measurement for charged particles within a jet. This improves the accuracy of the jet energy measurement, and improves the energy resolution by $\sim 20 \%$, and the dijet mass resolution for our Higgs boson signal by $\sim 7 \%$. This translates to an improvement in our expected cross section limit for $W H \rightarrow \ell \nu b \bar{b}$ of $\sim 5 \%$.

The next several years of Higgs boson physics will be devoted to measuring its properties to determine if we have found the standard model Higgs boson, or if we have found a Higgs boson that simply looks like the standard model Higgs boson. While we can currently measure many of the Higgs boson's properties, such as its mass, its spin, and its couplings to other particles; it will be important to improve the precision of these measurements to truly understand the nature of this particle. 
APPENDICES 


\section{Appendix A Multijet MVA Input Distributions}

Shown below in Figs. A.1 to A.3 are the variables used to train our BDT that discriminates between signal and multijet events. Figures A.4 to A.6 show the same variables with the

total background and signal normalized to have the same area. The variables are described in Table 6.8 in the main text. 

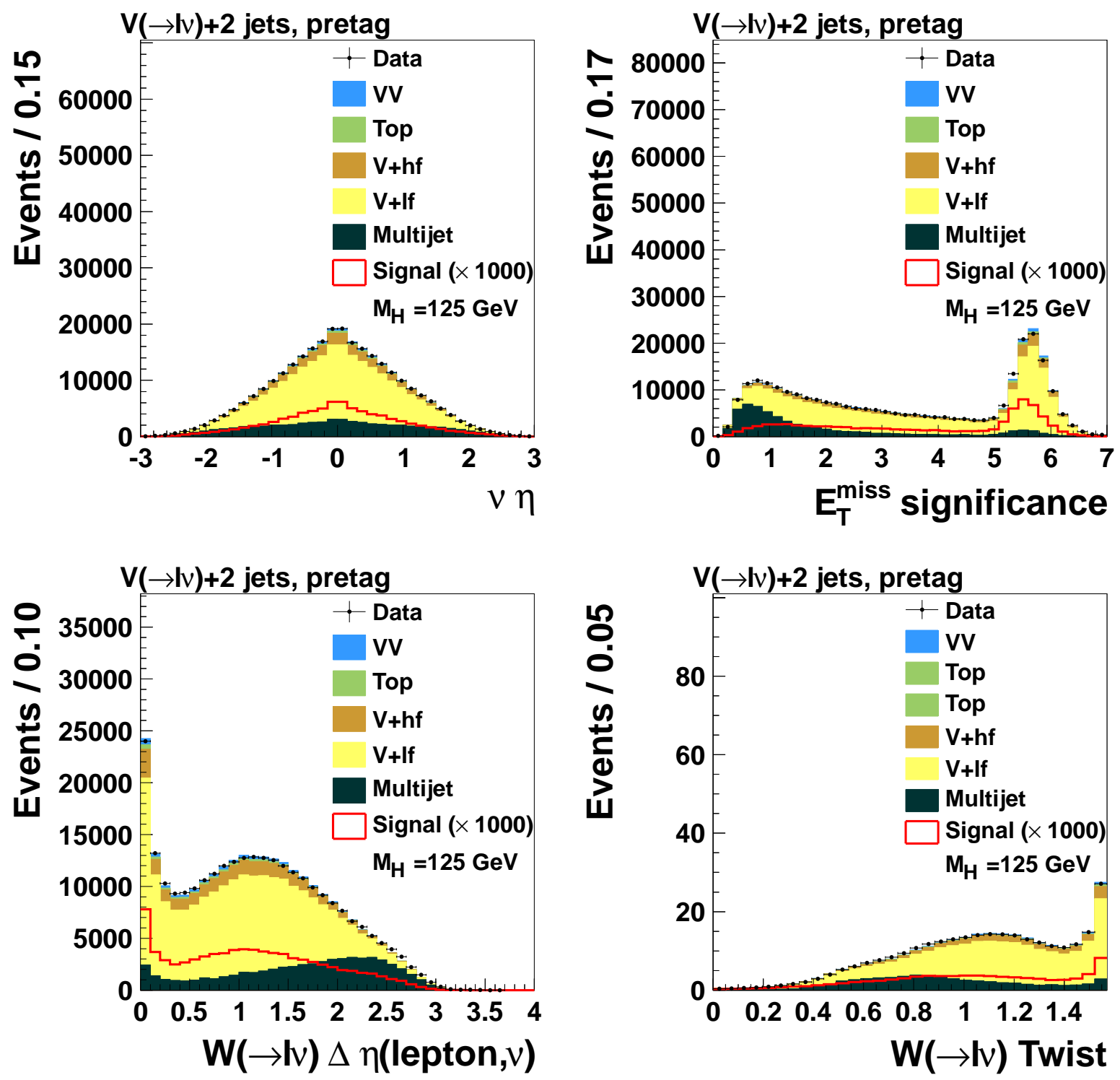

Figure A.1: Input variables to the multijet BDT shown for all events containing two jets: (top left) $\eta$ of the $E_{T}$, (top right) the $E_{T}$ significance, (bottom left) $\Delta \eta(\ell, \nu)$, and (bottom right) the twist of the $\ell-\nu$ system. The signal is shown for $M_{H}=125 \mathrm{GeV}$ multiplied by a factor of 1000. Overflow events are added to the last bin. 

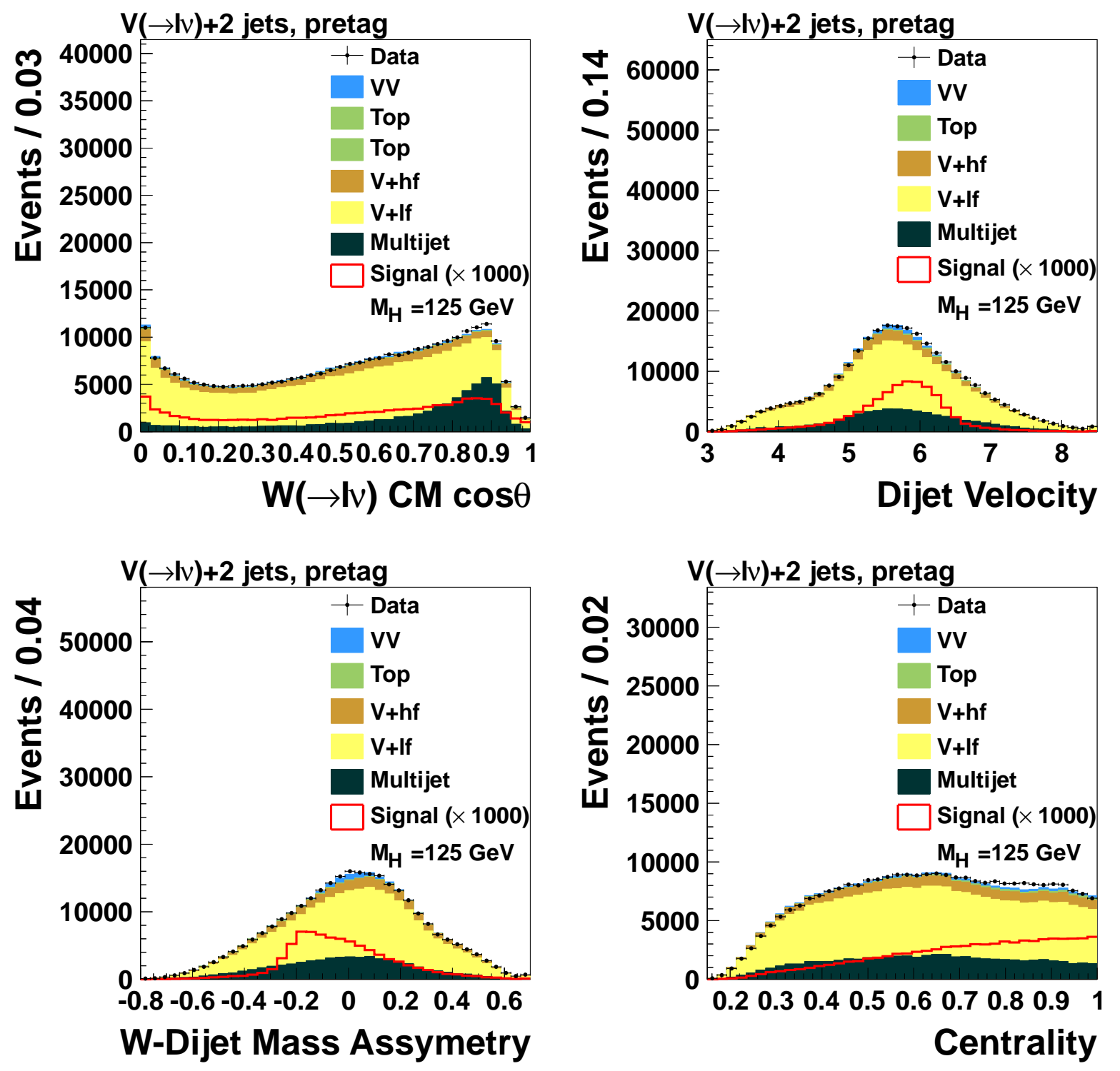

Figure A.2: Input variables to the multijet BDT shown for all events containing two jets: (top left) $\cos (\theta)$ in the $\ell \nu$ center-of-momentum frame, (top right) the velocity of the dijet system, (bottom left) the mass asymmetry between the dijet and the $\ell \nu$ system, and (bottom right) the centrality of the $\ell \nu j j$ system. The signal is shown for $M_{H}=125 \mathrm{GeV}$ multiplied by a factor of 1000 . Overflow events are added to the last bin. 

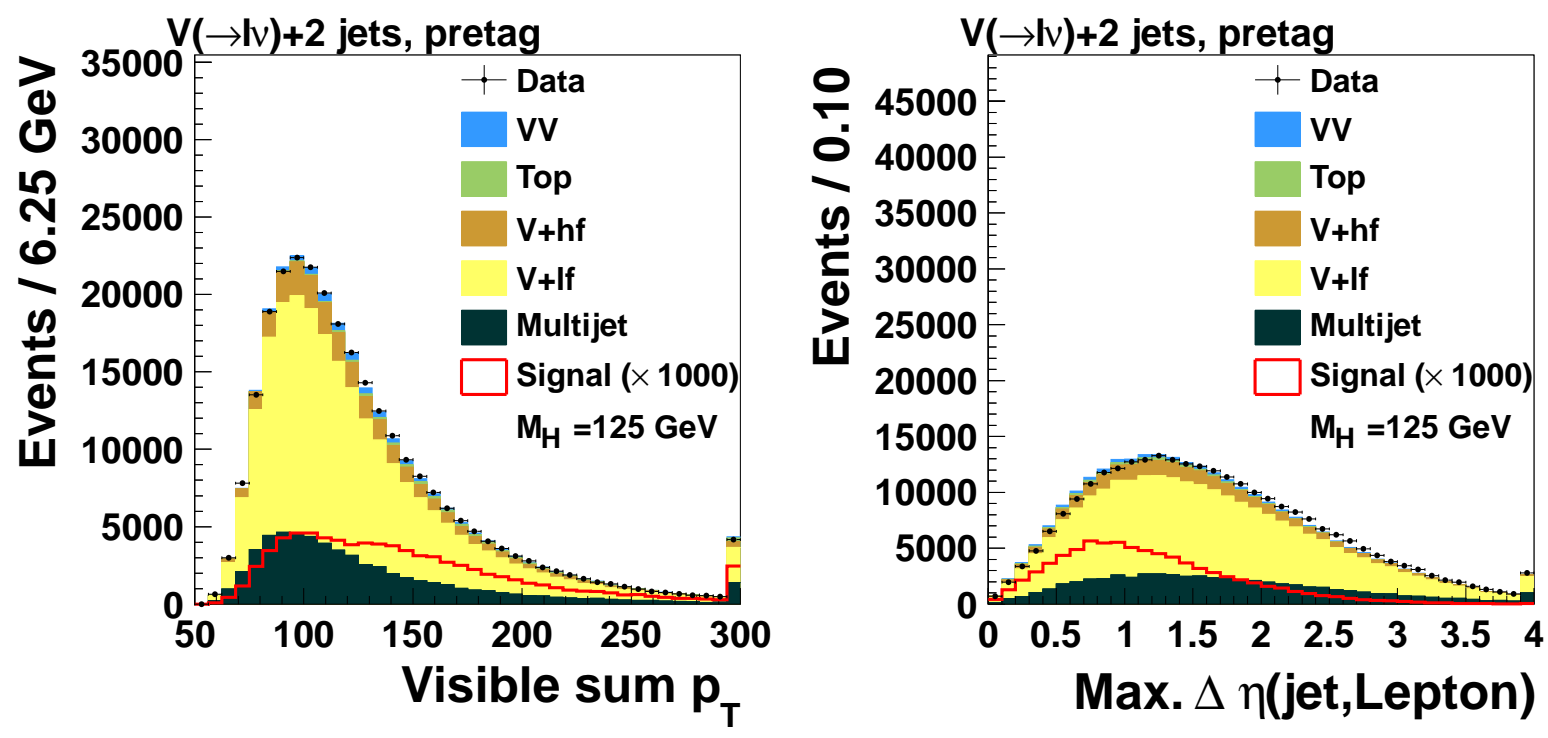

Figure A.3: Input variables to the multijet BDT shown for all events containing two jets: (left) the magnitude of the vector sum of $p_{T}$ for all visible particles, and (right) the maximum $\Delta \eta(\ell, j)$. The signal is shown for $M_{H}=125 \mathrm{GeV}$ multiplied by a factor of 1000 . Overflow events are added to the last bin. 

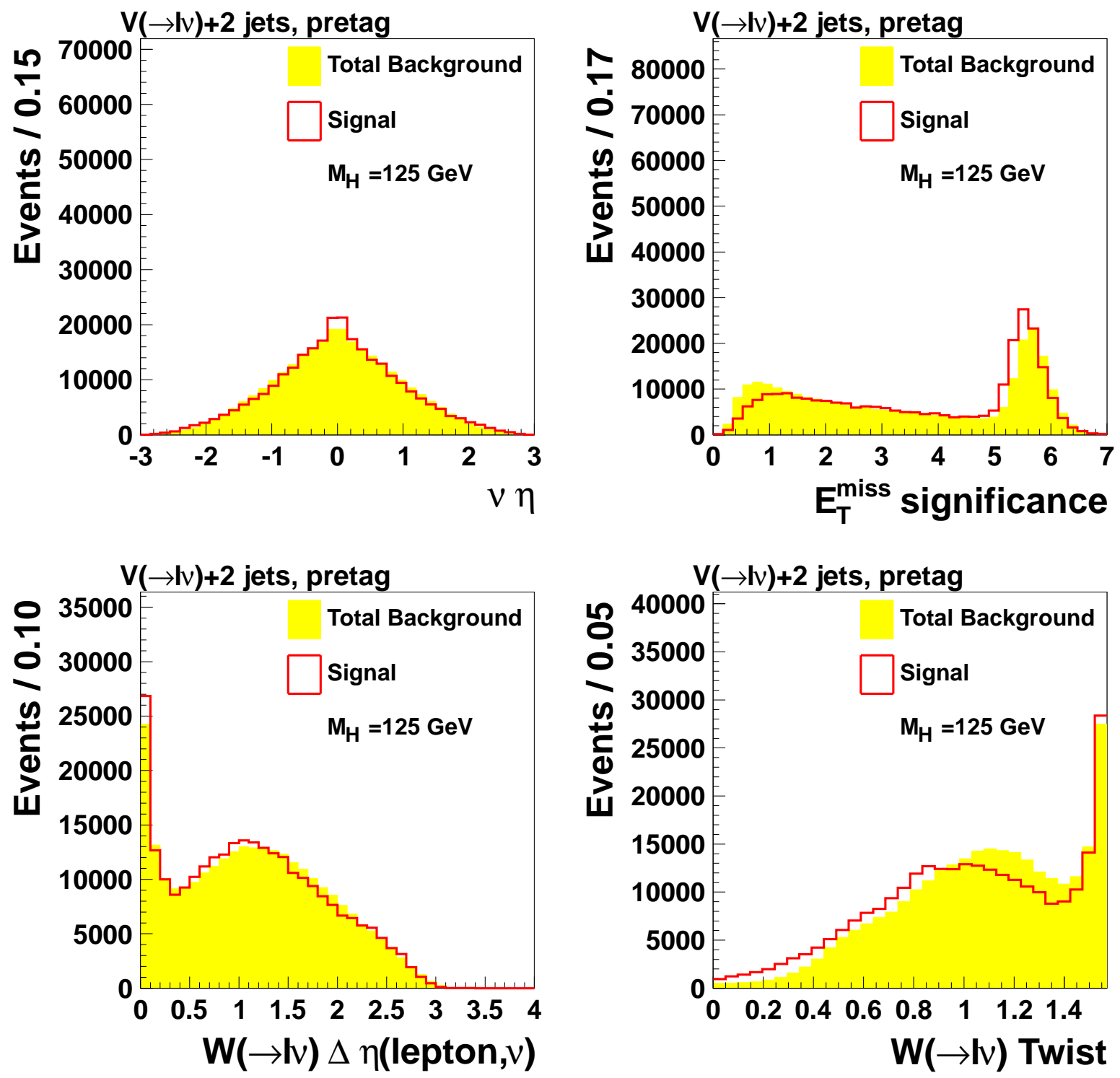

Figure A.4: Input variables to the multijet BDT shown for all events containing two jets: (top left) $\eta$ of the $E_{T}$, (top right) the $E_{T}$ significance, (bottom left) $\Delta \eta(\ell, \nu)$, and (bottom right) the twist of the $\ell-\nu$ system. The signal is shown for $M_{H}=125 \mathrm{GeV}$. The signal is normalized to the sum of the backgrounds. Overflow events are added to the last bin. 

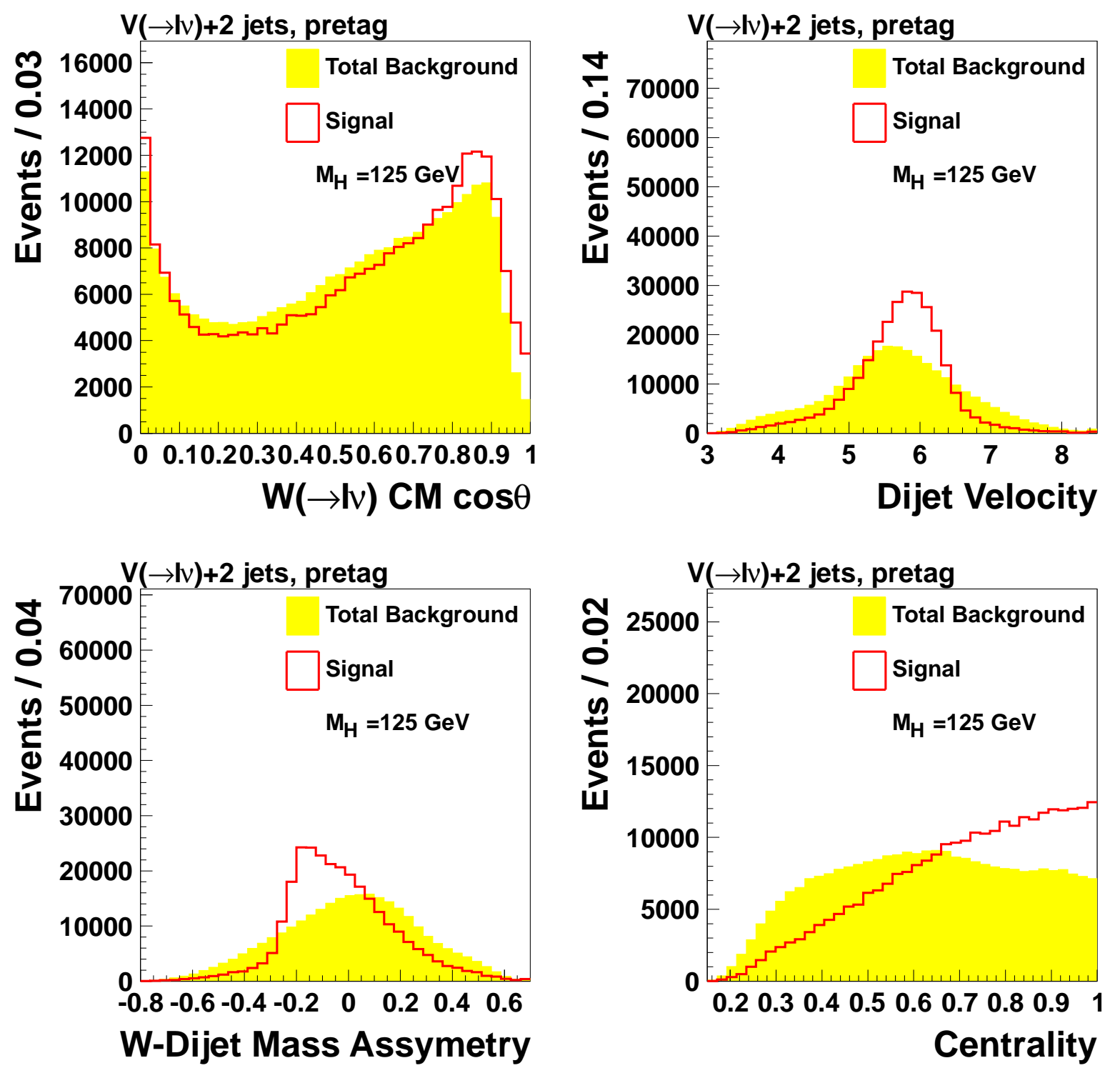

Figure A.5: Input variables to the multijet BDT shown for all events containing two jets: (top left) $\cos (\theta)$ in the $\ell \nu$ center-of-momentum frame, (top right) the velocity of the dijet system, (bottom left) the mass asymmetry between the dijet and the $\ell \nu$ system, and (bottom right) the centrality of the $\ell \nu j j$ system. The signal is shown for $M_{H}=125 \mathrm{GeV}$. The signal is normalized to the sum of the backgrounds. Overflow events are added to the last bin. 

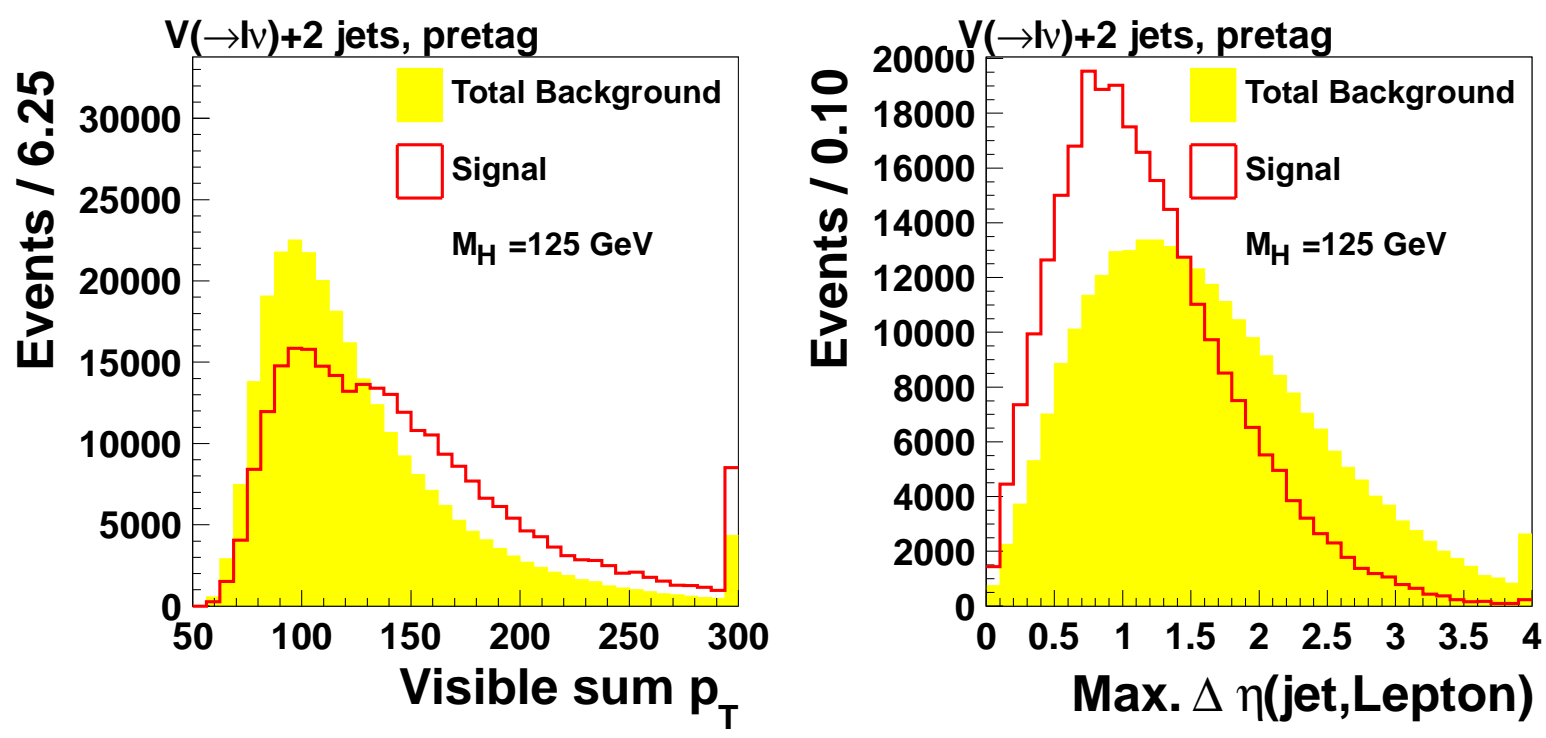

Figure A.6: Input variables to the multijet BDT shown for all events containing two jets: (left) the magnitude of the vector sum of $p_{T}$ for all visible particles, and (right) the maximum $\Delta \eta(\ell, j)$. The signal is shown for $M_{H}=125 \mathrm{GeV}$. The signal is normalized to the sum of the backgrounds. Overflow events are added to the last bin. 


\section{Appendix B Final BDT Input and Output Distributions}

Shown here are the input variables to the BDT trained to distinguish signal from all backgrounds in Figs. B.1 to B.14, and the output BDT distributions that were not included in the text in Figs. B.15 to B.38. Additionally, the input variables and BDT output distributions are shown with the signal and sum of backgrounds normalized to the same area in Figs. B.39 to B.84. The BDT input variables are described in Table 6.9 in the main text. 

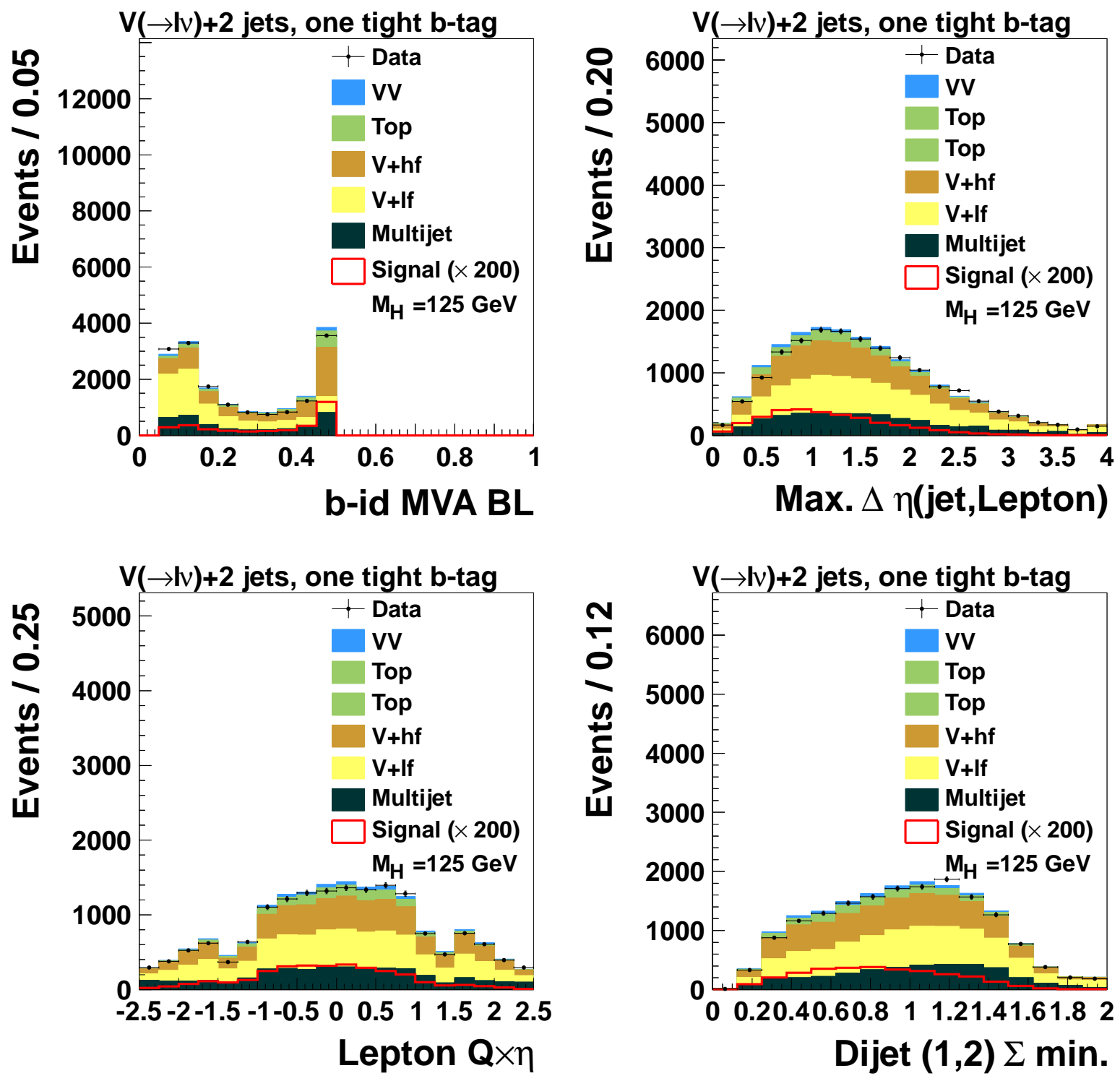

Figure B.1: Input variables to the BDT trained to distinguish signal from all backgrounds in events with two jets and one tight b-tag: (top left) the bid MVA output, (top right) the maximum $\Delta \eta(\ell, j)$, (bottom left) $q^{\ell} \times \eta^{\ell}$, and (bottom right) the minimum significance of the dijet system. The signal is shown for $M_{H}=125 \mathrm{GeV}$, and is multiplied by a factor of 200. Overflow events are added to the last bin. 

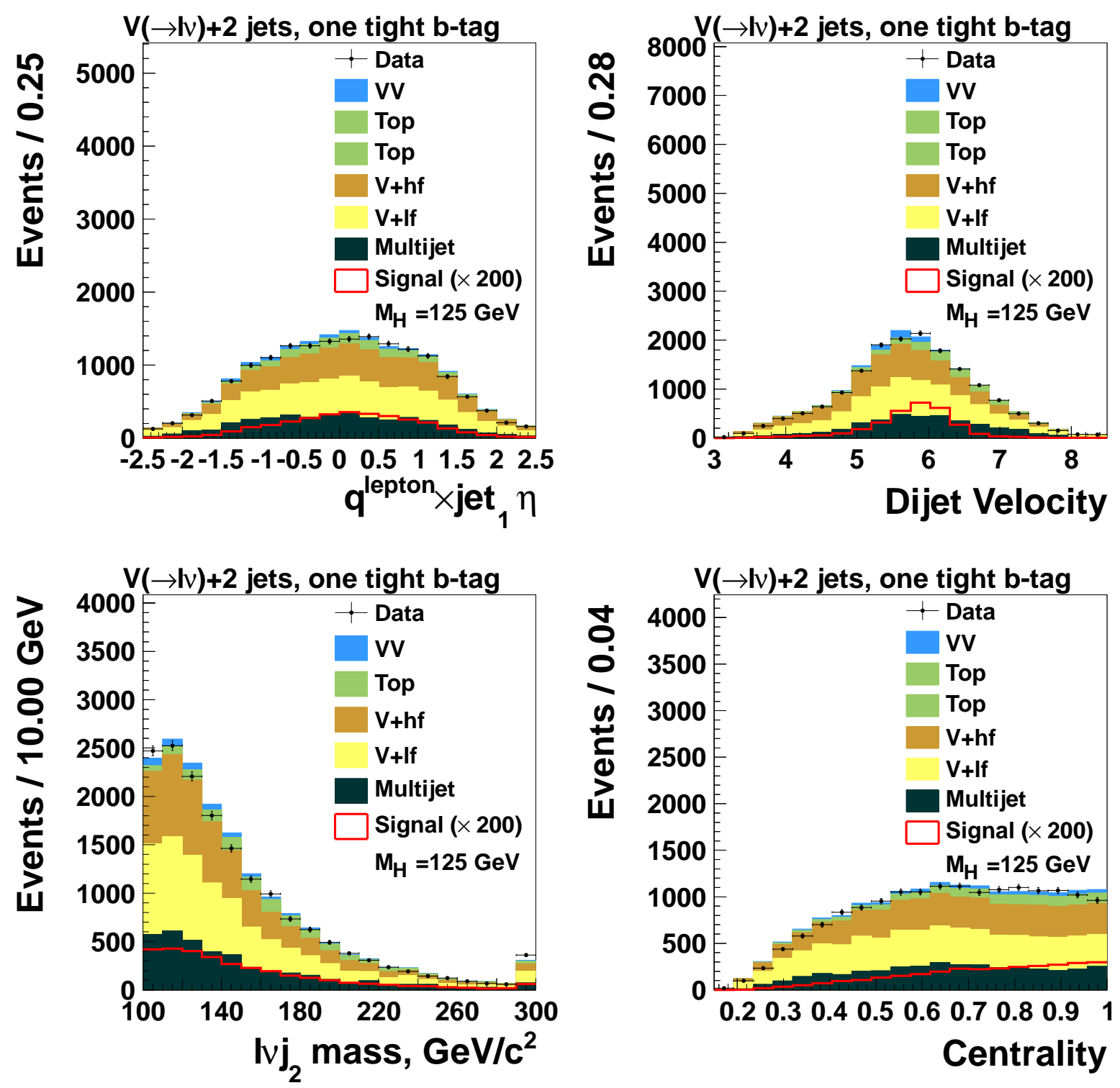

Figure B.2: Input variables to the BDT trained to distinguish signal from all backgrounds in events with two jets and one tight b-tag. The signal is shown for $M_{H}=125 \mathrm{GeV}$, and is multiplied by a factor of 200. Overflow events are added to the last bin. 

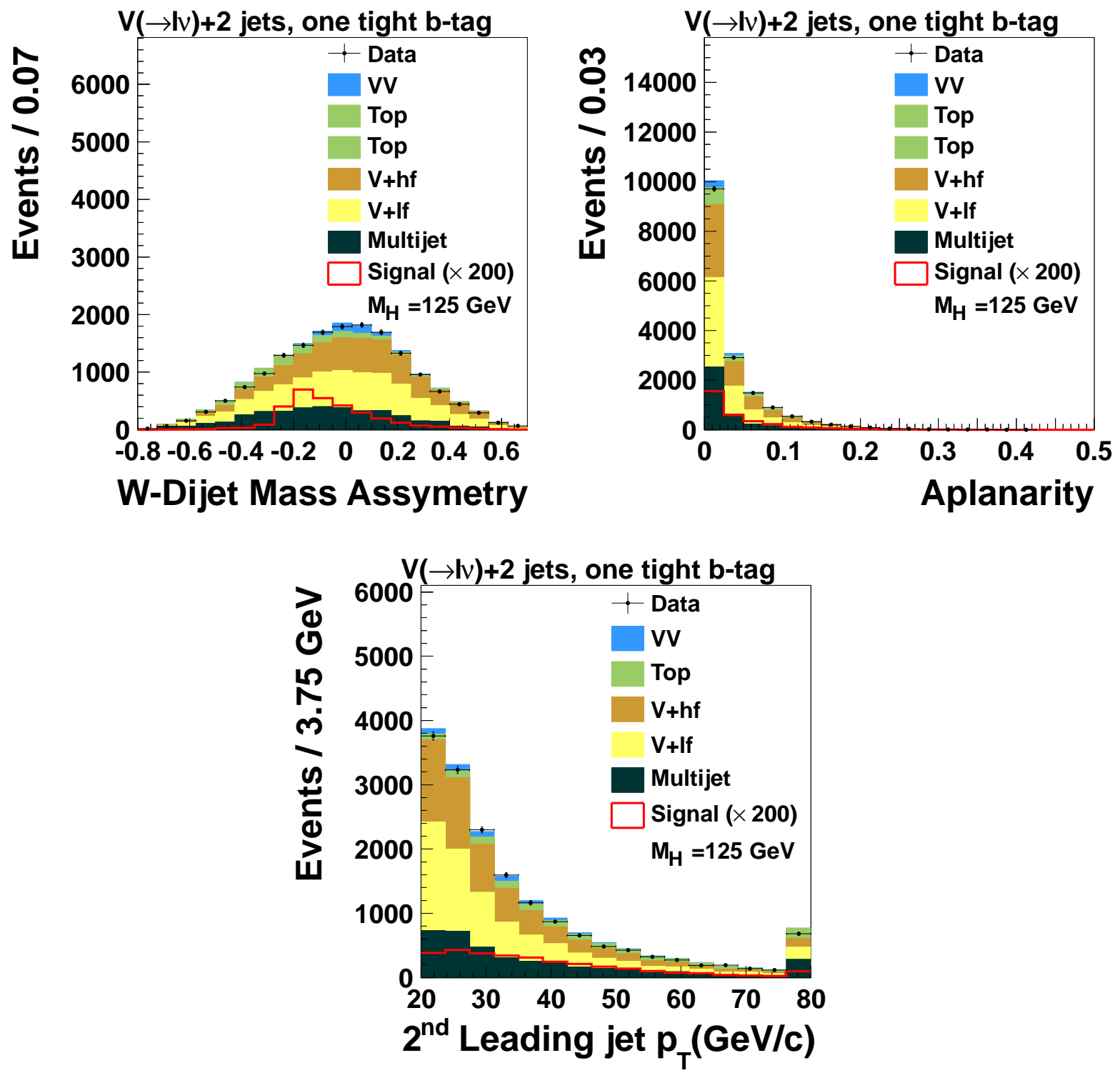

Figure B.3: Input variables to the BDT trained to distinguish signal from all backgrounds in events with two jets and one tight b-tag: (top left) the mass asymmetry between the $\ell \nu$ and dijet systems, (top right) aplanarity, and (bottom) the second leading jet $p_{T}$. The signal is shown for $M_{H}=125 \mathrm{GeV}$, and is multiplied by a factor of 200 . Overflow events are added to the last bin. 

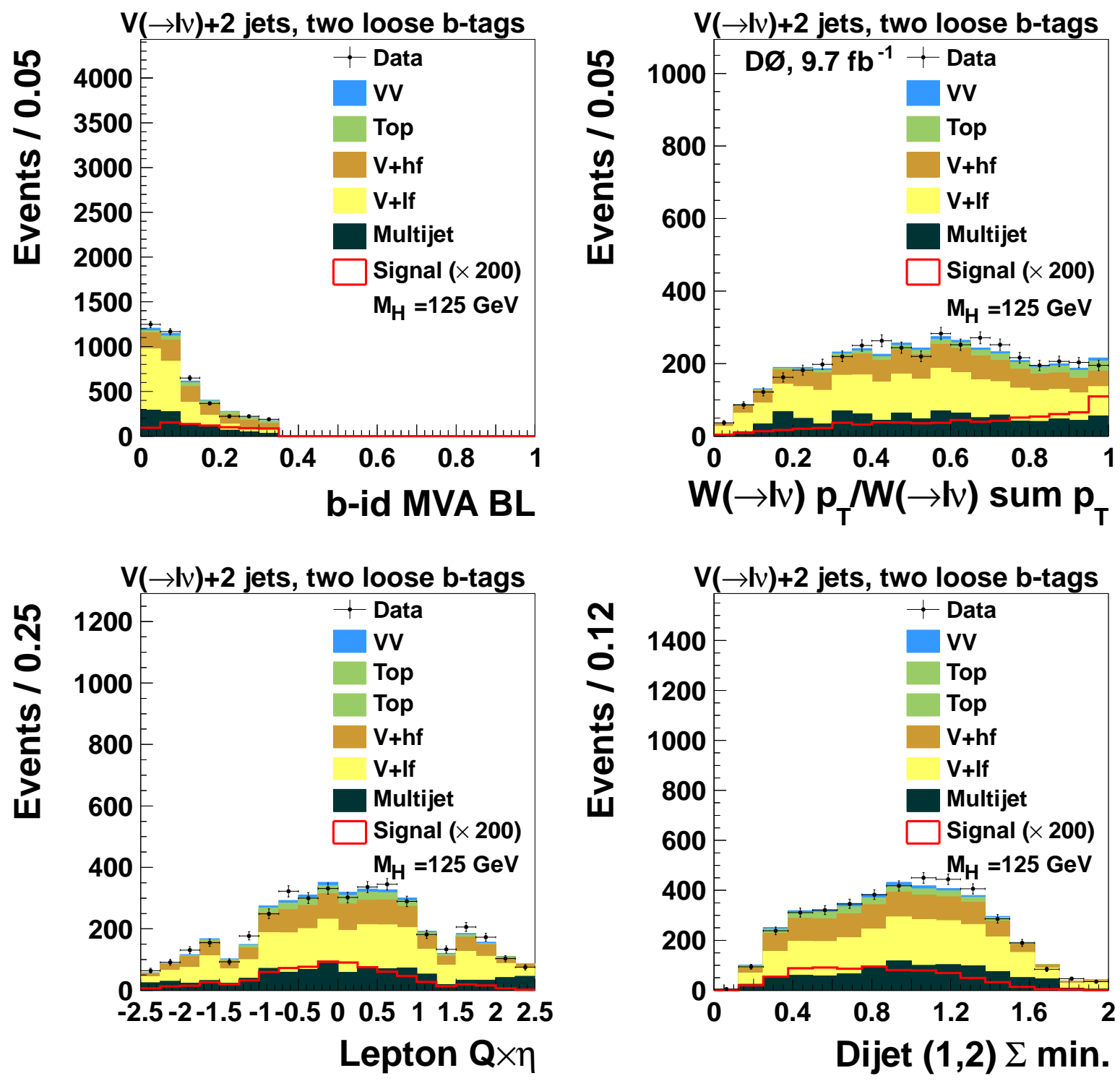

Figure B.4: Input variables to the BDT trained to distinguish signal from all backgrounds in events with two jets and two loose b-tag: (top left) the bid MVA output, (top right) the maximum $\Delta \eta(\ell, j)$, (bottom left) $q^{\ell} \times \eta^{\ell}$, and (bottom right) the minimum significance of the dijet system. The signal is shown for $M_{H}=125 \mathrm{GeV}$, and is multiplied by a factor of 200. Overflow events are added to the last bin. 

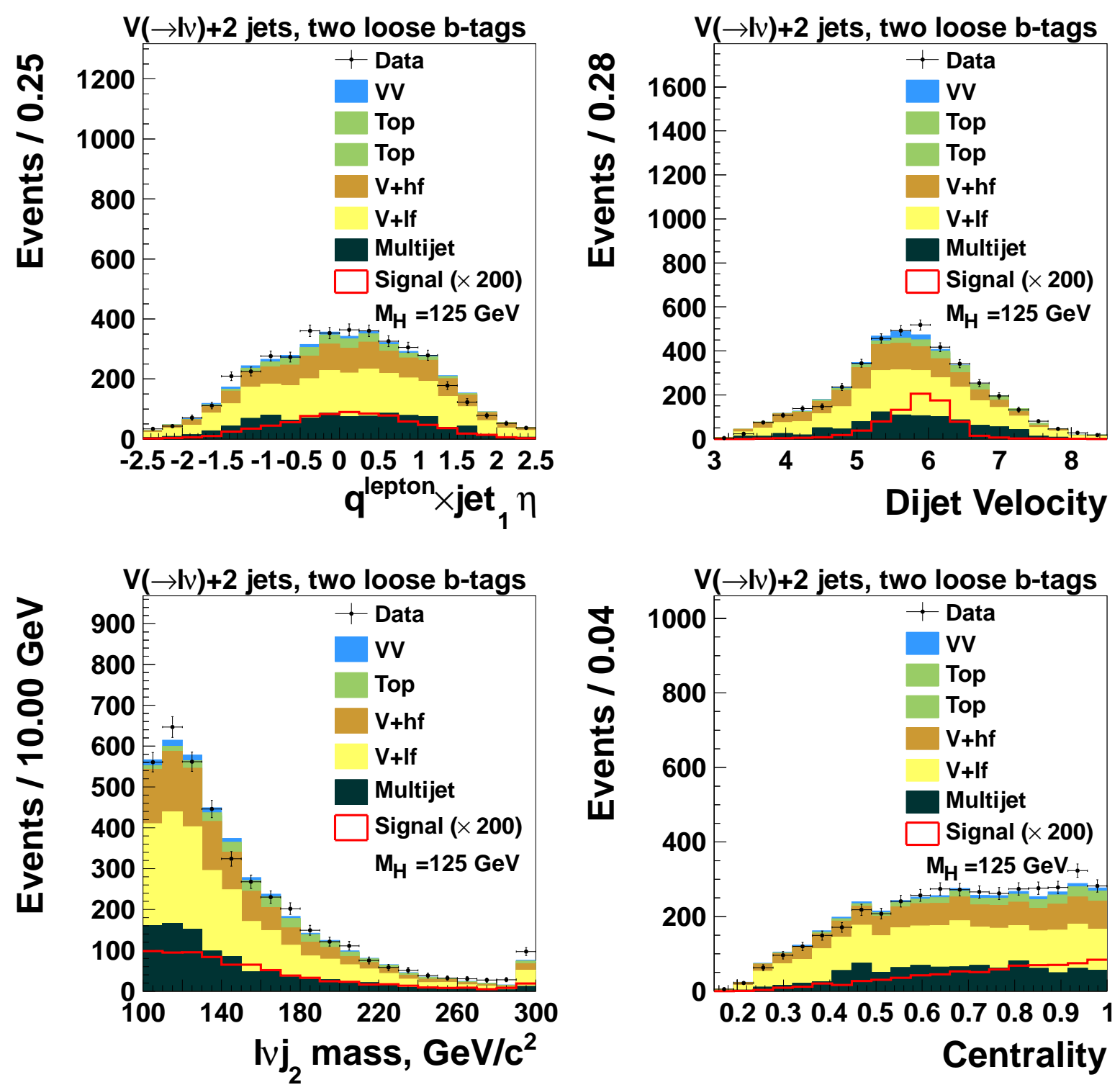

Figure B.5: Input variables to the BDT trained to distinguish signal from all backgrounds in events with two jets and two loose b-tag. The signal is shown for $M_{H}=125 \mathrm{GeV}$, and is multiplied by a factor of 200. Overflow events are added to the last bin. 

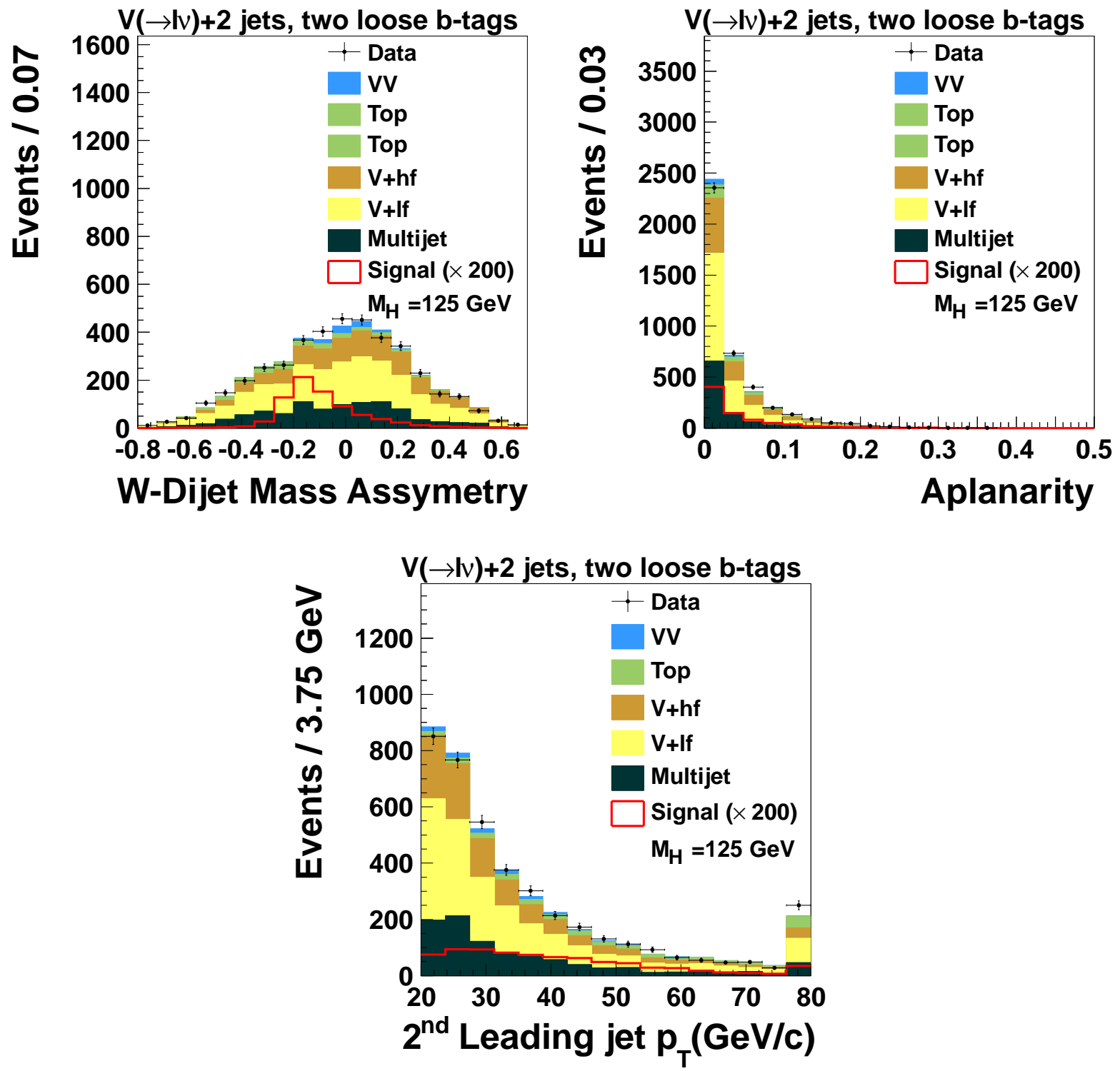

Figure B.6: Input variables to the BDT trained to distinguish signal from all backgrounds in events with two jets and two loose b-tag: (top left) the mass asymmetry between the $\ell \nu$ and dijet systems, (top right) aplanarity, and (bottom) the second leading jet $p_{T}$. The signal is shown for $M_{H}=125 \mathrm{GeV}$, and is multiplied by a factor of 200 . Overflow events are added to the last bin. 

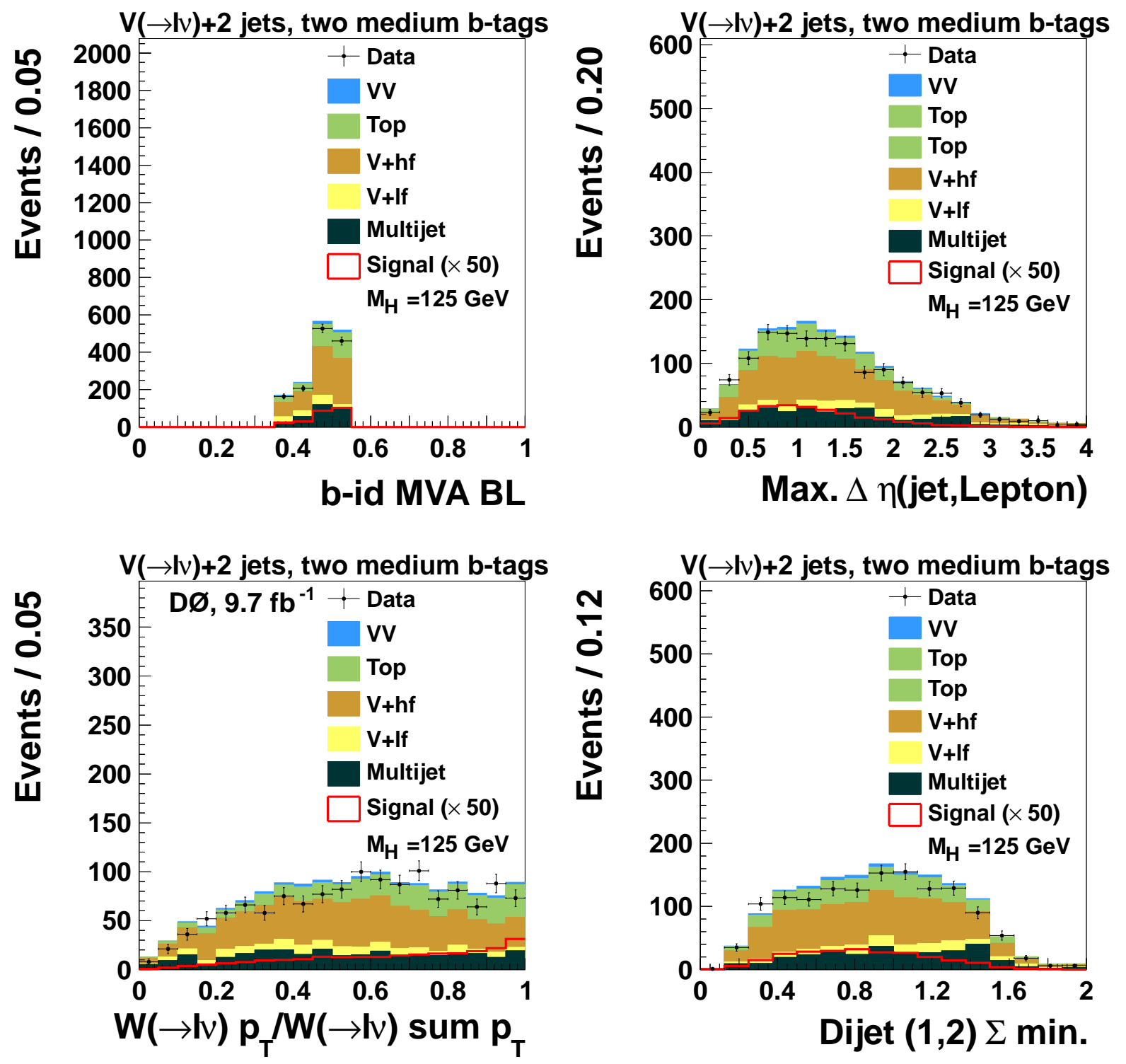

Figure B.7: Input variables to the BDT trained to distinguish signal from all backgrounds in events with two jets and two medium b-tag: (top left) the bid MVA output, (top right) the maximum $\Delta \eta(\ell, j)$, (bottom left) $q^{\ell} \times \eta^{\ell}$, and (bottom right) the minimum significance of the dijet system. The signal is shown for $M_{H}=125 \mathrm{GeV}$, and is multiplied by a factor of 50. Overflow events are added to the last bin. 

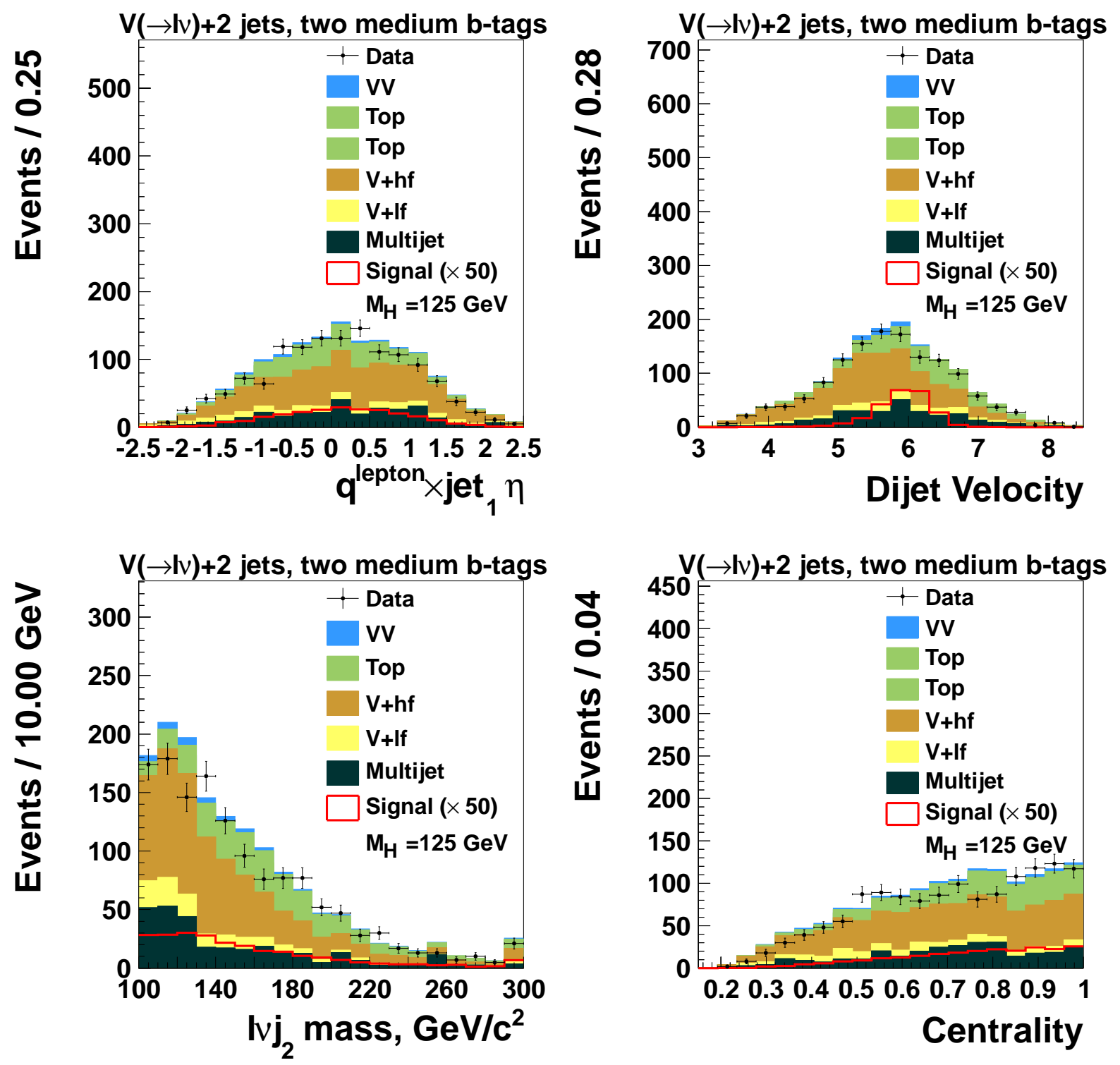

Figure B.8: Input variables to the BDT trained to distinguish signal from all backgrounds in events with two jets and two medium b-tag. The signal is shown for $M_{H}=125 \mathrm{GeV}$, and is multiplied by a factor of 50. Overflow events are added to the last bin. 

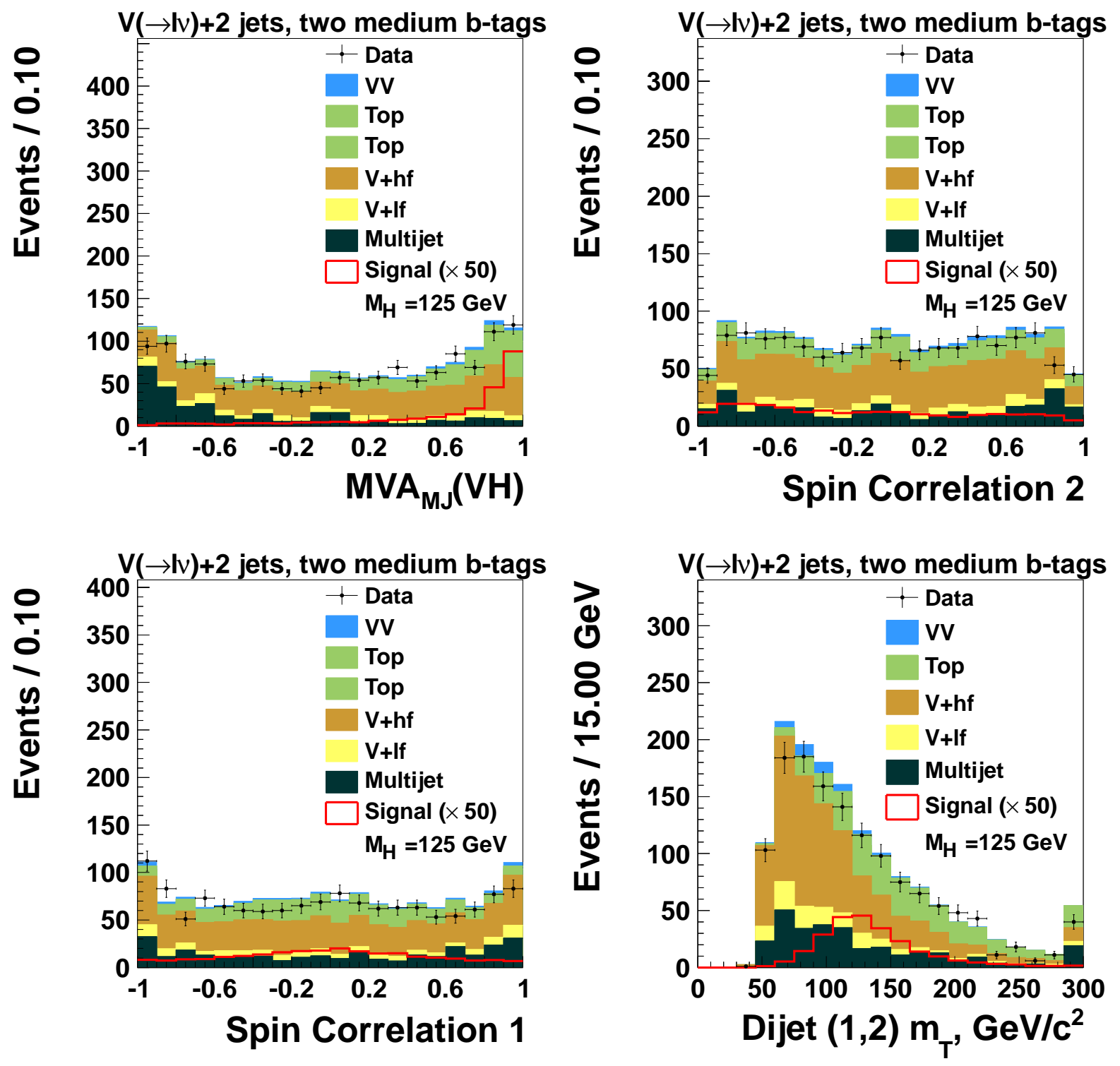

Figure B.9: Input variables to the BDT trained to distinguish signal from all backgrounds in events with two jets and two medium b-tag: (top left) the multijet MVA output, (top right) $\cos \left(\chi^{*}\right)$, (bottom left) $\cos \left(\theta^{*}\right.$, and (bottom right) the dijet transverse mass. The signal is shown for $M_{H}=125 \mathrm{GeV}$, and is multiplied by a factor of 50 . Overflow events are added to the last bin. 


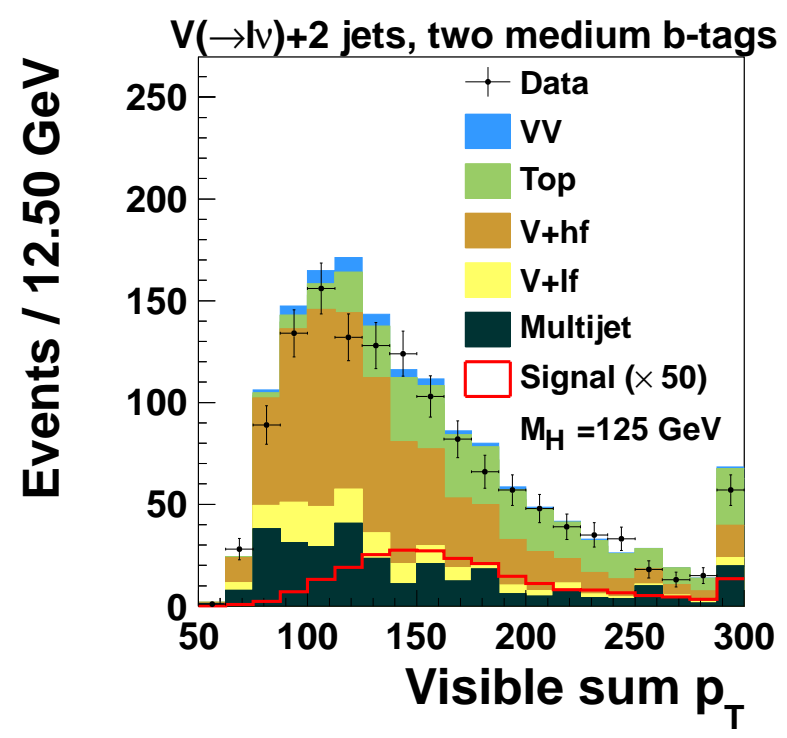

Figure B.10: Input variables to the BDT trained to distinguish signal from all backgrounds in events with two jets and two medium b-tags: the magnitude of the vector $p_{T}$ sum for all visible particles. The signal is shown for $M_{H}=125 \mathrm{GeV}$, and is multiplied by a factor of 50. Overflow events are added to the last bin. 

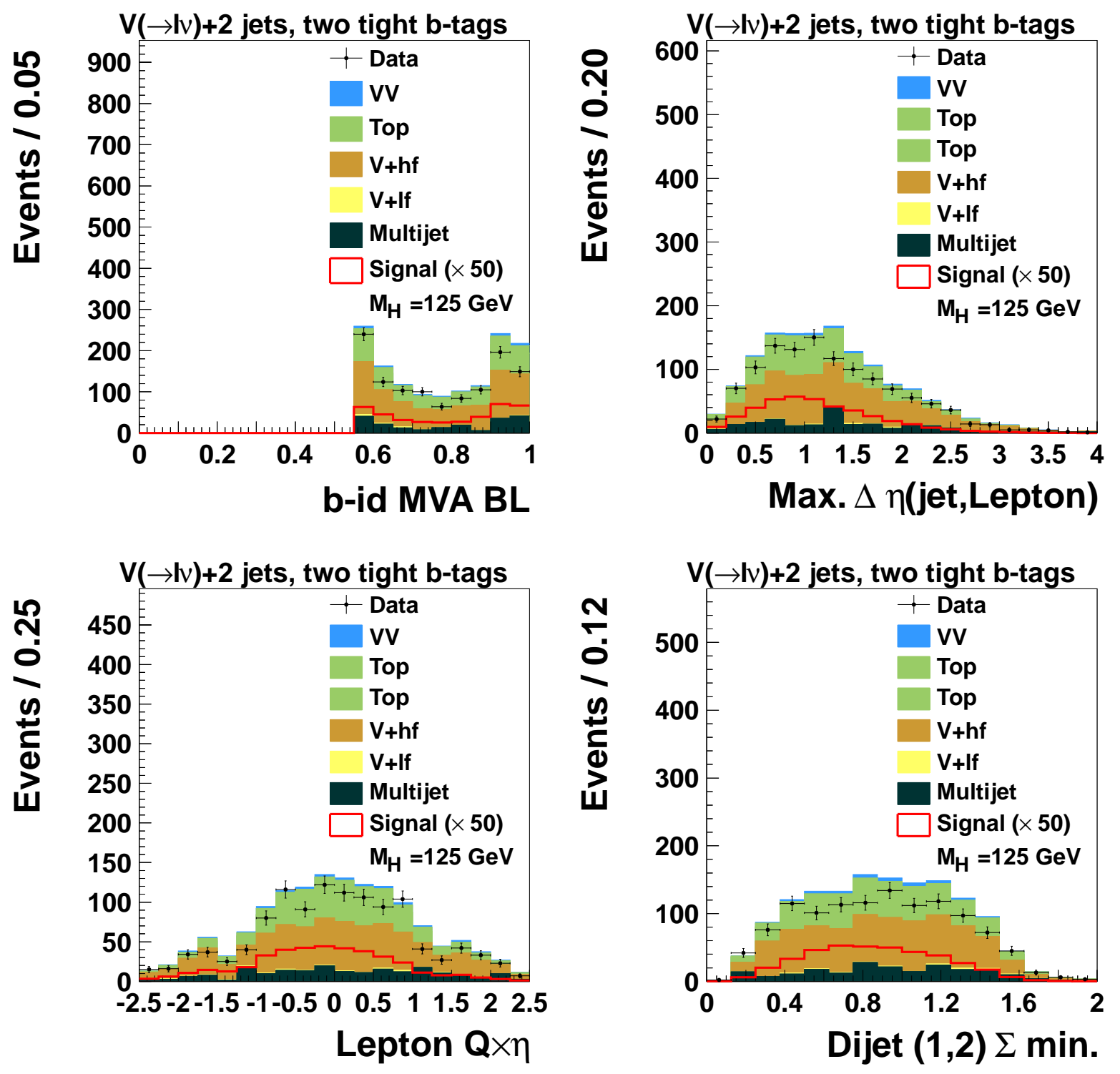

Figure B.11: Input variables to the BDT trained to distinguish signal from all backgrounds in events with two jets and two tight b-tag: (top left) the bid MVA output, (top right) the maximum $\Delta \eta(\ell, j)$, (bottom left) $q^{\ell} \times \eta^{\ell}$, and (bottom right) the minimum significance of the dijet system. The signal is shown for $M_{H}=125 \mathrm{GeV}$, and is multiplied by a factor of 50. Overflow events are added to the last bin. 

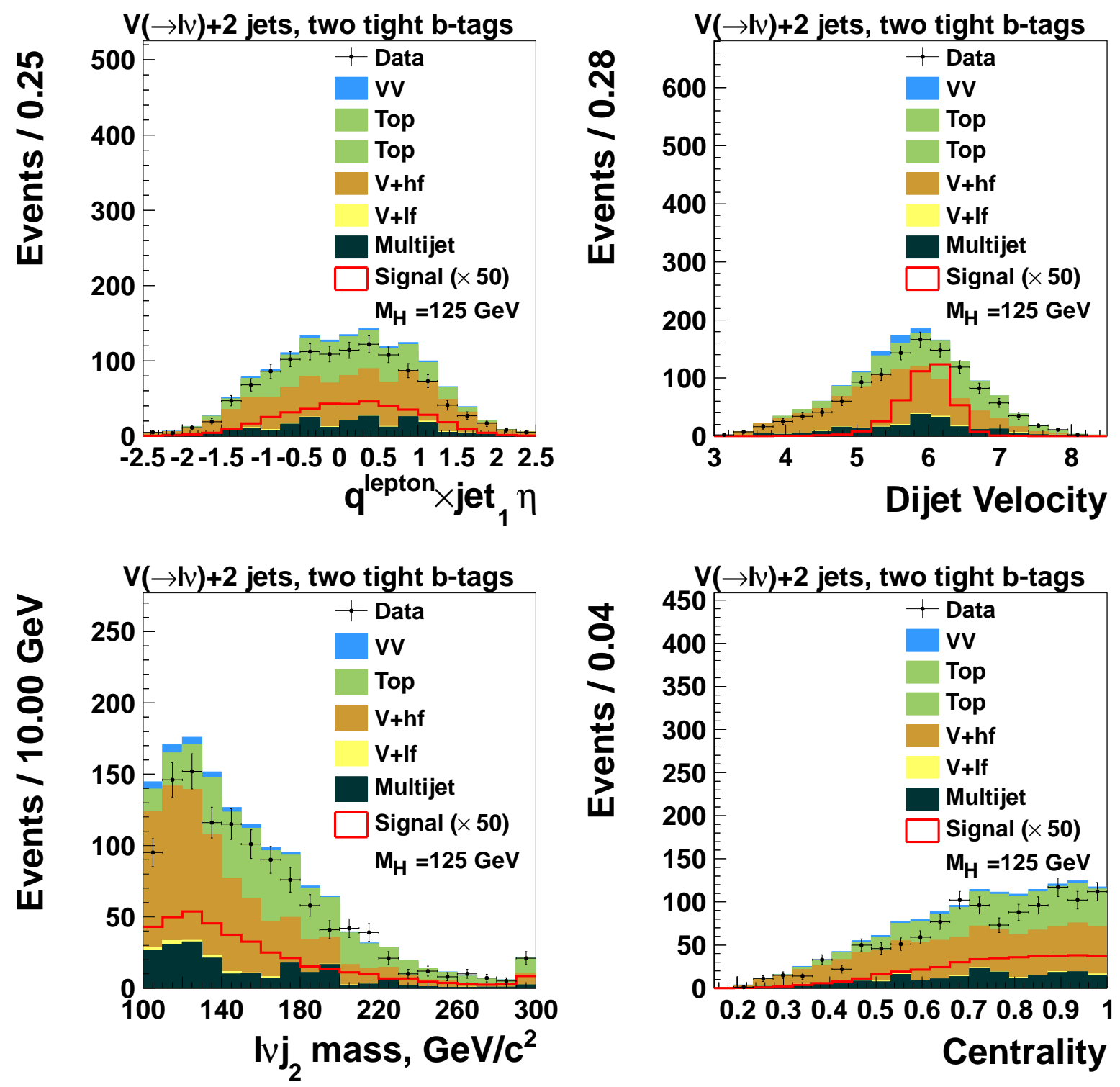

Figure B.12: Input variables to the BDT trained to distinguish signal from all backgrounds in events with two jets and two tight b-tag. The signal is shown for $M_{H}=125 \mathrm{GeV}$, and is multiplied by a factor of 50. Overflow events are added to the last bin. 

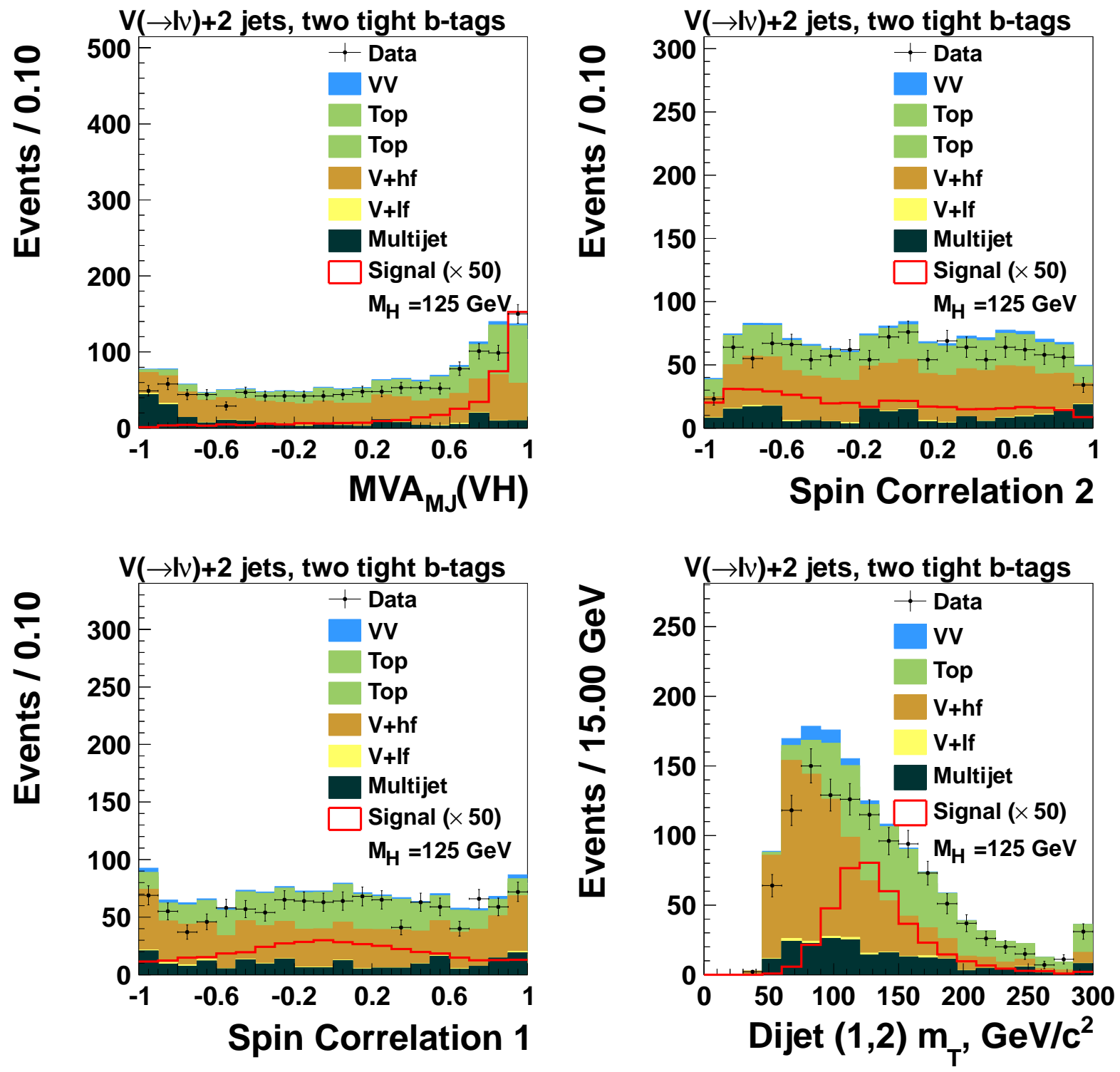

Figure B.13: Input variables to the BDT trained to distinguish signal from all backgrounds in events with two jets and two tight b-tag: (top left) the multijet MVA output, (top right) $\cos \left(\chi^{*}\right)$, (bottom left) $\cos \left(\theta^{*}\right.$, and (bottom right) the dijet transverse mass. The signal is shown for $M_{H}=125 \mathrm{GeV}$, and is multiplied by a factor of 50 . Overflow events are added to the last bin. 


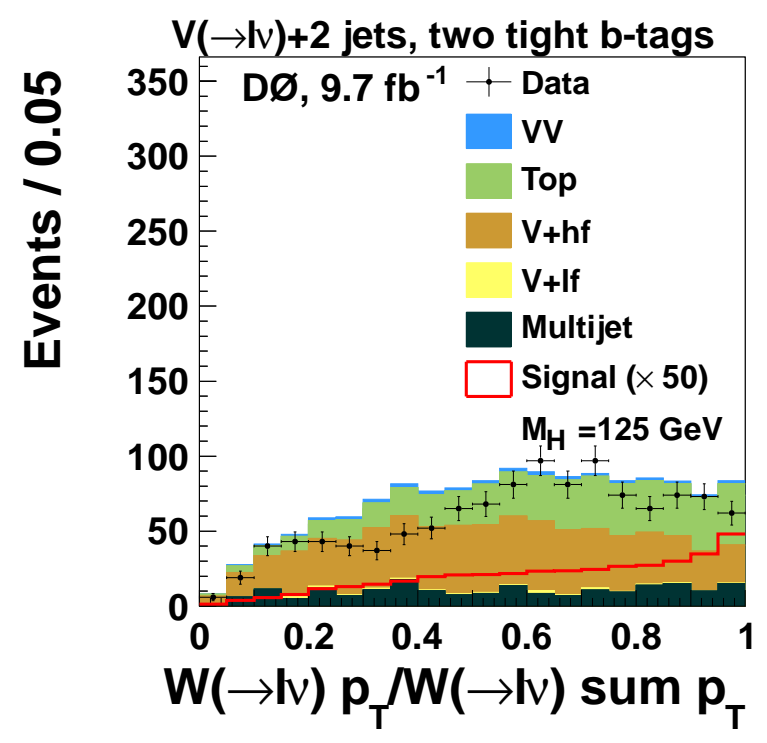

Figure B.14: Input variables to the BDT trained to distinguish signal from all backgrounds in events with two jets and two tight b-tag: the ratio of the $W p_{T}$ to the sum of the lepton $p_{T}$ and $E_{T}$. The signal is shown for $M_{H}=125 \mathrm{GeV}$, and is multiplied by a factor of 50 . Overflow events are added to the last bin. 

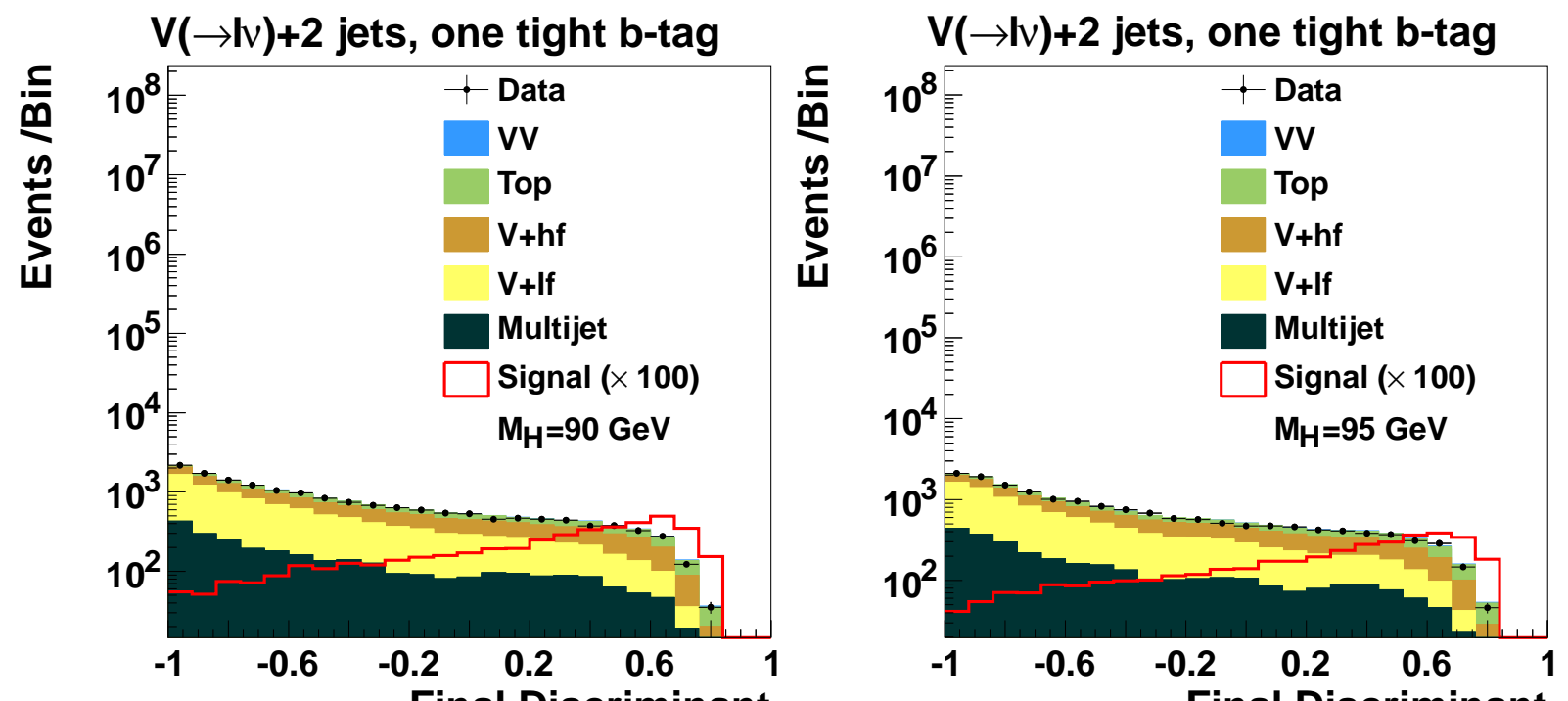

Final Discriminant

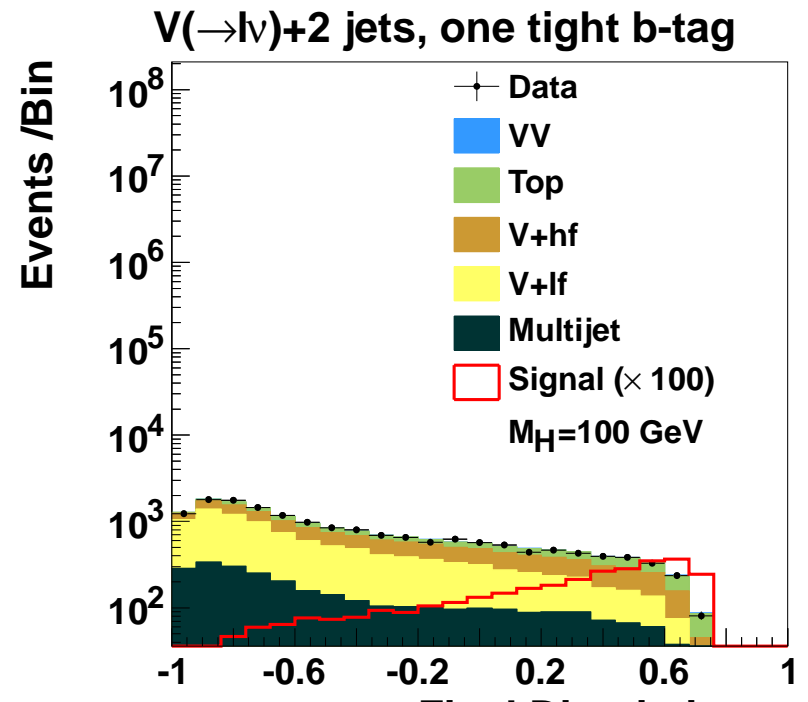

Final Discriminant

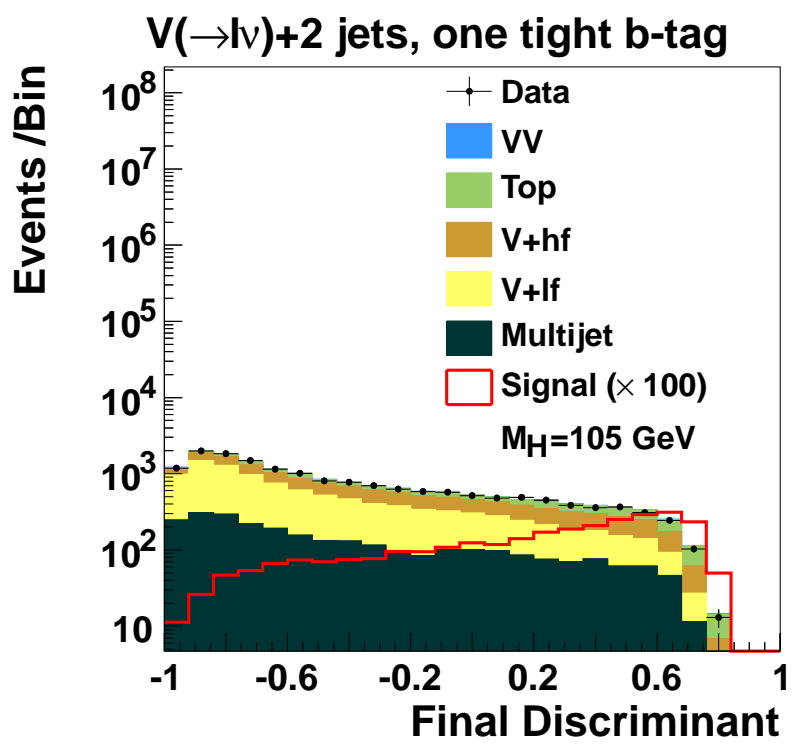

Figure B.15: The final MVA output for events with two jets and one tight b-tag for (top left) $M_{H}=90 \mathrm{GeV}$, (top right) $M_{H}=95 \mathrm{GeV}$, (bottom left) $M_{H}=100 \mathrm{GeV}$, and (bottom right) $M_{H}=105 \mathrm{GeV}$. 

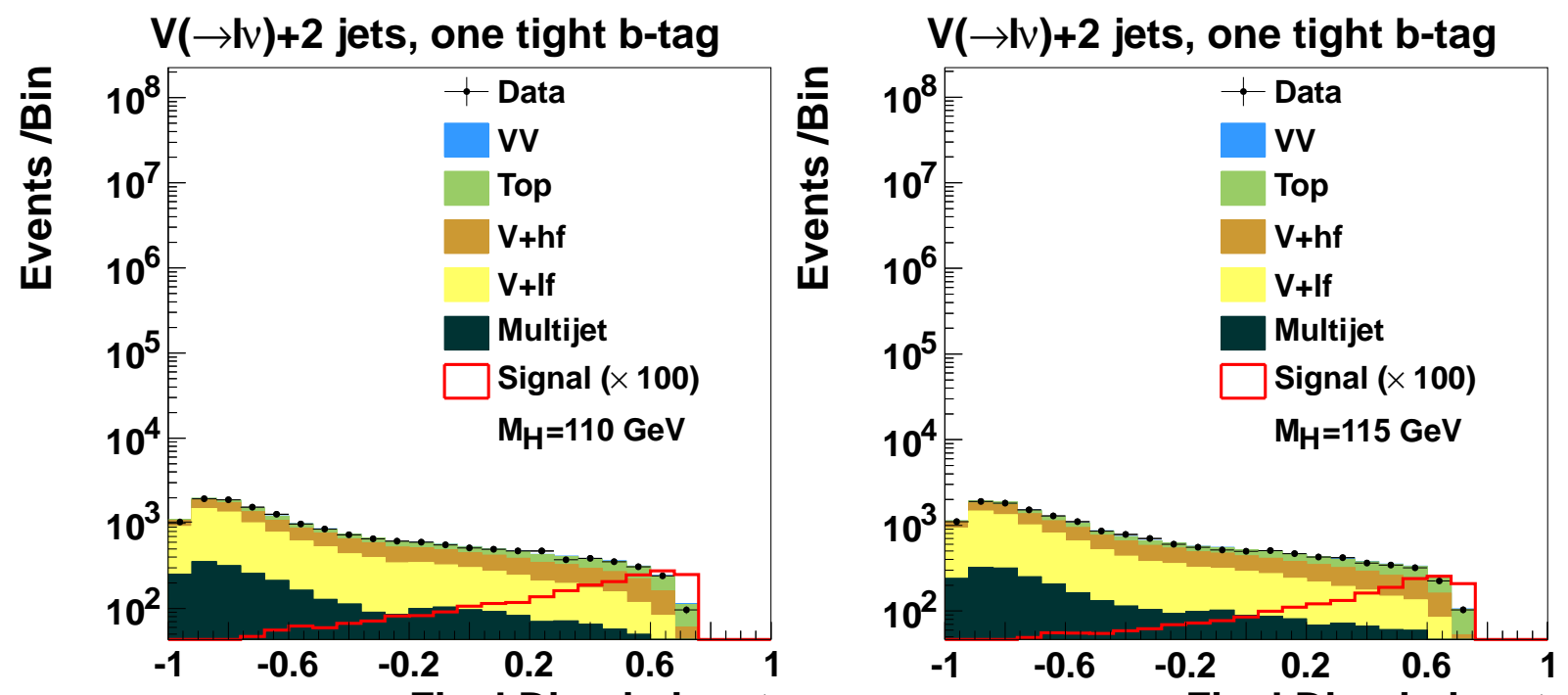

Final Discriminant

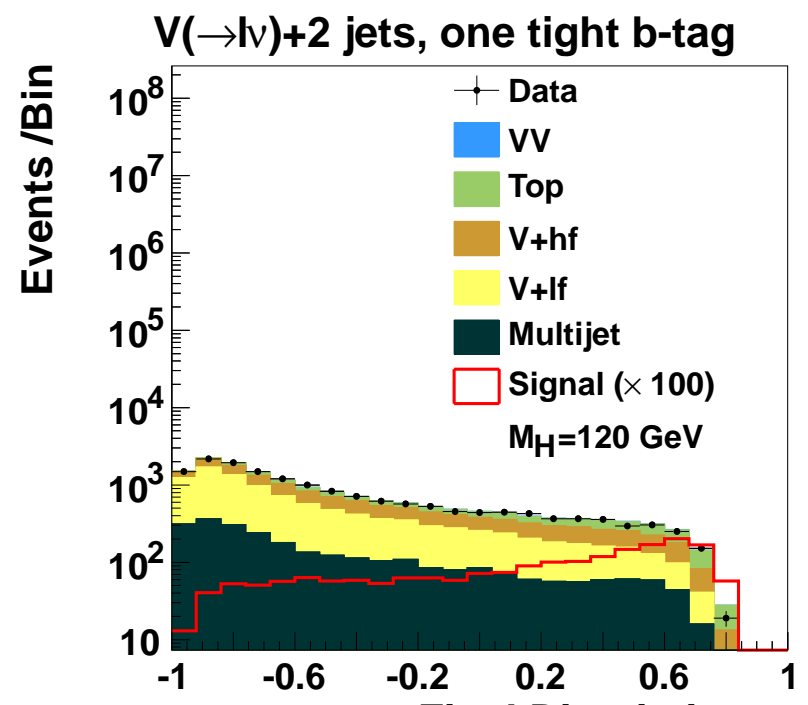

Final Discriminant

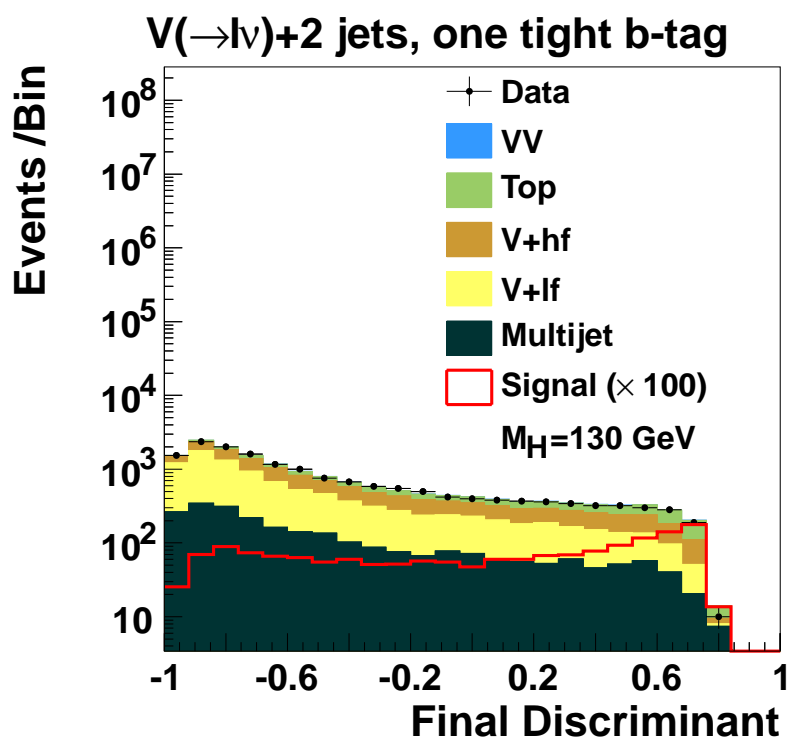

Figure B.16: The final MVA output for events with two jets and one tight b-tag for (top left) $M_{H}=110 \mathrm{GeV}$, (top right) $M_{H}=115 \mathrm{GeV}$, (bottom left) $M_{H}=120 \mathrm{GeV}$, and (bottom right) $M_{H}=130 \mathrm{GeV}$. 


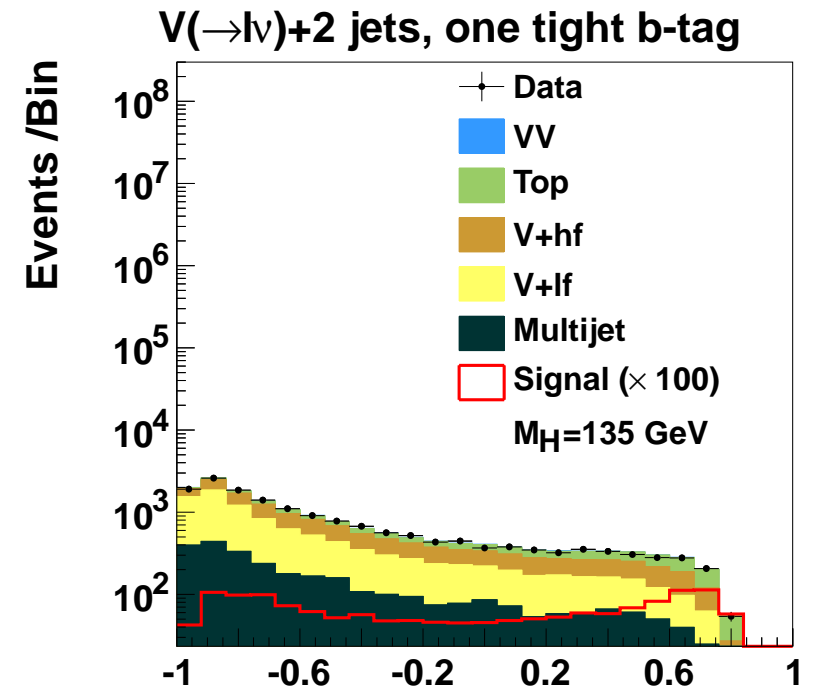

Final Discriminant

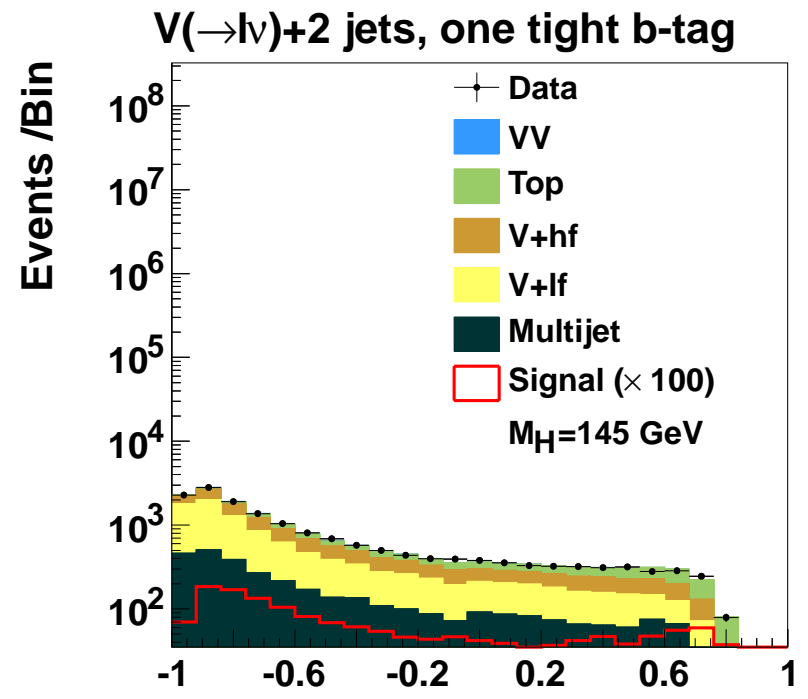

Final Discriminant

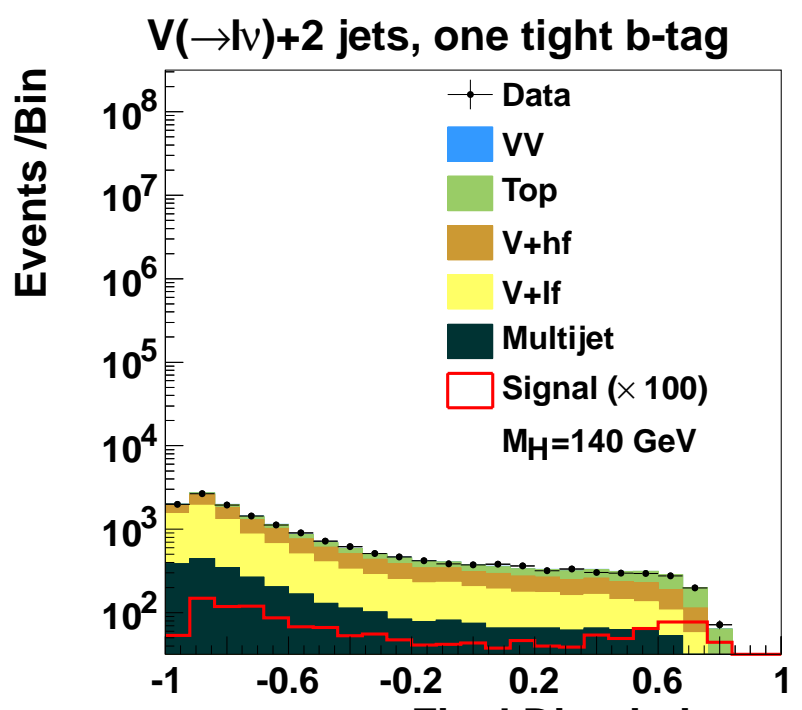

Final Discriminant

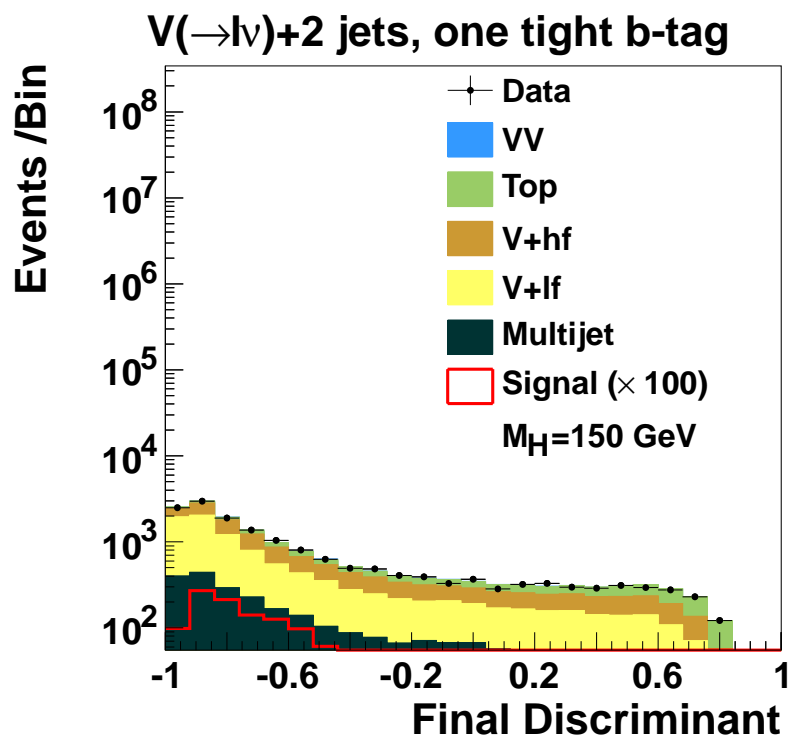

Figure B.17: The final MVA output for events with two jets and one tight b-tag for (top left) $M_{H}=135 \mathrm{GeV}$, (top right) $M_{H}=140 \mathrm{GeV}$, (bottom left) $M_{H}=145 \mathrm{GeV}$, and (bottom right) $M_{H}=150 \mathrm{GeV}$. 

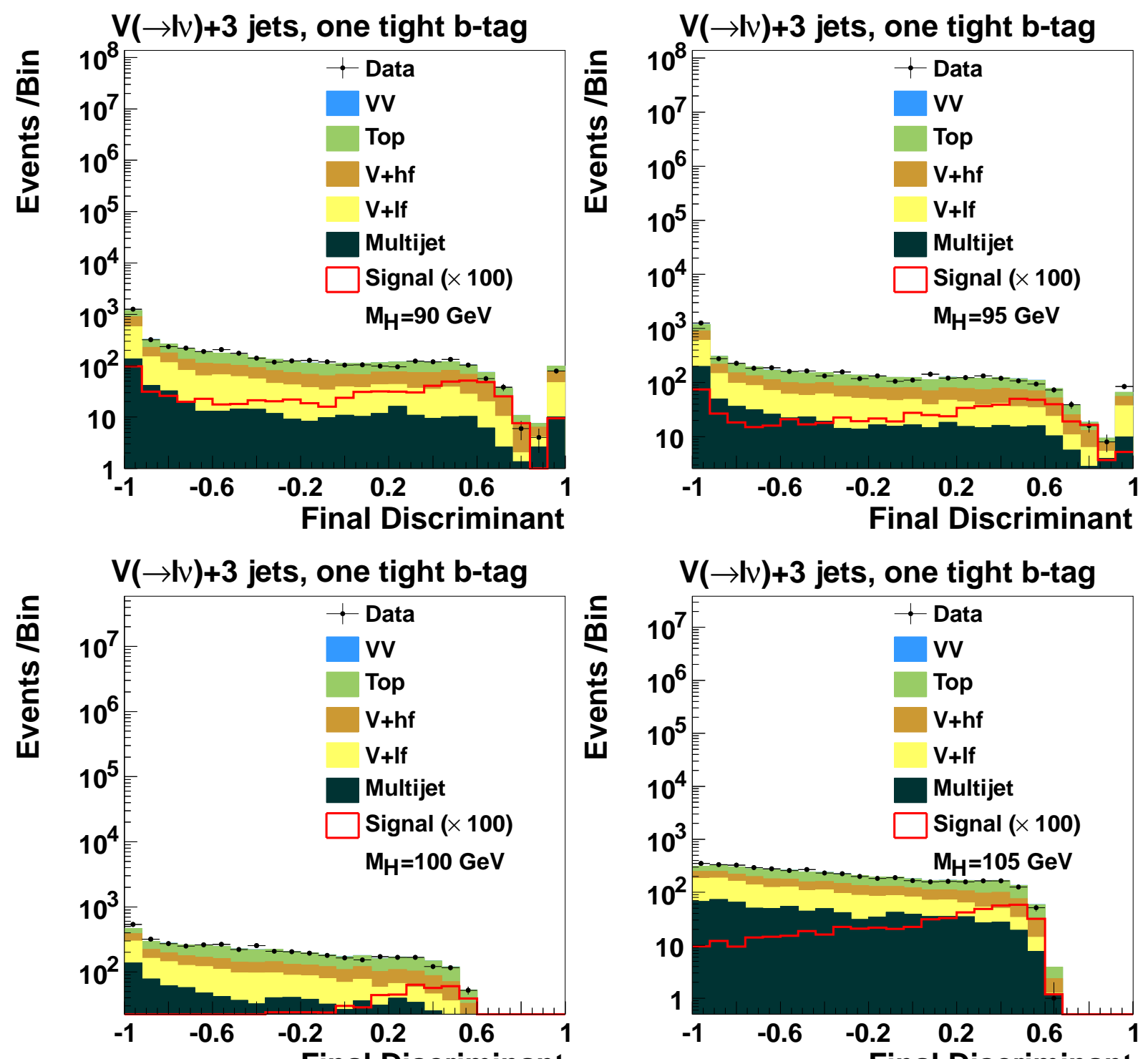

Final Discriminant

Final Discriminant

Figure B.18: The final MVA output for events with three jets and one tight b-tag for (top left) $M_{H}=90 \mathrm{GeV}$, (top right) $M_{H}=95 \mathrm{GeV}$, (bottom left) $M_{H}=100 \mathrm{GeV}$, and (bottom right) $M_{H}=105 \mathrm{GeV}$. 

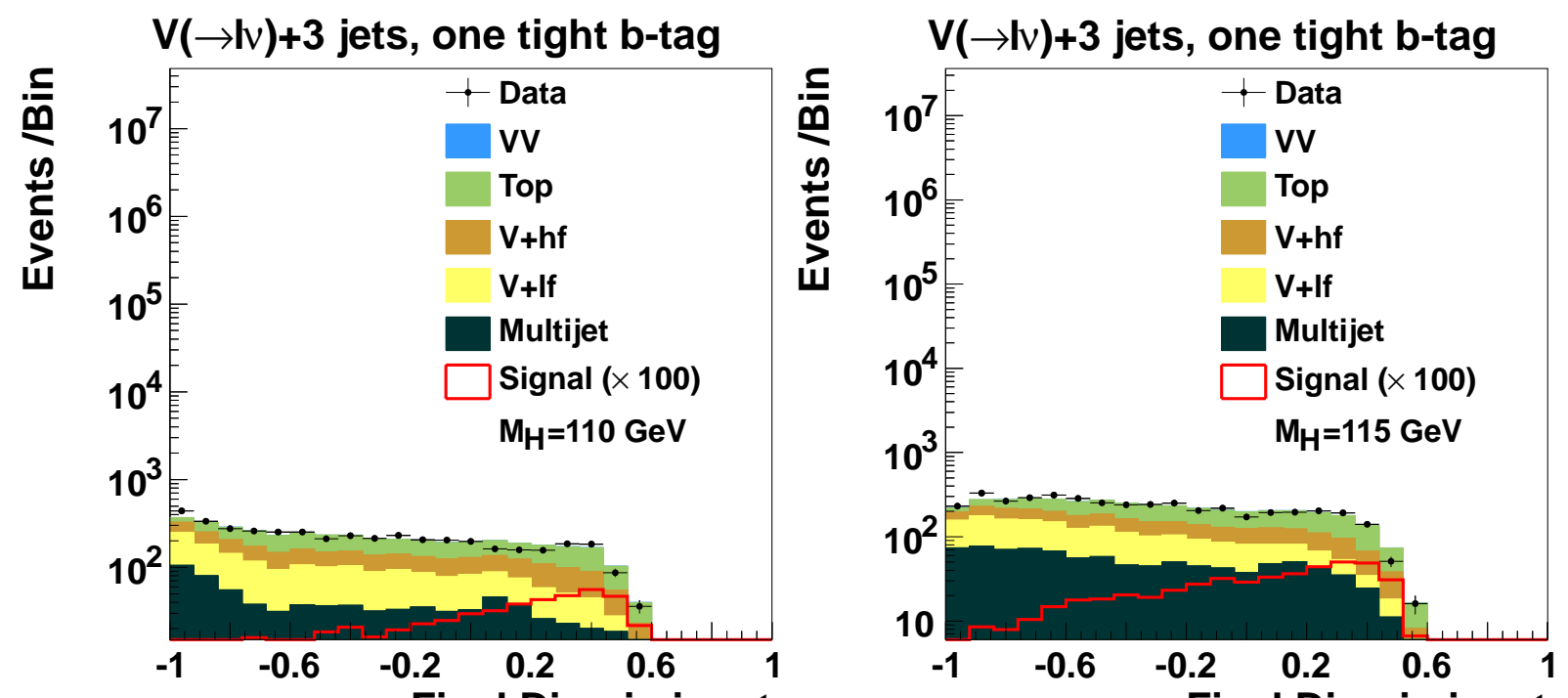

Final Discriminant

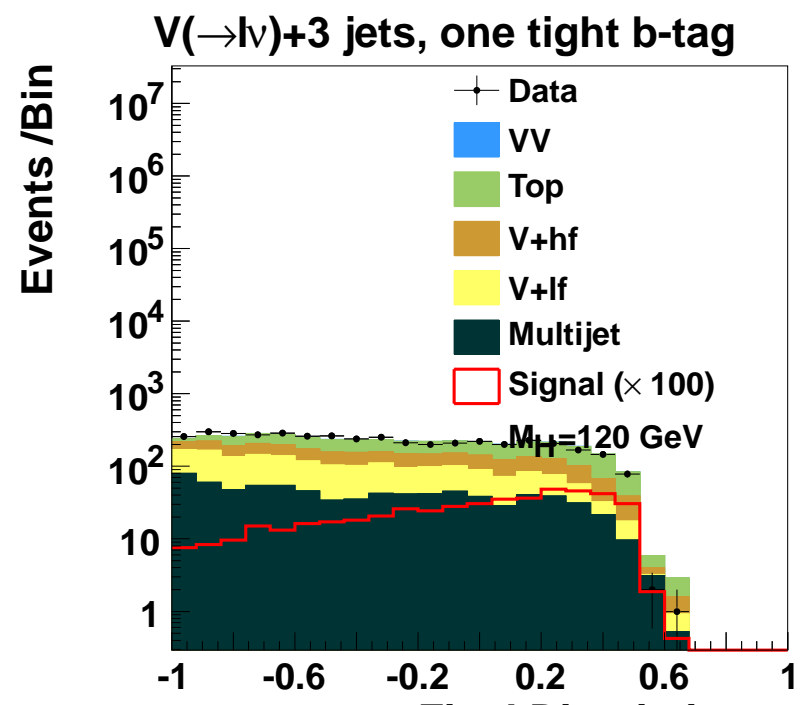

Final Discriminant

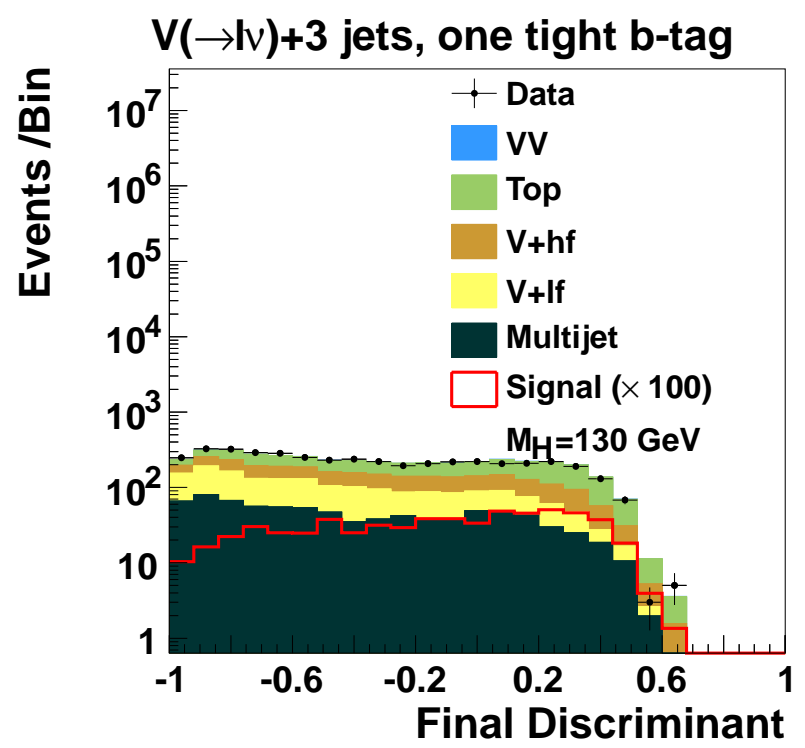

Figure B.19: The final MVA output for events with three jets and one tight b-tag for (top left) $M_{H}=110 \mathrm{GeV}$, (top right) $M_{H}=115 \mathrm{GeV}$, (bottom left) $M_{H}=120 \mathrm{GeV}$, and (bottom right) $M_{H}=130 \mathrm{GeV}$. 

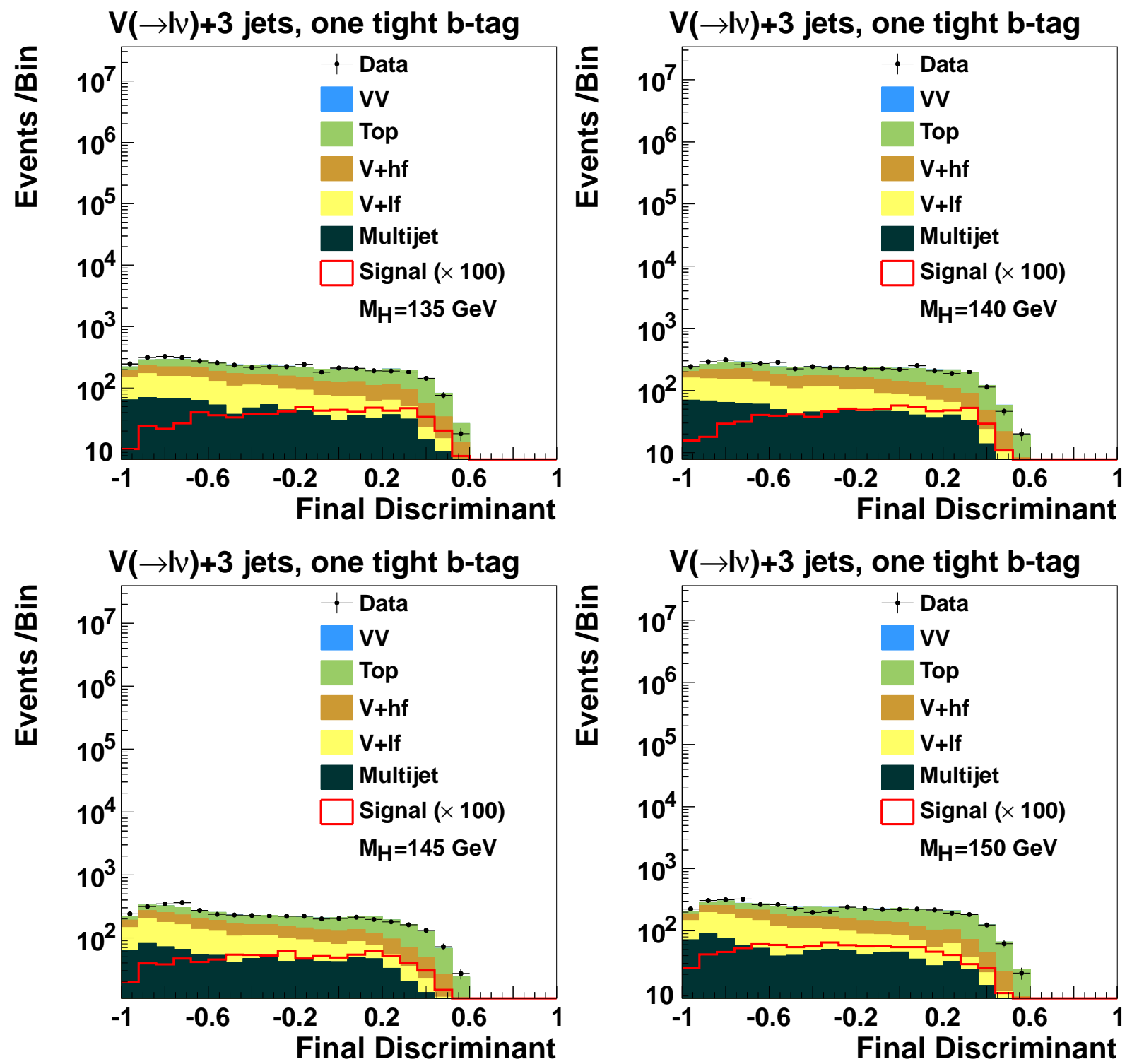

Figure B.20: The final MVA output for events with three jets and one tight b-tag for (top left) $M_{H}=135 \mathrm{GeV}$, (top right) $M_{H}=140 \mathrm{GeV}$, (bottom left) $M_{H}=145 \mathrm{GeV}$, and (bottom right) $M_{H}=150 \mathrm{GeV}$. 

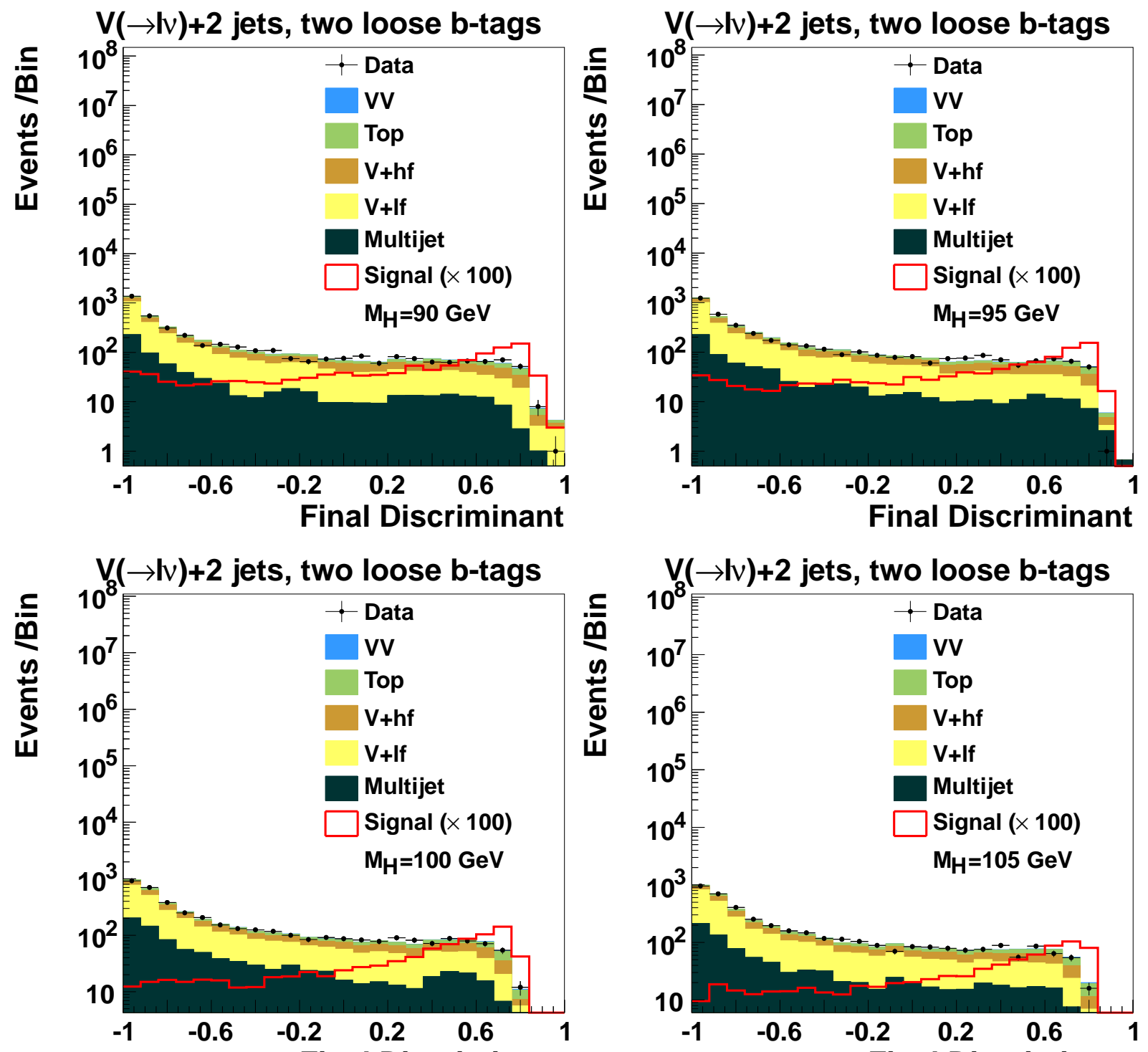

Final Discriminant

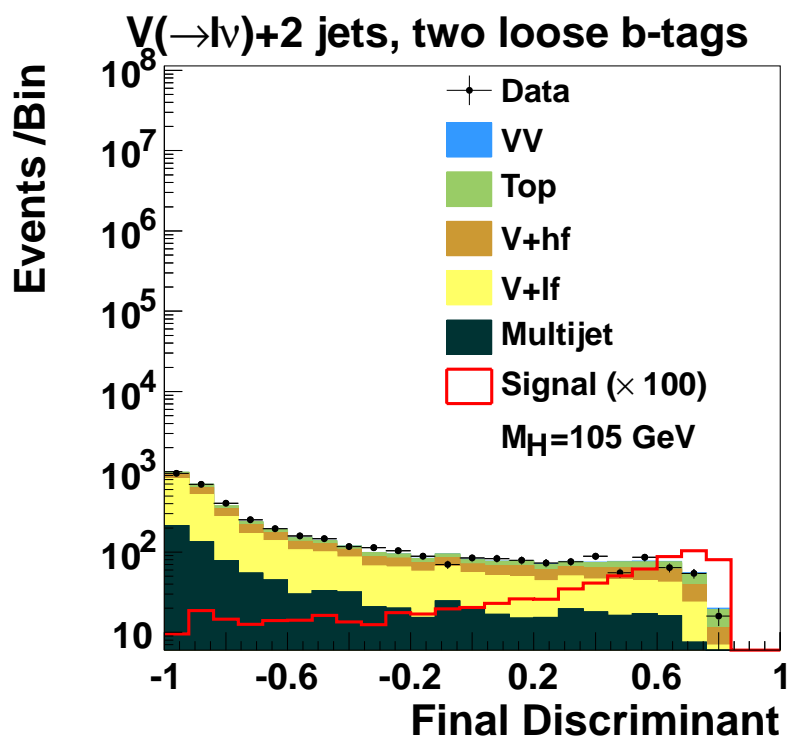

Figure B.21: The final MVA output for events with two jets and two loose b-tags for (top left) $M_{H}=90 \mathrm{GeV}$, (top right) $M_{H}=95 \mathrm{GeV}$, (bottom left) $M_{H}=100 \mathrm{GeV}$, and (bottom right) $M_{H}=105 \mathrm{GeV}$. 

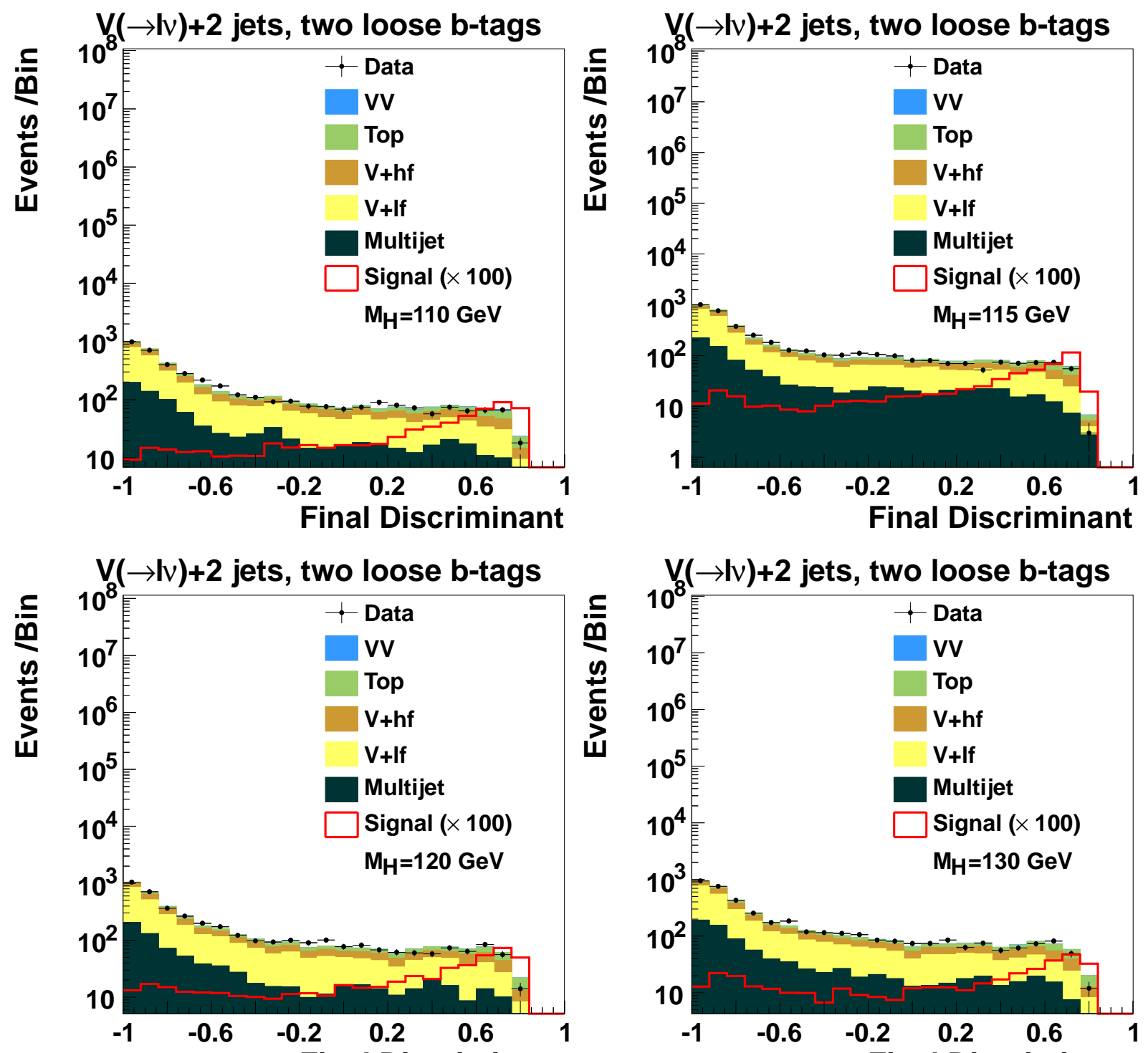

Final Discriminant

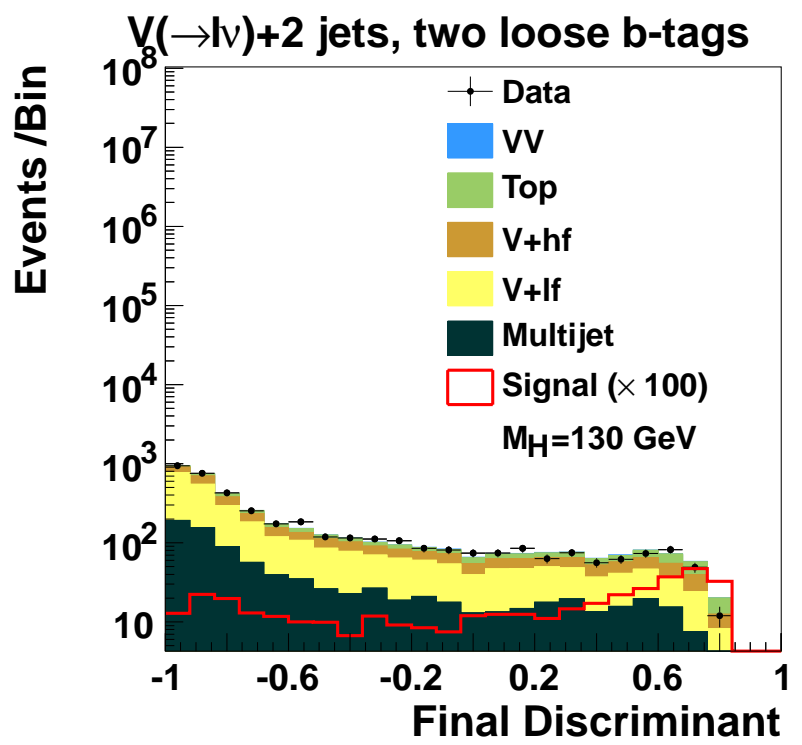

Figure B.22: The final MVA output for events with two jets and two loose b-tags for (top left) $M_{H}=110 \mathrm{GeV}$, (top right) $M_{H}=115 \mathrm{GeV}$, (bottom left) $M_{H}=120 \mathrm{GeV}$, and (bottom right) $M_{H}=130 \mathrm{GeV}$. 

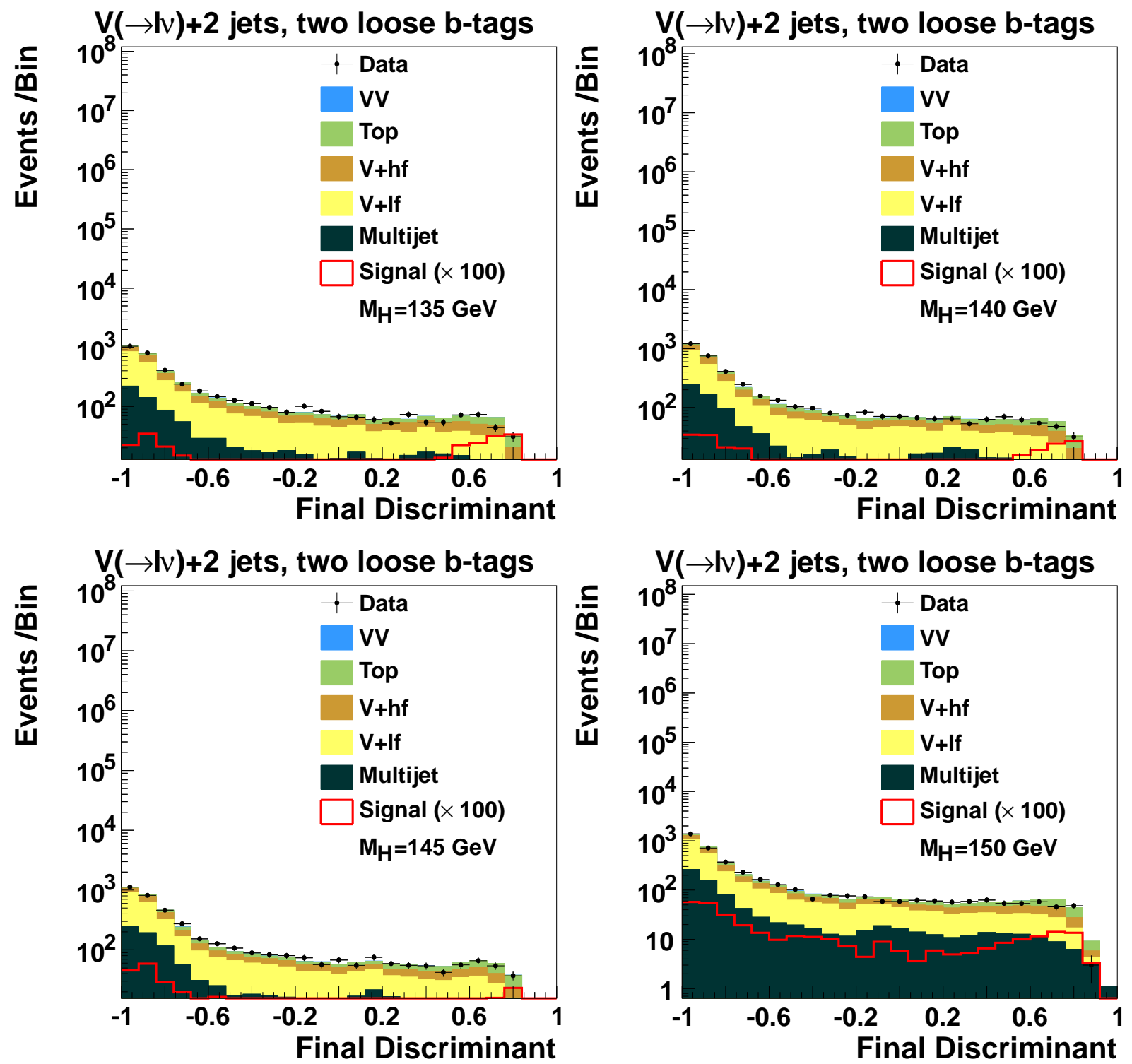

Figure B.23: The final MVA output for events with two jets and two loose b-tags for (top left) $M_{H}=135 \mathrm{GeV}$, (top right) $M_{H}=140 \mathrm{GeV}$, (bottom left) $M_{H}=145 \mathrm{GeV}$, and (bottom right) $M_{H}=150 \mathrm{GeV}$. 

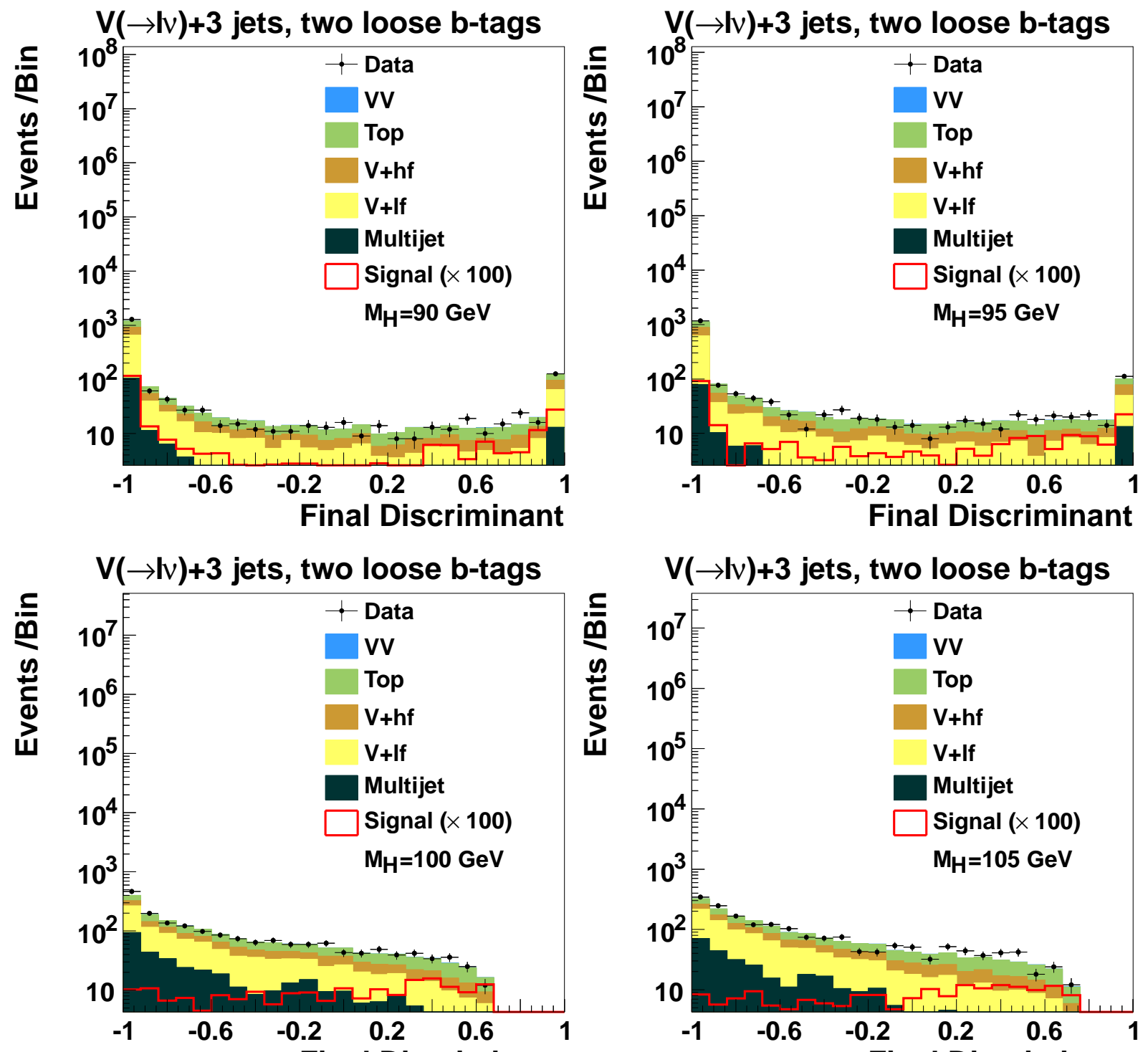

Final Discriminant

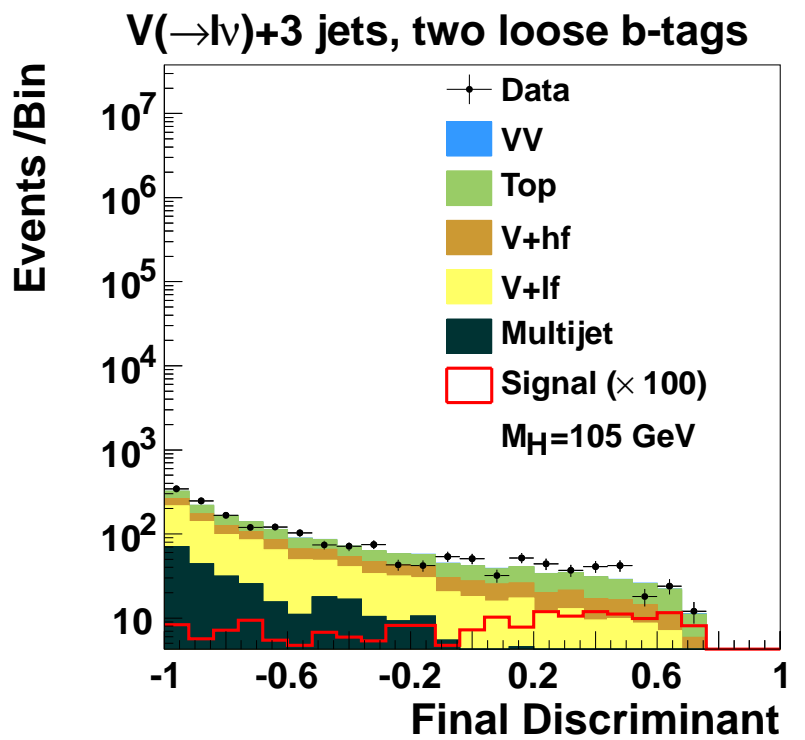

Figure B.24: The final MVA output for events with three jets and two loose b-tags for (top left) $M_{H}=90 \mathrm{GeV}$, (top right) $M_{H}=95 \mathrm{GeV}$, (bottom left) $M_{H}=100 \mathrm{GeV}$, and (bottom right) $M_{H}=105 \mathrm{GeV}$. 

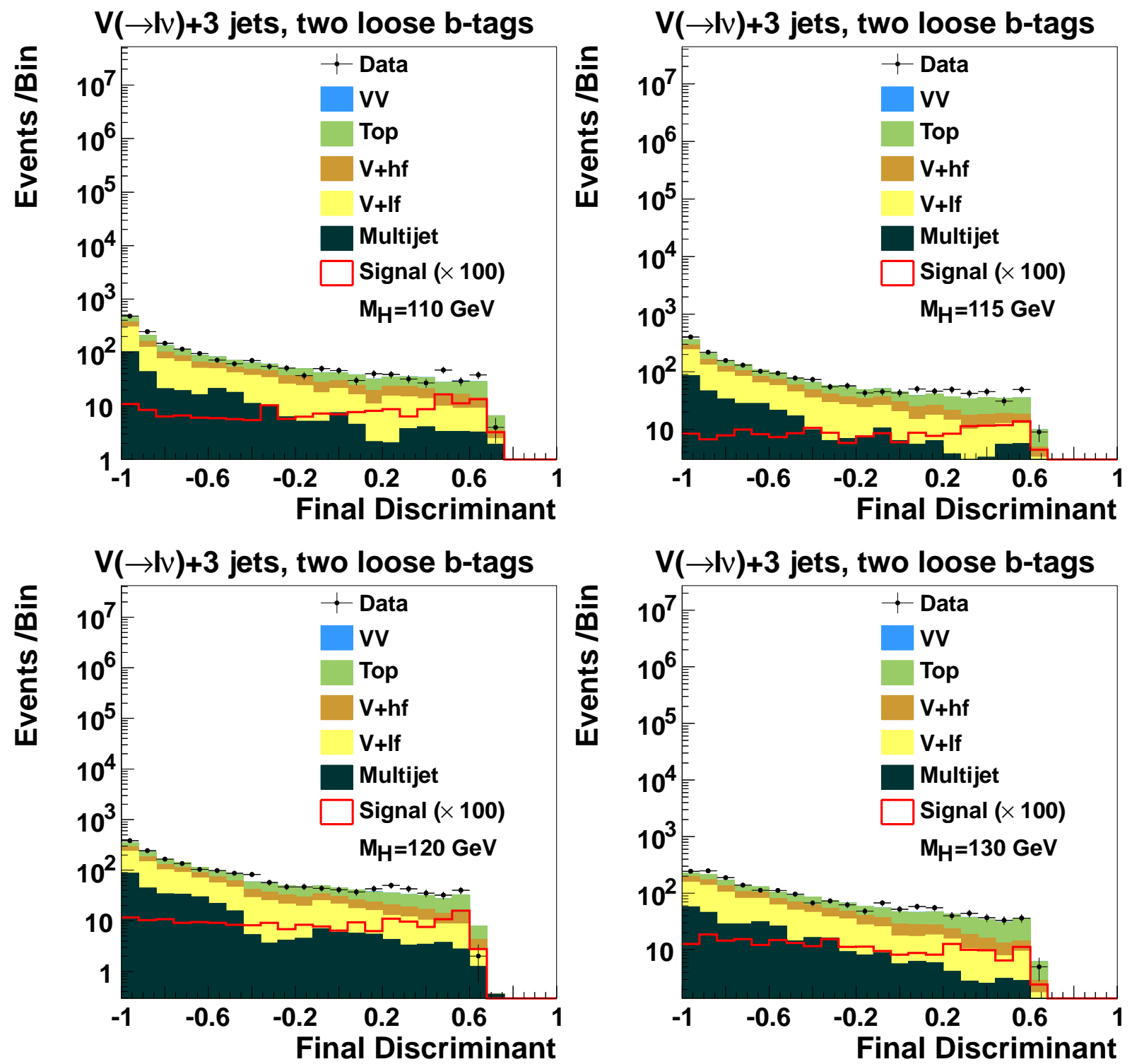

Figure B.25: The final MVA output for events with three jets and two loose b-tags for (top left) $M_{H}=110 \mathrm{GeV}$, (top right) $M_{H}=115 \mathrm{GeV}$, (bottom left) $M_{H}=120 \mathrm{GeV}$, and (bottom right) $M_{H}=130 \mathrm{GeV}$. 

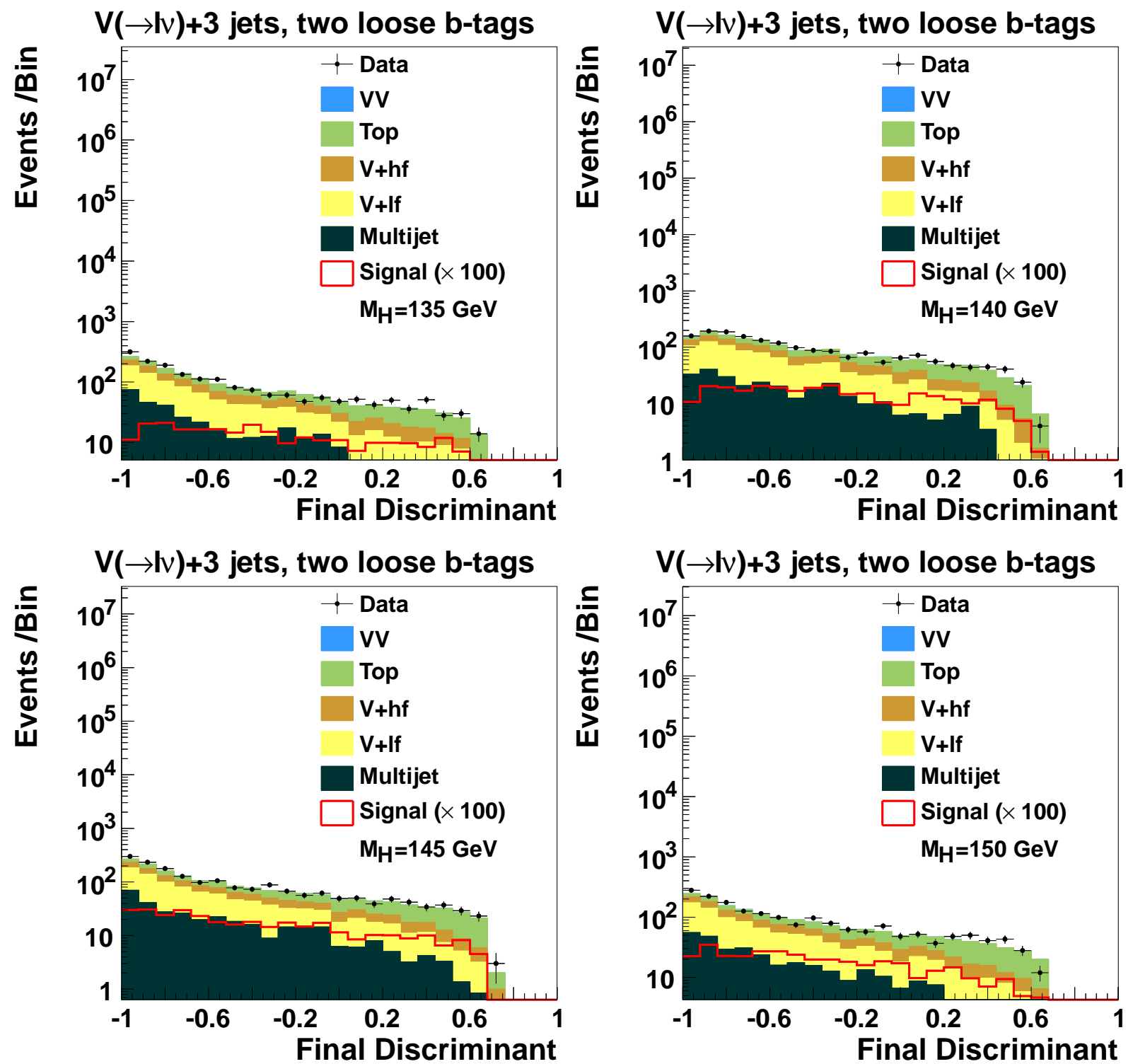

Figure B.26: The final MVA output for events with three jets and two loose b-tags for (top left) $M_{H}=135 \mathrm{GeV}$, (top right) $M_{H}=140 \mathrm{GeV}$, (bottom left) $M_{H}=145 \mathrm{GeV}$, and (bottom right) $M_{H}=150 \mathrm{GeV}$. 


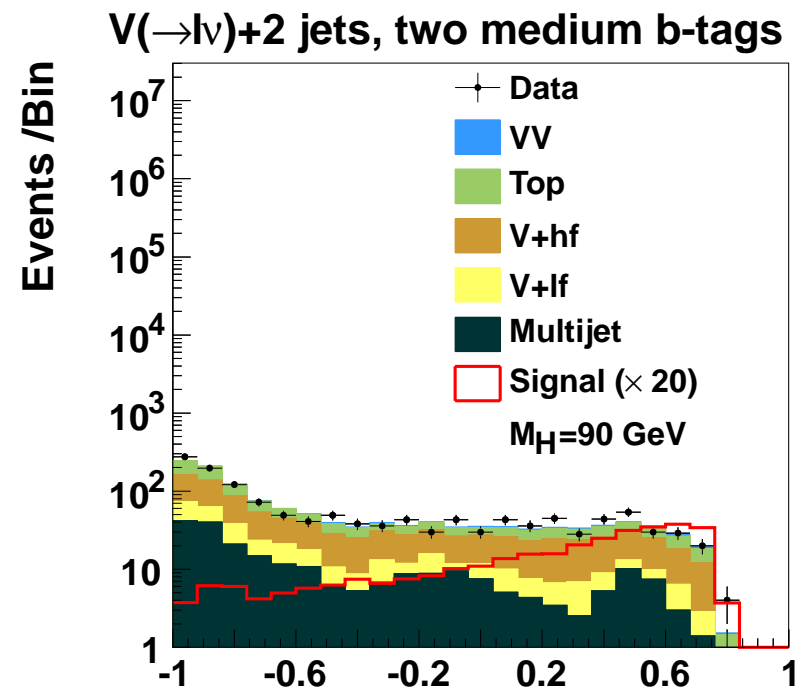

Final Discriminant

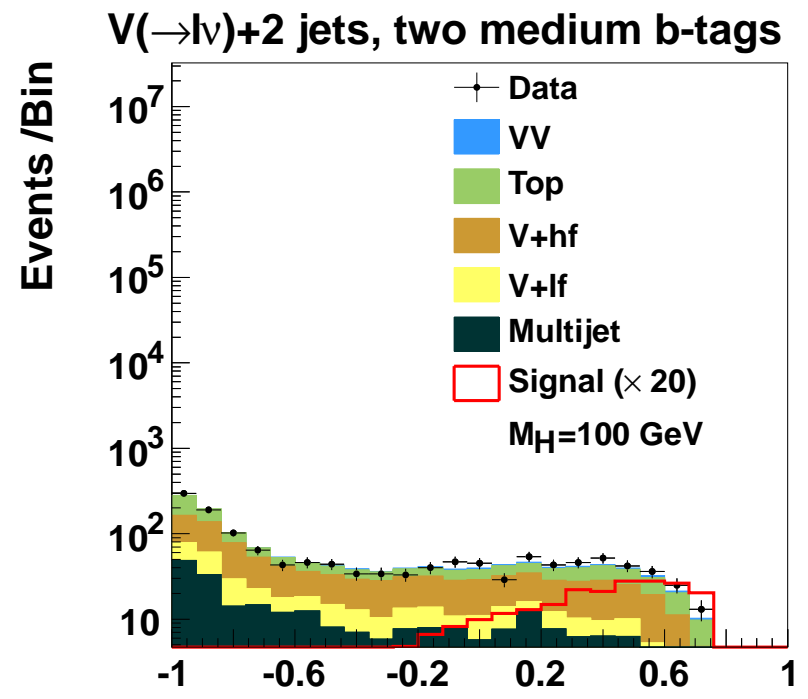

Final Discriminant

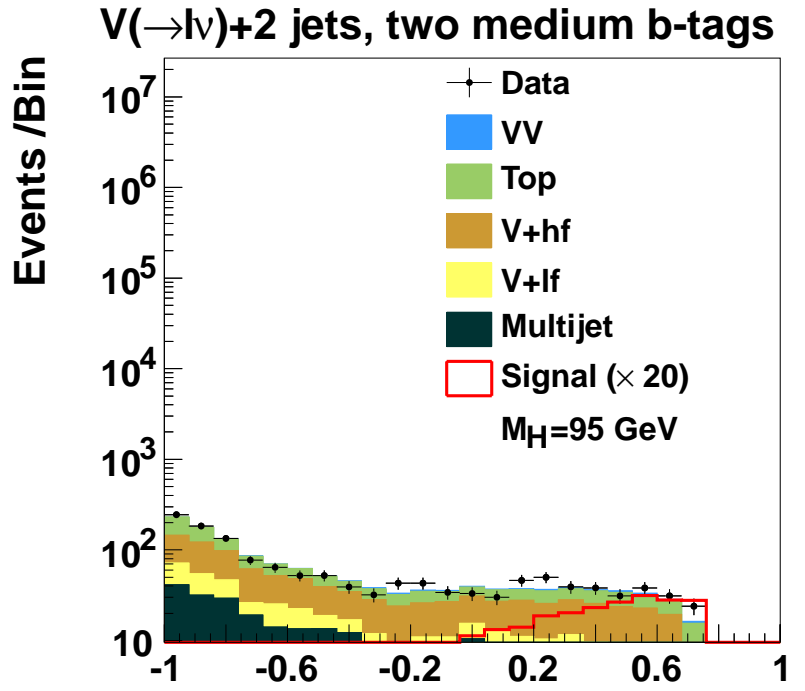

Final Discriminant

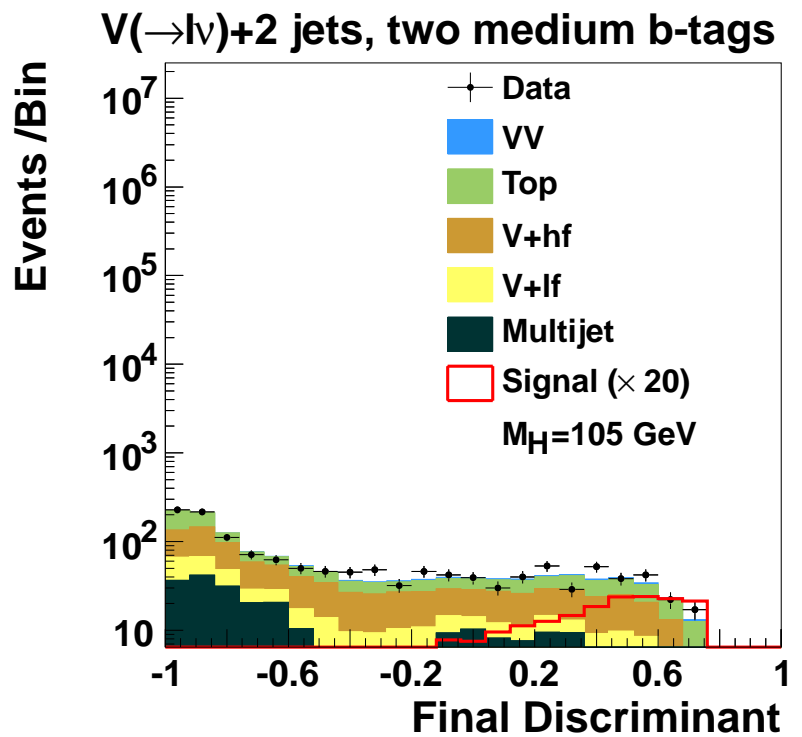

Figure B.27: The final MVA output for events with two jets and two medium b-tags for (top left) $M_{H}=90 \mathrm{GeV}$, (top right) $M_{H}=95 \mathrm{GeV}$, (bottom left) $M_{H}=100 \mathrm{GeV}$, and (bottom right) $M_{H}=105 \mathrm{GeV}$. 

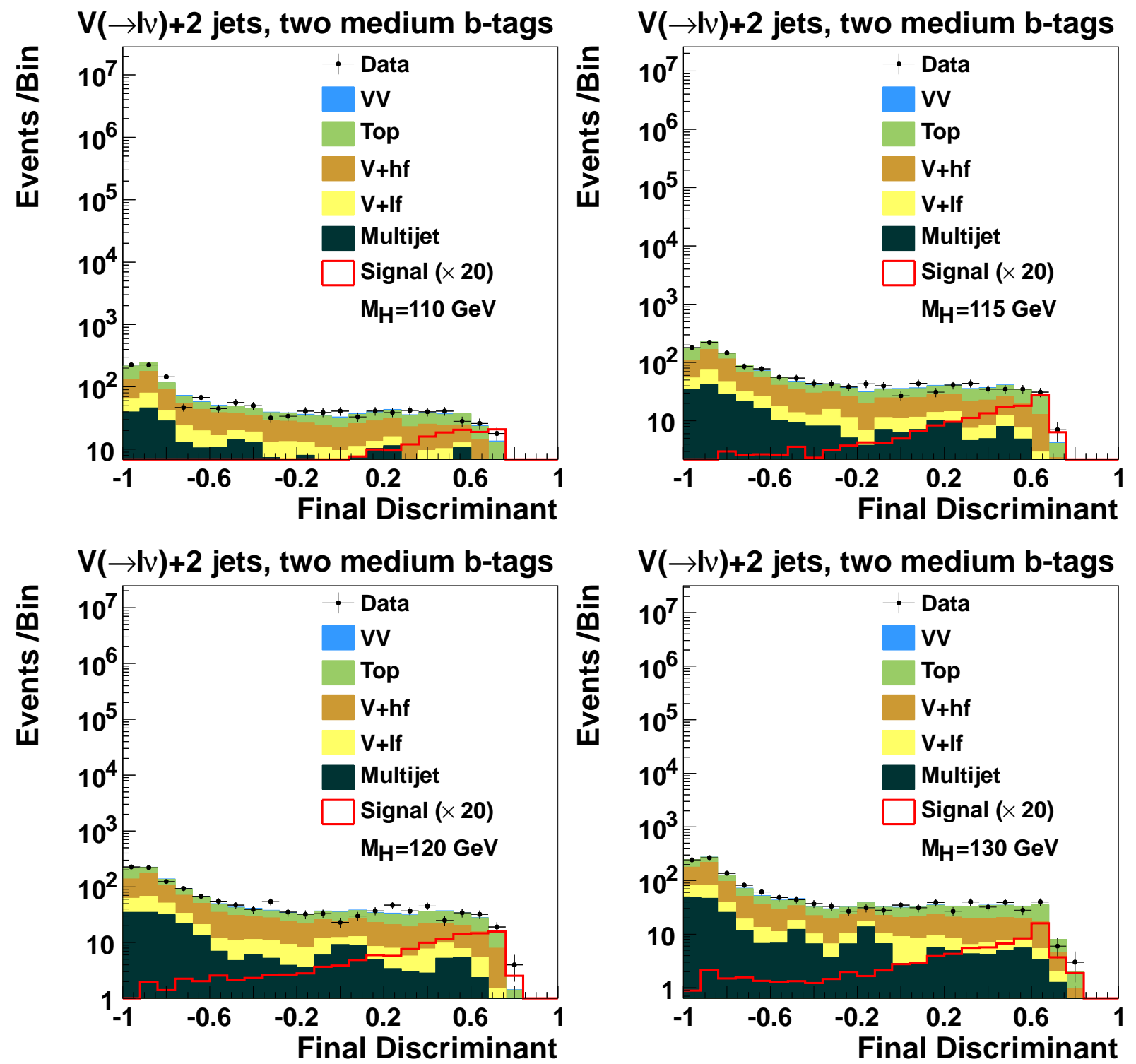

Figure B.28: The final MVA output for events with two jets and two medium b-tags for (top left) $M_{H}=110 \mathrm{GeV}$, (top right) $M_{H}=115 \mathrm{GeV}$, (bottom left) $M_{H}=120 \mathrm{GeV}$, and (bottom right) $M_{H}=130 \mathrm{GeV}$. 

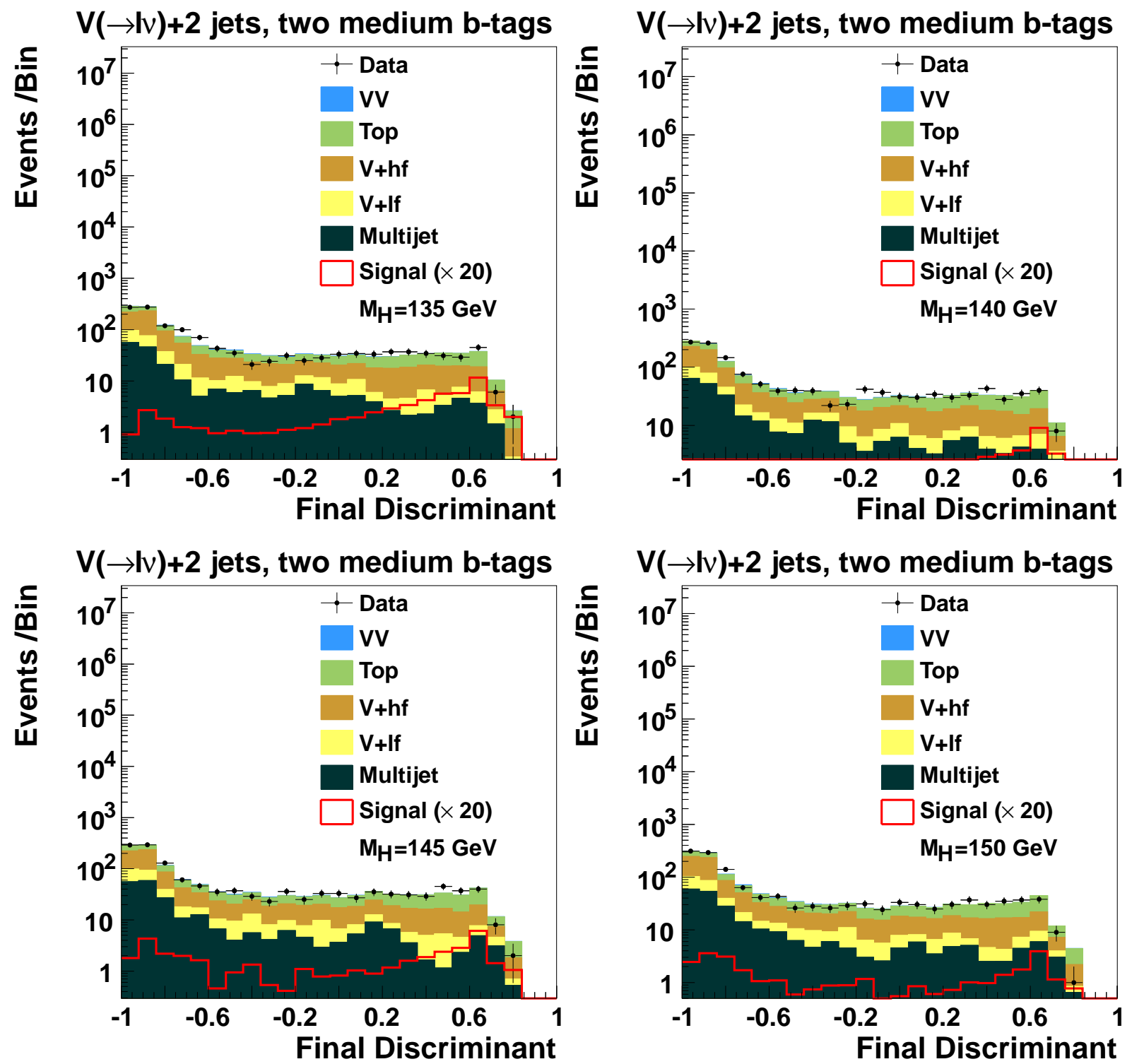

Figure B.29: The final MVA output for events with two jets and two medium b-tags for (top left) $M_{H}=135 \mathrm{GeV}$, (top right) $M_{H}=140 \mathrm{GeV}$, (bottom left) $M_{H}=145 \mathrm{GeV}$, and (bottom right) $M_{H}=150 \mathrm{GeV}$. 


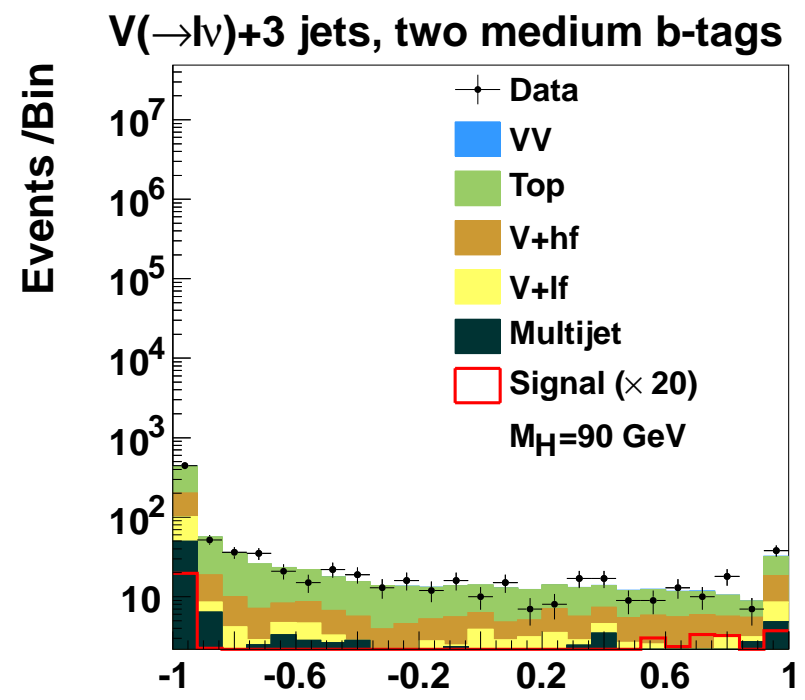

Final Discriminant

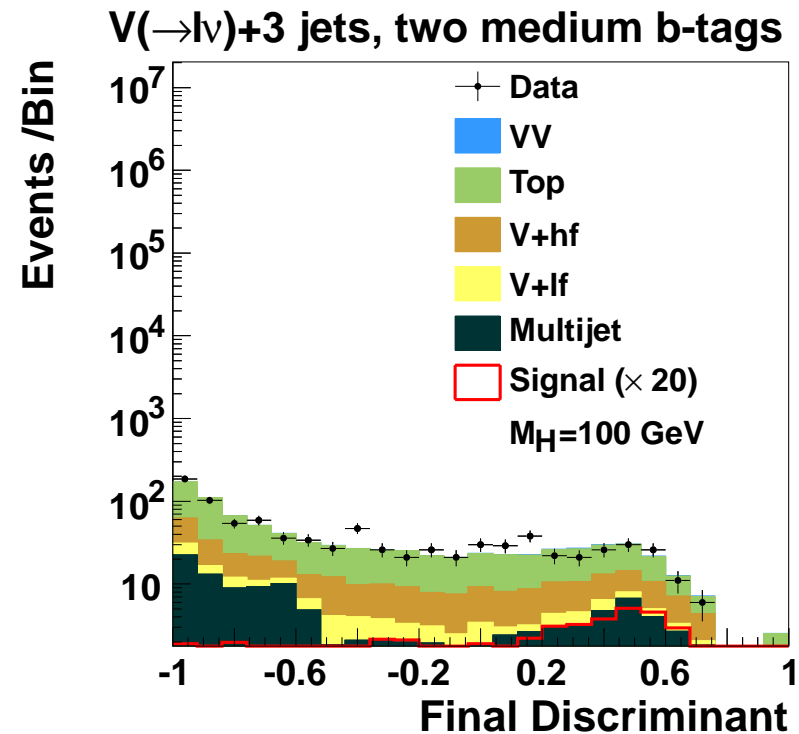

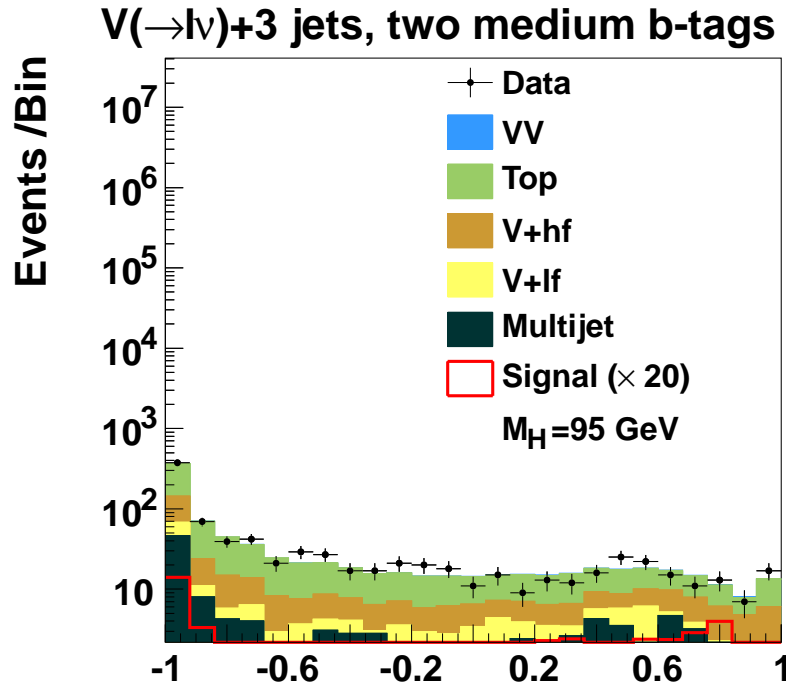

Final Discriminant

$\mathrm{V}(\rightarrow \mathrm{N})+3$ jets, two medium b-tags

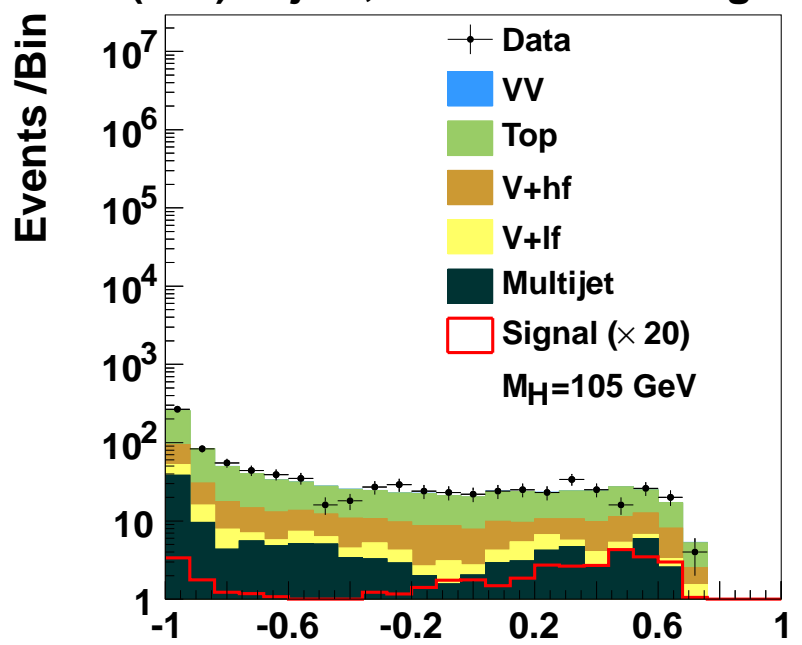

Final Discriminant

Figure B.30: The final MVA output for events with three jets and two medium b-tags for (top left) $M_{H}=90 \mathrm{GeV}$, (top right) $M_{H}=95 \mathrm{GeV}$, (bottom left) $M_{H}=100 \mathrm{GeV}$, and (bottom right) $M_{H}=105 \mathrm{GeV}$. 

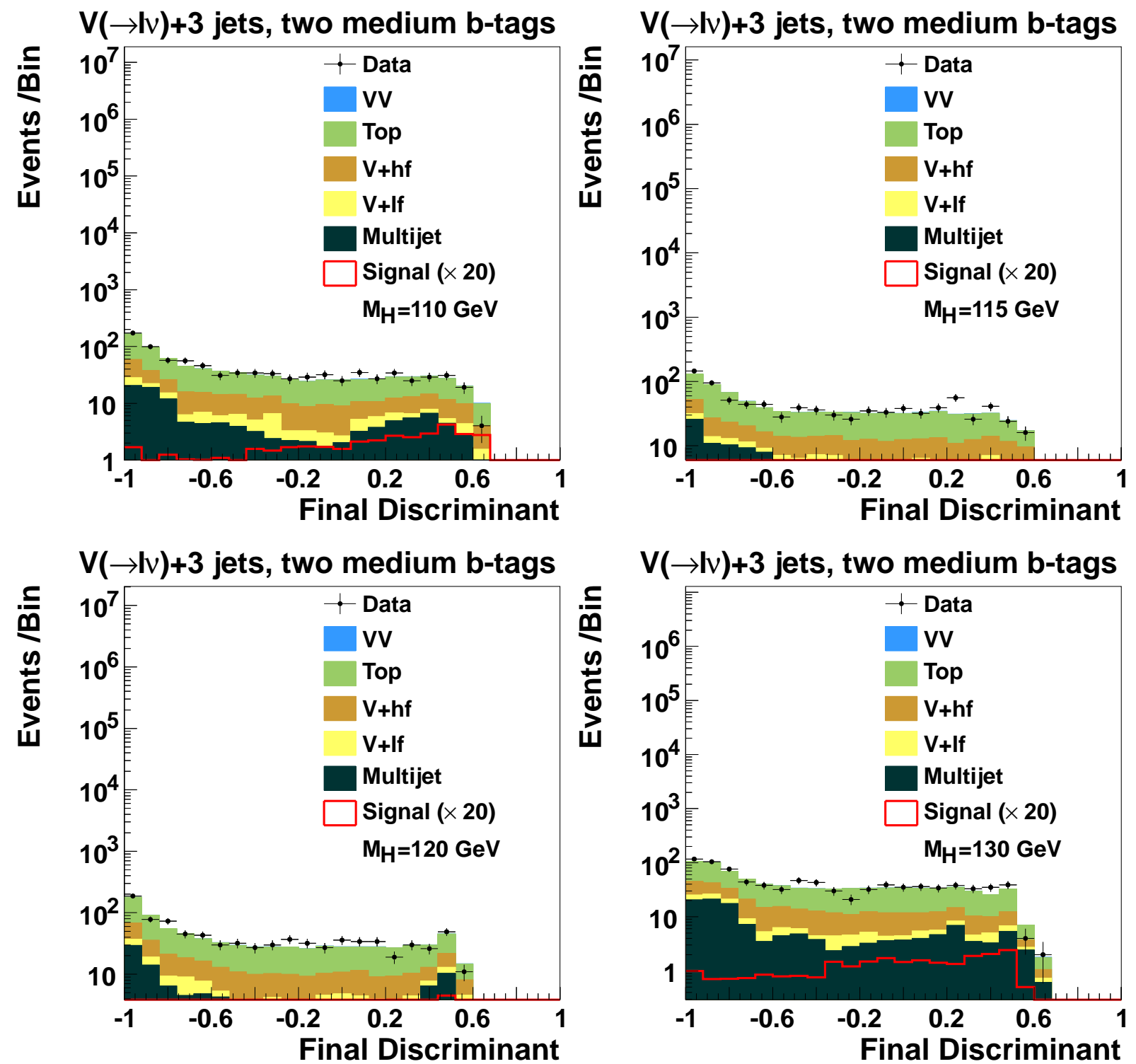

Figure B.31: The final MVA output for events with three jets and two medium b-tags for (top left) $M_{H}=110 \mathrm{GeV}$, (top right) $M_{H}=115 \mathrm{GeV}$, (bottom left) $M_{H}=120 \mathrm{GeV}$, and (bottom right) $M_{H}=130 \mathrm{GeV}$. 

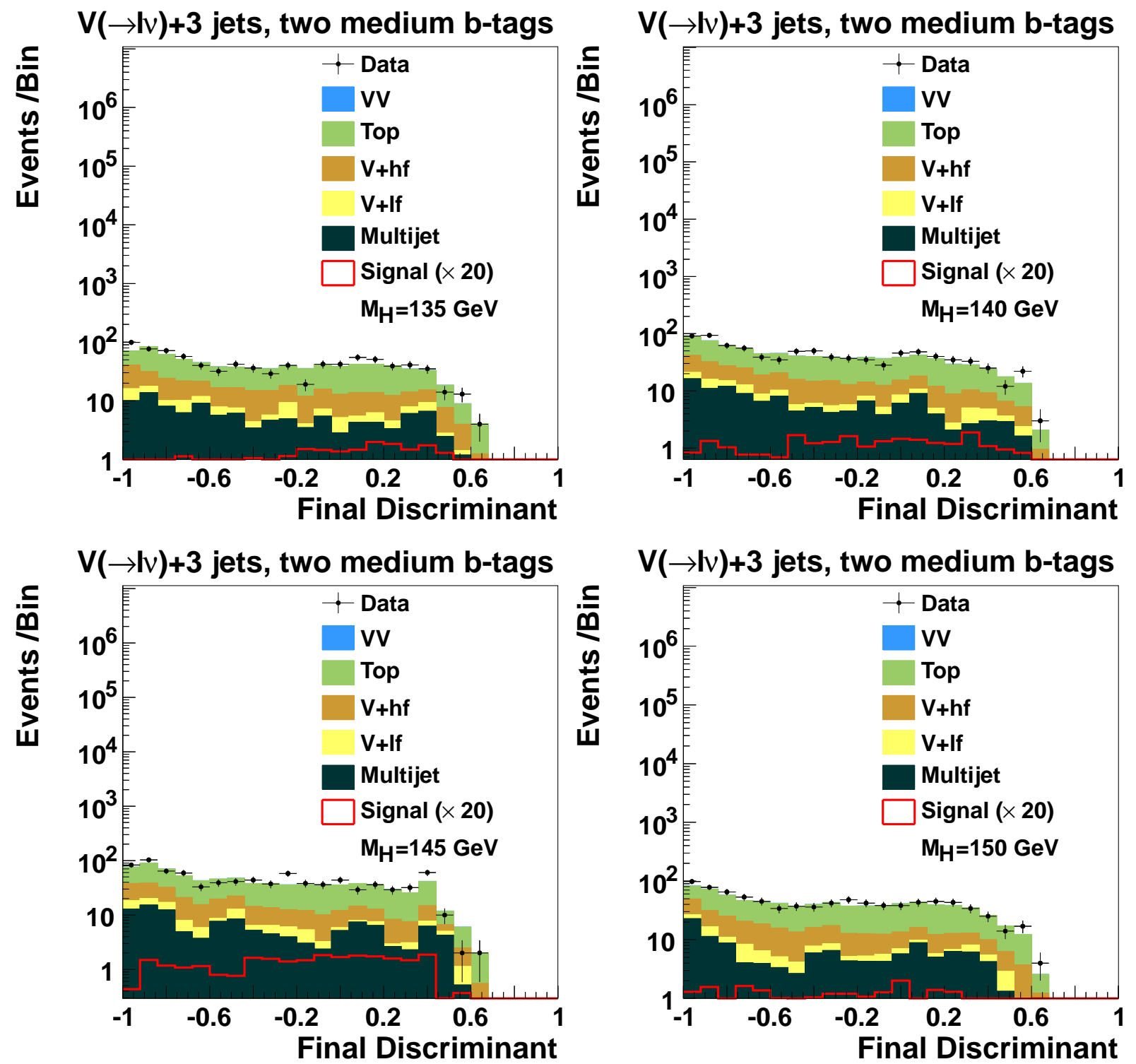

Figure B.32: The final MVA output for events with three jets and two medium b-tags for (top left) $M_{H}=135 \mathrm{GeV}$, (top right) $M_{H}=140 \mathrm{GeV}$, (bottom left) $M_{H}=145 \mathrm{GeV}$, and (bottom right) $M_{H}=150 \mathrm{GeV}$. 

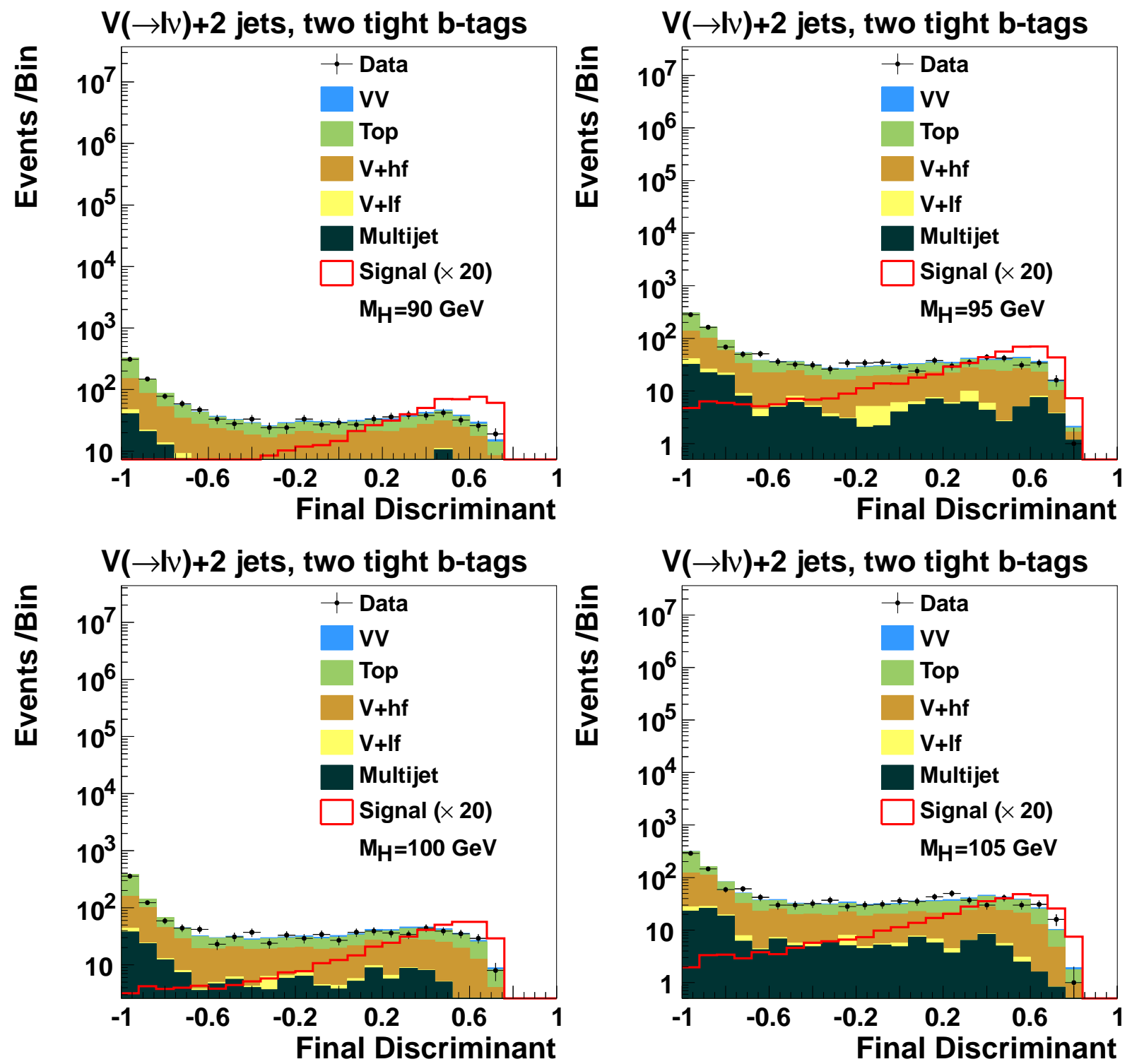

Figure B.33: The final MVA output for events with two jets and two tight b-tags for (top left) $M_{H}=90 \mathrm{GeV}$, (top right) $M_{H}=95 \mathrm{GeV}$, (bottom left) $M_{H}=100 \mathrm{GeV}$, and (bottom right) $M_{H}=105 \mathrm{GeV}$. 

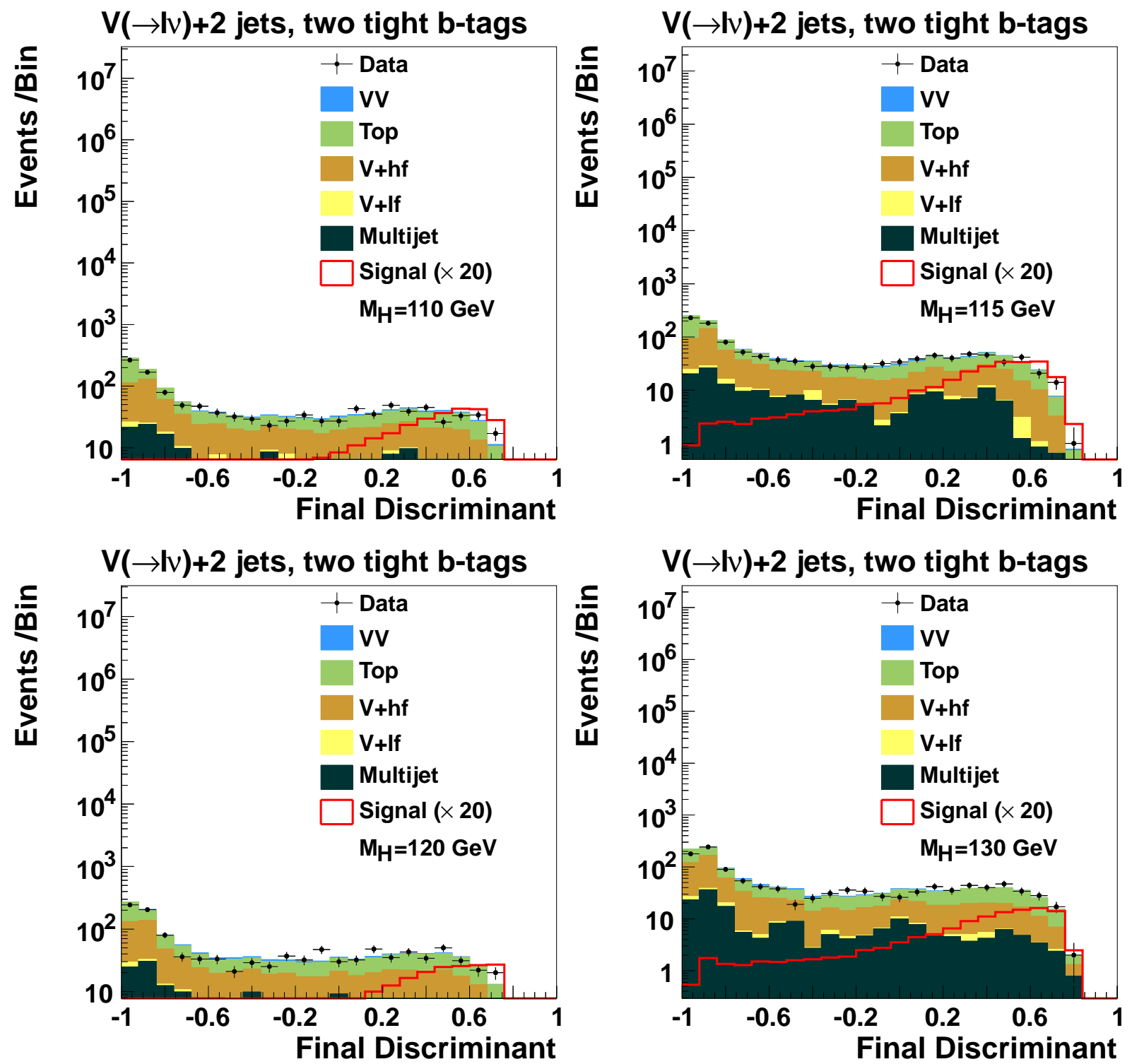

Figure B.34: The final MVA output for events with two jets and two tight b-tags for (top left) $M_{H}=110 \mathrm{GeV}$, (top right) $M_{H}=115 \mathrm{GeV}$, (bottom left) $M_{H}=120 \mathrm{GeV}$, and (bottom right) $M_{H}=130 \mathrm{GeV}$. 

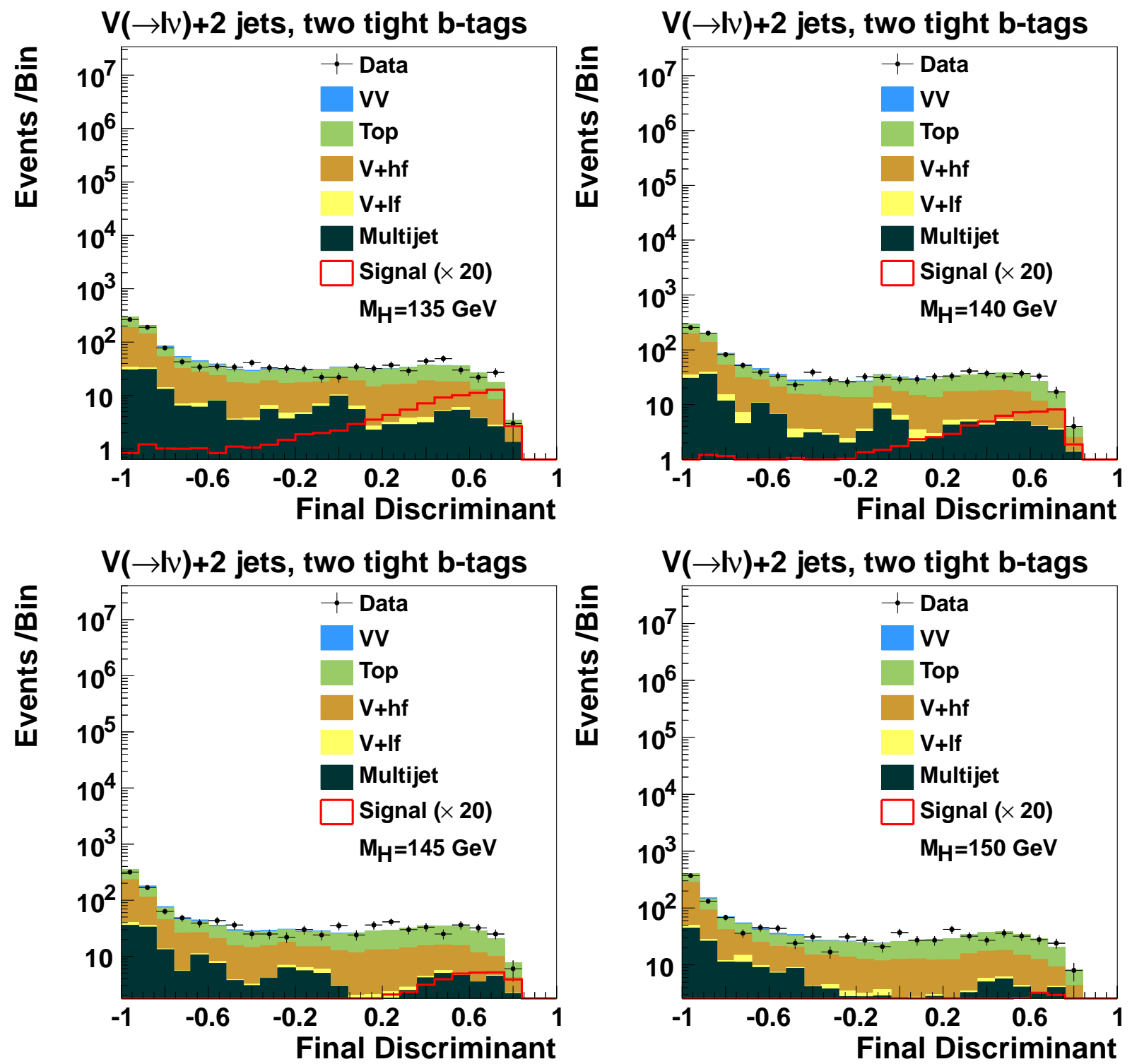

Figure B.35: The final MVA output for events with two jets and two tight b-tags for (top left) $M_{H}=135 \mathrm{GeV}$, (top right) $M_{H}=140 \mathrm{GeV}$, (bottom left) $M_{H}=145 \mathrm{GeV}$, and (bottom right) $M_{H}=150 \mathrm{GeV}$. 

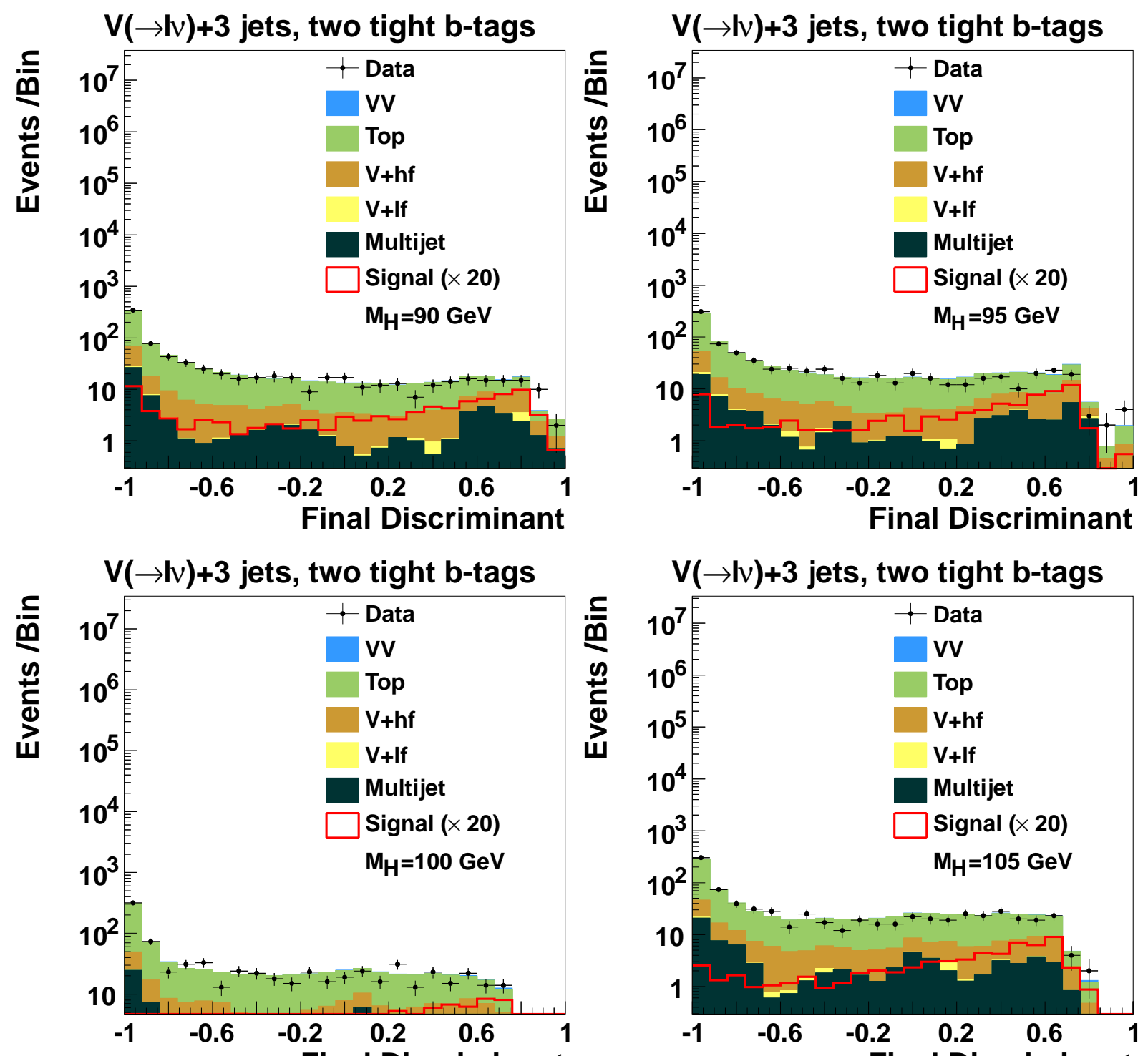

Final Discriminant

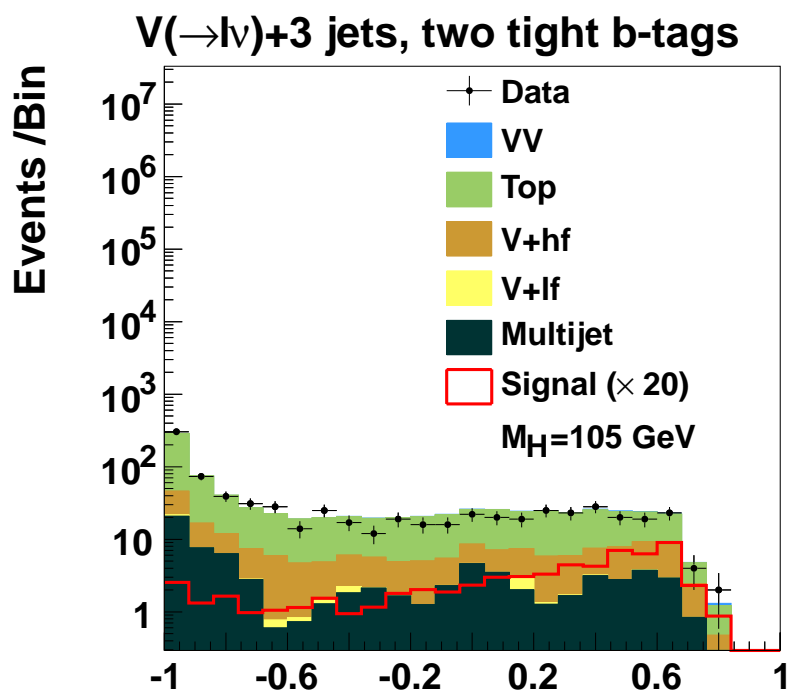

Final Discriminant

Figure B.36: The final MVA output for events with three jets and two tight b-tags for (top left) $M_{H}=90 \mathrm{GeV}$, (top right) $M_{H}=95 \mathrm{GeV}$, (bottom left) $M_{H}=100 \mathrm{GeV}$, and (bottom right) $M_{H}=105 \mathrm{GeV}$. 

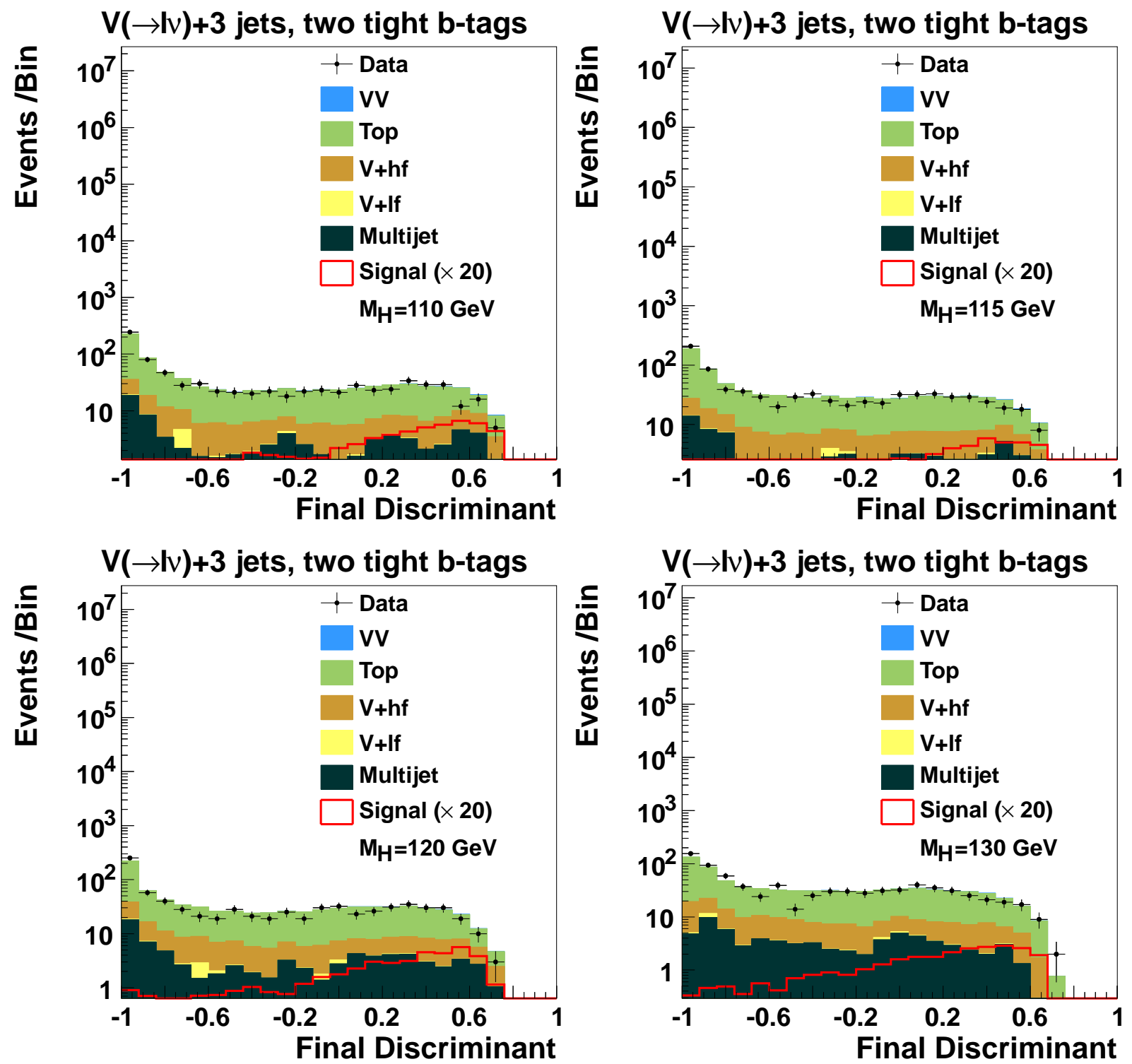

Figure B.37: The final MVA output for events with three jets and two tight b-tags for (top left) $M_{H}=110 \mathrm{GeV}$, (top right) $M_{H}=115 \mathrm{GeV}$, (bottom left) $M_{H}=120 \mathrm{GeV}$, and (bottom right) $M_{H}=130 \mathrm{GeV}$. 

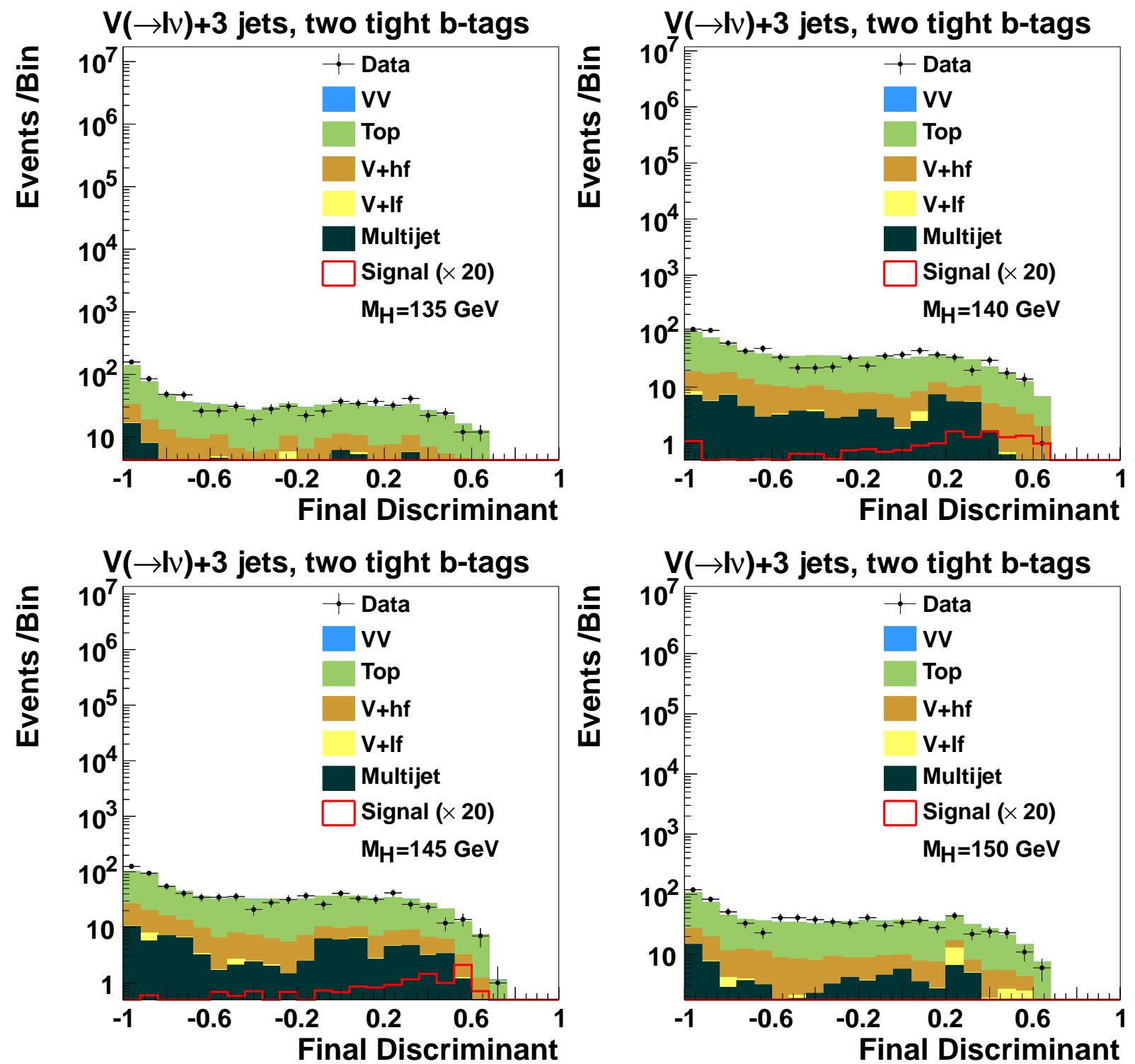

Figure B.38: The final MVA output for events with three jets and two tight b-tags for (top left) $M_{H}=135 \mathrm{GeV}$, (top right) $M_{H}=140 \mathrm{GeV}$, (bottom left) $M_{H}=145 \mathrm{GeV}$, and (bottom right) $M_{H}=150 \mathrm{GeV}$. 

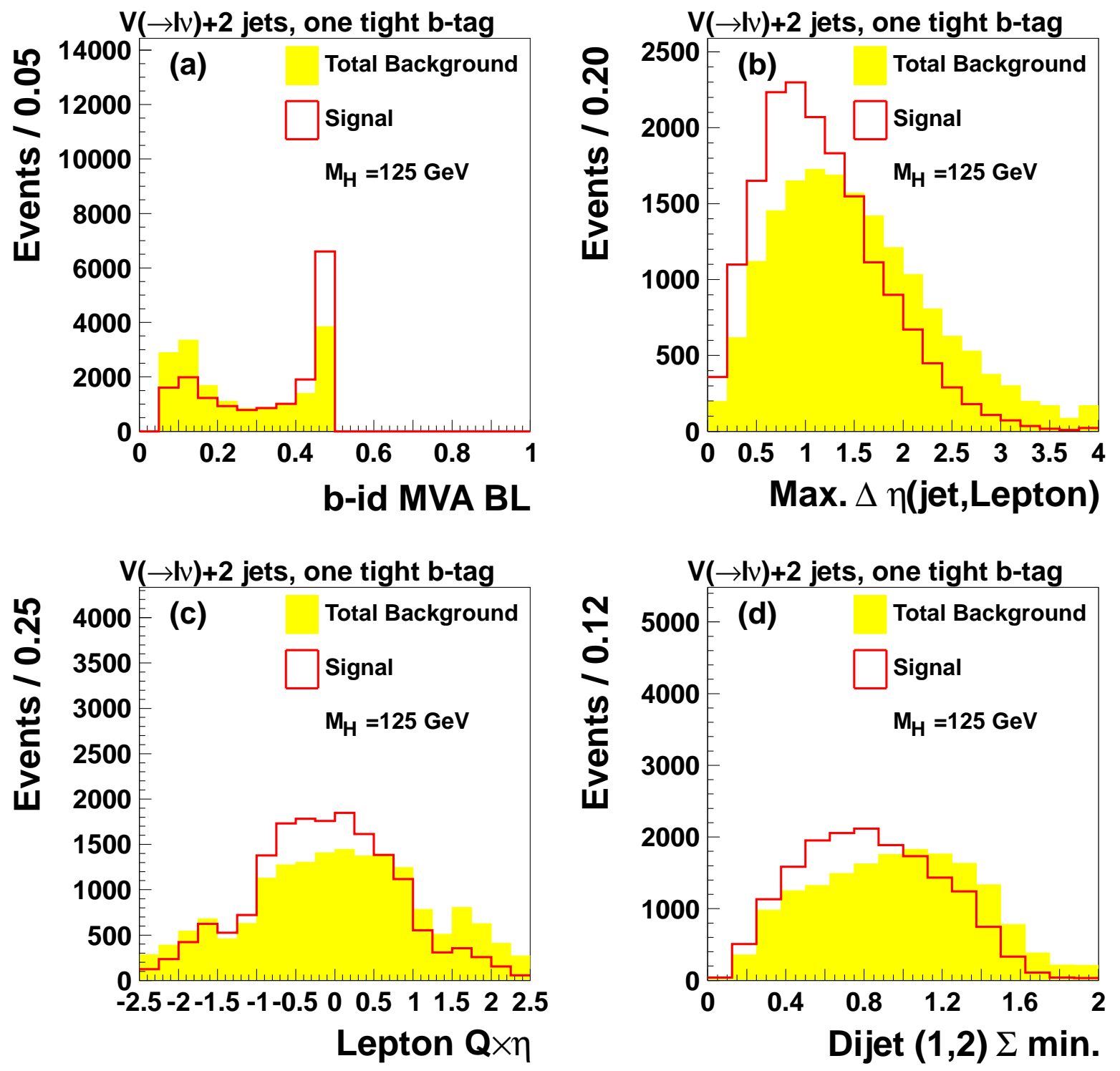

Figure B.39: Input variables to the BDT trained to distinguish signal from all backgrounds in events with two jets and one tight b-tag: (top left) the bid MVA output, (top right) the maximum $\Delta \eta(\ell, j)$, (bottom left) $q^{\ell} \times \eta^{\ell}$, and (bottom right) the minimum significance of the dijet system. The signal is shown for $M_{H}=125 \mathrm{GeV}$, and is normalized to the sum of the backgrounds. Overflow events are added to the last bin. 

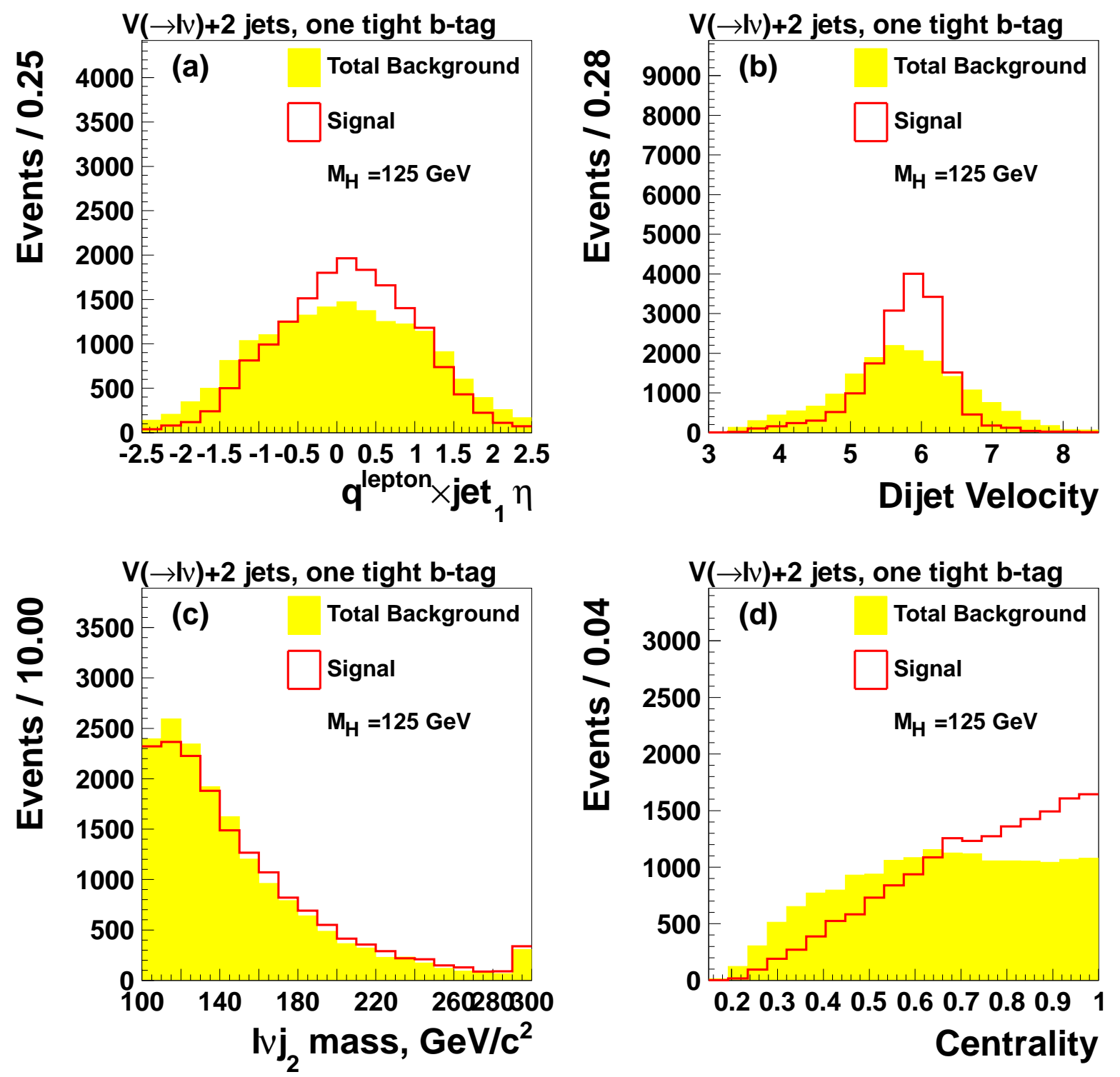

Figure B.40: Input variables to the BDT trained to distinguish signal from all backgrounds in events with two jets and one tight b-tag. The signal is shown for $M_{H}=125 \mathrm{GeV}$, and is normalized to the sum of the backgrounds. Overflow events are added to the last bin. 

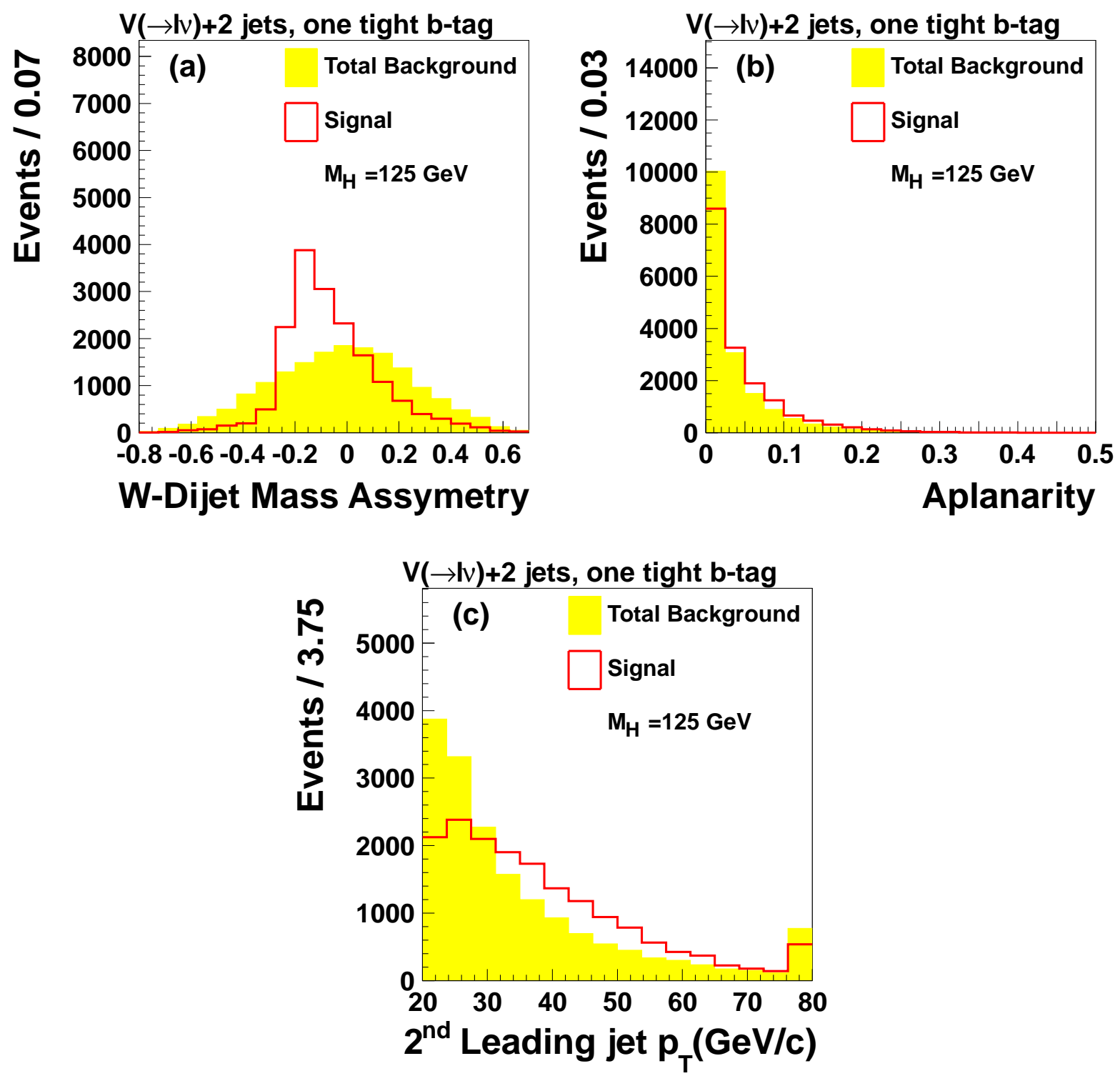

Figure B.41: Input variables to the BDT trained to distinguish signal from all backgrounds in events with two jets and one tight b-tag: (top left) the mass asymmetry between the $\ell \nu$ and dijet systems, (top right) aplanarity, and (bottom) the second leading jet $p_{T}$. The signal is shown for $M_{H}=125 \mathrm{GeV}$, and is normalized to the sum of the backgrounds. Overflow events are added to the last bin. 

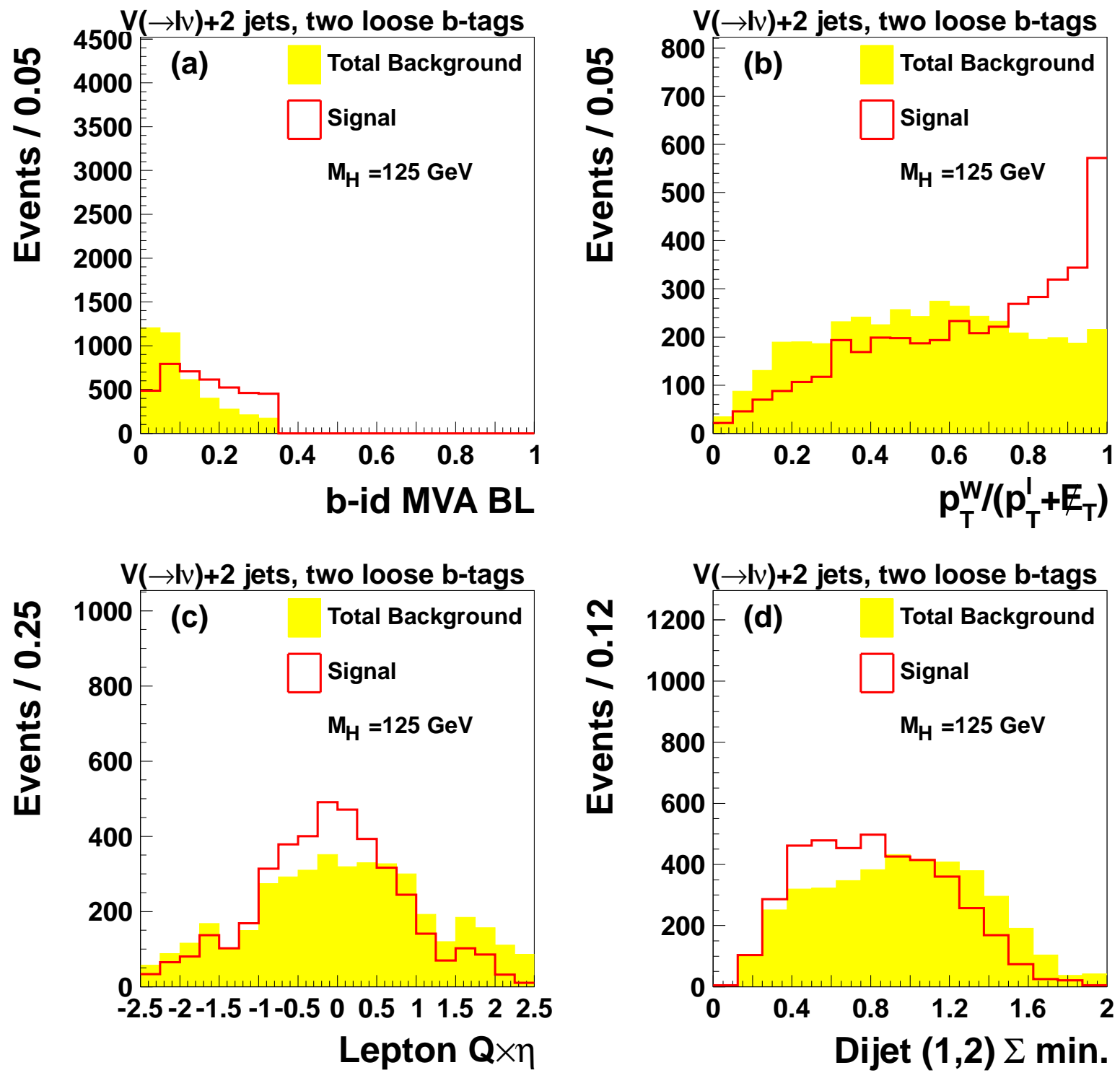

Figure B.42: Input variables to the BDT trained to distinguish signal from all backgrounds in events with two jets and two loose b-tag: (top left) the bid MVA output, (top right) the maximum $\Delta \eta(\ell, j)$, (bottom left) $q^{\ell} \times \eta^{\ell}$, and (bottom right) the minimum significance of the dijet system. The signal is shown for $M_{H}=125 \mathrm{GeV}$, and is normalized to the sum of the backgrounds. Overflow events are added to the last bin. 

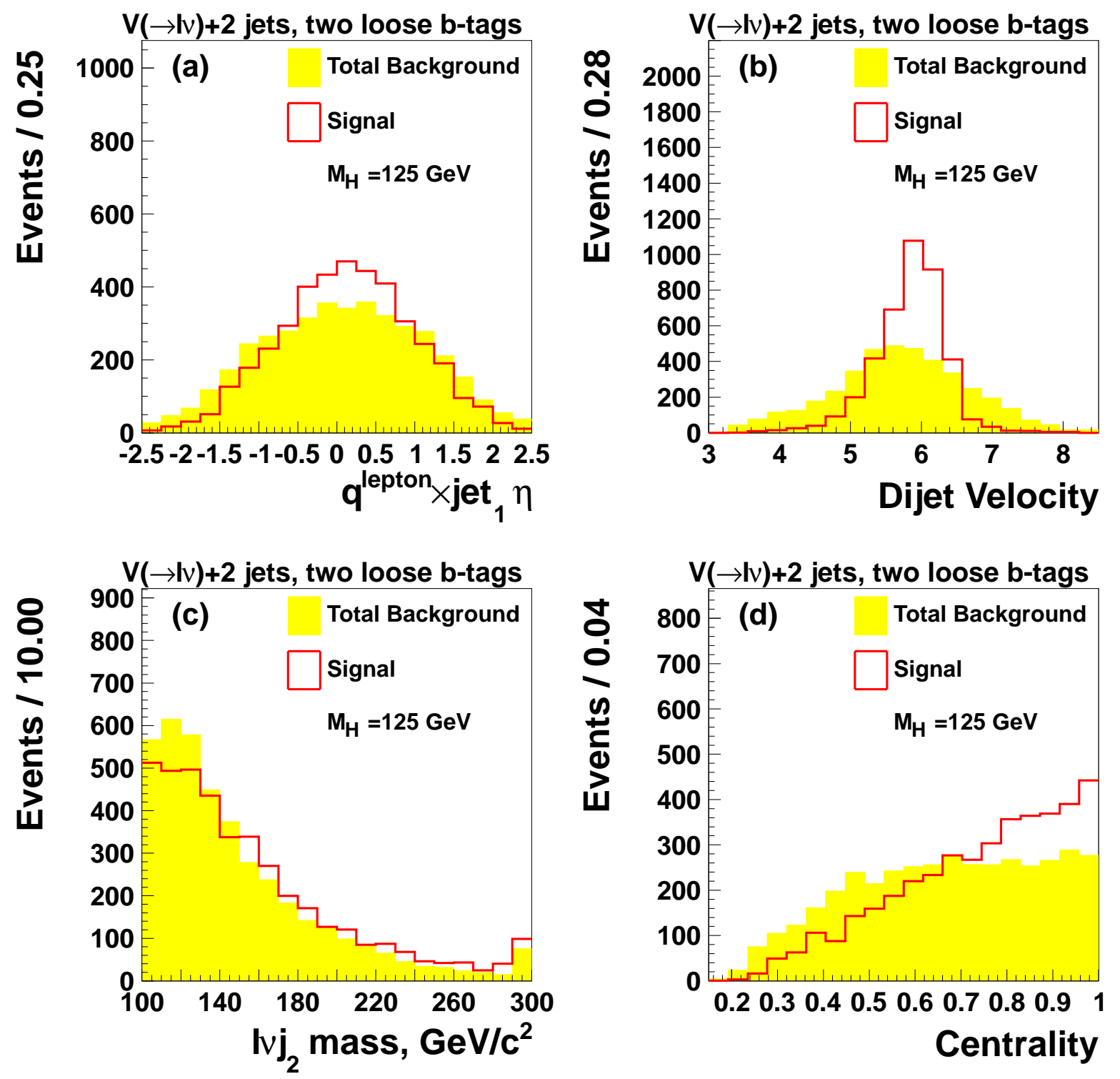

Figure B.43: Input variables to the BDT trained to distinguish signal from all backgrounds in events with two jets and two loose b-tag. The signal is shown for $M_{H}=125 \mathrm{GeV}$, and is normalized to the sum of the backgrounds. Overflow events are added to the last bin. 

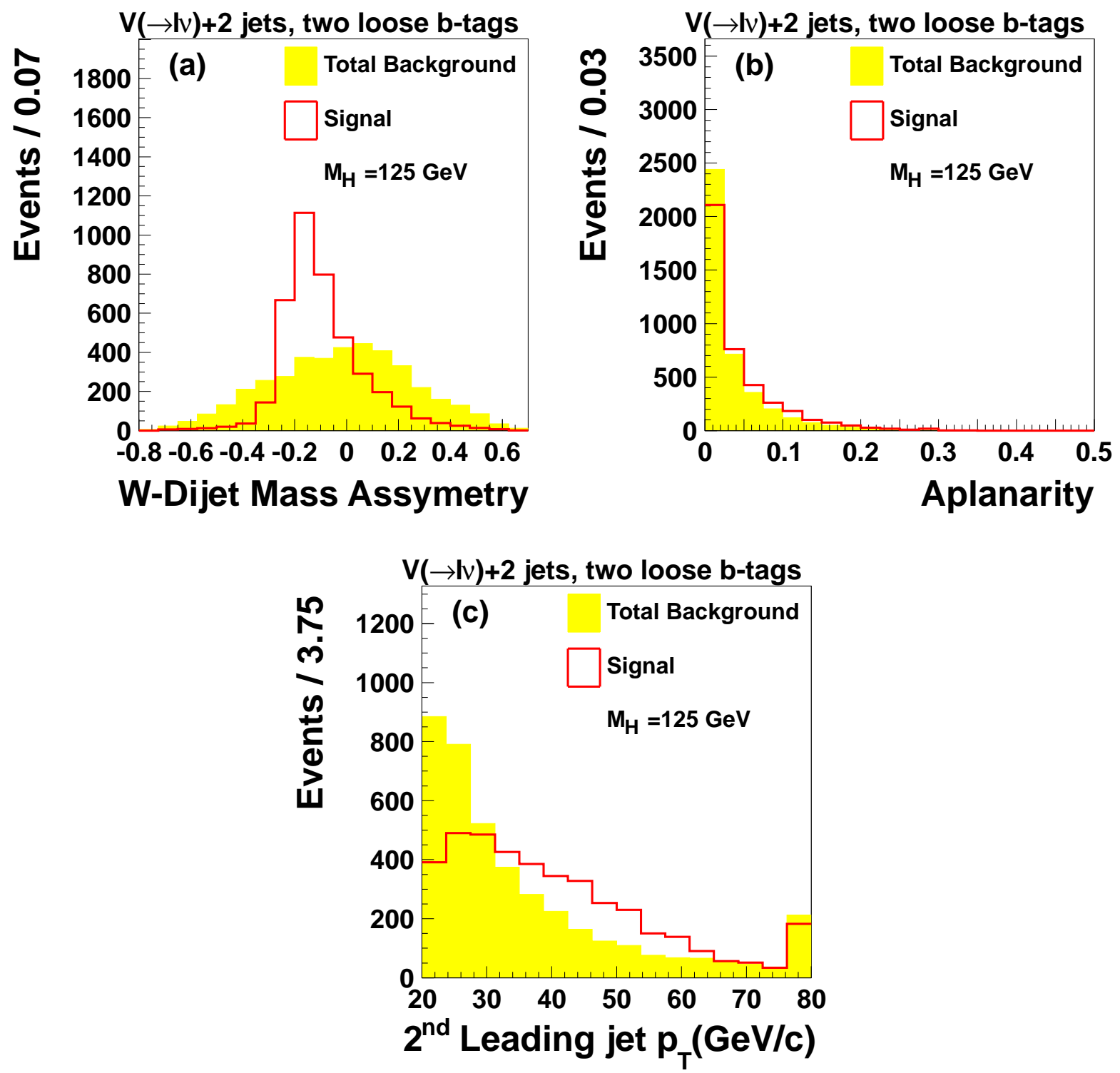

Figure B.44: Input variables to the BDT trained to distinguish signal from all backgrounds in events with two jets and two loose b-tag: (top left) the mass asymmetry between the $\ell \nu$ and dijet systems, (top right) aplanarity, and (bottom) the second leading jet $p_{T}$. The signal is shown for $M_{H}=125 \mathrm{GeV}$, and is normalized to the sum of the backgrounds. Overflow events are added to the last bin. 

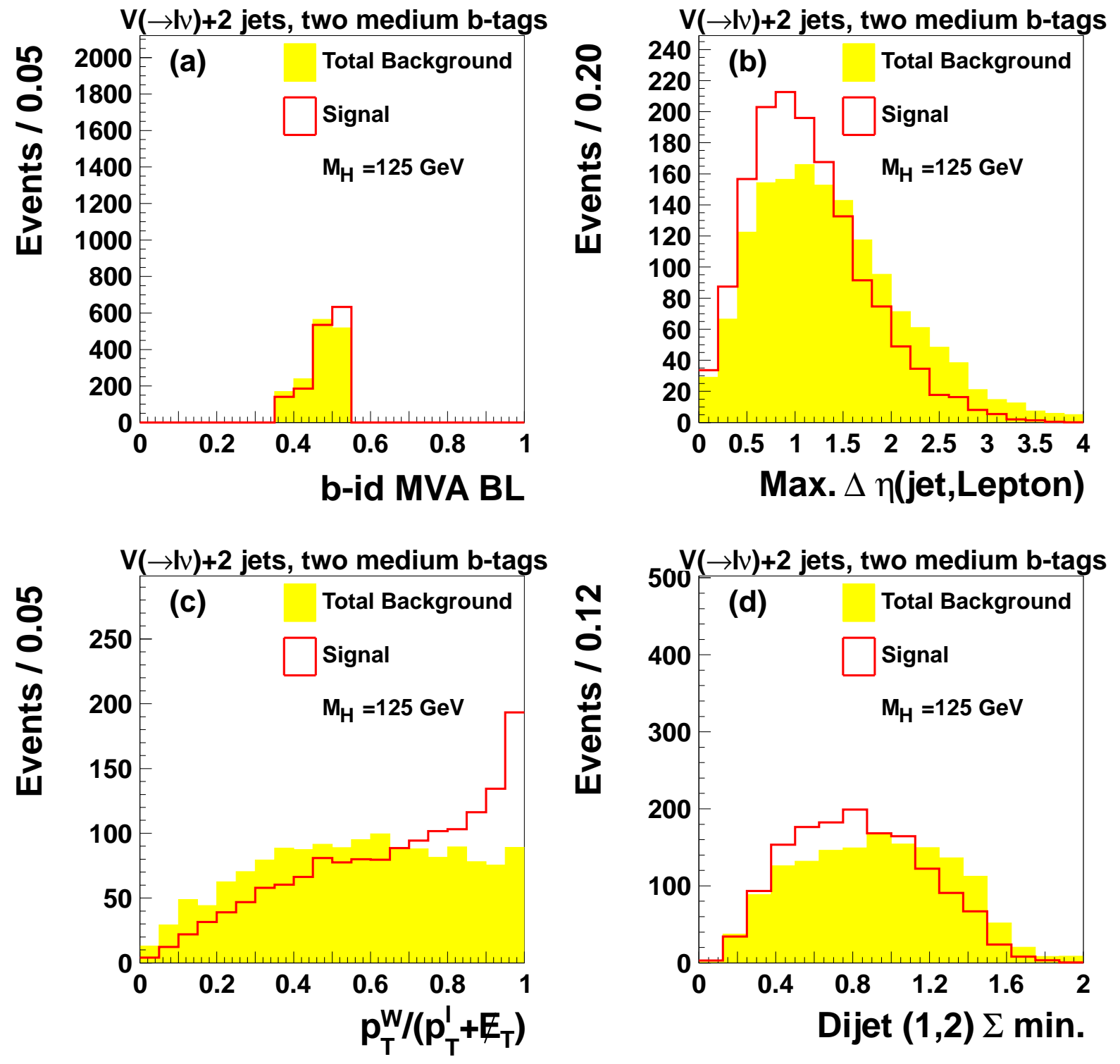

Figure B.45: Input variables to the BDT trained to distinguish signal from all backgrounds in events with two jets and two medium b-tag: (top left) the bid MVA output, (top right) the maximum $\Delta \eta(\ell, j)$, (bottom left) $q^{\ell} \times \eta^{\ell}$, and (bottom right) the minimum significance of the dijet system. The signal is shown for $M_{H}=125 \mathrm{GeV}$, and is normalized to the sum of the backgrounds. Overflow events are added to the last bin. 

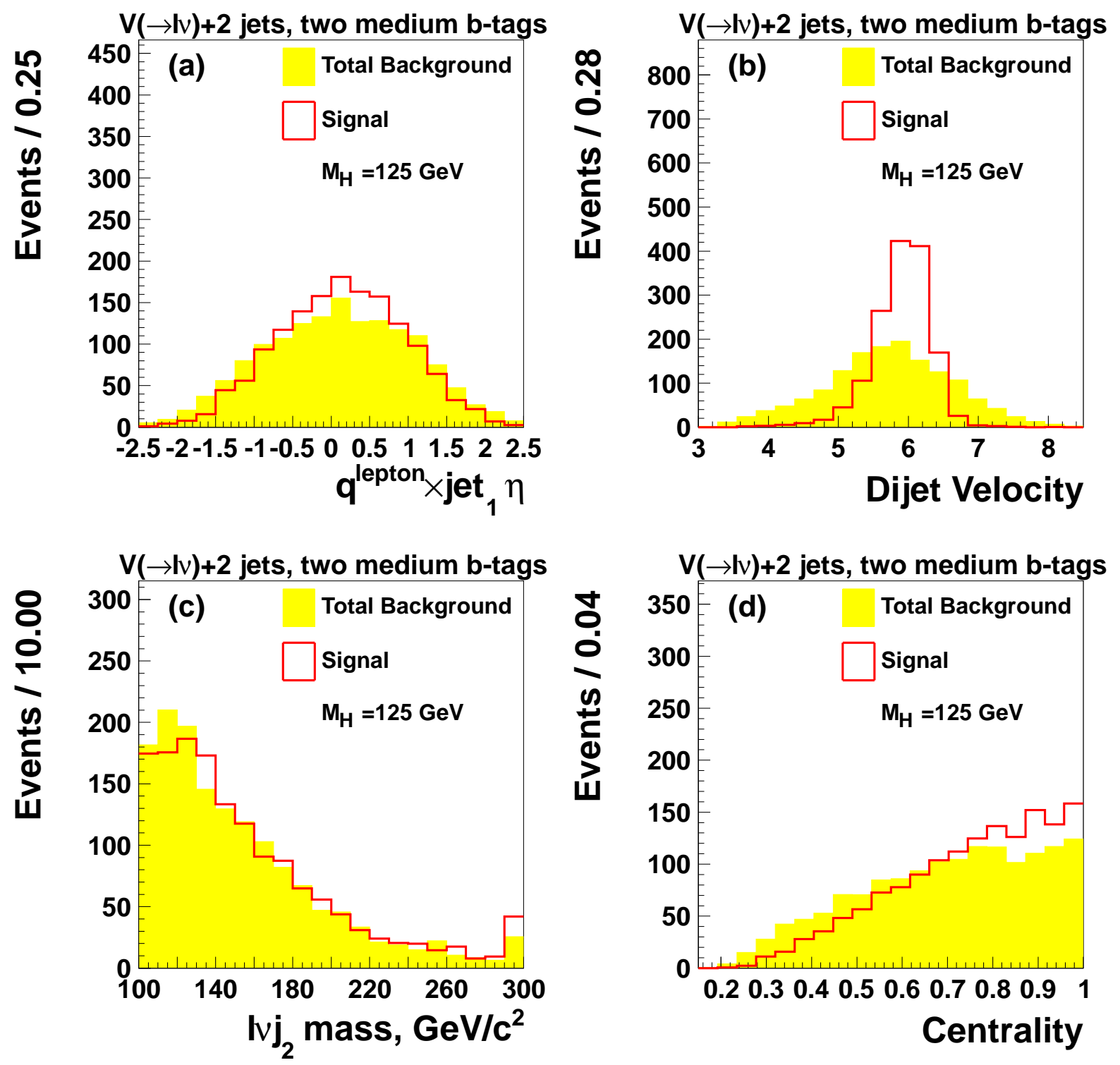

Figure B.46: Input variables to the BDT trained to distinguish signal from all backgrounds in events with two jets and two medium b-tag. The signal is shown for $M_{H}=125 \mathrm{GeV}$, and is normalized to the sum of the backgrounds. Overflow events are added to the last bin. 

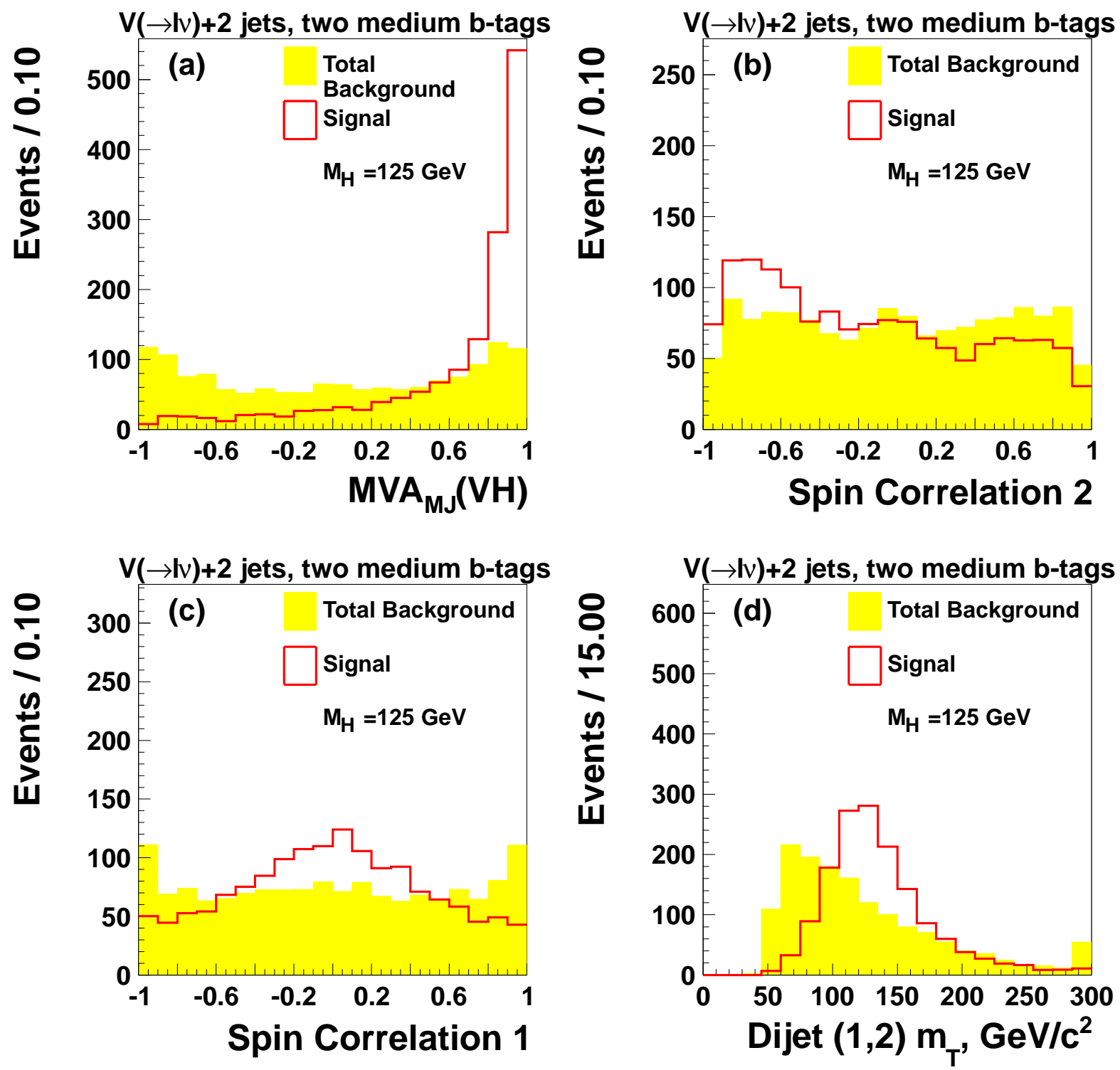

Figure B.47: Input variables to the BDT trained to distinguish signal from all backgrounds in events with two jets and two medium b-tag: (top left) the multijet MVA output, (top right) $\cos \left(\chi^{*}\right)$, (bottom left) $\cos \left(\theta^{*}\right.$, and (bottom right) the dijet transverse mass. The signal is shown for $M_{H}=125 \mathrm{GeV}$, and is normalized to the sum of the backgrounds. Overflow events are added to the last bin. 


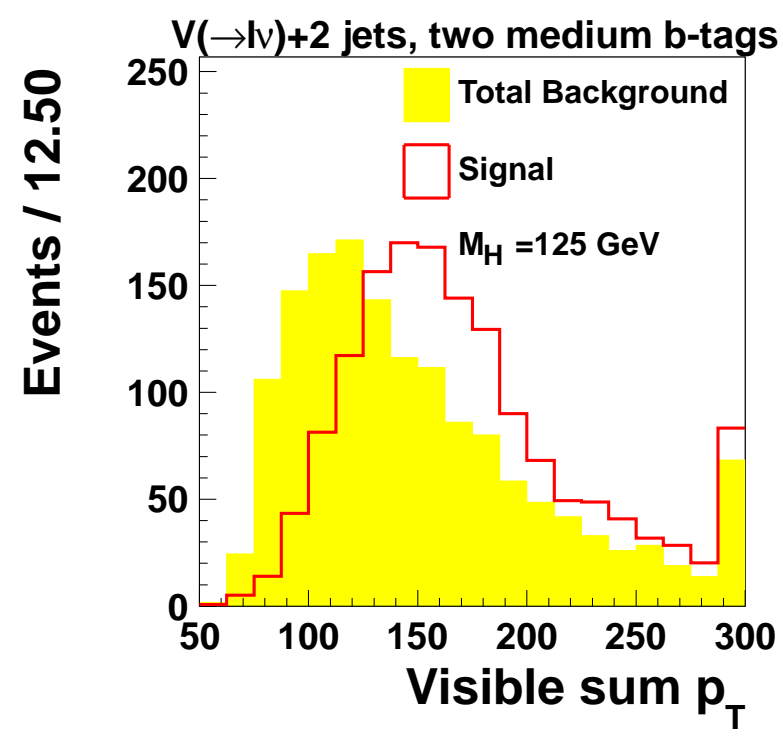

Figure B.48: Input variables to the BDT trained to distinguish signal from all backgrounds in events with two jets and two medium b-tags: the magnitude of the vector $p_{T}$ sum for all visible particles. The signal is shown for $M_{H}=125 \mathrm{GeV}$, and is normalized to the sum of the backgrounds. Overflow events are added to the last bin. 

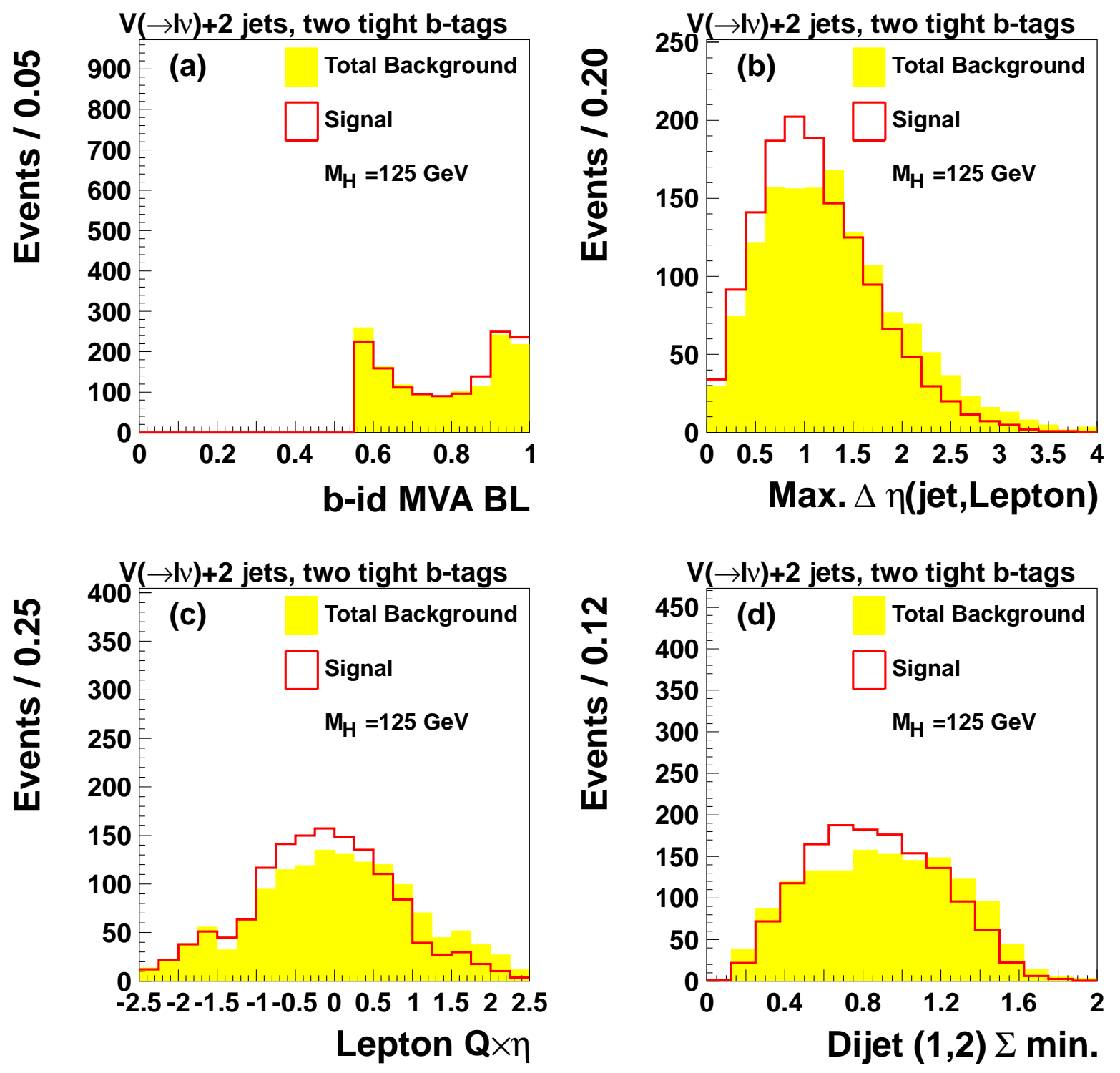

Figure B.49: Input variables to the BDT trained to distinguish signal from all backgrounds in events with two jets and two tight b-tag: (top left) the bid MVA output, (top right) the maximum $\Delta \eta(\ell, j)$, (bottom left) $q^{\ell} \times \eta^{\ell}$, and (bottom right) the minimum significance of the dijet system. The signal is shown for $M_{H}=125 \mathrm{GeV}$, and is normalized to the sum of the backgrounds. Overflow events are added to the last bin. 

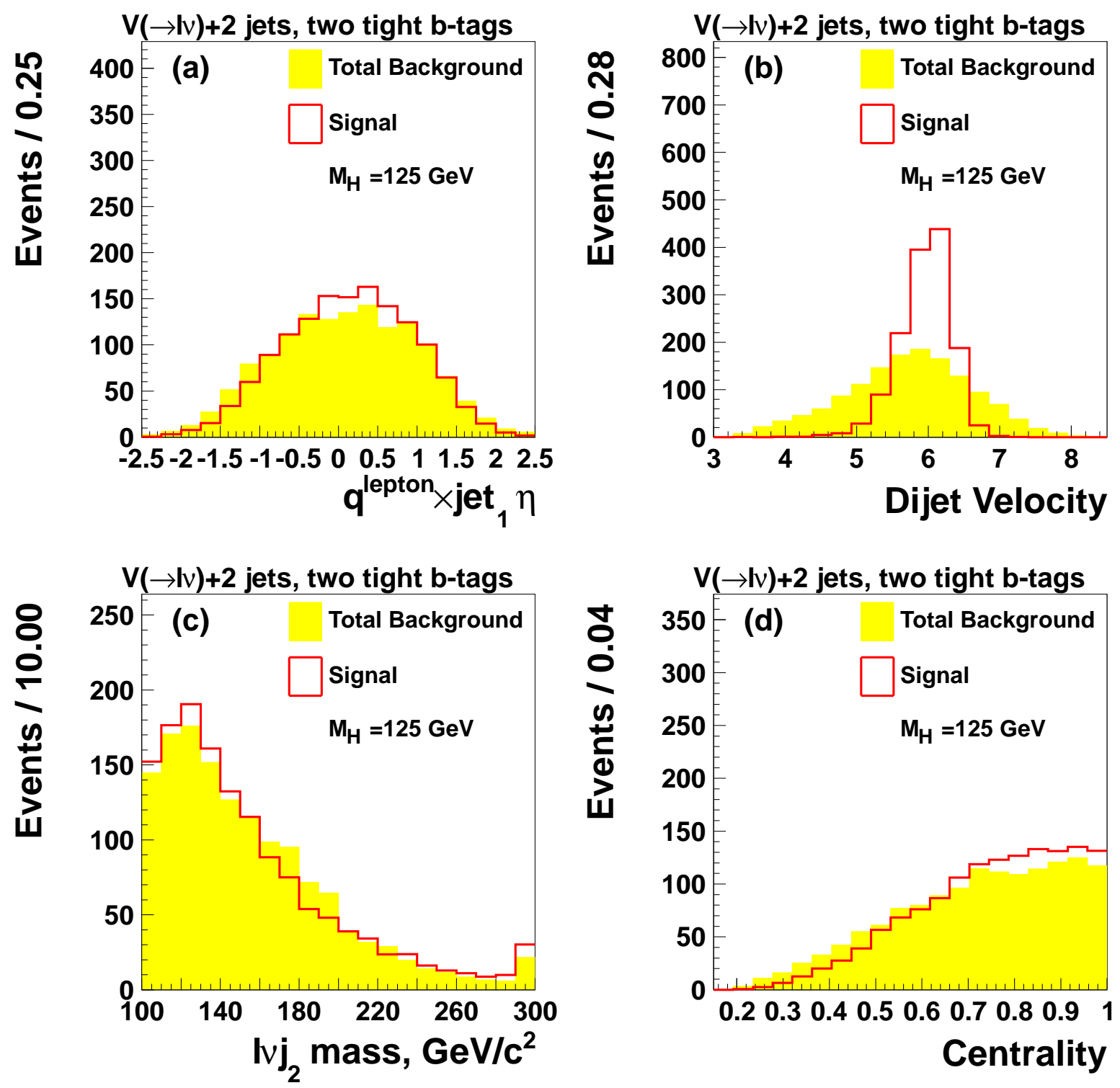

Figure B.50: Input variables to the BDT trained to distinguish signal from all backgrounds in events with two jets and two tight b-tag. The signal is shown for $M_{H}=125 \mathrm{GeV}$, and is normalized to the sum of the backgrounds. Overflow events are added to the last bin. 

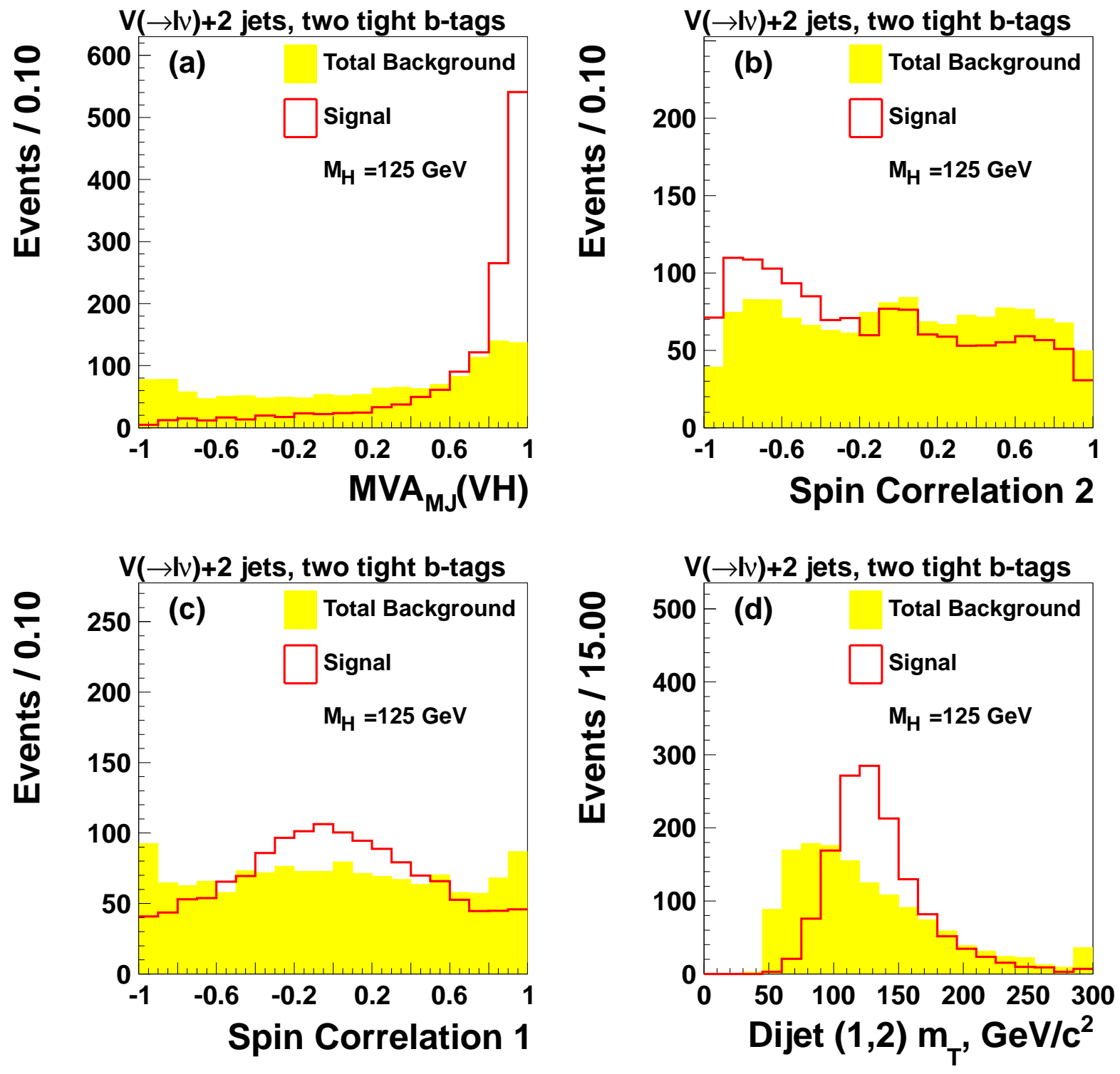

Figure B.51: Input variables to the BDT trained to distinguish signal from all backgrounds in events with two jets and two tight b-tag: (top left) the multijet MVA output, (top right) $\cos \left(\chi^{*}\right)$, (bottom left) $\cos \left(\theta^{*}\right.$, and (bottom right) the dijet transverse mass. The signal is shown for $M_{H}=125 \mathrm{GeV}$, and is normalized to the sum of the backgrounds. Overflow events are added to the last bin. 


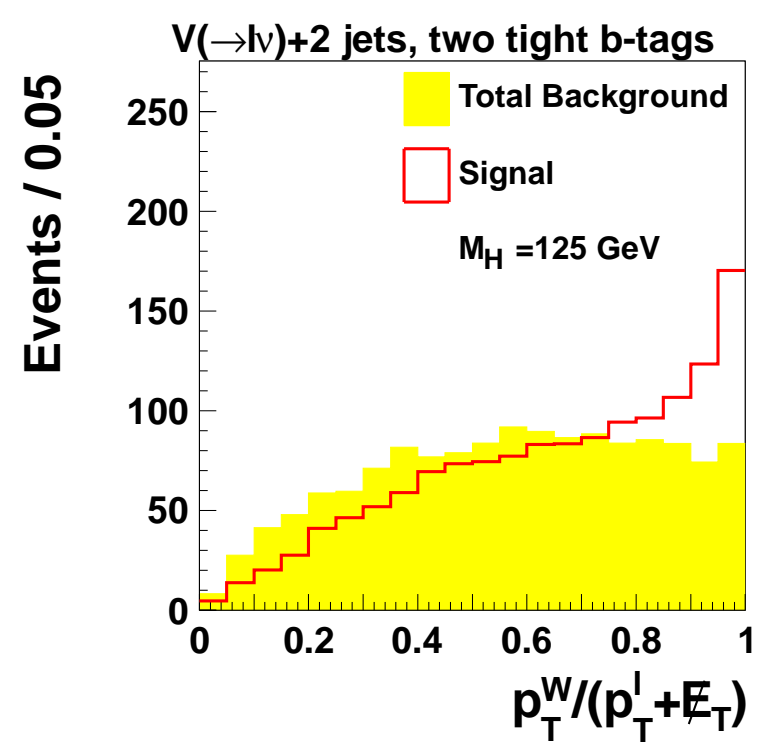

Figure B.52: Input variables to the BDT trained to distinguish signal from all backgrounds in events with two jets and two tight b-tag: the ratio of the $W p_{T}$ to the sum of the lepton $p_{T}$ and $E_{T}$. The signal is shown for $M_{H}=125 \mathrm{GeV}$, and is normalized to the sum of the backgrounds. Overflow events are added to the last bin. 

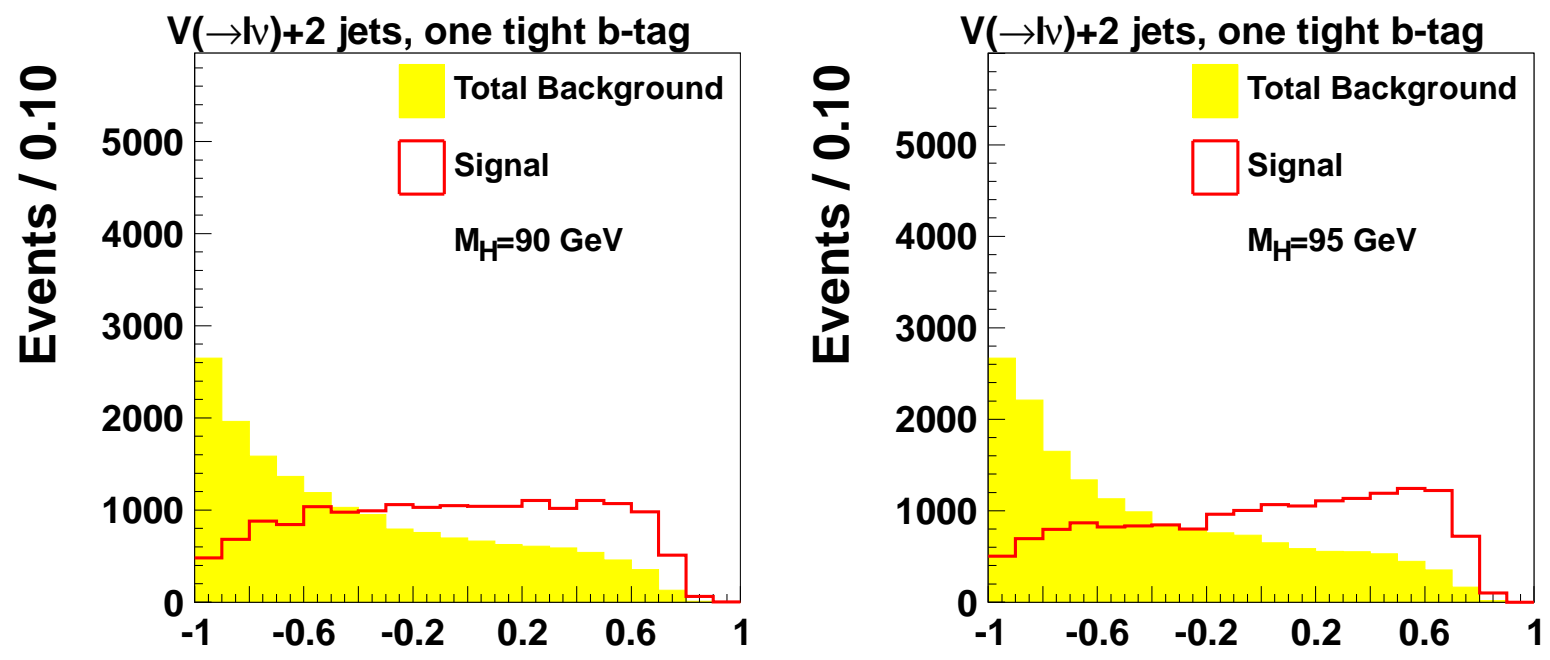

Final Discriminant

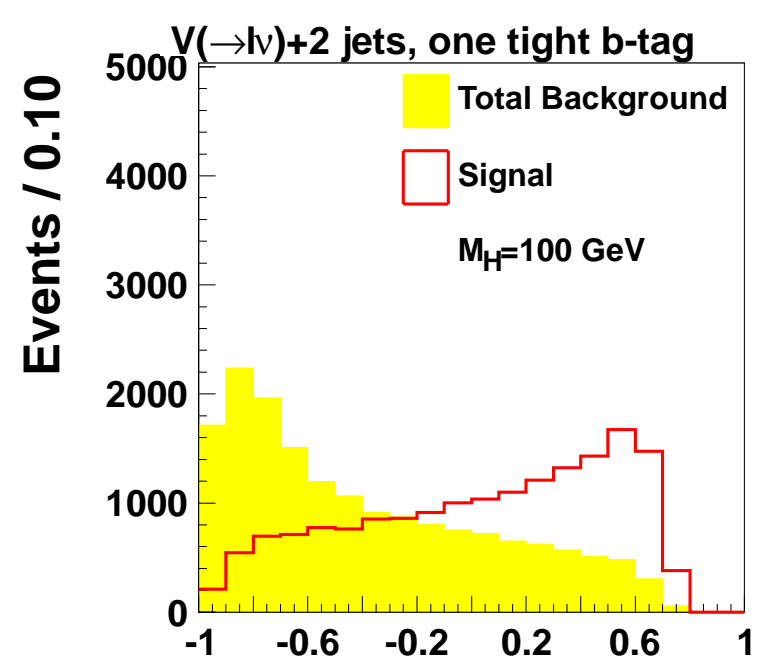

Final Discriminant

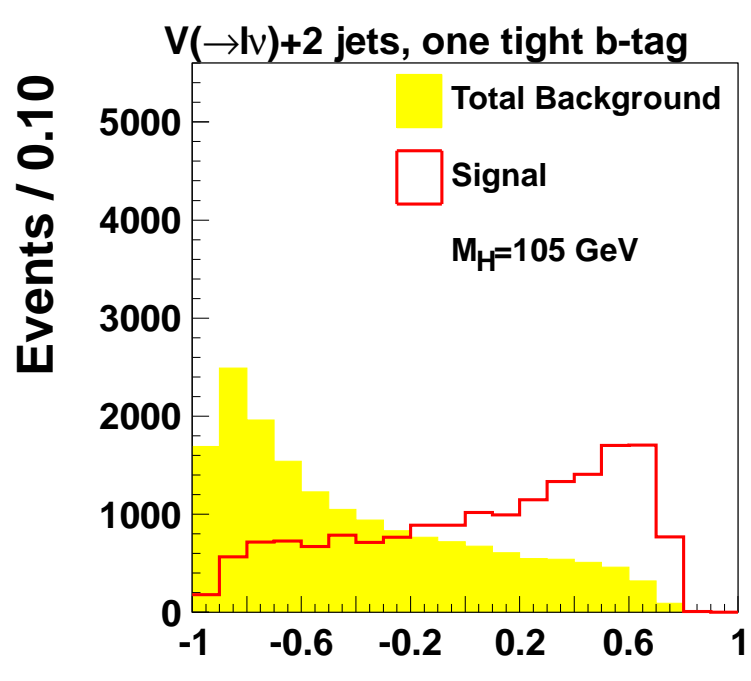
Final Discriminant

Figure B.53: The final MVA output for events with two jets and one tight b-tag for (top left) $M_{H}=90 \mathrm{GeV}$, (top right) $M_{H}=95 \mathrm{GeV}$, (bottom left) $M_{H}=100 \mathrm{GeV}$, and (bottom right) $M_{H}=105 \mathrm{GeV}$. The signal is normalized to the sum of the backgrounds. 


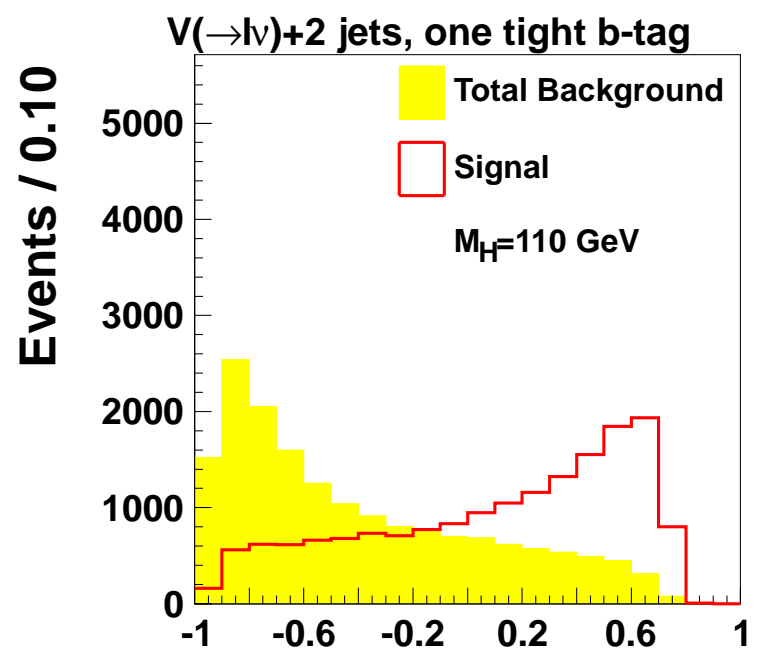

Final Discriminant

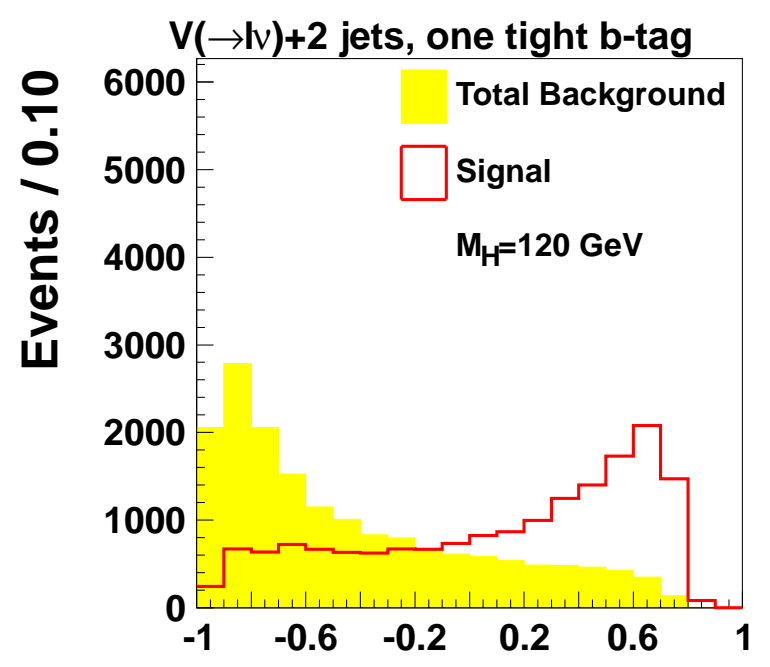

Final Discriminant

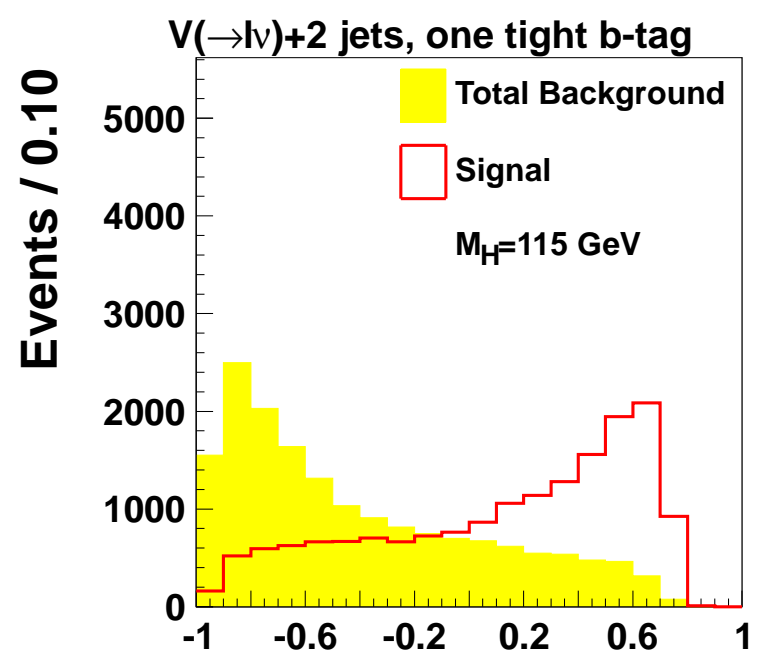

Final Discriminant

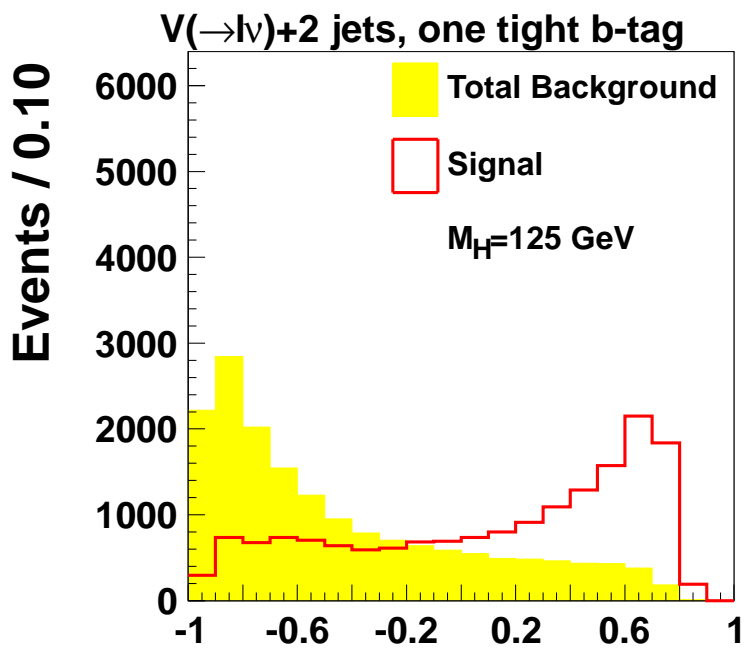

Final Discriminant

Figure B.54: The final MVA output for events with two jets and one tight b-tag for (top left) $M_{H}=110 \mathrm{GeV}$, (top right) $M_{H}=115 \mathrm{GeV}$, (bottom left) $M_{H}=120 \mathrm{GeV}$, and (bottom right) $M_{H}=125 \mathrm{GeV}$. The signal is normalized to the sum of the backgrounds. 


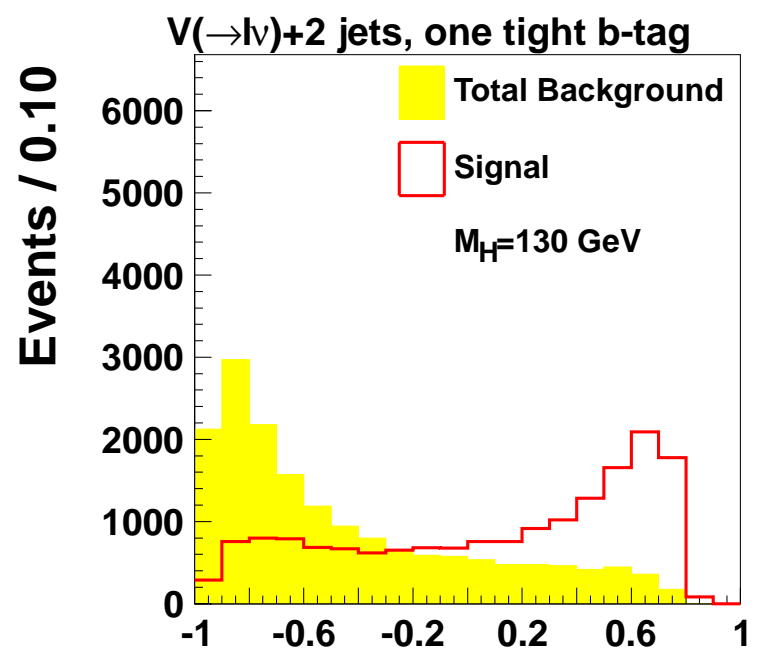

Final Discriminant

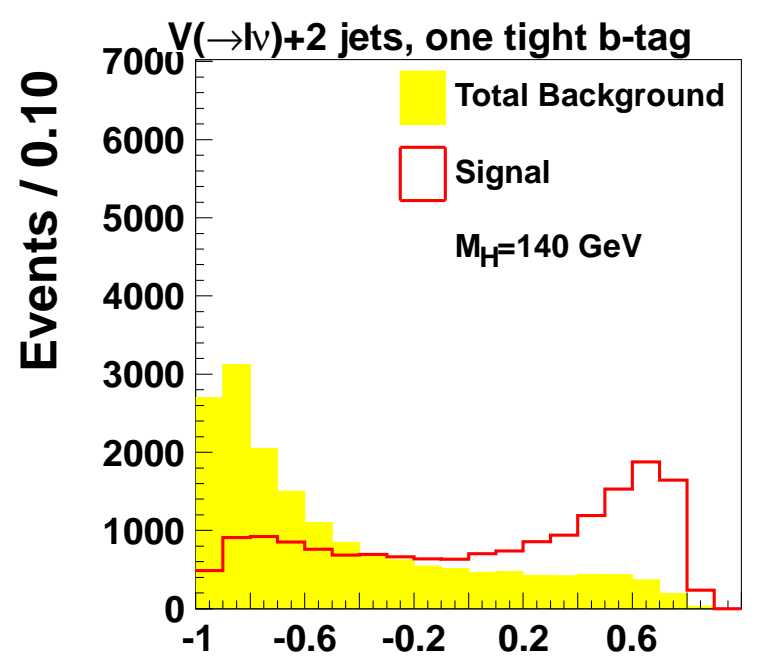

Final Discriminant

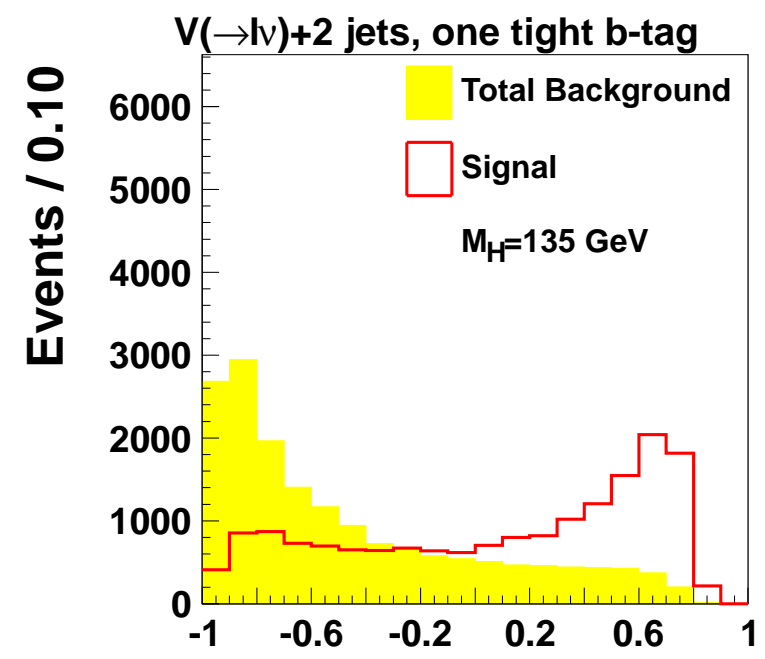

Final Discriminant

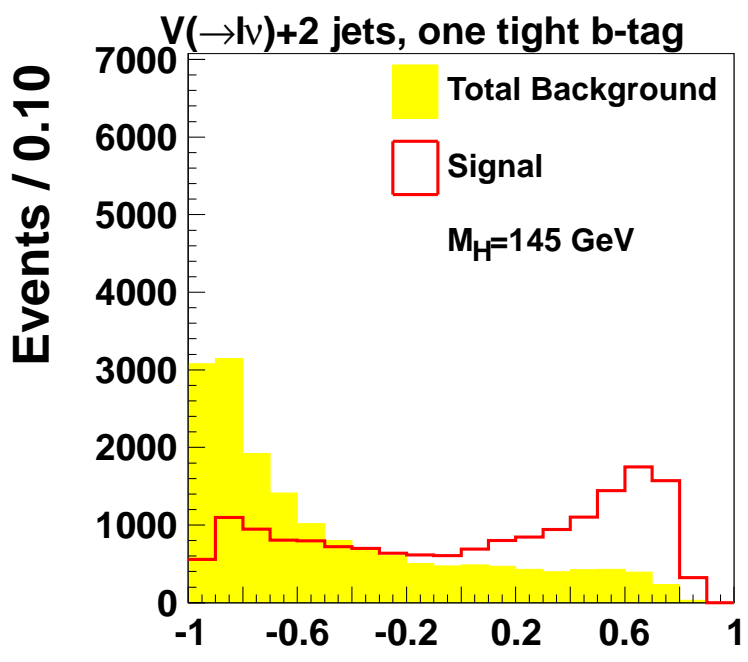

Final Discriminant

Figure B.55: The final MVA output for events with two jets and one tight b-tag for (top left) $M_{H}=130 \mathrm{GeV}$, (top right) $M_{H}=135 \mathrm{GeV}$, (bottom left) $M_{H}=140 \mathrm{GeV}$, and (bottom right) $M_{H}=145 \mathrm{GeV}$. The signal is normalized to the sum of the backgrounds. 


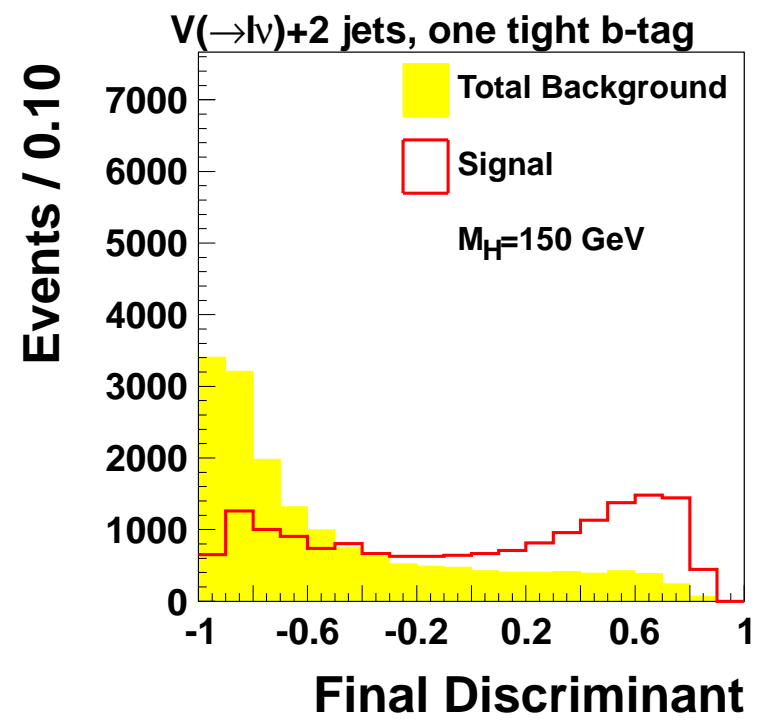

Figure B.56: The final MVA output for events with two jets and one tight b-tag for $M_{H}=$ $150 \mathrm{GeV}$. The signal is normalized to the sum of the backgrounds. 


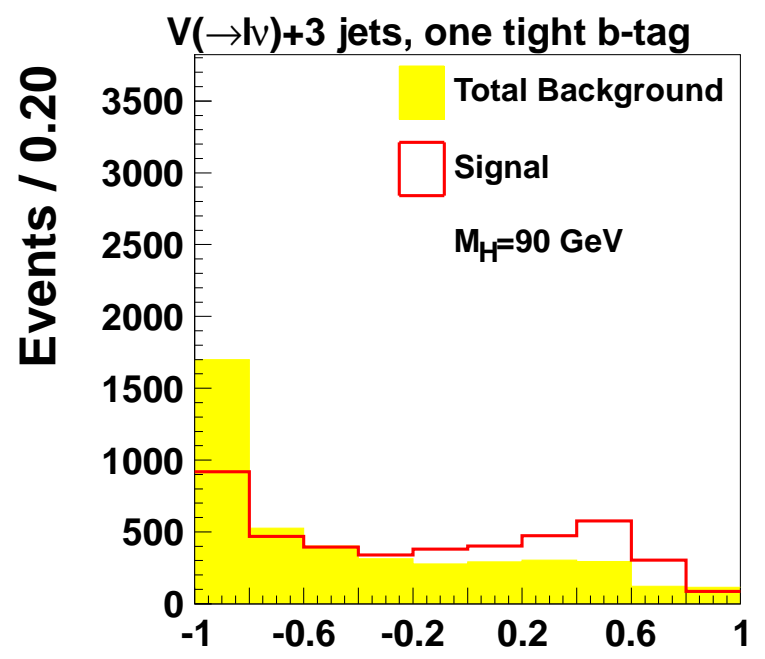

Final Discriminant

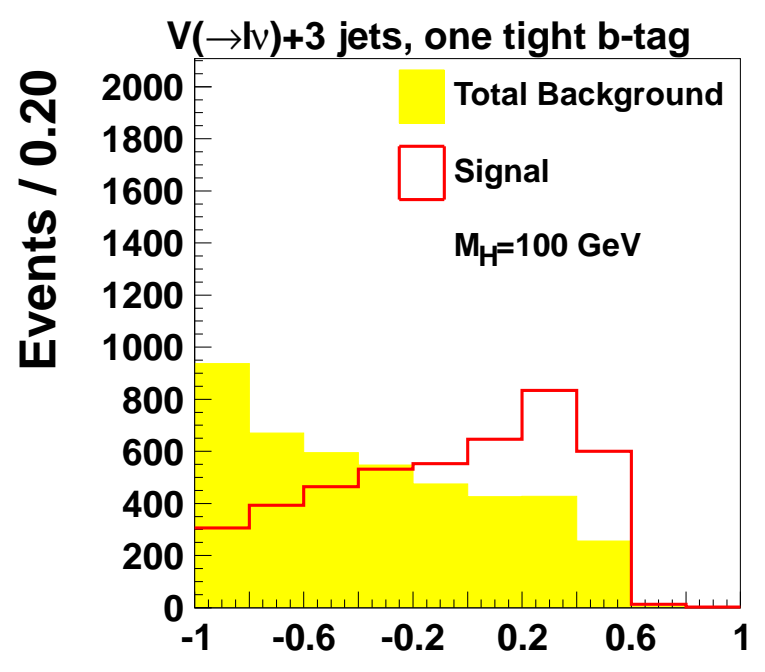

Final Discriminant

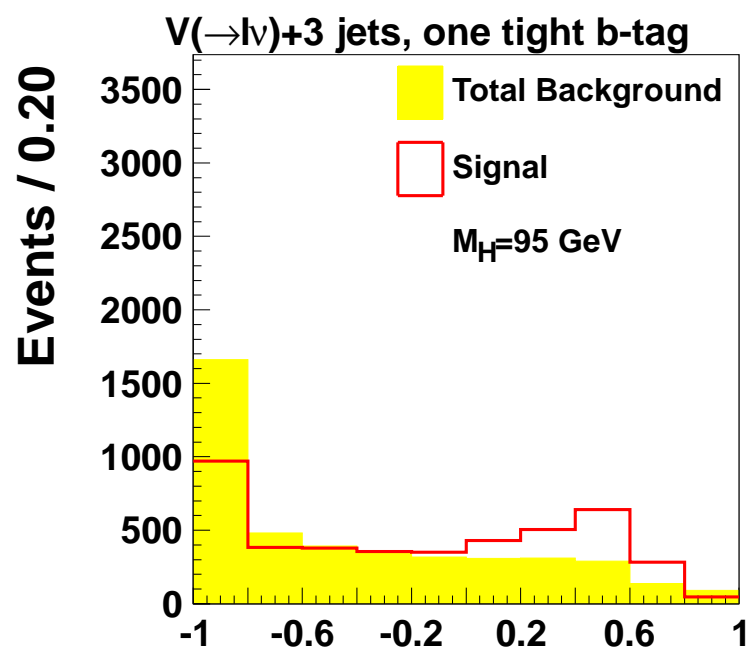

Final Discriminant

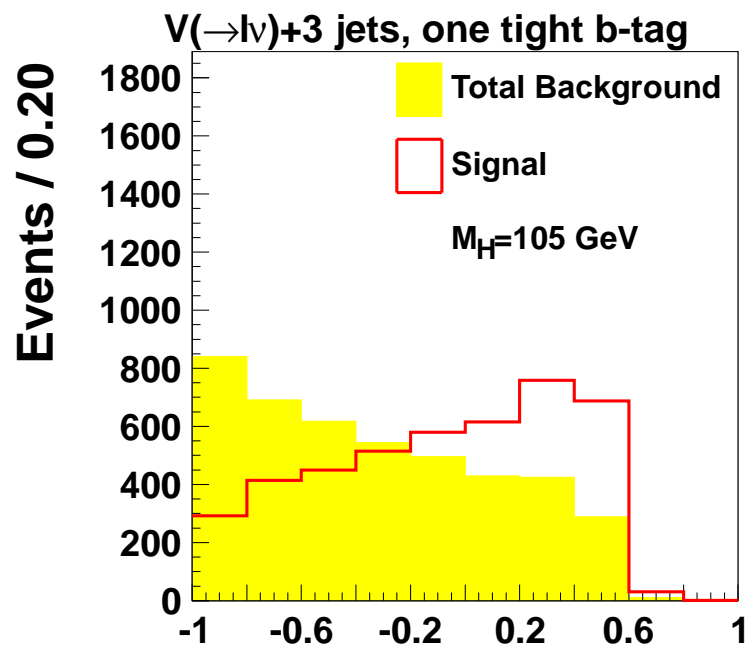

Final Discriminant

Figure B.57: The final MVA output for events with three jets and one tight b-tag for (top left) $M_{H}=90 \mathrm{GeV}$, (top right) $M_{H}=95 \mathrm{GeV}$, (bottom left) $M_{H}=100 \mathrm{GeV}$, and (bottom right) $M_{H}=105 \mathrm{GeV}$. The signal is normalized to the sum of the backgrounds. 


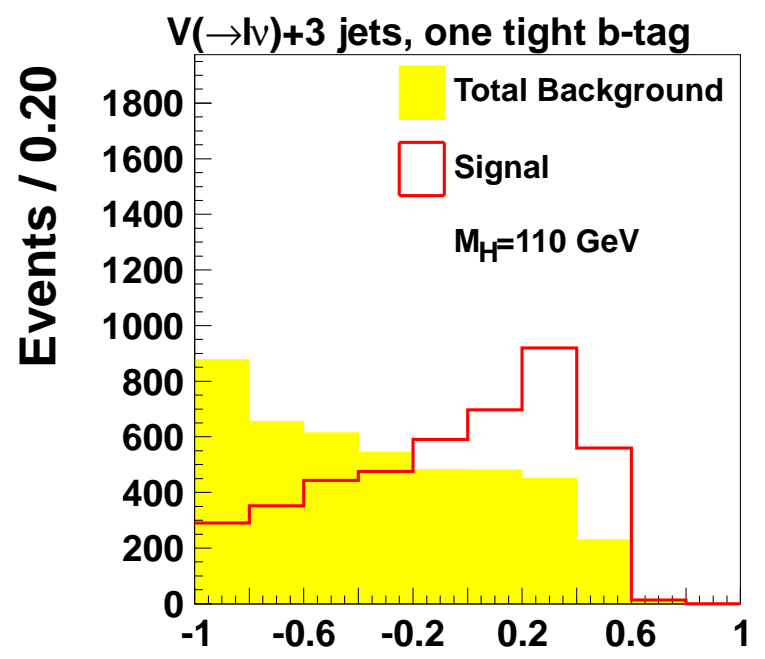

Final Discriminant

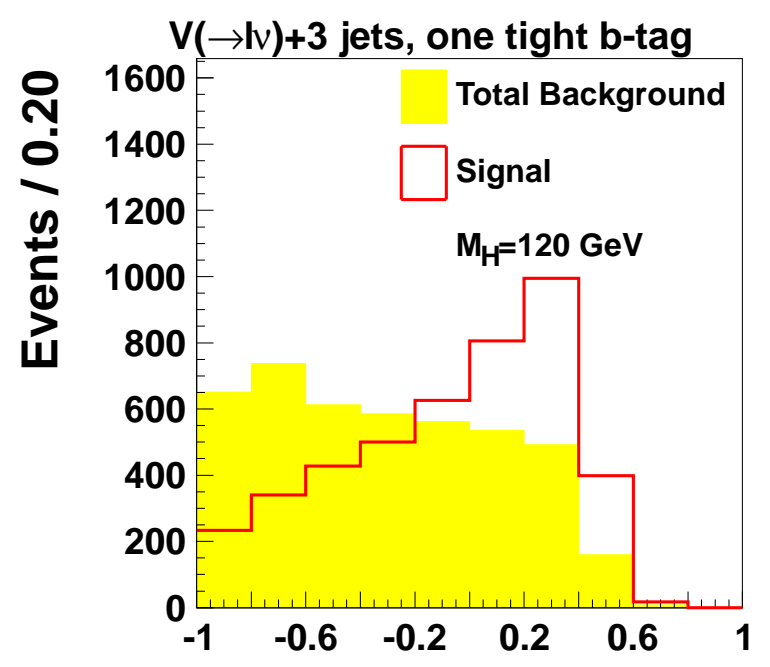

Final Discriminant

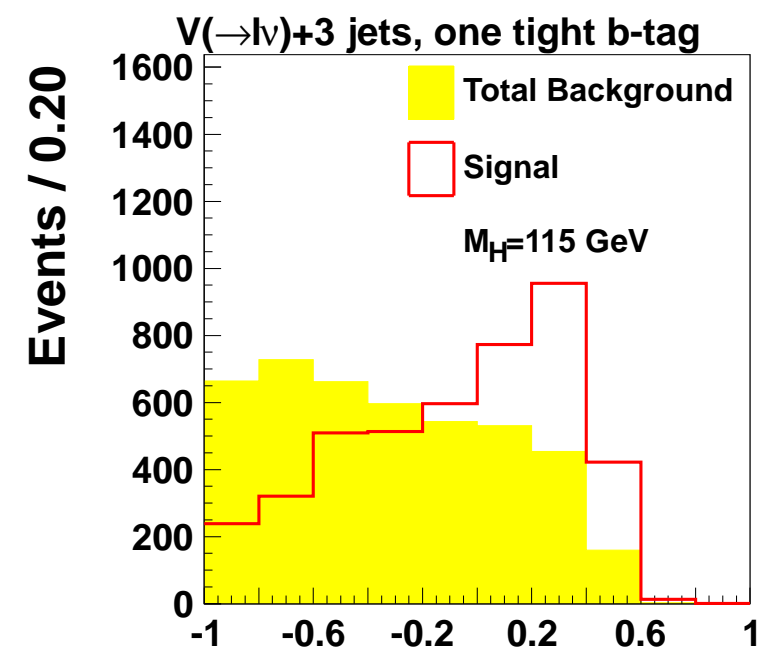

Final Discriminant

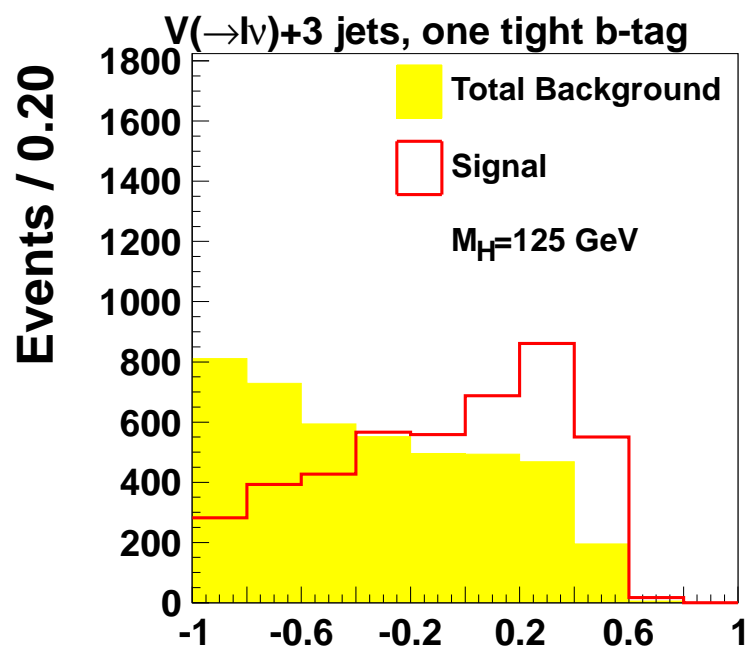

Final Discriminant

Figure B.58: The final MVA output for events with three jets and one tight b-tag for (top left) $M_{H}=110 \mathrm{GeV}$, (top right) $M_{H}=115 \mathrm{GeV}$, (bottom left) $M_{H}=120 \mathrm{GeV}$, and (bottom right) $M_{H}=125 \mathrm{GeV}$. The signal is normalized to the sum of the backgrounds. 


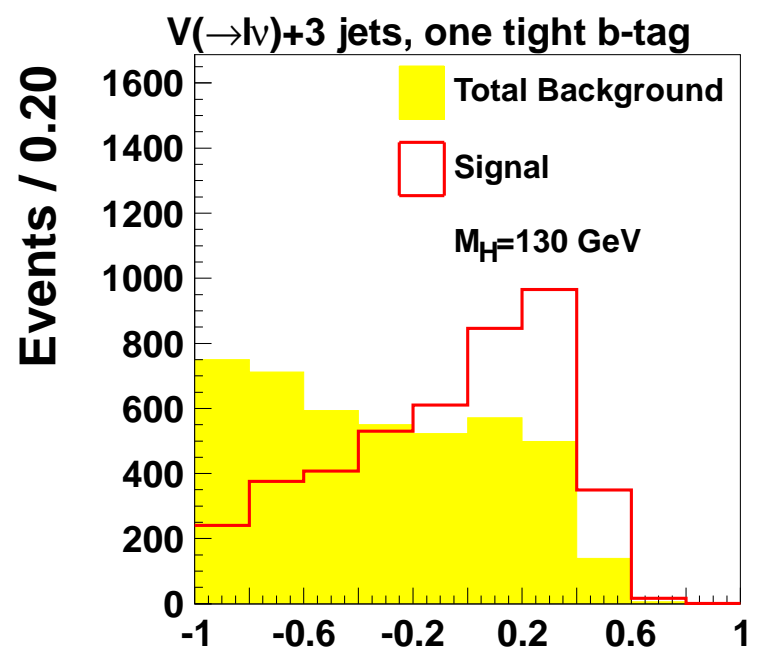

Final Discriminant

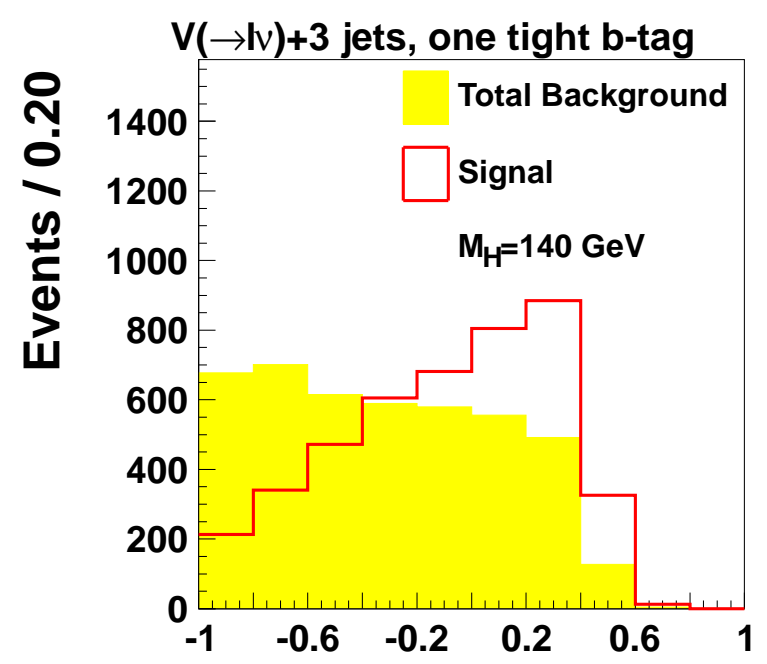

Final Discriminant

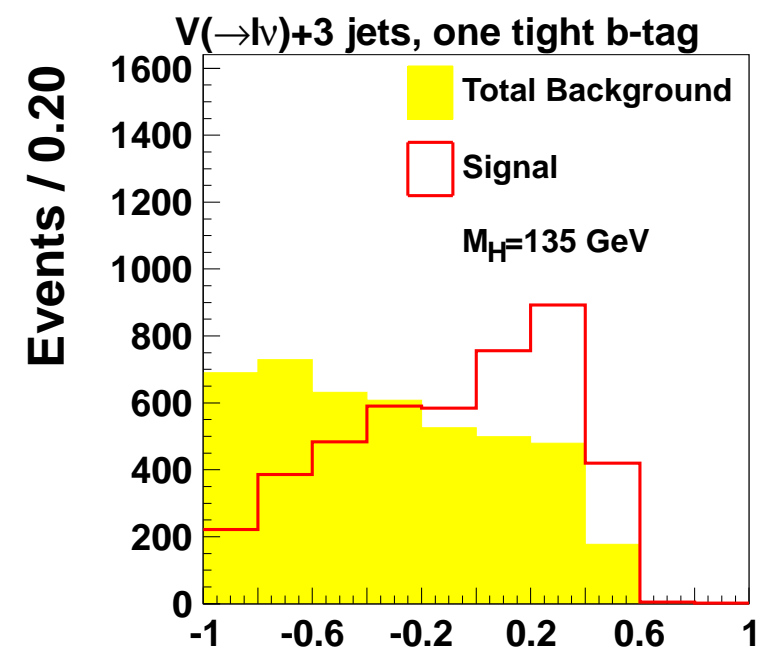

Final Discriminant

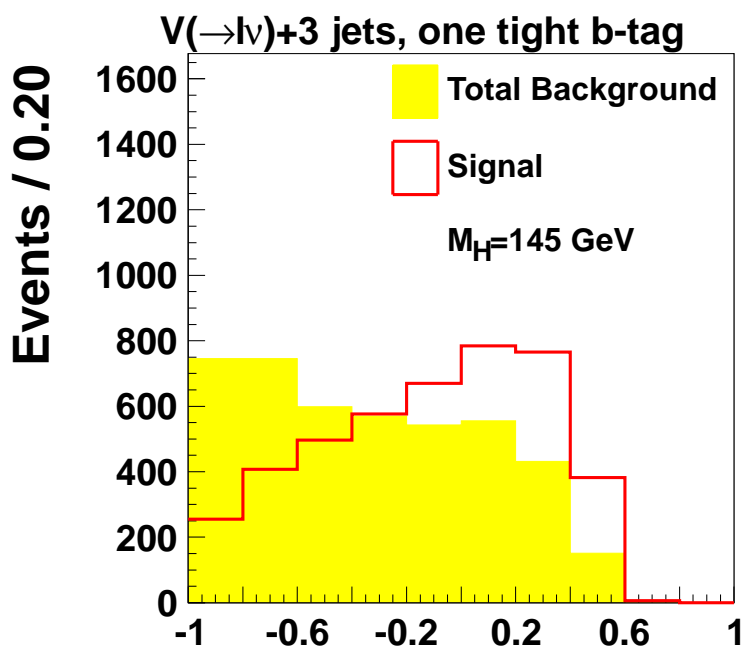

Final Discriminant

Figure B.59: The final MVA output for events with three jets and one tight b-tag for (top left) $M_{H}=130 \mathrm{GeV}$, (top right) $M_{H}=135 \mathrm{GeV}$, (bottom left) $M_{H}=140 \mathrm{GeV}$, and (bottom right) $M_{H}=145 \mathrm{GeV}$. The signal is normalized to the sum of the backgrounds. 


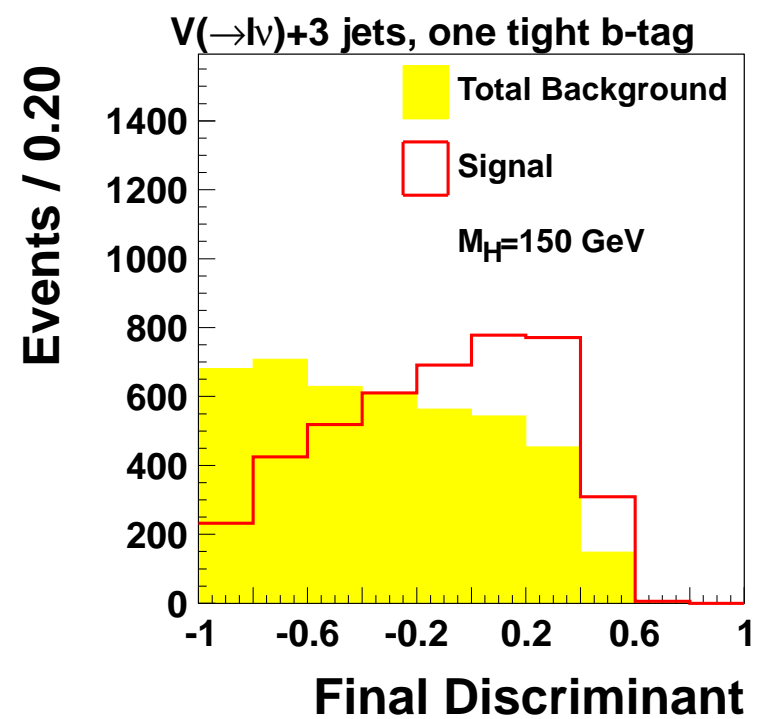

Figure B.60: The final MVA output for events with three jets and one tight b-tag for $M_{H}=150 \mathrm{GeV}$. The signal is normalized to the sum of the backgrounds. 


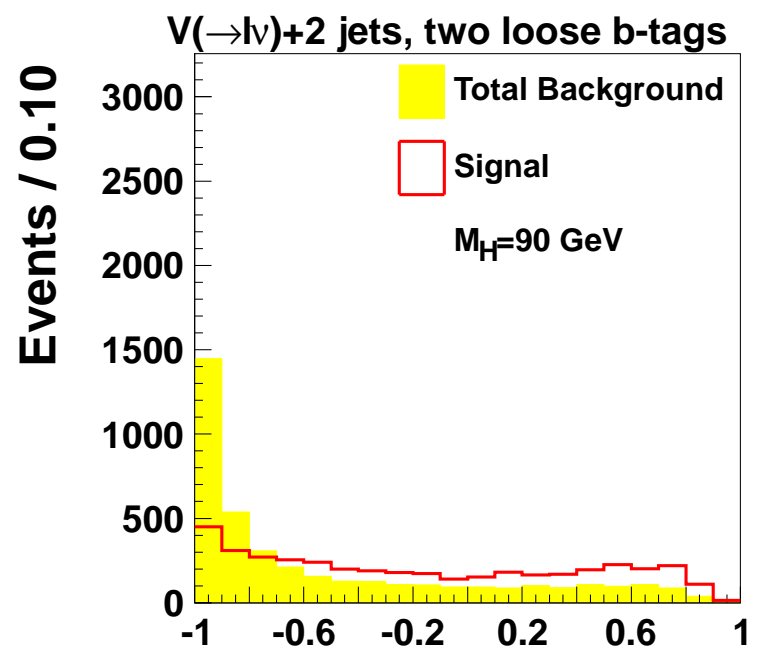

Final Discriminant

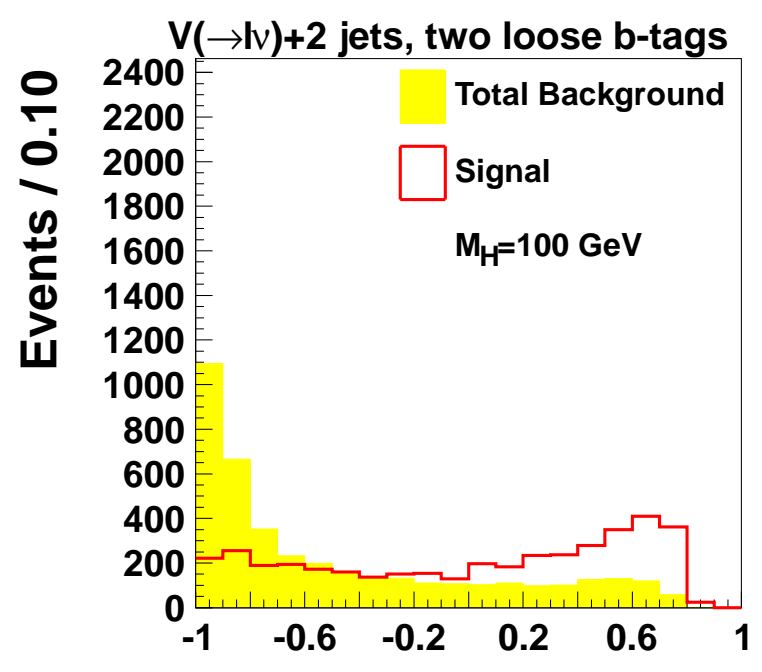

Final Discriminant

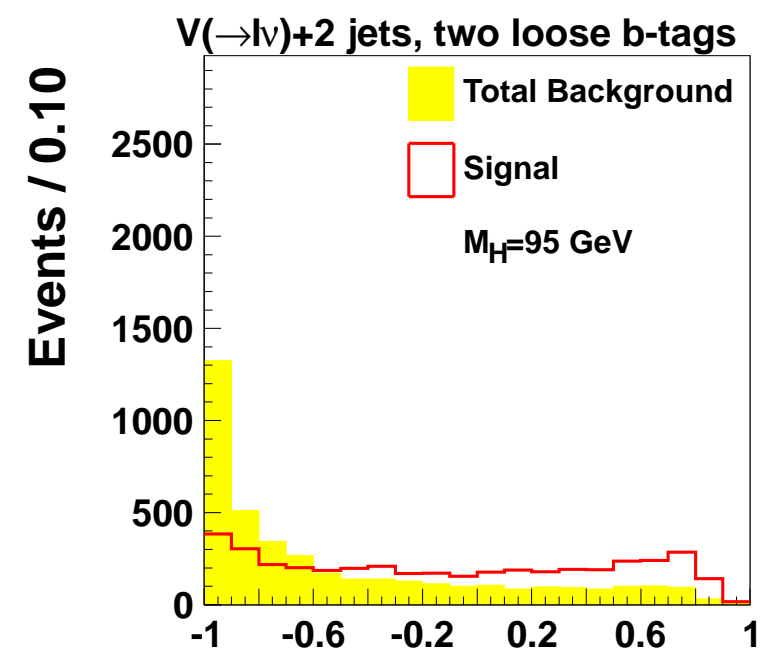

Final Discriminant

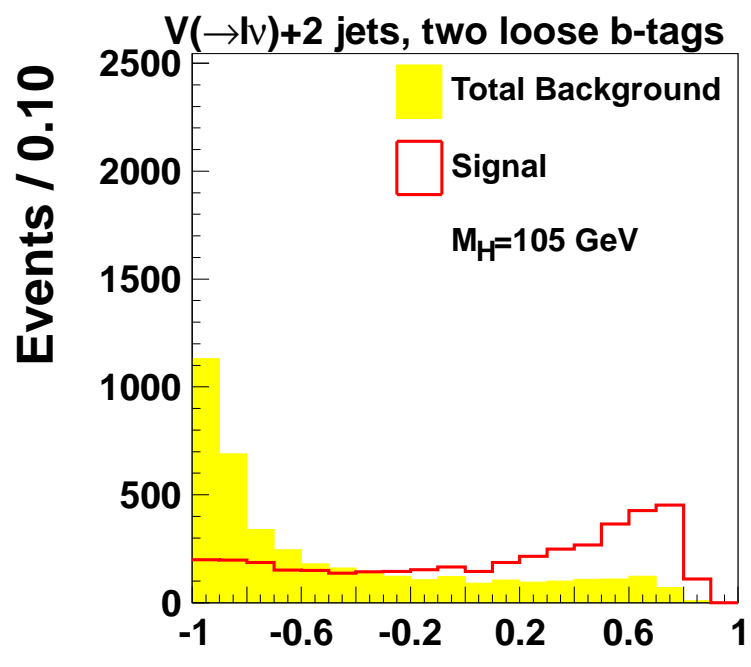

Final Discriminant

Figure B.61: The final MVA output for events with two jets and two loose b-tags for (top left) $M_{H}=90 \mathrm{GeV}$, (top right) $M_{H}=95 \mathrm{GeV}$, (bottom left) $M_{H}=100 \mathrm{GeV}$, and (bottom right) $M_{H}=105 \mathrm{GeV}$. The signal is normalized to the sum of the backgrounds. 


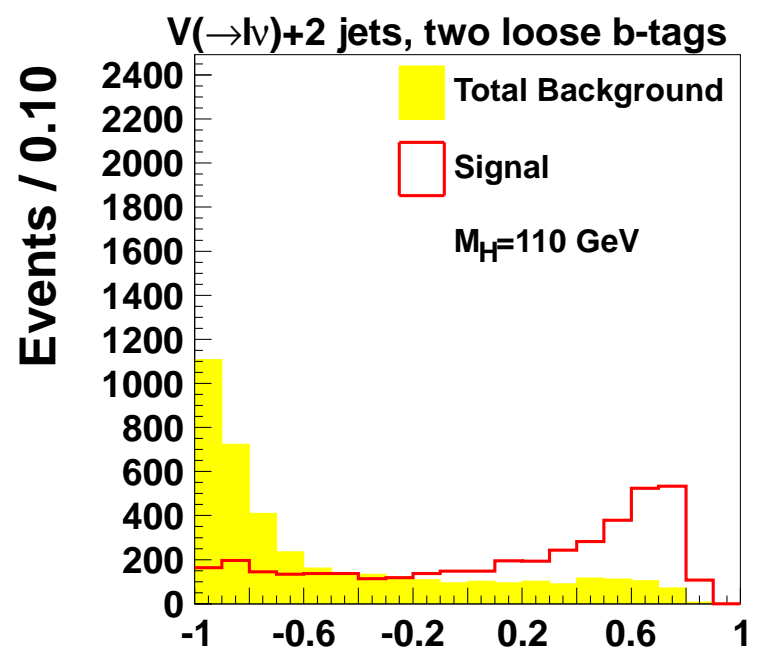

Final Discriminant

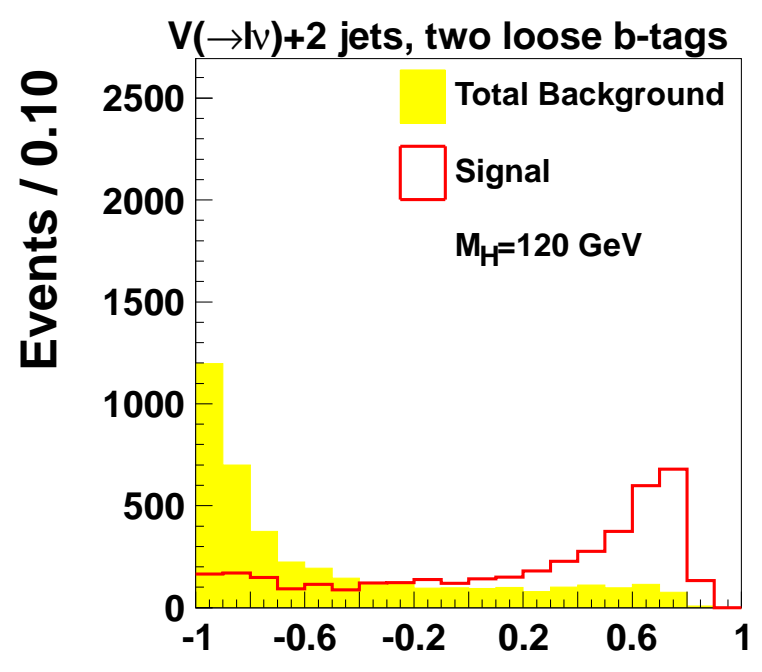

Final Discriminant

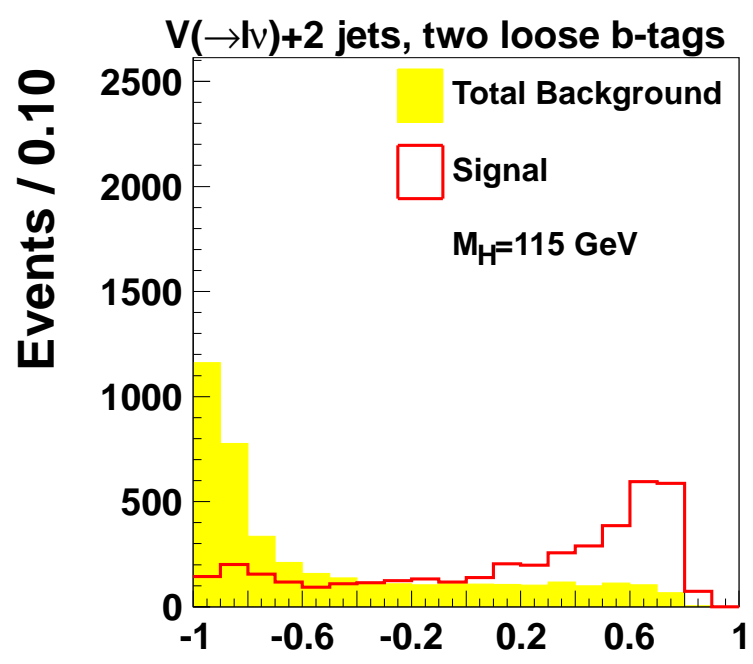

Final Discriminant

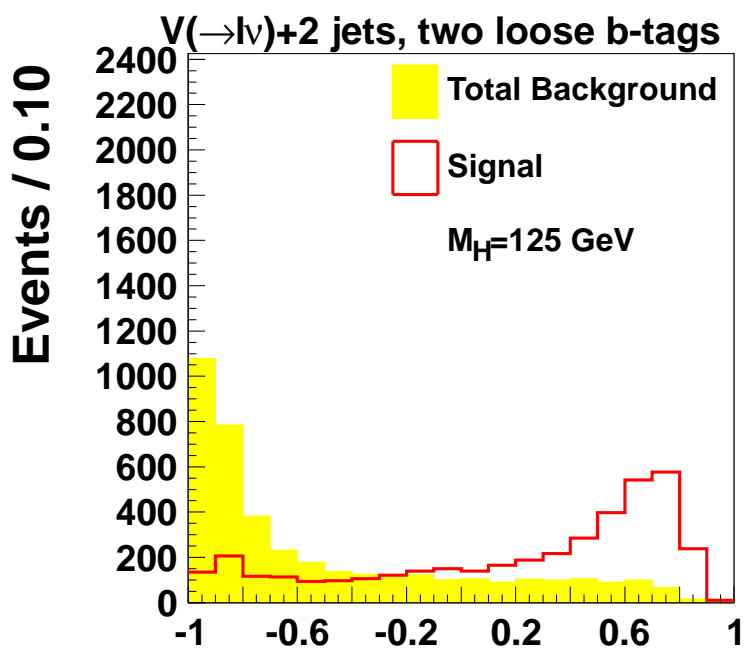

Final Discriminant

Figure B.62: The final MVA output for events with two jets and two loose b-tags for (top left) $M_{H}=110 \mathrm{GeV}$, (top right) $M_{H}=115 \mathrm{GeV}$, (bottom left) $M_{H}=120 \mathrm{GeV}$, and (bottom right) $M_{H}=125 \mathrm{GeV}$. The signal is normalized to the sum of the backgrounds. 


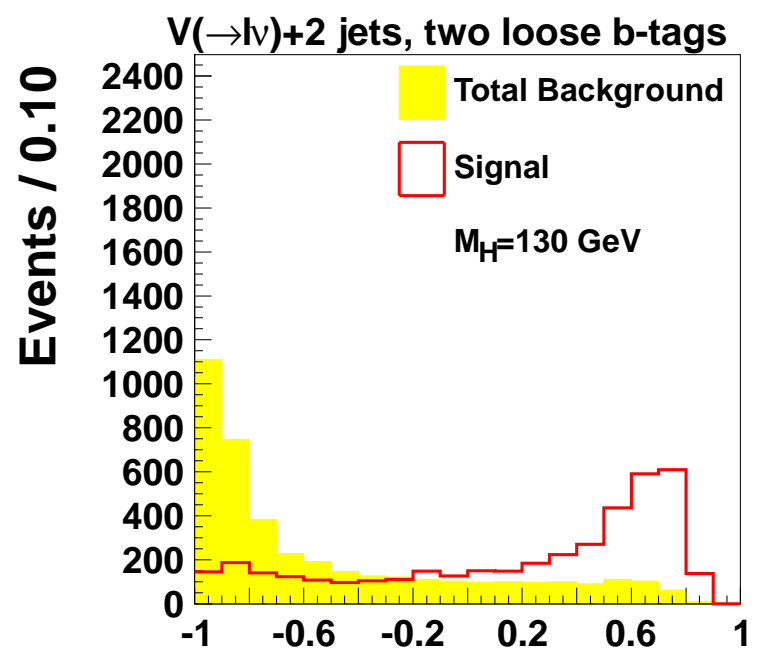

Final Discriminant

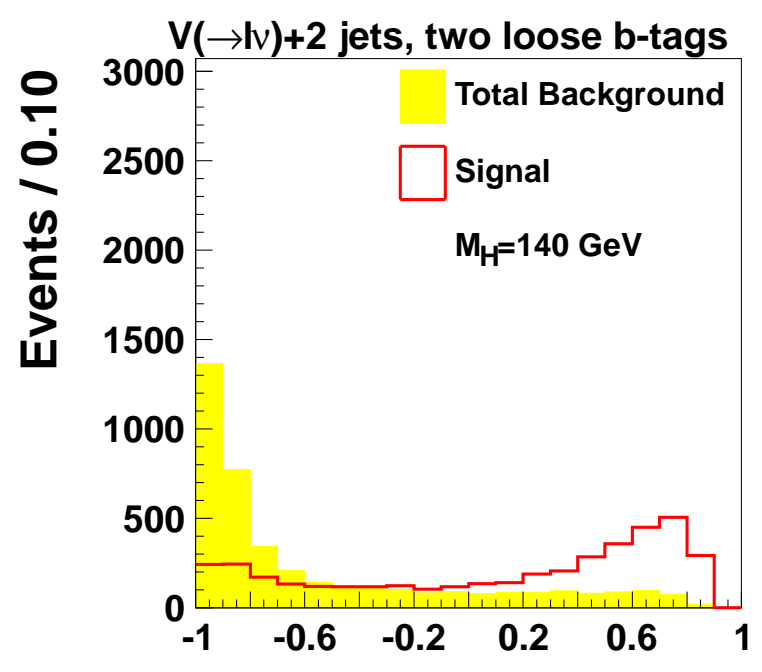

Final Discriminant

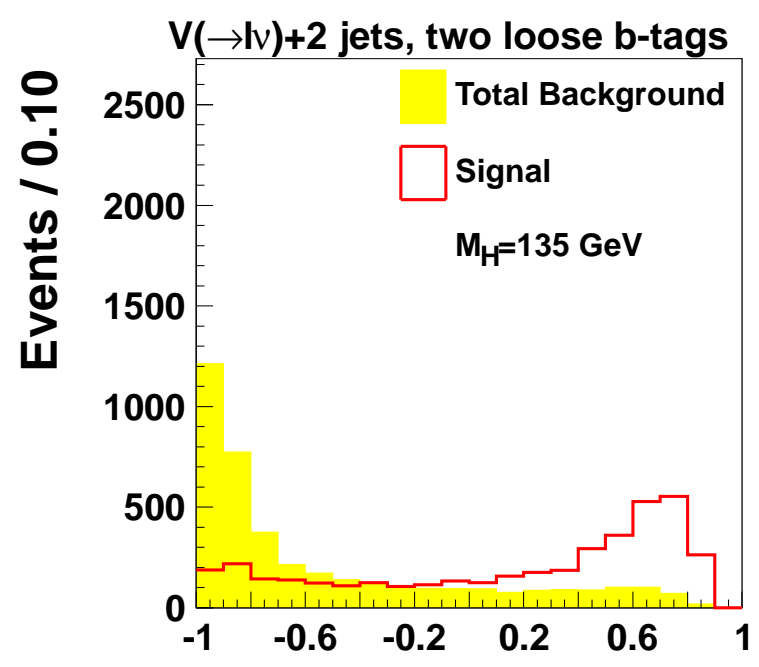

Final Discriminant

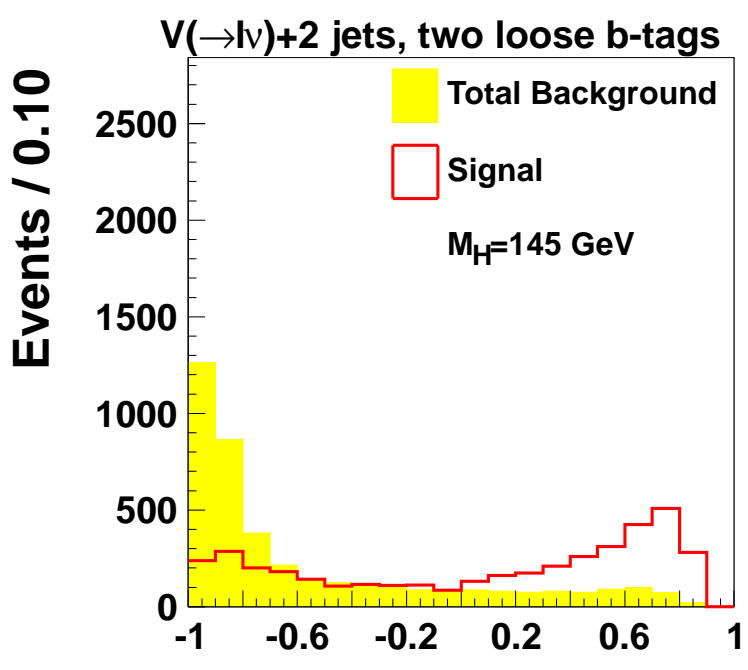

Final Discriminant

Figure B.63: The final MVA output for events with two jets and two loose b-tags for (top left) $M_{H}=130 \mathrm{GeV}$, (top right) $M_{H}=135 \mathrm{GeV}$, (bottom left) $M_{H}=140 \mathrm{GeV}$, and (bottom right) $M_{H}=145 \mathrm{GeV}$. The signal is normalized to the sum of the backgrounds. 


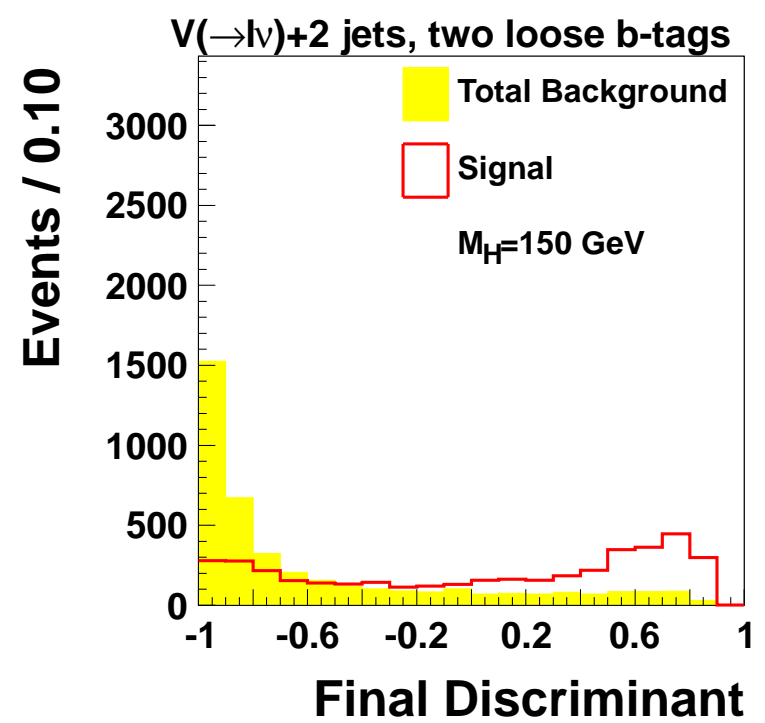

Figure B.64: The final MVA output for events with two jets and two loose b-tags for $M_{H}=$ $150 \mathrm{GeV}$. The signal is normalized to the sum of the backgrounds. 


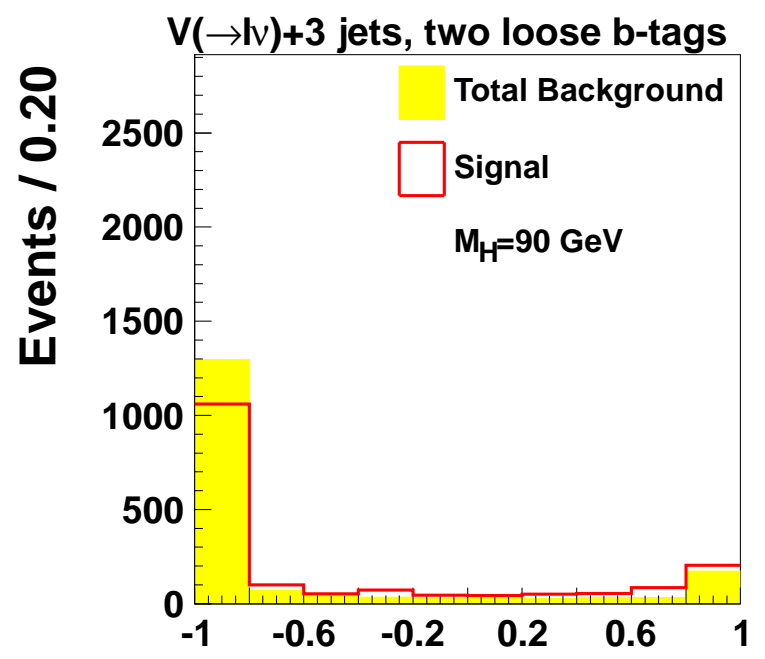

Final Discriminant

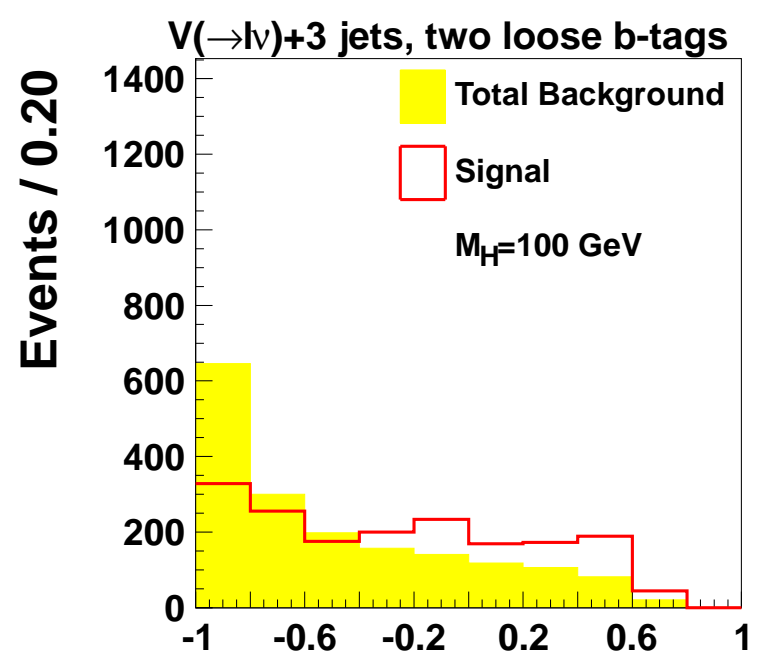

Final Discriminant

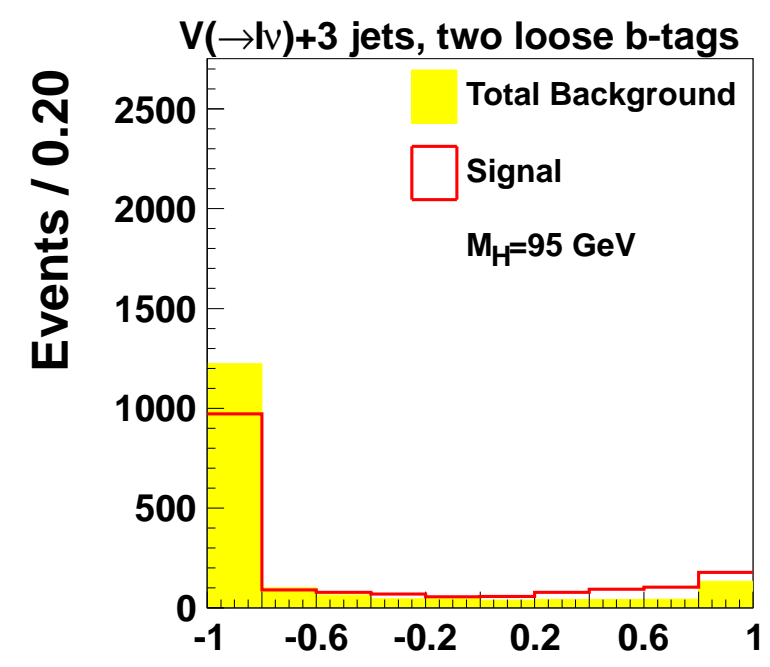

Final Discriminant

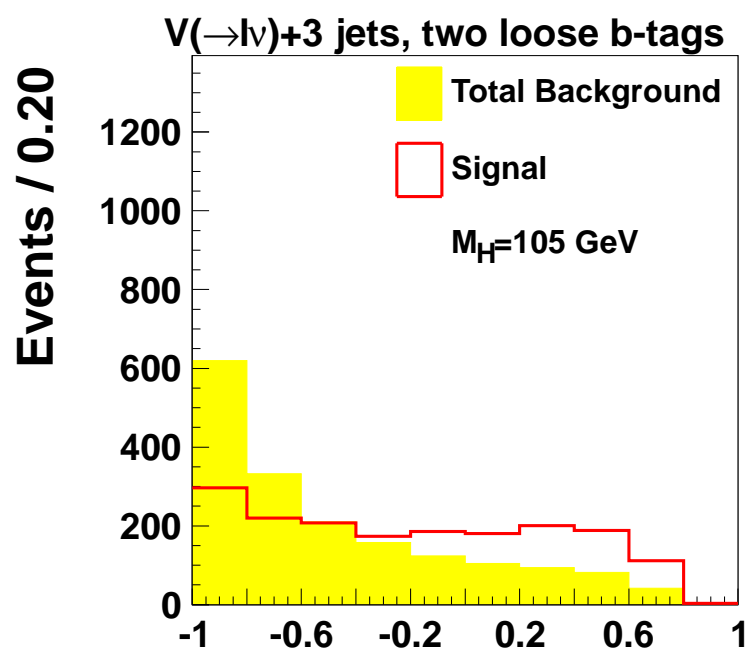

Final Discriminant

Figure B.65: The final MVA output for events with three jets and two loose b-tags for (top left) $M_{H}=90 \mathrm{GeV}$, (top right) $M_{H}=95 \mathrm{GeV}$, (bottom left) $M_{H}=100 \mathrm{GeV}$, and (bottom right) $M_{H}=105 \mathrm{GeV}$. The signal is normalized to the sum of the backgrounds. 


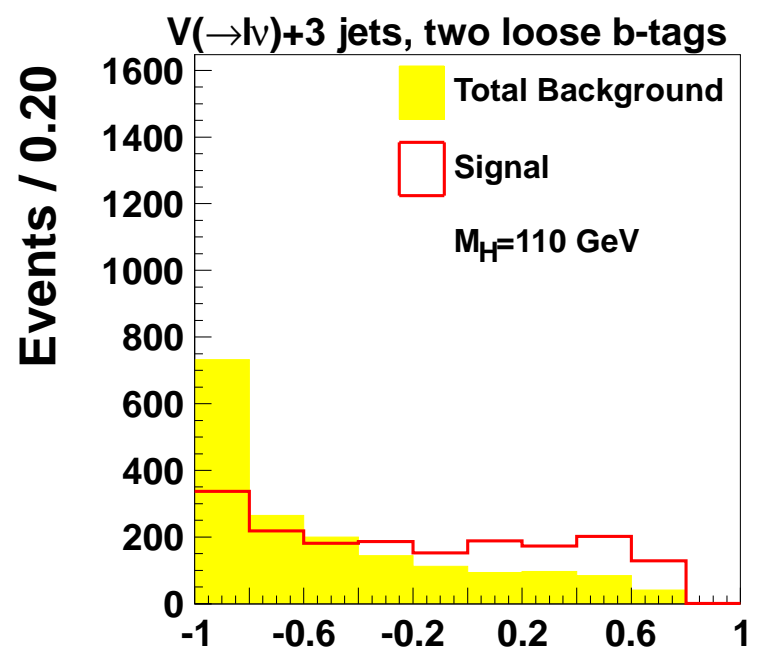

Final Discriminant

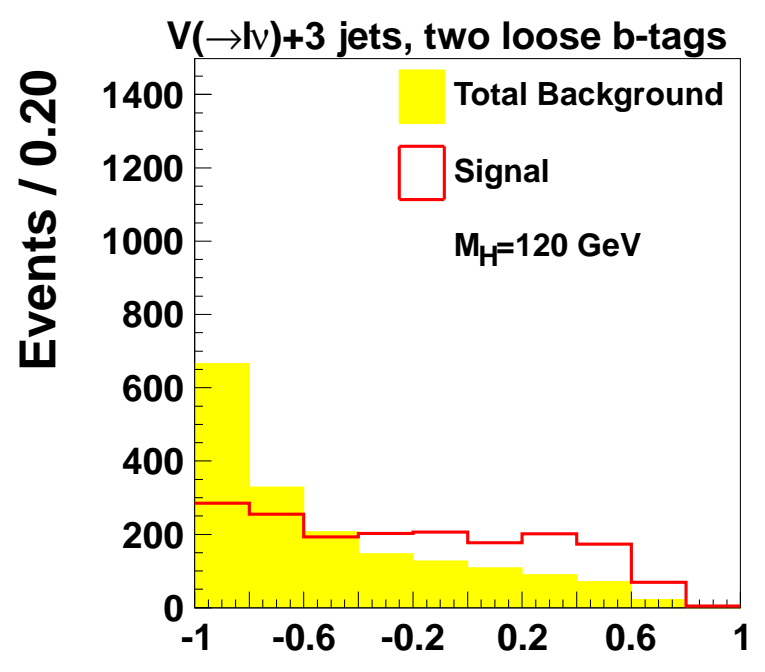

Final Discriminant

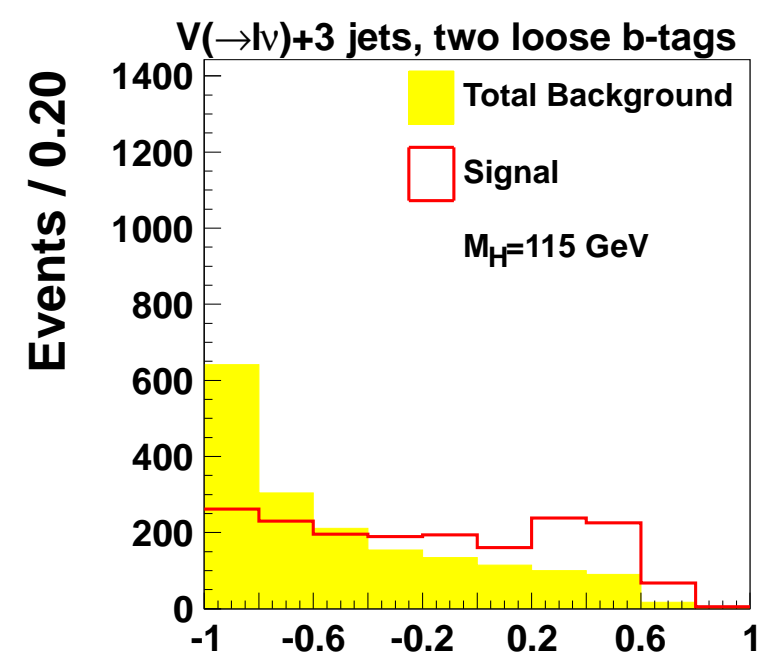

Final Discriminant

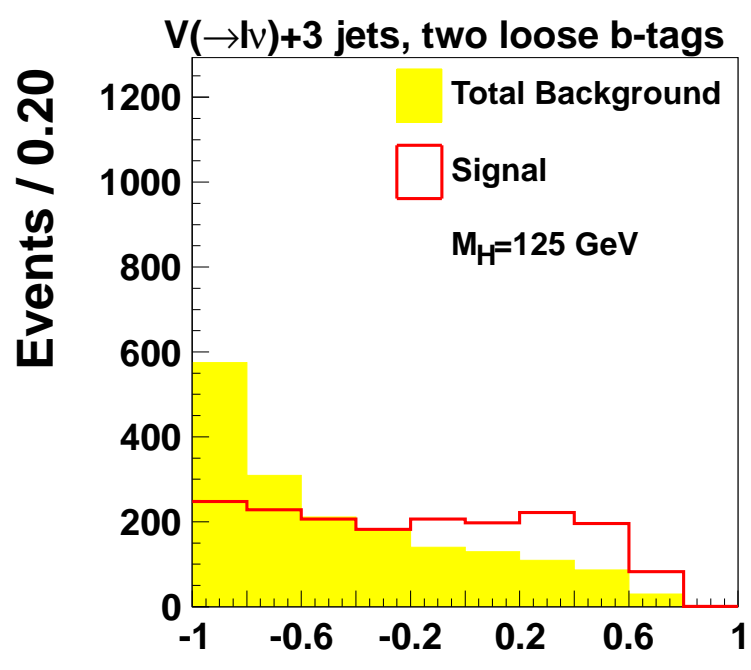
Final Discriminant

Figure B.66: The final MVA output for events with three jets and two loose b-tags for (top left) $M_{H}=110 \mathrm{GeV}$, (top right) $M_{H}=115 \mathrm{GeV}$, (bottom left) $M_{H}=120 \mathrm{GeV}$, and (bottom right) $M_{H}=125 \mathrm{GeV}$. The signal is normalized to the sum of the backgrounds. 


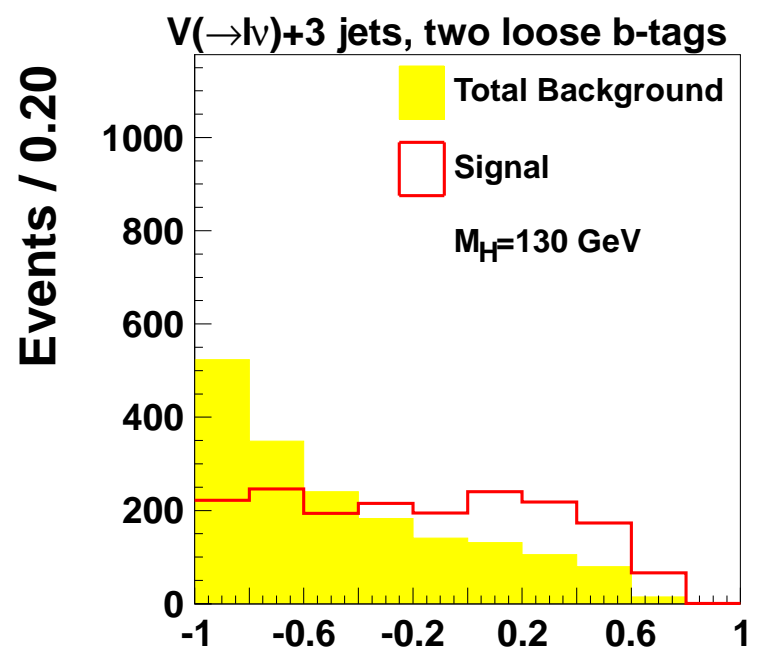

Final Discriminant

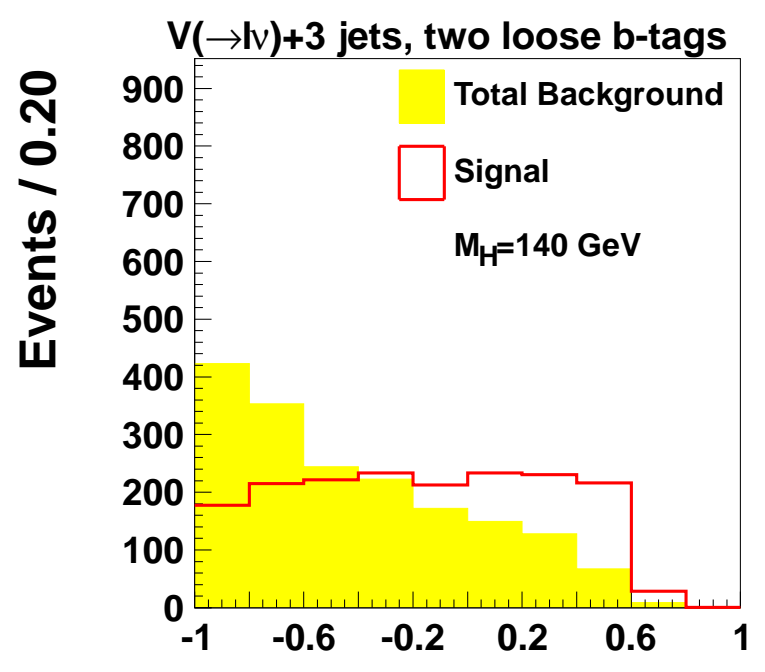

Final Discriminant

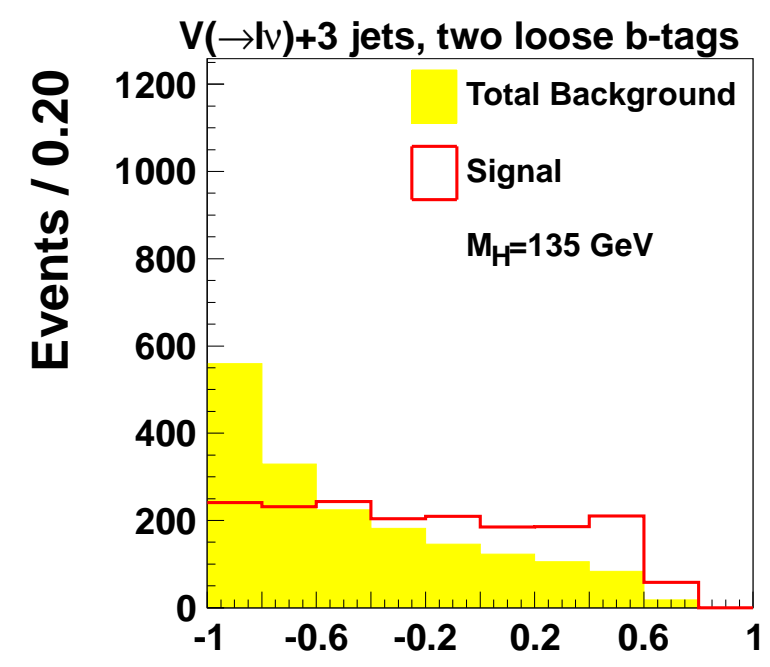

Final Discriminant

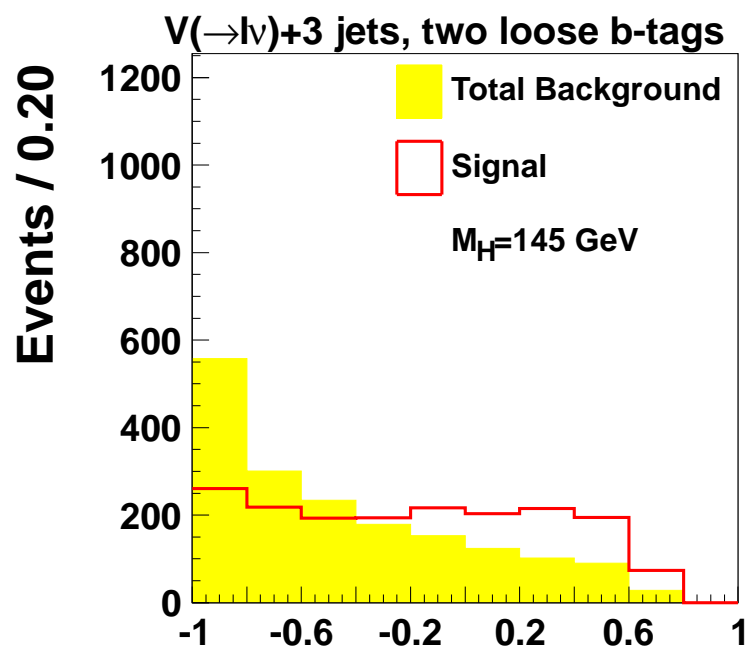

Final Discriminant

Figure B.67: The final MVA output for events with three jets and two loose b-tags for (top left) $M_{H}=130 \mathrm{GeV}$, (top right) $M_{H}=135 \mathrm{GeV}$, (bottom left) $M_{H}=140 \mathrm{GeV}$, and (bottom right) $M_{H}=145 \mathrm{GeV}$. The signal is normalized to the sum of the backgrounds. 


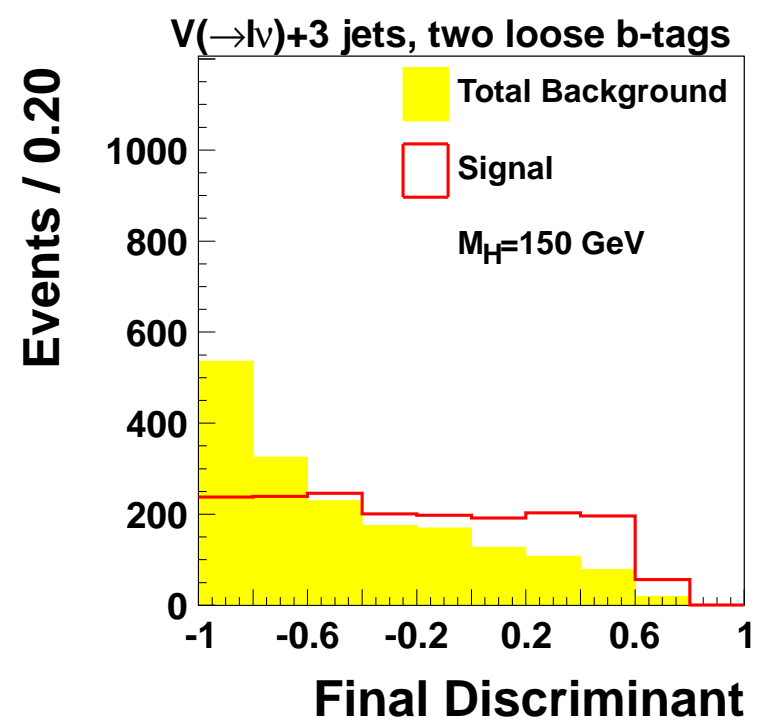

Figure B.68: The final MVA output for events with three jets and two loose b-tags for $M_{H}=150 \mathrm{GeV}$. The signal is normalized to the sum of the backgrounds. 


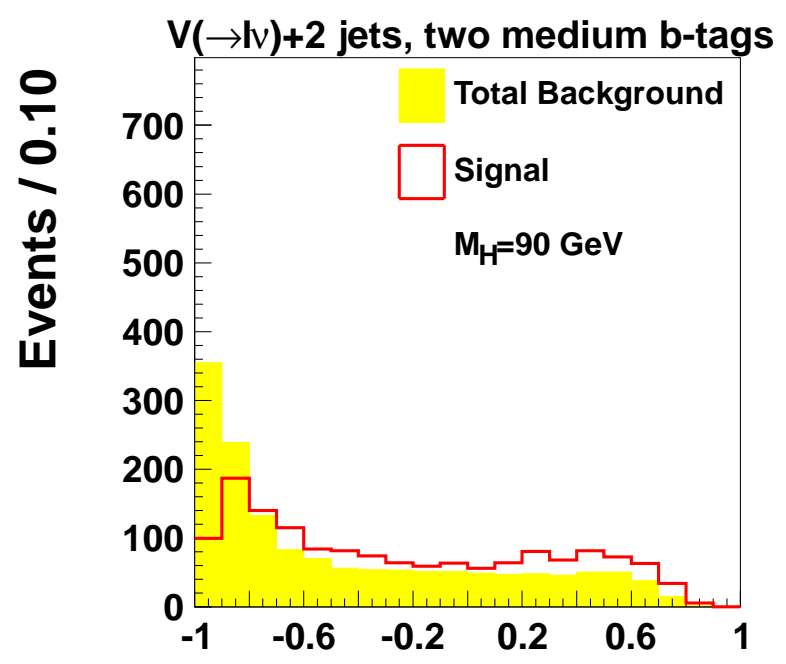

Final Discriminant

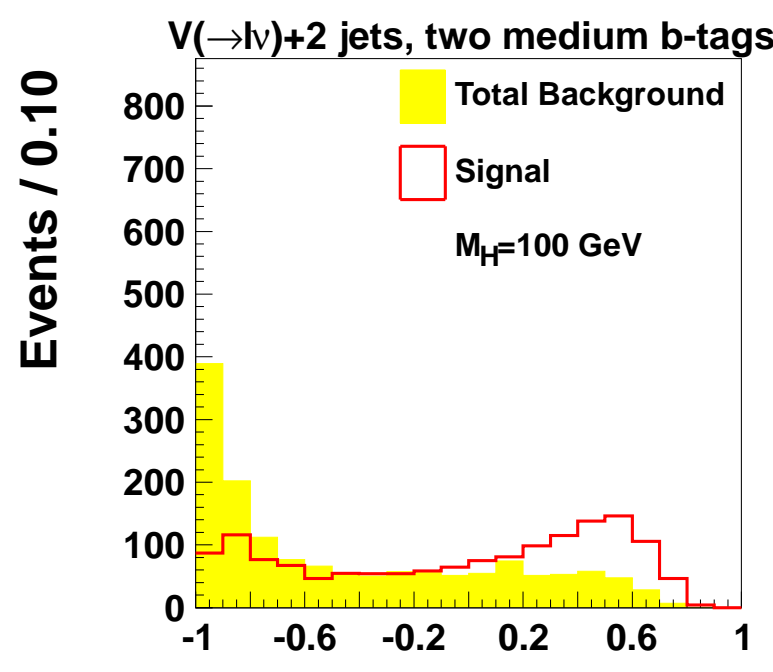

Final Discriminant

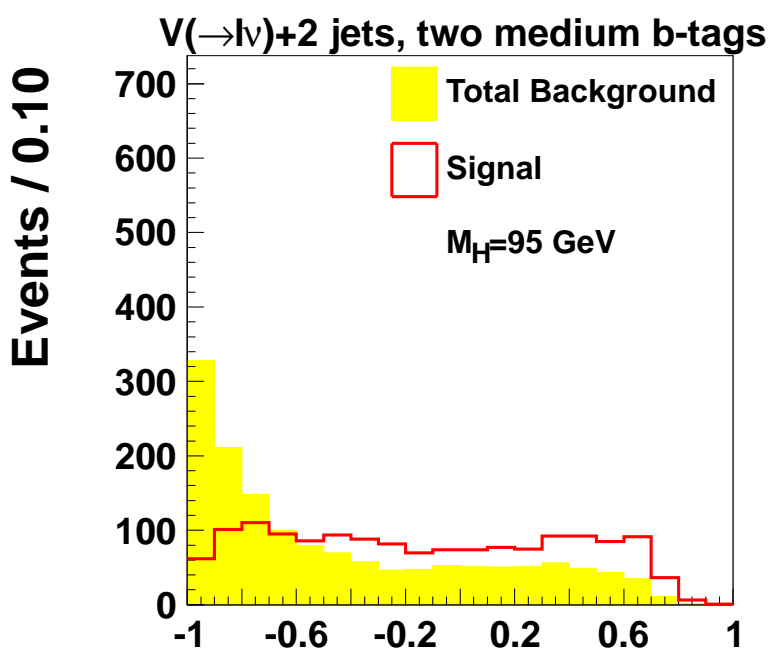

Final Discriminant

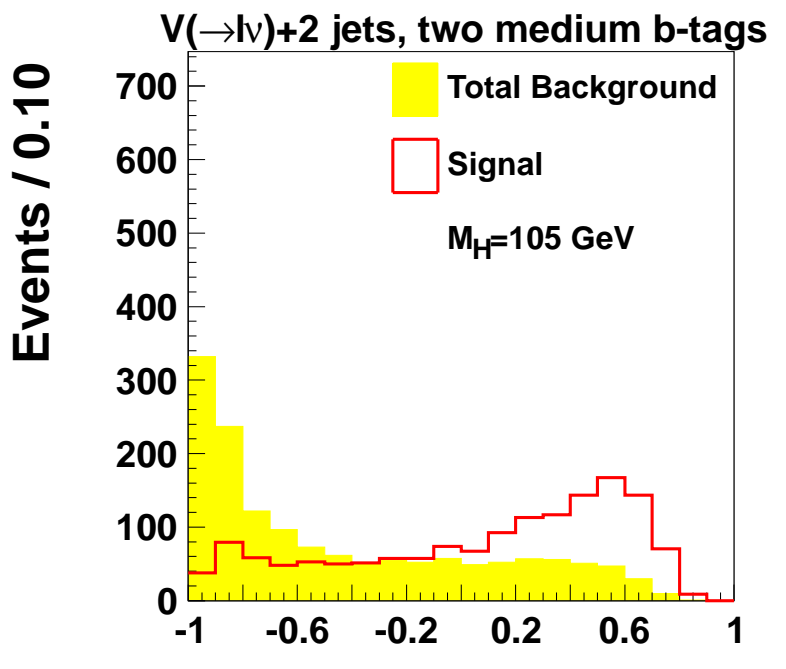

Final Discriminant

Figure B.69: The final MVA output for events with two jets and two medium b-tags for (top left) $M_{H}=90 \mathrm{GeV}$, (top right) $M_{H}=95 \mathrm{GeV}$, (bottom left) $M_{H}=100 \mathrm{GeV}$, and (bottom right) $M_{H}=105 \mathrm{GeV}$. The signal is normalized to the sum of the backgrounds. 


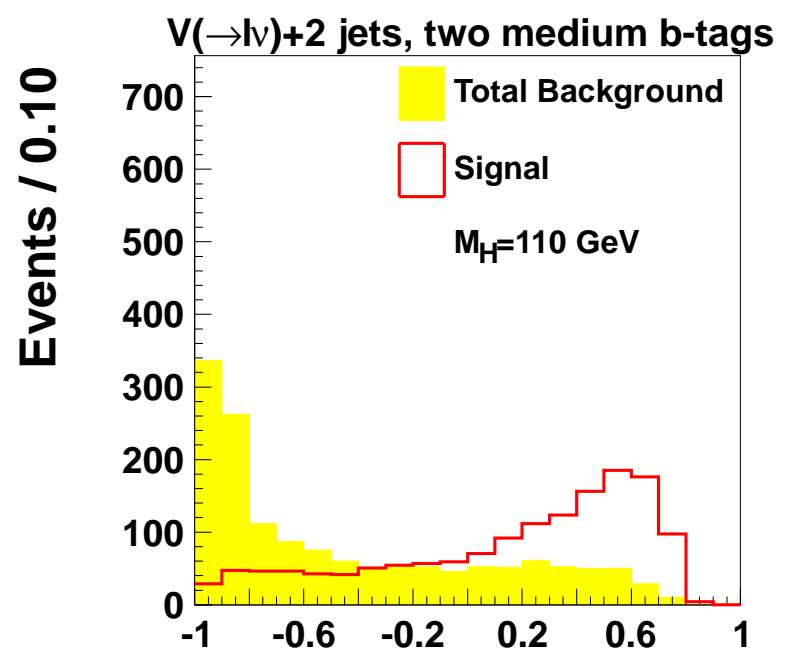

Final Discriminant

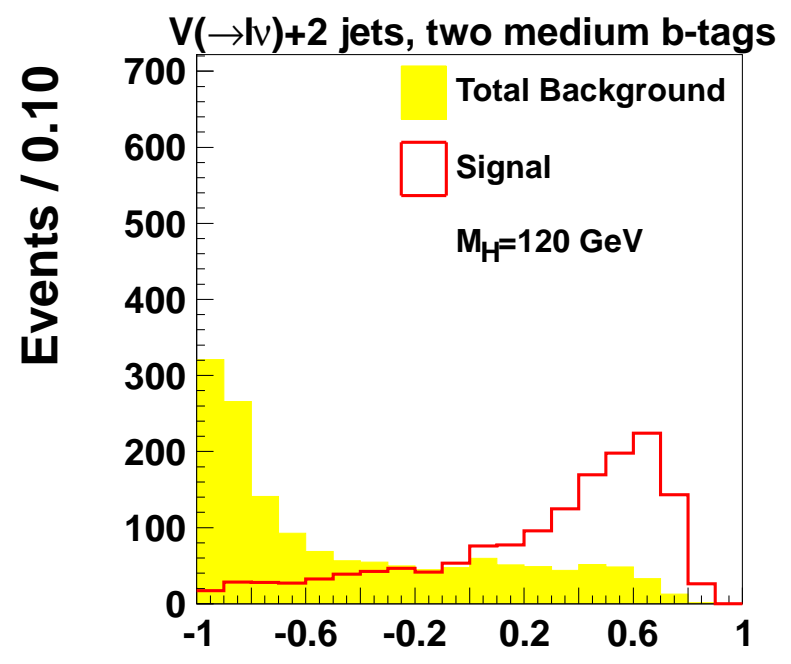

Final Discriminant

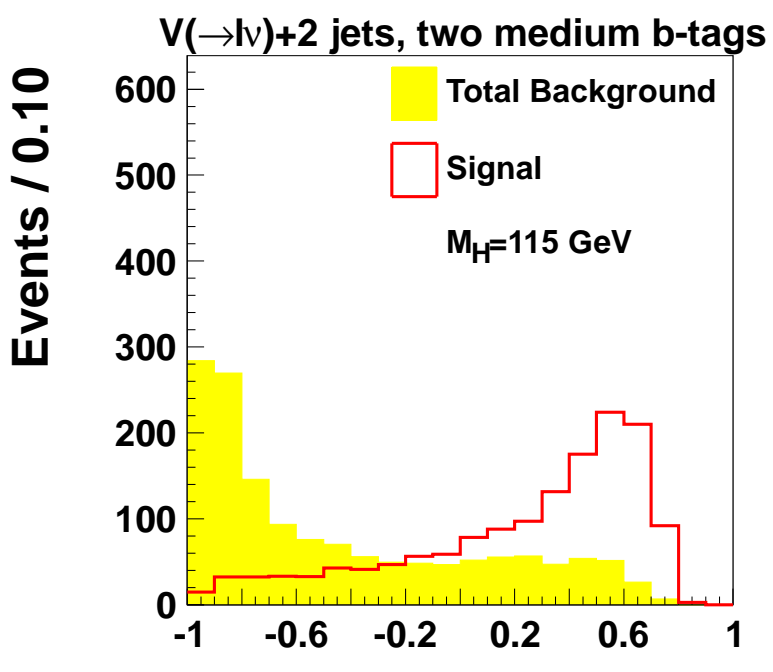

Final Discriminant

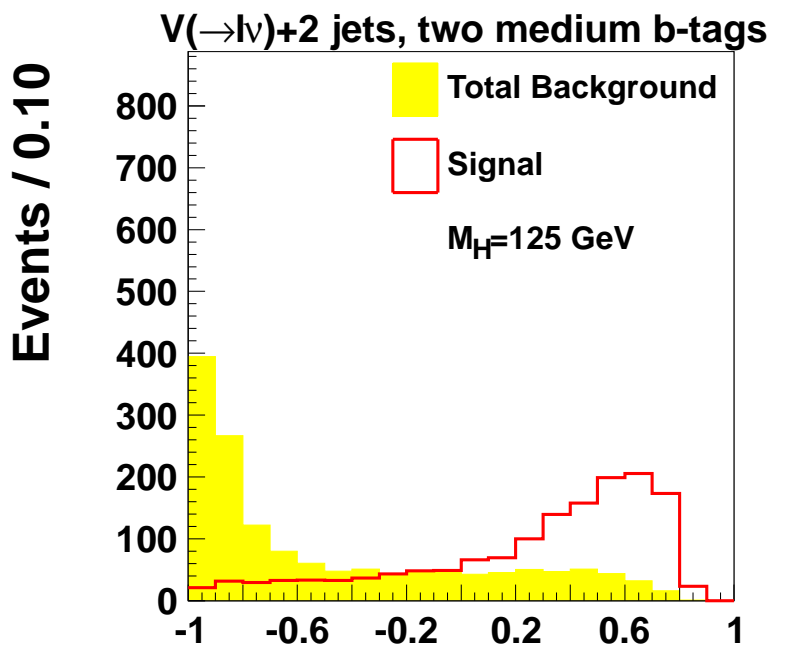

Final Discriminant

Figure B.70: The final MVA output for events with two jets and two medium b-tags for (top left) $M_{H}=110 \mathrm{GeV}$, (top right) $M_{H}=115 \mathrm{GeV}$, (bottom left) $M_{H}=120 \mathrm{GeV}$, and (bottom right) $M_{H}=125 \mathrm{GeV}$. The signal is normalized to the sum of the backgrounds. 


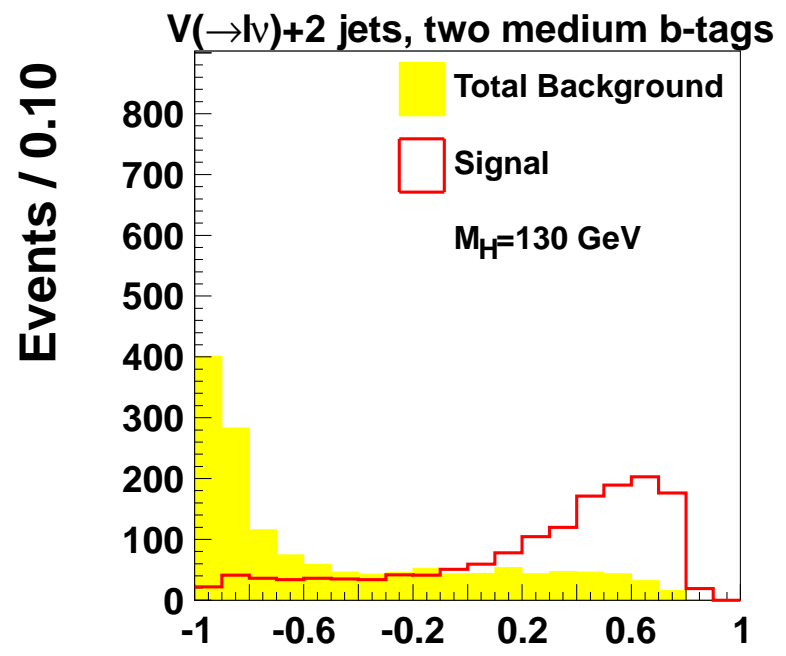

Final Discriminant

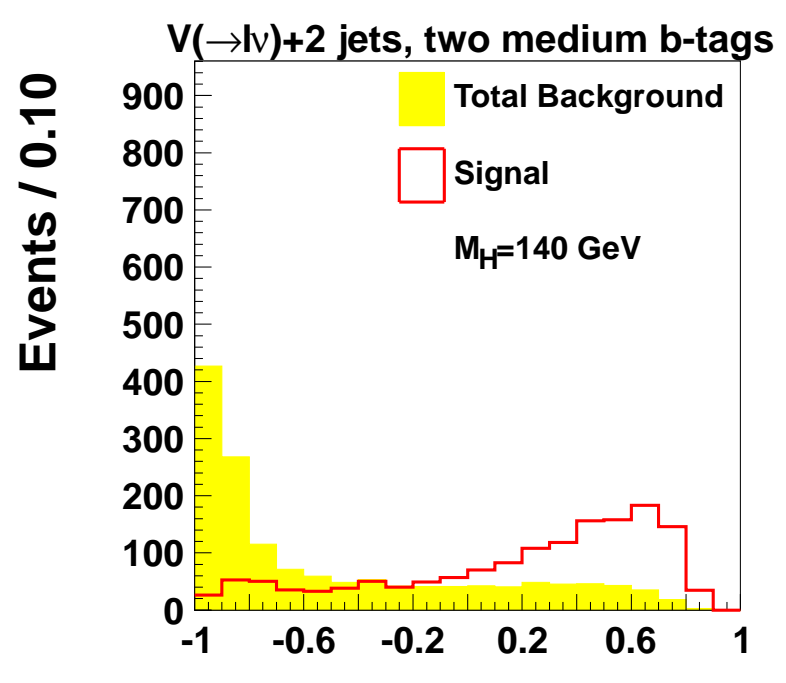

Final Discriminant

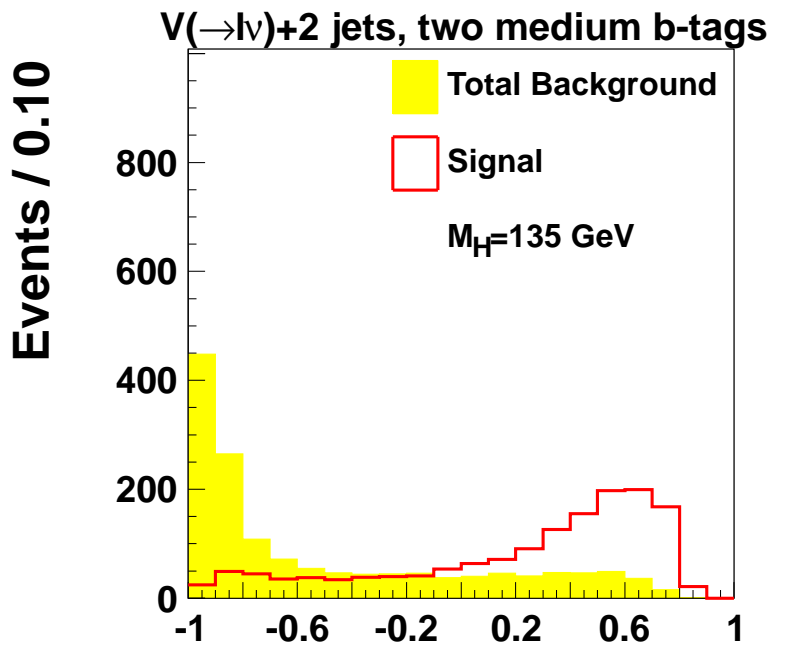

Final Discriminant

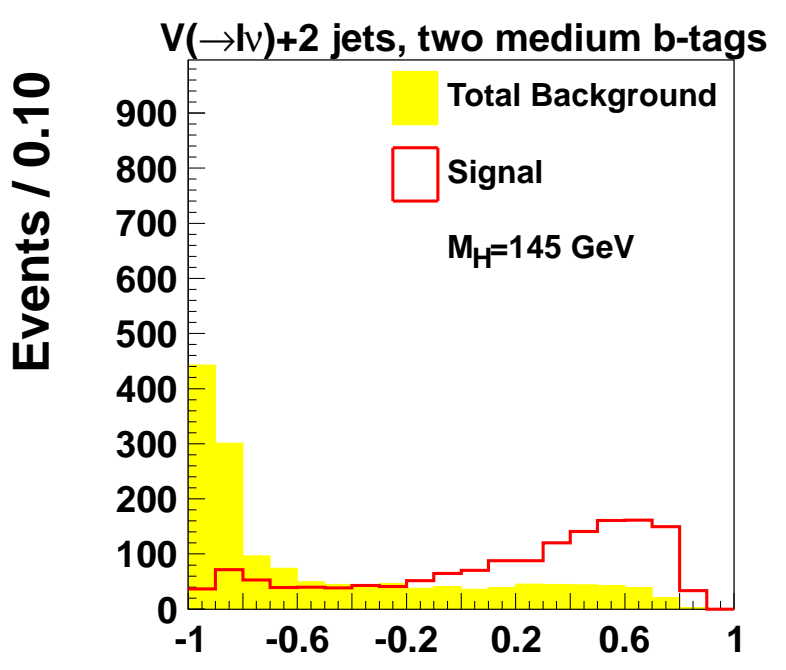

Final Discriminant

Figure B.71: The final MVA output for events with two jets and two medium b-tags for (top left) $M_{H}=130 \mathrm{GeV}$, (top right) $M_{H}=135 \mathrm{GeV}$, (bottom left) $M_{H}=140 \mathrm{GeV}$, and (bottom right) $M_{H}=145 \mathrm{GeV}$. The signal is normalized to the sum of the backgrounds. 


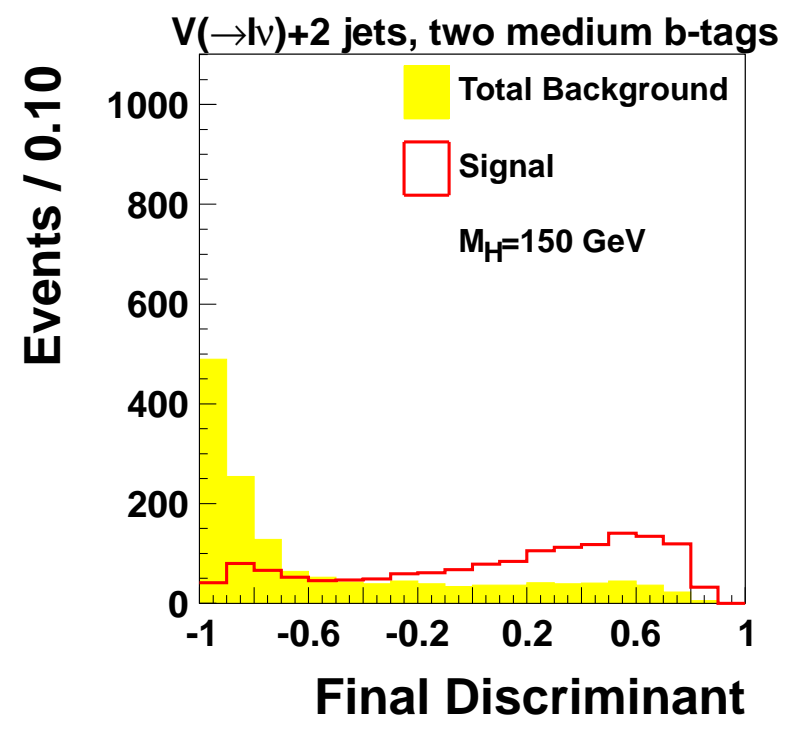

Figure B.72: The final MVA output for events with two jets and two medium b-tags for $M_{H}=150 \mathrm{GeV}$. The signal is normalized to the sum of the backgrounds. 


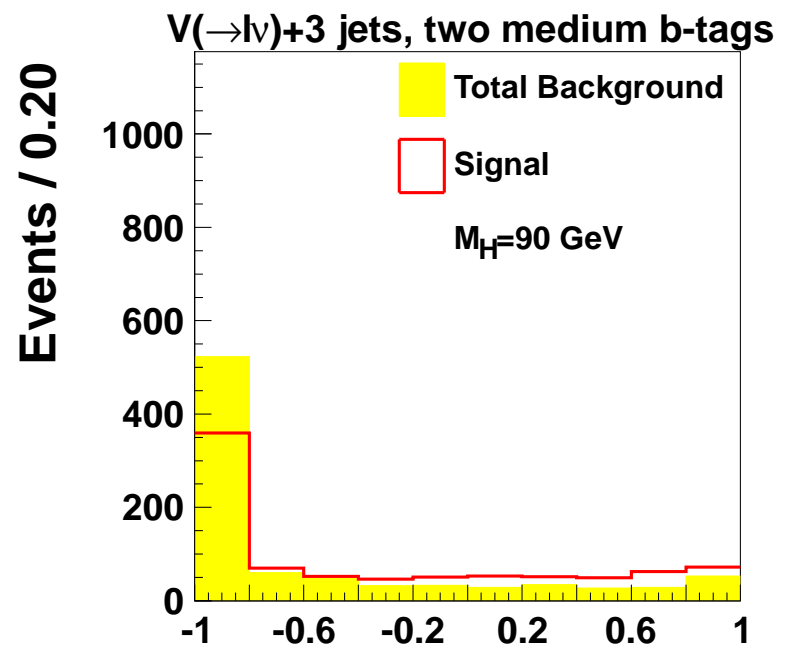

Final Discriminant

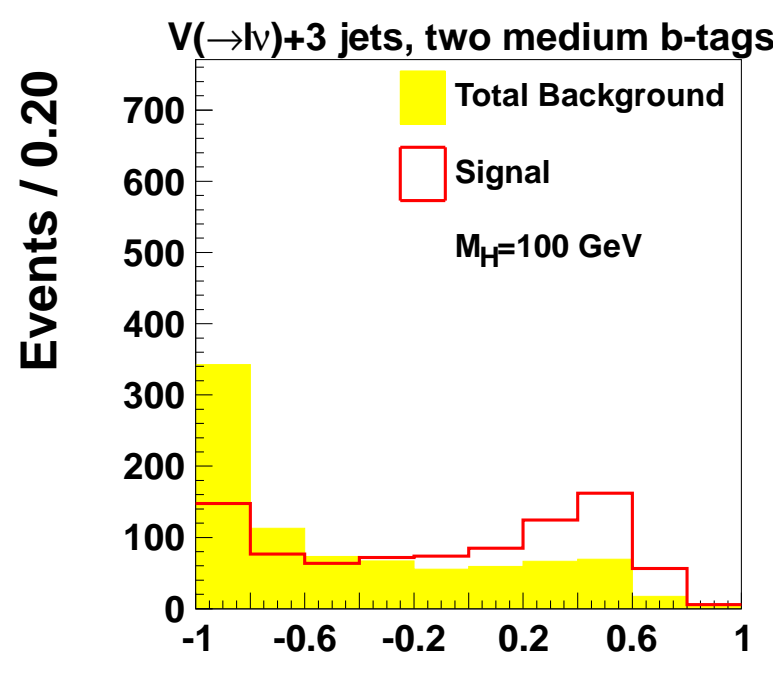

Final Discriminant

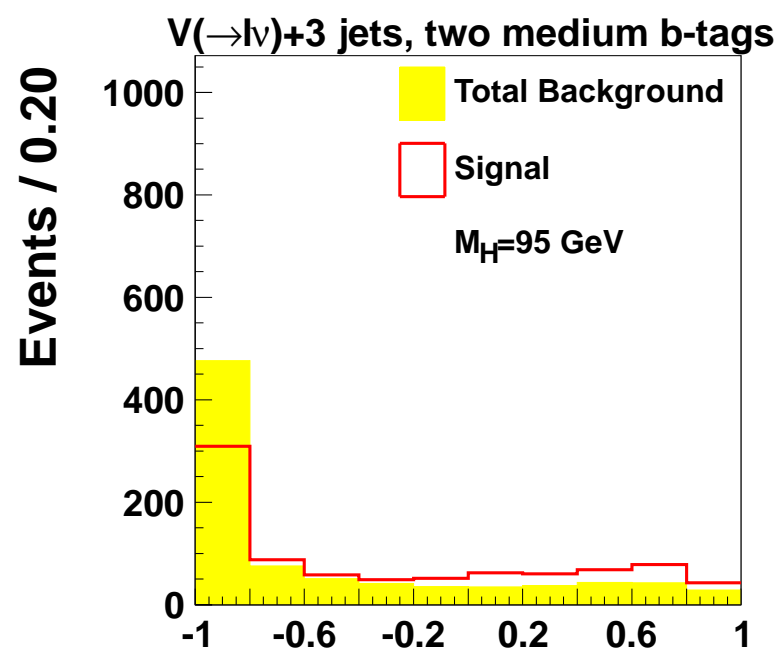

Final Discriminant

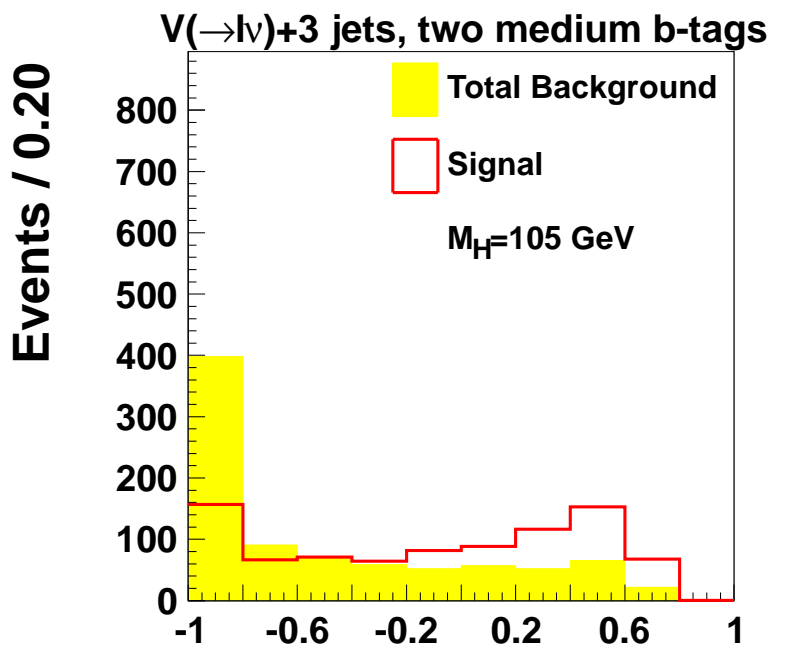

Final Discriminant

Figure B.73: The final MVA output for events with three jets and two medium b-tags for (top left) $M_{H}=90 \mathrm{GeV}$, (top right) $M_{H}=95 \mathrm{GeV}$, (bottom left) $M_{H}=100 \mathrm{GeV}$, and (bottom right) $M_{H}=105 \mathrm{GeV}$. The signal is normalized to the sum of the backgrounds. 


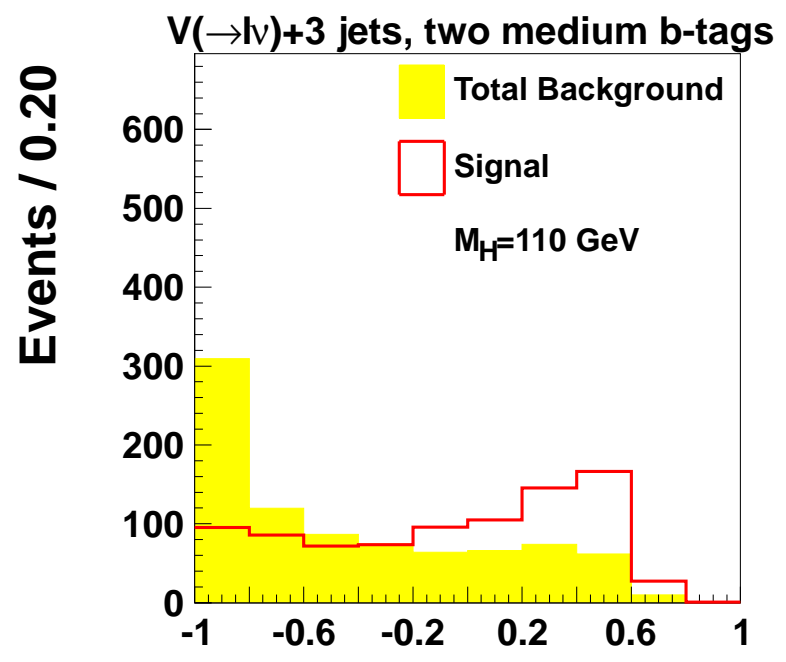

Final Discriminant

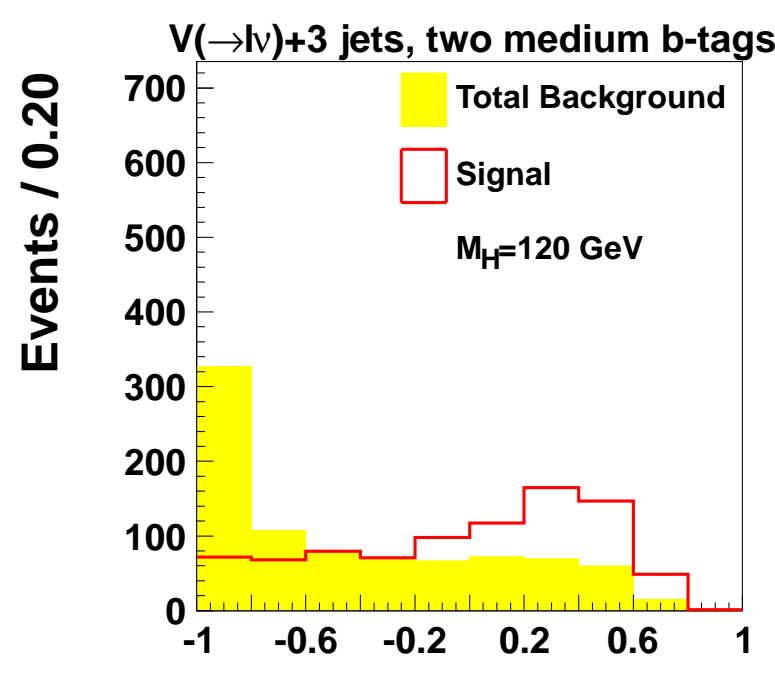

Final Discriminant

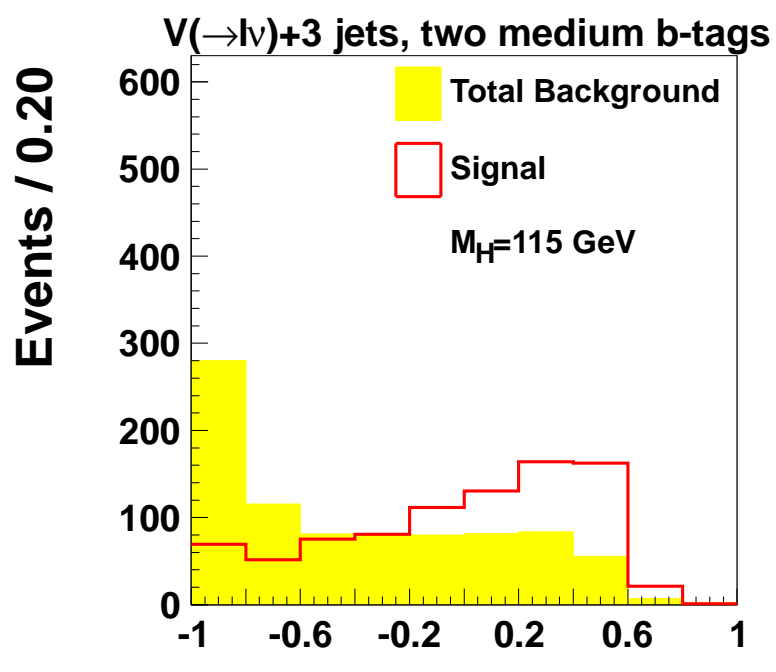

Final Discriminant

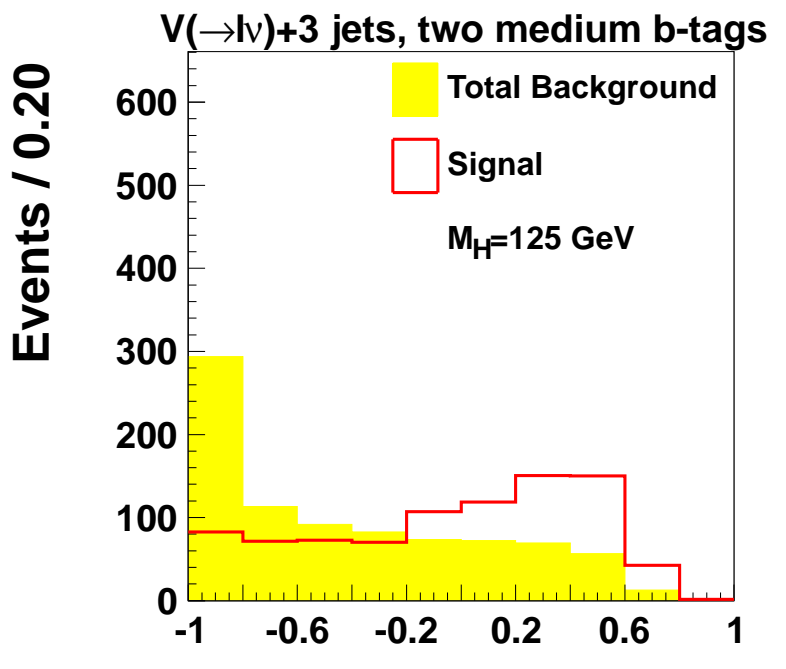

Final Discriminant

Figure B.74: The final MVA output for events with three jets and two medium b-tags for (top left) $M_{H}=110 \mathrm{GeV}$, (top right) $M_{H}=115 \mathrm{GeV}$, (bottom left) $M_{H}=120 \mathrm{GeV}$, and (bottom right) $M_{H}=125 \mathrm{GeV}$. The signal is normalized to the sum of the backgrounds. 


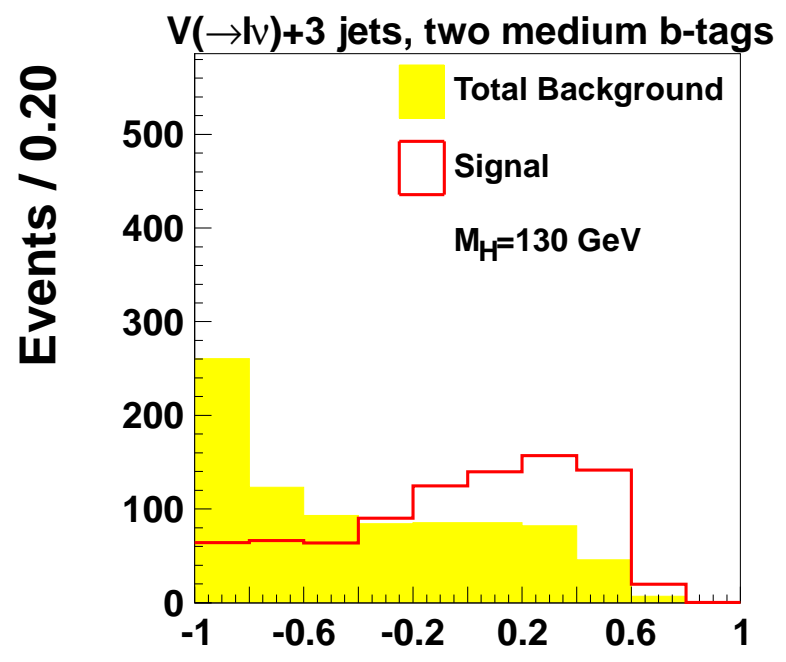

Final Discriminant

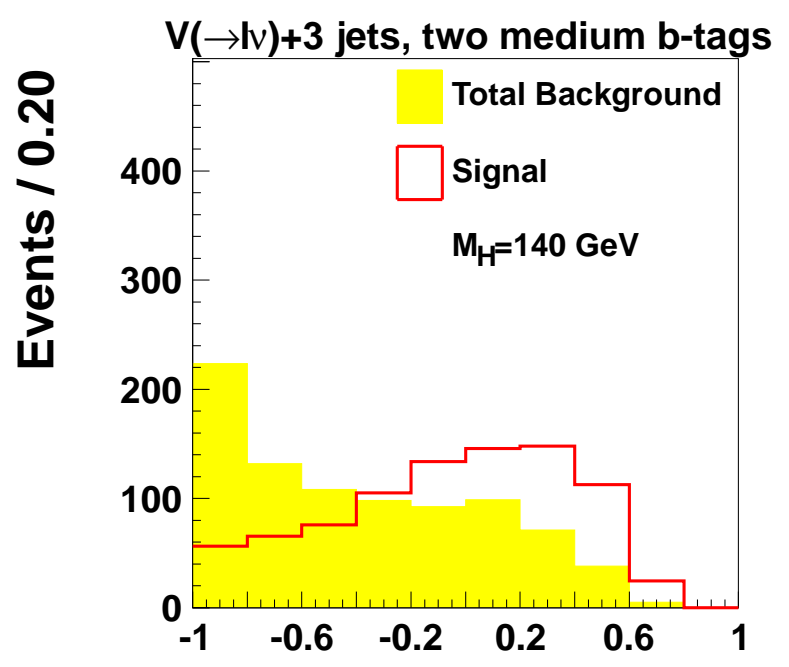

Final Discriminant

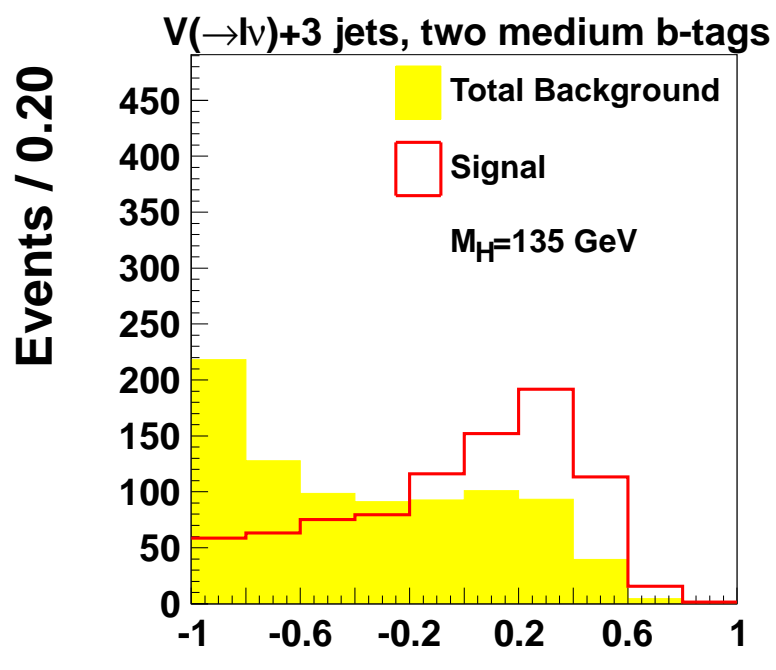

Final Discriminant

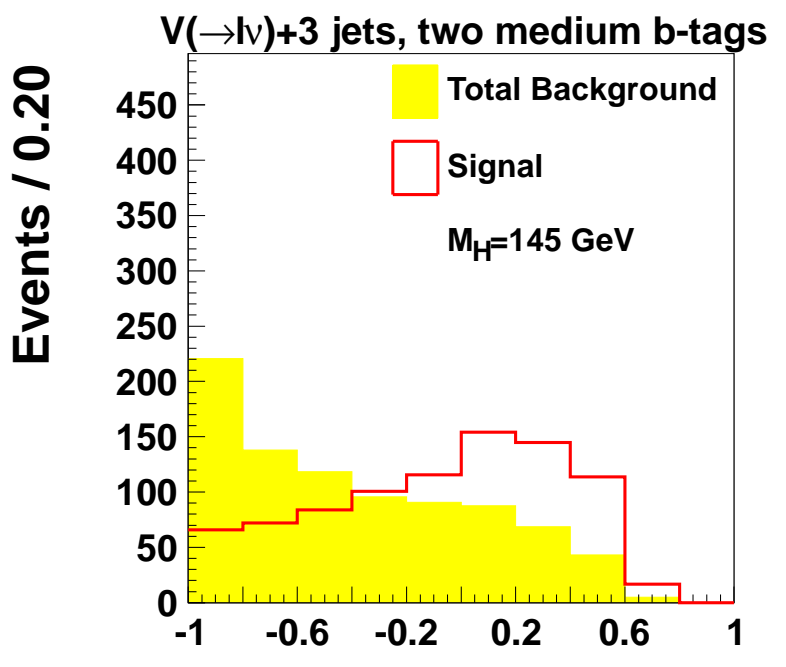

Final Discriminant

Figure B.75: The final MVA output for events with three jets and two medium b-tags for (top left) $M_{H}=130 \mathrm{GeV}$, (top right) $M_{H}=135 \mathrm{GeV}$, (bottom left) $M_{H}=140 \mathrm{GeV}$, and (bottom right) $M_{H}=145 \mathrm{GeV}$. The signal is normalized to the sum of the backgrounds. 


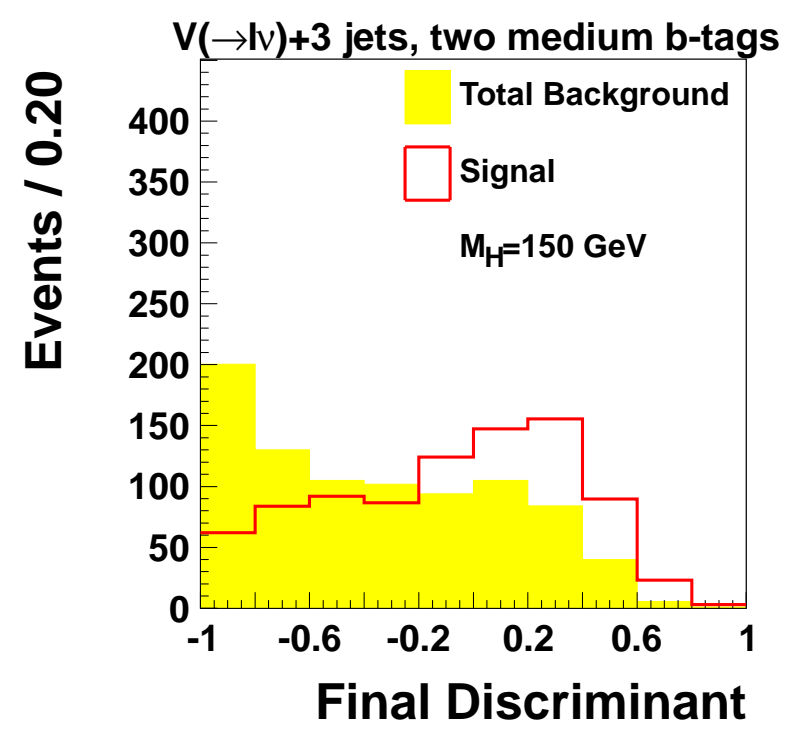

Figure B.76: The final MVA output for events with three jets and two medium b-tags for $M_{H}=150 \mathrm{GeV}$. The signal is normalized to the sum of the backgrounds. 

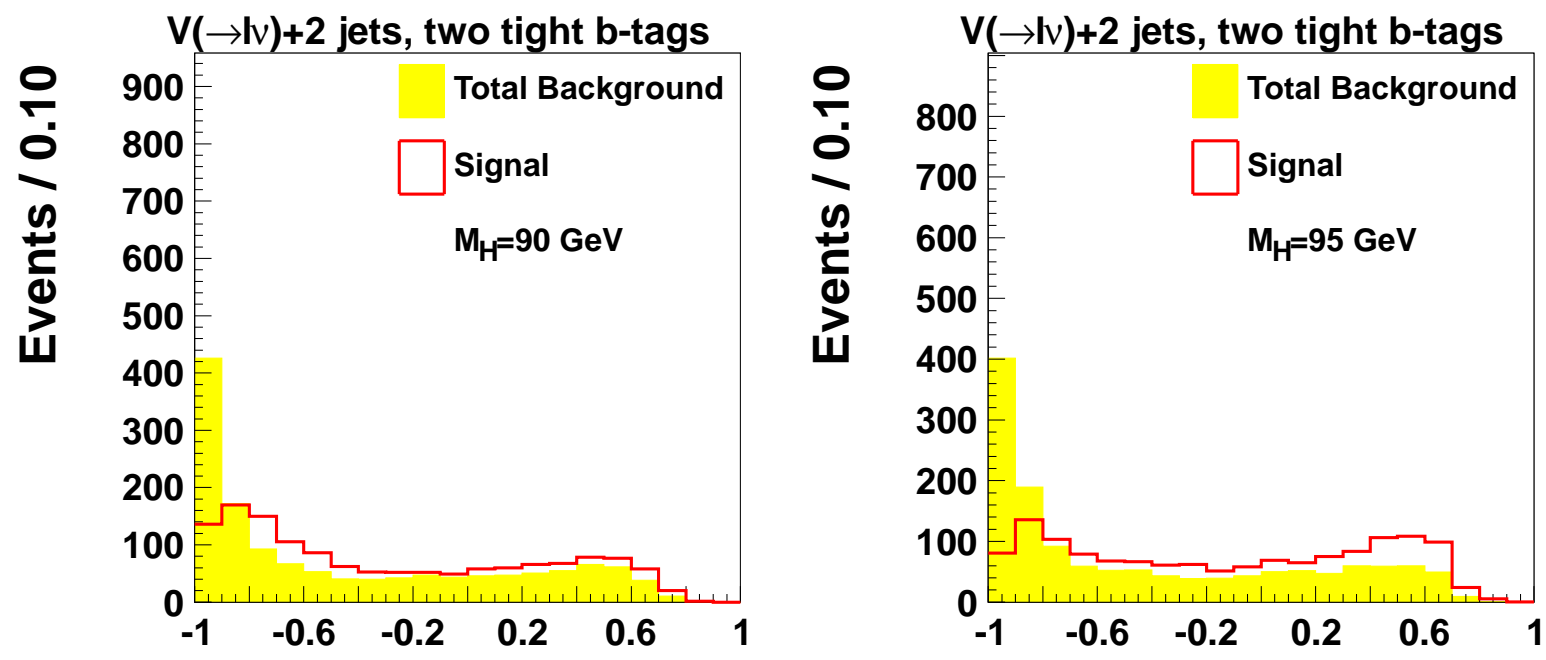

Final Discriminant
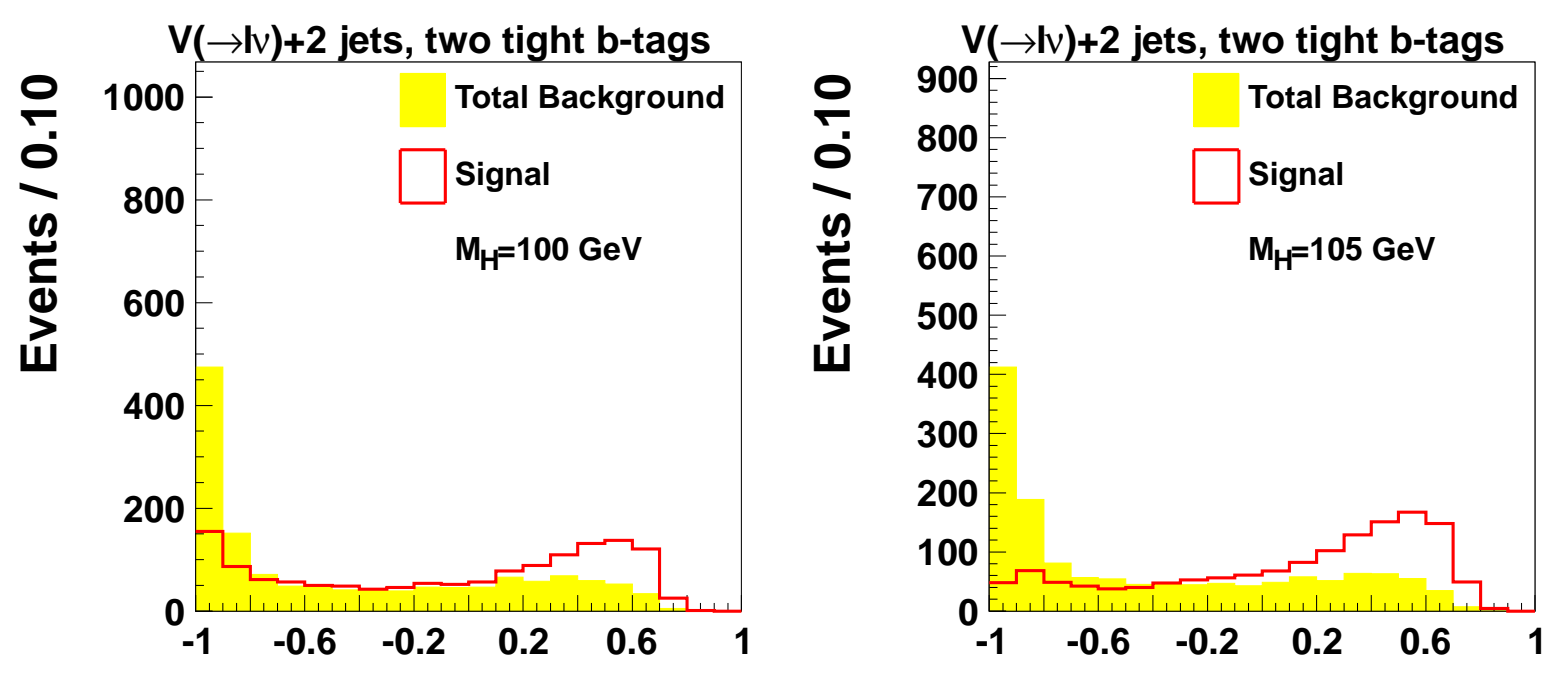

Final Discriminant Final Discriminant

Figure B.77: The final MVA output for events with two jets and two tight b-tags for (top left) $M_{H}=90 \mathrm{GeV}$, (top right) $M_{H}=95 \mathrm{GeV}$, (bottom left) $M_{H}=100 \mathrm{GeV}$, and (bottom right) $M_{H}=105 \mathrm{GeV}$. The signal is normalized to the sum of the backgrounds. 


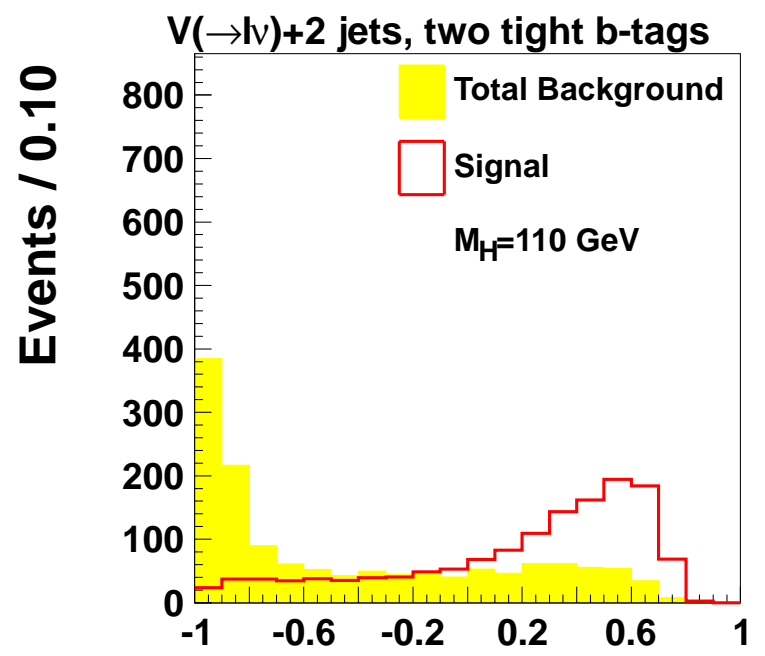

Final Discriminant

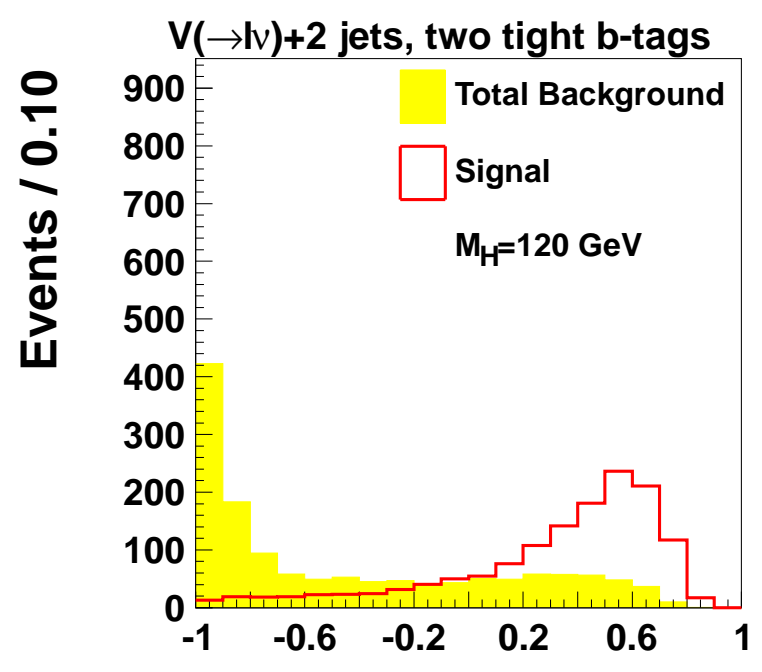

Final Discriminant

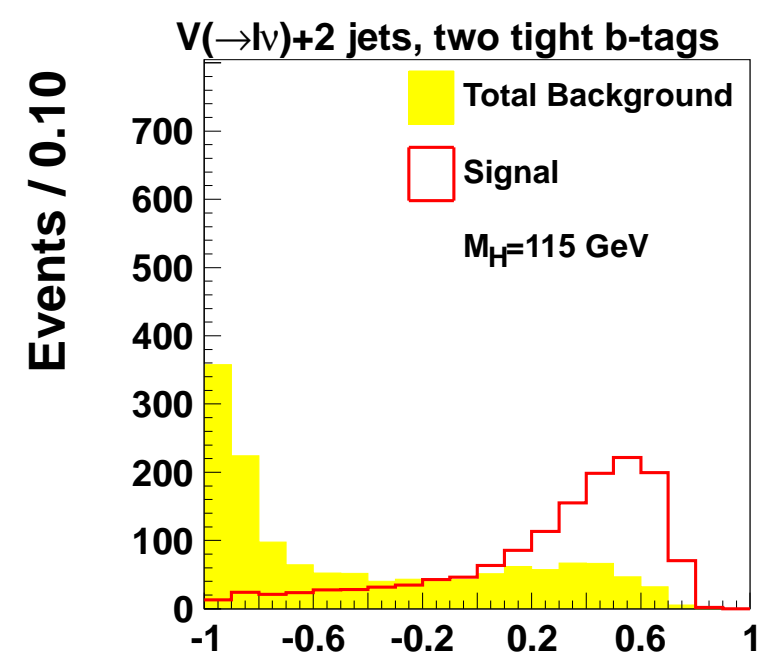

Final Discriminant

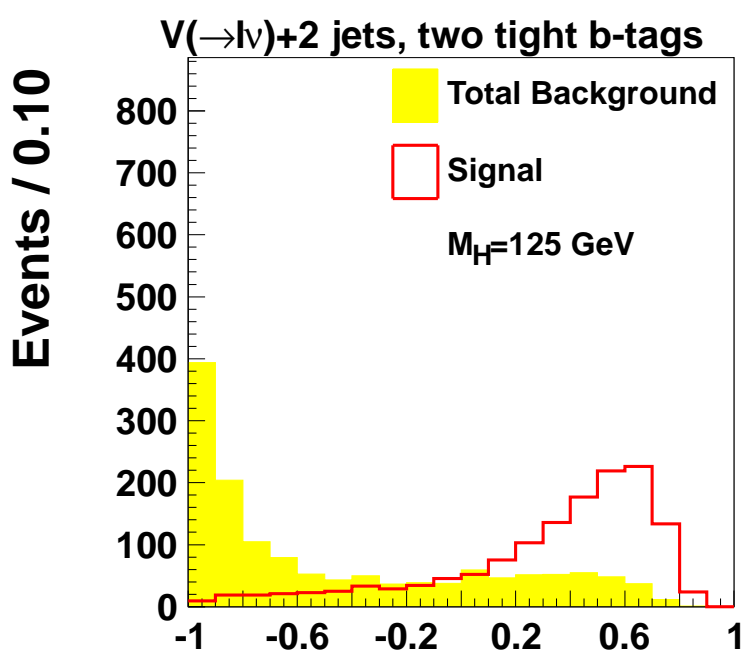

Final Discriminant

Figure B.78: The final MVA output for events with two jets and two tight b-tags for (top left) $M_{H}=110 \mathrm{GeV}$, (top right) $M_{H}=115 \mathrm{GeV}$, (bottom left) $M_{H}=120 \mathrm{GeV}$, and (bottom right) $M_{H}=125 \mathrm{GeV}$. The signal is normalized to the sum of the backgrounds. 


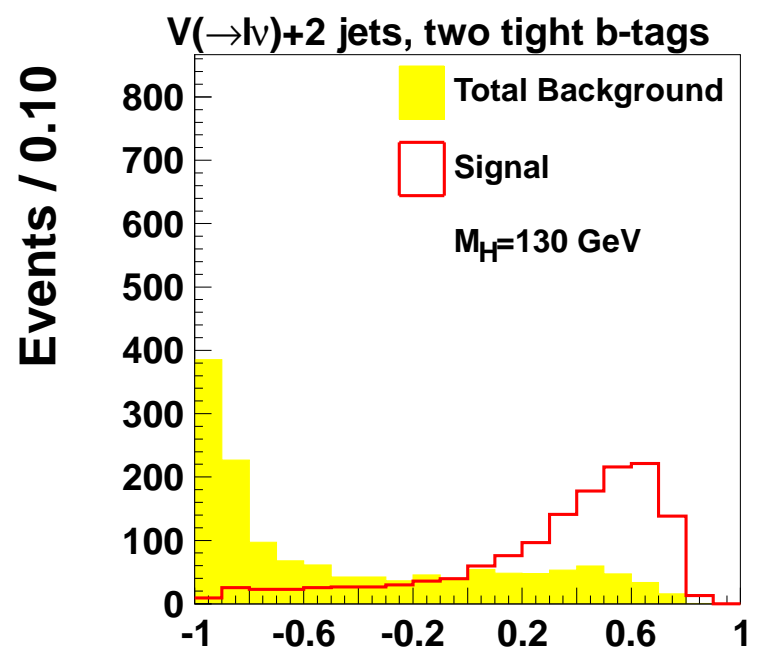

Final Discriminant

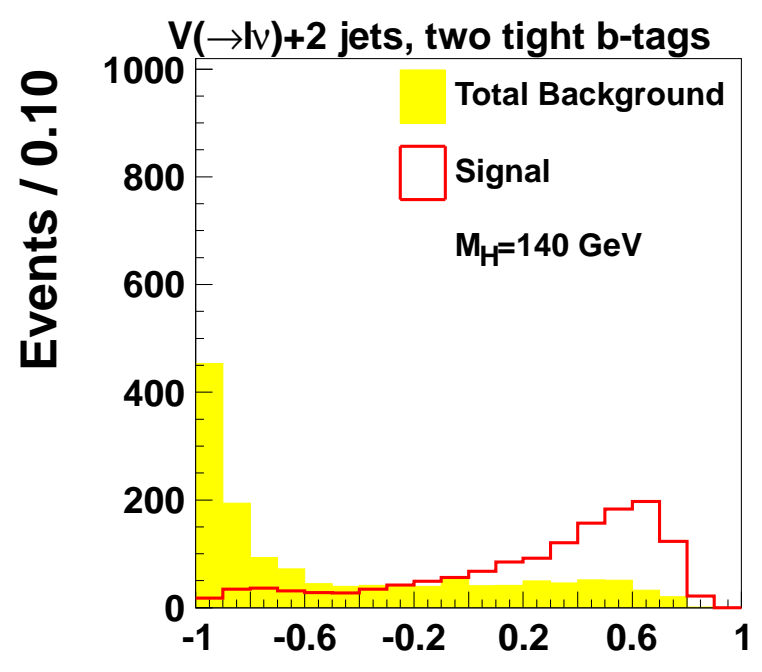

Final Discriminant

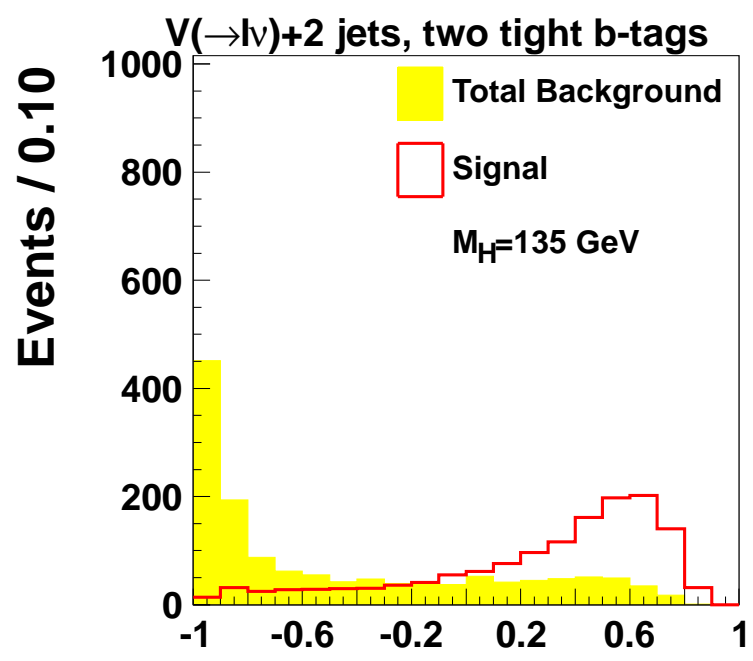

Final Discriminant

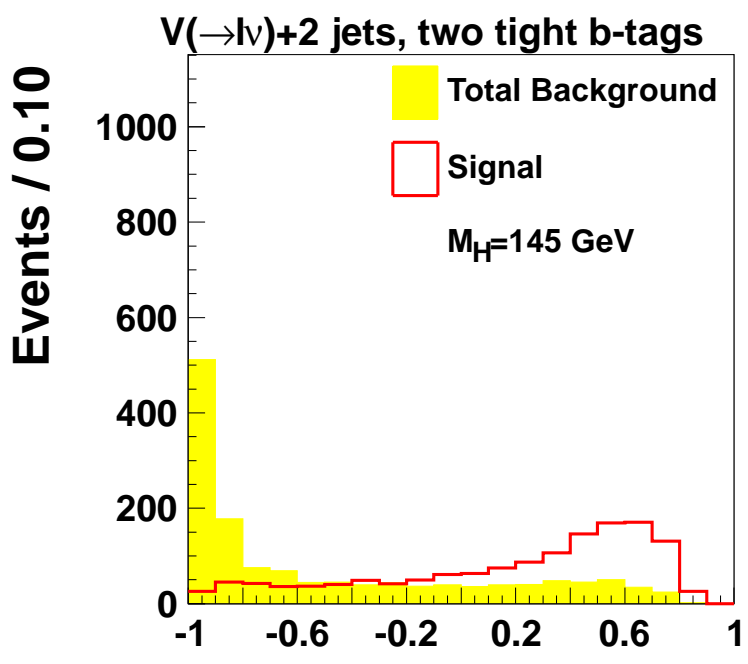

Final Discriminant

Figure B.79: The final MVA output for events with two jets and two tight b-tags for (top left) $M_{H}=130 \mathrm{GeV}$, (top right) $M_{H}=135 \mathrm{GeV}$, (bottom left) $M_{H}=140 \mathrm{GeV}$, and (bottom right) $M_{H}=145 \mathrm{GeV}$. The signal is normalized to the sum of the backgrounds. 


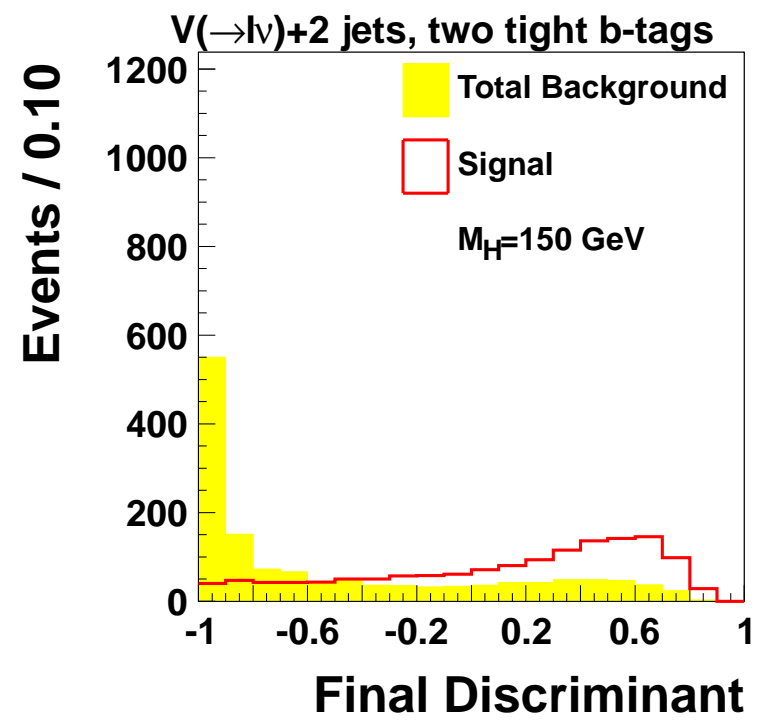

Figure B.80: The final MVA output for events with two jets and two tight b-tags for $M_{H}=$ $150 \mathrm{GeV}$. The signal is normalized to the sum of the backgrounds. 

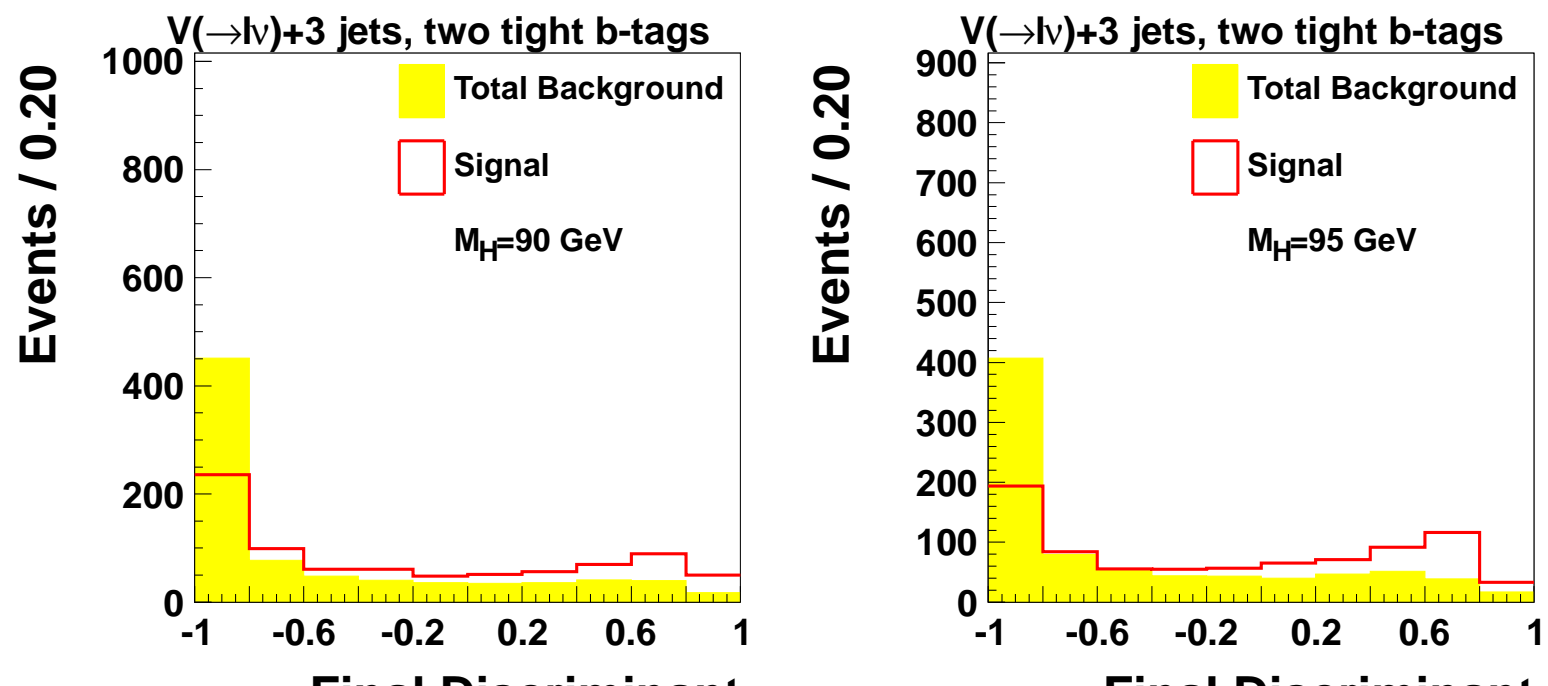

Final Discriminant
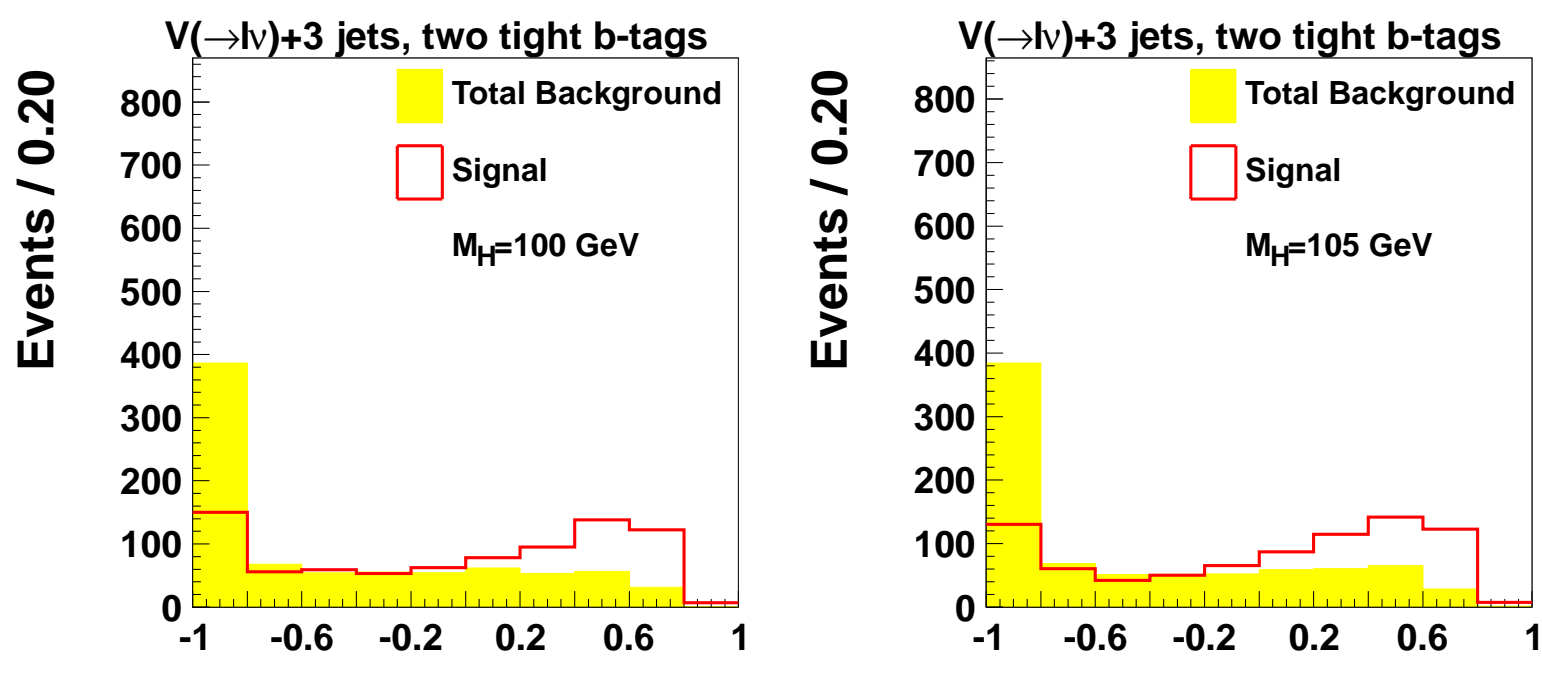

Final Discriminant Final Discriminant

Figure B.81: The final MVA output for events with three jets and two tight b-tags for (top left) $M_{H}=90 \mathrm{GeV}$, (top right) $M_{H}=95 \mathrm{GeV}$, (bottom left) $M_{H}=100 \mathrm{GeV}$, and (bottom right) $M_{H}=105 \mathrm{GeV}$. The signal is normalized to the sum of the backgrounds. 


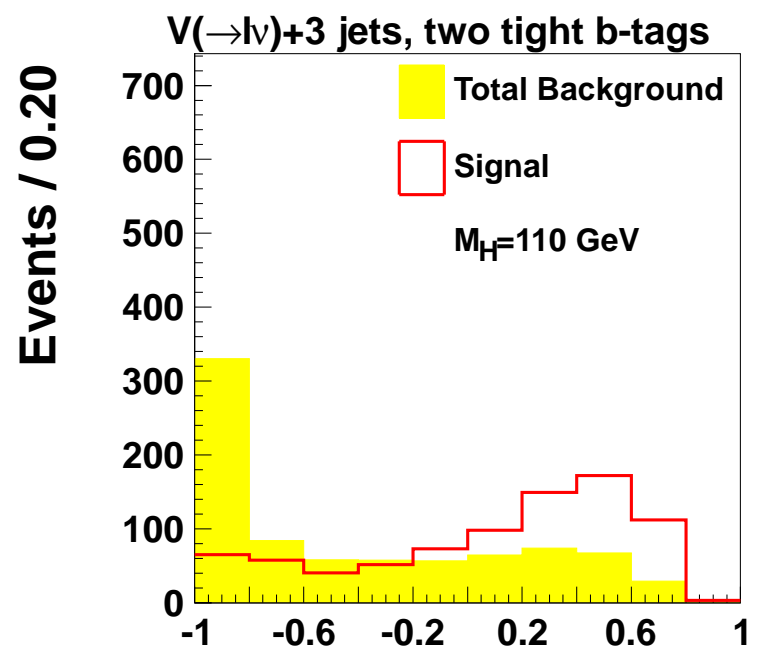

Final Discriminant

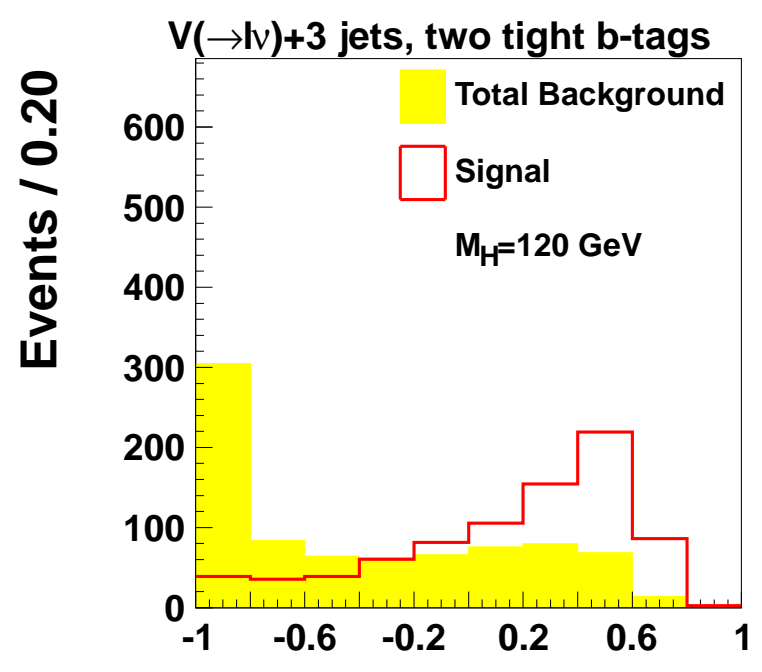

Final Discriminant

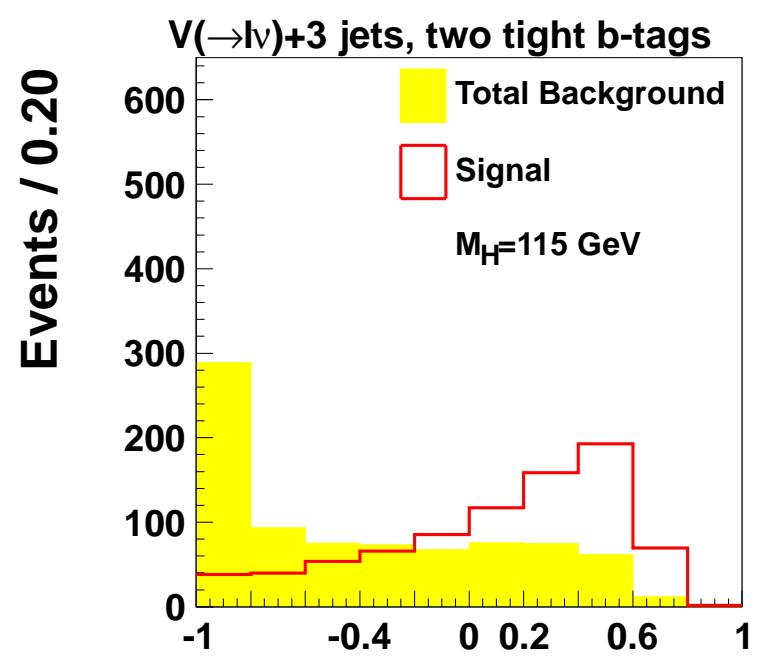

Final Discriminant

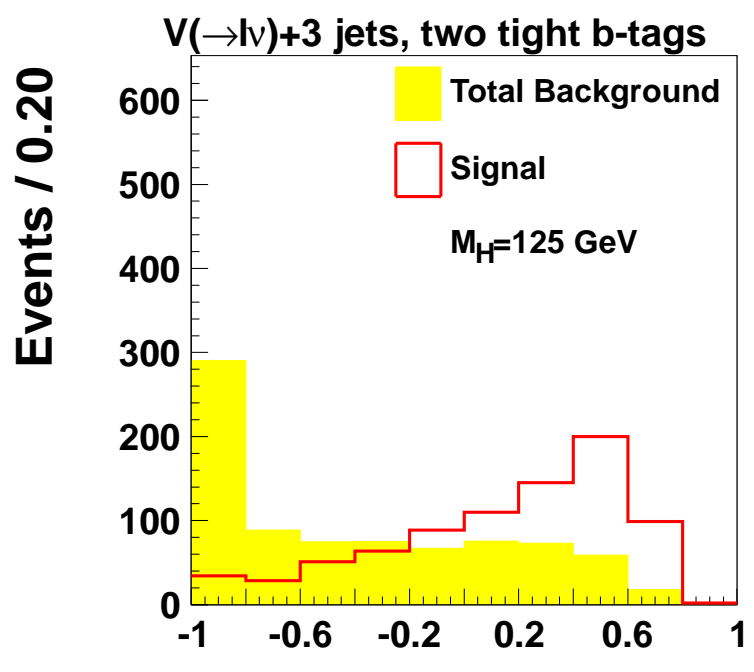

Final Discriminant

Figure B.82: The final MVA output for events with three jets and two tight b-tags for (top left) $M_{H}=110 \mathrm{GeV}$, (top right) $M_{H}=115 \mathrm{GeV}$, (bottom left) $M_{H}=120 \mathrm{GeV}$, and (bottom right) $M_{H}=125 \mathrm{GeV}$. The signal is normalized to the sum of the backgrounds. 


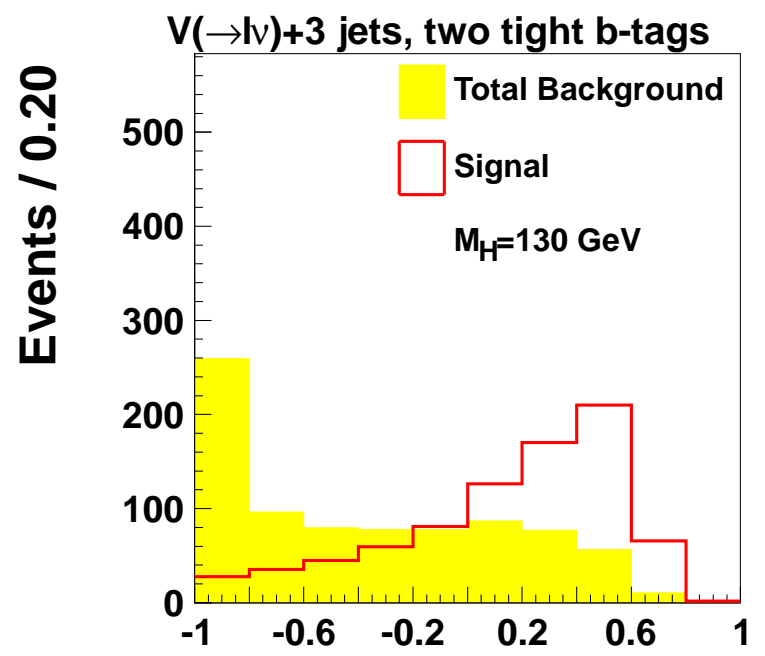

Final Discriminant

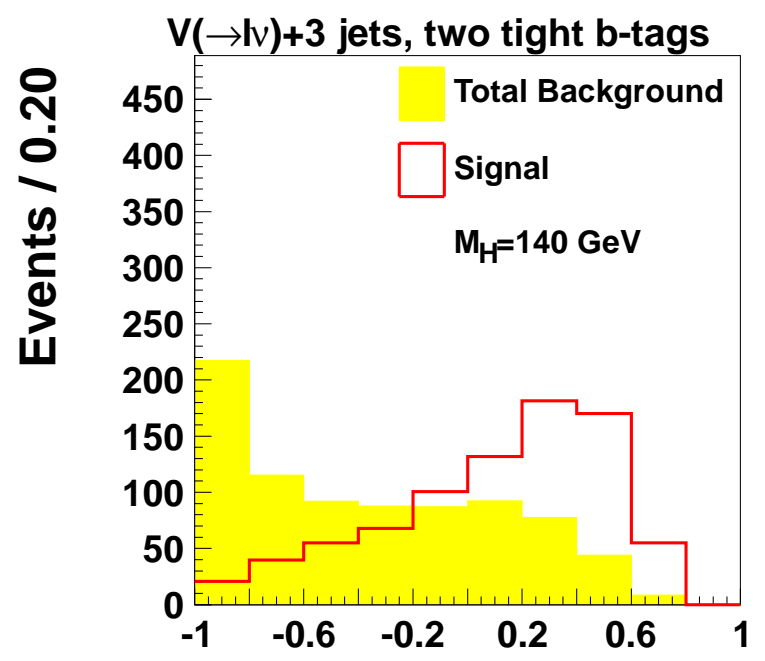

Final Discriminant

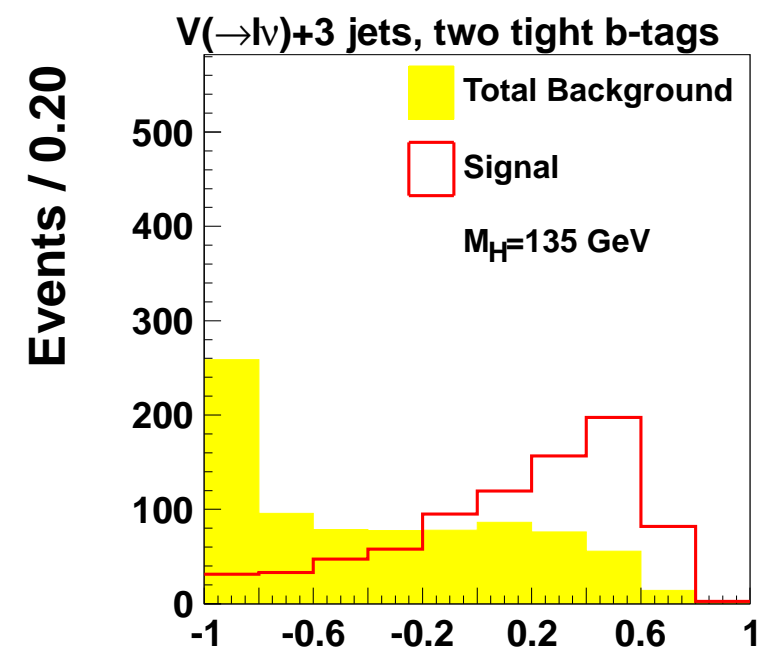

Final Discriminant

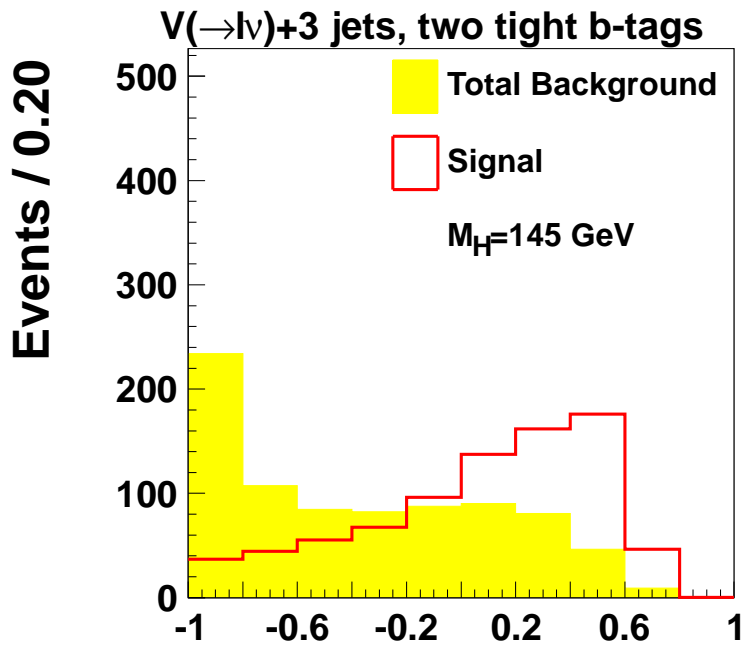

Final Discriminant

Figure B.83: The final MVA output for events with three jets and two tight b-tags for (top left) $M_{H}=130 \mathrm{GeV}$, (top right) $M_{H}=135 \mathrm{GeV}$, (bottom left) $M_{H}=140 \mathrm{GeV}$, and (bottom right) $M_{H}=145 \mathrm{GeV}$. The signal is normalized to the sum of the backgrounds. 


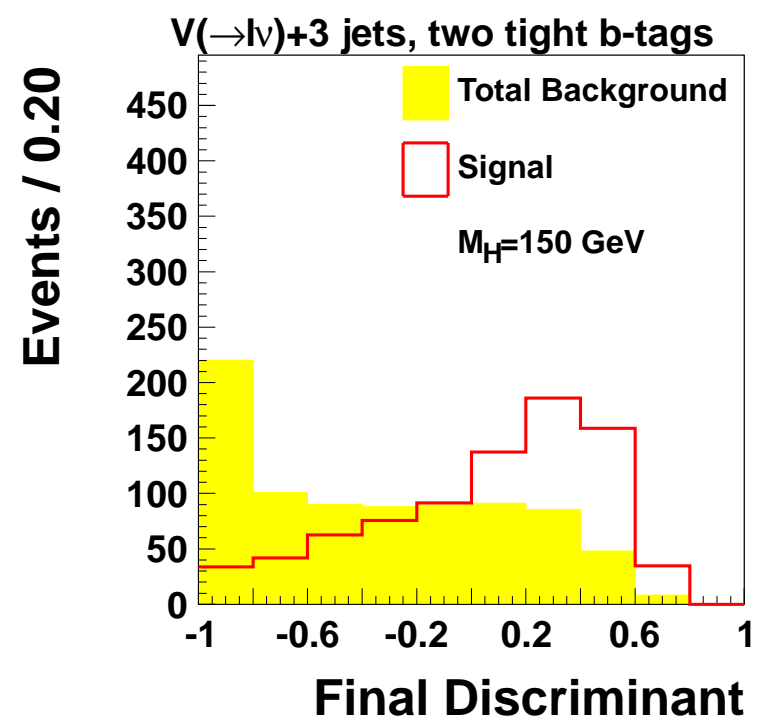

Figure B.84: The final MVA output for events with three jets and two tight b-tags for $M_{H}=150 \mathrm{GeV}$. The signal is normalized to the sum of the backgrounds. 
BIBLIOGRAPHY 


\section{BIBLIOGRAPHY}

[1] J. Beringer et al., (The Particle Data Group), Phys. Rev. D 86, 010001 (2012).

[2] V. M. Abazov et al., (D0 Collaboration), Phys. Rev. D 88, 052008 (2013).

[3] M. Baak et al., Gfitter - a generic fitter project for hep model testing, http:// project-gfitter.web.cern.ch/project-gfitter/.

[4] G. Aad et al., (ATLAS Collaboration), Phys. Rev. D 86, 032003 (2012).

[5] S. Chatrchyan et al., (CMS Collaboration), Phys. Lett. B 710, 26 (2012).

[6] V. M. Abazov et al., Nucl. Instrum. Methods. Phys. Res. A 565, 463 (2006).

[7] V. M. Abazov et al., (D0 Collaboration), Phys. Rev. D 88, 052011 (2013).

[8] T. Aaltonen et al., (CDF and D0 Collaborations), Phys. Rev. D 88, 052014 (2013).

[9] F. Halze and A. Martin, Quarks and Leptons: An Introductory Course in Modern Particle Physics (Wiley, 1984).

[10] D. Griffiths, Introduction to Elementary Particles (John Wiley \& Sons Inc, 1987).

[11] M. E. Peskin and D. V. Schroeder, An Introduction To Quantum Field Theory (Westview Press, 1995).

[12] F. Englert and R. Brout, Phys. Rev. Lett. 13, 321 (1964).

[13] P. W. Higgs, Phys. Rev. Lett. 13, 508 (1964).

[14] G. S. Guralnik, C. R. Hagen, and T. W. B. Kibble, Phys. Rev. Lett. 13, 585 (1964).

[15] http://www.nobelprize.org/nobel_prizes/physics/.

[16] R. Barate et al., (LEP Working Group for Higgs boson searches), Phys. Lett. B 565, 61 (2003). 
[17] T. Aaltonen et al., (CDF and D0 Collaborations), Phys. Rev. Lett. 109, 071804 (2012).

[18] R. Wilson, Phys. Today 30N10, 23 (1977).

[19] Accelerators - Fermilab's Chain of Accelerators.

[20] S. N. Ahemed et al., Nucl. Instrum. Methods in Phys. Res. Sect. A 634, 8 (2011).

[21] V. Abazov et al., Nucl. Instrum. Methods in Phys. Res. Sect. A 552, 372 (2005).

[22] M. Abolins, IEEE Trans Nucl. Sci. 52, 340 (2004).

[23] V. Abazov et al., The D0 Collaboration, Nucl. Inst. A , arXiv:1307.5202, Submitted on July 19, 2013.

[24] B. Abbott et al., Nucl. Instrum. Methods. Phys. Res. A 424, 352 (1999).

[25] L. Breiman, Mach. Learn. 45, 5 (2001).

[26] T. Sjöstrand, S. Mrenna, and P. Z. Skands, J. High Energy Phys. 05, 026 (2006).

[27] H. L. Lai et al., Phys. Rev. D 55, 1280 (1997).

[28] J. Pumplin, D. Stump, J. Huston, N. P. Lai, H.-L., and W.-K. Tung, J. High Energy Phys. 07, 012 (2002).

[29] M. L. Mangano, M. Moretti, F. Piccinini, R. Pittau, and A. D. Polosa, J. High Energy Phys. 07, 001 (2003).

[30] J. Alwall et al., Eur. Phys. J. C 53, 473 (2007).

[31] E. Boos et al., Nucl. Instrum. Methods Phys. Res. A 534, 250 (2004).

[32] E. Boos, V. Bunichev, L. Dudko, V. Savrin, and V. Sherstnev, Phys. Atom. Nucl. 69, 1317 (2006).

[33] R. Brun and F. Carminati, GEANT Detector Description and Simulation Tool, CERN Program Library Long Writeup W5013, 1993 (unpublished). 
[34] V. M. Abazov et al., (D0 Collaboration), Phys. Rev. D 76, 012003 (2007).

[35] T. Junk, Nucl. Instrum. Methods Phys. Res. A 434, 435 (1999).

[36] A. L. Read, J. Phys. G 28, 2693 (2002).

[37] W. Fisher, FERMILAB-TM-2386-E (2007).

[38] I. Narsky, arXiv:physics/0507157, (2005).

[39] L. Breiman, J. Friedman, R. Olshen, and C. Stone, Classification and Regression Trees (Wadsworth \& Brooks/Cole Advanced Books and Software, Pacific Grove, CA, 1984).

[40] R. E. Schapire, The Boosting Approach to Machine Learning: An Overview, MSRI Workshop on Nonlinear Estimation and Classification, Berkeley, CA, USA, 2001.

[41] Y. Freund and R. E. Schapire, J. Japanese Society for Artificial Intelligence 14, 771 (1999), (in Japanese, translation by Naoki Abe).

[42] J. H. Friedman, eConf C030908, WEAT003 (2003).

[43] A. Hoecker et al., PoS ACAT, 040 (2007), we use version 4.1.0.

[44] S. J. Parke and S. Veseli, Phys. Rev. D 60, 093003 (1999).

[45] K. Black et al., arxiv:hep-ph/1010.3698v2, (2011).

[46] U. Langenfeld, S. Moch, and P. Uwer, Phys. Rev. D 80, 054009 (2009).

[47] N. Kidonakis, Phys. Rev. D 74, 114012 (2006).

[48] J. M. Campbell and R. K. Ellis, Phys. Rev. D 60, 113006 (1999).

[49] J. M. Campbell, arXiv:hep-ph/0105226, (2001).

[50] J. M. Campbell, R. K. Ellis, and C. Williams, MCFM - Monte Carlo for FeMtobarn processes, http://mcfm.fnal.gov/.

[51] T. Andeen et al., FERMILAB-TM-2365 (2007). 
[52] S. Chatrchyan et al., (CMS Collaboration), Phys. Lett. B 716, 30 (2012).

[53] G. Aad et al., (ATLAS Collaboration), Phys. Lett. B 726, 88 (2013), arXiv:1307.1427.

[54] G. Aad et al., (ATLAS Collaboration), Phys. Lett. B 726, 120 (2013), arXiv:1307.1432.

[55] S. Chatrchyan et al., (CMS Collaboration), Phys. Rev. Lett. 110, 081803 (2013), arXiv:1212.6639.

[56] The D0 Collaboration, Constraints on the JP $=2+$ hypothesis for the $125 \mathrm{GeV}$ boson in $\mathrm{W} / \mathrm{Z}+$ bb final states, D0 conference note 6387, 2013.

[57] The D0 Collaboration, Constraints on the JP $=0$ - hypothesis for the Higgs boson in $\mathrm{W} / \mathrm{Z}+$ bb final states at the D0 Experiment, D0 conference note 6406, 2013. 